\title{
Process Design Concepts for Stabilization of High Level Waste Calcine
}

Thomas R. Thomas

Alan K. Herbst

June 2005

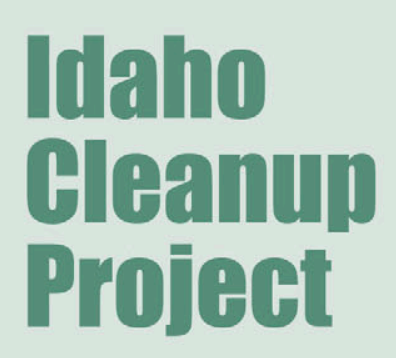


ICP/EXT-05-00925

Project No. 23582

\title{
Process Design Concepts for Stabilization of High Level Waste Calcine
}

\author{
Thomas R. Thomas \\ Alan K. Herbst \\ June 2005 \\ Idaho Cleanup Project \\ Idaho Falls, Idaho 83415
}

Prepared for the

U.S. Department of Energy

Assistant Secretary for Environmental Management

Under DOE-NE Idaho Operations Office

Contract DE-AC07-05ID14516 


\section{ABSTRACT}

The current baseline assumption is that packaging "as is" and direct disposal of high level waste (HLW) calcine in a Monitored Geologic Repository will be allowed. The fall back position is to develop a stabilized waste form for the HLW calcine, that will meet repository waste acceptance criteria currently in place, in case regulatory initiatives are unsuccessful. A decision between direct disposal or a stabilization alternative is anticipated by June 2006 .

The purposes of this Engineering Design File (EDF) are to provide a pre-conceptual design on three low temperature processes under development for stabilization of high level waste calcine (i.e., the grout, hydroceramic grout, and iron phosphate ceramic processes) and to support a down selection among the three candidates. The key assumptions for the pre-conceptual design assessment are that a) a waste treatment plant would operate over eight years for 200 days a year, b) a design processing rate of $3.67 \mathrm{~m}^{3} /$ day or $4670 \mathrm{~kg} /$ day of HLW calcine would be needed, and c) the performance of waste form would remove the HLW calcine from the hazardous waste category, and d) the waste form loadings would range from about $21-25 \mathrm{wt} \%$ calcine.

The conclusions of this EDF study are that:

- $\quad$ To date, the grout formulation appears to be the best candidate stabilizer among the three being tested for HLW calcine and appears to be the easiest to mix, pour, and cure.

- $\quad$ Only minor differences would exist between the process steps of the grout and hydroceramic grout stabilization processes. If temperature control of the mixer at about $80^{\circ} \mathrm{C}$ is required, it would add a major level of complexity to the iron phosphate stabilization process.

- It is too early in the development program to determine which stabilizer will produce the minimum amount of stabilized waste form for the entire HLW inventory, but the volume is assumed to be within the range of 12,250 to $14,470 \mathrm{~m}^{3}$.

- The stacked vessel height of the hot process vessels in the hydroceramic grout process (i.e., $21 \mathrm{~m}$ ) appears to be about the same as that estimated by the Direct Cementitious Waste Process in 1998, for which a conceptual design was developed. Some of the conceptual design efforts in the 1998 study may be applicable to the stabilizer processes addressed in this EDF.

- The gamma radiation fields near the process vessels handling HLW calcine would vary from a range of about $300-350 \mathrm{R} / \mathrm{hr}$ at a distance of $2.5 \mathrm{~cm}$ from the side of the vessels to a range of about $50-170 \mathrm{R} / \mathrm{hr}$ at a distance of $100 \mathrm{~cm}$ from the side of the vessels. The calculations were made for combined calcine, which was defined as the total HLW calcine inventory uniformly mixed.

- The gamma radiation fields near the stabilized waste in canisters would range from about $25-170 \mathrm{R} / \mathrm{hr}$ at $2.5 \mathrm{~cm}$ from the side of the canister and $5-35 \mathrm{R} / \mathrm{hr}$ at $100 \mathrm{~cm}$ from the side of the canister, depending on the which bin set was the source of calcine. 
The recommendations from this EDF study are:

- A value engineering session and cost benefit study should be done to determine if redundant process vessel trains would be needed. Due to the nature of remote operations and maintenance, a second process train may be needed to provide a greater certainty that production goals would be met in case process upsets were to occur.

- $\quad$ Pre-blended dry stabilizer ingredients should be used where possible to reduce the number of process vessels that would be required to deliver the cold ingredients (i.e., the number of bulk, day, and batch vessels required) to the mixer. In the case of the grout process, which uses three ingredients, a pre-blended mix would reduce the number of cold process vessels for the dry solids from nine to three.

- If sampling is required, a minimum of two day vessels for the HLW calcine would be required to allow one to undergo sampling and analysis while the other is feeding the grout mixer. An assessment of analytical turn around time for sample analysis should be done to determine the number of day vessels that will be required. The waste treatment plant should have a dedicated hot laboratory for required analytical services.

- $\quad$ Single batch vessels for solids and liquids should be used in the process vessel train to prevent overfilling the mixer and to better control the formulation for the HLW calcine and stabilizer ingredients.

- $\quad$ Pilot-scale studies with automated remote process steps are needed to test mixer designs, and to demonstrate process control during the addition of ingredients to the mixer, the mixing process, and transfer of the mix to the canister.

- As a starting point for gravity feed hopper design, a critical friction angle of $20^{\circ}$ should be used for the calcine hoppers. However, this friction angle does not take into consideration the effects of fines on the calcine flow and additional testing should be done to determine this effect. 


\section{ACKNOWLEDGMENTS}

The authors would like to thank the CH2M-WG Idaho subject matter experts listed below for participating in a value engineering session on March 9, 2005 to agree on an outline of subject mater for this document and/or participating in the review cycles of the document.

Richard D. Adams

Jerry D. Barker

Jim T. Beck

Kim F. Childs

Ken C. Coughlan

Christy M. Frazee

Clark B. Millet

Cliff W. Olsen

Mike W. Patterson

Mike C. Swenson 


\section{CONTENTS}

ABSTRACT iii

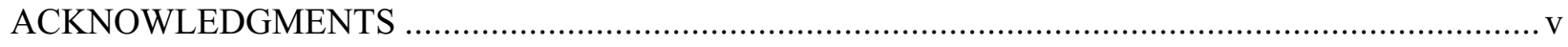

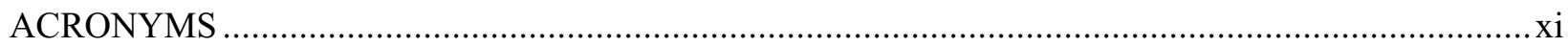

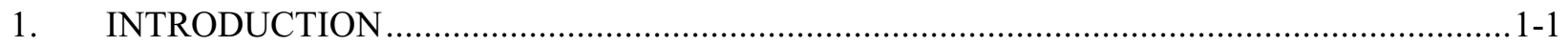

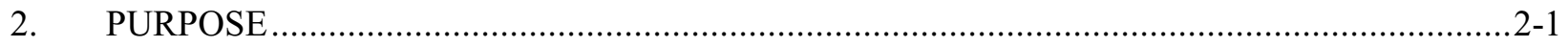

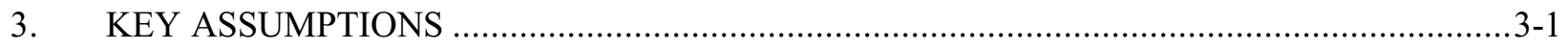

3.1 HLW Calcine Processing Rate ....................................................................... 3-1

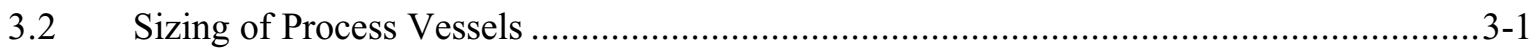

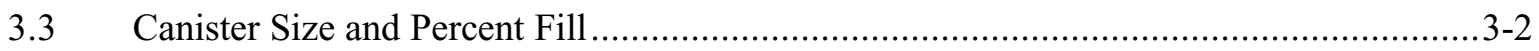

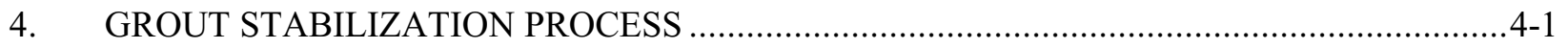

4.1 Mass Balance Flow Sheet ...............................................................................

4.2 Major Process Steps and Equipment Design ....................................................... $4-1$

4.2.1 Bulk Receiving Vessels .....................................................................4-2

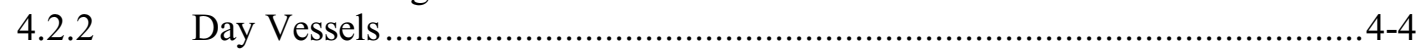

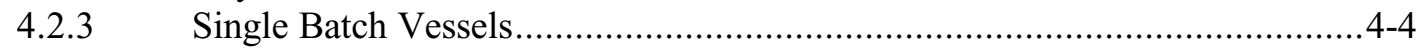

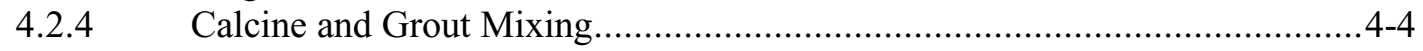

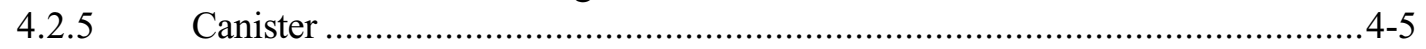

4.3 Process Monitors and Sampling Steps ............................................................... $4-5$

4.3.1 Bulk Vessel Process Monitors........................................................................ $4-5$

4.3.2 Day Vessel Process Monitors....................................................................4-7

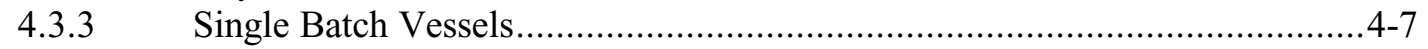

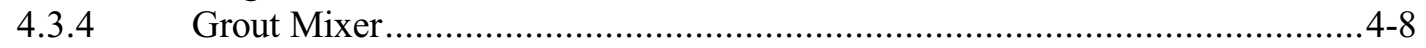

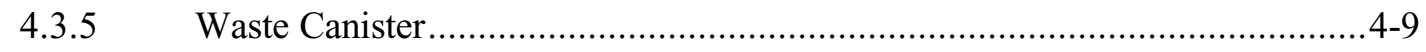

5. HYDROCERAMIC GROUT STABILIZATION PROCESS ........................................... $5-1$

5.1 Mass Balance Flow Sheet ................................................................................. $5-1$

5.2 Major Process Steps and Equipment Design ........................................................ $5-1$

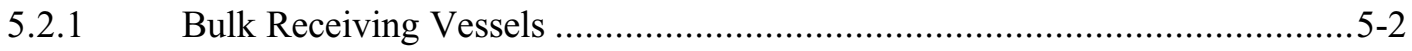

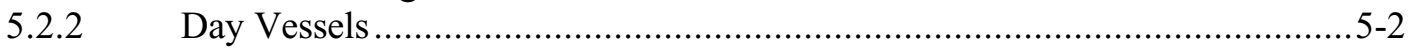

5.2.3 Single Batch Vessels................................................................. $5-4$

5.2.4 Calcine and Hydroceramic Grout Mixing ...................................................

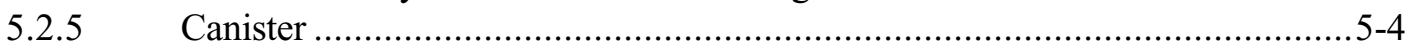


5.3 Process Monitors and Sampling Steps ..............................................................

5.3.1 Bulk Vessel Process Monitors.................................................................... $5-5$

5.3.2 Day Vessel Process Monitors.............................................................. 5-5

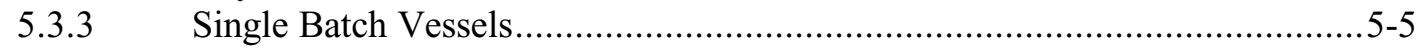

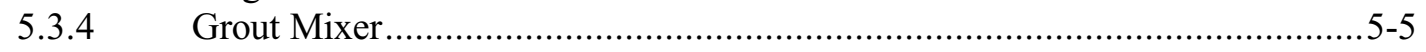

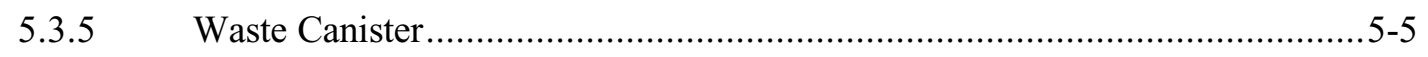

6. IRON PHOSPHATE CERAMIC STABILIZATION PROCESS …......................................... $6-1$

6.1 Mass Balance Flow Sheet ................................................................................. $6-1$

6.2 Major Process Steps and Equipment Design ..................................................... 6-1

6.2.1 Bulk Receiving Vessels .................................................................6-2

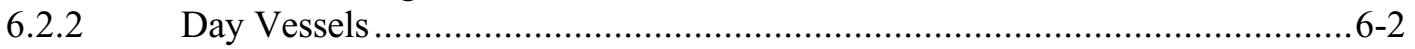

6.2.3 Single Batch Vessels......................................................................... $6-2$

6.2.4 Calcine and Iron Phosphate Ceramic Mixing ..........................................6-2

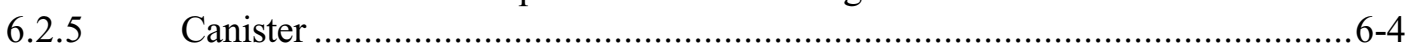

6.3 Process Monitors and Sampling Steps ................................................................. 6-4

7. COMPARISON OF STABILIZATION PROCESSES …..................................................

7.1 Waste Form Performance Data to Date.................................................................. $7-1$

Relative Process Complexity …....................................................................

7.3 Number of Waste Canisters Produced........................................................................

7.4 Comparison to the Direct Cementitious Waste Option ...........................................

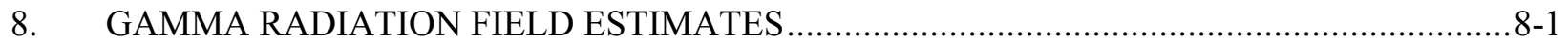

8.1 Process Vessel Radiation Field Estimates ................................................................ 8-1

8.2 Waste Canister Radiation Field Estimates ............................................................... 8-2

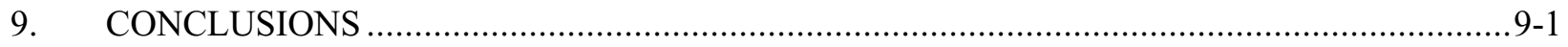

10. DESIGN ISSUES BEYOND SCOPE OF DOCUMENT …....................................... 10-1

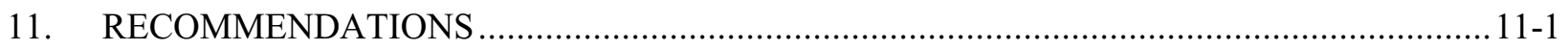

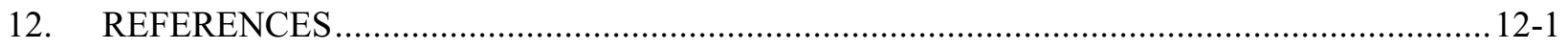

Appendix A—Method for Sizing Funnel Flow Hoppers ........................................................... A-1

Appendix B-1-Mass Flow Sheet Calculations for the Grout Stabilization Process ......................... B-1-1

Appendix B-2-Estimated Process Vessel Sizes for Grout Stabilization Process ............................ B-2-1 
Appendix C-1-Mass Flow Sheet Calculations for the Hydroceramic Grout Stabilization Process .... C-1-1

Appendix C-2-Estimated Process Vessel Sized for the Hydroceramic Grout Stabilization

Process

Appendix D-1-Mass Flow Sheet Calculations for the Iron Phosphate Ceramic Grout

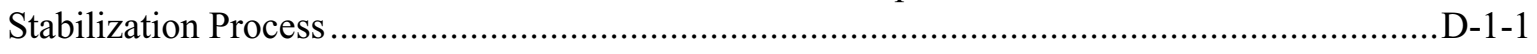

Appendix D-2-Estimated Process Vessel Sizes for the Iron Phosphate Ceramic Grout

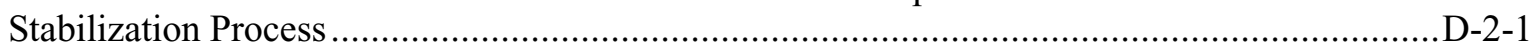

Appendix E-MicroShield Calculations for Gamma Field Radiation Estimate ................................ E-1

\section{FIGURES}

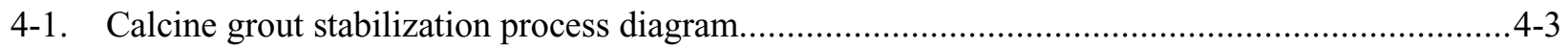

4-2. Process monitoring steps for the grout stabilization process.................................................. $4-6$

5-1. Calcine hydroceramic cement stabilization process diagram............................................5-3

6-1. Calcine iron phosphate ceramic stabilization process diagram .........................................6-3

8-1. Gamma radiation field from the side of the grouting process vessels and canister for combined calcine

8-2. Gamma radiation fields from waste canister filled with various grouted calcines

8-3. Gamma radiation fields from above a filled canister for combined calcine.

\section{TABLES}

4-1. Calcine waste form composition and recipe for $1.0 \mathrm{~m}^{3}$ of grouted waste.................................4-1

5-1. Calcine waste form composition and recipe for $1.0 \mathrm{~m}^{3}$ of hydroceramic waste..........................5-1

6-1. Calcine waste form composition and recipe for $1.0 \mathrm{~m}^{3}$ of iron phosphate waste.......................6-1

8-1. Gamma radiation fields $(\mathrm{R} / \mathrm{hr})$ from grouting process vessels for combined calcine .................. $8-1$

8-2. Gamma radiation fields $(\mathrm{R} / \mathrm{hr})$ from calcine in waste canister grouted at $21.5 \mathrm{wt} \% \ldots \ldots \ldots \ldots \ldots \ldots . . .8-3$

8-3. Gamma radiation fields $(\mathrm{R} / \mathrm{hr})$ from above top of waste canister....................................... $8-4$ 


\section{ACRONYMS}

\begin{tabular}{|c|c|}
\hline BBWI & Bechtel BWXT Idaho, LLC \\
\hline BFS & Blast Furnace Slag \\
\hline CSSFs & Calcined Solids Storage Facilities \\
\hline DIAL-MSU & Diagnostics Instrumentation and Analytical Laboratory-Mississippi State University \\
\hline $\mathrm{DOE} / \mathrm{ID}$ & Department of Energy Idaho Operations Office \\
\hline $\mathrm{DOE} / \mathrm{HQ}$ & Department of Energy Headquarters \\
\hline $\mathrm{DOE} / \mathrm{EM}$ & Department of Energy Environmental Management \\
\hline DOE/NE-ID & Department of Energy Idaho Operations Office-Nuclear Energy \\
\hline DWPF & Defense Waste Processing Facility \\
\hline DWCO & Direct Cementitious Waste Option \\
\hline EA & Environmental Assessment \\
\hline EDF & Engineering Design File \\
\hline FA & Fly Ash from Coal Fired Power Plant \\
\hline FY & Fiscal Year \\
\hline $\mathrm{HC}$ & Hydroceramic Process \\
\hline HLW & High Level Waste \\
\hline ICP & Idaho Cleanup Project \\
\hline INL & Idaho National Laboratory \\
\hline INTEC & Idaho Nuclear Technology and Engineering Center \\
\hline $\mathrm{OPC}$ & Ordinary Portland Cement \\
\hline PCT & Product Consistency Test \\
\hline RCRA & Resource Conservation and Recovery Act \\
\hline S\&A & Sample and Analysis \\
\hline SBW & Sodium Bearing Waste \\
\hline SNF & Spent Nuclear Fuel \\
\hline
\end{tabular}


TCLP Toxicity Characteristic Leaching Procedure

UTS Universal Treatment Standards

WAPS Waste Acceptance Performance Specifications

WASRD Waste Acceptance Systems Requirements Document 


\section{Process Design Concepts for Stabilization of High Level Waste Calcine}

\section{INTRODUCTION}

Spent nuclear fuel (SNF) was reprocessed until 1992 at the Idaho Chemical Processing Plant (now called the Idaho Nuclear Technology and Engineering Center [INTEC]) located at the Idaho National Laboratory (INL). Reprocessing produced liquid high level waste (HLW) that was subsequently converted to a dry granular solid waste by a process called calcination. The solid waste, called HLW calcine, is primarily composed of various metal oxides, calcium fluoride, and sodium and potassium nitrates depending on the type of SNF that was reprocessed. Calcine is highly radioactive and also meets the Resource Conservation and Recovery Act (RCRA) definition of a hazardous waste because it exhibits the RCRA hazardous characteristic of toxicity for one or more toxic metal constituents and contains listed constituents associated with the RCRA hazardous waste numbers F001, F002, F005, and U134 (PLN-1144 2002). A Calcine Disposition Project baseline assumption is that implementation of a strategy to effectively work within the current RCRA regulatory framework to remove the calcined HLW from RCRA regulation as a hazardous waste is necessary to allow disposal at the Monitored Geologic Repository at Yucca Mountain, Nevada. The current DOE prohibition of disposing RCRA hazardous waste in the repository drives the need to either pursue specific exclusion of calcined HLW from regulation as hazardous waste or develop effective treatment to remove the waste from regulation as hazardous waste.

An estimated $4400 \mathrm{~m}^{3}$ of granular HLW calcine is stored at INTEC in six Calcined Solids Storage Facilities (CSSFs) of varying designs, which contain from 3-12 stainless steel bins housed in concrete vaults (Staiger and Swenson 2005). The bins range from 20 to $68 \mathrm{ft}$ in height and up to $13.5 \mathrm{ft}$ in diameter. As of 2005, the storage periods of the calcine range from about 41 years for the oldest calcine in CSSF I to about 5 years for some of the calcine in the CSSF VI. The major types of calcine in the CSSFs are: alumina in CSSF I; alumina and zirconia in CSSF II; mostly zirconia and some zirconia blended with sodium bearing waste (SBW) in CSSF III; and zirconia blended with increasing amounts of SBW waste in CSSFs IV-VI. Some of the key milestones in the Department of Energy Idaho Operations Office (DOE/ID) Performance Management Plan for Accelerating Cleanup of the INEEL regarding the HLW calcine are to (DOE/ID 2002):

- Complete a sample retrieval and characterization demonstration by September 2007

- Issue a record of decision on calcine treatment path forward by December 2009

- Submit an application for a RCRA Part B Permit for calcine treatment, retrieval, and packaging process by a date to be determined (note, per the DOE Request for Proposals for the Idaho Cleanup Project (ICP) contract, this date is September 30, 2009)

- Complete retrieval, packaging, alternative treatment, and shipping to a repository by December 2035. 
The Calcine Disposition Project was initiated in fiscal year (FY) 2003. In support of the DOE/ID Performance Management Plan, the Calcine Disposition Project is developing design concepts for retrieval, treatment, and packaging of the calcine and conducting scientific and engineering studies to support the design concepts. The current baseline assumption being used for planning and scheduling is that packaging "as is" and direct disposal of HLW calcine in a Monitored Geologic Repository will be allowed (DOE/NE-ID 2005). The fall back position is to develop a stabilized waste form for the HLW calcine, that will meet repository waste acceptance criteria currently in place, in case regulatory initiatives are unsuccessful. 


\section{PURPOSE}

With DOE/ID guidance that vitrification was no longer the preferred option as a waste form (Frei 2002), the Calcine Disposition Project evaluated 56 candidate stabilizers for potential application to the HLW calcine in FY 2004. As a result of this evaluation, three low-temperature stabilizers were selected as potential candidates for development (Thomas and Childs 2004). These three candidates (iron phosphate ceramic, hydroceramic grout and grout cement) are currently under development (Plodinec et. al. 2004) by the Diagnostic Instrumentation and Analysis Laboratory at Mississippi State University (DIAL-MSU). The purpose of this Engineering Design File (EDF) is to:

- $\quad$ Provide pre-conceptual process designs for the stabilizers under development

- $\quad$ Compare the process complexities among the three candidate stabilizers

- $\quad$ Estimate the volumes of stabilized waste that would be generated

- $\quad$ Provide engineering guidance in down selecting a stabilizer if more than one of the processes appear promising based on the DIAL-MSU studies

- $\quad$ Support the project schedule to down select a reference stabilization process for development by June 2006, if approval of "as is" packaging of HLW calcine is not obtained. 
2-2 


\section{KEY ASSUMPTIONS}

The key assumptions for calcine processing rate, sizing of process vessels, canister size and percent fill are given in Sections 3.1, 3.2, and 3.3, respectively.

\subsection{HLW Calcine Processing Rate}

The draft Mission Need Statement for the Calcine Disposition Project assumes that all of the HLW calcine will be retrieved and packaged for shipment to a Monitored Geologic Repository over a period of eight years between 2015 and 2022 (DOE/NE-ID 2005). In this document, it is assumed that a plant designed to stabilize the HLW calcine would operate 24 hours per day, 200 days per year, which would correspond to a plant uptime of 55\%. The remaining $45 \%$ downtime would be used for maintenance and recovery from potential process upsets. The total number of operating days of a calcine treatment plant would equate to 1600 days based on the above assumptions of an eight year operating period and 200 days per year.

The total volume and mass of HLW calcine stored in the calcine bin sets at INTEC are estimated to be $4400 \mathrm{~m}^{3}$ and $5.6 \times 10^{6} \mathrm{~kg}$, respectively (Staiger and Swenson 2005). Dividing these inventory numbers by 1600 days of operation equates to an average required processing rate that can be expressed either as $2.75 \mathrm{~m}^{3} /$ day or $3500 \mathrm{~kg} / \mathrm{day}$. A further assumption is that the design capacity of the calcine treatment plant should be $33 \%$ greater than the required average processing rate capacity. This design philosophy provides a contingency for processing at greater rates, if process upsets cause operations to fall below the average required rate. Adding $33 \%$ to the average required processing rate equates to a design processing rate that can be expressed either as $3.67 \mathrm{~m}^{3} /$ day or $4670 \mathrm{~kg} / \mathrm{day}$. The design volume processing rate was used in this document for sizing vessels. The design mass processing rate was used for developing the process mass balance.

\subsection{Sizing of Process Vessels}

An evaluation of type, size, and the basic shape of process vessels was undertaken for each of the stabilization options and the results are given in Sections 4 through 7. The evaluation in this document is preconceptual in nature and not intended to be viewed as an optimized approach for process vessel arrays and configurations. For example, two bulk calcine receipt vessels may be needed instead of one, if one is being filled while the other is feeding the calcine treatment plant (i.e., these operations may not be compatible for the same vessel at the same time). As indicated in Section 3.1, the sizes of the vessels were based on a design calcine processing rate of $3.67 \mathrm{~m}^{3} /$ day. Some of the higher level assumptions used to select and size the vessels were:

- $\quad$ The bulk calcine receipt and stabilizer ingredient vessels would be sized to contain enough calcine feed (i.e., $25.7 \mathrm{~m}^{3}$ of calcine) and stabilizer feed for a seven-day operating period

- The bulk vessels would feed day vessels, which would be sized to hold enough feed for a 24-hour operating period (i.e., $3.67 \mathrm{~m}^{3}$ of calcine)

- $\quad$ The day vessels would feed single batch vessels, which would be sized to hold enough feed to prepare one canister of stabilized waste

- $\quad$ The batch vessels would feed the grout mixer vessel, which would be sized to mix and transfer one canister batch of stabilized calcine. 
The basic shape chosen for the process vessels to handle solids was a funnel flow hopper design, which consists of a cylindrical top section and a conical bottom section. Liquid vessels would be traditional cylindrical shaped vessels. The grout mixer would be based on commercially available mixers which are described in Section 4.2 of this document. For the cylindrical section of the funnel flow hopper, a height to diameter ratio of about $2: 1$ was selected. The dimensions of the conical section were constrained by three parameters:

- The diameter of the cylindrical section above it

- A critical hopper friction angle of $20^{\circ}$ from the vertical axis of the cylindrical section

- A bottom hopper opening with a radius of $0.152 \mathrm{~m}$ (6 in.).

The critical hopper friction angle is based on tests done in 1991 by Johanson and Johanson on calcine flow properties in hoppers (Wanke 1993). The results were based on tests done with bed particles of cold calcine simulants with the caveat that the addition of fines would significantly alter the observed performance and that tests should be repeated with fines. An evaluation in 2004 also identified the need to determine the minimum conical hopper slope to ensure solids flow as a major development need for calcine retrieval technology (Thomas 2004). In this document, the critical friction angle was used for all the vessels that handled dry solids with the understanding that more testing is required to better define the critical angles for both the radioactive and cold ingredients. The Johanson and Johanson study assumed a hopper outlet radius of $0.152 \mathrm{~m}$, which is also assumed in this document. An illustration of the method and the equations for sizing the solids process vessels is given in Appendix A, using the above set of assumptions.

\subsection{Canister Size and Percent Fill}

The current version of the DOE Waste Acceptance System Requirements Document (WASRD) allows for the repository use of HLW canisters with an outside diameter of $64 \mathrm{~cm}$ and two optional lengths of 301 or $451 \mathrm{~cm}$ (DOE/RW 2002), which are commonly referred to as the $2 \mathrm{ft}$ by $10 \mathrm{ft}$ and $2 \mathrm{ft}$ by $15 \mathrm{ft}$ canisters. The WASRD has deleted the requirement for percent fill of the canister, but a requirement of $\geq 80 \%$ fill still resides in the current version of the Waste Acceptance Product Specifications for Vitrified High-Level Waste Forms (DOE/EM 1996). A recent study on the trade off of canister sizes versus number of canisters produced via packaging calcine "as is" assumed a $98 \%$ fill for the $2 \mathrm{ft}$ by $10 \mathrm{ft}$ and $2 \mathrm{ft}$ by $15 \mathrm{ft}$ canisters (Kerr 2005). For this document, the $2 \mathrm{ft}$ by $15 \mathrm{ft}$ canister with a $90 \%$ fill was assumed to estimate the number of canisters that would be produced by the stabilization options under consideration. The $90 \%$ fill requires less process control in the quantity of stabilized waste delivered to the canister and reduces the process potential for an overfill situation. Based on an empty volume of $1.1 \mathrm{~m}^{3}$ for the $2 \mathrm{ft}$ by $15 \mathrm{ft}$ canister with a $1.27 \mathrm{~cm}$ wall thickness (Rahl 2003), a 90\% fill equates to about $1.0 \mathrm{~m}^{3}$. The empty canister volume cited by Rahl is based on a length less than $451 \mathrm{~cm}$ due to the impact plates and skirts at both ends in the canister design. A waste volume of $1.0 \mathrm{~m}^{3}$ per canister at $90 \%$ fill is used in this document to estimate the number of canisters that would be produced by each of the candidate processes if the entire inventory of HLW calcine were stabilized. 


\section{GROUT STABILIZATION PROCESS}

The grout formulation and process described in Sections 4.1 and 4.2 are based on studies to develop cement waste forms for sodium bearing waste and the low level waste fraction of HLW calcine for application at INTEC (Herbst 1997, Herbst et. al. 1998). A grout formulation consisting of equal portions of ordinary Portland Cement (OPC), ground blast furnace slag (BFS), Class F fly ash (FA), and a water-to-total solids ratio of 0.40 was developed for calcined SBW and zirconia and alumina simulants to make waste form loadings of 7-24 weight percent (wt $\%$ ). The total solids was defined as the sum of the weight for the waste, OPC, BFS, and FA. The wt $\%$ waste loadings were based on the ratio of the weight of the calcine to the weight of the total solids and water used in the formulation. The general observation was that for waste loadings up to $17 \mathrm{wt} \%$, the grout was fluid and could be poured into molds. For waste loadings between 21-24 wt\%, the grout piled up in the molds rather than leveling off. Formulation development is currently underway at DIAL-MSU to optimize calcine waste loadings while maintaining a low enough viscosity for mixing and meeting waste form performance standards. It is anticipated that the formulation of equal parts OPC:BFS:FA and the water required will need to be modified for application to HLW calcine. However, for flow sheet development in this document, a $21.5 \mathrm{wt} \%$ waste loading is assumed with a stabilizer consisting of equal parts OPC:BFS:FA and the prior target water-to-total solids ratio of 0.40 .

\subsection{Mass Balance Flow Sheet}

Stabilization of calcine via grouting would be accomplished by mixing the calcine with a blend of OPC, BFS, and FA and water to make a slurry mixture. Further, a reductant, such as sodium sulfide, could be added to promote stabilization of the RCRA toxic metals. Table 4-1 presents the mass balance flow sheet by $\mathrm{wt} \%$ for a batch large enough to fill one canister to $1.0 \mathrm{~m}^{3}$ (see Section 3.3 for canister description). These components are combined batch wise in a grout mixer, mixed, and then poured into a waste disposal canister. This formulation was used to size the vessels discussed below in Section 4.2 and as the starting point in the flow sheet calculations given in Appendix B-1 for the grouting process steps.

\subsection{Major Process Steps and Equipment Design}

As noted in Section 3.1 "HLW Calcine Processing Rate", the grouting process is designed to process $4,670 \mathrm{~kg}$ of calcine per day. This would meet the production rate required to complete calcine treatment in eight years. It is recommended a value engineering or other cost-benefit studies be done to determine if a redundant train is needed to meet production goals if the first train were down for a few

Table 4-1. Calcine waste form composition and recipe for $1.0 \mathrm{~m}^{3}$ of grouted waste.

\begin{tabular}{lcc}
\hline \multicolumn{1}{c}{ Component } & $\begin{array}{c}\text { Weight Percent } \\
(\mathrm{wt} \%)\end{array}$ & $\begin{array}{c}\text { One Batch Mass } \\
(\mathrm{kg})\end{array}$ \\
\hline Calcine & $21.5 \%$ & 383.1 \\
Portland Cement & $16.6 \%$ & 295.8 \\
Blast Furnace Slag & $16.6 \%$ & 295.8 \\
Coal Fly Ash & $16.6 \%$ & 295.8 \\
Water & $28.7 \%$ & 508.2 \\
Sodium Sulfide & $<1 \%$ & $<18$ \\
\hline
\end{tabular}


weeks for any reason. Due to the nature of remote operations and maintenance, the need for a second grout train is highly recommended. Due to the hygroscopic nature of the calcine and solids used in the grouting process, the solid ingredients should not be exposed to humid gases prior to their addition to the grout mixing vessel. The solids process vessels will most likely require a small purge of dry air or nitrogen. The major process steps and vessel sizes are described below and illustrated in Figure 4-1. The process steps follow the five major feed steps described in Section 3.2 where the calcine and feed material move through: (a) bulk vessels to (b) day vessels to (c) single batch vessels to (d) the grout mixer, and to (e) the canister. The vessel dimensions are based on the methods described in Section 3.2 and Appendix A. Also per Appendix A, the critical friction angle of the hopper for the bulk, day, and single batch vessels was based on the most conservative value of $20^{\circ}$ recommended in the Johanson and Johanson study (Wanke 1993). The dimensions of the process vessels, which are discussed below, are summarized in Appendix B-2.

\subsubsection{Bulk Receiving Vessels}

For the grouting process, bulk vessels will have capacities for a seven day supply of calcine and stabilizer powders; thus, sufficient feed will be needed to fill a total of about 85 canisters. To provide this capacity, the calcine bulk vessel will be $30 \mathrm{~m}^{3}$ and the bulk stabilizer vessel will be $60 \mathrm{~m}^{3}$.

The bulk calcine vessel will receive calcine from the calcine retrieval system. This vessel will need to be a hopper design described in Section 3.2 to handle the calcine. As noted in Appendix B-2, the vessel would be about $2.7 \mathrm{~m}$ in diameter and $7.4 \mathrm{~m}$ high. As indicated in Section 4.2, the outlet angle of this hopper should be no greater than $20^{\circ}$ from the vertical. This steep angle is needed to prevent bridging or blockage of the calcine. It is further anticipated that the vessel will need an auger at the base to control delivery to the day vessels.

The $60 \mathrm{~m}^{3}$ bulk stabilizer vessel will also need to be a hopper design to handle the cement powders. Per calculations in Appendix B-2, the approximate dimensions are $3.4 \mathrm{~m}$ in diameter by $9.4 \mathrm{~m}$ high. The vessel would also use an auger system to deliver powders to the day vessel. Since the OPC, BFS, and FA are all commercially available products, it is recommended that they be purchased from a cement vendor premixed to the required blend. Current experiments show a blend of equal measures of each stabilizer as noted in Table 4-1. The pre-blending eliminates the need for an individual bulk tank for each stabilizer.

To supply the needed $60 \mathrm{~m}^{3}$ of stabilizer per week, a railroad hopper car of blended OPC, BFS, and FA powders would be needed each week. A common hopper car can handle about $100,000 \mathrm{~kg}$ or $115 \mathrm{~m}^{3}$ of materials. Assuming a bulk density of $1500 \mathrm{~kg} / \mathrm{m}^{3}$ for the blended stabilizers, $60 \mathrm{~m}^{3}$ would weigh $90,000 \mathrm{~kg}$. Thus, the hopper car would be about one half full, but would be near maximum weight capacity. A method of transferring the stabilizers from the railroad hopper to the bulk vessel would be needed in the facility. To prepare the stabilizers, a vendor would need the capacity to blend $60 \mathrm{~m}^{3}$ $\left(20 \mathrm{~m}^{3}\right.$ each) and transport the material to the INL weekly. If such a vendor is not available, it would be necessary to have individual bulk tanks. Then a train of three hopper cars with $60 \mathrm{~m}^{3}$ of each stabilizer ingredient would provide the facility with a three week supply. If this latter method is selected, the facility would need a system to blend the stabilizer ingredients. Preblending the stabilizer ingredients eliminates the need to control four separate powder feed lines to the grout mixer and reduces the probability of a formulation being out of specification. 


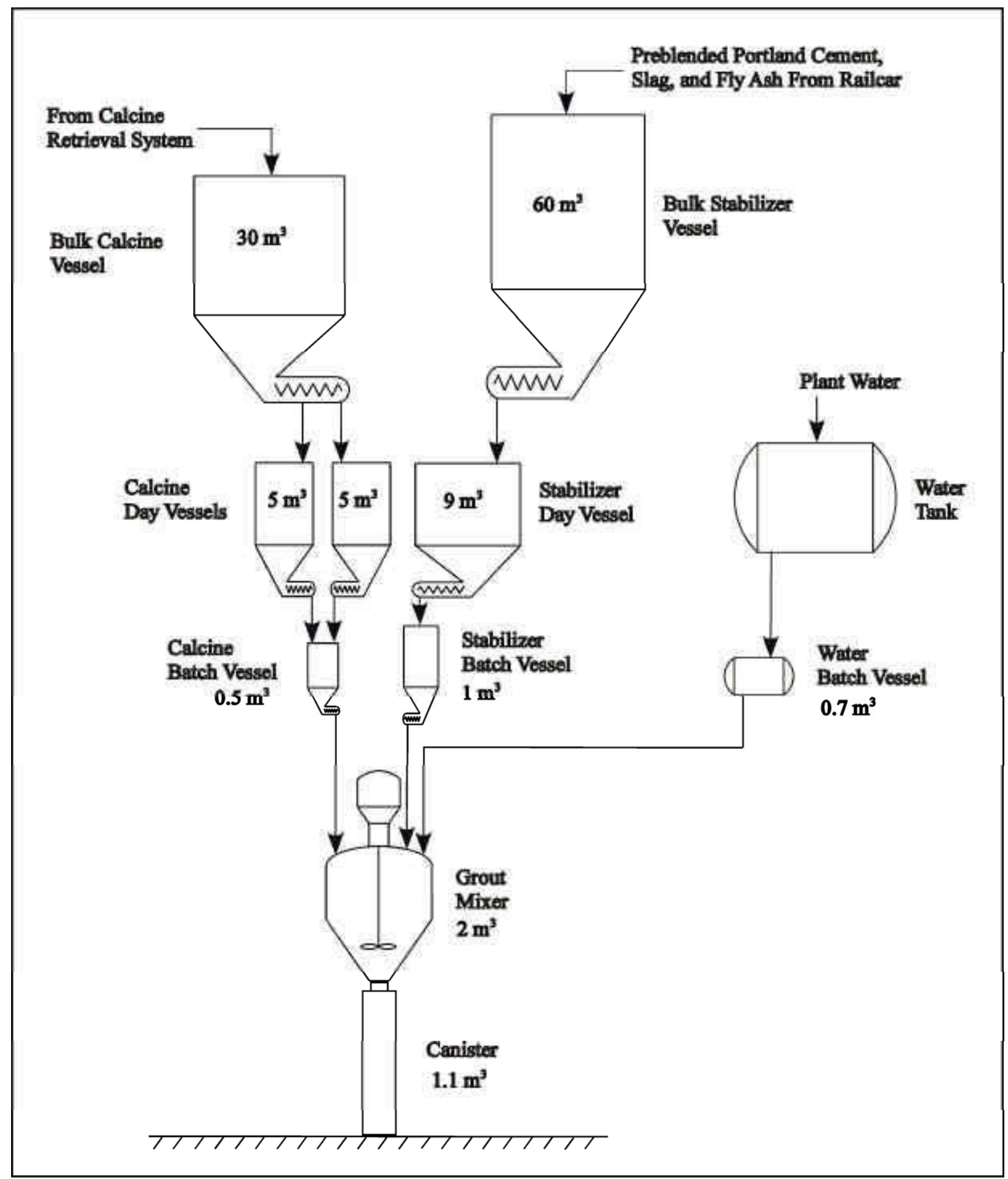

Figure 4-1. Calcine grout stabilization process diagram. 


\subsubsection{Day Vessels}

Day vessels would be sized to hold sufficient materials for 12 batches. For calcine, it is recommended to have a minimum of two day vessels. This would provide the opportunity to sample and analyze one vessel, if required, while the other is being used as feed for processing. If this does not provide enough analytical time, then more day tanks may be needed. It is anticipated that the treatment facility would have its own dedicated hot laboratory for analytical services to expedite turn around time in the analyses. Per calculations in Appendix B-2, the calcine day vessels would be $5 \mathrm{~m}^{3}$ hoppers about $1.5 \mathrm{~m}$ in diameter by $3.9 \mathrm{~m}$ high each. Both day vessels would supply calcine via augers to a single batch vessel. These vessels also require the steep hopper angle.

The stabilizer day vessel would be a $9 \mathrm{~m}^{3}$ hopper about $1.8 \mathrm{~m}$ in diameter and $4.8 \mathrm{~m}$ high. This vessel will also supply a single batch vessel as noted in Figure 4-1. If there are no requirements to sample the day stabilizer vessel and if the feed controls are accurate, the stabilizer day vessel may not be needed as the bulk vessel could feed the single batch vessel directly.

A water supply tank capable of holding one or more days of process water will be needed. This is recommended in the event of an unexpected water or power outage and water is needed in the mixer vessel to prevent in-mixer solidification. The water supply vessel will also feed a single batch tank.

\subsubsection{Single Batch Vessels}

Single batch vessels for solids and water are recommended in order to prevent overfilling the mixer and to control the grout/calcine mix formulation. Each batch vessel is thus sized to provide enough feed for one canister batch as noted in Table 4-1 and additional head space to accommodate variation in solids densities. For example, the estimated average densities of HLW calcine categorized by bin range from 740 to $1,410 \mathrm{~kg} / \mathrm{m}^{3}$ (Staiger and Swenson 2005). The calcine batch vessel would be about $0.5 \mathrm{~m}^{3}$, the stabilizer batch vessel would be about $1 \mathrm{~m}^{3}$, and the water batch tank would be about $0.7 \mathrm{~m}^{3}$. These solids vessels would need to meter the desired material to the mixer at a rate to provide good mixing. For example, the solids feed rate should be slow enough to allow the mixer to blend the solids with the water and not have solids build up on the grout surface or the mixer walls. If a chemical reductant, such as sodium sulfide, is needed for RCRA toxic metal stabilization, this chemical would be dissolved in water and added to the water batch tank as part of the required water.

\subsubsection{Calcine and Grout Mixing}

The calcine, stabilizer, and water batch vessels would each feed the grout mixer. As currently envisioned, the mixer could be a 1.5 to $2 \mathrm{~m}^{3}(400+$ gal $)$ planetary batch mixer with a bottom discharge to the disposal canister such as a Ross DPM 400 mixer (Charles and Ross and Son Co 2005). The mixer will need to be covered to prevent fugitive dust during the filling and mixing operations. The cover will need entry points for the calcine, stabilizer, and water and a vessel off-gas exit for displaced air and dust. The mixer should be instrumented with paddle torque as noted in Section 4.3.

The full amount of water required would be added first to the mixer. With the mixer running, calcine and stabilizer powders could then be metered in over a period of time (approximately 15 to 30 minutes) to permit adequate mixing and blending. When fully mixed, the grouted waste would be discharged into the canister mounted directly below the mixer. As soon as the mixer is empty, the next batch of water should immediately be added to prevent drying or caking of any remaining grout in the mixer. This water would then be used for the next batch of calcine grout. 
To facilitate a batch mixer, the "wet" grouted waste viscosity must be fluid enough to allow bottom discharge and easy filling of the canister. The DIAL-MSU studies will provide this information and later pilot-scale studies are recommended. Depending on the final waste form mixing viscosity, the selection of a continuous mixer may be more applicable. If the grout is more viscous, a continuous processor mixer may be considered, such as those made by Autocon or Readco (Autocon 2005, Readco 2005). This would necessitate changes in the batch vessel design and controls. Pilot scale testing is recommended to determine if the mixer can be adapted to remote control operation.

\subsubsection{Canister}

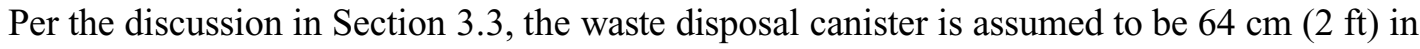
diameter by $451 \mathrm{~cm}(15 \mathrm{ft})$ high. It is planned to make the canister out of $1.27 \mathrm{~cm}(0.5 \mathrm{in})$ thick 316 stainless steel; thus, the canister mass would be about $930 \mathrm{~kg}(2050 \mathrm{lb})$ (Rahl 2003). A method of handling and positioning the empty canisters under the mixer will need to be developed. Once the canister is filled with $1.0 \mathrm{~m}^{3}$ of calcine grout at $1800 \mathrm{~kg}(3960 \mathrm{lb})$, the total mass of the canister and grout will be about $2,730 \mathrm{~kg}(6010 \mathrm{lb})$. The filled canister would then be moved to a curing area.

It is anticipated that initial set (i.e., hardening) of the grout should take place within 24 hours; thus, the curing area would need to stage up to 12 canisters for one day of processing. After the first day, the canister should be inspected for bleed water. The grout formulation should be designed to avoid bleed water, but the process must allow for such an event. A desiccant such as silica gel could be added to fill the canister void space. This would absorb bleed water and control any later condensation from the grout. At this point the canister could be capped.

Following capping, the canister would be moved to a decontamination area where it would be cleaned and surveyed. Once decontaminated, the canister would be ready for shipment. It would be moved to the packaging area where the canisters are placed in shipping casks. An interim storage area may be needed to allow for shipping delays or receiving delays at the repository. Such an area would need to hold up to 85 canisters for a week or up to 340 for a month. If the interim area were full, processing would need to be halted until shipping could continue.

\subsection{Process Monitors and Sampling Steps}

The process monitors and sampling steps illustrated in Figure 4-2 are discussed below and are preconceptual in nature. No decisions have been made on the level of inventory tracking, the need for solids mixing in process vessels, the need for sampling the calcine feed or waste form product, or the need to monitor the filling of the canister. It is assumed that these decisions will be addressed once a stabilizer process has been developed, selected as the preferred treatment alternative, and taken through the conceptual design process. The discussion below starts with the potential monitoring needs of the bulk vessels and steps through the grout stabilization process by describing the potential process monitoring needs of the day vessels, the single batch vessels, the grout mixer, and the waste canister, respectively.

\subsubsection{Bulk Vessel Process Monitors}

The process monitoring needs for the large seven-day supply bulk vessels will be to avoid overfilling them during solids transfers and tracking the amount left as they are used to feed the day vessels. As indicated in Figure 4-2, examples of process monitors that could fulfill these functions are paddle detectors and load cells. Dry material level switches using a stainless steel shaft with paddle blades, which are turned by an externally mounted motor, are commercially available for this application (OMEGA 1992a). The detectors can be mounted vertically on the side or horizontally on top of vessels. 


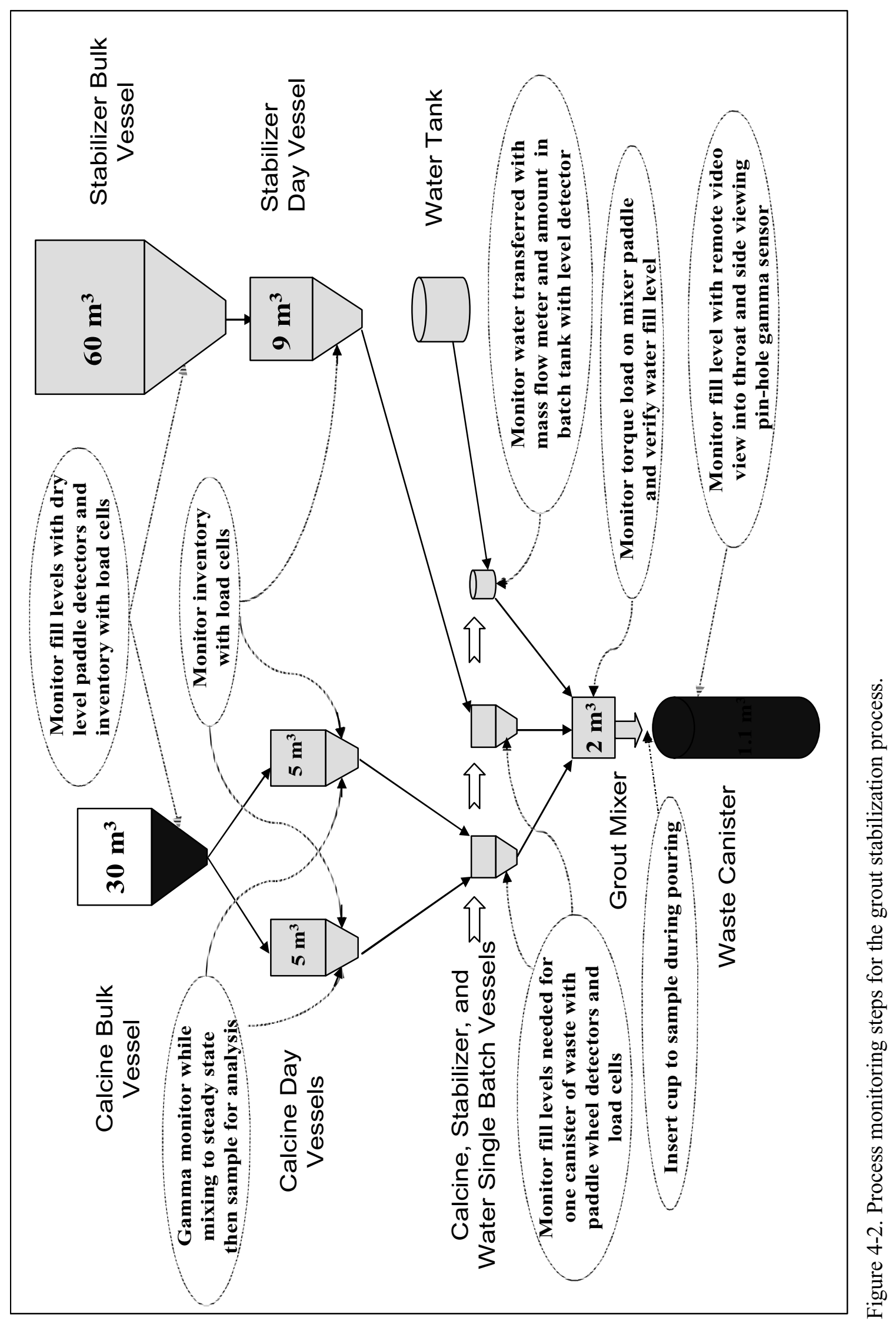

$\dot{1}$
$\dot{1}$ 
Side mounted paddle detectors could serve to indicate when the fill level is at a point that the vessel should be refilled. They work on the principal of turning the paddle with a low-torque, slow-speed motor, which actuates a cut off switch if resistance from solids is encountered and they resume turning when the resistance ceases. Their specific application is to prevent vessels from overflowing and they are commonly found in the chemical, food, mining, plastics, and ceramics industries. Because the motors reside outside the vessel, they could be shielded to avoid radiation damage and they are designed for replacement, if they fail.

For tracking inventory or solids fill level, internal detectors mounted inside the top of the bulk vessel such as a non-contact ultrasonic sensor or a laser level detector may have potential application but their suitability to a highly radioactive and/or dusty environment was not evaluated. External sensors are preferable to avoid the hostile environment inside the vessel. Double ended shear beam load cells are specifically designed for industrial application to process vessels, hoppers, tanks and trucks. They come with a flat mounting plate upon which the legs of the process vessels and tanks can be bolted. The process vessel should be totally supported by the load cells with the weight as uniformly distributed as possible. Vendor information indicates that load cells constructed with nickel-plated steel for corrosive environments and full scale ranges between 5000 and 150,000 lb are available with full scale accuracies of $0.03 \%$ (OMEGA 1992b). Because of the wide variety of full-scale ranges available, they could be applied to most of the process vessels and tanks where inventory tracking is required.

\subsubsection{Day Vessel Process Monitors}

The process needs for the calcine and stabilizer day vessels will be to accurately monitor inventory. An additional need for the calcine day vessels may be to monitor the approach to steady state mixing of the calcine prior to sampling and analysis (S\&A), if feed characterization for process control is required. An option for monitoring the approach to steady state mixing may be a pin-hole gamma monitor (i.e., a field of view with a small volume component relative to the vessel volume) positioned at the side of the day vessel. An alternative to gamma monitoring would be to experimentally determine the minimum time required for steady state mixing based on the mixing mechanism, vessel configuration, and calcine type. Although requirements for the frequency of sampling and which constituents will need to be analyzed are undefined at this point in time, it is anticipated some level of S\&A would be required for quality and process control in a future grouting plant.

A similar assumption was made in developing a conceptual design for a hydroceramic grouting plant (Dafoe and Losinski 1998) for HLW calcine, which was done to support the Idaho High-Level \& Facilities Disposition Environmental Impact Statement. The prior conceptual design assumed that the production control samples would be taken from a large $50 \mathrm{~m}^{3}$ batch vessel, which would hold enough calcine for a one week production schedule. As with the bulk vessels, the load cells described in Section 4.3.1 could be used to determine inventory in the day vessels prior to S\&A, track the inventory, and track the amount of feed delivered to the single batch vessels.

\subsubsection{Single Batch Vessels}

The process monitoring needs for the single batch vessels (i.e., sized to contain the ingredients for mixing one waste canister of grouted waste) would be to avoid overfilling and to verify the quantity of feed that was delivered from the day vessels. This is a crucial process monitoring step to avoid the potential for making a batch of grouted waste that could overfill the waste canister, which has $0.1 \mathrm{~m}^{3}$ of headspace available at $90 \%$ fill to handle some minor variations in the volume of ingredients delivered through the process. As indicated in Section 4.2.3, the batch volumes will vary due to the variability in calcine density and a determination of a proper batch size needs to be based on weight rather than volume. The weight of calcine and stabilizer removed from the day vessels would be compared to the 
weight gain in the single batch vessels. If an excessive amount of ingredients were determined to be in the single batch vessels, the load cells could be used as a final control step to track the required amount of solids being added to the grout mixer. Paddle wheel detectors could also be mounted in the top of the single batch vessels to detect a process upset state of completely filling the vessels.

The water required for the grout formulations would be metered in via a mass flow meter between the day water tank and the single batch tank. The water transferred to the single batch tank would be the amount required to mix one canister of grouted waste. The water inventory in the batch tank would be monitored via some sort of level detector. Since this tank will be in a non-radioactive zone, there are a number of different types of level detectors that could be used such as mechanical floats, radiofrequency probes, ultrasonic probes, capacitance probes, pressure differential bubblers, and non-contact level indicators using ultrasonic and laser sensors. An example of the type of accuracy available is the ability to measure the level of a liquid within 1/32" for ultrasonic level probes and 1.25 " for non-contact ultrasonic level detectors (OMEGA 1992a). The accuracy for measuring the liquid volume with level detectors would depend, in part, on the geometry of the vessel and the depth of the liquid. For example, using a level detector with a 1.25 " accuracy in a cylindrical vessel with $508 \mathrm{~L}$ of water (i.e., $508 \mathrm{~kg}$ per Table 4-1) and a height two times the diameter (i.e., $137.2 \mathrm{~cm}$ by $68.6 \mathrm{~cm}$ ) would equate to a volume accuracy of about $2.3 \%$.

\subsubsection{Grout Mixer}

The main process monitoring need for the grout mixer would be to monitor the viscosity of mix to ensure that it was not becoming too viscous for the pouring step. The process mixing time for acceptable mixing and pouring viscosities would be based on cold and hot full-scale qualification runs. The process correction to an unexpected high viscosity would be to immediately add more water. This is a crucial process control step to avoid an irreversible process upset in which the grout becomes unmixable and hardens in the mixer. As indicated in Figure 4-2, the torque on the mixer paddle would be monitored to avoid the upset situation.

A secondary process monitoring need would be to verify the water fill level in the mixer if it were in a standby mode for several days. As indicated in Section 4.2.3, it is anticipated that the water input to the mixer would be monitored via mass balance measurements on the single batch water tank during normal operation. However, if the mixer were placed in standby mode, water loss could occur via evaporation to the off-gas system or leakage through the pour valve to the canister. Because the mixer vessel will be subjected to the forces of vibration and torque from the mixing action, load cells are not considered the best choice for determining water inventory. In addition the load cells would not be able to discriminate between the weight of the residual grout left in mixer and the water content. The use of internal contact or non-contact level sensors that were discussed in Section 4.3.4 are also not recommended due to the vessel vibration and the anticipation that the inside of the vessel will eventually be coated to some degree by residual grout.

To verify the amount of water in the mixer prior to restart after an extended shutdown, a gamma densitometer using cobalt-60 as the radiation source and gamma detector(s) on the opposite side of the vessel could be used as an external, non-intrusive, non-contact detector to measure the difference in the gamma attenuation depending on whether it is passing through water or air in the vessel. Cobalt-60 has three predominant photo peaks at 1.17, 1.33, and 1.63 million electron volts (Mev) which could be measured in the presence of the $0.66 \mathrm{Mev}$ Cs- 137 photo peak. The cobalt- 60 radiation source and a gamma detector could be designed to track up and down the vertical axis of the mixer vessel to determine the location of the liquid level-headspace interface in the vessel. An alternative approach would be to fix the cobalt- 60 radiation source on one side of the vessel and fix several gamma detectors on the opposite side and calibrate the gamma detector readings based on the fill level of the mixer. 


\subsubsection{Waste Canister}

The crucial process monitoring need for the waste canister is to avoid overfilling and having to recover, crush, and recycle the grouted waste. As indicated in Figure 4-2, two methods are proposed to monitor the filling process. A pin-hole gamma sensor could be positioned on a vertical rack, which allows it to track up and down the length of the canister. It could be initially positioned near the bottom and rise vertically with the grout level as the canister is being filled. An alternative approach would be to position several gamma sensors along the length of the canister. The coupling mechanism of the pour spout to the canister and the availability of viewing space down the neck are still undefined. Neck diameters of 3, 4, and 6 in. are under consideration for the canister design (Rahl 2003). If the neck designs allow sufficient viewing space, a small fiber optic cable connected to a video camera could be used to view the progress of filling also.

It is anticipated that some sort of insertable cup would be designed to allow small pour samples to be taken as a final check on quality control of the product. This sampling need was also envisioned in the conceptual design of the hydroceramic grouting plant although the sample would be taken from the grout mixer rather than during the pouring operation (Dafoe and Losinski 1998). A precedent for sampling the final HLW product has been set by the Defense Waste Processing Facility at the Savannah River Site, which designed and used a stainless steel cup to take molten glass samples during the filling operation of Canisters 50 and 61 with HLW in borosilicate glass (Bibler et. al. 1998). 


\section{HYDROCERAMIC GROUT STABILIZATION PROCESS}

Hydroceramic (HC) cements are a class of geopolymeric concretes made from a mixture of waste material, calcined clay, sodium hydroxide, water, and minor additives. The HC stabilizer additives are designed to chemically combine with the calcine such that the major components of aluminum, silicon, and sodium produce a sodalite with the generic formula $\left(\mathrm{NaAlSiO}_{4}\right)_{3} \cdot \mathrm{NaX}$ where $\mathrm{X}$ can be a sulfate, nitrate or chloride. There must be sufficient caustic (sodium hydroxide) in the mix to dissolve the alumina and silica in the stabilizer additives. The grout formulation and process described in Sections 5.1 and 5.2 are based on studies to develop the HC cement waste forms for application to sodium bearing waste and HLW calcine located at INTEC (Siemer 2002, Siemer et. al. 2002). The conceptual design proposed for application of the HC grouting process to HLW calcine (Dafoe and Losinski 1998) would produce an extremely viscous paste that would require kneading for mixing and extrusion for transfer from the mixer to the canister. Curing would require an autoclaving step at about $275^{\circ} \mathrm{C}$, which would be followed by a dewatering step at $250^{\circ} \mathrm{C}$ (Dafoe and Losinski 1998). To expedite mixing and pouring, studies are underway at DIAL-MSU to developed HC grouts that are more fluid and can be cured below $80^{\circ} \mathrm{C}$ (Plodinec et. al. 2004). For flow sheet development in this document, a formulation similar to prior work (i.e., a silica additive has been substituted for vermiculite additive) and a $24.4 \mathrm{wt} \%$ waste loading is assumed.

\subsection{Mass Balance Flow Sheet}

Stabilization of calcine via hydroceramic grouting would be accomplished by mixing the calcine with a blend of metakaolin clay, silica fume, and sodium hydroxide ( $30 \mathrm{wt} \%$ solution). A reductant, sodium sulfide, will be added to promote stabilization of the RCRA toxic metals. Table 5-1 presents the mass balance flow sheet by weight percent and kilograms for a batch large enough to fill one canister to $1.0 \mathrm{~m}^{3}$. As indicated in the table, the final waste loading would be $24.4 \mathrm{wt} \%$. These components would be combined batchwise in a grout mixer, mixed, and then poured into a waste disposal canister. This formulation was also used to size the vessels discussed below in Section 5.2 and as the starting point in the flow sheet calculations given in Appendix C-1 for the hydroceramic grouting process steps.

\subsection{Major Process Steps and Equipment Design}

As noted in Section 3.1 "HLW Calcine Processing Rate," the hydroceramic process would be designed to grout $4760 \mathrm{~kg}$ of calcine per day to produce about 11 canisters of waste per day. This would meet the production rate required to complete calcine treatment in eight years. It is recommended a value engineering or other cost-benefit studies be done to determine if a redundant train is needed to meet

Table 5-1. Calcine Waste Form Composition and Recipe for $1.0 \mathrm{~m}^{3}$ of Hydroceramic Waste.

\begin{tabular}{lcc}
\hline \multicolumn{1}{c}{ Component } & $\begin{array}{c}\text { Weight Percent } \\
(\mathrm{wt} \%)\end{array}$ & $\begin{array}{c}\text { One Batch Mass } \\
(\mathrm{kg})\end{array}$ \\
\hline Calcine & $24.4 \%$ & 410.7 \\
Metakaolin Clay & $40.7 \%$ & 685.0 \\
Silica Fume & $7.3 \%$ & 122.9 \\
Sodium Hydroxide & $26.8 \%$ & 451.0 \\
(30 wt\% solution) & & \\
Sodium Sulfide & $0.8 \%$ & 13.5 \\
\hline
\end{tabular}


production goals if the first train were down for a few weeks for any reason. Due to the nature of remote operations and maintenance, the need for a second grout train is highly recommended. Due to the hygroscopic nature of the calcine and solids used in the hydroceramic process, the solid ingredients should not be exposed to humid gases prior to their addition to the grout mixing vessel. The solids process vessels will most likely require a small purge of dry air or nitrogen. The major process steps and vessel sizes are described below and illustrated in Figure 5-1. The process steps follow the five major feed steps described in Section 3.2 where the calcine and feed material move through: (a) bulk vessels to (b) day vessels to (c) batch vessels to (d) the grout mixer, and to e) the canister. The vessel dimensions are based on the methods described in Section 3.2 and Appendix A. Also per Appendix A, the critical friction angle of the hopper for the bulk, day, and single batch vessels was based on the most conservative value of $20^{\circ}$ recommended in the Johanson and Johanson study (Wanke 1993). The dimensions of the process vessels, which are discussed below, are summarized in Appendix C-2.

\subsubsection{Bulk Receiving Vessels}

The hydroceramic process bulk vessels will have capacity for a seven day supply of calcine and stabilizer powders. To provide this capacity, the calcine bulk vessel will be $30 \mathrm{~m}^{3}$ and the clay and silica bulk stabilizer vessel will need to be $140 \mathrm{~m}^{3}$ due to the material's low density. For the hydroceramic process, the bulk calcine vessel will be the same as in the grout process (see Section 4.2.1). The $140 \mathrm{~m}^{3}$ bulk stabilizer vessel will also need to be a hopper design to handle the clay and silica fume. The approximate dimensions would be $4.5 \mathrm{~m}$ in diameter by $12.6 \mathrm{~m}$ high. The vessel would also use an auger system to deliver powders to the day vessel. Metakaolin clay and silica fume are commercially available products. It is recommended that they be purchased from a vendor that will pre-blend the needed mixture. Preblending the powders eliminates the need for an individual bulk tank for each stabilizer.

To supply the needed $130 \mathrm{~m}^{3}$ of stabilizer per week, two railroad hopper cars of blended clay and silica would be needed each week. A method of transferring the stabilizers from the railroad hopper to the bulk vessel would be needed in the facility. To prepare the stabilizers, a vendor would need the capacity to blend $110 \mathrm{~m}^{3}$ clay with $20 \mathrm{~m}^{3}$ silica and transport the material to the INL weekly. If such a vendor is not available, it would be necessary to have individual bulk vessels. The individual bulk vessels could replace the $140 \mathrm{~m}^{3}$ bulk vessel. Both bulk vessels would then feed the day vessel. In this case, the day vessel would need mixing capability to blend and homogenize the clay and silica.

The bulk sodium hydroxide would be stored in a $35 \mathrm{~m}^{3}$ vessel along with the needed sodium sulfide. A supply of $30 \mathrm{wt} \%$ liquid sodium hydroxide will be needed, approximately $28 \mathrm{~m}^{3}(37,100 \mathrm{~kg})$ each week. As an alternative, 11,450 kg of solid sodium hydroxide could be supplied and mixed with $25,100 \mathrm{~kg}$ water on site to prepare the needed $30 \mathrm{wt} \%$ solution. Additionally, $1,070 \mathrm{~kg}$ of sodium sulfide will need to be added to the sodium hydroxide solution. It would be best if the sodium sulfide could be pre-blended with the $30 \mathrm{wt} \%$ sodium hydroxide as a liquid; otherwise, such liquid/solid blending equipment will be needed in the bulk vessel.

\subsubsection{Day Vessels}

Day vessels are sized to hold sufficient materials to process $4760 \mathrm{~kg}$ of calcine per day and produce about 11 canisters of waste per day. For calcine, it is recommended to have a minimum of two day vessels as noted in Section 4.2.2. This provides the opportunity to sample and analyze one vessel while the other is being used for processing. If this does not provide enough analytical time, then more day tanks may be needed. The calcine day vessels would be $5 \mathrm{~m}^{3}$ hoppers about $1.5 \mathrm{~m}$ in diameter by $3.9 \mathrm{~m}$ high each. Both day vessels would supply calcine via augers to a single batch vessel. These vessels also require the steep hopper angle. 


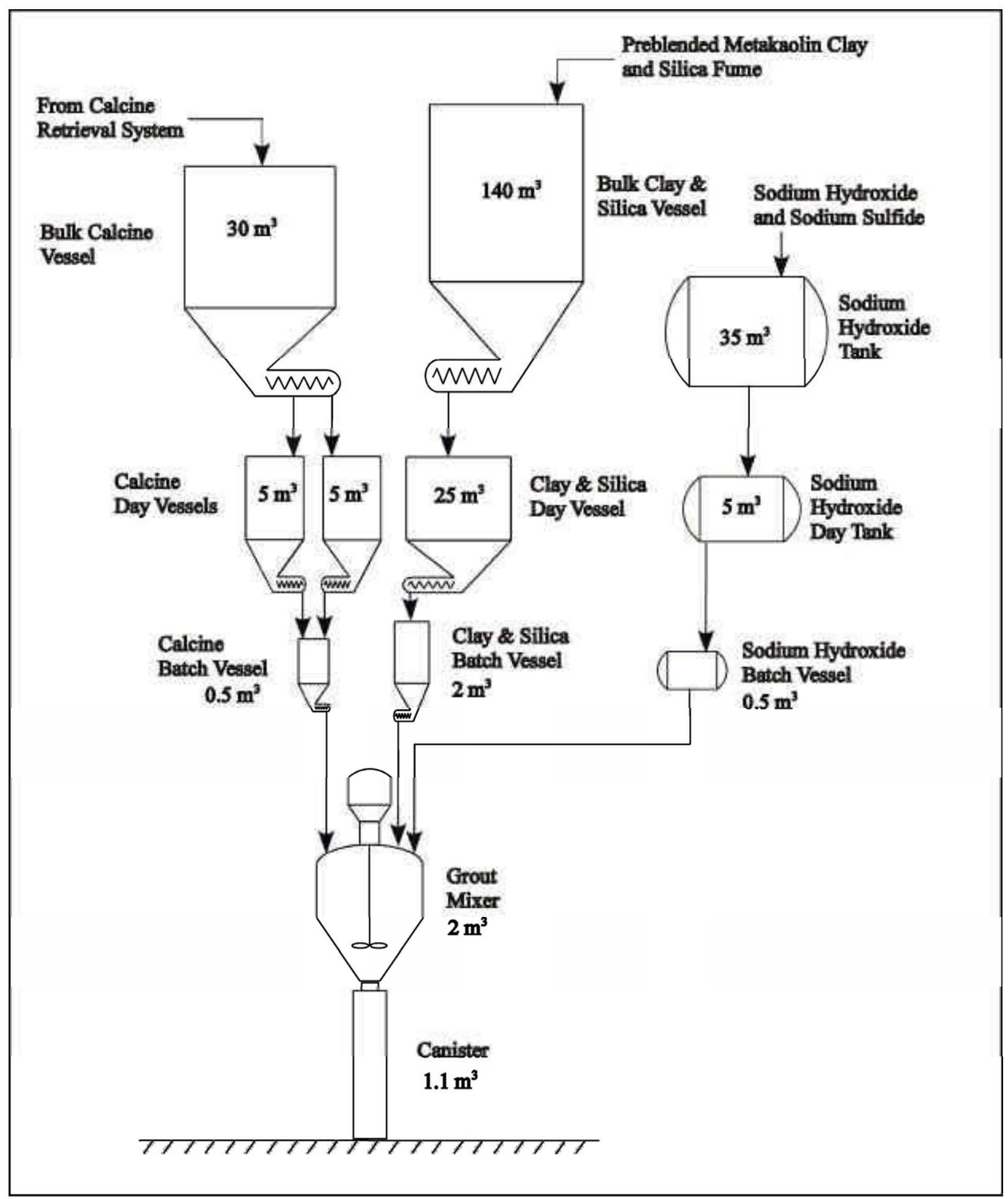

Figure 5-1. Calcine hydroceramic cement stabilization process diagram. 
The clay and silica day vessel would be a $25 \mathrm{~m}^{3}$ hopper about $2.5 \mathrm{~m}$ in diameter and $6.9 \mathrm{~m}$ high. This vessel will also supply a single batch vessel as shown in Figure 5-1. As noted in Section 5.2.1, this day vessel may need mixing capability if pre-blended clay and silica are not available. A $5 \mathrm{~m}^{3}$ day vessel is needed for about 11 batches of the $30 \mathrm{wt} \%$ sodium hydroxide and sodium sulfide solution. This vessel will also supply a single batch sodium hydroxide tank.

\subsubsection{Single Batch Vessels}

Single batch vessels for solids and liquids are recommended in order to prevent overfilling the mixer and to control the grout/calcine mix formulation. Each batch vessel needs to be capable of providing the needed amount for one canister batch as noted in Table 5-1. The calcine batch vessel would be about $0.5 \mathrm{~m}^{3}$, the clay and silica stabilizer batch vessel would be about $2 \mathrm{~m}^{3}$, and the sodium hydroxide batch tank would be about $0.5 \mathrm{~m}^{3}$. The solids vessels would need to meter the desired material to the mixer at a rate to provide good mixing. The calcine single batch vessel needs the same design angle to prevent bridging as in the other calcine vessels.

\subsubsection{Calcine and Hydroceramic Grout Mixing}

The calcine, clay and silica stabilizers, and sodium hydroxide batch vessels would each feed the grout mixer. As currently envisioned, the mixer could be a 1.5 to $2 \mathrm{~m}^{3}(400+$ gallon) planetary batch mixer with a bottom discharge to the disposal canister such as Ross DPM 400 mixer (Charles and Ross and Son Co 2005). The mixer will need to be covered to prevent fugitive dust during the filling and mixing operations. The cover will need entry points for the calcine, stabilizers, and water and a vessel off-gas exit for displaced air and dust. The mixer should be instrumented with paddle torque as noted in Section 5.3.

The full amount of sodium hydroxide would be added first to the mixer. With the mixer running, calcine and stabilizers could then be metered in over a period of time (approximately 15 to 30 minutes) to permit adequate mixing and blending. When fully mixed, the grouted waste would be discharged into the canister mounted directly below the mixer. As soon as the mixer is empty, the next batch of sodium hydroxide should immediately be added to prevent drying or caking of any remaining grout in the mixer. This sodium hydroxide would then be used for the next batch of calcine grout. If a shutdown or maintenance were anticipated, water could be added to the mixer rather than sodium hydroxide. Thus, a water inlet to the mixer is needed for rinse and flush. This would further present a need to deal with any rinse water.

To facilitate a batch mixer, the waste mixture's viscosity must be fluid enough to allow bottom discharge and easy filling of the canister. Section 5 noted that the original hydroceramic formulation was very viscous and required a mixer extruder. DIAL-MSU is working to develop a fluid "wet" grout. If the hydroceramic grout is more viscous, a continuous processor mixer may be considered, such as those made by Autocon or Readco (Autocon 2005, Readco 2005). This would necessitate changes in the batch vessel design and controls.

\subsubsection{Canister}

The waste disposal canisters used in this process would be the same as described in Section 4.2.5 as well as the curing, capping, decontamination, and shipping areas. 


\subsection{Process Monitors and Sampling Steps}

Most of the process monitors and sampling steps illustrated in Figure 4-2 and described in Sections 4.3.1 through 4.3.5 for the grout stabilization process would also be applicable to the hydroceramic grout stabilization process. The different or additional requirements for the hydroceramic grout stabilization process are described below.

\subsubsection{Bulk Vessel Process Monitors}

The additional requirement beyond monitoring the fill levels and inventories of the bulk calcine and stabilizer vessels described in Section 4.3.1 would be the need to monitor the liquid level of the bulk sodium hydroxide tank shown in Figure 5-1. A mass flow meter would also be required to monitor the amount of caustic transferred to the sodium hydroxide day tank. Since the bulk sodium hydroxide tank will be in a non-radioactive zone, there are a number of different types of level detectors that could be used such as mechanical floats, radiofrequency probes, ultrasonic probes, capacitance probes, pressure differential bubblers, and non-contact level indicators using ultrasonic and laser sensors. An example of the type of accuracy available is the ability to measure the level of a liquid within 1/32" for ultrasonic level probes and 1.25" for non-contact ultrasonic level detectors (OMEGA 1992a). Materials of construction such as stainless steel would be required for components of instrumentation in direct contact with the caustic solution.

\subsubsection{Day Vessel Process Monitors}

The additional requirement beyond monitoring the fill levels and inventories of the day calcine and stabilizer vessels described in Section 4.3.2 would be the need to monitor the liquid level of the day sodium hydroxide tank shown in Figure 5-1. A mass flow meter would also be required to monitor the amount of caustic transferred to the sodium hydroxide batch tank. As indicated in Section 5.3.1, there are a number of different types of level detectors that could be used.

\subsubsection{Single Batch Vessels}

The additional requirement beyond monitoring the fill levels and inventories of the single batch calcine and stabilizer vessels described in Section 4.3.3 would be the need to monitor the liquid level of the single batch sodium hydroxide tank shown in Figure 5-1. As indicated in Section 5.3.1, there are a number of different types of level detectors that could be used.

\subsubsection{Grout Mixer}

There are no different or additional monitoring requirements anticipated for this step between the grout and hydroceramic stabilization processes.

\subsubsection{Waste Canister}

There are no different or additional monitoring requirements anticipated for this step between the grout and hydroceramic stabilization processes. 


\section{IRON PHOSPHATE CERAMIC STABILIZATION PROCESS}

The iron phosphate ceramic process described in Sections 6.1 and 6.2 is based on studies to develop it as a stabilization agent for mixed low level waste and transuranic waste (Medvedev et. al. 1998, Aloy et. al. 2000). The process results not only in micro- and macro-encapsulation of contaminants, but also in chemical binding of the waste in the form of phosphates. Phosphate binders are a heterogeneous system consisting of a powder with metal oxides and a solution of phosphoric acid. A chemical reaction between these constituents leads to hydrated salts of orthophosphoric acid that can be characterized as an inorganic polymer. The use of the iron phosphate ceramic process for application to HLW calcine simulants has not been reported. Studies are currently underway at DIAL-MSU to develop a formulation and method that will stabilize HLW calcine simulants (Plodinec 2004) and, to date, the studies indicate that the reported formulation for other types of simulants will have to be modified for application to HLW calcine. However, for the flow sheet development in this document the literature values (Medvedev et. al. 1998, Aloy et. al. 2000) of about $13 \mathrm{wt} \%$ magnetite mixed with $25 \mathrm{wt} \%$ simulant and added to $62 \mathrm{wt} \%$ of $50 \%$ phosphoric acid are used.

\subsection{Mass Balance Flow Sheet}

Stabilization of calcine via the iron phosphate ceramic process would be accomplished by mixing the calcine with magnetite (black iron oxide, $\mathrm{Fe}_{3} \mathrm{O}_{4}$ ) and then adding the solid ingredients to phosphoric acid (50 wt $\%$ solution). Based on current DIAL-MSU studies, the phosphoric acid must be heated to about $60^{\circ} \mathrm{C}$ before adding the solid ingredients. Once the exothermic chemical reaction begins, the temperature must be maintained between 60 to $80^{\circ} \mathrm{C}$ during mixing. Curing in the canister may also require maintenance of $80^{\circ} \mathrm{C}$ for up to a week. Table 6-1 presents the mass balance flow sheet by weight percent and kilograms for a batch large enough to fill one canister to $1.0 \mathrm{~m}^{3}$. As indicated in the table, the final waste loading would be $25.4 \mathrm{wt} \%$. The calcine and powdered magnetite would first be blended together as solids. Next, the calcine and magnetite mixture would be combined batchwise with phosphoric acid in a grout mixer, mixed, and then poured into a waste disposal canister. This formulation was also used to size the vessels discussed below in Section 6.2 and as the starting point in the flow sheet calculations given in Appendix D-1 for the iron phosphate ceramic process steps.

\subsection{Major Process Steps and Equipment Design}

As noted in Section 3.1 "HLW Calcine Processing Rate", the iron phosphate ceramic process would be designed to process $4760 \mathrm{~kg}$ calcine per day and produce about 10 canisters of waste per day. This would meet the production rate required to complete calcine treatment in 8 years. It is recommended a value engineering or other cost-benefit studies be done to determine if a redundant train is needed to meet production goals if the first train were down for a few weeks for any reason. Due to the nature of remote operations and maintenance, the need for a second process train is highly recommended. Due to the hygroscopic nature of the calcine and solids used in the iron phosphate ceramic process, the solid

Table 6-1. Calcine waste form composition and recipe for $1.0 \mathrm{~m}^{3}$ of iron phosphate waste.

\begin{tabular}{lcc}
\hline \multicolumn{1}{c}{ Component } & $\begin{array}{c}\text { Weight Percent } \\
(\mathrm{wt} \%)\end{array}$ & $\begin{array}{c}\text { One Batch Mass } \\
(\mathrm{kg})\end{array}$ \\
\hline Calcine & $25.4 \%$ & 452.6 \\
Iron Oxide (Magnetite) & $12.7 \%$ & 226.3 \\
Phosphoric Acid & $61.9 \%$ & 1103.1 \\
\hline
\end{tabular}


ingredients should not be exposed to humid gases prior to their addition to the grout mixing vessel. The solids process vessels will most likely require a small purge of dry air or nitrogen. The major process steps and vessel sizes are described below and illustrated in Figure 6-1. The process steps follow the five major feed steps described in Section 3.2 where the calcine and feed material move through (a) bulk vessels to (b) day vessels to (c) batch vessels to (d) the grout mixer, and to (e) the canister. The vessel dimensions found in Appendix D-1 are based on the methods described in Section 3.2 and Appendix A.

\subsubsection{Bulk Receiving Vessels}

The iron phosphate process bulk vessels will also have capacity for a seven day supply of calcine and stabilizers. To provide this capacity, the calcine bulk vessel will be $30 \mathrm{~m}^{3}$, the bulk magnetite vessel will be $8 \mathrm{~m}^{3}$, and the phosphoric acid bulk tank will be $70 \mathrm{~m}^{3}$. The bulk calcine vessel will be the same as described in the grout process (Section 4.2.1). The $8 \mathrm{~m}^{3}$ bulk magnetite vessel will also need to be a hopper design to handle the powdered magnetite. The approximate dimensions would be $1.7 \mathrm{~m}$ in diameter by $4.6 \mathrm{~m}$ high. The vessel would also use an auger system to deliver magnetite to both calcine day vessels. The bulk phosphoric acid tank would be $70 \mathrm{~m}^{3}$ with an approximate diameter of $3.5 \mathrm{~m}$ and $7.1 \mathrm{~m}$ in length. A supply of approximately $60 \mathrm{~m}^{3}(80,300 \mathrm{~kg})$ each week of $50 \mathrm{wt} \%$ phosphoric acid will be needed.

\subsubsection{Day Vessels}

Day vessels are sized to hold sufficient materials to meet the daily process rate of $4760 \mathrm{~kg}$ calcine per day. For calcine, it is recommended to have a minimum of two day vessels as noted in Section 4.2.2. This provides the opportunity to sample and analyze one vessel while the other is being used for processing. If this does not provide enough analytical time, then more day vessels would be needed. The calcine day vessels would be $6 \mathrm{~m}^{3}$ hoppers about $1.6 \mathrm{~m}$ in diameter by $4.1 \mathrm{~m}$ high each. Both day vessels would supply calcine via augers to a single batch vessel. These vessels also require the steep hopper angle. For the iron phosphate process, the calcine day vessel must be able to blend calcine with magnetite.

Calcine blending was noted in the other processes to provide an average mixture of calcine for analyses, but here, it is essential to obtain a homogenous mixture of calcine and magnetite to provide the proper amount of stabilizer to the calcine. A $10 \mathrm{~m}^{3}$ day vessel is needed for about 10 batches of the $50 \mathrm{wt} \%$ phosphoric acid. This vessel will also supply a single batch tank.

\subsubsection{Single Batch Vessels}

Single batch vessels for solids and liquids are recommended in order to prevent overfilling the mixer and to control the mix formulation. Each batch vessel needs to be capable of providing the needed amount for one canister batch as noted in Table 6-1. The calcine/magnetite batch vessel would be about $0.7 \mathrm{~m}^{3}$ and the phosphoric acid batch tank would be about $1.0 \mathrm{~m}^{3}$. The solids vessel would need to meter the desired material to the mixer at a rate to provide good mixing. The calcine/magnetite single batch vessel should have the same design angle to prevent bridging as in the other calcine vessels.

\subsubsection{Calcine and Iron Phosphate Ceramic Mixing}

The calcine/magnetite and phosphoric acid batch vessels will each feed the grout mixer. As currently envisioned, the mixer could be a 1.5 to $2 \mathrm{~m}^{3}$ (400+ gallon) planetary batch mixer with a bottom discharge to the disposal canister (such as Ross DPM 400 mixer). For this process, the mixer must have heating/cooling capability and it needs to be covered to prevent fugitive dust during the filling and mixing 


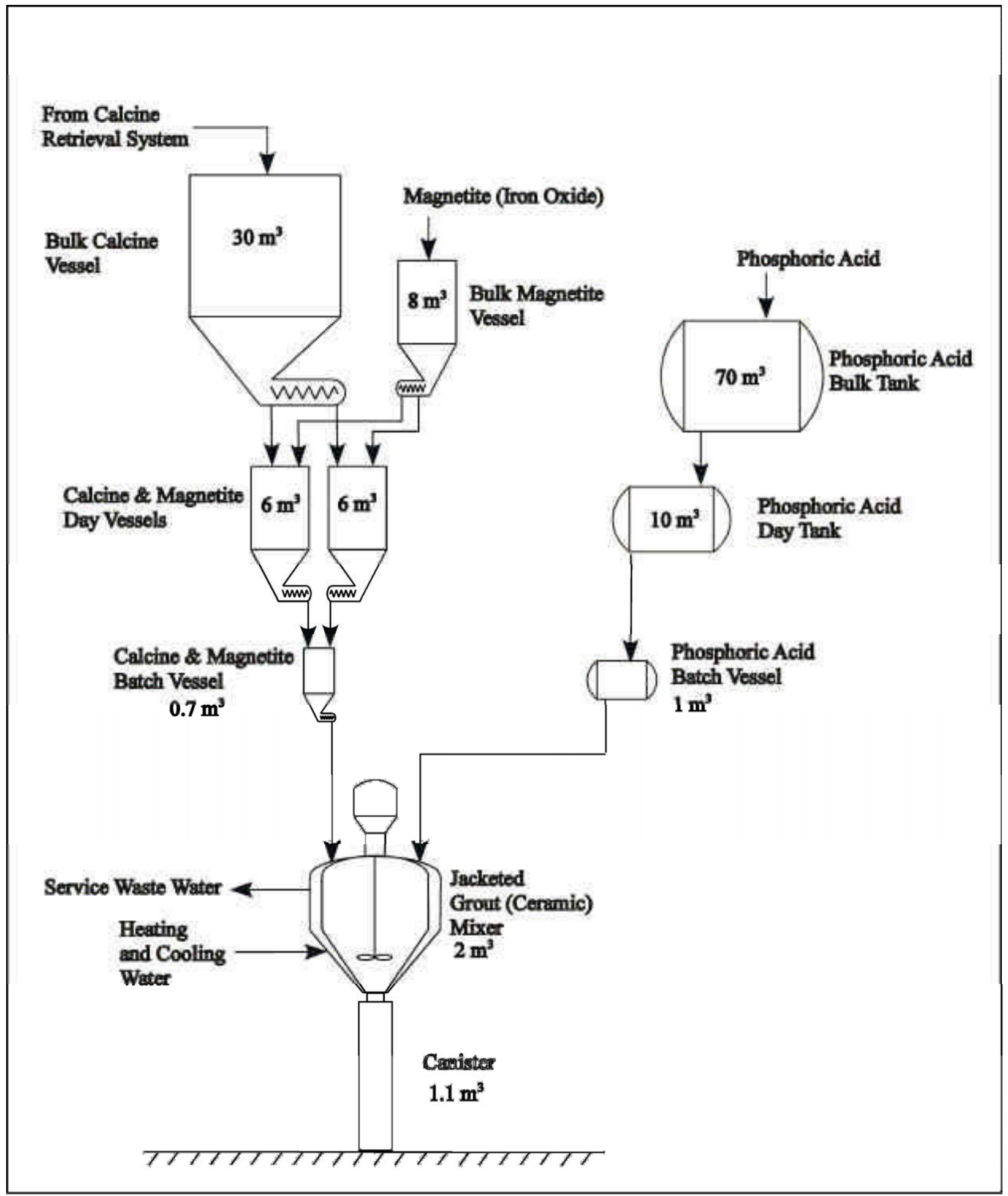

Figure 6-1. Calcine iron phosphate ceramic stabilization process diagram. 
operations. The cover will need entry points for the calcine/magnetite and the acid and a vessel off-gas exit for displaced air and dust. The mixer should be instrumented with temperature control and paddle torque.

The full amount of phosphoric acid would be added first to the mixer and while stirring the acid would be heated to $60^{\circ} \mathrm{C}$. Then the calcine/magnetite mixture could be metered in over a period of time (approximately 15 to 30 minutes) to permit adequate mixing and blending. During the mixing phase, the mixture temperature must be kept under $80^{\circ} \mathrm{C}$. When fully mixed, the grouted waste would be discharged into the canister mounted directly below the mixer. As soon as the mixer is empty, the next batch of phosphoric acid should immediately be added to prevent drying or caking of any remaining grout in the mixer. This phosphoric acid would then be used for the next batch of calcine grout. If a shutdown were anticipated, water should be added to the mixer rather than phosphoric acid. Thus, a water inlet to the mixer is needed for rinse and flush. This would further present a need to deal with any rinse water.

To facilitate a batch mixer, the waste mixture's viscosity must be fluid enough to allow bottom discharge and easy filling of the canister. If the iron phosphate ceramic is more viscous, a continuous processor mixer may be considered, such as those made by Autocon or Readco. This would necessitate changes in the batch vessel design and controls.

\subsubsection{Canister}

The waste disposal canisters used in this process will be the same as described in Section 4.2.5. For the iron phosphate ceramic, the capped canister would need to be placed in a curing room for a week where the temperature is maintained at $80^{\circ} \mathrm{C}$ to allow the waste ceramic cure properly. (Laboratory studies show the waste ceramic remains a sludge without the heated curing.)

\subsection{Process Monitors and Sampling Steps}

The process monitoring requirements for the iron phosphate ceramic stabilization process shown in Figure 6-1 would be virtually identical to the hydroceramic stabilization process shown in Figure 5-1 and described in Sections 5.3.1 through 5.3.5. The major exception would be the need to monitor and maintain the temperature of reaction between $60-80^{\circ} \mathrm{C}$ in the ceramic mixer. Although, a process variation occurs in which the magnetite would be mixed with the HLW calcine prior to addition to the ceramic mixer, the requirement to sample and analyze the day vessels is common to all three stabilizer processes and would not introduce an additional process monitoring requirement for the iron phosphate ceramic process. 


\section{COMPARISON OF STABILIZATION PROCESSES}

\subsection{Waste Form Performance Data to Date}

As indicated in Section 2, the three candidate stabilizer processes described in Sections 4 through 6 are currently under development. Per a collaborative DIAL/BBWI test plan for the period of June 2004 through February 2006 (Plodinec et. al. 2004), the main objectives are to develop stabilized waste forms that:

- $\quad$ Have compressive strengths $>500$ psi

- $\quad$ Meet the Universal Treatment Standard (UTS) limits for RCRA metals in the toxicity characteristic leaching procedure (TCLP)

- $\quad$ Perform as well as the Environmental Assessment (EA) borosilicate glass in the product consistency test (PCT)

- $\quad$ Have a minimum waste loading of $25 \mathrm{wt} \%$ on a dry weight basis

- $\quad$ Optimize the waste form loadings while maintaining mixing and pouring properties that would be compatible with large scale remote operations.

An assessment of these objectives based on test data generated through April 2005 indicated the following (Thomas 2005):

- Grout and hydroceramic grout waste forms have been developed which have average compressive strengths of 2800 and 760 psi, respectively. Formulations for the iron phosphate waste forms have not been found which can meet the $>500$ psi criterion.

- $\quad$ Grout formulations have been developed which can meet the UTS limits for $\mathrm{Cd}, \mathrm{Cr}, \mathrm{Hg}$, and $\mathrm{Ni}$ in the TCLP for waste loadings tested (i.e., between 25 and 35 dry wt $\%$ ). At the $35 \mathrm{dry} w \mathrm{wt} \%$ loadings, the concentrations of $\mathrm{Cd}$ and $\mathrm{Cr}$ in the TCLP were close to the UTS limits. The hydroceramic grout and iron phosphate ceramic waste forms exceeded the UTS limits for one or more of the four RCRA metals tested in the TCLP.

- $\quad$ All three waste forms exceeded the EA borosilicate glass leach limits in the PCT for Na (i.e., failed PCT leach limit). Test data to date indicate that none of the stabilizers under development may be able to pass the PCT.

- Waste loadings of $>25 \mathrm{wt} \%$ on a dry weight basis was obtained for all three waste forms.

- $\quad$ Preliminary results indicate that about 35 dry wt $\%$ waste loadings in grout will be the upper limit for the waste loading at which the mixture becomes too viscous to mix and pour. Based on a water-to-total solids ratio of 0.4 used as the reference formulation (i.e., see Section 4), 35 dry wt $\%$ corresponds to waste loading of $25 \mathrm{wt} \%$ when the water is factored in.

Tests to optimize waste form loadings and improve the leaching characteristics of the waste forms in the TCLP and PCT are planned for May through September, 2005. To date, the grout formulation appears to be the best candidate stabilizer among the three being tested for HLW calcine. This assessment 
is based on its ability to pass the compression test, TCLP and waste loading performance criteria. In addition, the formulation appears to be the easiest to mix, pour, and cure. However, additional testing with the grout and other two stabilizers must be completed before a candidate is selected for cold mock-up studies and hot bench scale tests.

\subsection{Relative Process Complexity}

Based on the illustrations of the grout, hydroceramic grout and iron phosphate ceramic stabilization processes given in Figures 4-1, 5-1, and 6-1, only minor differences would exist among the process steps between the bulk feed vessels and the waste canister. With the exception that sodium hydroxide is used in place of water, there is no difference between the grout and the hydroceramic grout processes in the process flow diagrams. The iron phosphate ceramic process requires that one of the stabilizer reagents (i.e., the magnetite) be added and mixed with the calcine feed in the day vessels rather than each constituent being fed directly to the grout or ceramic mixer. This process variation should not add process complexity given that mixing and sampling is envisioned for the day vessels in all three cases. Formulation development to date indicates that the mixing step of the iron phosphate step may require maintaining the ceramic mixer between $60^{\circ} \mathrm{C}$ to $80^{\circ} \mathrm{C}$ (Thomas 2005). If so, this will be a major process complexity relative to running the grout mixer at ambient cell temperature. Development to date, indicates that the curing step for the waste in the canister may have to be elevated from room temperature to about $80^{\circ} \mathrm{C}$ for both the hydroceramic grout and iron phosphate ceramic waste forms, with the elevation being done in two steps involving room and the higher temperature for the hydroceramic grout (Thomas 2005). If so, this will also add significant process complexity for these two processes relative to the grout process which would use room temperature for curing.

\subsection{Number of Waste Canisters Produced}

As indicated in the Appendices B-1, C-1, and D-1, the waste loadings for the mass balance flow sheets were assumed to be $21.5,24.4$, and $25.4 \mathrm{wt} \%$ (based on all constituents added) for the grout, hydroceramic grout, and iron phosphate ceramic stabilization processes, respectively. These waste loadings are values that have been reported in the literature and were used as a starting point for the DIAL-MSU stabilizer development work (Plodinec 2004). As indicated in Section 7.1, the studies to optimize the waste loadings with regard to performance parameters remain to be done. There will be little or no variation in the numbers of canisters produced by the three stabilizer processes due to waste form density because the densities are assumed to be about the same (i.e., 1700 or $1800 \mathrm{~kg} / \mathrm{m}^{3}$ ). To estimate the number of waste canisters that would be produced, it is assumed that the waste loadings will be between 21.5 and $25.4 \mathrm{wt} \%$ and that the waste form density will be $1800 \mathrm{~kg} / \mathrm{m}^{3}$, regardless of which process is chosen. As indicated in Section 3.1, the assumed total HLW calcine inventory is $5.6 \times 10^{6} \mathrm{~kg}$. Per Section 3.3, the assumed volume of stabilized waste per canister is $1.0 \mathrm{~m}^{3}$. The volume of waste that would be produced at waste loadings of 21.5 and $25.4 \mathrm{wt} \%$ are, respectively:

$$
\begin{aligned}
& {\left[\left(5.6 \times 10^{6} \mathrm{~kg} \text { calcine }\right) /(0.215)\right] /\left(1800 \mathrm{~kg} \text { waste } / \mathrm{m}^{3}\right)=14,470 \mathrm{~m}^{3}} \\
& {\left[\left(5.6 \times 10^{6} \mathrm{~kg} \text { calcine }\right) /(0.254)\right] /\left(1800 \mathrm{~kg} \text { waste } / \mathrm{m}^{3}\right)=12,250 \mathrm{~m}^{3}}
\end{aligned}
$$

These volumes also equate to the number of canisters that would be produced assuming each canister contains $1.0 \mathrm{~m}^{3}$ of waste product. 


\subsection{Comparison to the Direct Cementitious Waste Option}

In 1997, a conceptual design for application of the Direct Cementitious Waste Option (DCWO) to HLW calcine was developed (Dafoe and Losinski 1998) to provide input to the Idaho HLW \& Facilities Disposition Environmental Impact Statement (DOE/EIS 2002). The hydroceramic grout development work at DIAL-MSU is based, in part, on the formulation and process flow sheet that was developed for the DCWO conceptual design. The DCWO process is different in that the stabilizer and calcine would be mixed as a thick paste rather than a pourable slurry, it would involve the use of kneading and extrusion operations in and from the mixer, and the curing process would require high temperature autoclaving and dewatering steps between $250-300^{\circ} \mathrm{C}$.

The processing rate used for sizing the process vessels in the DCWO conceptual design assumed all of the HLW calcine would be stabilized in five years, which is similar to the eight year processing rate period assumed in this document. Some of the features of the DCWO waste treatment facility illustrated in drawing DCWO-A-13 of the conceptual design report are (Dafoe and Losinski 1998):

- $\quad$ One $80 \mathrm{~m}^{3}$ calcine receipt vessel (4 $\mathrm{m}$ diameter by $15 \mathrm{~m}$ tall) designed to hold a two week supply of calcine feed and supply the calcine dry process lot tank

- $\quad$ One $50 \mathrm{~m}^{3}$ calcine dry process lot tank ( $3 \mathrm{~m}$ diameter by $4 \mathrm{~m}$ tall) that would:

- $\quad$ Provide a one week supply of calcine feed to the calcine batch vessels

- $\quad$ Be mechanically mixed

- $\quad$ Be sampled, analyzed, and assigned a process lot number

- $\quad$ Provide feed to the calcine batch vessels

- $\quad$ Four $1.5 \mathrm{~m}^{3}$ calcine batch vessels ( $1 \mathrm{~m}$ diameter by $2 \mathrm{~m}$ tall) sized to hold the single batch feed to the mixer

- $\quad$ Four $3.6 \mathrm{~m}^{3}$ kneader mixers ( $2 \mathrm{~m}$ wide by $2 \mathrm{~m}$ long by $3 \mathrm{~m}$ high) for mixing and extruding the stabilized waste mixture

- $\quad$ Four canister fill stations with canisters $(0.61 \mathrm{~m}$ diameter by $3 \mathrm{~m}$ high), which are fed by the kneader mixers

- $\quad$ Process vessels and tanks to add the dry stabilizer and sodium hydroxide to the kneader mixers.

The vessel sequence, sizing, and functions of the DCWO are similar to the hydroceramic grout stabilization process shown in Figure 5-1 of this document in which the calcine would be:

- $\quad$ Received by a receipt bulk vessel $\left(30 \mathrm{~m}^{3}\right)$, which holds a one week supply of feed

- $\quad$ Transferred to calcine day vessels $\left(5 \mathrm{~m}^{3}\right)$, which would hold a one day supply of feed, be mechanically mixed, be sampled and analyzed, and hold a one day supply of feed

- $\quad$ Transferred to a calcine batch vessel $\left(0.5 \mathrm{~m}^{3}\right)$, which would hold a one canister batch supply of feed 
- $\quad$ Transferred to the grout mixer $\left(2 \mathrm{~m}^{3}\right)$, which would also receive the dry and liquid stabilizer reagents for required for one canister of waste

- Transferred to the canister for curing.

The conceptual design of the DCWO waste treatment facility illustrated in drawing DCWO-A-13 indicates that the calcine process vessels and canisters would be stacked in five levels of shielded cells, which would require a building height of about $48 \mathrm{~m}(156 \mathrm{ft})$. The stacked height of the vessels and the canister without consideration of the separation by the shielded cells and piping (i.e., the sum of the vessels and canister heights cited above) would be about $27 \mathrm{~m}(88 \mathrm{ft})$. The DWCO stacked vessel height estimate is similar to that for hydroceramic grout stabilization process given in Appendix B-2 of this

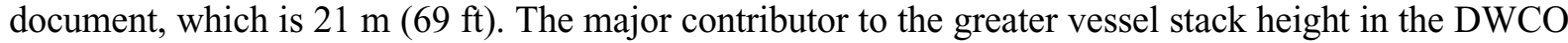
process is the use of a calcine receipt vessel to hold a two weeks supply of calcine rather that a one week supply assumed here. If the $15 \mathrm{~m}$ high vessel in the DWCO process were reduced to a one week supply vessel (i.e., reduced to a $7.5 \mathrm{~m}$ height), there would be no apparent difference between the estimates for the stacked vessel height of the DWCO conceptual design and the hydroceramic grout stabilization process described here. 


\section{GAMMA RADIATION FIELD ESTIMATES}

Several MicroShield calculations were completed to provide gamma radiation field estimates for the calcine in the grout stabilization process. MicroShield 6.10 is a radiation shielding software program that is validated for ICP/INL use. To calculate the radiation estimates, calcine and stabilizer compositions, calcine source data, vessel sizes, waste loadings, and waste densities were needed. Stabilizer compositions are given in Appendices B-1, C-1, and D-1. Calcine chemical compositions were taken from Table 6-1 in EDF-4504 (Dahl et. al. 2004). Calcine source data were taken from INEEL/EXT-98-00455 Rev. 2 (Staiger 2005). Specifically, Table A14 provided each CSSF curie activity by radionuclide decayed to January 2016 and Table A17 provided bulk density, mass, and volume for each CSSF. A spreadsheet was developed to calculate the curie concentration for the calcine in each CSSF and the average curie concentration for the total calcine inventory. In the calculations, the total radionuclide curie activity in each CSSF and the total calcine inventory was divided by the applicable calcine volume to obtain an average $\mu \mathrm{Ci} / \mathrm{m}^{3}$ or divided by the mass to obtain an average $\mathrm{Ci} / \mathrm{kg}$. Based on the calcine $\mathrm{wt} \%$ waste loading in the grout, the radioactivity for the grout was calculated in terms of $\mu \mathrm{Ci} / \mathrm{m}^{3}$. Sample calculations are provided in Appendix E as well as the source term spreadsheets. The calcine and grout source concentrations were then entered into MicroShield to estimate the radiation field from the calcine in the process vessels and in the waste canister. MicroShield uses the calcine and grout densities as the source material density. The case results are included in Appendix E. Since the vessel sizes are only preliminary estimates, the gamma radiation field results are not to be used for final design calculations. These preliminary results are provided as initial input for conceptual design.

\subsection{Process Vessel Radiation Field Estimates}

As noted in Figure 4-1 for the grout process, calcine is collected in the bulk vessel $\left(30 \mathrm{~m}^{3}\right)$ from the retrieval system. The calcine is then transferred to a day vessel $\left(5 \mathrm{~m}^{3}\right)$. Next the calcine goes to an individual batch vessel $\left(0.5 \mathrm{~m}^{3}\right)$ and then to the grout mixer $\left(2 \mathrm{~m}^{3}\right)$ where the stabilizer cements are added. Finally, the grouted calcine is dispensed to the waste canister $\left(1.1 \mathrm{~m}^{3}\right)$. The estimated dimensions for each of these process vessels are noted in Appendix B-2. For the process vessels, it was assumed the vessel will be constructed of 3/8-in. 316 stainless steel; thus, this steel provides the shielding in these calculations. Each vessel has a hopper section that is conical; however, since MicroShield can not handle multiple geometries, the vessel was treated as a complete cylinder for the height and diameter. Additionally, the vessel was considered "full" in that the entire volume was filled with source material. The waste canister was modeled as 90\% full. Table 8-1 and Figure 8-1 below show the MicroShield

Table 8-1. Gamma radiation fields (R/hr) from grouting process vessels for combined calcine. ${ }^{\mathrm{a}}$

\begin{tabular}{|c|c|c|c|c|}
\hline \multirow[b]{2}{*}{ Vessel } & \multirow[b]{2}{*}{ Material } & \multicolumn{3}{|c|}{$\mathrm{R} / \mathrm{hr}$ at Distance from Vessel Source } \\
\hline & & $2.5 \mathrm{~cm}$ & $30.5 \mathrm{~cm}$ & $100 \mathrm{~cm}$ \\
\hline Bulk & Calcine & 349.7 & 257.4 & 167.2 \\
\hline Day & Calcine & 331.5 & 215.8 & 114.4 \\
\hline Batch & Calcine & 299.9 & 145.1 & 46.3 \\
\hline Mixer & Grouted Calcine & 75.9 & 44.8 & 20.3 \\
\hline Canister & Grouted Calcine & 57.8 & 28.6 & 12.0 \\
\hline
\end{tabular}




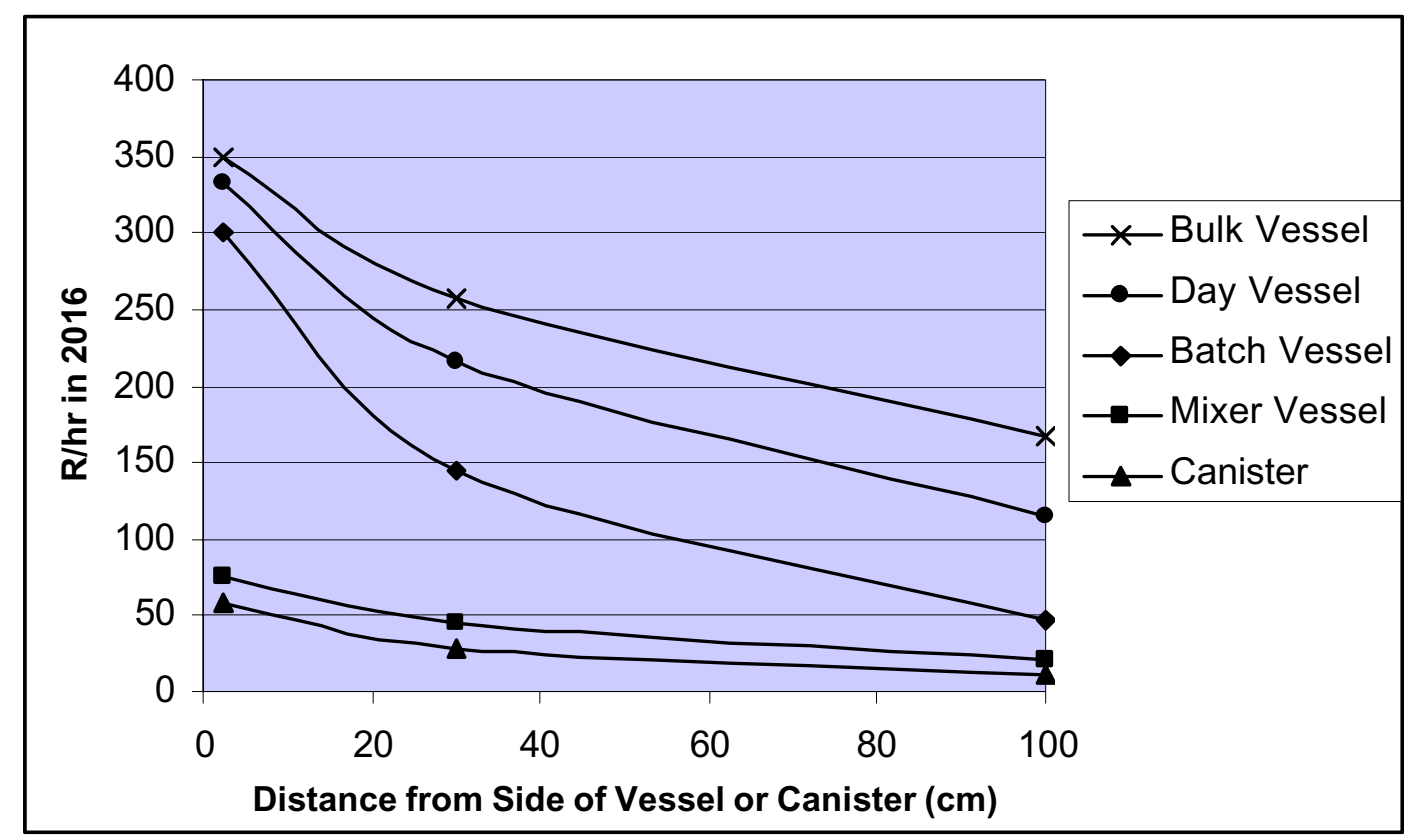

Figure 8-1. Gamma radiation field from the side of the grouting process vessels and canister for combined calcine (i.e., total HLW calcine inventory uniformly mixed).

results in terms of Roentgen per hour $(\mathrm{R} / \mathrm{hr})$ at three points from the vertical center of the vessel, specifically, $2.5 \mathrm{~cm}, 30.5 \mathrm{~cm}$, and $100 \mathrm{~cm}$ (corresponding to $1 \mathrm{in} ., 1 \mathrm{ft}$, and $1 \mathrm{~m}$ from the side of the vessel). For these calculations, the average radionuclide activities for the total HLW calcine inventory (i.e., combined calcine) were used. From Table 8-1, it is readily noted that these vessels will have high radiation fields and will need to be in shielded cells. Since various instrumentation and controls and solids handling equipment will be on or near these vessels, remote maintenance will be needed to reduce/prevent personnel exposure. Process control instrumentation may need to be radiation hardened or shielded to operate next to or near the process vessels and canister.

For comparison purposes a gamma radiation field estimate was made on the bulk calcine using $2 \mathrm{ft}$ of concrete as the vessel wall. With the concrete shielding, the radiation field dropped to milli-Roentgen (mR) levels with $7.1 \mathrm{mR} / \mathrm{hr}$ at $2.54 \mathrm{~cm}$ and $4.6 \mathrm{mR} / \mathrm{hr}$ at $1 \mathrm{~m}$. As a design consideration, perhaps the bulk vessel could be constructed as a concrete vault. The radiation field due to the bulk vessel would be greatly reduced by the concrete; however, the calcine transfer auger at the base of the vessel would still contain calcine that would add to the radiation fields.

\subsection{Waste Canister Radiation Field Estimates}

Gamma radiation field estimates were calculated for each CSSF bin set calcine. As indicated in Section 3.3, the canister is assumed to be $90 \%$ full and contain $1.0 \mathrm{~m}^{3}$ of grouted waste. Using the 24-in. diameter and the $90 \%$ grout fill, the height for the MicroShield calculations was $337.86 \mathrm{~cm}(11.1 \mathrm{ft})$. (Note: The fill portion of the $15 \mathrm{ft}$ canister height is reduced by the crush sections on the top and bottom to $12.3 \mathrm{ft}$; then, multiplied by $90 \%$ yields $11.1 \mathrm{ft}$.) For the canister, 1/2-in. 316 stainless steel was used as the material of construction and the radiation shield. The MicroShield cases are based on the grout stabilization flowsheet at $21.5 \mathrm{wt} \%$ waste loading and a waste density of $1800 \mathrm{~kg} / \mathrm{m}^{3}$. The radiation field results are given in Table 8-2 and graphed in Figure 8-2. It is readily noted, that CSSF I has the "hottest" calcine and CSSF VI the "coolest". At $2.5 \mathrm{~cm}$ the radiation field varies from a maximum of about 
$169 \mathrm{R} / \mathrm{hr}$ to a minimum of $27 \mathrm{R} / \mathrm{hr}$ with the combined calcine at $58 \mathrm{R} / \mathrm{hr}$. Remote handling will be required for the filled canisters. The case reports are included in Appendix E.

Table 8-2. Gamma radiation fields (R/hr) from calcine in waste canister grouted at $21.5 \mathrm{wt} \%$.

\begin{tabular}{|c|c|c|c|}
\hline \multirow{2}{*}{$\begin{array}{l}\text { Calcine } \\
\text { Source }\end{array}$} & \multicolumn{3}{|c|}{$\mathrm{R} / \mathrm{hr}$ at Distance from Canister } \\
\hline & $2.5 \mathrm{~cm}$ & $30.5 \mathrm{~cm}$ & $100 \mathrm{~cm}$ \\
\hline CSSF I & 169.0 & 83.5 & 35.1 \\
\hline CSSF II & 63.4 & 31.3 & 13.2 \\
\hline CSSF III & 51.1 & 25.3 & 10.6 \\
\hline CSSF IV & 64.5 & 31.9 & 13.4 \\
\hline CSSF V & 62.9 & 31.0 & 13.1 \\
\hline CSSF VI & 26.8 & 13.3 & 5.7 \\
\hline Combined $^{\mathrm{a}}$ & 57.8 & 28.6 & 12.0 \\
\hline
\end{tabular}

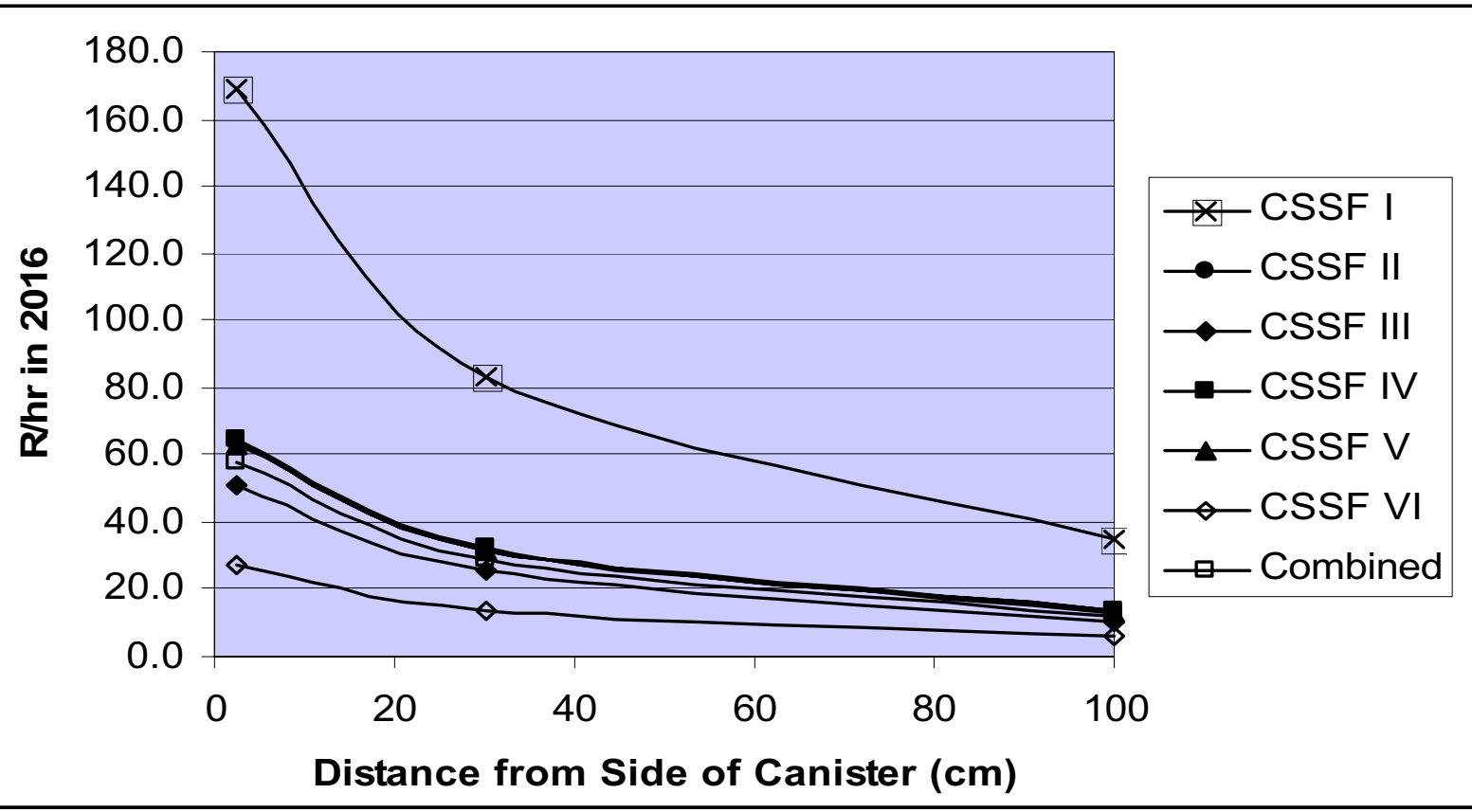

Figure 8-2. Gamma radiation fields from waste canister filled with various grouted calcines (combined calcine is defined as the total HLW calcine inventory uniformly mixed). 
An attempt was made to estimate the radiation field above the filled waste canister that would be experienced by a video camera if such a device were used to view the filling process. MicroShield is not able to model a shield with a hole in it as a canister cap with an open inlet nozzle; thus, radiation estimates were made with and without a cap. The actual results would be somewhere in between. However, if the cap has a 6-in. open hole, the results may not be much lower than the open top results. The estimates are given in Table 8-3 and Figure 8-3. The contact radiation fields between the open and closed canister are quite different (double); however, the $30.5 \mathrm{~cm}$ and $1 \mathrm{~m}$ levels are very similar. These estimates do not include the radiation from the grout mixer which would be positioned above the canister.

Table 8-3. Gamma radiation fields ( $\mathrm{R} / \mathrm{hr}$ ) from above top of waste canister.

\begin{tabular}{ccccccc}
\hline & \multicolumn{3}{c}{ R/hr with Open Top } & \multicolumn{3}{c}{ R/hr with Closed Top } \\
\cline { 2 - 7 } Material & $2.5 \mathrm{~cm}$ & $30.5 \mathrm{~cm}$ & $100 \mathrm{~cm}$ & $2.5 \mathrm{~cm}$ & $30.5 \mathrm{~cm}$ & $100 \mathrm{~cm}$ \\
\hline Grouted Calcine & 122.8 & 32.9 & 6.1 & 62.5 & 22.3 & 4.3 \\
\hline
\end{tabular}

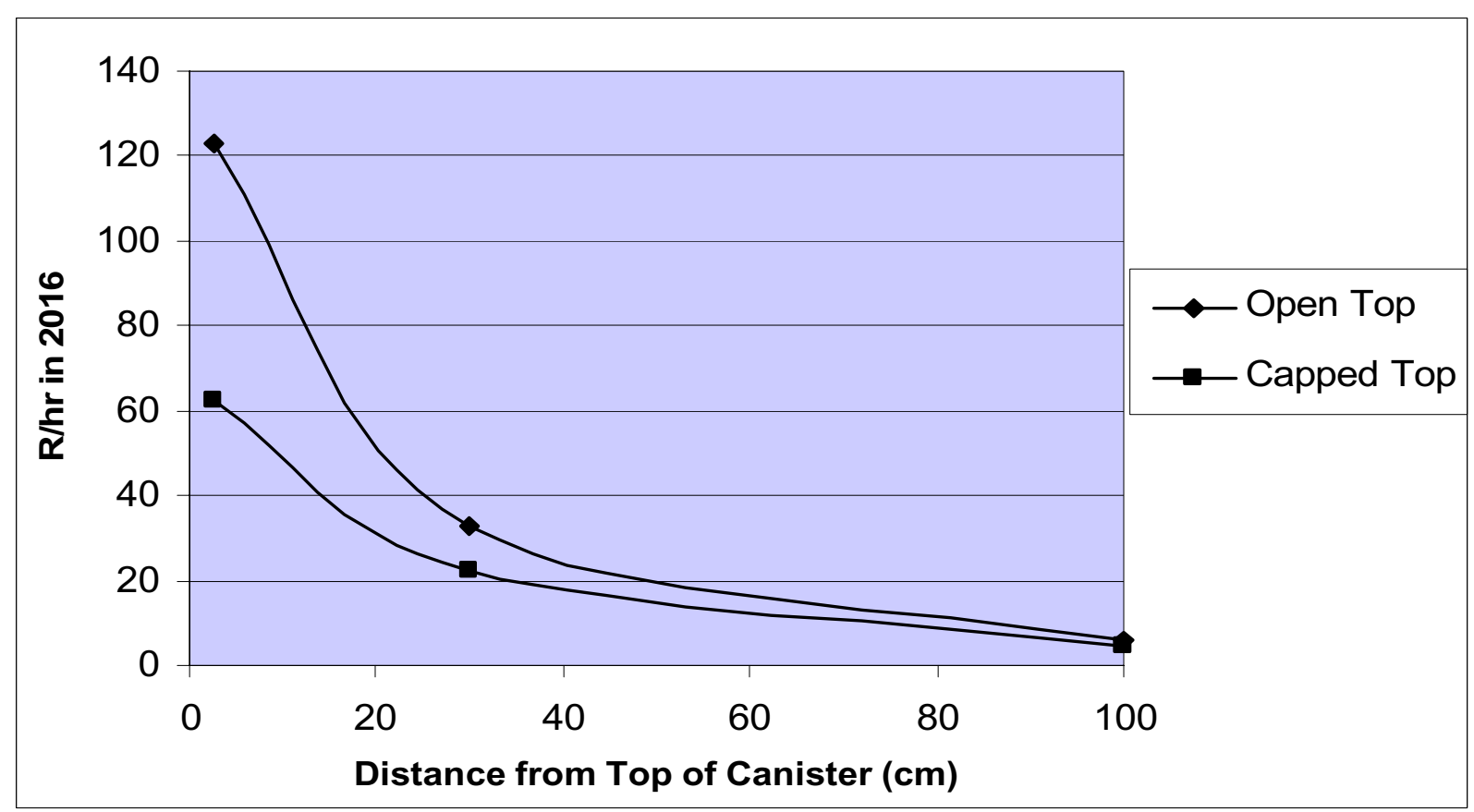

Figure 8-3. Gamma radiation fields from above a filled canister for combined calcine (i.e., the total HLW calcine inventory uniformly mixed). 


\section{CONCLUSIONS}

Based on the information presented in this EDF, the following conclusions can be made regarding the grout, hydroceramic grout, and iron phosphate ceramic stabilization processes under development:

- $\quad$ To date, the grout formulation appears to be the best candidate stabilizer among the three being tested for HLW calcine. This assessment is based on its ability to pass the compression test, TCLP and waste loading performance criteria. In addition, the formulation appears to be the easiest to mix, pour, and cure.

- Test data to date indicate that none of the stabilizers under development may be able to pass the PCT.

- $\quad$ Only minor differences would exist between the process steps of the grout and hydroceramic grout stabilization processes. If temperature control of the mixer at about $80^{\circ} \mathrm{C}$ is required, it would add a major level of complexity to the iron phosphate stabilization process. If not, only minor differences would exist among the three candidate processes up to the point of pouring the waste into a canister. If temperature control at about $80^{\circ} \mathrm{C}$ is required during the curing step of the hydroceramic grout and iron phosphate ceramic stabilization processes, it would add an additional level of process complexity also.

- It is too early in the development program to determine which stabilizer will produce the minimum amount of waste but, in this EDF, the volume is assumed to be within the range of 12,250 to $14,470 \mathrm{~m}^{3}$.

- $\quad$ The stacked vessel height of the hot process vessels in the hydroceramic grout process (i.e., $21 \mathrm{~m}$ ) appears to be about the same as that estimated the DCWO process in 1998, for which a conceptual design was developed. Therefore, some of the conceptual design efforts in the 1998 study may be applicable to the stabilizer processes addressed in this EDF.

- $\quad$ The gamma radiation fields near the process vessels handling HLW calcine would vary from a range of about 300 to $350 \mathrm{R} / \mathrm{hr}$ at a distance of $2.5 \mathrm{~cm}$ from the side of the vessels to a range of about 50 to 170 at a distance of $100 \mathrm{~cm}$ from the side of the vessels. The calculations were made for combined calcine, which was defined as the HLW calcine inventory uniformly mixed.

- $\quad$ The gamma radiation fields near the stabilized waste in canisters would range from about 25 to $170 \mathrm{R} / \mathrm{hr}$ at $2.5 \mathrm{~cm}$ from the side of the canister and 5 to $35 \mathrm{R} / \mathrm{hr}$ at $100 \mathrm{~cm}$ from the side of the canister, depending on the CSSF source of calcine. 
9-2 


\section{DESIGN ISSUES BEYOND SCOPE OF DOCUMENT}

During the review cycle of this document, several engineering questions were raised which were considered beyond the scope of this pre-conceptual design document. These questions and reasons why they were not addressed are given below. Once a stabilizer has been selected as the preferred alternative and follow-on mock-up, hot bench scale, pilot plant studies and conceptual design efforts conducted, these questions will be addressed.

- What methods would be used to avoid buildup of stabilizer residue in the grout/ceramic mixer shown in Figures 4-1 through 6-1, especially in the headspace, and what methods would be used to clean the residue? How would secondary waste streams be handled?

Response: To determine this, the stabilizer needs to be selected and the mixing properties of viscosity and set up time need to be established. If the process has a low viscosity, the liquid for the next batch could be introduced as a high pressure spray to wash down deposits. Or it may be determined that any deposit build up would become self limiting in thickness and not a problem. The amount of secondary liquid waste streams is unknown but it is anticipated they would be sent to a hold tank and recycled into the mixer as a liquid stream.

- What would be the process for filling bulk stabilizer vessels from rail cars or trucks?

Response: It is anticipated that pneumatic air lift or conveyer belt lift would be used.

- What should be done with mixer if an abrupt shutdown is required and it still contains grout?

Response: Backup or standby power must be available to avoid abrupt shutdowns and allow an orderly discharge of grout to the canister and addition of make water to the mixer for the next batch.

- What should be done to design calcine receipt lag storage capacity beyond one week into the treatment plant to avoid shut down after a given bin has been emptied? It may take much longer to move the pneumatic retrieval apparatus from bin to bin or bin set to bin set which would require that the treatment plant be shut down.

Response: Options include larger or more bulk receipt vessels or scheduling treatment plant maintenance in conjunction with relocation of pneumatic retrieval equipment. Once the treatment and pneumatic retrieval processes enter the conceptual design phase, they should be designed together with this issue in mind.

- $\quad$ Is the 6-in. radius opening on the bottom of the hoppers the correct size for adequate flow or should a different size be selected? There should be a huge amount of expertise in handling similar dry solids with lots of fines that one could draw on for sizing vessels, openings, and cone angles.

Response: Commercial vendor information should be used to design the hoppers and openings for the cold stabilizer ingredients, for which ample information should be available. However, only one study regarding the flow properties of calcine simulants in hoppers was found (Wanke 1993) and that study indicated that additional studies were needed to design the hopper. This need is noted in Section 11 on Recommendations. 
- $\quad$ Frequent sampling and analysis seems to be the wrong way to deal with calcine. The process should have a recipe that envelops the major types of calcine that will be encountered in the process so one does not have to sample. Sampling means mixing to get uniformity which is another major complication to be avoided if possible.

Response: The treatment process should be designed to be as generic as possible for all types of major calcines. The need for sampling and analysis should be determined during the conceptual design phase. No attempt was made to resolve the need for sampling and analysis in this document other than to show where sampling capabilities might be built into the process design.

- $\quad$ The use of desiccants to absorb bleed water from the waste form in a canister will not be acceptable unless they are stable at repository temperatures and if radiolysis of the water in the desiccant and stabilizer will be a problem. This needs to be determined before any stabilizer using water is selected. Drying may be required to get the residual water content low enough to avoid excessive radiolysis and pressurization of the waste canister.

Response: The objective is to develop a stabilizer formulation that does not bleed water. The comment with regard to the use of a desiccant was stated as a potential solution to an off-normal process event. The effect of high temperature storage will be tested using waste forms with calcine simulants and a literature review of studies on radiolysis is planned. Radiolysis and container pressurization at repository temperatures are issues that require the stabilization of actual radioactive waste calcine, which are planned for the hot bench scale studies.

- Why didn't this document address detailed operating scenarios for the process steps, determine the credible failures that could occur at each step, then determined the desired prevention or mitigation method for each failure mode?

Response: It is too early in the stabilizer development process to address design information at this level of detail. A stabilizer should be developed to the point that it is down selected as the preferred treatment alternative and the issues addressed during the conceptual design stage.

- How would it be determined if out-of-spec grout has been produced and, if it is, how would the grouted waste be recycled and reprocessed?

Response: Periodic grout sampling as it is being poured was proposed as a method to check for out-of-spec grout. Definitions for what is considered out-of-spec and provisions for recycling the waste as feed to the grout mixer would be addressed during the conceptual design phase.

- Is a coupling device needed between the grout mixer and the canister for the pouring operation? The risk of contamination spread should be small if the fill nozzle is a short distance above the canister opening. This would avoid the remote operations complexity to mate a coupling or insert a tapered nozzle into the canister.

Response: As indicated in Section 4.3.5, the coupling mechanism of the pour spout to the canister is still undefined. This issue would be addressed during the conceptual design phase, especially with regard to how contamination free the canister throat and top needs to be for the curing period and the capping operation. 
- $\quad$ Taking samples of the grouted waste product should be avoided if at all possible. Qualification of the waste form should be done by qualifying the stabilizer recipe.

Response: Sampling requirements for verification that the stabilized waste form meets product specifications is an issue that will have to be negotiated and resolved with the regulatory acceptance agency. Given this may occur long after a conceptual design is issued and the plant is built, it would be a good contingency plan to build the product sampling capability into the treatment plant as was done for the Defense Waste Treatment Plant at the Savannah River Site.

- If the hopper opening or auger becomes blocked, how will the solids vessel be emptied? What about seismic safety issues associated with stacking process vessels, especially those containing HLW calcine?

Response: These issues will be addressed during the conceptual design phase.

- Vessel off-gas dust control systems and secondary wastes generated seem to be significant process issues that were not addressed in this document. What kind of off-gas control systems would be required?

Response: It is anticipated that a small dry gas purge will be required in all the solids vessels to keep the materials dry and free flowing. This comment was made in Sections 4.2, 5.2, and 6.2. It is also anticipated that vessel off-gas would be vented through HEPA filters for dust control. Detailed consideration of the off-gas issue and secondary waste generated will be addressed during the conceptual design phase. 
10-4 


\section{RECOMMENDATIONS}

Based on the information presented in this EDF, the following recommendations can be made regarding the grout, hydroceramic grout, and iron phosphate ceramic stabilization processes under development:

- $\quad$ A value engineering session and cost benefit study should be done to determine if redundant process vessel trains would be needed. Due to the nature of remote operations and maintenance, a second process train may be needed to provide a greater certainty that production goals would be met in case process upsets were to occur.

- $\quad$ Pre-blended dry stabilizer ingredients should be used where possible to reduce the number of process vessels that would be required to deliver the cold ingredients (i.e., the number of bulk, day, and batch vessels required) to the mixer. In the case of the grout process, which uses three ingredients, a pre-blended mix would reduce the number of cold process vessels for the dry solids from nine to three.

- A minimum of two day vessels for the HLW calcine would be required to allow one to undergo sampling and analysis while the other is feeding the grout mixer. An assessment of analytical turn around time for sample analysis should be done to determine the number of day vessels that will be required. The waste treatment plant should have a dedicated hot laboratory for required analytical services.

- $\quad$ Single batch vessels for solids and liquids should be used in the process vessel train to prevent overfilling the mixer and to better control the formulation for the HLW calcine and stabilizer ingredients.

- $\quad$ Pilot-scale studies with automated process steps are needed to test mixer designs and to demonstrate process control during the addition of ingredients to the mixer, the mixing process, and transfer of the mix to the canister.

- $\quad$ As a starting point for gravity feed hopper design, a critical friction angle of $20^{\circ}$ should be used for the calcine hoppers. However, this friction angle does not take into consideration the effects of fines on the calcine flow properties and additional testing should be done to determine this effect. 
11-2 


\section{REFERENCES}

Aloy, A. S., Kovarskaya, E. N., Kotsova, T. I., Marcheret, Y., Medvedev, P. G., and Todd, T. A., 2000, Iron Phosphate Ceramics for Solidification of Mixed Low-Level Waste, United States Patent \# 6,075,176, June 13, 2000.

Autocon Continuous Processing Systems, 2005, 2360 Vallejo Street, St. Helena, CA 94574, www.autoconsystems.com (visited March 21, 2005).

Bibler, N. E., Ray, J. W., Fellinger, T. L., Hodoh, O. B., Beck, R. S., and Lien, O. G., 1998, "Characterization of the Radioactive Glass Currently being Produced by the Defense Waste Processing Facility at the Savannah River Site", Proceedings of Waste Management 98, Session 14, paper 04, Tucson, Arizona, March 1-5, 1998.

Charles Ross \& Son Co, 2005, 710 Old Willets Path, Hauppauge, NY 11788, www.mixers.com (visted March 21, 2005).

Dahl, C. A., Frazee, C. M., Loo, H. H., Beck, J. T., and Tisdale, W. S., 2004, A Summary of Properties Used to Evaluate INEEL Calcine Disposal in the Yucca Mountain Repository, EDF-4504, March 10, 2004.

Dafoe, R. E. and Losinski, S. J., 1998, Direct Cementitious Waste Option Study Report, INEEL/EXT-97-01399, February 1998.

DOE/EIS, 2002, Idaho High-Level Waste \& Facilities Disposition Final Environmental Impact Statement, DOE/EIS-0287, September 2002.

DOE/ID, 2002, Environmental Management Performance Management Plan for Accelerating Cleanup of the Idaho National Engineering and Environmental Laboratory, DOE/ID-11006, July 2002.

DOE/EM, 1996, U. S. Department of Energy Office of Environmental Waste Management, Waste Acceptance Product Specifications for Vitrified High-Level Waste Forms, EM-WAPS Rev. 02, December 1996.

DOE/NE-ID, 2005, draft Mission Need Statement: Calcine Disposition Project, Major Systems Acquisition Product, draft DOE/NE-ID-XXXXX, January 2005.

DOE/RW, 2002, Office of Civilian Radioactive Waste Management, Civilian Radioactive Waste Management System Waste Acceptance System Requirements Document, DOE/RW-0351, Revision 4, January 2002.

Frei, M. W., DOE/ID Memorandum to J. H. Roberson, DOE/HQ, January 17, 2002, "Path Forward for Idaho High-Level Waste and Facilities Disposition Final Environmental Impact Statement", CCN 29211 (EDMS Reference Number: HLW-103-877).

Herbst, A. K., 1997, "Optimization of Hydraulic Cement Admixture Waste Forms for Sodium-Bearing, High Aluminum, and High Zirconia Waste," Proceedings of the $18^{\text {th }}$ U.S. DOE Low-Level Radioactive Waste Management Conference, Salt Lake City, May 20-22, 1997.

Herbst, A. K., Marshall, D. W. and McCray, J. A., 1998, ICPP Low-Activity Waste Grout Stabilization Development Program FY-97 Status Report, INEEL/EXT-98-00116, February 1998. 
Medvedev, P., Johnson, S. G., Frank, S. M. and Macheret, Y., 1998, "Iron Phosphate Ceramic as a Candidate Encapsulate Wasteform for Transuranic Waste: A Preliminary Study with an Emphasis on Setting Kinetics and Waste Loading", Radioactive Waste Management and Environmental Research, pp. 259-278, November 1998.

OMEGA, 1992a, The Flow and Level Handbook, Vol 28, OMEGA Engineering Inc, 1992.

OMEGA, 1992b, The Pressure, Strain and Force Handbook, Vol 28, OMEGA Engineering Inc, 1992.

PLN-1144, 2002, Calcine Waste Form and Packaging Requirement, December 5, 2002.

Plodinec, M. J., Giordana, A. Bricka, M., Leone, T., Beck, J. T., Thomas, T. R., and Tisdale, W. S., 2004, DIAL/MSU Test Plan to Develop Stabilizers for High Level Waste Calcine, DIAL/ICP-SP3-TP-001, May 18, 2004 (EDMS Job Id 1593908).

Rahl, T. E., 2003, FY 2003 Conceptual Design Effort for the High Level Waste Disposal Canister, EDF-4096, August 21, 2003.

Readco Manufacturing, Inc., 2005, 901 S. Richland Ave., York, PA 17405, www.readco.com, (visited March 21, 2005).

Siemer, D. D., 2002 "Performance of Hydroceramic Concretes on Radwaste Leach Tests", Ceramic Transactions, Vol 132, pp 369-373, 2002.

Siemer, D. D., Roy D. M., Grutzeck, M. W. and Scheetz, B. E., 2002 "Cementitious Solidification of DOE's Legacy Reprocessing Waste", Proceedings of the $9^{\text {th }}$ Biennial International Spectrum Conference, Reno, NV, August 4-8, 2002.

Staiger, M. D. and Swenson, M. C., 2005, Calcined Waste Storage at the Idaho Nuclear Technology and Engineering Center, INEEL/EXT-98-00455, Rev. 2, January 2005.

Thomas, K. A., 2005, Cost Comparison for the Transfer of Select Calcined Waste Canisters to the Monitored Geologic Repository at Yucca Mountain, Nevada, ICP/EXT-05-00824, March 2005.

Thomas, T. R., 2004, Technology Development Needs for the Calcine Disposition Project, EDF-5107, August 16, 2004.

Thomas, T. R. and Childs, K. F., 2004, Down Selection of Candidate Technologies for Calcine Stabilization Studies, EDF-4753, April 21, 2004.

Thomas, T. R., 2005, An Assessment of Phase 1 DIAL-MSU Data on the Development of Stabilizers for HLW Calcine, BBWI letter to J. T. Beck, Trt-2-05, April 28, 2005.

Wanke., C. S., 1993, Summary of Calcine Product Flow Properties, Westinghouse Idaho Nuclear Company letter to J. D. Herzog, Wan-1-93, January 26, 1993 (EDMS Id; HLW-REF-5152/WAN-1-93). 
Appendix A

Method for Sizing Funnel Flow Hoppers 


$$
\text { A-2 }
$$




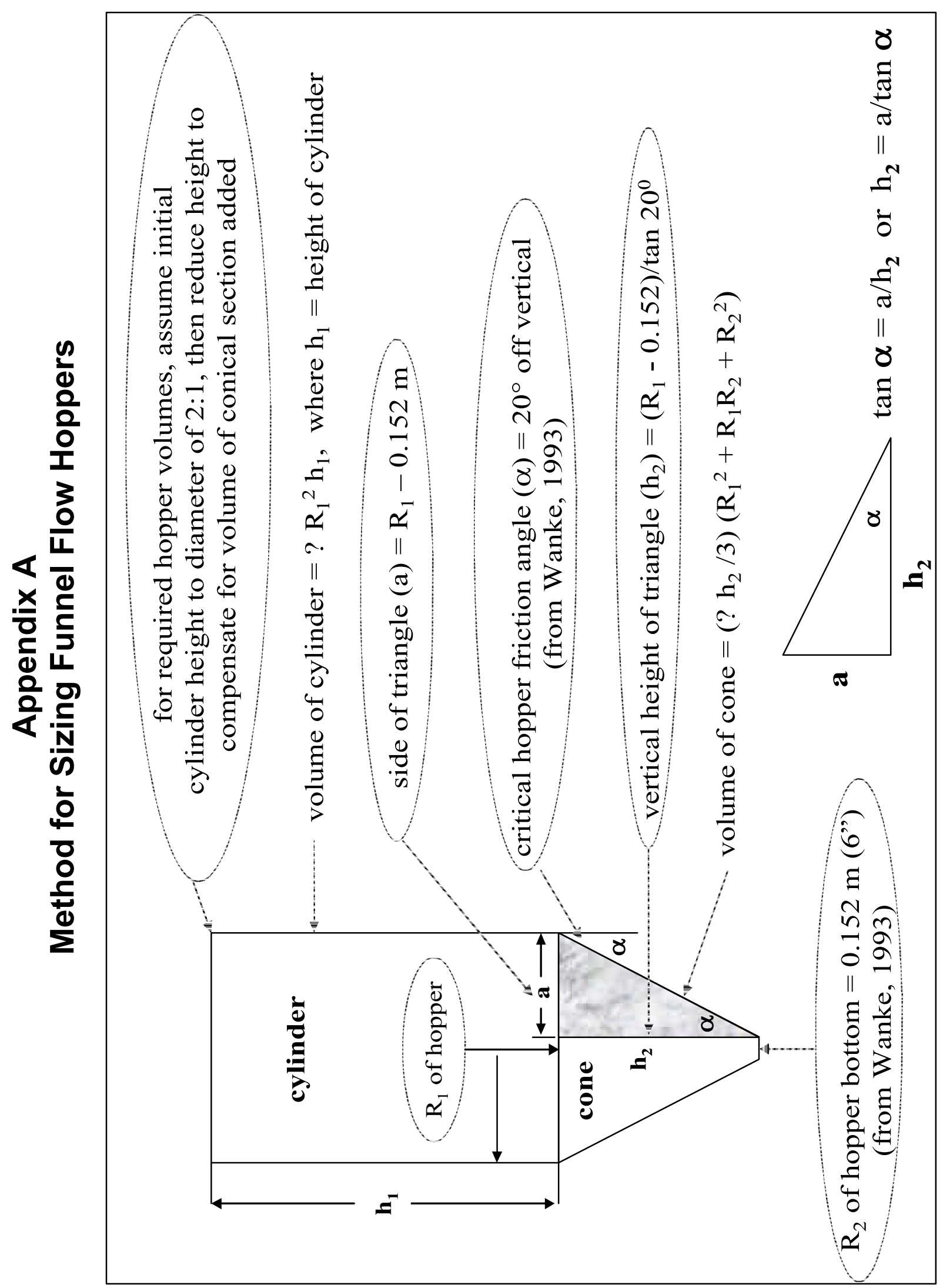


Appendix B-1

\section{Mass Flow Sheet Calculations for the Grout Stabilization Process}




$$
\text { B-1-2 }
$$




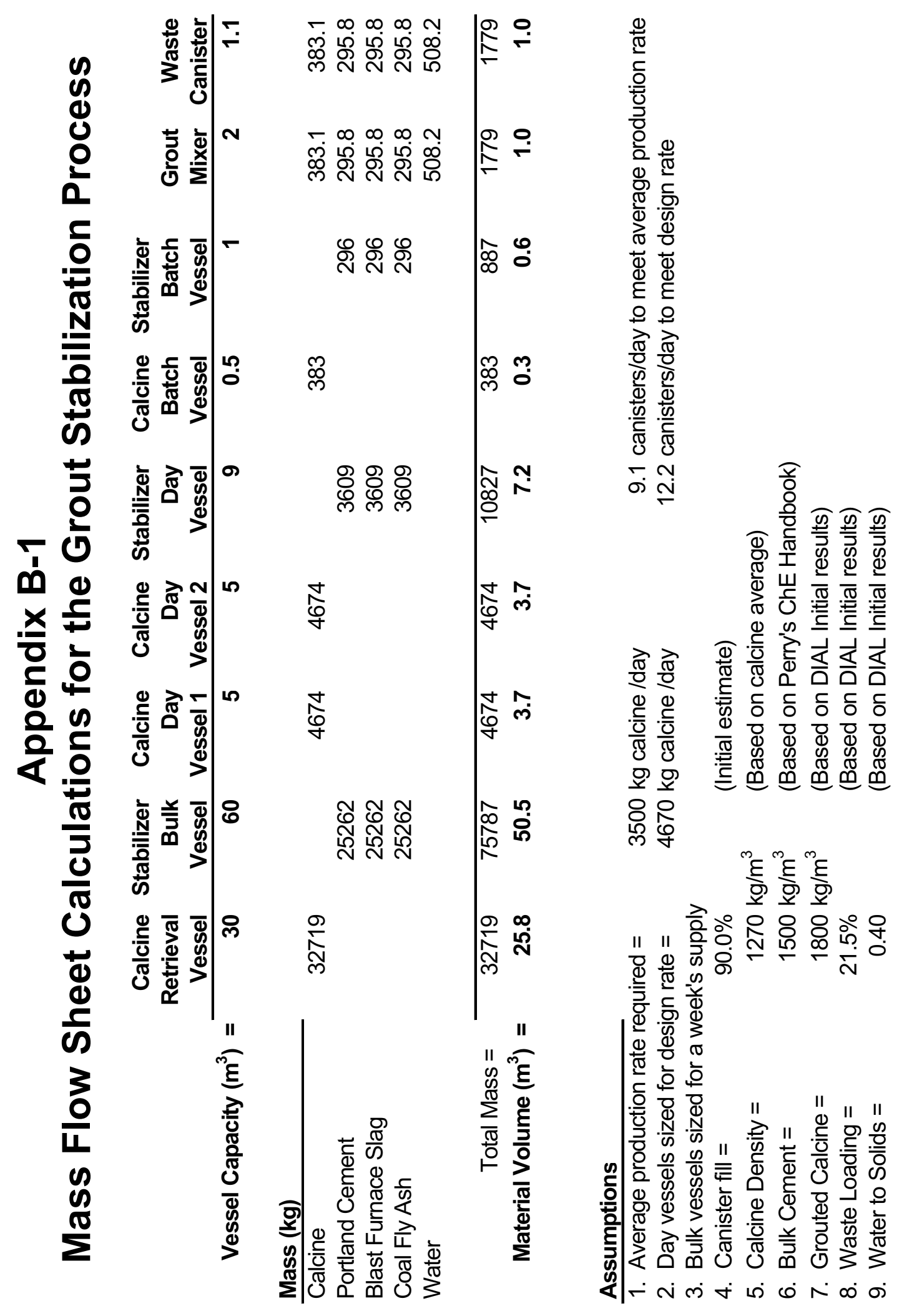


B-1-4 


\section{Appendix B-2}

\section{Estimated Process Vessel Sizes for Grout Stabilization Process}




$$
\text { B-2-2 }
$$




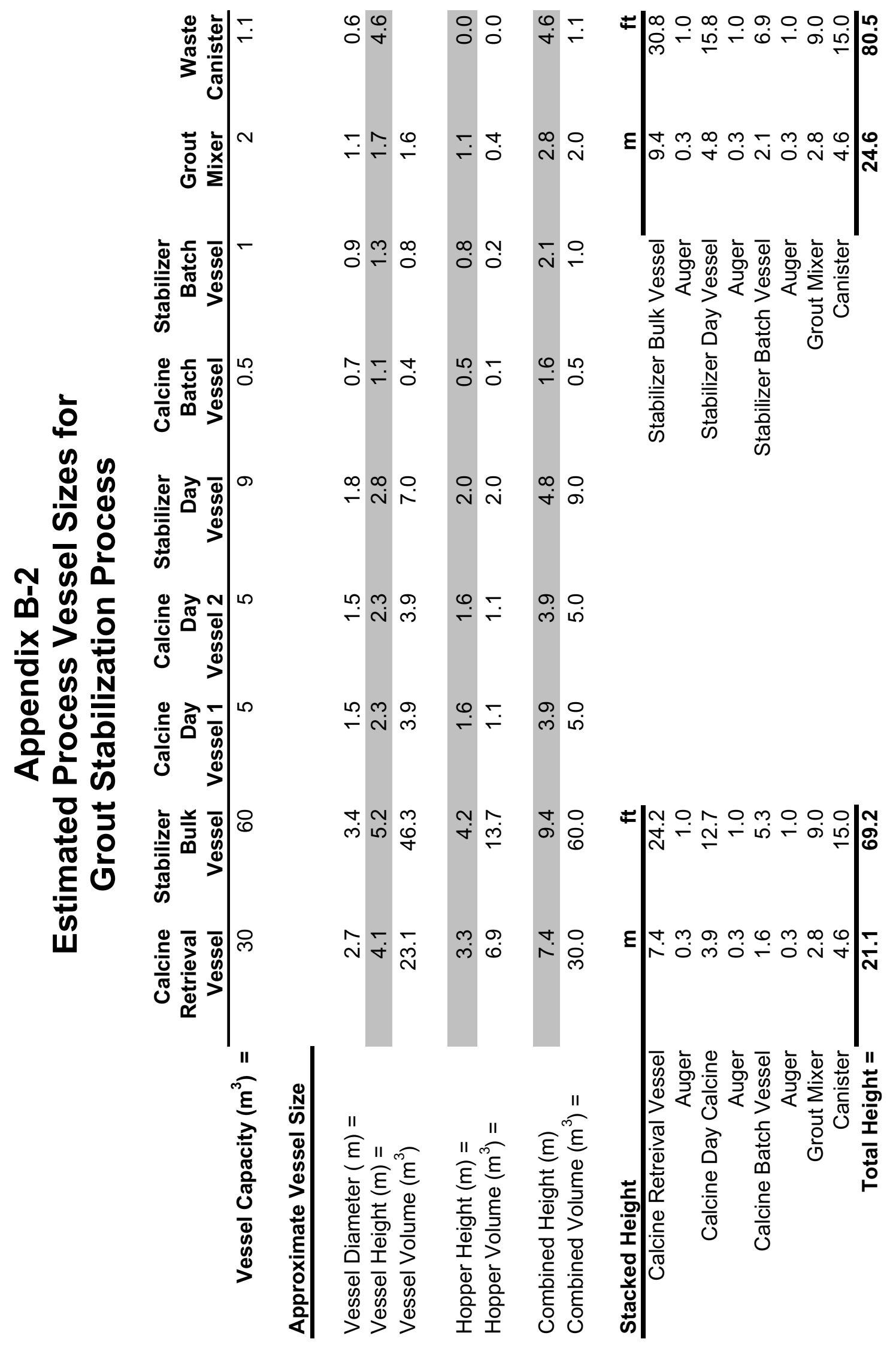


B-2-4 


\begin{abstract}
Appendix C-1
Mass Flow Sheet Calculations for the Hydroceramic Grout Stabilization Process
\end{abstract}


C-1-2 


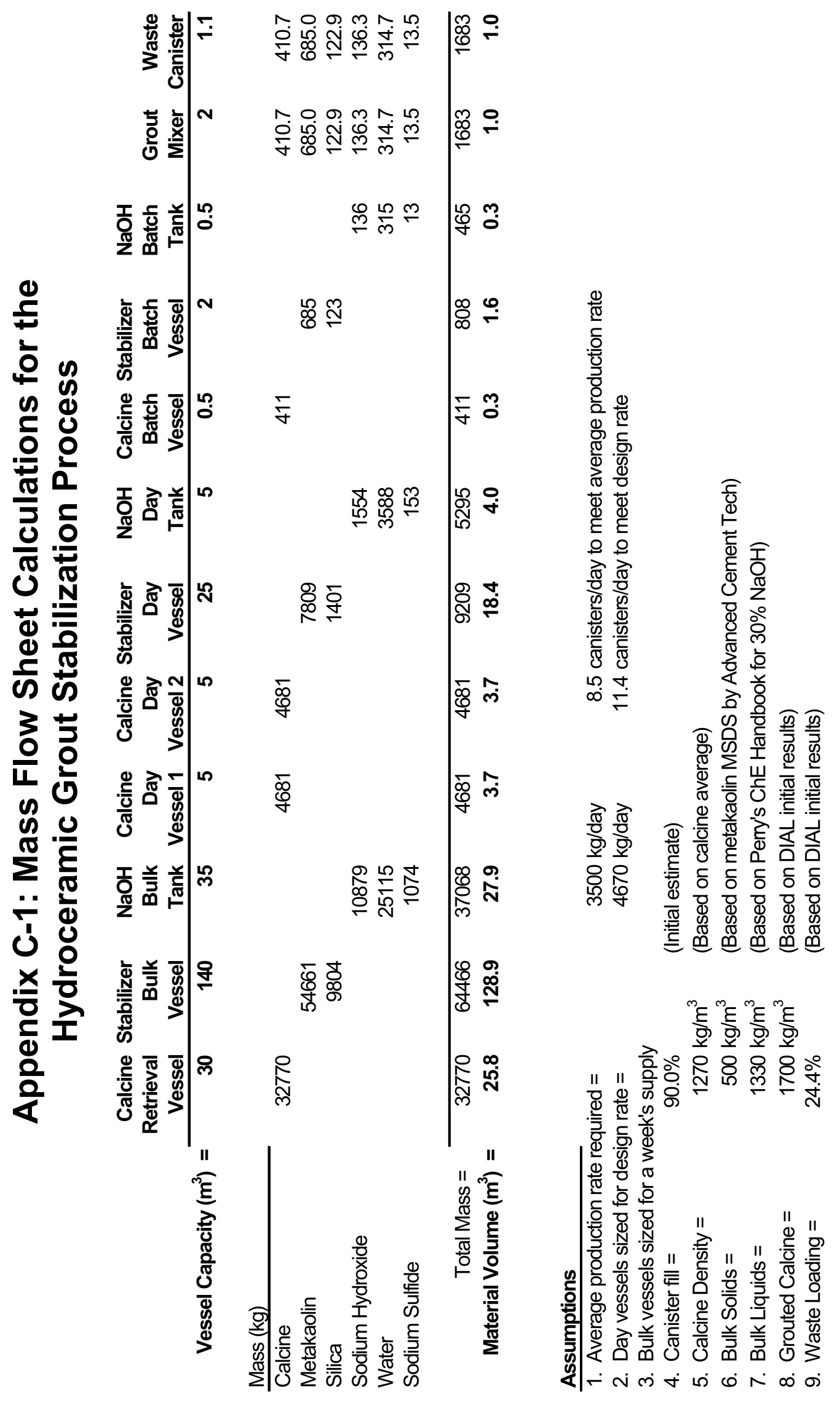


C-1-4 


\begin{abstract}
Appendix C-2
Estimated Process Vessel Sized for the Hydroceramic Grout Stabilization Process
\end{abstract}


C-2-2 


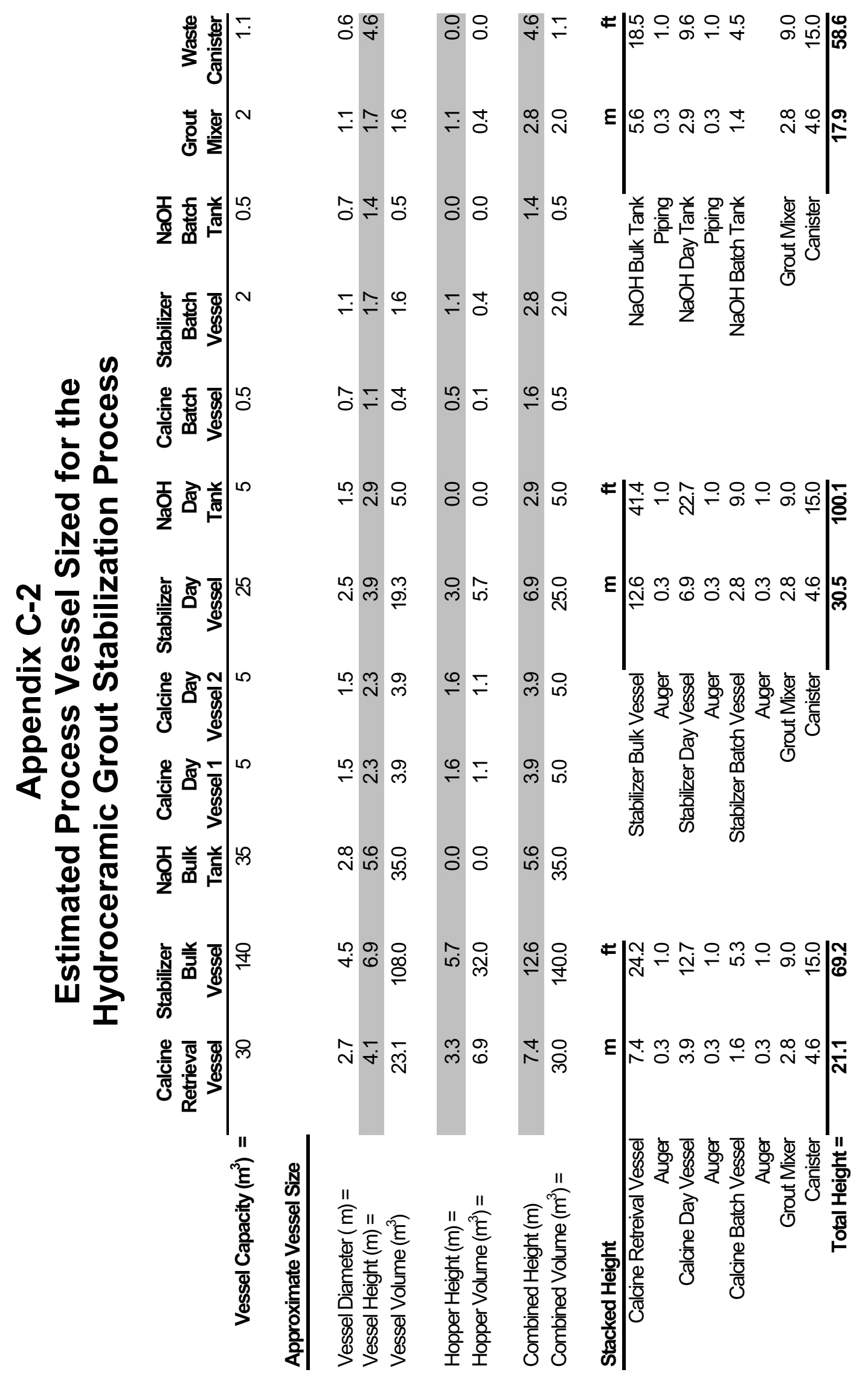


C-2-4 


\section{Appendix D-1}

Mass Flow Sheet Calculations for the Iron Phosphate Ceramic Grout Stabilization Process 
D-1-2 


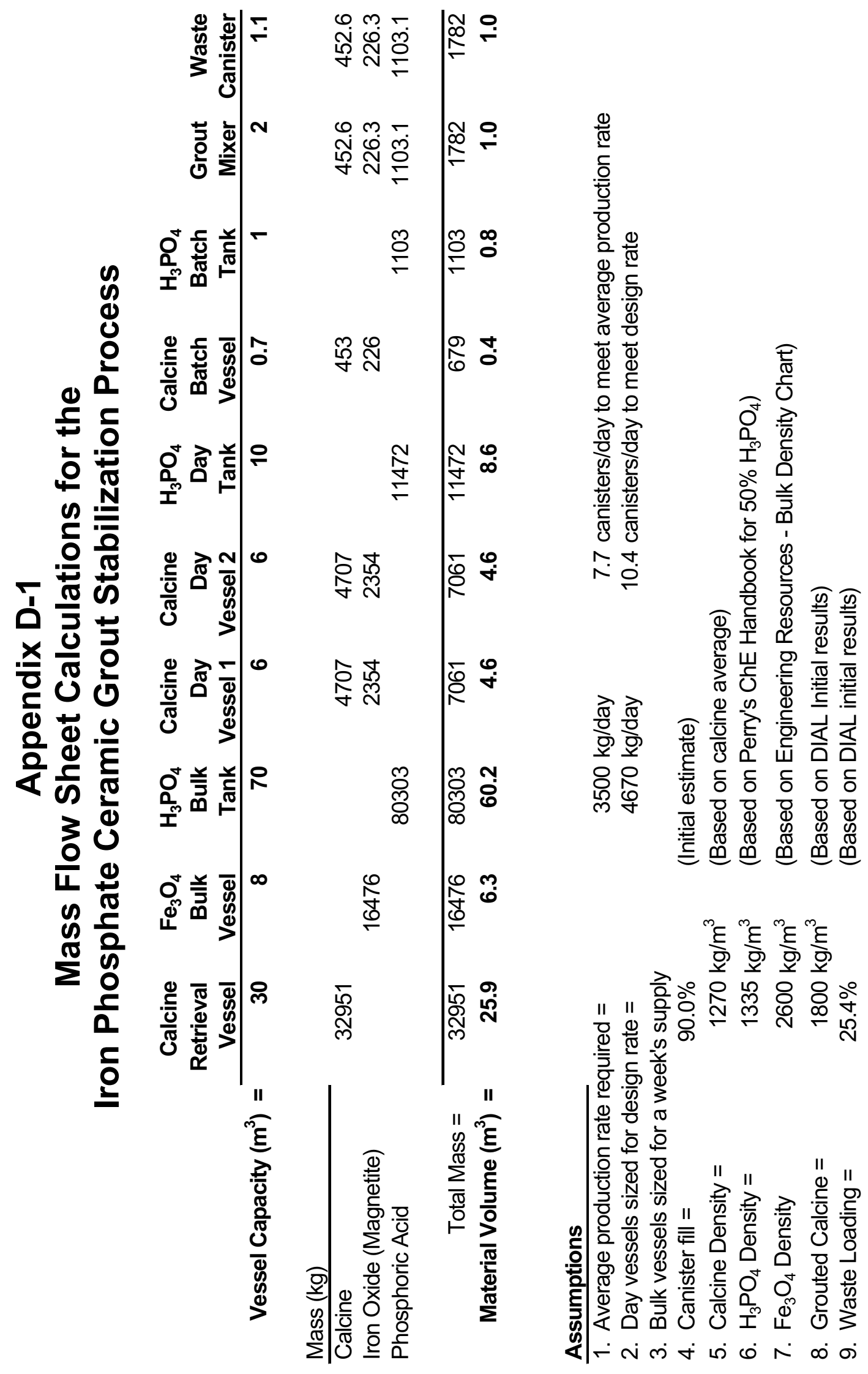




$$
\text { D-2-4 }
$$




\begin{abstract}
Appendix D-2
Estimated Process Vessel Sizes for the Iron Phosphate Ceramic Grout Stabilization Process
\end{abstract}




$$
\text { D-2-2 }
$$




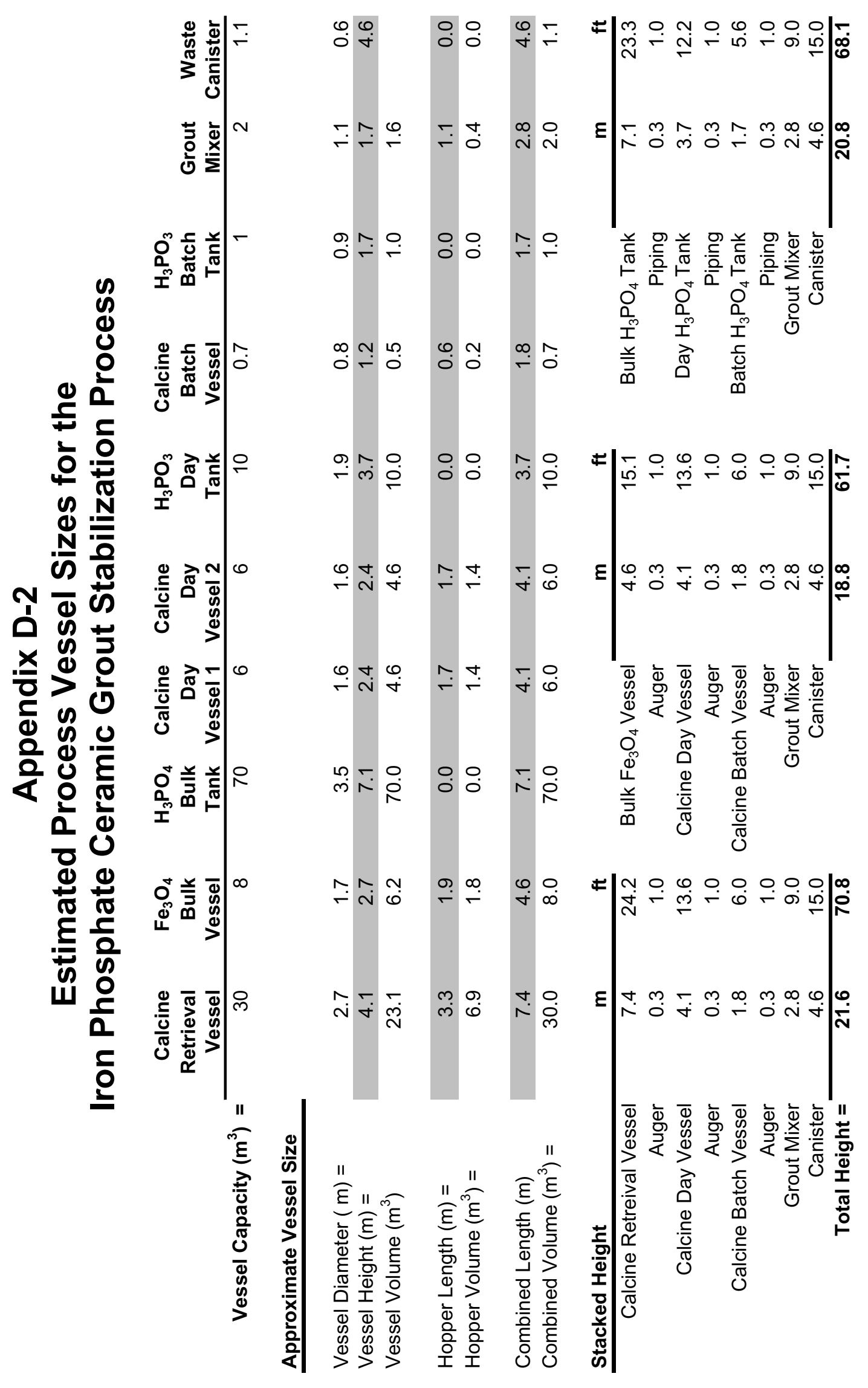




$$
\text { D-2-4 }
$$




\section{Appendix E \\ MicroShield Calculations for Gamma Field Radiation Estimate}


E-2 


\section{SAMPLE CALCULATIONS}

Curie Concentration in Calcine and Calcine Grout

Used in MicroShield Dose Calculations

Example: CSSF 1, Am-241

Table A14 lists Am-241 at 7.15E+01 curies for Bin Set 1 in January 2016

Table A17 lists CSSF 1 volume as $2.22 \mathrm{E}+05$ liters $=222 \mathrm{~m}^{3}$

Table A17 lists CSSF 1 mass as $1.83 \mathrm{E}+05 \mathrm{~kg}$

From DIAL work grout waste loading is $21.5 \mathrm{wt} \%$

From DIAL work grout density is $1.80 \mathrm{~g} / \mathrm{cm}^{3}=1800 \mathrm{~kg} / \mathrm{m}^{3}$

Usable canister volume $=66860 \mathrm{in}^{3}$ with 12 in radius

Canister filled to $90 \%$

Curie Concentration in Calcine:

$(7.15 \mathrm{E}+01 \mathrm{Ci})\left(10^{6} \mu \mathrm{Ci} / \mathrm{Ci}\right)=3.22 \mathrm{E}-01 \mu \mathrm{Ci} / \mathrm{cm}^{3}$

$\left(222 \mathrm{~m}^{3}\right)\left(10^{6} \mathrm{~cm}^{3} / \mathrm{m}^{3}\right)$

Curie Concentration in Grout:

$(7.15 \mathrm{E}+01 \mathrm{Ci})(21.5 \mathrm{~kg}$ calcine $/ 100 \mathrm{~kg}$ grout $)\left(1800 \mathrm{~kg}\right.$ grout $\left./ \mathrm{m}^{3}\right)\left(10^{6} \mu \mathrm{Ci} / \mathrm{Ci}\right)=1.51 \mathrm{E}-1 \mu \mathrm{Ci} / \mathrm{cm}^{3}$ $(1.83 \mathrm{E}+05 \mathrm{~kg}$ calcine $)\left(10^{6} \mathrm{~cm}^{3} / \mathrm{m}^{3}\right)$

90\% Canister Fill Level:

$\mathrm{Lf}=\left(\underline{\left.66860 \mathrm{in}^{3}\right)(0.90)}=133.01 \mathrm{in}=337.86 \mathrm{~cm}\right.$

$(3.14)(12 \text { in })^{2}$ 
CSSF 1 Calcine Source Term

Based on INEEL/EXT-98-00455 Rev. 2

Table A14 and Table A17

$\begin{array}{rc}\text { CSSF 1 Calcine Volume }= & 222 \mathrm{~m}^{3} \\ \text { Calcine Mass }= & 1.83 \mathrm{E}+05 \mathrm{~kg} \\ \text { Grout Waste Loading }= & 21.50 \% \\ \text { Grout Waste Density }= & 1800 \mathrm{~kg} / \mathrm{m}^{3}\end{array}$

\begin{tabular}{|c|c|c|c|c|c|c|c|}
\hline Nuclide & $\begin{array}{r}\text { Year } 2016 \\
\text { Curies }\end{array}$ & $\begin{array}{r}\text { Calcine } \\
\mathrm{Ci} / \mathrm{m}^{3}\end{array}$ & $\begin{array}{l}\text { Calcine } \\
\mathrm{uCi} / \mathrm{cm}^{3}\end{array}$ & $\begin{array}{r}\text { Calcine } \\
\text { Ci } / \mathrm{kg}\end{array}$ & $\begin{array}{l}\text { Grout } \\
\mathrm{Ci} / \mathrm{kg}\end{array}$ & $\begin{array}{l}\text { Grout } \\
\mathrm{Ci} / \mathrm{m}^{3}\end{array}$ & $\begin{array}{r}\text { Grout } \\
\mathrm{uCi} / \mathrm{cm}^{3}\end{array}$ \\
\hline Am-241 & $7.15 \mathrm{E}+01$ & 3.22E-01 & 3.22E-01 & 3.91E-04 & 8.40E-05 & $1.51 \mathrm{E}-01$ & $1.51 \mathrm{E}-01$ \\
\hline Am-243 & 8.68E-03 & 3.91E-05 & 3.91E-05 & 4.74E-08 & 1.02E-08 & 1.84E-05 & $1.84 \mathrm{E}-05$ \\
\hline Ba-137m & $7.68 \mathrm{E}+05$ & $3.46 \mathrm{E}+03$ & $3.46 \mathrm{E}+03$ & $4.20 \mathrm{E}+00$ & $9.02 \mathrm{E}-01$ & $1.62 E+03$ & $1.62 \mathrm{E}+03$ \\
\hline Ce-144 & $3.74 \mathrm{E}-14$ & $1.68 \mathrm{E}-16$ & $1.68 \mathrm{E}-16$ & 2.04E-19 & 4.39E-20 & 7.91E-17 & $7.91 \mathrm{E}-17$ \\
\hline $\mathrm{Cm}-242$ & 7.24E-03 & 3.26E-05 & 3.26E-05 & 3.96E-08 & $8.51 \mathrm{E}-09$ & $1.53 \mathrm{E}-05$ & $1.53 \mathrm{E}-05$ \\
\hline $\mathrm{Cm}-244$ & 1.36E-02 & $6.13 E-05$ & $6.13 \mathrm{E}-05$ & 7.43E-08 & $1.60 \mathrm{E}-08$ & 2.88E-05 & $2.88 \mathrm{E}-05$ \\
\hline Co-60 & 3.83E-01 & 1.73E-03 & 1.73E-03 & 2.09E-06 & 4.50E-07 & 8.10E-04 & 8.10E-04 \\
\hline Cs-134 & $5.12 \mathrm{E}-03$ & 2.31E-05 & 2.31E-05 & $2.80 \mathrm{E}-08$ & $6.02 \mathrm{E}-09$ & $1.08 \mathrm{E}-05$ & $1.08 \mathrm{E}-05$ \\
\hline Cs-135 & $1.08 \mathrm{E}+01$ & 4.86E-02 & 4.86E-02 & 5.90E-05 & 1.27E-05 & $2.28 \mathrm{E}-02$ & $2.28 \mathrm{E}-02$ \\
\hline Cs-137 & $8.12 \mathrm{E}+05$ & $3.66 E+03$ & $3.66 E+03$ & $4.44 \mathrm{E}+00$ & 9.54E-01 & $1.72 E+03$ & $1.72 \mathrm{E}+03$ \\
\hline Eu-152 & 7.00E+00 & 3.15E-02 & 3.15E-02 & 3.83E-05 & 8.22E-06 & 1.48E-02 & 1.48E-02 \\
\hline Eu-154 & $4.33 E+02$ & $1.95 E+00$ & $1.95 E+00$ & $2.37 \mathrm{E}-03$ & $5.09 \mathrm{E}-04$ & $9.16 \mathrm{E}-01$ & $9.16 \mathrm{E}-01$ \\
\hline Eu-155 & $2.26 \mathrm{E}+01$ & 1.02E-01 & 1.02E-01 & $1.23 \mathrm{E}-04$ & 2.66E-05 & 4.78E-02 & 4.78E-02 \\
\hline I-129 & 8.97E-02 & 4.04E-04 & 4.04E-04 & 4.90E-07 & 1.05E-07 & $1.90 \mathrm{E}-04$ & 1.90E-04 \\
\hline $\mathrm{Ni}-63$ & $0.00 \mathrm{E}+00$ & $0.00 \mathrm{E}+00$ & $0.00 \mathrm{E}+00$ & $0.00 \mathrm{E}+00$ & $0.00 \mathrm{E}+00$ & $0.00 E+00$ & $0.00 \mathrm{E}+00$ \\
\hline $\mathrm{Np}-237$ & $1.09 \mathrm{E}+00$ & 4.91E-03 & 4.91E-03 & 5.96E-06 & $1.28 \mathrm{E}-06$ & $2.31 \mathrm{E}-03$ & 2.31E-03 \\
\hline $\mathrm{Pa}-233$ & 1.09E+00 & 4.91E-03 & 4.91E-03 & 5.96E-06 & 1.28E-06 & 2.31E-03 & 2.31E-03 \\
\hline $\mathrm{Pm}-147$ & $2.98 \mathrm{E}+00$ & 1.34E-02 & 1.34E-02 & 1.63E-05 & 3.50E-06 & 6.30E-03 & 6.30E-03 \\
\hline Pr-144 & 3.74E-14 & $1.68 \mathrm{E}-16$ & $1.68 \mathrm{E}-16$ & 2.04E-19 & 4.39E-20 & 7.91E-17 & $7.91 \mathrm{E}-17$ \\
\hline Pu-238 & $3.17 \mathrm{E}+02$ & $1.43 E+00$ & $1.43 \mathrm{E}+00$ & $1.73 \mathrm{E}-03$ & 3.72E-04 & $6.70 \mathrm{E}-01$ & $6.70 \mathrm{E}-01$ \\
\hline Pu-239 & $4.28 \mathrm{E}+01$ & 1.93E-01 & 1.93E-01 & 2.34E-04 & 5.03E-05 & $9.05 \mathrm{E}-02$ & $9.05 E-02$ \\
\hline Pu-240 & $1.71 \mathrm{E}+01$ & 7.70E-02 & 7.70E-02 & $9.34 \mathrm{E}-05$ & 2.01E-05 & $3.62 \mathrm{E}-02$ & $3.62 \mathrm{E}-02$ \\
\hline Pu-241 & 1.19E+02 & 5.36E-01 & 5.36E-01 & $6.50 \mathrm{E}-04$ & 1.40E-04 & 2.52E-01 & 2.52E-01 \\
\hline Pu-242 & 9.89E-03 & 4.45E-05 & 4.45E-05 & 5.40E-08 & 1.16E-08 & 2.09E-05 & $2.09 \mathrm{E}-05$ \\
\hline Ru-106 & $1.90 \mathrm{E}-10$ & 8.56E-13 & 8.56E-13 & $1.04 \mathrm{E}-15$ & $2.23 \mathrm{E}-16$ & $4.02 \mathrm{E}-13$ & $4.02 E-13$ \\
\hline Sb-125 & 1.22E-01 & $5.50 \mathrm{E}-04$ & $5.50 \mathrm{E}-04$ & 6.67E-07 & $1.43 \mathrm{E}-07$ & 2.58E-04 & $2.58 \mathrm{E}-04$ \\
\hline Sb-126 & $1.54 \mathrm{E}+00$ & $6.94 \mathrm{E}-03$ & 6.94E-03 & $8.42 \mathrm{E}-06$ & 1.81E-06 & $3.26 \mathrm{E}-03$ & $3.26 \mathrm{E}-03$ \\
\hline $\mathrm{Sb}-126 \mathrm{~m}$ & 1.10E+01 & 4.95E-02 & 4.95E-02 & 6.01E-05 & 1.29E-05 & 2.33E-02 & $2.33 \mathrm{E}-02$ \\
\hline Sm-151 & 1.65E+04 & $7.43 E+01$ & $7.43 \mathrm{E}+01$ & 9.02E-02 & 1.94E-02 & $3.49 E+01$ & $3.49 \mathrm{E}+01$ \\
\hline Sr-90 & $6.93 E+05$ & $3.12 E+03$ & $3.12 \mathrm{E}+03$ & $3.79 E+00$ & 8.14E-01 & $1.47 E+03$ & 1.47E+03 \\
\hline Tc-99 & 4.26E+02 & $1.92 \mathrm{E}+00$ & $1.92 \mathrm{E}+00$ & 2.33E-03 & $5.00 \mathrm{E}-04$ & $9.01 \mathrm{E}-01$ & $9.01 \mathrm{E}-01$ \\
\hline Th-230 & 1.15E-01 & 5.18E-04 & 5.18E-04 & $6.28 \mathrm{E}-07$ & 1.35E-07 & 2.43E-04 & 2.43E-04 \\
\hline Th-231 & 2.39E-02 & $1.08 \mathrm{E}-04$ & $1.08 \mathrm{E}-04$ & 1.31E-07 & 2.81E-08 & 5.05E-05 & $5.05 \mathrm{E}-05$ \\
\hline U-232 & 8.13E-05 & 3.66E-07 & 3.66E-07 & $4.44 \mathrm{E}-10$ & $9.55 \mathrm{E}-11$ & $1.72 \mathrm{E}-07$ & $1.72 \mathrm{E}-07$ \\
\hline U-233 & 1.57E-04 & 7.07E-07 & 7.07E-07 & $8.58 \mathrm{E}-10$ & $1.84 \mathrm{E}-10$ & 3.32E-07 & 3.32E-07 \\
\hline$U-234$ & $3.44 \mathrm{E}+00$ & $1.55 \mathrm{E}-02$ & 1.55E-02 & 1.88E-05 & 4.04E-06 & 7.27E-03 & 7.27E-03 \\
\hline U-235 & 2.39E-02 & $1.08 \mathrm{E}-04$ & $1.08 \mathrm{E}-04$ & $1.31 \mathrm{E}-07$ & 2.81E-08 & 5.05E-05 & 5.05E-05 \\
\hline U-236 & 5.55E-02 & $2.50 \mathrm{E}-04$ & $2.50 \mathrm{E}-04$ & 3.03E-07 & 6.52E-08 & 1.17E-04 & 1.17E-04 \\
\hline$U-237$ & 3.90E-03 & 1.76E-05 & 1.76E-05 & 2.13E-08 & 4.58E-09 & 8.25E-06 & 8.25E-06 \\
\hline U-238 & 1.35E-03 & 6.08E-06 & 6.08E-06 & 7.38E-09 & 1.59E-09 & 2.85E-06 & $2.85 \mathrm{E}-06$ \\
\hline$Y-90$ & $6.93 \mathrm{E}+05$ & $3.12 E+03$ & $3.12 E+03$ & $3.79 E+00$ & 8.14E-01 & 1.47E+03 & 1.47E+03 \\
\hline Total & 2.98 & & & & & & \\
\hline
\end{tabular}


CSSF 2 Calcine Source Term

Based on INEEL/EXT-98-00455 Rev. 2

Table A14 and Table A17

$\begin{array}{rc}\text { CSSF 2 Calcine Volume }= & 851 \mathrm{~m}^{3} \\ \text { Calcine Mass }= & 1.03 \mathrm{E}+06 \mathrm{~kg} \\ \text { Grout Waste Loading }= & 21.50 \% \\ \text { Grout Waste Density }= & 1800 \mathrm{~kg} / \mathrm{m}^{3}\end{array}$

\begin{tabular}{|c|c|c|c|c|c|c|c|}
\hline Nuclide & $\begin{array}{r}\text { Year } 2016 \\
\text { Curies }\end{array}$ & $\begin{array}{r}\text { Calcine } \\
\mathrm{Ci} / \mathrm{m}^{3}\end{array}$ & $\begin{array}{l}\text { Calcine } \\
\mathrm{uCi} / \mathrm{cm}^{3}\end{array}$ & $\begin{array}{r}\text { Calcine } \\
\text { Ci } / \mathrm{kg}\end{array}$ & $\begin{array}{l}\text { Grout } \\
\mathrm{Ci} / \mathrm{kg}\end{array}$ & $\begin{array}{l}\text { Grout } \\
\mathrm{Ci} / \mathrm{m}^{3}\end{array}$ & $\begin{array}{r}\text { Grout } \\
\mathrm{uCi} / \mathrm{cm}^{3}\end{array}$ \\
\hline Am-241 & $1.05 \mathrm{E}+03$ & $1.23 E+00$ & $1.23 \mathrm{E}+00$ & 1.02E-03 & $2.19 \mathrm{E}-04$ & $3.95 \mathrm{E}-01$ & $3.95 \mathrm{E}-01$ \\
\hline Am-243 & 8.96E-01 & 1.05E-03 & 1.05E-03 & 8.70E-07 & 1.87E-07 & 3.37E-04 & 3.37E-04 \\
\hline Ba-137m & $1.62 \mathrm{E}+06$ & $1.90 E+03$ & $1.90 \mathrm{E}+03$ & $1.57 \mathrm{E}+00$ & 3.38E-01 & $6.09 E+02$ & $6.09 \mathrm{E}+02$ \\
\hline Ce-144 & 7.47E-12 & $8.78 \mathrm{E}-15$ & $8.78 \mathrm{E}-15$ & $7.25 \mathrm{E}-18$ & $1.56 \mathrm{E}-18$ & $2.81 \mathrm{E}-15$ & $2.81 \mathrm{E}-15$ \\
\hline $\mathrm{Cm}-242$ & $2.59 \mathrm{E}+00$ & $3.04 \mathrm{E}-03$ & 3.04E-03 & $2.51 \mathrm{E}-06$ & $5.41 \mathrm{E}-07$ & $9.73 E-04$ & $9.73 \mathrm{E}-04$ \\
\hline $\mathrm{Cm}-244$ & $9.00 \mathrm{E}-01$ & 1.06E-03 & 1.06E-03 & 8.74E-07 & 1.88E-07 & 3.38E-04 & $3.38 \mathrm{E}-04$ \\
\hline Co-60 & 2.27E+01 & 2.67E-02 & 2.67E-02 & $2.20 \mathrm{E}-05$ & 4.74E-06 & 8.53E-03 & 8.53E-03 \\
\hline Cs-134 & 4.02E-01 & $4.72 \mathrm{E}-04$ & 4.72E-04 & $3.90 \mathrm{E}-07$ & 8.39E-08 & $1.51 \mathrm{E}-04$ & 1.51E-04 \\
\hline Cs-135 & $2.66 \mathrm{E}+01$ & 3.13E-02 & 3.13E-02 & 2.58E-05 & 5.55E-06 & 9.99E-03 & 9.99E-03 \\
\hline Cs-137 & $1.71 \mathrm{E}+06$ & $2.01 \mathrm{E}+03$ & $2.01 \mathrm{E}+03$ & $1.66 \mathrm{E}+00$ & 3.57E-01 & $6.42 \mathrm{E}+02$ & $6.42 \mathrm{E}+02$ \\
\hline Eu-152 & 4.06E+01 & 4.77E-02 & 4.77E-02 & 3.94E-05 & 8.47E-06 & $1.53 \mathrm{E}-02$ & 1.53E-02 \\
\hline Eu-154 & $2.87 E+03$ & $3.37 E+00$ & $3.37 E+00$ & $2.79 \mathrm{E}-03$ & $5.99 \mathrm{E}-04$ & $1.08 E+00$ & $1.08 \mathrm{E}+00$ \\
\hline Eu-155 & 1.64E+02 & $1.93 \mathrm{E}-01$ & 1.93E-01 & $1.59 \mathrm{E}-04$ & $3.42 E-05$ & 6.16E-02 & $6.16 \mathrm{E}-02$ \\
\hline I-129 & 1.65E-01 & 1.94E-04 & 1.94E-04 & $1.60 \mathrm{E}-07$ & $3.44 \mathrm{E}-08$ & 6.20E-05 & $6.20 \mathrm{E}-05$ \\
\hline $\mathrm{Ni}-63$ & $5.77 \mathrm{E}+04$ & $6.78 E+01$ & $6.78 \mathrm{E}+01$ & $5.60 \mathrm{E}-02$ & $1.20 \mathrm{E}-02$ & $2.17 E+01$ & $2.17 \mathrm{E}+01$ \\
\hline Np-237 & $1.29 \mathrm{E}+00$ & $1.52 \mathrm{E}-03$ & 1.52E-03 & $1.25 \mathrm{E}-06$ & 2.69E-07 & $4.85 \mathrm{E}-04$ & 4.85E-04 \\
\hline $\mathrm{Pa}-233$ & $1.29 \mathrm{E}+00$ & 1.52E-03 & 1.52E-03 & $1.25 \mathrm{E}-06$ & 2.69E-07 & 4.85E-04 & 4.85E-04 \\
\hline $\mathrm{Pm}-147$ & $5.14 \mathrm{E}+01$ & 6.04E-02 & 6.04E-02 & 4.99E-05 & 1.07E-05 & 1.93E-02 & 1.93E-02 \\
\hline Pr-144 & 7.47E-12 & 8.78E-15 & 8.78E-15 & $7.25 \mathrm{E}-18$ & $1.56 \mathrm{E}-18$ & $2.81 \mathrm{E}-15$ & $2.81 \mathrm{E}-15$ \\
\hline Pu-238 & $9.01 \mathrm{E}+03$ & $1.06 \mathrm{E}+01$ & $1.06 \mathrm{E}+01$ & 8.75E-03 & $1.88 \mathrm{E}-03$ & $3.39 E+00$ & $3.39 \mathrm{E}+00$ \\
\hline Pu-239 & $1.81 \mathrm{E}+02$ & 2.13E-01 & 2.13E-01 & 1.76E-04 & $3.78 \mathrm{E}-05$ & 6.80E-02 & 6.80E-02 \\
\hline Pu-240 & $1.52 \mathrm{E}+02$ & 1.79E-01 & 1.79E-01 & $1.48 \mathrm{E}-04$ & 3.17E-05 & 5.71E-02 & 5.71E-02 \\
\hline Pu-241 & $4.58 \mathrm{E}+03$ & $5.38 E+00$ & $5.38 \mathrm{E}+00$ & 4.45E-03 & $9.56 \mathrm{E}-04$ & $1.72 E+00$ & $1.72 \mathrm{E}+00$ \\
\hline Pu-242 & 3.55E-01 & 4.17E-04 & 4.17E-04 & 3.45E-07 & 7.41E-08 & 1.33E-04 & 1.33E-04 \\
\hline Ru-106 & 2.82E-07 & $3.31 \mathrm{E}-10$ & 3.31E-10 & $2.74 \mathrm{E}-13$ & $5.89 E-14$ & $1.06 \mathrm{E}-10$ & $1.06 \mathrm{E}-10$ \\
\hline Sb-125 & $7.48 \mathrm{E}+01$ & 8.79E-02 & 8.79E-02 & 7.26E-05 & 1.56E-05 & 2.81E-02 & $2.81 \mathrm{E}-02$ \\
\hline Sb-126 & $2.87 \mathrm{E}+00$ & 3.37E-03 & 3.37E-03 & $2.79 \mathrm{E}-06$ & 5.99E-07 & 1.08E-03 & $1.08 \mathrm{E}-03$ \\
\hline $\mathrm{Sb}-126 \mathrm{~m}$ & $2.05 E+01$ & $2.41 \mathrm{E}-02$ & 2.41E-02 & 1.99E-05 & 4.28E-06 & 7.70E-03 & 7.70E-03 \\
\hline Sm-151 & $2.46 \mathrm{E}+04$ & $2.89 E+01$ & $2.89 \mathrm{E}+01$ & 2.39E-02 & 5.13E-03 & $9.24 \mathrm{E}+00$ & $9.24 \mathrm{E}+00$ \\
\hline Sr-90 & $1.49 \mathrm{E}+06$ & $1.75 E+03$ & $1.75 \mathrm{E}+03$ & $1.45 E+00$ & $3.11 \mathrm{E}-01$ & $5.60 \mathrm{E}+02$ & $5.60 \mathrm{E}+02$ \\
\hline Tc-99 & 7.77E+02 & 9.13E-01 & 9.13E-01 & 7.54E-04 & 1.62E-04 & 2.92E-01 & 2.92E-01 \\
\hline Th-230 & 1.48E-01 & $1.74 \mathrm{E}-04$ & 1.74E-04 & $1.44 \mathrm{E}-07$ & $3.09 E-08$ & 5.56E-05 & 5.56E-05 \\
\hline Th-231 & 5.97E-02 & 7.02E-05 & 7.02E-05 & $5.80 \mathrm{E}-08$ & $1.25 \mathrm{E}-08$ & 2.24E-05 & $2.24 \mathrm{E}-05$ \\
\hline U-232 & 9.18E-03 & 1.08E-05 & 1.08E-05 & 8.91E-09 & 1.92E-09 & $3.45 \mathrm{E}-06$ & $3.45 \mathrm{E}-06$ \\
\hline$U-233$ & 2.12E-03 & 2.49E-06 & 2.49E-06 & 2.06E-09 & $4.43 E-10$ & 7.97E-07 & 7.97E-07 \\
\hline U-234 & $2.19 \mathrm{E}+01$ & 2.57E-02 & 2.57E-02 & 2.13E-05 & 4.57E-06 & 8.23E-03 & 8.23E-03 \\
\hline U-235 & 5.59E-02 & 6.57E-05 & 6.57E-05 & 5.43E-08 & 1.17E-08 & 2.10E-05 & 2.10E-05 \\
\hline U-236 & $1.55 \mathrm{E}-01$ & 1.82E-04 & 1.82E-04 & $1.50 \mathrm{E}-07$ & $3.24 \mathrm{E}-08$ & 5.82E-05 & 5.82E-05 \\
\hline U-237 & $1.25 \mathrm{E}-01$ & 1.47E-04 & 1.47E-04 & $1.21 \mathrm{E}-07$ & 2.61E-08 & 4.70E-05 & 4.70E-05 \\
\hline U-238 & 3.39E-03 & 3.98E-06 & 3.98E-06 & 3.29E-09 & 7.08E-10 & 1.27E-06 & $1.27 \mathrm{E}-06$ \\
\hline$Y-90$ & $1.49 \mathrm{E}+06$ & $1.75 E+03$ & $1.75 E+03$ & $1.45 E+00$ & $3.11 \mathrm{E}-01$ & $5.60 E+02$ & $5.60 \mathrm{E}+02$ \\
\hline Total & 6.41 & & & & & & \\
\hline
\end{tabular}


CSSF 3 Calcine Source Term

Based on INEEL/EXT-98-00455 Rev. 2

Table A14 and Table A17

$\begin{array}{rc}\text { CSSF 3 Calcine Volume }= & 1120 \mathrm{~m}^{3} \\ \text { Calcine Mass }= & 1.44 \mathrm{E}+06 \mathrm{~kg} \\ \text { Grout Waste Loading }= & 21.50 \% \\ \text { Grout Waste Density }= & 1800 \mathrm{~kg} / \mathrm{m}^{3}\end{array}$

\begin{tabular}{|c|c|c|c|c|c|c|c|}
\hline Nuclide & $\begin{array}{r}\text { Year } 2016 \\
\text { Curies }\end{array}$ & $\begin{array}{r}\text { Calcine } \\
\mathrm{Ci} / \mathrm{m}^{3}\end{array}$ & $\begin{array}{l}\text { Calcine } \\
\mathrm{uCi} / \mathrm{cm}^{3}\end{array}$ & $\begin{array}{r}\text { Calcine } \\
\text { Ci } / \mathrm{kg}\end{array}$ & $\begin{array}{l}\text { Grout } \\
\mathrm{Ci} / \mathrm{kg}\end{array}$ & $\begin{array}{l}\text { Grout } \\
\mathrm{Ci} / \mathrm{m}^{3}\end{array}$ & $\begin{array}{r}\text { Grout } \\
\mathrm{uCi} / \mathrm{cm}^{3}\end{array}$ \\
\hline Am-241 & $2.33 \mathrm{E}+03$ & $2.08 \mathrm{E}+00$ & $2.08 \mathrm{E}+00$ & 1.62E-03 & $3.48 \mathrm{E}-04$ & $6.26 \mathrm{E}-01$ & $6.26 \mathrm{E}-01$ \\
\hline Am-243 & $1.54 \mathrm{E}+00$ & 1.38E-03 & 1.38E-03 & 1.07E-06 & 2.30E-07 & 4.14E-04 & $4.14 \mathrm{E}-04$ \\
\hline Ba-137m & $1.83 \mathrm{E}+06$ & $1.63 \mathrm{E}+03$ & $1.63 E+03$ & $1.27 \mathrm{E}+00$ & $2.73 \mathrm{E}-01$ & $4.92 E+02$ & $4.92 \mathrm{E}+02$ \\
\hline Ce-144 & $1.50 \mathrm{E}-09$ & $1.34 \mathrm{E}-12$ & 1.34E-12 & $1.04 \mathrm{E}-15$ & $2.24 \mathrm{E}-16$ & $4.03 E-13$ & $4.03 E-13$ \\
\hline $\mathrm{Cm}-242$ & $3.72 E+00$ & 3.32E-03 & 3.32E-03 & $2.58 \mathrm{E}-06$ & $5.55 \mathrm{E}-07$ & $1.00 \mathrm{E}-03$ & $1.00 \mathrm{E}-03$ \\
\hline $\mathrm{Cm}-244$ & $1.27 \mathrm{E}+00$ & 1.13E-03 & $1.13 \mathrm{E}-03$ & 8.82E-07 & 1.90E-07 & 3.41E-04 & $3.41 \mathrm{E}-04$ \\
\hline Co-60 & 4.14E+01 & 3.70E-02 & 3.70E-02 & 2.88E-05 & $6.18 \mathrm{E}-06$ & 1.11E-02 & 1.11E-02 \\
\hline Cs-134 & 8.93E-01 & 7.97E-04 & 7.97E-04 & $6.20 \mathrm{E}-07$ & 1.33E-07 & $2.40 \mathrm{E}-04$ & 2.40E-04 \\
\hline Cs-135 & $3.69 E+01$ & $3.29 \mathrm{E}-02$ & 3.29E-02 & 2.56E-05 & 5.51E-06 & 9.92E-03 & 9.92E-03 \\
\hline Cs-137 & $1.93 E+06$ & $1.72 \mathrm{E}+03$ & $1.72 \mathrm{E}+03$ & $1.34 \mathrm{E}+00$ & $2.88 \mathrm{E}-01$ & $5.19 E+02$ & $5.19 E+02$ \\
\hline Eu-152 & 7.07E+01 & 6.31E-02 & 6.31E-02 & 4.91E-05 & 1.06E-05 & $1.90 \mathrm{E}-02$ & 1.90E-02 \\
\hline Eu-154 & $2.24 \mathrm{E}+03$ & $2.00 \mathrm{E}+00$ & $2.00 \mathrm{E}+00$ & 1.56E-03 & 3.34E-04 & $6.02 \mathrm{E}-01$ & 6.02E-01 \\
\hline Eu-155 & $1.79 \mathrm{E}+02$ & $1.60 \mathrm{E}-01$ & 1.60E-01 & $1.24 \mathrm{E}-04$ & 2.67E-05 & 4.81E-02 & 4.81E-02 \\
\hline I-129 & 1.67E-01 & 1.49E-04 & 1.49E-04 & 1.16E-07 & $2.49 \mathrm{E}-08$ & 4.49E-05 & 4.49E-05 \\
\hline $\mathrm{Ni}-63$ & $1.20 \mathrm{E}+05$ & $1.07 \mathrm{E}+02$ & $1.07 \mathrm{E}+02$ & 8.33E-02 & 1.79E-02 & $3.23 E+01$ & $3.23 E+01$ \\
\hline $\mathrm{Np}-237$ & 7.68E+00 & 6.86E-03 & 6.86E-03 & 5.33E-06 & $1.15 \mathrm{E}-06$ & 2.06E-03 & 2.06E-03 \\
\hline $\mathrm{Pa}-233$ & $7.68 \mathrm{E}+00$ & 6.86E-03 & 6.86E-03 & 5.33E-06 & 1.15E-06 & 2.06E-03 & $2.06 \mathrm{E}-03$ \\
\hline $\mathrm{Pm}-147$ & $7.42 \mathrm{E}+01$ & 6.63E-02 & 6.63E-02 & 5.15E-05 & 1.11E-05 & 1.99E-02 & 1.99E-02 \\
\hline Pr-144 & 1.50E-09 & $1.34 \mathrm{E}-12$ & 1.34E-12 & $1.04 \mathrm{E}-15$ & $2.24 \mathrm{E}-16$ & 4.03E-13 & $4.03 E-13$ \\
\hline Pu-238 & 4.07E+04 & $3.63 E+01$ & $3.63 E+01$ & 2.83E-02 & $6.08 \mathrm{E}-03$ & $1.09 E+01$ & $1.09 \mathrm{E}+01$ \\
\hline Pu-239 & $3.28 \mathrm{E}+02$ & 2.93E-01 & 2.93E-01 & 2.28E-04 & $4.90 \mathrm{E}-05$ & 8.82E-02 & 8.82E-02 \\
\hline Pu-240 & $4.05 E+02$ & $3.62 \mathrm{E}-01$ & 3.62E-01 & 2.81E-04 & $6.05 E-05$ & 1.09E-01 & 1.09E-01 \\
\hline Pu-241 & 1.09E+04 & $9.73 E+00$ & $9.73 E+00$ & 7.57E-03 & 1.63E-03 & $2.93 E+00$ & $2.93 \mathrm{E}+00$ \\
\hline Pu-242 & $1.02 \mathrm{E}+00$ & 9.11E-04 & 9.11E-04 & 7.08E-07 & 1.52E-07 & 2.74E-04 & 2.74E-04 \\
\hline Ru-106 & 4.63E-07 & 4.13E-10 & $4.13 \mathrm{E}-10$ & $3.22 \mathrm{E}-13$ & $6.91 \mathrm{E}-14$ & $1.24 \mathrm{E}-10$ & $1.24 \mathrm{E}-10$ \\
\hline Sb-125 & $4.50 \mathrm{E}+00$ & $4.02 \mathrm{E}-03$ & 4.02E-03 & $3.13 \mathrm{E}-06$ & $6.72 \mathrm{E}-07$ & 1.21E-03 & $1.21 \mathrm{E}-03$ \\
\hline Sb-126 & $2.96 \mathrm{E}+00$ & 2.64E-03 & 2.64E-03 & $2.06 \mathrm{E}-06$ & 4.42E-07 & 7.96E-04 & 7.96E-04 \\
\hline $\mathrm{Sb}-126 \mathrm{~m}$ & $2.11 \mathrm{E}+01$ & $1.88 \mathrm{E}-02$ & 1.88E-02 & 1.47E-05 & $3.15 E-06$ & 5.67E-03 & 5.67E-03 \\
\hline Sm-151 & $1.43 E+04$ & $1.28 \mathrm{E}+01$ & $1.28 \mathrm{E}+01$ & 9.93E-03 & 2.14E-03 & $3.84 \mathrm{E}+00$ & $3.84 \mathrm{E}+00$ \\
\hline Sr-90 & $1.72 \mathrm{E}+06$ & $1.54 \mathrm{E}+03$ & $1.54 \mathrm{E}+03$ & $1.19 \mathrm{E}+00$ & 2.57E-01 & $4.62 E+02$ & $4.62 E+02$ \\
\hline Tc-99 & 7.76E+02 & 6.93E-01 & 6.93E-01 & 5.39E-04 & 1.16E-04 & 2.09E-01 & 2.09E-01 \\
\hline Th-230 & 2.89E-02 & $2.58 \mathrm{E}-05$ & 2.58E-05 & 2.01E-08 & 4.31E-09 & 7.77E-06 & 7.77E-06 \\
\hline Th-231 & 7.49E-02 & 6.69E-05 & 6.69E-05 & $5.20 \mathrm{E}-08$ & $1.12 \mathrm{E}-08$ & 2.01E-05 & $2.01 \mathrm{E}-05$ \\
\hline U-232 & 9.17E-02 & 8.19E-05 & 8.19E-05 & 6.37E-08 & 1.37E-08 & 2.46E-05 & $2.46 \mathrm{E}-05$ \\
\hline U-233 & 5.04E-03 & 4.50E-06 & 4.50E-06 & 3.50E-09 & $7.53 \mathrm{E}-10$ & $1.35 \mathrm{E}-06$ & $1.35 \mathrm{E}-06$ \\
\hline$U-234$ & $1.59 \mathrm{E}+01$ & 1.42E-02 & 1.42E-02 & 1.10E-05 & 2.37E-06 & 4.27E-03 & 4.27E-03 \\
\hline U-235 & 4.07E-02 & 3.63E-05 & 3.63E-05 & 2.83E-08 & 6.08E-09 & 1.09E-05 & 1.09E-05 \\
\hline U-236 & 1.09E-01 & $9.73 \mathrm{E}-05$ & 9.73E-05 & 7.57E-08 & 1.63E-08 & 2.93E-05 & $2.93 \mathrm{E}-05$ \\
\hline U-237 & 2.66E-01 & 2.38E-04 & 2.38E-04 & 1.85E-07 & 3.97E-08 & 7.15E-05 & 7.15E-05 \\
\hline U-238 & 3.61E-03 & 3.22E-06 & $3.22 \mathrm{E}-06$ & 2.51E-09 & 5.39E-10 & 9.70E-07 & $9.70 \mathrm{E}-07$ \\
\hline$Y-90$ & $1.72 \mathrm{E}+06$ & $1.54 \mathrm{E}+03$ & $1.54 \mathrm{E}+03$ & 1.19E+00 & 2.57E-01 & $4.62 E+02$ & $4.62 E+02$ \\
\hline Total & 7.39 & & & & & & \\
\hline
\end{tabular}


CSSF 4 Calcine Source Term

Based on INEEL/EXT-98-00455 Rev. 2

Table A14 and Table A17

$\begin{array}{rc}\text { CSSF 4 Calcine Volume }= & 486 \mathrm{~m}^{3} \\ \text { Calcine Mass }= & 6.46 \mathrm{E}+05 \mathrm{~kg} \\ \text { Grout Waste Loading }= & 21.50 \% \\ \text { Grout Waste Density }= & 1800 \mathrm{~kg} / \mathrm{m}^{3}\end{array}$

\begin{tabular}{|c|c|c|c|c|c|c|c|}
\hline Nuclide & $\begin{array}{r}\text { Year } 2016 \\
\text { Curies }\end{array}$ & $\begin{array}{r}\text { Calcine } \\
\mathrm{Ci} / \mathrm{m}^{3}\end{array}$ & $\begin{array}{l}\text { Calcine } \\
\mathrm{uCi} / \mathrm{cm}^{3}\end{array}$ & $\begin{array}{r}\text { Calcine } \\
\text { Ci } / \mathrm{kg}\end{array}$ & $\begin{array}{l}\text { Grout } \\
\mathrm{Ci} / \mathrm{kg}\end{array}$ & $\begin{array}{l}\text { Grout } \\
\mathrm{Ci} / \mathrm{m}^{3}\end{array}$ & $\begin{array}{r}\text { Grout } \\
\mathrm{uCi} / \mathrm{cm}^{3}\end{array}$ \\
\hline Am-241 & $1.56 \mathrm{E}+03$ & $3.21 \mathrm{E}+00$ & $3.21 \mathrm{E}+00$ & $2.41 \mathrm{E}-03$ & $5.19 \mathrm{E}-04$ & $9.35 \mathrm{E}-01$ & $9.35 \mathrm{E}-01$ \\
\hline Am-243 & 8.59E-01 & 1.77E-03 & 1.77E-03 & 1.33E-06 & 2.86E-07 & $5.15 \mathrm{E}-04$ & $5.15 \mathrm{E}-04$ \\
\hline Ba-137m & $1.03 E+06$ & $2.12 E+03$ & $2.12 \mathrm{E}+03$ & $1.59 \mathrm{E}+00$ & $3.43 \mathrm{E}-01$ & $6.17 E+02$ & $6.17 \mathrm{E}+02$ \\
\hline Ce-144 & 2.37E-08 & $4.88 \mathrm{E}-11$ & $4.88 \mathrm{E}-11$ & $3.67 \mathrm{E}-14$ & $7.89 \mathrm{E}-15$ & $1.42 \mathrm{E}-11$ & $1.42 \mathrm{E}-11$ \\
\hline $\mathrm{Cm}-242$ & $1.98 \mathrm{E}+00$ & 4.07E-03 & 4.07E-03 & 3.07E-06 & $6.59 \mathrm{E}-07$ & 1.19E-03 & $1.19 \mathrm{E}-03$ \\
\hline $\mathrm{Cm}-244$ & $5.80 \mathrm{E}-01$ & 1.19E-03 & 1.19E-03 & 8.98E-07 & 1.93E-07 & 3.47E-04 & 3.47E-04 \\
\hline Co-60 & $3.62 E+01$ & 7.45E-02 & 7.45E-02 & 5.60E-05 & 1.20E-05 & 2.17E-02 & 2.17E-02 \\
\hline Cs-134 & $2.72 \mathrm{E}+00$ & $5.60 \mathrm{E}-03$ & 5.60E-03 & $4.21 \mathrm{E}-06$ & $9.05 \mathrm{E}-07$ & 1.63E-03 & $1.63 \mathrm{E}-03$ \\
\hline Cs-135 & $2.15 \mathrm{E}+01$ & 4.42E-02 & 4.42E-02 & 3.33E-05 & 7.16E-06 & 1.29E-02 & 1.29E-02 \\
\hline Cs-137 & $1.09 E+06$ & $2.24 \mathrm{E}+03$ & $2.24 \mathrm{E}+03$ & $1.69 \mathrm{E}+00$ & 3.63E-01 & $6.53 E+02$ & $6.53 \mathrm{E}+02$ \\
\hline Eu-152 & $4.09 E+01$ & 8.42E-02 & 8.42E-02 & 6.33E-05 & 1.36E-05 & 2.45E-02 & $2.45 \mathrm{E}-02$ \\
\hline Eu-154 & $3.40 E+03$ & $7.00 E+00$ & $7.00 \mathrm{E}+00$ & $5.26 \mathrm{E}-03$ & 1.13E-03 & $2.04 \mathrm{E}+00$ & $2.04 \mathrm{E}+00$ \\
\hline Eu-155 & $1.47 E+02$ & $3.02 E-01$ & 3.02E-01 & $2.28 \mathrm{E}-04$ & 4.89E-05 & 8.81E-02 & 8.81E-02 \\
\hline I-129 & 9.34E-02 & 1.92E-04 & 1.92E-04 & $1.45 \mathrm{E}-07$ & $3.11 \mathrm{E}-08$ & 5.60E-05 & 5.60E-05 \\
\hline $\mathrm{Ni}-63$ & $7.09 E+04$ & $1.46 \mathrm{E}+02$ & $1.46 \mathrm{E}+02$ & 1.10E-01 & 2.36E-02 & $4.25 E+01$ & $4.25 \mathrm{E}+01$ \\
\hline Np-237 & $1.86 \mathrm{E}+01$ & 3.83E-02 & 3.83E-02 & 2.88E-05 & 6.19E-06 & $1.11 \mathrm{E}-02$ & 1.11E-02 \\
\hline $\mathrm{Pa}-233$ & $1.86 \mathrm{E}+01$ & 3.83E-02 & 3.83E-02 & 2.88E-05 & 6.19E-06 & 1.11E-02 & 1.11E-02 \\
\hline $\mathrm{Pm}-147$ & $3.81 E+01$ & 7.84E-02 & 7.84E-02 & 5.90E-05 & 1.27E-05 & 2.28E-02 & 2.28E-02 \\
\hline Pr-144 & 2.37E-08 & $4.88 \mathrm{E}-11$ & 4.88E-11 & $3.67 \mathrm{E}-14$ & $7.89 E-15$ & $1.42 \mathrm{E}-11$ & $1.42 \mathrm{E}-11$ \\
\hline Pu-238 & $1.70 \mathrm{E}+04$ & $3.50 E+01$ & $3.50 \mathrm{E}+01$ & 2.63E-02 & 5.66E-03 & $1.02 E+01$ & $1.02 E+01$ \\
\hline Pu-239 & $4.08 E+02$ & 8.40E-01 & 8.40E-01 & 6.32E-04 & 1.36E-04 & $2.44 \mathrm{E}-01$ & 2.44E-01 \\
\hline Pu-240 & $2.28 \mathrm{E}+02$ & 4.69E-01 & 4.69E-01 & $3.53 \mathrm{E}-04$ & 7.59E-05 & 1.37E-01 & 1.37E-01 \\
\hline Pu-241 & $6.36 \mathrm{E}+03$ & $1.31 \mathrm{E}+01$ & $1.31 \mathrm{E}+01$ & 9.85E-03 & $2.12 \mathrm{E}-03$ & $3.81 \mathrm{E}+00$ & $3.81 \mathrm{E}+00$ \\
\hline Pu-242 & 5.87E-01 & $1.21 \mathrm{E}-03$ & 1.21E-03 & 9.09E-07 & $1.95 \mathrm{E}-07$ & $3.52 \mathrm{E}-04$ & 3.52E-04 \\
\hline Ru-106 & 4.28E-06 & 8.81E-09 & 8.81E-09 & 6.63E-12 & $1.42 \mathrm{E}-12$ & 2.56E-09 & 2.56E-09 \\
\hline Sb-125 & $6.37 E+00$ & 1.31E-02 & 1.31E-02 & $9.86 \mathrm{E}-06$ & $2.12 \mathrm{E}-06$ & 3.82E-03 & $3.82 \mathrm{E}-03$ \\
\hline Sb-126 & $1.66 \mathrm{E}+00$ & $3.42 \mathrm{E}-03$ & $3.42 \mathrm{E}-03$ & $2.57 \mathrm{E}-06$ & 5.52E-07 & 9.94E-04 & 9.94E-04 \\
\hline $\mathrm{Sb}-126 \mathrm{~m}$ & $1.19 E+01$ & $2.45 \mathrm{E}-02$ & 2.45E-02 & $1.84 \mathrm{E}-05$ & $3.96 \mathrm{E}-06$ & 7.13E-03 & 7.13E-03 \\
\hline Sm-151 & $7.00 \mathrm{E}+03$ & $1.44 \mathrm{E}+01$ & $1.44 \mathrm{E}+01$ & 1.08E-02 & 2.33E-03 & $4.19 E+00$ & $4.19 \mathrm{E}+00$ \\
\hline Sr-90 & $9.39 E+05$ & $1.93 E+03$ & $1.93 E+03$ & $1.45 E+00$ & $3.13 E-01$ & $5.63 E+02$ & $5.63 E+02$ \\
\hline Tc-99 & $4.34 \mathrm{E}+02$ & 8.93E-01 & 8.93E-01 & $6.72 \mathrm{E}-04$ & $1.44 \mathrm{E}-04$ & 2.60E-01 & $2.60 \mathrm{E}-01$ \\
\hline Th-230 & 4.62E-03 & 9.51E-06 & 9.51E-06 & 7.15E-09 & 1.54E-09 & 2.77E-06 & 2.77E-06 \\
\hline Th-231 & 2.98E-02 & $6.13 \mathrm{E}-05$ & $6.13 \mathrm{E}-05$ & 4.61E-08 & 9.92E-09 & 1.79E-05 & 1.79E-05 \\
\hline U-232 & $6.26 \mathrm{E}-02$ & 1.29E-04 & 1.29E-04 & 9.69E-08 & 2.08E-08 & 3.75E-05 & 3.75E-05 \\
\hline$U-233$ & 3.06E-03 & 6.30E-06 & 6.30E-06 & 4.74E-09 & 1.02E-09 & 1.83E-06 & 1.83E-06 \\
\hline U-234 & $1.53 E+01$ & 3.15E-02 & 3.15E-02 & 2.37E-05 & 5.09E-06 & 9.17E-03 & 9.17E-03 \\
\hline U-235 & 2.98E-02 & $6.13 \mathrm{E}-05$ & $6.13 \mathrm{E}-05$ & 4.61E-08 & 9.92E-09 & 1.79E-05 & 1.79E-05 \\
\hline U-236 & 8.82E-02 & 1.81E-04 & 1.81E-04 & 1.37E-07 & $2.94 \mathrm{E}-08$ & $5.28 \mathrm{E}-05$ & $5.28 \mathrm{E}-05$ \\
\hline U-237 & $1.56 \mathrm{E}-01$ & $3.21 \mathrm{E}-04$ & $3.21 \mathrm{E}-04$ & $2.41 \mathrm{E}-07$ & 5.19E-08 & 9.35E-05 & 9.35E-05 \\
\hline U-238 & $2.14 \mathrm{E}-03$ & 4.40E-06 & 4.40E-06 & 3.31E-09 & $7.12 \mathrm{E}-10$ & 1.28E-06 & $1.28 \mathrm{E}-06$ \\
\hline$Y-90$ & $9.39 \mathrm{E}+05$ & $1.93 E+03$ & $1.93 E+03$ & $1.45 E+00$ & $3.13 \mathrm{E}-01$ & $5.63 E+02$ & $5.63 E+02$ \\
\hline Total & 4.11 & & & & & & \\
\hline
\end{tabular}


CSSF 5 Calcine Source Term

Based on INEEL/EXT-98-00455 Rev. 2

Table A14 and Table A17

$\begin{array}{rc}\text { CSSF } 5 \text { Calcine Volume }= & 1010 \mathrm{~m}^{3} \\ \text { Calcine Mass }= & 1.41 \mathrm{E}+06 \mathrm{~kg} \\ \text { Grout Waste Loading }= & 21.50 \% \\ \text { Grout Waste Density }= & 1800 \mathrm{~kg} / \mathrm{m}^{3}\end{array}$

\begin{tabular}{|c|c|c|c|c|c|c|c|}
\hline Nuclide & $\begin{array}{r}\text { Year } 2016 \\
\text { Curies }\end{array}$ & $\begin{array}{r}\text { Calcine } \\
\mathrm{Ci} / \mathrm{m}^{3}\end{array}$ & $\begin{array}{l}\text { Calcine } \\
\mathrm{uCi} / \mathrm{cm}^{3}\end{array}$ & $\begin{array}{r}\text { Calcine } \\
\text { Ci } / \mathrm{kg}\end{array}$ & $\begin{array}{l}\text { Grout } \\
\mathrm{Ci} / \mathrm{kg}\end{array}$ & $\begin{array}{l}\text { Grout } \\
\mathrm{Ci} / \mathrm{m}^{3}\end{array}$ & $\begin{array}{r}\text { Grout } \\
\mathrm{uCi} / \mathrm{cm}^{3}\end{array}$ \\
\hline Am-241 & $3.24 \mathrm{E}+03$ & $3.21 \mathrm{E}+00$ & $3.21 \mathrm{E}+00$ & $2.30 \mathrm{E}-03$ & $4.94 \mathrm{E}-04$ & 8.89E-01 & 8.89E-01 \\
\hline Am-243 & $1.93 \mathrm{E}+00$ & 1.91E-03 & 1.91E-03 & 1.37E-06 & 2.94E-07 & 5.30E-04 & 5.30E-04 \\
\hline Ba-137m & $2.19 \mathrm{E}+06$ & $2.17 E+03$ & $2.17 \mathrm{E}+03$ & $1.55 \mathrm{E}+00$ & 3.34E-01 & $6.01 \mathrm{E}+02$ & $6.01 \mathrm{E}+02$ \\
\hline Ce-144 & 9.57E-06 & $9.48 \mathrm{E}-09$ & $9.48 \mathrm{E}-09$ & $6.79 \mathrm{E}-12$ & 1.46E-12 & 2.63E-09 & $2.63 \mathrm{E}-09$ \\
\hline $\mathrm{Cm}-242$ & $4.91 \mathrm{E}+00$ & $4.86 \mathrm{E}-03$ & 4.86E-03 & $3.48 \mathrm{E}-06$ & 7.49E-07 & $1.35 \mathrm{E}-03$ & $1.35 \mathrm{E}-03$ \\
\hline $\mathrm{Cm}-244$ & $1.75 \mathrm{E}+00$ & 1.73E-03 & $1.73 \mathrm{E}-03$ & $1.24 \mathrm{E}-06$ & 2.67E-07 & 4.80E-04 & $4.80 \mathrm{E}-04$ \\
\hline Co-60 & $7.58 \mathrm{E}+02$ & 7.50E-01 & 7.50E-01 & 5.38E-04 & 1.16E-04 & 2.08E-01 & 2.08E-01 \\
\hline Cs-134 & $3.95 \mathrm{E}+01$ & 3.91E-02 & 3.91E-02 & $2.80 \mathrm{E}-05$ & $6.02 \mathrm{E}-06$ & $1.08 \mathrm{E}-02$ & 1.08E-02 \\
\hline Cs-135 & $4.30 \mathrm{E}+01$ & 4.26E-02 & 4.26E-02 & 3.05E-05 & 6.56E-06 & 1.18E-02 & 1.18E-02 \\
\hline Cs-137 & $2.32 \mathrm{E}+06$ & $2.30 E+03$ & $2.30 \mathrm{E}+03$ & $1.65 \mathrm{E}+00$ & 3.54E-01 & $6.37 E+02$ & $6.37 \mathrm{E}+02$ \\
\hline Eu-152 & $8.40 \mathrm{E}+01$ & 8.32E-02 & 8.32E-02 & 5.96E-05 & 1.28E-05 & 2.31E-02 & 2.31E-02 \\
\hline Eu-154 & $7.08 \mathrm{E}+03$ & $7.01 \mathrm{E}+00$ & $7.01 \mathrm{E}+00$ & 5.02E-03 & 1.08E-03 & $1.94 \mathrm{E}+00$ & $1.94 \mathrm{E}+00$ \\
\hline Eu-155 & $6.14 \mathrm{E}+02$ & 6.08E-01 & 6.08E-01 & 4.35E-04 & 9.36E-05 & $1.69 \mathrm{E}-01$ & 1.69E-01 \\
\hline I-129 & 2.00E-01 & $1.98 \mathrm{E}-04$ & 1.98E-04 & $1.42 \mathrm{E}-07$ & 3.05E-08 & 5.49E-05 & 5.49E-05 \\
\hline $\mathrm{Ni}-63$ & $1.41 \mathrm{E}+05$ & $1.40 E+02$ & $1.40 \mathrm{E}+02$ & $1.00 \mathrm{E}-01$ & 2.15E-02 & $3.87 E+01$ & $3.87 E+01$ \\
\hline $\mathrm{Np}-237$ & $3.84 \mathrm{E}+01$ & $3.80 \mathrm{E}-02$ & 3.80E-02 & 2.72E-05 & 5.86E-06 & $1.05 E-02$ & 1.05E-02 \\
\hline $\mathrm{Pa}-233$ & $3.84 \mathrm{E}+01$ & 3.80E-02 & 3.80E-02 & 2.72E-05 & 5.86E-06 & $1.05 \mathrm{E}-02$ & 1.05E-02 \\
\hline $\mathrm{Pm}-147$ & $9.76 \mathrm{E}+01$ & 9.66E-02 & 9.66E-02 & 6.92E-05 & 1.49E-05 & 2.68E-02 & 2.68E-02 \\
\hline Pr-144 & 9.57E-06 & 9.48E-09 & 9.48E-09 & 6.79E-12 & $1.46 \mathrm{E}-12$ & 2.63E-09 & 2.63E-09 \\
\hline Pu-238 & $2.86 \mathrm{E}+04$ & $2.83 E+01$ & $2.83 E+01$ & $2.03 \mathrm{E}-02$ & 4.36E-03 & $7.85 E+00$ & $7.85 \mathrm{E}+00$ \\
\hline Pu-239 & $9.42 \mathrm{E}+02$ & 9.33E-01 & 9.33E-01 & 6.68E-04 & 1.44E-04 & $2.59 \mathrm{E}-01$ & 2.59E-01 \\
\hline Pu-240 & $4.43 E+02$ & 4.39E-01 & 4.39E-01 & $3.14 \mathrm{E}-04$ & $6.75 \mathrm{E}-05$ & $1.22 \mathrm{E}-01$ & 1.22E-01 \\
\hline Pu-241 & 1.27E+04 & $1.26 \mathrm{E}+01$ & $1.26 \mathrm{E}+01$ & 9.01E-03 & 1.94E-03 & $3.49 E+00$ & $3.49 E+00$ \\
\hline Pu-242 & $1.09 \mathrm{E}+00$ & $1.08 \mathrm{E}-03$ & 1.08E-03 & 7.73E-07 & 1.66E-07 & 2.99E-04 & $2.99 \mathrm{E}-04$ \\
\hline Ru-106 & 3.22E-04 & 3.19E-07 & 3.19E-07 & $2.28 \mathrm{E}-10$ & 4.91E-11 & 8.84E-08 & 8.84E-08 \\
\hline Sb-125 & $3.84 \mathrm{E}+01$ & 3.80E-02 & 3.80E-02 & 2.72E-05 & 5.86E-06 & $1.05 \mathrm{E}-02$ & 1.05E-02 \\
\hline Sb-126 & $3.55 \mathrm{E}+00$ & 3.51E-03 & $3.51 \mathrm{E}-03$ & $2.52 \mathrm{E}-06$ & 5.41E-07 & 9.74E-04 & $9.74 \mathrm{E}-04$ \\
\hline $\mathrm{Sb}-126 \mathrm{~m}$ & $2.53 E+01$ & $2.50 \mathrm{E}-02$ & 2.50E-02 & 1.79E-05 & 3.86E-06 & 6.94E-03 & 6.94E-03 \\
\hline Sm-151 & $1.88 \mathrm{E}+04$ & $1.86 \mathrm{E}+01$ & $1.86 \mathrm{E}+01$ & 1.33E-02 & 2.87E-03 & $5.16 \mathrm{E}+00$ & $5.16 \mathrm{E}+00$ \\
\hline Sr-90 & $2.12 \mathrm{E}+06$ & $2.10 \mathrm{E}+03$ & $2.10 \mathrm{E}+03$ & $1.50 \mathrm{E}+00$ & 3.23E-01 & $5.82 \mathrm{E}+02$ & $5.82 E+02$ \\
\hline Tc-99 & $9.33 E+02$ & 9.24E-01 & $9.24 \mathrm{E}-01$ & 6.62E-04 & $1.42 \mathrm{E}-04$ & 2.56E-01 & $2.56 \mathrm{E}-01$ \\
\hline Th-230 & 5.51E-02 & 5.46E-05 & 5.46E-05 & 3.91E-08 & 8.40E-09 & $1.51 \mathrm{E}-05$ & 1.51E-05 \\
\hline Th-231 & $9.88 \mathrm{E}-02$ & $9.78 \mathrm{E}-05$ & $9.78 \mathrm{E}-05$ & 7.01E-08 & 1.51E-08 & 2.71E-05 & $2.71 \mathrm{E}-05$ \\
\hline U-232 & 8.55E-02 & 8.47E-05 & 8.47E-05 & $6.06 \mathrm{E}-08$ & 1.30E-08 & $2.35 \mathrm{E}-05$ & $2.35 \mathrm{E}-05$ \\
\hline U-233 & $5.66 \mathrm{E}-03$ & $5.60 \mathrm{E}-06$ & $5.60 \mathrm{E}-06$ & 4.01E-09 & $8.63 \mathrm{E}-10$ & $1.55 \mathrm{E}-06$ & $1.55 \mathrm{E}-06$ \\
\hline$U-234$ & $9.52 \mathrm{E}+00$ & 9.43E-03 & 9.43E-03 & 6.75E-06 & $1.45 \mathrm{E}-06$ & 2.61E-03 & 2.61E-03 \\
\hline U-235 & $1.25 \mathrm{E}-01$ & $1.24 \mathrm{E}-04$ & 1.24E-04 & 8.87E-08 & 1.91E-08 & 3.43E-05 & $3.43 \mathrm{E}-05$ \\
\hline U-236 & 4.91E-01 & 4.86E-04 & 4.86E-04 & $3.48 \mathrm{E}-07$ & 7.49E-08 & 1.35E-04 & 1.35E-04 \\
\hline U-237 & 3.10E-01 & 3.07E-04 & 3.07E-04 & 2.20E-07 & 4.73E-08 & 8.51E-05 & 8.51E-05 \\
\hline U-238 & 4.19E-02 & 4.15E-05 & 4.15E-05 & 2.97E-08 & 6.39E-09 & 1.15E-05 & 1.15E-05 \\
\hline$Y-90$ & $2.12 E+06$ & $2.10 E+03$ & $2.10 \mathrm{E}+03$ & $1.50 \mathrm{E}+00$ & 3.23E-01 & $5.82 E+02$ & $5.82 E+02$ \\
\hline Total & 8.97 & & & & & & \\
\hline
\end{tabular}


CSSF 6 Calcine Source Term

Based on INEEL/EXT-98-00455 Rev. 2

Table A14 and Table A17

$\begin{array}{rc}\text { CSSF 6 Calcine Volume }= & 724 \mathrm{~m}^{3} \\ \text { Calcine Mass }= & 8.83 \mathrm{E}+05 \mathrm{~kg} \\ \text { Grout Waste Loading }= & 21.50 \% \\ \text { Grout Waste Density }= & 1800 \mathrm{~kg} / \mathrm{m}^{3}\end{array}$

\begin{tabular}{|c|c|c|c|c|c|c|c|}
\hline Nuclide & $\begin{array}{r}\text { Year } 2016 \\
\text { Curies }\end{array}$ & $\begin{array}{r}\text { Calcine } \\
\mathrm{Ci} / \mathrm{m}^{3}\end{array}$ & $\begin{array}{l}\text { Calcine } \\
\mathrm{uCi} / \mathrm{cm}^{3}\end{array}$ & $\begin{array}{r}\text { Calcine } \\
\mathrm{Ci} / \mathrm{kg}\end{array}$ & $\begin{array}{l}\text { Grout } \\
\mathrm{Ci} / \mathrm{kg}\end{array}$ & $\begin{array}{l}\text { Grout } \\
\mathrm{Ci} / \mathrm{m}^{3}\end{array}$ & $\begin{array}{r}\text { Grout } \\
\mathrm{uCi} / \mathrm{cm}^{3}\end{array}$ \\
\hline Am-241 & $6.00 \mathrm{E}+02$ & 8.29E-01 & 8.29E-01 & 6.80E-04 & $1.46 \mathrm{E}-04$ & $2.63 \mathrm{E}-01$ & $2.63 \mathrm{E}-01$ \\
\hline Am-243 & $5.76 \mathrm{E}-01$ & 7.96E-04 & 7.96E-04 & $6.52 \mathrm{E}-07$ & 1.40E-07 & $2.52 \mathrm{E}-04$ & $2.52 \mathrm{E}-04$ \\
\hline Ba-137m & $5.87 \mathrm{E}+05$ & $8.11 \mathrm{E}+02$ & $8.11 \mathrm{E}+02$ & $6.65 \mathrm{E}-01$ & 1.43E-01 & $2.57 E+02$ & $2.57 \mathrm{E}+02$ \\
\hline Ce-144 & 4.17E-06 & 5.76E-09 & 5.76E-09 & $4.72 \mathrm{E}-12$ & $1.02 \mathrm{E}-12$ & 1.83E-09 & $1.83 \mathrm{E}-09$ \\
\hline $\mathrm{Cm}-242$ & $1.49 \mathrm{E}+00$ & 2.06E-03 & 2.06E-03 & $1.69 \mathrm{E}-06$ & 3.63E-07 & $6.53 \mathrm{E}-04$ & $6.53 \mathrm{E}-04$ \\
\hline $\mathrm{Cm}-244$ & 9.04E-01 & 1.25E-03 & 1.25E-03 & 1.02E-06 & 2.20E-07 & 3.96E-04 & $3.96 \mathrm{E}-04$ \\
\hline Co-60 & 1.10E+02 & $1.52 \mathrm{E}-01$ & $1.52 \mathrm{E}-01$ & 1.25E-04 & 2.68E-05 & 4.82E-02 & $4.82 \mathrm{E}-02$ \\
\hline Cs-134 & $9.13 \mathrm{E}+00$ & 1.26E-02 & 1.26E-02 & 1.03E-05 & $2.22 \mathrm{E}-06$ & $4.00 \mathrm{E}-03$ & $4.00 \mathrm{E}-03$ \\
\hline Cs-135 & $1.08 \mathrm{E}+01$ & 1.49E-02 & 1.49E-02 & 1.22E-05 & 2.63E-06 & 4.73E-03 & $4.73 \mathrm{E}-03$ \\
\hline Cs-137 & $6.20 \mathrm{E}+05$ & $8.56 \mathrm{E}+02$ & $8.56 \mathrm{E}+02$ & 7.02E-01 & $1.51 \mathrm{E}-01$ & $2.72 E+02$ & $2.72 \mathrm{E}+02$ \\
\hline Eu-152 & $2.23 E+01$ & 3.08E-02 & 3.08E-02 & 2.53E-05 & $5.43 E-06$ & 9.77E-03 & 9.77E-03 \\
\hline Eu-154 & $1.41 \mathrm{E}+03$ & $1.95 E+00$ & $1.95 E+00$ & 1.60E-03 & $3.43 E-04$ & $6.18 \mathrm{E}-01$ & 6.18E-01 \\
\hline Eu-155 & 1.63E+02 & $2.25 \mathrm{E}-01$ & 2.25E-01 & 1.85E-04 & 3.97E-05 & 7.14E-02 & 7.14E-02 \\
\hline I-129 & $9.13 E+00$ & 1.26E-02 & 1.26E-02 & 1.03E-05 & 2.22E-06 & 4.00E-03 & 4.00E-03 \\
\hline $\mathrm{Ni}-63$ & $3.40 \mathrm{E}+04$ & $4.70 E+01$ & $4.70 \mathrm{E}+01$ & $3.85 \mathrm{E}-02$ & $8.28 \mathrm{E}-03$ & $1.49 E+01$ & $1.49 \mathrm{E}+01$ \\
\hline $\mathrm{Np}-237$ & $6.22 \mathrm{E}+00$ & 8.59E-03 & 8.59E-03 & 7.04E-06 & $1.51 \mathrm{E}-06$ & $2.73 \mathrm{E}-03$ & 2.73E-03 \\
\hline $\mathrm{Pa}-233$ & $6.22 \mathrm{E}+00$ & 8.59E-03 & 8.59E-03 & 7.04E-06 & 1.51E-06 & 2.73E-03 & $2.73 \mathrm{E}-03$ \\
\hline $\mathrm{Pm}-147$ & $3.66 \mathrm{E}+01$ & 5.06E-02 & 5.06E-02 & 4.14E-05 & 8.91E-06 & $1.60 \mathrm{E}-02$ & 1.60E-02 \\
\hline Pr-144 & 4.17E-06 & 5.76E-09 & 5.76E-09 & 4.72E-12 & $1.02 \mathrm{E}-12$ & 1.83E-09 & $1.83 \mathrm{E}-09$ \\
\hline Pu-238 & $4.59 \mathrm{E}+03$ & $6.34 \mathrm{E}+00$ & $6.34 \mathrm{E}+00$ & 5.20E-03 & 1.12E-03 & $2.01 \mathrm{E}+00$ & $2.01 \mathrm{E}+00$ \\
\hline Pu-239 & $3.91 \mathrm{E}+02$ & 5.40E-01 & 5.40E-01 & 4.43E-04 & 9.52E-05 & $1.71 \mathrm{E}-01$ & 1.71E-01 \\
\hline Pu-240 & 1.15E+02 & 1.59E-01 & 1.59E-01 & 1.30E-04 & 2.80E-05 & $5.04 \mathrm{E}-02$ & 5.04E-02 \\
\hline Pu-241 & $3.25 \mathrm{E}+03$ & $4.49 E+00$ & $4.49 E+00$ & 3.68E-03 & 7.91E-04 & $1.42 \mathrm{E}+00$ & $1.42 \mathrm{E}+00$ \\
\hline Pu-242 & $1.81 \mathrm{E}+02$ & 2.50E-01 & $2.50 \mathrm{E}-01$ & 2.05E-04 & 4.41E-05 & 7.93E-02 & 7.93E-02 \\
\hline Ru-106 & 1.64E-04 & 2.27E-07 & 2.27E-07 & $1.86 \mathrm{E}-10$ & $3.99 E-11$ & 7.19E-08 & 7.19E-08 \\
\hline Sb-125 & $1.31 \mathrm{E}+01$ & 1.81E-02 & 1.81E-02 & $1.48 \mathrm{E}-05$ & 3.19E-06 & $5.74 \mathrm{E}-03$ & $5.74 \mathrm{E}-03$ \\
\hline Sb-126 & $9.39 \mathrm{E}-01$ & 1.30E-03 & 1.30E-03 & $1.06 \mathrm{E}-06$ & 2.29E-07 & 4.12E-04 & $4.12 \mathrm{E}-04$ \\
\hline $\mathrm{Sb}-126 \mathrm{~m}$ & $6.71 \mathrm{E}+00$ & 9.27E-03 & 9.27E-03 & 7.60E-06 & 1.63E-06 & 2.94E-03 & 2.94E-03 \\
\hline Sm-151 & $5.74 \mathrm{E}+03$ & $7.93 E+00$ & $7.93 \mathrm{E}+00$ & $6.50 \mathrm{E}-03$ & 1.40E-03 & $2.52 E+00$ & $2.52 \mathrm{E}+00$ \\
\hline Sr-90 & $5.84 \mathrm{E}+05$ & 8.07E+02 & 8.07E+02 & 6.61E-01 & $1.42 \mathrm{E}-01$ & $2.56 \mathrm{E}+02$ & $2.56 \mathrm{E}+02$ \\
\hline Tc-99 & $2.47 E+02$ & $3.41 \mathrm{E}-01$ & 3.41E-01 & 2.80E-04 & $6.01 \mathrm{E}-05$ & $1.08 \mathrm{E}-01$ & $1.08 \mathrm{E}-01$ \\
\hline Th-230 & 2.37E-02 & 3.27E-05 & 3.27E-05 & 2.68E-08 & 5.77E-09 & $1.04 \mathrm{E}-05$ & 1.04E-05 \\
\hline Th-231 & 7.51E-02 & $1.04 \mathrm{E}-04$ & 1.04E-04 & $8.51 \mathrm{E}-08$ & 1.83E-08 & $3.29 \mathrm{E}-05$ & $3.29 \mathrm{E}-05$ \\
\hline U-232 & 1.26E-02 & 1.74E-05 & 1.74E-05 & $1.43 \mathrm{E}-08$ & 3.07E-09 & 5.52E-06 & 5.52E-06 \\
\hline$U-233$ & 1.34E-03 & $1.85 \mathrm{E}-06$ & 1.85E-06 & 1.52E-09 & $3.26 \mathrm{E}-10$ & 5.87E-07 & 5.87E-07 \\
\hline U-234 & $3.17 \mathrm{E}+00$ & 4.38E-03 & 4.38E-03 & 3.59E-06 & 7.72E-07 & 1.39E-03 & 1.39E-03 \\
\hline U-235 & 8.12E-02 & 1.12E-04 & 1.12E-04 & $9.20 \mathrm{E}-08$ & $1.98 \mathrm{E}-08$ & 3.56E-05 & 3.56E-05 \\
\hline U-236 & 1.92E-01 & $2.65 \mathrm{E}-04$ & 2.65E-04 & $2.17 \mathrm{E}-07$ & 4.67E-08 & 8.41E-05 & 8.41E-05 \\
\hline U-237 & 8.36E-02 & 1.15E-04 & 1.15E-04 & 9.47E-08 & 2.04E-08 & 3.66E-05 & 3.66E-05 \\
\hline U-238 & 8.05E-02 & 1.11E-04 & 1.11E-04 & $9.12 \mathrm{E}-08$ & 1.96E-08 & 3.53E-05 & 3.53E-05 \\
\hline$Y-90$ & $5.84 \mathrm{E}+05$ & 8.07E+02 & 8.07E+02 & 6.61E-01 & $1.42 \mathrm{E}-01$ & $2.56 \mathrm{E}+02$ & $2.56 \mathrm{E}+02$ \\
\hline Total & 2.43 & & & & & & \\
\hline
\end{tabular}


Combined Calcine Source Term

Based on INEEL/EXT-98-00455 Rev. 2

Table A14 and Table A17 $\begin{array}{rc}\text { Total Calcine Volume }= & 4411 \mathrm{~m}^{3} \\ \text { Total Calcine Mass }= & 5.59 \mathrm{E}+06 \mathrm{~kg} \\ \text { Grout Waste Loading }= & 21.50 \% \\ \text { Grout Waste Density }= & 1800 \mathrm{~kg} / \mathrm{m}^{3}\end{array}$

\begin{tabular}{|c|c|c|c|c|c|c|c|}
\hline & $\begin{array}{r}\text { Year } 2016 \\
\text { Curies }\end{array}$ & $\begin{array}{r}\text { Calcine } \\
\mathrm{Ci} / \mathrm{m}^{3}\end{array}$ & $\begin{array}{l}\text { Calcine } \\
\mathrm{uCi} / \mathrm{cm}^{3}\end{array}$ & $\begin{array}{r}\text { Calcine } \\
\mathrm{Ci} / \mathrm{kg}\end{array}$ & $\begin{array}{l}\text { Grout } \\
\mathrm{Ci} / \mathrm{kg}\end{array}$ & $\begin{array}{l}\text { Grout } \\
\mathrm{Ci} / \mathrm{m}^{3}\end{array}$ & $\begin{array}{r}\text { Grout } \\
\mathrm{uCi} / \mathrm{cm}^{3}\end{array}$ \\
\hline Am-241 & $8.85 \mathrm{E}+03$ & $2.01 \mathrm{E}+00$ & $2.01 \mathrm{E}+00$ & $1.58 \mathrm{E}-03$ & $3.40 \mathrm{E}-04$ & $6.12 \mathrm{E}-01$ & $.12 \mathrm{E}-01$ \\
\hline Am-243 & $81 \mathrm{E}+00$ & $.32 \mathrm{E}-03$ & 1.32E-03 & 1.04E-06 & 2.23E-07 & 4.02E-04 & 02E-04 \\
\hline$-137 m$ & $02 E+06$ & $1.82 E+03$ & $1.82 E+03$ & $1.43 E+00$ & 3.08E-01 & $5.55 E+02$ & $5.55 \mathrm{E}+02$ \\
\hline e-144 & 38E-05 & 3.13E-09 & 3.13E-09 & $2.47 \mathrm{E}-12$ & $5.31 \mathrm{E}-13$ & $9.55 \mathrm{E}-10$ & $.55 \mathrm{E}-$ \\
\hline$m-242$ & $47 E+01$ & 3.33E-03 & 3.33E-03 & $2.63 E-06$ & $5.65 \mathrm{E}-07$ & 1.02E-03 & $1.02 \mathrm{E}-03$ \\
\hline Cm-244 & $5.42 \mathrm{E}+00$ & 1.23E-03 & 1.23E-03 & 9.69E-07 & 2.08E-07 & 3.75E-04 & 3.75E-04 \\
\hline Co-60 & $9.69 \mathrm{E}+02$ & 2.20E-01 & 2.20E-01 & 1.73E-04 & 3.73E-05 & 6.71E-02 & 6.71E-02 \\
\hline Cs-134 & $5.27 \mathrm{E}+01$ & 1.19E-02 & 1.19E-02 & $9.42 \mathrm{E}-06$ & $2.03 E-06$ & $3.65 \mathrm{E}-03$ & $3.65 \mathrm{E}-$ \\
\hline Cs-135 & $1.49 E+02$ & 3.38E-02 & 3.38E-02 & 2.66E-05 & 5.73E-06 & 1.03E-02 & 1.03E-02 \\
\hline Cs-137 & $8.48 \mathrm{E}+06$ & $1.92 \mathrm{E}+03$ & $1.92 E+03$ & $1.52 \mathrm{E}+00$ & 3.26E-01 & $5.87 \mathrm{E}+02$ & $5.87 E+02$ \\
\hline$u-152$ & $2.66 \mathrm{E}+02$ & 6.03E-02 & 6.03E-02 & 4.76E-05 & 1.02E-05 & $1.84 \mathrm{E}-02$ & $1.84 \mathrm{E}-02$ \\
\hline ג-154 & $1.74 \mathrm{E}+04$ & $3.94 E+00$ & $3.94 \mathrm{E}+00$ & 3.11E-03 & 6.69E-04 & $1.20 \mathrm{E}+00$ & $1.20 \mathrm{E}+0 \mathrm{C}$ \\
\hline$d-1$ & $E+03$ & 2.92E-01 & 2.92E-01 & 2.31E-04 & $6 \mathrm{E}-05$ & 8.93E-02 & 8.93E-0 \\
\hline 129 & $\mathrm{E}-01$ & 1.74E-04 & 1.74E-04 & 1.37E-07 & 2.95E-08 & 5.32E-05 & $5.32 \mathrm{E}-0$ \\
\hline i-63 & $E+05$ & $9.59 \mathrm{E}+01$ & $.59 E+01$ & 7.56E-02 & 1.63E-02 & $2.93 E+01$ & $2.93 E+0$ \\
\hline -237 & $7.33 E+01$ & 1.66E-02 & 1.66E-02 & 1.31E-05 & 2.82E-06 & 5.07E-03 & 5.07E-0 \\
\hline$a-233$ & $7.33 \mathrm{E}+01$ & 1.66E-02 & 1.66E-02 & 1.31E-05 & 2.82E-06 & 5.07E-03 & 5.07E-03 \\
\hline$m-147$ & $3.01 \mathrm{E}+02$ & 6.82E-02 & $6.82 \mathrm{E}-02$ & 5.38E-05 & 1.16E-05 & 2.08E-02 & $2.08 \mathrm{E}-02$ \\
\hline$r-144$ & 1.38E-05 & 3.13E-09 & 3.13E-09 & $2.47 \mathrm{E}-12$ & 5.31E-13 & $9.55 \mathrm{E}-10$ & $9.55 \mathrm{E}-10$ \\
\hline$u-238$ & $1.00 \mathrm{E}+05$ & $2.27 E+01$ & $2.27 E+01$ & 1.79E-02 & 3.84E-03 & $6.92 E+00$ & $6.92 \mathrm{E}+0 \mathrm{C}$ \\
\hline$u-239$ & $2.29 E+03$ & 5.19E-01 & 5.19E-01 & 4.10E-04 & 05 & $1.58 \mathrm{E}-01$ & $1.58 \mathrm{E}-$ \\
\hline$u-24$ & $E+03$ & $3.08 \mathrm{E}-01$ & $3.08 \mathrm{E}-01$ & $2.43 E-04$ & 5.23E-05 & $9.41 \mathrm{E}-02$ & $9.41 \mathrm{E}-0$ \\
\hline$u-241$ & $3.79 \mathrm{E}+04$ & $8.59 \mathrm{E}+00$ & $8.59 E+00$ & $6.78 \mathrm{E}-03$ & 1.46E-03 & $2.62 \mathrm{E}+00$ & $2.62 \mathrm{E}+0 \mathrm{C}$ \\
\hline u-242 & $1.84 \mathrm{E}+02$ & 4.17E-02 & 4.17E-02 & 3.29E-05 & 7.07E-06 & 1.27E-02 & 1.27E-0 \\
\hline-1 & -04 & 1.11E-07 & -07 & 8.78E-11 & $1.89 \mathrm{E}-11$ & 3.40E-08 & $3.40 \mathrm{E}-08$ \\
\hline 5 & +02 & 3.11E-02 & -02 & $2.45 \mathrm{E}-05$ & 5.27E-06 & $9.48 \mathrm{E}-03$ & $9.48 \mathrm{E}-03$ \\
\hline-126 & +01 & 3.06E-03 & $=-03$ & $2.41 \mathrm{E}-06$ & $E-07$ & 9.34E-04 & 9.34E-04 \\
\hline b-126m & $9.66 \mathrm{E}+01$ & 2.19E-02 & 2.19E-02 & 1.73E-05 & 3.71E-06 & 6.69E-03 & 6.69E-03 \\
\hline Sm-151 & $8.69 \mathrm{E}+04$ & 1.97E+01 & $1.97 E+01$ & 1.55E-02 & 3.34E-03 & $6.01 \mathrm{E}+00$ & $6.01 \mathrm{E}+00$ \\
\hline Sr-90 & $7.55 E+06$ & $1.71 \mathrm{E}+03$ & $1.71 \mathrm{E}+03$ & $1.35 E+00$ & 2.90E-01 & $5.23 E+02$ & $5.23 E+02$ \\
\hline Tc-99 & $3.59 \mathrm{E}+03$ & 8.14E-01 & 8.14E-01 & $6.42 \mathrm{E}-04$ & 1.38E-04 & 2.48E-01 & $2.48 \mathrm{E}-0$ \\
\hline Th-230 & 3.75E-01 & 8.50E-05 & 8.50E-05 & 6.71E-08 & 1.44E-08 & 2.60E-05 & $2.60 \mathrm{E}-0$ \\
\hline h-2 & 3.62E-01 & $8.21 \mathrm{E}-05$ & $8.21 \mathrm{E}-05$ & $6.47 \mathrm{E}-08$ & 1.39E-08 & $2.51 \mathrm{E}-05$ & $2.51 \mathrm{E}-0$ \\
\hline U-23 & 2.62E-01 & 5.94E-05 & 5.94E-05 & 4.69E-08 & 1.01E-08 & $1.81 \mathrm{E}-05$ & $1.81 \mathrm{E}-05$ \\
\hline U-23 & 4E-02 & 3.94E-06 & 3.94E-06 & 3.11E-09 & 6.69E-10 & $1.20 \mathrm{E}-06$ & $1.20 \mathrm{E}-0$ \\
\hline $\mathrm{J}-23$ & $6.92 \mathrm{E}+01$ & 1.57E-02 & 1.57E-02 & $1.24 \mathrm{E}-05$ & 2.66E-06 & 4.79E-03 & $4.79 \mathrm{E}-0$ \\
\hline $\mathrm{J}-235$ & E-01 & 8.07E-05 & 8.07E-05 & 6.37E-08 & 1.37E-08 & 2.46E-05 & $2.46 \mathrm{E}-\mathrm{C}$ \\
\hline U-236 & $1.09 \mathrm{E}+00$ & 2.47E-04 & 2.47E-04 & $1.95 \mathrm{E}-07$ & 4.19E-08 & 7.54E-05 & 7.54E-0 \\
\hline U-237 & $9.45 \mathrm{E}-01$ & 2.14E-04 & 2.14E-04 & 1.69E-07 & 3.63E-08 & 6.54E-05 & $6.54 \mathrm{E}-0$ \\
\hline U-238 & 1.33E-01 & $3.02 \mathrm{E}-05$ & 3.02E-05 & $2.38 \mathrm{E}-08$ & 5.11E-09 & $9.20 \mathrm{E}-06$ & $9.20 \mathrm{E}-06$ \\
\hline Y-90 & $7.55 E+06$ & $1.71 \mathrm{E}+03$ & $1.71 E+03$ & $1.35 E+00$ & 2.90E-01 & $5.23 E+02$ & $5.23 \mathrm{E}+0$ \\
\hline otal & & & & & & & \\
\hline
\end{tabular}


Page : 1

DOS File : Combined Calcine in Bulk Tank.ms6

Run Date: May 3, 2005

Run Time: 2:38:06 PM

Duration : 00:01:05
File Ref:

Date:

By

Checked:

Case Title: Combined Calcine

Description: Combined Calcine in Bulk Retrieval Vessel (3/8" 316 SS) Geometry: 7 - Cylinder Volume - Side Shields

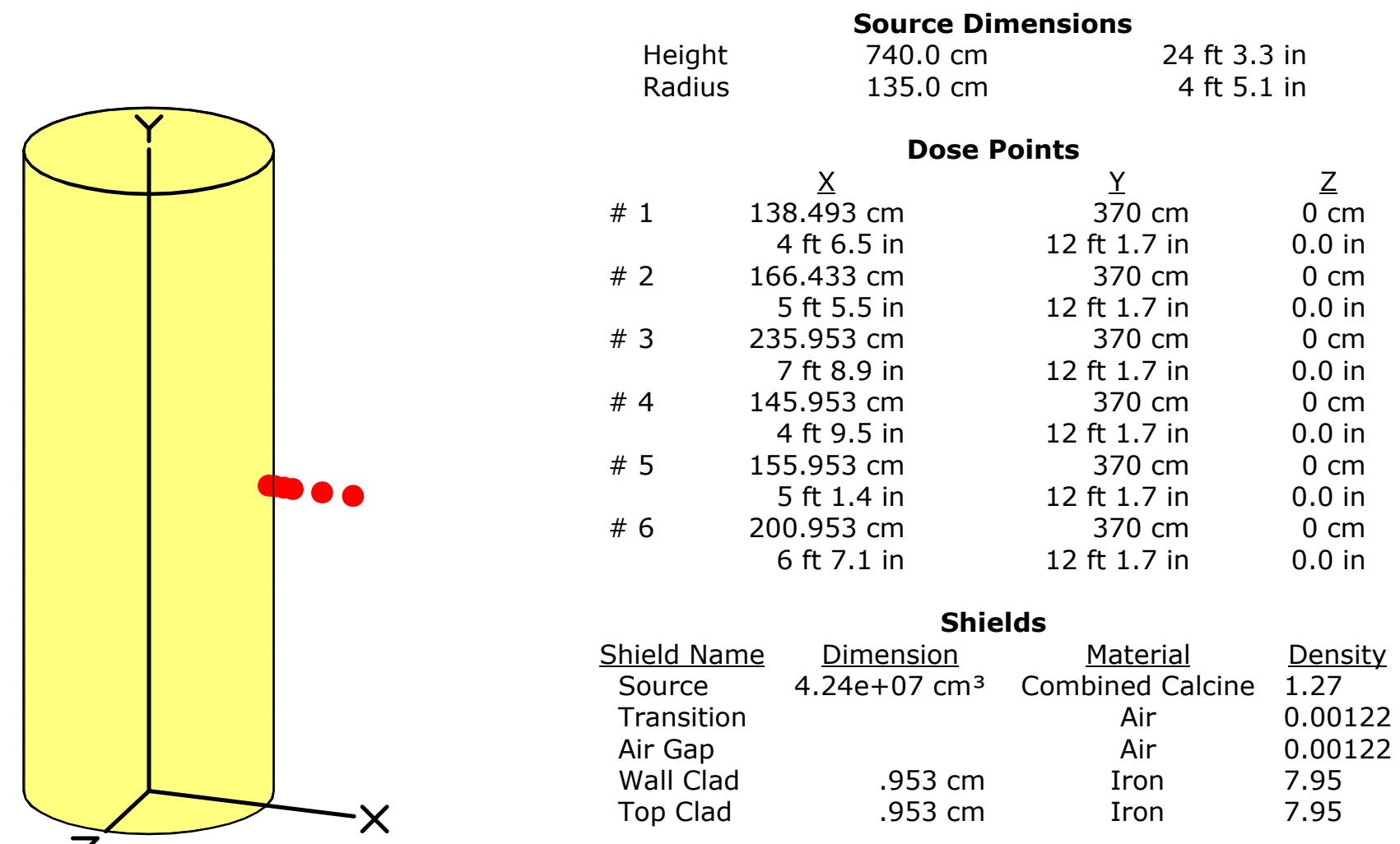

Source Input

Grouping Method : Standard Indices

Number of Groups : 25

Lower Energy Cutoff : 0.015

Photons < 0.015 : Included

Library : ICRP-38

$\begin{array}{lc}\frac{\text { Nuclide }}{\text { Am-241 }} & \underline{\text { curies }} \\ \text { Am-243 } & 8.5162 e+001 \\ \text { Ba-137m } & 7.5927 e-002 \\ \text { Ce-144 } & 1.3262 e-007 \\ \text { Cm-242 } & 1.4109 e-001 \\ \text { Cm-244 } & 5.2114 e-002 \\ \text { Co-60 } & 9.3212 e+000 \\ \text { Cs-134 } & 5.0419 e-001 \\ \text { Cs-135 } & 1.4321 e+000 \\ \text { Cs-137 } & 8.1349 e+004 \\ \text { Eu-152 } & 2.5549 e+000 \\ \text { Eu-154 } & 1.6693 e+002 \\ \text { Eu-155 } & 1.2372 e+001 \\ \text { I-129 } & 7.3722 e-003 \\ \mathrm{Ni}-63 & 4.0632 e+003 \\ \mathrm{~Np}-237 & 7.0333 e-001 \\ \mathrm{~Pa}-233 & 7.0333 e-001 \\ \mathrm{Pm}-147 & 2.8896 e+000\end{array}$

becquerels

$3.1510 \mathrm{e}+012$

$2.0693 e+009$

$2.8531 e+015$

$4.9068 \mathrm{e}+003$

$5.2203 e+009$

$1.9282 e+009$

$3.4488 \mathrm{e}+011$

$1.8655 \mathrm{e}+010$

$5.2987 e+010$

$3.0099 e+015$

$9.4530 \mathrm{e}+010$

$6.1766 \mathrm{e}+012$

$4.5776 e+011$

$2.7277 e+008$

$1.5034 \mathrm{e}+014$

$2.6023 e+010$

$2.6023 e+010$

$1.0691 e+011$ $\mu \mathrm{Ci} / \mathrm{cm}^{3}$

$2.0100 \mathrm{e}+000$

$1.3200 \mathrm{e}-003$

$1.8200 \mathrm{e}+003$

3.1300e-009

3.3300e-003

$1.2300 \mathrm{e}-003$

2.2000e-001

1.1900e-002

3.3800e-002

$1.9200 \mathrm{e}+003$

6.0300e-002

$3.9400 \mathrm{e}+000$

2.9200e-001

$1.7400 \mathrm{e}-004$

$9.5900 \mathrm{e}+001$

$1.6600 \mathrm{e}-002$

$1.6600 \mathrm{e}-002$

6.8200e-002
$\mathrm{Bq} / \mathrm{cm}^{3}$

$7.4370 \mathrm{e}+004$

$4.8840 \mathrm{e}+001$

$6.7340 e+007$

$1.1581 \mathrm{e}-004$

$1.2321 e+002$

$4.5510 e+001$

$8.1400 e+003$

$4.4030 \mathrm{e}+002$

$1.2506 \mathrm{e}+003$

$7.1040 \mathrm{e}+007$

$2.2311 e+003$

$1.4578 \mathrm{e}+005$

$1.0804 \mathrm{e}+004$

$6.4380 \mathrm{e}+000$

$3.5483 e+006$

$6.1420 \mathrm{e}+002$

$6.1420 \mathrm{e}+002$

$2.5234 \mathrm{e}+003$ 
Page : 2

DOS File : Combined Calcine in Bulk Tank.ms6

Run Date: May 3, 2005

Run Time: 2:38:06 PM

Duration : 00:01:05

\begin{tabular}{|c|c|c|c|c|}
\hline Nuclide & curies & becquerels & $\mu \mathrm{Ci} / \mathrm{cm}^{3}$ & $\underline{\mathrm{Bq} / \mathrm{cm}^{3}}$ \\
\hline Pr-144 & $1.3 \overline{262 \mathrm{e}-007}$ & $4.9068 e+003$ & $3.1300 \mathrm{e}-009$ & $1 . \overline{1581 \mathrm{e}-004}$ \\
\hline Pu-238 & $9.6178 e+002$ & $3.5586 e+013$ & $2.2700 \mathrm{e}+001$ & $8.3990 e+005$ \\
\hline Pu-239 & $2.1990 \mathrm{e}+001$ & $8.1361 e+011$ & $5.1900 \mathrm{e}-001$ & $1.9203 e+004$ \\
\hline Pu-240 & $1.3050 \mathrm{e}+001$ & $4.8284 e+011$ & $3.0800 e-001$ & $1.1396 \mathrm{e}+004$ \\
\hline Pu-241 & $3.6395 e+002$ & $1.3466 \mathrm{e}+013$ & $8.5900 e+000$ & $3.1783 e+005$ \\
\hline Pu-242 & $1.7668 \mathrm{e}+000$ & $6.5371 \mathrm{e}+010$ & $4.1700 \mathrm{e}-002$ & $1.5429 e+003$ \\
\hline Ru-106 & $4.7030 e-006$ & $1.7401 e+005$ & $1.1100 \mathrm{e}-007$ & $4.1070 \mathrm{e}-003$ \\
\hline Sb-125 & $1.3177 e+000$ & $4.8754 \mathrm{e}+010$ & $3.1100 \mathrm{e}-002$ & $1.1507 e+003$ \\
\hline Sb-126 & $1.2965 \mathrm{e}-001$ & $4.7970 e+009$ & $3.0600 \mathrm{e}-003$ & $1.1322 \mathrm{e}+002$ \\
\hline Sb-126m & $9.2788 \mathrm{e}-001$ & $3.4332 e+010$ & $2.1900 \mathrm{e}-002$ & $8.1030 e+002$ \\
\hline Sm-151 & $8.3467 e+002$ & $3.0883 e+013$ & $1.9700 e+001$ & $7.2890 e+005$ \\
\hline Sr-90 & $7.2451 e+004$ & $2.6807 e+015$ & $1.7100 e+003$ & $6.3270 e+007$ \\
\hline Tc-99 & $3.4488 \mathrm{e}+001$ & $1.2761 \mathrm{e}+012$ & $8.1400 \mathrm{e}-001$ & $3.0118 e+004$ \\
\hline Th-230 & $3.6014 \mathrm{e}-003$ & $1.3325 e+008$ & $8.5000 e-005$ & $3.1450 \mathrm{e}+000$ \\
\hline Th-231 & $3.4785 e-003$ & $1.2870 \mathrm{e}+008$ & $8.2100 \mathrm{e}-005$ & $3.0377 e+000$ \\
\hline$U-232$ & $2.5167 e-003$ & $9.3119 e+007$ & $5.9400 \mathrm{e}-005$ & $2.1978 e+000$ \\
\hline$U-233$ & $1.6693 e-004$ & $6.1766 e+006$ & $3.9400 e-006$ & $1.4578 \mathrm{e}-001$ \\
\hline$U-234$ & $6.6519 \mathrm{e}-001$ & $2.4612 e+010$ & $1.5700 \mathrm{e}-002$ & $5.8090 e+002$ \\
\hline U-235 & $3.4192 \mathrm{e}-003$ & $1.2651 \mathrm{e}+008$ & $8.0700 e-005$ & $2.9859 e+000$ \\
\hline$U-236$ & $1.0465 \mathrm{e}-002$ & $3.8721 e+008$ & $2.4700 \mathrm{e}-004$ & $9.1390 e+000$ \\
\hline$U-237$ & $9.0670 \mathrm{e}-003$ & $3.3548 e+008$ & $2.1400 \mathrm{e}-004$ & $7.9180 e+000$ \\
\hline$U-238$ & $1.2795 \mathrm{e}-003$ & $4.7343 e+007$ & $3.0200 e-005$ & $1.1174 \mathrm{e}+000$ \\
\hline Y-90 & $7.2451 e+004$ & $2.6807 e+015$ & $1.7100 e+003$ & $6.3270 e+007$ \\
\hline
\end{tabular}

\section{Integration Parameters}

Radial 24

Circumferential 24

Y Direction (axial) $\quad 24$

$\begin{array}{cc}\frac{\begin{array}{c}\text { Energy } \\ \text { MeV }\end{array}}{} & \begin{array}{c}\text { Activity } \\ \text { photons/sec }\end{array} \\ 0.015 & \\ 0.02 & 2.603 \mathrm{e}+12 \\ 0.03 & 1.450 \mathrm{e}+12 \\ 0.04 & 1.729 \mathrm{e}+14 \\ 0.05 & 4.257 \mathrm{e}+13 \\ 0.06 & 3.767 \mathrm{e}+11 \\ 0.08 & 1.132 \mathrm{e}+12 \\ 0.1 & 1.488 \mathrm{e}+11 \\ 0.15 & 2.636 \mathrm{e}+12 \\ 0.2 & 8.425 \mathrm{e}+09 \\ 0.3 & 4.358 \mathrm{e}+11 \\ 0.4 & 5.185 \mathrm{e}+10 \\ 0.5 & 1.057 \mathrm{e}+11 \\ 0.6 & 3.368 \mathrm{e}+10 \\ 0.8 & 2.562 \mathrm{e}+15 \\ 1.0 & 2.363 \mathrm{e}+12 \\ 1.5 & 2.308 \mathrm{e}+12 \\ 2.0 & 2.786 \mathrm{e}+12 \\ 3.0 & 1.173 \mathrm{e}+08 \\ & 1.242 \mathrm{e}+04\end{array}$

Results - Dose Point \# 1 - $(138.493,370,0) \mathbf{c m}$

\begin{tabular}{|c|c|c|c|}
\hline$\frac{\text { Fluence Rate }}{\mathrm{MeV} / \mathrm{cm}^{2} / \mathrm{sec}}$ & $\frac{\text { Fluence Rate }}{\mathrm{MeV} / \mathrm{cm}^{2} / \mathrm{sec}}$ & $\frac{\text { Exposure Rate }}{\mathrm{mR} / \mathrm{hr}}$ & $\frac{\text { Exposure Rate }}{\mathrm{mR} / \mathrm{hr}}$ \\
\hline No Buildup & With Buildup & No Buildup & With Buildup \\
\hline $2.274 \mathrm{e}-236$ & $8.613 e-22$ & $1.951 \mathrm{e}-237$ & $7.387 e-23$ \\
\hline $1.370 \mathrm{e}-124$ & $6.085 e-22$ & $4.745 e-126$ & $2.108 e-23$ \\
\hline $6.676 e-36$ & $1.394 \mathrm{e}-19$ & $6.616 e-38$ & $1.382 \mathrm{e}-21$ \\
\hline $5.235 \mathrm{e}-14$ & $7.858 \mathrm{e}-14$ & $2.315 \mathrm{e}-16$ & $3.475 \mathrm{e}-16$ \\
\hline $1.032 \mathrm{e}-07$ & $1.931 \mathrm{e}-07$ & $2.748 \mathrm{e}-10$ & $5.143 e-10$ \\
\hline $1.985 \mathrm{e}-03$ & $4.496 \mathrm{e}-03$ & $3.944 \mathrm{e}-06$ & $8.930 e-06$ \\
\hline $3.980 \mathrm{e}-01$ & $1.163 e+00$ & $6.298 e-04$ & $1.840 \mathrm{e}-03$ \\
\hline $1.244 \mathrm{e}+02$ & $4.185 e+02$ & $1.903 e-01$ & $6.403 e-01$ \\
\hline $5.600 e+00$ & $2.150 e+01$ & $9.222 \mathrm{e}-03$ & $3.541 \mathrm{e}-02$ \\
\hline $8.152 e+02$ & $3.313 e+03$ & $1.439 e+00$ & $5.847 e+00$ \\
\hline $2.693 e+02$ & $1.085 e+03$ & $5.108 \mathrm{e}-01$ & $2.057 e+00$ \\
\hline $9.978 e+02$ & $3.812 e+03$ & $1.944 \mathrm{e}+00$ & $7.428 e+00$ \\
\hline $4.908 e+02$ & $1.760 e+03$ & $9.634 \mathrm{e}-01$ & $3.456 \mathrm{e}+00$ \\
\hline $5.277 e+07$ & $1.779 e+08$ & $1.030 e+05$ & $3.472 e+05$ \\
\hline $8.346 e+04$ & $2.537 e+05$ & $1.587 e+02$ & $4.826 e+02$ \\
\hline $1.236 \mathrm{e}+05$ & $3.465 e+05$ & $2.278 \mathrm{e}+02$ & $6.387 e+02$ \\
\hline $3.154 \mathrm{e}+05$ & $7.649 e+05$ & $5.306 e+02$ & $1.287 e+03$ \\
\hline $2.206 e+01$ & $4.923 e+01$ & $3.411 \mathrm{e}-02$ & $7.613 e-02$ \\
\hline $4.570 \mathrm{e}-03$ & $9.088 \mathrm{e}-03$ & $6.201 \mathrm{e}-06$ & $1.233 \mathrm{e}-05$ \\
\hline
\end{tabular}


Page : 3

DOS File : Combined Calcine in Bulk Tank.ms6

Run Date: May 3, 2005

Run Time: 2:38:06 PM

Duration : 00:01:05

\begin{tabular}{|c|c|}
\hline$\frac{\text { Energy }}{\underline{\text { MeV }}}$ & $\frac{\text { Activity }}{\text { photons/sec }}$ \\
\hline TOTALS: & $2.794 e+15$ \\
\hline$\frac{\text { Energy }}{\mathrm{MeV}}$ & $\begin{array}{c}\text { Activity } \\
\text { photons/sec }\end{array}$ \\
\hline $\begin{array}{c}0.015 \\
0.02\end{array}$ & $\begin{array}{l}2.603 e+12 \\
1.450 e+12\end{array}$ \\
\hline 0.03 & $1.729 \mathrm{e}+14$ \\
\hline 0.04 & $4.257 e+13$ \\
\hline 0.05 & $3.767 e+11$ \\
\hline 0.06 & $1.132 \mathrm{e}+12$ \\
\hline 0.08 & $1.488 \mathrm{e}+11$ \\
\hline 0.1 & $2.636 \mathrm{e}+12$ \\
\hline 0.15 & $8.425 e+09$ \\
\hline 0.2 & $4.358 e+11$ \\
\hline 0.3 & $5.185 e+10$ \\
\hline 0.4 & $1.057 e+11$ \\
\hline 0.5 & $3.368 \mathrm{e}+10$ \\
\hline 0.6 & $2.562 e+15$ \\
\hline 0.8 & $2.363 e+12$ \\
\hline 1.0 & $2.308 \mathrm{e}+12$ \\
\hline 1.5 & $2.786 e+12$ \\
\hline 2.0 & $1.173 e+08$ \\
\hline 3.0 & $1.242 \mathrm{e}+04$ \\
\hline
\end{tabular}

$\begin{array}{ccc}\frac{\text { Fluence Rate }}{\text { MeV/cm } / \mathrm{sec}} & \frac{\text { Fluence Rate }}{\text { MeV/cm } 2 / \mathrm{sec}} & \frac{\text { Exposure Rate }}{\mathrm{mR} / \mathrm{hr}} \\ \frac{\text { No Buildup }}{5.329 \mathrm{e}+07} & \frac{\text { With Buildup }}{1.793 \mathrm{e}+08} & \frac{\text { No Buildup }}{1.039 \mathrm{e}+05}\end{array}$

Exposure Rate $\mathrm{mR} / \mathrm{hr}$

With Buildup

$3.497 e+05$

\section{Results - Dose Point \# 2 - $(166.433,370,0) \mathbf{c m}$}

\begin{tabular}{|c|c|c|c|}
\hline Fluence Rate & Fluence Rate & Exposure Rate & Exposure Rate \\
\hline $\mathrm{MeV} / \mathrm{cm}^{2} / \mathrm{sec}$ & $\mathrm{MeV} / \mathrm{cm}^{2} / \mathrm{sec}$ & $\underline{\mathrm{mR} / \mathrm{hr}}$ & $\mathrm{mR} / \mathrm{hr}$ \\
\hline No Buildup & With Buildup & No Buildup & With Buildup \\
\hline $5.145 e-189$ & $6.070 e-22$ & $4.413 e-190$ & $5.207 e-23$ \\
\hline $9.883 e-88$ & $4.289 e-22$ & $3.423 e-89$ & $1.486 \mathrm{e}-23$ \\
\hline $2.597 e-25$ & $9.828 e-20$ & $2.574 \mathrm{e}-27$ & $9.740 \mathrm{e}-22$ \\
\hline $3.755 e-10$ & $5.504 \mathrm{e}-10$ & $1.661 \mathrm{e}-12$ & $2.434 \mathrm{e}-12$ \\
\hline $3.345 e-06$ & $5.933 e-06$ & $8.912 \mathrm{e}-09$ & $1.580 \mathrm{e}-08$ \\
\hline $7.339 e-03$ & $1.553 \mathrm{e}-02$ & $1.458 \mathrm{e}-05$ & $3.085 e-05$ \\
\hline $3.685 e-01$ & $1.010 \mathrm{e}+00$ & $5.832 \mathrm{e}-04$ & $1.598 \mathrm{e}-03$ \\
\hline $8.749 e+01$ & $2.849 e+02$ & $1.339 \mathrm{e}-01$ & $4.359 \mathrm{e}-01$ \\
\hline $3.833 e+00$ & $1.503 e+01$ & $6.312 \mathrm{e}-03$ & $2.476 \mathrm{e}-02$ \\
\hline $5.726 e+02$ & $2.397 e+03$ & $1.011 \mathrm{e}+00$ & $4.230 e+00$ \\
\hline $1.932 \mathrm{e}+02$ & $7.965 e+02$ & $3.666 \mathrm{e}-01$ & $1.511 \mathrm{e}+00$ \\
\hline $7.221 e+02$ & $2.806 e+03$ & $1.407 e+00$ & $5.468 e+00$ \\
\hline $3.568 e+02$ & $1.296 \mathrm{e}+03$ & $7.004 \mathrm{e}-01$ & $2.544 \mathrm{e}+00$ \\
\hline $3.847 e+07$ & $1.309 e+08$ & $7.509 e+04$ & $2.556 e+05$ \\
\hline $6.103 e+04$ & $1.865 e+05$ & $1.161 \mathrm{e}+02$ & $3.547 e+02$ \\
\hline $9.050 e+04$ & $2.544 \mathrm{e}+05$ & $1.668 e+02$ & $4.689 e+02$ \\
\hline $2.312 \mathrm{e}+05$ & $5.602 e+05$ & $3.889 e+02$ & $9.425 e+02$ \\
\hline $1.617 e+01$ & $3.599 e+01$ & $2.500 e-02$ & $5.566 \mathrm{e}-02$ \\
\hline $3.348 \mathrm{e}-03$ & $6.639 e-03$ & $4.542 \mathrm{e}-06$ & $9.007 e-06$ \\
\hline $3.885 e+07$ & $1.319 e+08$ & $7.576 e+04$ & $2.574 \mathrm{e}+05$ \\
\hline
\end{tabular}

TOTALS: $\quad 2.794 \mathrm{e}+15$

Results - Dose Point \# 3 - $(235.953,370,0) \mathbf{c m}$

$\begin{array}{cc}\begin{array}{cc}\text { Energy } \\ \text { MeV }\end{array} & \begin{array}{c}\text { Activity } \\ \text { photons/sec }\end{array} \\ 0.015 & \\ 0.02 & 2.603 \mathrm{e}+12 \\ 0.03 & 1.450 \mathrm{e}+12 \\ 0.04 & 1.729 \mathrm{e}+14 \\ 0.05 & 4.257 \mathrm{e}+13 \\ 0.06 & 3.767 \mathrm{e}+11 \\ 0.08 & 1.132 \mathrm{e}+12 \\ 0.1 & 1.488 \mathrm{e}+11 \\ 0.15 & 2.636 \mathrm{e}+12 \\ 0.2 & 8.425 \mathrm{e}+09 \\ 0.3 & 4.358 \mathrm{e}+11 \\ 0.4 & 5.185 \mathrm{e}+10 \\ 0.5 & 1.057 \mathrm{e}+11 \\ 0.6 & 3.368 \mathrm{e}+10 \\ 0.8 & 2.562 \mathrm{e}+15 \\ 1.0 & 2.363 \mathrm{e}+12 \\ 1.5 & 2.308 \mathrm{e}+12 \\ 2.0 & 2.786 \mathrm{e}+12 \\ 3.0 & 1.173 \mathrm{e}+08 \\ & 1.242 \mathrm{e}+04\end{array}$

Fluence Rate

$\mathrm{MeV} / \mathrm{cm}^{2} / \mathrm{sec}$

No Buildup

$1.243 e-188$

$5.226 \mathrm{e}-88$

$1.380 \mathrm{e}-25$

$2.603 e-10$

$2.378 \mathrm{e}-06$

5.073e-03

$2.498 \mathrm{e}-01$

$5.881 \mathrm{e}+01$

$2.550 \mathrm{e}+00$

$3.790 \mathrm{e}+02$

$1.273 e+02$

$4.745 e+02$

$2.341 \mathrm{e}+02$

$2.520 \mathrm{e}+07$

$3.988 \mathrm{e}+04$

$5.901 \mathrm{e}+04$

$1.501 \mathrm{e}+05$

$1.046 \mathrm{e}+01$

2.158e-03

$2.545 e+07$
Fluence Rate

$\mathrm{MeV} / \mathrm{cm}^{2} / \mathrm{sec}$

With Buildup

$3.475 \mathrm{e}-22$

$2.455 \mathrm{e}-22$

$5.625 \mathrm{e}-20$

$3.818 \mathrm{e}-10$

$4.217 \mathrm{e}-06$

$1.073 \mathrm{e}-02$

6.843e-01

$1.914 \mathrm{e}+02$

$9.983 e+00$

$1.580 \mathrm{e}+03$

$5.214 \mathrm{e}+02$

$1.831 \mathrm{e}+03$

$8.436 \mathrm{e}+02$

$8.505 e+07$

$1.208 \mathrm{e}+05$

$1.643 e+05$

$3.603 e+05$

$2.308 \mathrm{e}+01$

4.241e-03

$8.570 e+07$
Exposure Rate $\mathrm{mR} / \mathrm{hr}$

No Buildup

$1.066 \mathrm{e}-189$

$1.810 \mathrm{e}-89$

$1.368 \mathrm{e}-27$

$1.151 \mathrm{e}-12$

6.336e-09

$1.008 \mathrm{e}-05$

3.954e-04

$8.998 \mathrm{e}-02$

4.200e-03

6.690e-01

2.415e-01

9.246e-01

4.594e-01

$4.918 \mathrm{e}+04$

$7.585 \mathrm{e}+01$

$1.088 \mathrm{e}+02$

$2.525 \mathrm{e}+02$

$1.618 \mathrm{e}-02$

2.928e-06

$4.962 e+04$
Exposure Rate $\mathrm{mR} / \mathrm{hr}$

With Buildup

$2.980 \mathrm{e}-23$

$8.504 \mathrm{e}-24$

$5.575 \mathrm{e}-22$

$1.689 \mathrm{e}-12$

$1.123 \mathrm{e}-08$

$2.130 \mathrm{e}-05$

$1.083 \mathrm{e}-03$

$2.929 \mathrm{e}-01$

$1.644 \mathrm{e}-02$

$2.789 \mathrm{e}+00$

$9.891 \mathrm{e}-01$

$3.567 e+00$

$1.656 \mathrm{e}+00$

$1.660 \mathrm{e}+05$

$2.297 \mathrm{e}+02$

$3.029 \mathrm{e}+02$

$6.061 \mathrm{e}+02$

$3.568 \mathrm{e}-02$

5.754e-06

$1.672 \mathrm{e}+05$

Results - Dose Point \# 4 - $(145.953,370,0) \mathrm{cm}$ 
Page : 4

DOS File : Combined Calcine in Bulk Tank.ms6

Run Date: May 3, 2005

Run Time: 2:38:06 PM

Duration : 00:01:05

$\begin{array}{cc}\frac{\text { Energy }}{\underline{\mathrm{MeV}}} & \begin{array}{c}\text { Activity } \\ \text { photons/sec }\end{array} \\ 0.015 & \\ 0.02 & 2.603 \mathrm{e}+12 \\ 0.03 & 1.450 \mathrm{e}+12 \\ 0.04 & 1.729 \mathrm{e}+14 \\ 0.05 & 4.257 \mathrm{e}+13 \\ 0.06 & 3.767 \mathrm{e}+11 \\ 0.08 & 1.132 \mathrm{e}+12 \\ 0.1 & 1.488 \mathrm{e}+11 \\ 0.15 & 2.636 \mathrm{e}+12 \\ 0.2 & 8.425 \mathrm{e}+09 \\ 0.3 & 4.358 \mathrm{e}+11 \\ 0.4 & 5.185 \mathrm{e}+10 \\ 0.5 & 1.057 \mathrm{e}+11 \\ 0.6 & 3.368 \mathrm{e}+10 \\ 0.8 & 2.562 \mathrm{e}+15 \\ 1.0 & 2.363 \mathrm{e}+12 \\ 1.5 & 2.308 \mathrm{e}+12 \\ 2.0 & 2.786 \mathrm{e}+12 \\ 3.0 & 1.173 \mathrm{e}+08 \\ & 1.242 \mathrm{e}+04\end{array}$

\begin{tabular}{|c|c|c|}
\hline Fluence Rate & Fluence Rate & Exposure Rate \\
\hline $\mathrm{MeV} / \mathrm{cm}^{2} / \mathrm{sec}$ & $\mathrm{MeV} / \mathrm{cm}^{2} / \mathrm{sec}$ & $\mathrm{mR} / \mathrm{hr}$ \\
\hline No Buildup & With Buildup & No Buildup \\
\hline $2.804 \mathrm{e}-198$ & $7.681 \mathrm{e}-22$ & $2.405 e-199$ \\
\hline $1.415 e-91$ & $5.427 e-22$ & $4.902 e-93$ \\
\hline $6.049 e-26$ & $1.244 \mathrm{e}-19$ & $5.995 e-28$ \\
\hline $4.773 e-10$ & $7.013 e-10$ & $2.111 \mathrm{e}-12$ \\
\hline $5.449 \mathrm{e}-06$ & $9.661 \mathrm{e}-06$ & $1.452 \mathrm{e}-08$ \\
\hline $1.092 \mathrm{e}-02$ & $2.295 e-02$ & $2.169 \mathrm{e}-05$ \\
\hline $4.612 \mathrm{e}-01$ & $1.248 \mathrm{e}+00$ & $7.298 \mathrm{e}-04$ \\
\hline $1.039 e+02$ & $3.358 e+02$ & $1.590 \mathrm{e}-01$ \\
\hline $4.515 e+00$ & $1.776 e+01$ & $7.435 e-03$ \\
\hline $6.780 e+02$ & $2.858 \mathrm{e}+03$ & $1.197 \mathrm{e}+00$ \\
\hline $2.302 e+02$ & $9.576 e+02$ & $4.367 \mathrm{e}-01$ \\
\hline $8.631 e+02$ & $3.387 e+03$ & $1.682 \mathrm{e}+00$ \\
\hline $4.275 e+02$ & $1.568 \mathrm{e}+03$ & $8.391 \mathrm{e}-01$ \\
\hline $4.617 e+07$ & $1.587 e+08$ & $9.013 e+04$ \\
\hline $7.346 e+04$ & $2.265 e+05$ & $1.397 e+02$ \\
\hline $1.092 e+05$ & $3.094 e+05$ & $2.012 e+02$ \\
\hline $2.799 e+05$ & $6.830 \mathrm{e}+05$ & $4.709 e+02$ \\
\hline $1.962 \mathrm{e}+01$ & $4.394 e+01$ & $3.034 \mathrm{e}-02$ \\
\hline $4.072 \mathrm{e}-03$ & $8.115 \mathrm{e}-03$ & $5.525 \mathrm{e}-06$ \\
\hline $4.664 \mathrm{e}+07$ & $1.599 \mathrm{e}+08$ & $9.094 e+04$ \\
\hline
\end{tabular}

Exposure Rate $\mathrm{mR} / \mathrm{hr}$

With Buildup

$6.588 \mathrm{e}-23$

$1.880 \mathrm{e}-23$

$1.232 \mathrm{e}-21$

$3.102 \mathrm{e}-12$

$2.574 \mathrm{e}-08$

$4.558 \mathrm{e}-05$

$1.975 \mathrm{e}-03$

$5.137 \mathrm{e}-01$

$2.924 \mathrm{e}-02$

$5.044 \mathrm{e}+00$

$1.817 \mathrm{e}+00$

$6.600 \mathrm{e}+00$

$3.078 \mathrm{e}+00$

$3.097 \mathrm{e}+05$

$4.308 \mathrm{e}+02$

$5.703 e+02$

$1.149 \mathrm{e}+03$

$6.795 \mathrm{e}-02$

$1.101 \mathrm{e}-05$

TOTALS: $\quad 2.794 \mathrm{e}+15$

Results - Dose Point \# 5 - $(155.953,370,0) \mathrm{cm}$

\begin{tabular}{|c|c|}
\hline $\begin{array}{c}\text { Energy } \\
\underline{\mathrm{MeV}}\end{array}$ & $\begin{array}{c}\text { Activity } \\
\text { photons/sec }\end{array}$ \\
\hline 0.015 & $2.603 e+12$ \\
\hline 0.02 & $1.450 e+12$ \\
\hline 0.03 & $1.729 e+14$ \\
\hline 0.04 & $4.257 e+13$ \\
\hline 0.05 & $3.767 e+11$ \\
\hline 0.06 & $1.132 \mathrm{e}+12$ \\
\hline 0.08 & $1.488 e+11$ \\
\hline 0.1 & $2.636 e+12$ \\
\hline 0.15 & $8.425 e+09$ \\
\hline 0.2 & $4.358 e+11$ \\
\hline 0.3 & $5.185 e+10$ \\
\hline 0.4 & $1.057 e+11$ \\
\hline 0.5 & $3.368 e+10$ \\
\hline 0.6 & $2.562 e+15$ \\
\hline 0.8 & $2.363 e+12$ \\
\hline 1.0 & $2.308 e+12$ \\
\hline 1.5 & $2.786 e+12$ \\
\hline 2.0 & $1.173 e+08$ \\
\hline 3.0 & $1.242 \mathrm{e}+04$ \\
\hline
\end{tabular}

Fluence Rate

$\mathrm{MeV} / \mathrm{cm}^{2} / \mathrm{sec}$

No Buildup

$1.571 \mathrm{e}-190$

$2.973 \mathrm{e}-88$

$3.002 \mathrm{e}-25$

$5.088 \mathrm{e}-10$

$3.859 \mathrm{e}-06$

$7.799 \mathrm{e}-03$

$3.912 \mathrm{e}-01$

$9.388 \mathrm{e}+01$

Fluence Rate

$\mathrm{MeV} / \mathrm{cm}^{2} / \mathrm{sec}$

With Buildup

$6.789 \mathrm{e}-22$

4.797e-22

$1.099 \mathrm{e}-19$

$7.454 \mathrm{e}-10$

$6.825 \mathrm{e}-06$

$1.647 \mathrm{e}-02$

$1.073 e+00$

Exposure Rate $\mathrm{mR} / \mathrm{hr}$

No Buildup

$1.347 \mathrm{e}-191$

$1.030 \mathrm{e}-89$

$2.975 \mathrm{e}-27$

2.250e-12

$1.028 \mathrm{e}-08$

$1.549 \mathrm{e}-05$

6.191e-04

$1.436 \mathrm{e}-01$

$4.155 \mathrm{e}+00$

$3.064 \mathrm{e}+02$

$6.843 \mathrm{e}-03$

$6.224 \mathrm{e}+02$

$1.634 \mathrm{e}+01$

$1.098 \mathrm{e}+00$

$2.104 \mathrm{e}+02$

$3.992 \mathrm{e}-01$

$7.872 \mathrm{e}+02$

$1.534 \mathrm{e}+00$

$3.893 e+02$

7.641e-01

$4.200 \mathrm{e}+07$

$6.669 \mathrm{e}+04$

$8.197 e+04$

$9.898 \mathrm{e}+04$

$1.269 \mathrm{e}+02$

$2.532 \mathrm{e}+05$

$1.825 \mathrm{e}+02$

$4.259 \mathrm{e}+02$

$1.772 \mathrm{e}+01$

2.740e-02

$3.672 \mathrm{e}-03$

4.982e-06

$3.118 \mathrm{e}+05$

TOTALS: $\quad 2.794 \mathrm{e}+15$

$4.242 \mathrm{e}+07$

$1.445 \mathrm{e}+08$

$8.271 e+04$

Exposure Rate $\mathrm{mR} / \mathrm{hr}$

With Buildup

$5.824 \mathrm{e}-23$

$1.662 \mathrm{e}-23$

$1.089 \mathrm{e}-21$

$3.297 \mathrm{e}-12$

$1.818 \mathrm{e}-08$

$3.270 \mathrm{e}-05$

$1.699 \mathrm{e}-03$

$4.688 \mathrm{e}-01$

$2.690 \mathrm{e}-02$

$4.608 \mathrm{e}+00$

$1.650 \mathrm{e}+00$

$5.981 \mathrm{e}+00$

$2.786 \mathrm{e}+00$

$2.800 \mathrm{e}+05$

$3.889 \mathrm{e}+02$

$5.144 \mathrm{e}+02$

$1.035 \mathrm{e}+03$

$6.114 \mathrm{e}-02$

9.897e-06

$2.819 e+05$

$\begin{array}{cc}\frac{\underline{\text { Energy }}}{\text { MeV }} & \begin{array}{c}\text { Activity } \\ \text { photons/sec }\end{array} \\ 0.015 & 2.603 \mathrm{e}+12 \\ 0.02 & 1.450 \mathrm{e}+12\end{array}$

Results - Dose Point \# 6 - $(200.953,370,0) \mathrm{cm}$

$\begin{array}{ccc}\frac{\text { Fluence Rate }}{\text { MeV/cm } 2 / \mathrm{sec}} & \frac{\text { Fluence Rate }}{\text { MeV/cm } \mathrm{cm}^{2} / \mathrm{sec}} & \frac{\text { Exposure Rate }}{\mathrm{mR} / \mathrm{hr}} \\ \frac{\text { No Buildup }}{1.845 \mathrm{e}-188} & \frac{\text { With Buildup }}{4.476 \mathrm{e}-22} & \text { No Buildup } \\ 8.272 \mathrm{e}-88 & 3.162 \mathrm{e}-22 & 1.583 \mathrm{e}-189 \\ & & 2.865 \mathrm{e}-89\end{array}$

Exposure Rate $\mathrm{mR} / \mathrm{hr}$

With Buildup

$3.839 \mathrm{e}-23$

$1.095 \mathrm{e}-23$

E-14 
Page : 5

DOS File : Combined Calcine in Bulk Tank.ms6

Run Date: May 3, 2005

Run Time: 2:38:06 PM

Duration : 00:01:05

$\begin{array}{ccc}\begin{array}{cc}\text { Energy } \\ \text { MeV }\end{array} & & \begin{array}{c}\text { Activity } \\ \text { photons } / \mathrm{sec}\end{array} \\ 0.03 & & 1.729 \mathrm{e}+14 \\ 0.04 & & 4.257 \mathrm{e}+13 \\ 0.05 & & 3.767 \mathrm{e}+11 \\ 0.06 & & 1.132 \mathrm{e}+12 \\ 0.08 & & 1.488 \mathrm{e}+11 \\ 0.1 & & 2.636 \mathrm{e}+12 \\ 0.15 & & 8.425 \mathrm{e}+09 \\ 0.2 & & 4.358 \mathrm{e}+11 \\ 0.3 & & 5.185 \mathrm{e}+10 \\ 0.4 & & 1.057 \mathrm{e}+11 \\ 0.5 & 3.368 \mathrm{e}+10 \\ 0.6 & 2.562 \mathrm{e}+15 \\ 0.8 & 2.363 \mathrm{e}+12 \\ 1.0 & 2.308 \mathrm{e}+12 \\ 1.5 & 2.786 \mathrm{e}+12 \\ 2.0 & 1.173 \mathrm{e}+08 \\ 3.0 & 1.242 \mathrm{e}+04\end{array}$

\begin{tabular}{|c|c|}
\hline$\frac{\text { Fluence Rate }}{\mathrm{MeV} / \mathrm{cm}^{2} / \mathrm{sec}}$ & $\frac{\text { Fluence Rate }}{\mathrm{MeV} / \mathrm{cm}^{2} / \mathrm{sec}}$ \\
\hline & $\frac{\mathrm{m} / \mathrm{ev} / \mathrm{cm} / \mathrm{sec}}{\text { With Buildup }}$ \\
\hline$\frac{\text { No Buildup }}{1.537 e-25}$ & $7.246 \mathrm{e}-20$ \\
\hline $3.120 \mathrm{e}-10$ & $4.575 e-10$ \\
\hline $2.830 \mathrm{e}-06$ & $5.016 e-06$ \\
\hline $6.037 e-03$ & $1.276 \mathrm{e}-02$ \\
\hline $2.981 \mathrm{e}-01$ & $8.164 \mathrm{e}-01$ \\
\hline $7.038 e+01$ & $2.291 \mathrm{e}+02$ \\
\hline $3.061 \mathrm{e}+00$ & $1.199 \mathrm{e}+01$ \\
\hline $4.559 e+02$ & $1.903 e+03$ \\
\hline $1.534 \mathrm{e}+02$ & $6.305 e+02$ \\
\hline $5.727 e+02$ & $2.217 \mathrm{e}+03$ \\
\hline $2.827 e+02$ & $1.023 e+03$ \\
\hline $3.046 e+07$ & $1.032 \mathrm{e}+08$ \\
\hline $4.826 e+04$ & $1.468 e+05$ \\
\hline $7.148 \mathrm{e}+04$ & $1.999 \mathrm{e}+05$ \\
\hline $1.822 \mathrm{e}+05$ & $4.393 e+05$ \\
\hline $1.272 \mathrm{e}+01$ & $2.818 e+01$ \\
\hline $2.629 \mathrm{e}-03$ & $5.189 \mathrm{e}-03$ \\
\hline $3.076 e+07$ & $1.040 e+08$ \\
\hline
\end{tabular}

$\frac{\text { Exposure Rate }}{\mathrm{mR/hr}}$

No Buildup

$1.523 \mathrm{e}-27$

$1.380 \mathrm{e}-12$

$7.538 \mathrm{e}-09$

$1.199 \mathrm{e}-05$

$4.718 \mathrm{e}-04$

$1.077 \mathrm{e}-01$

5.041e-03

8.046e-01

2.911e-01

$1.116 \mathrm{e}+00$

5.549e-01

$5.945 e+04$

$9.179 \mathrm{e}+01$

$1.318 \mathrm{e}+02$

$3.065 \mathrm{e}+02$

$1.967 \mathrm{e}-02$

3.566e-06

$5.998 e+04$
Exposure Rate $\mathrm{mR} / \mathrm{hr}$

With Buildup

$7.182 \mathrm{e}-22$

$2.023 \mathrm{e}-12$

$1.336 \mathrm{e}-08$

$2.535 \mathrm{e}-05$

$1.292 \mathrm{e}-03$

$3.504 \mathrm{e}-01$

$1.974 \mathrm{e}-02$

$3.360 \mathrm{e}+00$

$1.196 \mathrm{e}+00$

$4.321 \mathrm{e}+00$

$2.008 \mathrm{e}+00$

$2.015 \mathrm{e}+05$

$2.792 \mathrm{e}+02$

$3.686 \mathrm{e}+02$

$7.391 \mathrm{e}+02$

$4.358 \mathrm{e}-02$

7.040e-06

$2.029 e+05$ 
Page : 1

DOS File : Combined Calcine in Day Tank.ms6

Run Date: April 5, 2005

Run Time: 1:17:38 PM

Duration : 00:01:04
File Ref:

Date:

By:

Checked:

\section{Case Title: Combined Calcine \\ Description: Combined Calcine in Day Tank (3/8" 316 SS) Geometry: 7 - Cylinder Volume - Side Shields}

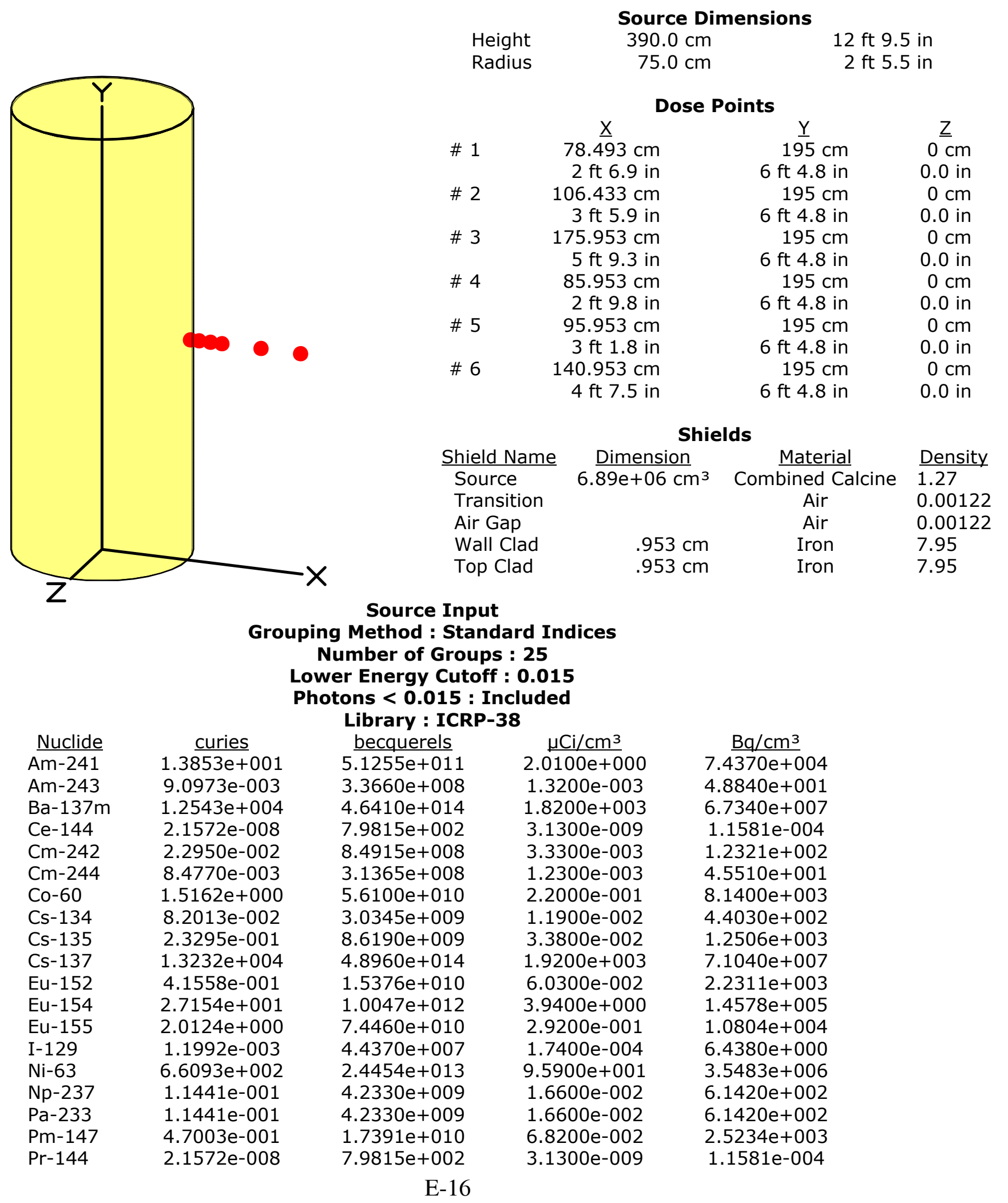


Page : 2

DOS File : Combined Calcine in Day Tank.ms6

Run Date: April 5, 2005

Run Time: 1:17:38 PM

Duration : 00:01:04

\begin{tabular}{|c|c|c|c|c|}
\hline Nuclide & curies & becquerels & $\mu \mathrm{Ci} / \mathrm{cm}^{3}$ & $\mathrm{~Bq} / \mathrm{cm}^{3}$ \\
\hline Pu-238 & $1.5645 \mathrm{e}+002$ & $5 . \overline{7885 e+012}$ & $2.2700 \mathrm{e}+001$ & $8.3990 \mathrm{e}+005$ \\
\hline Pu-239 & $3.5769 e+000$ & $1.3234 \mathrm{e}+011$ & $5.1900 \mathrm{e}-001$ & $1.9203 e+004$ \\
\hline Pu-240 & $2.1227 \mathrm{e}+000$ & $7.8540 e+010$ & $3.0800 \mathrm{e}-001$ & $1.1396 \mathrm{e}+004$ \\
\hline Pu-241 & $5.9201 e+001$ & $2.1904 \mathrm{e}+012$ & $8.5900 e+000$ & $3.1783 e+005$ \\
\hline Pu-242 & $2.8739 \mathrm{e}-001$ & $1.0633 e+010$ & $4.1700 \mathrm{e}-002$ & $1.5429 \mathrm{e}+003$ \\
\hline Ru-106 & $7.6500 \mathrm{e}-007$ & $2.8305 e+004$ & $1.1100 \mathrm{e}-007$ & $4.1070 \mathrm{e}-003$ \\
\hline Sb-125 & $2.1434 \mathrm{e}-001$ & $7.9305 e+009$ & $3.1100 \mathrm{e}-002$ & $1.1507 e+003$ \\
\hline Sb-126 & $2.1089 \mathrm{e}-002$ & $7.8030 e+008$ & $3.0600 \mathrm{e}-003$ & $1.1322 \mathrm{e}+002$ \\
\hline Sb-126m & $1.5093 \mathrm{e}-001$ & $5.5845 e+009$ & $2.1900 \mathrm{e}-002$ & $8.1030 e+002$ \\
\hline Sm-151 & $1.3577 \mathrm{e}+002$ & $5.0235 e+012$ & $1.9700 \mathrm{e}+001$ & $7.2890 e+005$ \\
\hline Sr-90 & $1.1785 e+004$ & $4.3605 e+014$ & $1.7100 e+003$ & $6.3270 e+007$ \\
\hline Tc-99 & $5.6100 e+000$ & $2.0757 e+011$ & $8.1400 e-001$ & $3.0118 e+004$ \\
\hline Th-230 & $5.8581 \mathrm{e}-004$ & $2.1675 \mathrm{e}+007$ & $8.5000 \mathrm{e}-005$ & $3.1450 \mathrm{e}+000$ \\
\hline Th-231 & $5.6582 e-004$ & $2.0935 e+007$ & $8.2100 \mathrm{e}-005$ & $3.0377 e+000$ \\
\hline$U-232$ & $4.0938 \mathrm{e}-004$ & $1.5147 \mathrm{e}+007$ & $5.9400 \mathrm{e}-005$ & $2.1978 \mathrm{e}+000$ \\
\hline$U-233$ & $2.7154 \mathrm{e}-005$ & $1.0047 e+006$ & $3.9400 \mathrm{e}-006$ & $1.4578 \mathrm{e}-001$ \\
\hline$U-234$ & $1.0820 \mathrm{e}-001$ & $4.0035 e+009$ & $1.5700 \mathrm{e}-002$ & $5.8090 e+002$ \\
\hline U-235 & $5.5617 e-004$ & $2.0578 \mathrm{e}+007$ & $8.0700 e-005$ & $2.9859 e+000$ \\
\hline$U-236$ & $1.7023 \mathrm{e}-003$ & $6.2985 e+007$ & $2.4700 \mathrm{e}-004$ & $9.1390 e+000$ \\
\hline$U-237$ & $1.4749 \mathrm{e}-003$ & $5.4570 e+007$ & $2.1400 \mathrm{e}-004$ & $7.9180 e+000$ \\
\hline$U-238$ & $2.0813 e-004$ & $7.7010 e+006$ & $3.0200 e-005$ & $1.1174 \mathrm{e}+000$ \\
\hline Y-90 & $1.1785 e+004$ & $4.3605 e+014$ & $1.7100 e+003$ & $6.3270 e+007$ \\
\hline
\end{tabular}

\begin{tabular}{|c|c|}
\hline $\begin{array}{c}\text { Energy } \\
\text { MeV }\end{array}$ & $\begin{array}{c}\frac{\text { Activity }}{\text { photons/sec }} \\
\end{array}$ \\
\hline 0.015 & $4.234 \mathrm{e}+11$ \\
\hline 0.02 & $2.358 e+11$ \\
\hline 0.03 & $2.812 \mathrm{e}+13$ \\
\hline 0.04 & $6.925 e+12$ \\
\hline 0.05 & $6.128 \mathrm{e}+10$ \\
\hline 0.06 & $1.842 \mathrm{e}+11$ \\
\hline 0.08 & $2.420 e+10$ \\
\hline 0.1 & $4.288 e+11$ \\
\hline 0.15 & $1.370 \mathrm{e}+09$ \\
\hline 0.2 & $7.089 e+1$ \\
\hline 0.3 & $8.435 e+0$ \\
\hline 0.4 & $1.720 e+1$ \\
\hline 0.5 & $5.478 e+0$ \\
\hline 0.6 & $4.167 e+1$ \\
\hline 0.8 & $3.844 e+1$ \\
\hline 1.0 & $3.754 \mathrm{e}+1$ \\
\hline 1.5 & $4.532 e+1$ \\
\hline 2.0 & $1.908 \mathrm{e}+0$ \\
\hline 3.0 & $2.020 e+0$ \\
\hline
\end{tabular}

Radial

Integration Parameters

Y Direction (axial) $\quad 24$

\begin{tabular}{|c|c|c|c|}
\hline \\
\hline Fluence Rate & Fluence Rate & Exposure Rate & Exposure Rate \\
\hline $\mathrm{MeV} / \mathrm{cm}^{2} / \mathrm{sec}$ & $\mathrm{MeV} / \mathrm{cm}^{2} / \mathrm{sec}$ & $\mathrm{mR} / \mathrm{hr}$ & $\mathrm{mR} / \mathrm{hr}$ \\
\hline No Buildup & With Buildup & No Buildup & With Buildup \\
\hline $4.730 \mathrm{e}-211$ & $4.514 \mathrm{e}-22$ & $4.057 e-212$ & $3.872 \mathrm{e}-23$ \\
\hline $1.063 e-97$ & $3.190 \mathrm{e}-22$ & $3.683 e-99$ & $1.105 \mathrm{e}-23$ \\
\hline $9.535 e-28$ & $7.309 e-20$ & $9.450 e-30$ & $7.244 \mathrm{e}-22$ \\
\hline $1.030 \mathrm{e}-10$ & $1.522 \mathrm{e}-10$ & $4.553 e-13$ & $6.731 \mathrm{e}-13$ \\
\hline $3.450 \mathrm{e}-06$ & $6.196 \mathrm{e}-06$ & $9.190 \mathrm{e}-09$ & $1.650 \mathrm{e}-08$ \\
\hline $1.101 \mathrm{e}-02$ & $2.352 \mathrm{e}-02$ & $2.186 \mathrm{e}-05$ & $4.672 \mathrm{e}-05$ \\
\hline $5.590 \mathrm{e}-01$ & $1.512 \mathrm{e}+00$ & $8.845 e-04$ & $2.393 e-03$ \\
\hline $1.200 \mathrm{e}+02$ & $3.816 e+02$ & $1.837 \mathrm{e}-01$ & $5.838 \mathrm{e}-01$ \\
\hline $4.905 e+00$ & $1.903 e+01$ & $8.077 e-03$ & $3.134 \mathrm{e}-02$ \\
\hline $7.281 e+02$ & $3.043 e+03$ & $1.285 e+00$ & $5.370 e+00$ \\
\hline $2.459 e+02$ & $1.018 e+03$ & $4.664 \mathrm{e}-01$ & $1.931 \mathrm{e}+00$ \\
\hline $9.204 \mathrm{e}+02$ & $3.601 e+03$ & $1.793 e+00$ & $7.017 e+00$ \\
\hline $4.555 e+02$ & $1.667 e+03$ & $8.941 \mathrm{e}-01$ & $3.272 \mathrm{e}+00$ \\
\hline $4.918 e+07$ & $1.687 e+08$ & $9.599 e+04$ & $3.292 e+05$ \\
\hline $7.819 e+04$ & $2.406 e+05$ & $1.487 e+02$ & $4.576 e+02$ \\
\hline $1.161 \mathrm{e}+05$ & $3.282 e+05$ & $2.141 e+02$ & $6.050 e+02$ \\
\hline $2.973 e+05$ & $7.215 e+05$ & $5.001 e+02$ & $1.214 \mathrm{e}+03$ \\
\hline $2.079 e+01$ & $4.621 e+01$ & $3.215 e-02$ & $7.145 e-02$ \\
\hline $4.297 e-03$ & $8.466 \mathrm{e}-03$ & $5.830 \mathrm{e}-06$ & $1.149 \mathrm{e}-05$ \\
\hline $4.967 e+07$ & $1.700 e+08$ & $9.685 e+04$ & $3.315 e+05$ \\
\hline
\end{tabular}

Results - Dose Point \# 2 - $(106.433,195,0) \mathrm{cm}$ 
Page : 3

DOS File : Combined Calcine in Day Tank.ms6

Run Date: April 5, 2005

Run Time: 1:17:38 PM

Duration : 00:01:04

\begin{tabular}{|c|c|}
\hline$\frac{\text { Energy }}{\text { MeV }}$ & $\frac{\text { Activity }}{\text { photons/sec }}$ \\
\hline 0.015 & $4.234 e+11$ \\
\hline 0.02 & $2.358 e+11$ \\
\hline 0.03 & $2.812 e+13$ \\
\hline 0.04 & $6.925 \mathrm{e}+12$ \\
\hline 0.05 & $6.128 \mathrm{e}+10$ \\
\hline 0.06 & $1.842 \mathrm{e}+11$ \\
\hline 0.08 & $2.420 \mathrm{e}+10$ \\
\hline 0.1 & $4.288 e+11$ \\
\hline 0.15 & $1.370 e+09$ \\
\hline 0.2 & $7.089 e+10$ \\
\hline 0.3 & $8.435 e+09$ \\
\hline 0.4 & $1.720 e+10$ \\
\hline 0.5 & $5.478 e+09$ \\
\hline 0.6 & $4.167 e+14$ \\
\hline 0.8 & $3.844 \mathrm{e}+11$ \\
\hline 1.0 & $3.754 e+11$ \\
\hline 1.5 & $4.532 e+11$ \\
\hline 2.0 & $1.908 e+07$ \\
\hline 3.0 & $2.020 e+03$ \\
\hline
\end{tabular}

\begin{tabular}{|c|c|c|}
\hline Fluence Rate & Fluence Rate & Exposure Rate \\
\hline $\mathrm{MeV} / \mathrm{cm}^{2} / \mathrm{sec}$ & $\mathrm{MeV} / \mathrm{cm}^{2} / \mathrm{sec}$ & $\mathrm{mR} / \mathrm{hr}$ \\
\hline No Buildup & With Buildup & No Buildup \\
\hline $9.000 \mathrm{e}-188$ & $2.636 \mathrm{e}-22$ & $7.720 \mathrm{e}-189$ \\
\hline $3.835 e-86$ & $1.862 \mathrm{e}-22$ & $1.328 e-87$ \\
\hline $3.910 \mathrm{e}-25$ & $4.267 e-20$ & $3.875 e-27$ \\
\hline $3.844 \mathrm{e}-10$ & $5.631 \mathrm{e}-10$ & $1.700 \mathrm{e}-12$ \\
\hline $3.052 \mathrm{e}-06$ & $5.405 e-06$ & $8.130 \mathrm{e}-09$ \\
\hline $6.418 e-03$ & $1.356 \mathrm{e}-02$ & $1.275 \mathrm{e}-05$ \\
\hline $3.171 \mathrm{e}-01$ & $8.681 \mathrm{e}-01$ & $5.019 \mathrm{e}-04$ \\
\hline $7.493 e+01$ & $2.437 e+02$ & $1.146 \mathrm{e}-01$ \\
\hline $3.263 e+00$ & $1.277 e+01$ & $5.374 \mathrm{e}-03$ \\
\hline $4.861 e+02$ & $2.027 e+03$ & $8.579 \mathrm{e}-01$ \\
\hline $1.636 \mathrm{e}+02$ & $6.713 e+02$ & $3.103 e-01$ \\
\hline $6.104 e+02$ & $2.360 e+03$ & $1.189 \mathrm{e}+00$ \\
\hline $3.012 e+02$ & $1.088 \mathrm{e}+03$ & $5.913 e-01$ \\
\hline $3.245 e+07$ & $1.098 e+08$ & $6.333 e+04$ \\
\hline $5.139 e+04$ & $1.559 \mathrm{e}+05$ & $9.774 \mathrm{e}+01$ \\
\hline $7.607 e+04$ & $2.121 \mathrm{e}+05$ & $1.402 \mathrm{e}+02$ \\
\hline $1.935 \mathrm{e}+05$ & $4.641 \mathrm{e}+05$ & $3.256 \mathrm{e}+02$ \\
\hline $1.349 \mathrm{e}+01$ & $2.965 e+01$ & $2.085 e-02$ \\
\hline $2.775 \mathrm{e}-03$ & $5.418 \mathrm{e}-03$ & $3.765 e-06$ \\
\hline $3.277 e+07$ & $1.106 \mathrm{e}+08$ & $6.390 e+04$ \\
\hline
\end{tabular}

Exposure Rate $\mathrm{mR} / \mathrm{hr}$

With Buildup

$2.261 \mathrm{e}-23$

$6.451 \mathrm{e}-24$

$4.229 \mathrm{e}-22$

$2.491 \mathrm{e}-12$

$1.440 \mathrm{e}-08$

$2.694 \mathrm{e}-05$

$1.374 \mathrm{e}-03$

$3.729 \mathrm{e}-01$

$2.102 \mathrm{e}-02$

$3.578 \mathrm{e}+00$

$1.273 \mathrm{e}+00$

$4.599 \mathrm{e}+00$

$2.136 \mathrm{e}+00$

$2.143 e+05$

$2.966 \mathrm{e}+02$

$3.910 \mathrm{e}+02$

$7.809 \mathrm{e}+02$

$4.585 \mathrm{e}-02$

$7.350 \mathrm{e}-06$

TOTALS: $\quad 4.545 \mathrm{e}+14$

\section{Results - Dose Point \# 3 - $(175.953,195,0)$ cm}

\begin{tabular}{|c|c|}
\hline$\frac{\text { Energy }}{\mathrm{MeV}}$ & $\begin{array}{c}\text { Activity } \\
\text { photons/sec }\end{array}$ \\
\hline 0.015 & $4.234 e+11$ \\
\hline 0.02 & $2.358 e+11$ \\
\hline 0.03 & $2.812 e+13$ \\
\hline 0.04 & $6.925 e+12$ \\
\hline 0.05 & $6.128 \mathrm{e}+10$ \\
\hline 0.06 & $1.842 e+11$ \\
\hline 0.08 & $2.420 \mathrm{e}+10$ \\
\hline 0.1 & $4.288 e+11$ \\
\hline 0.15 & $1.370 e+09$ \\
\hline 0.2 & $7.089 e+10$ \\
\hline 0.3 & $8.435 e+09$ \\
\hline 0.4 & $1.720 e+10$ \\
\hline 0.5 & $5.478 e+09$ \\
\hline 0.6 & $4.167 e+14$ \\
\hline 0.8 & $3.844 \mathrm{e}+11$ \\
\hline 1.0 & $3.754 e+11$ \\
\hline 1.5 & $4.532 e+11$ \\
\hline 2.0 & $1.908 e+07$ \\
\hline 3.0 & $2.020 e+03$ \\
\hline
\end{tabular}

\begin{tabular}{|c|c|c|}
\hline $\begin{array}{l}\text { Fluence Rate } \\
\mathrm{MeV} / \mathrm{cm}^{2} / \mathrm{sec}\end{array}$ & $\begin{array}{l}\text { Fluence Rate } \\
\mathrm{MeV} / \mathrm{cm}^{2} / \mathrm{sec}\end{array}$ & $\begin{array}{c}\text { Exposure Rate } \\
\mathrm{mR} / \mathrm{hr}\end{array}$ \\
\hline No Buildup & With Buildup & No Buildup \\
\hline $3.143 e-188$ & $1.168 \mathrm{e}-22$ & $2.696 \mathrm{e}-189$ \\
\hline $1.589 \mathrm{e}-86$ & $8.256 e-23$ & $5.504 e-88$ \\
\hline $2.465 e-25$ & $1.892 \mathrm{e}-20$ & $2.443 e-27$ \\
\hline $2.265 e-10$ & $3.317 e-10$ & $1.002 \mathrm{e}-12$ \\
\hline $1.792 \mathrm{e}-06$ & $3.174 \mathrm{e}-06$ & $4.773 e-09$ \\
\hline $3.770 e-03$ & $7.969 \mathrm{e}-03$ & $7.489 e-06$ \\
\hline $1.849 \mathrm{e}-01$ & $5.061 \mathrm{e}-01$ & $2.925 e-04$ \\
\hline $4.332 \mathrm{e}+01$ & $1.408 e+02$ & $6.628 \mathrm{e}-02$ \\
\hline $1.853 e+00$ & $7.197 e+00$ & $3.051 \mathrm{e}-03$ \\
\hline $2.729 e+02$ & $1.123 e+03$ & $4.816 \mathrm{e}-01$ \\
\hline $9.070 \mathrm{e}+01$ & $3.646 e+02$ & $1.721 \mathrm{e}-01$ \\
\hline $3.359 e+02$ & $1.268 \mathrm{e}+03$ & $6.545 e-01$ \\
\hline $1.649 \mathrm{e}+02$ & $5.805 e+02$ & $3.236 \mathrm{e}-01$ \\
\hline $1.768 \mathrm{e}+07$ & $5.823 e+07$ & $3.450 e+04$ \\
\hline $2.778 e+04$ & $8.201 \mathrm{e}+04$ & $5.283 e+01$ \\
\hline $4.086 e+04$ & $1.109 e+05$ & $7.531 e+01$ \\
\hline $1.027 e+05$ & $2.398 e+05$ & $1.727 e+02$ \\
\hline $7.092 \mathrm{e}+00$ & $1.520 e+01$ & $1.097 e-02$ \\
\hline $1.444 \mathrm{e}-03$ & $2.753 \mathrm{e}-03$ & $1.959 \mathrm{e}-06$ \\
\hline $1.785 e+07$ & $5.866 e+07$ & $3.480 e+04$ \\
\hline
\end{tabular}

$2.158 e+05$

TOTALS: $\quad 4.545 e+14$

Results - Dose Point \# 4 - $(85.953,195,0) \mathrm{cm}$

$\begin{array}{ll}\frac{\text { Energy }}{\text { MeV }} & \begin{array}{c}\text { Activity } \\ \text { photons/sec }\end{array} \\ 0.015 & 4.234 \mathrm{e}+11 \\ 0.02 & 2.358 \mathrm{e}+11 \\ 0.03 & 2.812 \mathrm{e}+13\end{array}$

\begin{tabular}{|c|c|c|}
\hline$\frac{\text { Fluence Rate }}{\mathrm{MeV} / \mathrm{cm}^{2} / \mathrm{sec}}$ & $\frac{\text { Fluence Rate }}{\mathrm{MeV} / \mathrm{cm}^{2} / \mathrm{sec}}$ & $\frac{\text { Exposure Rate }}{\mathrm{mR} / \mathrm{hr}}$ \\
\hline No Buildup & With Buildup & No Buildup \\
\hline $5.595 e-190$ & $3.776 \mathrm{e}-22$ & $4.799 \mathrm{e}-191$ \\
\hline $1.147 \mathrm{e}-86$ & $2.668 \mathrm{e}-22$ & $3.972 \mathrm{e}-88$ \\
\hline $7.400 e-25$ & $6.114 \mathrm{e}-20$ & $7.334 \mathrm{e}-27$ \\
\hline
\end{tabular}

Exposure Rate $\mathrm{mR} / \mathrm{hr}$

With Buildup

$1.002 \mathrm{e}-23$

$2.860 \mathrm{e}-24$

$1.875 \mathrm{e}-22$

$1.467 \mathrm{e}-12$

8.455e-09

$1.583 \mathrm{e}-05$

8.008e-04

2.154e-01

$1.185 \mathrm{e}-02$

$1.982 \mathrm{e}+00$

$6.916 \mathrm{e}-01$

$2.471 \mathrm{e}+00$

$1.139 \mathrm{e}+00$

$1.137 \mathrm{e}+05$

$1.560 \mathrm{e}+02$

$2.043 e+02$

$4.035 e+02$

2.351e-02

3.736e-06

$1.144 \mathrm{e}+05$

$\frac{\text { Exposure Rate }}{\mathrm{mR/hr}}$
$\frac{\text { With Buildup }}{3.239 \mathrm{e}-23}$
$9.242 \mathrm{e}-24$
$6.059 \mathrm{e}-22$


Page : 4

DOS File : Combined Calcine in Day Tank.ms6

Run Date: April 5, 2005

Run Time: 1:17:38 PM

Duration : 00:01:04

$\begin{array}{ccc}\frac{\text { Energy }}{\underline{\text { MeV }}} & & \begin{array}{c}\text { Activity } \\ \text { photons } / \mathrm{sec}\end{array} \\ 0.04 & & 6.925 \mathrm{e}+12 \\ 0.05 & & 6.128 \mathrm{e}+10 \\ 0.06 & & 1.842 \mathrm{e}+11 \\ 0.08 & & 2.420 \mathrm{e}+10 \\ 0.1 & & 4.288 \mathrm{e}+11 \\ 0.15 & & 1.370 \mathrm{e}+09 \\ 0.2 & & 7.089 \mathrm{e}+10 \\ 0.3 & & 8.435 \mathrm{e}+09 \\ 0.4 & 1.720 \mathrm{e}+10 \\ 0.5 & 5.478 \mathrm{e}+09 \\ 0.6 & 4.167 \mathrm{e}+14 \\ 0.8 & 3.844 \mathrm{e}+11 \\ 1.0 & 3.754 \mathrm{e}+11 \\ 1.5 & 4.532 \mathrm{e}+11 \\ 2.0 & 1.908 \mathrm{e}+07 \\ 3.0 & 2.020 \mathrm{e}+03\end{array}$

\begin{tabular}{|c|c|c|}
\hline Fluence Rate & Fluence Rate & Exposure Rate \\
\hline $\mathrm{MeV} / \mathrm{cm}^{2} / \mathrm{sec}$ & $\mathrm{MeV} / \mathrm{cm}^{2} / \mathrm{sec}$ & $\mathrm{mR} / \mathrm{hr}$ \\
\hline No Buildup & With Buildup & No Buildup \\
\hline $6.106 \mathrm{e}-10$ & $8.935 \mathrm{e}-10$ & $2.701 \mathrm{e}-12$ \\
\hline $3.956 \mathrm{e}-06$ & $6.991 \mathrm{e}-06$ & $1.054 \mathrm{e}-08$ \\
\hline $7.868 e-03$ & $1.661 \mathrm{e}-02$ & $1.563 e-05$ \\
\hline $3.974 \mathrm{e}-01$ & $1.091 \mathrm{e}+00$ & $6.289 e-04$ \\
\hline $9.549 e+01$ & $3.116 \mathrm{e}+02$ & $1.461 \mathrm{e}-01$ \\
\hline $4.224 e+00$ & $1.660 \mathrm{e}+01$ & $6.956 \mathrm{e}-03$ \\
\hline $6.326 e+02$ & $2.653 e+03$ & $1.117 e+00$ \\
\hline $2.139 e+02$ & $8.837 e+02$ & $4.057 e-01$ \\
\hline $8.001 e+02$ & $3.117 e+03$ & $1.559 \mathrm{e}+00$ \\
\hline $3.956 e+02$ & $1.440 \mathrm{e}+03$ & $7.765 e-01$ \\
\hline $4.267 e+07$ & $1.455 e+08$ & $8.328 e+04$ \\
\hline $6.773 e+04$ & $2.071 \mathrm{e}+05$ & $1.288 \mathrm{e}+02$ \\
\hline $1.005 e+05$ & $2.822 \mathrm{e}+05$ & $1.852 \mathrm{e}+02$ \\
\hline $2.564 \mathrm{e}+05$ & $6.193 e+05$ & $4.314 e+02$ \\
\hline $1.791 \mathrm{e}+01$ & $3.963 e+01$ & $2.769 \mathrm{e}-02$ \\
\hline $3.695 \mathrm{e}-03$ & $7.258 \mathrm{e}-03$ & $5.013 e-06$ \\
\hline $4.309 e+07$ & $1.466 \mathrm{e}+08$ & $8.403 e+04$ \\
\hline
\end{tabular}

Exposure Rate $\mathrm{mR} / \mathrm{hr}$

With Buildup

$3.952 \mathrm{e}-12$

$1.862 \mathrm{e}-08$

$3.299 \mathrm{e}-05$

$1.726 \mathrm{e}-03$

$4.767 \mathrm{e}-01$

$2.733 \mathrm{e}-02$

$4.682 \mathrm{e}+00$

$1.676 \mathrm{e}+00$

$6.073 e+00$

$2.827 \mathrm{e}+00$

$2.840 \mathrm{e}+05$

$3.939 \mathrm{e}+02$

$5.201 \mathrm{e}+02$

$1.042 \mathrm{e}+03$

$6.129 \mathrm{e}-02$

$9.847 \mathrm{e}-06$

TOTALS: $\quad 4.545 e+14$

Results - Dose Point \# 5 - $(95.953,195,0) \mathrm{cm}$

\begin{tabular}{|c|c|}
\hline $\begin{array}{l}\text { Energy } \\
\underline{\mathrm{MeV}}\end{array}$ & $\begin{array}{c}\text { Activity } \\
\text { photons/sec }\end{array}$ \\
\hline 0.015 & $4.234 \mathrm{e}+11$ \\
\hline 0.02 & $2.358 e+11$ \\
\hline 0.03 & $2.812 \mathrm{e}+13$ \\
\hline 0.04 & $6.925 e+12$ \\
\hline 0.05 & $6.128 e+10$ \\
\hline 0.06 & $1.842 e+11$ \\
\hline 0.08 & $2.420 e+10$ \\
\hline 0.1 & $4.288 e+11$ \\
\hline 0.15 & $1.370 e+09$ \\
\hline 0.2 & $7.089 e+10$ \\
\hline 0.3 & $8.435 e+09$ \\
\hline 0.4 & $1.720 e+10$ \\
\hline 0.5 & $5.478 e+09$ \\
\hline 0.6 & $4.167 e+14$ \\
\hline 0.8 & $3.844 e+11$ \\
\hline 1.0 & $3.754 e+11$ \\
\hline 1.5 & $4.532 e+11$ \\
\hline 2.0 & $1.908 e+07$ \\
\hline 3.0 & $2.020 e+03$ \\
\hline
\end{tabular}

Fluence Rate $\quad$ Fluence Rate

$\mathrm{MeV} / \mathrm{cm}^{2} / \mathrm{sec} \quad \mathrm{MeV} / \mathrm{cm}^{2} / \mathrm{sec}$

No Buildup

$5.539 \mathrm{e}-188$

With Buildup

$4.729 \mathrm{e}-86$

$3.123 \mathrm{e}-22$

$5.163 \mathrm{e}-25$

$2.206 \mathrm{e}-22$

$5.056 \mathrm{e}-20$

$5.937 \mathrm{e}-10$

$4.053 \mathrm{e}-10$

$5.959 \mathrm{e}-06$

$3.362 \mathrm{e}-06$
$7.194 \mathrm{e}-03$

$1.520 \mathrm{e}-02$

$3.560 \mathrm{e}-01$

$9.747 \mathrm{e}-01$

$8.430 \mathrm{e}+01$

$2.743 e+02$

$1.444 \mathrm{e}+01$

$3.687 e+00$

$2.299 \mathrm{e}+03$

Exposure Rate $\mathrm{mR} / \mathrm{hr}$

No Buildup

4.751e-189

$1.638 \mathrm{e}-87$

5.117e-27

$1.793 \mathrm{e}-12$

8.956e-09

$1.429 \mathrm{e}-05$

5.634e-04

$1.290 \mathrm{e}-01$

$6.072 \mathrm{e}-03$

$503 e+02$

$9.712 \mathrm{e}-01$

$7.627 \mathrm{e}+02$

$3.518 \mathrm{e}-01$

$6.925 \mathrm{e}+02$

$2.685 e+03$

$1.349 \mathrm{e}+00$

$3.420 \mathrm{e}+02$

$1.239 \mathrm{e}+03$

$6.713 \mathrm{e}-01$

$3.686 \mathrm{e}+07$

$7.194 \mathrm{e}+04$

$5.842 e+04$

$1.111 \mathrm{e}+02$

$8.656 e+04$

$1.596 \mathrm{e}+02$

$2.205 e+05$

$3.711 \mathrm{e}+02$

$1.538 \mathrm{e}+01$

$2.379 \mathrm{e}-02$

$3.171 \mathrm{e}-03$

4.302e-06

$2.859 e+05$

TOTALS: $\quad 4.545 e+14$

$3.722 \mathrm{e}+07$

$7.259 e+04$

Exposure Rate $\mathrm{mR} / \mathrm{hr}$

With Buildup

$2.678 \mathrm{e}-23$

$7.643 e-24$

$5.011 \mathrm{e}-22$

$2.626 \mathrm{e}-12$

$1.587 \mathrm{e}-08$

$3.020 \mathrm{e}-05$

$1.542 \mathrm{e}-03$

4.197e-01

$2.379 \mathrm{e}-02$

$4.057 \mathrm{e}+00$

$1.447 \mathrm{e}+00$

$5.231 \mathrm{e}+00$

$2.432 \mathrm{e}+00$

$2.441 \mathrm{e}+05$

$3.383 e+02$

$4.464 \mathrm{e}+02$

$8.930 \mathrm{e}+02$

$5.249 \mathrm{e}-02$

$8.426 \mathrm{e}-06$

$2.458 e+05$

$\begin{array}{ll}\frac{\text { Energy }}{\text { MeV }} & \begin{array}{c}\text { Activity } \\ \text { photons/sec }\end{array} \\ 0.015 & 4.234 \mathrm{e}+11 \\ 0.02 & 2.358 \mathrm{e}+11 \\ 0.03 & 2.812 \mathrm{e}+13 \\ 0.04 & 6.925 \mathrm{e}+12 \\ 0.05 & 6.128 \mathrm{e}+10 \\ 0.06 & 1.842 \mathrm{e}+11\end{array}$

Results - Dose Point \# 6 - $(140.953,195,0)$ cm

$\begin{array}{ccc}\frac{\text { Fluence Rate }}{\text { MeV/cm } / \mathrm{sec}} & \frac{\text { Fluence Rate }}{\text { MeV/cm } / \mathrm{sec}} & \begin{array}{c}\text { Exposure Rate } \\ \mathrm{mR} / \mathrm{hr}\end{array} \\ \frac{\text { No Buildup }}{4.789 \mathrm{e}-188} & \frac{\text { With Buildup }}{1.680 \mathrm{e}-22} & \frac{\text { No Buildup }}{4.108 \mathrm{e}-189} \\ 1.955 \mathrm{e}-86 & 1.187 \mathrm{e}-22 & 6.772 \mathrm{e}-88 \\ 3.145 \mathrm{e}-25 & 2.720 \mathrm{e}-20 & 3.117 \mathrm{e}-27 \\ 2.856 \mathrm{e}-10 & 4.183 \mathrm{e}-10 & 1.263 \mathrm{e}-12 \\ 2.263 \mathrm{e}-06 & 4.009 \mathrm{e}-06 & 6.029 \mathrm{e}-09 \\ 4.764 \mathrm{e}-03 & 1.007 \mathrm{e}-02 & 9.463 \mathrm{e}-06\end{array}$

Exposure Rate $\mathrm{mR} / \mathrm{hr}$

With Buildup

$1.441 \mathrm{e}-23$

$4.112 \mathrm{e}-24$

2.696e-22

$1.850 \mathrm{e}-12$

$1.068 \mathrm{e}-08$

2.000e-05 
Page : 5

DOS File : Combined Calcine in Day Tank.ms6

Run Date: April 5, 2005

Run Time: $1: 17: 38$ PM

Duration : 00:01:04

\begin{tabular}{|c|c|c|c|c|c|}
\hline$\frac{\text { Energy }}{\text { MeV }}$ & $\frac{\text { Activity }}{\text { photons/sec }}$ & $\frac{\text { Fluence Rate }}{\mathrm{MeV} / \mathrm{cm}^{2} / \mathrm{sec}}$ & $\frac{\text { Fluence Rate }}{\mathrm{MeV} / \mathrm{cm}^{2} / \mathrm{sec}}$ & $\frac{\text { Exposure Rate }}{\mathrm{mR} / \mathrm{hr}}$ & $\frac{\text { Exposure Rate }}{\mathrm{mR} / \mathrm{hr}}$ \\
\hline & & No Buildup & With Buildup & No Buildup & With Buildup \\
\hline 0.08 & $2.420 e+10$ & $2.341 \mathrm{e}-01$ & $6.408 \mathrm{e}-01$ & $3.705 e-04$ & $1.014 \mathrm{e}-03$ \\
\hline 0.1 & $4.288 e+11$ & $5.506 e+01$ & $1.790 e+02$ & $8.424 \mathrm{e}-02$ & $2.739 e-01$ \\
\hline 0.15 & $1.370 e+09$ & $2.381 e+00$ & $9.291 e+00$ & $3.921 \mathrm{e}-03$ & $1.530 \mathrm{e}-02$ \\
\hline 0.2 & $7.089 e+10$ & $3.531 \mathrm{e}+02$ & $1.465 \mathrm{e}+03$ & $6.231 \mathrm{e}-01$ & $2.586 \mathrm{e}+00$ \\
\hline 0.3 & $8.435 e+09$ & $1.182 \mathrm{e}+02$ & $4.813 e+02$ & $2.243 e-01$ & $9.130 \mathrm{e}-01$ \\
\hline 0.4 & $1.720 e+10$ & $4.399 e+02$ & $1.684 e+03$ & $8.571 \mathrm{e}-01$ & $3.282 e+00$ \\
\hline 0.5 & $5.478 e+09$ & $2.166 e+02$ & 7.741e+02 & $4.252 \mathrm{e}-01$ & $1.520 \mathrm{e}+00$ \\
\hline 0.6 & $4.167 e+14$ & $2.329 e+07$ & $7.787 e+07$ & $4.545 e+04$ & $1.520 \mathrm{e}+05$ \\
\hline 0.8 & $3.844 \mathrm{e}+11$ & $3.675 e+04$ & $1.101 \mathrm{e}+05$ & $6.991 \mathrm{e}+01$ & $2.095 e+02$ \\
\hline 1.0 & $3.754 \mathrm{e}+11$ & $5.425 e+04$ & $1.494 \mathrm{e}+05$ & $1.000 e+02$ & $2.753 e+02$ \\
\hline 1.5 & $4.532 e+11$ & $1.372 \mathrm{e}+05$ & $3.248 e+05$ & $2.309 e+02$ & $5.465 e+02$ \\
\hline 2.0 & $1.908 e+07$ & $9.520 e+00$ & $2.066 e+01$ & $1.472 \mathrm{e}-02$ & $3.195 e-02$ \\
\hline 3.0 & $2.020 e+03$ & $1.948 \mathrm{e}-03$ & $3.757 e-03$ & $2.643 e-06$ & $5.097 \mathrm{e}-06$ \\
\hline TOTALS & $4.545 e+14$ & $2.352 e+07$ & $7.846 e+07$ & $4.585 e+04$ & $1.530 \mathrm{e}+05$ \\
\hline
\end{tabular}


Page : 1

DOS File : Combined Calcine in Batch Tank.ms6

Run Date: April 5, 2005

Run Time: 1:20:38 PM

Duration : 00:01:03
File Ref:

Date:

By:

Checked:

Case Title: Combined Calcine

Description: Combined Calcine in Batch Tank (3/8" 316 SS) Geometry: 7 - Cylinder Volume - Side Shields

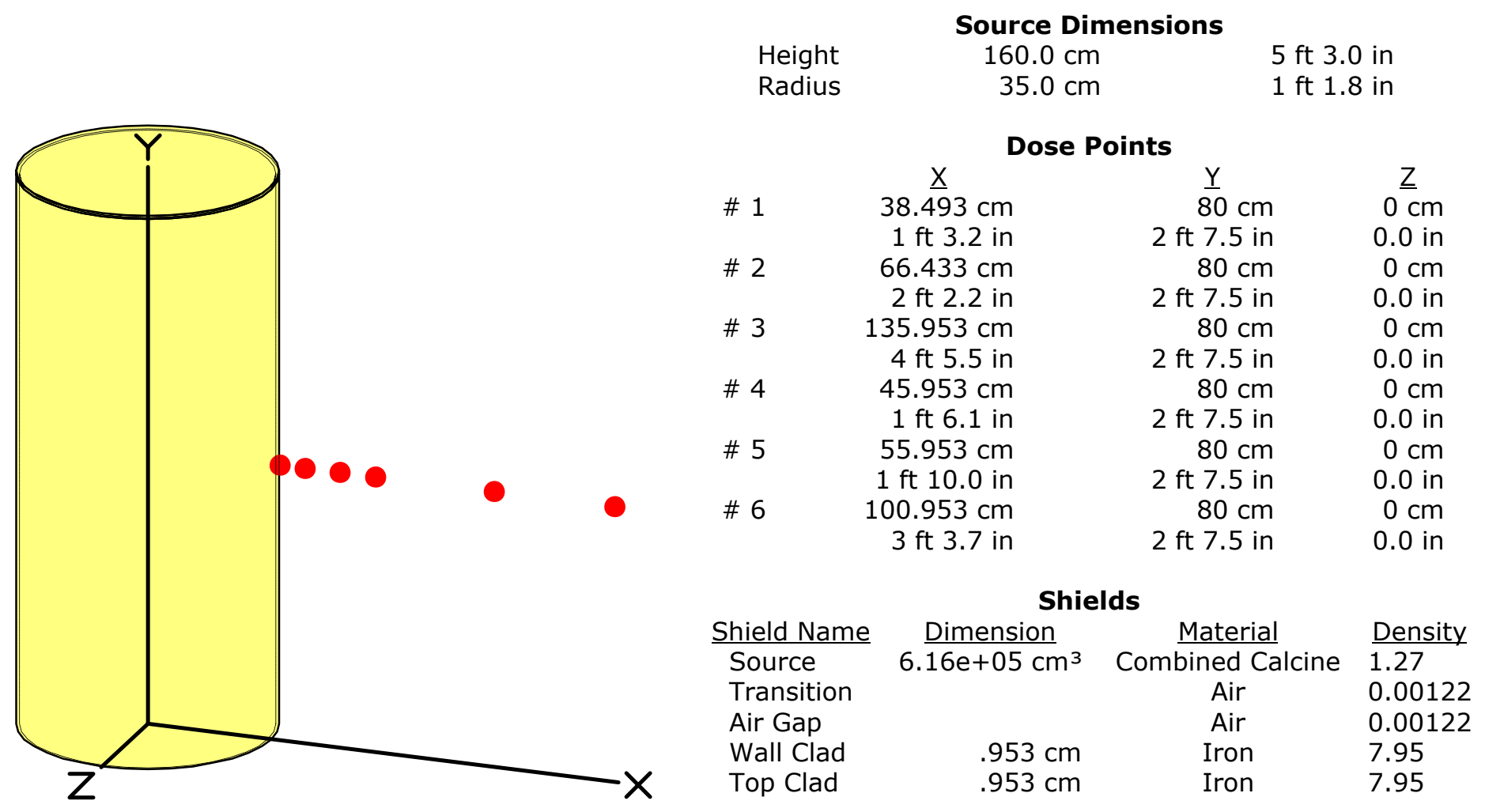

Source Input

Grouping Method : Standard Indices

Number of Groups : 25

Lower Energy Cutoff : 0.015

Photons < 0.015 : Included

Library : ICRP-38

\begin{tabular}{|c|c|}
\hline Nuclide & curies \\
\hline Am-241 & $1.2377 e+000$ \\
\hline Am-243 & $8.1279 e-004$ \\
\hline $\mathrm{Ba}-137 \mathrm{~m}$ & $1.1207 e+003$ \\
\hline $\mathrm{Ce}-144$ & $1.9273 \mathrm{e}-009$ \\
\hline $\mathrm{Cm}-242$ & $2.0505 e-003$ \\
\hline $\mathrm{Cm}-244$ & $7.5738 \mathrm{e}-004$ \\
\hline Co-60 & $1.3547 e-001$ \\
\hline Cs-134 & $7.3275 e-003$ \\
\hline Cs-135 & $2.0812 \mathrm{e}-002$ \\
\hline Cs-137 & $1.1822 \mathrm{e}+003$ \\
\hline Eu-152 & $3.7130 \mathrm{e}-002$ \\
\hline Eu-154 & $2.4261 e+000$ \\
\hline Eu-155 & $1.7980 \mathrm{e}-001$ \\
\hline I-129 & $1.0714 \mathrm{e}-004$ \\
\hline $\mathrm{Ni}-63$ & $5.9051 e+001$ \\
\hline$N p-237$ & $1.0221 \mathrm{e}-002$ \\
\hline Pa-233 & $1.0221 \mathrm{e}-002$ \\
\hline Pm-147 & 4.1994e-002 \\
\hline Pr-144 & $1.9273 e-009$ \\
\hline
\end{tabular}

becquerels

$4.5793 e+010$

$3.0073 e+007$

$4.1465 \mathrm{e}+013$

$7.1310 \mathrm{e}+001$

$7.5867 e+007$

$2.8023 e+007$

$5.0122 \mathrm{e}+009$

$2.7112 \mathrm{e}+008$

$7.7006 \mathrm{e}+008$

$4.3743 e+013$

$1.3738 \mathrm{e}+009$

$8.9764 \mathrm{e}+010$

$6.6526 \mathrm{e}+009$

$3.9642 \mathrm{e}+006$

$2.1849 e+012$

$3.7819 e+008$

$3.7819 e+008$

$1.5538 \mathrm{e}+009$

7.1310e+001 $\mu \mathrm{Ci} / \mathrm{cm}^{3}$

2.0100e+000

1.3200e-003

$1.8200 \mathrm{e}+003$

3.1300e-009

3.3300e-003

$1.2300 \mathrm{e}-003$

2.2000e-001

$1.1900 \mathrm{e}-002$

3.3800e-002

$1.9200 \mathrm{e}+003$

6.0300e-002

$3.9400 \mathrm{e}+000$

2.9200e-001

$1.7400 \mathrm{e}-004$

$9.5900 \mathrm{e}+001$

$1.6600 \mathrm{e}-002$

$1.6600 \mathrm{e}-002$

6.8200e-002

3.1300e-009
$\mathrm{Bq} / \mathrm{cm}^{3}$

$7.4370 \mathrm{e}+004$

$4.8840 \mathrm{e}+001$

$6.7340 \mathrm{e}+007$

$1.1581 \mathrm{e}-004$

$1.2321 e+002$

$4.5510 \mathrm{e}+001$

$8.1400 e+003$

$4.4030 \mathrm{e}+002$

$1.2506 \mathrm{e}+003$

$7.1040 \mathrm{e}+007$

$2.2311 \mathrm{e}+003$

$1.4578 \mathrm{e}+005$

$1.0804 \mathrm{e}+004$

$6.4380 e+000$

$3.5483 e+006$

$6.1420 \mathrm{e}+002$

$6.1420 \mathrm{e}+002$

$2.5234 \mathrm{e}+003$

1.1581e-004

E-21 
Page : 2

DOS File : Combined Calcine in Batch Tank.ms6

Run Date: April 5, 2005

Run Time: 1:20:38 PM

Duration : 00:01:03

\begin{tabular}{|c|c|c|c|c|}
\hline Nuclide & curies & becquerels & $\mu \mathrm{Ci} / \mathrm{cm}^{3}$ & $\mathrm{~Bq} / \mathrm{cm}^{3}$ \\
\hline Pu-238 & $1.3978 \mathrm{e}+001$ & $5 . \overline{1717 e+011}$ & $2.2700 \mathrm{e}+001$ & $8.3 \overline{990 e+005}$ \\
\hline Pu-239 & $3.1958 \mathrm{e}-001$ & $1.1824 \mathrm{e}+010$ & $5.1900 \mathrm{e}-001$ & $1.9203 e+004$ \\
\hline Pu-240 & $1.8965 \mathrm{e}-001$ & $7.0171 e+009$ & $3.0800 \mathrm{e}-001$ & $1.1396 \mathrm{e}+004$ \\
\hline Pu-241 & $5.2893 e+000$ & $1.9570 \mathrm{e}+011$ & $8.5900 e+000$ & $3.1783 e+005$ \\
\hline Pu-242 & $2.5677 e-002$ & $9.5004 \mathrm{e}+008$ & $4.1700 e-002$ & $1.5429 e+003$ \\
\hline Ru-106 & $6.8348 e-008$ & $2.5289 e+003$ & $1.1100 \mathrm{e}-007$ & $4.1070 e-003$ \\
\hline Sb-125 & $1.9150 \mathrm{e}-002$ & $7.0855 e+008$ & $3.1100 \mathrm{e}-002$ & $1.1507 e+003$ \\
\hline Sb-126 & $1.8842 \mathrm{e}-003$ & $6.9715 e+007$ & $3.0600 \mathrm{e}-003$ & $1.1322 \mathrm{e}+002$ \\
\hline Sb-126m & $1.3485 e-002$ & $4.9894 \mathrm{e}+008$ & $2.1900 \mathrm{e}-002$ & $8.1030 e+002$ \\
\hline Sm-151 & $1.2130 \mathrm{e}+001$ & $4.4882 \mathrm{e}+011$ & $1.9700 e+001$ & $7.2890 e+005$ \\
\hline Sr-90 & $1.0529 e+003$ & $3.8959 e+013$ & $1.7100 e+003$ & $6.3270 e+007$ \\
\hline Tc-99 & $5.0122 \mathrm{e}-001$ & $1.8545 e+010$ & $8.1400 \mathrm{e}-001$ & $3.0118 e+004$ \\
\hline Th-230 & $5.2339 e-005$ & $1.9365 e+006$ & $8.5000 e-005$ & $3.1450 \mathrm{e}+000$ \\
\hline Th-231 & $5.0553 e-005$ & $1.8705 e+006$ & $8.2100 \mathrm{e}-005$ & $3.0377 e+000$ \\
\hline$U-232$ & $3.6576 \mathrm{e}-005$ & $1.3533 e+006$ & $5.9400 \mathrm{e}-005$ & $2.1978 \mathrm{e}+000$ \\
\hline$U-233$ & $2.4261 \mathrm{e}-006$ & $8.9764 e+004$ & $3.9400 \mathrm{e}-006$ & $1.4578 \mathrm{e}-001$ \\
\hline$U-234$ & $9.6673 e-003$ & $3.5769 \mathrm{e}+008$ & $1.5700 \mathrm{e}-002$ & $5.8090 e+002$ \\
\hline U-235 & $4.9691 \mathrm{e}-005$ & $1.8386 \mathrm{e}+006$ & $8.0700 \mathrm{e}-005$ & $2.9859 e+000$ \\
\hline$U-236$ & $1.5209 \mathrm{e}-004$ & $5.6274 \mathrm{e}+006$ & $2.4700 e-004$ & $9.1390 e+000$ \\
\hline U-237 & $1.3177 \mathrm{e}-004$ & $4.8755 e+006$ & $2.1400 \mathrm{e}-004$ & $7.9180 e+000$ \\
\hline$U-238$ & $1.8596 \mathrm{e}-005$ & $6.8804 \mathrm{e}+005$ & $3.0200 \mathrm{e}-005$ & $1.1174 \mathrm{e}+000$ \\
\hline$Y-90$ & $1.0529 e+003$ & $3.8959 e+013$ & $1.7100 e+003$ & $6.3270 \mathrm{e}+007$ \\
\hline
\end{tabular}

$\begin{array}{ccc}\frac{\text { Energy }}{\text { MeV }} & & \begin{array}{c}\text { Activity } \\ \text { photons/sec }\end{array} \\ 0.015 & & 3.783 \mathrm{e}+10 \\ 0.02 & 2.107 \mathrm{e}+10 \\ 0.03 & 2.512 \mathrm{e}+12 \\ 0.04 & & 6.187 \mathrm{e}+11 \\ 0.05 & & 5.475 \mathrm{e}+09 \\ 0.06 & 1.646 \mathrm{e}+10 \\ 0.08 & 2.162 \mathrm{e}+09 \\ 0.1 & 3.831 \mathrm{e}+10 \\ 0.15 & 1.224 \mathrm{e}+08 \\ 0.2 & 6.333 \mathrm{e}+09 \\ 0.3 & 7.536 \mathrm{e}+08 \\ 0.4 & 1.536 \mathrm{e}+09 \\ 0.5 & 4.894 \mathrm{e}+08 \\ 0.6 & 3.723 \mathrm{e}+13 \\ 0.8 & 3.435 \mathrm{e}+10 \\ 1.0 & 3.354 \mathrm{e}+10 \\ 1.5 & 4.049 \mathrm{e}+10 \\ 2.0 & 1.705 \mathrm{e}+06 \\ 3.0 & 1.804 \mathrm{e}+02\end{array}$

Radial

Integration Parameters

Y Direction (axial) $\quad 24$

\begin{tabular}{|c|c|c|c|}
\hline \multicolumn{4}{|c|}{ esults - Do } \\
\hline Fluence Rate & Fluence Rate & Exposure Rate & Exposure Rate \\
\hline $\mathrm{MeV} / \mathrm{cm}^{2} / \mathrm{sec}$ & $\mathrm{MeV} / \mathrm{cm}^{2} / \mathrm{sec}$ & $\mathrm{mR} / \mathrm{hr}$ & $\mathrm{mR} / \mathrm{hr}$ \\
\hline No Buildup & With Buildup & No Buildup & With Buildup \\
\hline $5.468 \mathrm{e}-192$ & $1.842 \mathrm{e}-22$ & $4.690 \mathrm{e}-193$ & $1.580 \mathrm{e}-23$ \\
\hline $1.157 \mathrm{e}-86$ & $1.301 \mathrm{e}-22$ & $4.009 e-88$ & $4.508 e-24$ \\
\hline $7.586 \mathrm{e}-25$ & $2.982 \mathrm{e}-20$ & $7.518 \mathrm{e}-27$ & $2.955 e-22$ \\
\hline $7.442 \mathrm{e}-10$ & $1.089 \mathrm{e}-09$ & $3.291 \mathrm{e}-12$ & $4.818 e-12$ \\
\hline $4.833 e-06$ & $8.527 e-06$ & $1.288 \mathrm{e}-08$ & $2.271 \mathrm{e}-08$ \\
\hline $8.714 \mathrm{e}-03$ & $1.831 \mathrm{e}-02$ & $1.731 \mathrm{e}-05$ & $3.636 e-05$ \\
\hline $4.179 \mathrm{e}-01$ & $1.147 \mathrm{e}+00$ & $6.614 \mathrm{e}-04$ & $1.815 \mathrm{e}-03$ \\
\hline $1.010 e+02$ & $3.301 e+02$ & $1.546 \mathrm{e}-01$ & $5.051 \mathrm{e}-01$ \\
\hline $4.498 e+00$ & $1.771 \mathrm{e}+01$ & $7.408 e-03$ & $2.916 \mathrm{e}-02$ \\
\hline $6.752 e+02$ & $2.836 e+03$ & $1.192 \mathrm{e}+00$ & $5.006 e+00$ \\
\hline $2.285 e+02$ & $9.421 e+02$ & $4.334 \mathrm{e}-01$ & $1.787 e+00$ \\
\hline $8.539 e+02$ & $3.305 e+03$ & $1.664 \mathrm{e}+00$ & $6.440 e+00$ \\
\hline $4.216 e+02$ & $1.518 \mathrm{e}+03$ & $8.276 \mathrm{e}-01$ & $2.980 e+00$ \\
\hline $4.540 \mathrm{e}+07$ & $1.525 \mathrm{e}+08$ & $8.861 e+04$ & $2.976 \mathrm{e}+05$ \\
\hline $7.175 e+04$ & $2.146 \mathrm{e}+05$ & $1.365 e+02$ & $4.081 e+02$ \\
\hline $1.059 \mathrm{e}+05$ & $2.891 e+05$ & $1.952 \mathrm{e}+02$ & $5.328 e+02$ \\
\hline $2.664 e+05$ & $6.174 \mathrm{e}+05$ & $4.482 e+02$ & $1.039 e+03$ \\
\hline $1.834 \mathrm{e}+01$ & $3.862 e+01$ & $2.836 \mathrm{e}-02$ & $5.972 \mathrm{e}-02$ \\
\hline $3.691 \mathrm{e}-03$ & $6.839 \mathrm{e}-03$ & $5.008 \mathrm{e}-06$ & $9.279 \mathrm{e}-06$ \\
\hline $4.584 e+07$ & $1.536 e+08$ & $8.940 e+04$ & $2.996 e+05$ \\
\hline
\end{tabular}

Results - Dose Point \# 2 - $(66.433,80,0) \mathrm{cm}$

E-22 
Page : 3

DOS File : Combined Calcine in Batch Tank.ms6

Run Date: April 5, 2005

Run Time: 1:20:38 PM

Duration : 00:01:03

$\begin{array}{ccc}\frac{\text { Energy }}{\text { MeV }} & & \begin{array}{c}\text { Activity } \\ \text { photons/sec }\end{array} \\ 0.015 & & 3.783 \mathrm{e}+10 \\ 0.02 & 2.107 \mathrm{e}+10 \\ 0.03 & 2.512 \mathrm{e}+12 \\ 0.04 & 6.187 \mathrm{e}+11 \\ 0.05 & 5.475 \mathrm{e}+09 \\ 0.06 & 1.646 \mathrm{e}+10 \\ 0.08 & 2.162 \mathrm{e}+09 \\ 0.1 & 3.831 \mathrm{e}+10 \\ 0.15 & 1.224 \mathrm{e}+08 \\ 0.2 & 6.333 \mathrm{e}+09 \\ 0.3 & 7.536 \mathrm{e}+08 \\ 0.4 & 1.536 \mathrm{e}+09 \\ 0.5 & 4.894 \mathrm{e}+08 \\ 0.6 & 3.723 \mathrm{e}+13 \\ 0.8 & 3.435 \mathrm{e}+10 \\ 1.0 & 3.354 \mathrm{e}+10 \\ 1.5 & 4.049 \mathrm{e}+10 \\ 2.0 & 1.705 \mathrm{e}+06 \\ 3.0 & 1.804 \mathrm{e}+02\end{array}$

\begin{tabular}{|c|c|c|}
\hline$\frac{\text { Fluence Rate }}{\mathrm{MeV} / \mathrm{cm}^{2} / \mathrm{sec}}$ & $\frac{\text { Fluence Rate }}{\mathrm{MeV} / \mathrm{cm}^{2} / \mathrm{sec}}$ & $\frac{\text { Exposure Rate }}{\mathrm{mR} / \mathrm{hr}}$ \\
\hline No Buildup & With Buildup & No Buildup \\
\hline $9.148 \mathrm{e}-188$ & $7.246 e-23$ & $7.847 e-189$ \\
\hline $1.640 \mathrm{e}-85$ & $5.120 e-23$ & $5.680 e-87$ \\
\hline $4.168 \mathrm{e}-25$ & $1.173 \mathrm{e}-20$ & $4.131 \mathrm{e}-27$ \\
\hline $2.909 e-10$ & $4.260 \mathrm{e}-10$ & $1.287 e-12$ \\
\hline $2.277 \mathrm{e}-06$ & $4.032 \mathrm{e}-06$ & $6.064 \mathrm{e}-09$ \\
\hline $4.789 e-03$ & $1.012 \mathrm{e}-02$ & $9.512 e-06$ \\
\hline $2.352 \mathrm{e}-01$ & $6.435 \mathrm{e}-01$ & $3.722 \mathrm{e}-04$ \\
\hline $5.529 e+01$ & $1.796 e+02$ & $8.459 e-02$ \\
\hline $2.384 \mathrm{e}+00$ & $9.269 e+00$ & $3.925 e-03$ \\
\hline $3.523 e+02$ & $1.452 \mathrm{e}+03$ & $6.218 \mathrm{e}-01$ \\
\hline $1.173 e+02$ & $4.707 e+02$ & $2.226 \mathrm{e}-01$ \\
\hline $4.343 e+02$ & $1.629 e+03$ & $8.463 e-01$ \\
\hline $2.129 e+02$ & $7.410 e+02$ & $4.179 e-01$ \\
\hline $2.278 e+07$ & $7.385 e+07$ & $4.447 e+04$ \\
\hline $3.564 e+04$ & $1.027 e+05$ & $6.780 e+01$ \\
\hline $5.215 e+04$ & $1.370 e+05$ & $9.612 e+01$ \\
\hline $1.290 \mathrm{e}+05$ & $2.877 e+05$ & $2.170 \mathrm{e}+02$ \\
\hline $8.771 \mathrm{e}+00$ & $1.779 e+01$ & $1.356 \mathrm{e}-02$ \\
\hline $1.738 \mathrm{e}-03$ & $3.109 \mathrm{e}-03$ & $2.358 \mathrm{e}-06$ \\
\hline $2.300 e+07$ & $7.438 e+07$ & $4.485 e+04$ \\
\hline
\end{tabular}

Exposure Rate $\mathrm{mR} / \mathrm{hr}$

With Buildup

$6.215 e-24$

$1.773 \mathrm{e}-24$

$1.163 \mathrm{e}-22$

$1.884 \mathrm{e}-12$

$1.074 \mathrm{e}-08$

$2.010 \mathrm{e}-05$

$1.018 \mathrm{e}-03$

$2.747 \mathrm{e}-01$

$1.526 \mathrm{e}-02$

$2.562 \mathrm{e}+00$

$8.929 \mathrm{e}-01$

$3.173 e+00$

$1.454 \mathrm{e}+00$

$1.441 \mathrm{e}+05$

$1.953 \mathrm{e}+02$

$2.526 \mathrm{e}+02$

$4.841 \mathrm{e}+02$

$2.751 \mathrm{e}-02$

$4.219 \mathrm{e}-06$

TOTALS: $\quad 4.060 \mathrm{e}+13$

\section{Results - Dose Point \# 3 - $(135.953,80,0) \mathrm{cm}$}

\begin{tabular}{|c|c|}
\hline$\frac{\text { Energy }}{\mathrm{MeV}}$ & Activity \\
\hline 0.015 & $3.783 e+10$ \\
\hline 0.02 & $2.107 e+10$ \\
\hline 0.03 & $2.512 \mathrm{e}+12$ \\
\hline 0.04 & $6.187 e+11$ \\
\hline 0.05 & $5.475 e+09$ \\
\hline 0.06 & $1.646 \mathrm{e}+10$ \\
\hline 0.08 & $2.162 e+09$ \\
\hline 0.1 & $3.831 \mathrm{e}+10$ \\
\hline 0.15 & $1.224 \mathrm{e}+08$ \\
\hline 0.2 & $6.333 e+09$ \\
\hline 0.3 & $7.536 e+08$ \\
\hline 0.4 & $1.536 \mathrm{e}+09$ \\
\hline 0.5 & $4.894 e+08$ \\
\hline 0.6 & $3.723 e+13$ \\
\hline 0.8 & $3.435 e+10$ \\
\hline 1.0 & $3.354 \mathrm{e}+10$ \\
\hline 1.5 & $4.049 e+10$ \\
\hline 2.0 & $1.705 e+06$ \\
\hline 3.0 & $1.804 \mathrm{e}+02$ \\
\hline
\end{tabular}

\section{Fluence Rate Fluence Rate}

$\mathrm{MeV} / \mathrm{cm}^{2} / \mathrm{sec}$

No Buildup

$\mathrm{MeV} / \mathrm{cm}^{2} / \mathrm{sec}$

$3.567 \mathrm{e}-188$

$7.950 \mathrm{e}-86$

With Buildup

$1.964 \mathrm{e}-25$

$2.042 \mathrm{e}-23$

$1.443 \mathrm{e}-23$

$1.390 \mathrm{e}-10$

3.307e-21

$2.035 \mathrm{e}-10$

$1.084 \mathrm{e}-06$

2.247e-03

$1.919 \mathrm{e}-06$

$4.745 \mathrm{e}-03$

$1.041 \mathrm{e}-01$

$2.830 \mathrm{e}-01$

$2.301 \mathrm{e}+01$

$7.354 \mathrm{e}+01$

$9.034 \mathrm{e}-01$

$1.281 \mathrm{e}+02$

$3.388 \mathrm{e}+00$

$5.037 \mathrm{e}+02$

$4.116 \mathrm{e}+01$

$1.564 \mathrm{e}+02$

$1.496 \mathrm{e}+02$

$5.307 \mathrm{e}+02$

$7.239 e+01$

$2.385 e+02$

$7.671 \mathrm{e}+06$

$2.357 e+07$

$3.238 e+04$

$1.182 \mathrm{e}+04$

$4.286 \mathrm{e}+04$

$8.875 \mathrm{e}+04$

Exposure Rate $\mathrm{mR} / \mathrm{hr}$

No Buildup

3.059e-189

$2.754 \mathrm{e}-87$

$1.946 \mathrm{e}-27$

$6.146 \mathrm{e}-13$

2.886e-09

$4.464 \mathrm{e}-06$

$1.648 \mathrm{e}-04$

3.521e-02

$1.488 \mathrm{e}-03$

2.262e-01

7.809e-02

2.915e-01

$1.421 \mathrm{e}-01$

$1.497 \mathrm{e}+04$

$2.248 \mathrm{e}+01$

$4.143 e+04$

$3.151 \mathrm{e}+01$

$6.971 \mathrm{e}+01$

$2.783 e+00$

$4.304 \mathrm{e}-03$

$5.439 \mathrm{e}-04$

$5.443 e+00$

$7.379 \mathrm{e}-07$

TOTALS: $\quad 4.060 e+13$

$7.742 e+06$

$2.374 e+07$

$1.510 \mathrm{e}+04$

$1.451 \mathrm{e}+05$

Exposure Rate $\mathrm{mR} / \mathrm{hr}$

With Buildup

$1.752 \mathrm{e}-24$

$4.999 \mathrm{e}-25$

$3.277 \mathrm{e}-23$

$9.001 \mathrm{e}-13$

$5.113 e-09$

$9.424 \mathrm{e}-06$

$4.478 \mathrm{e}-04$

$1.125 \mathrm{e}-01$

$5.579 \mathrm{e}-03$

8.891e-01

2.968e-01

$1.034 \mathrm{e}+00$

$4.682 \mathrm{e}-01$

$4.601 \mathrm{e}+04$

$6.159 \mathrm{e}+01$

$7.901 \mathrm{e}+01$

$1.493 e+02$

$8.416 \mathrm{e}-03$

$1.279 \mathrm{e}-06$

$4.630 e+04$

$\begin{array}{ll}\frac{\text { Energy }}{\text { MeV }} & \begin{array}{c}\text { Activity } \\ \text { photons/sec }\end{array} \\ 0.015 & 3.783 \mathrm{e}+10 \\ 0.02 & 2.107 \mathrm{e}+10 \\ 0.03 & 2.512 \mathrm{e}+12\end{array}$

Results - Dose Point \# 4 - $(45.953,80,0) \mathrm{cm}$

\begin{tabular}{|c|c|c|}
\hline Fluence Rate & Fluence Rate & Exposure Rate \\
\hline $\mathrm{MeV} / \mathrm{cm}^{2} / \mathrm{sec}$ & $\mathrm{MeV} / \mathrm{cm}^{2} / \mathrm{sec}$ & $\mathrm{mR} / \mathrm{hr}$ \\
\hline No Buildup & With Buildup & No Buildup \\
\hline $1.621 \mathrm{e}-187$ & $1.339 \mathrm{e}-22$ & $1.391 \mathrm{e}-188$ \\
\hline $3.805 e-85$ & $9.459 e-23$ & $1.318 \mathrm{e}-86$ \\
\hline $5.862 e-25$ & $2.167 e-20$ & $5.810 e-27$ \\
\hline
\end{tabular}


Page : 4

DOS File : Combined Calcine in Batch Tank.ms6

Run Date: April 5, 2005

Run Time: 1:20:38 PM

Duration : 00:01:03

\begin{tabular}{|c|c|}
\hline$\frac{\text { Energy }}{\mathrm{MeV}}$ & $\begin{array}{c}\text { Activity } \\
\text { photons/sec }\end{array}$ \\
\hline 0.04 & $6.187 e+11$ \\
\hline 0.05 & $5.475 e+09$ \\
\hline 0.06 & $1.646 e+10$ \\
\hline 0.08 & $2.162 \mathrm{e}+09$ \\
\hline 0.1 & $3.831 e+10$ \\
\hline 0.15 & $1.224 \mathrm{e}+08$ \\
\hline 0.2 & $6.333 e+09$ \\
\hline 0.3 & $7.536 e+08$ \\
\hline 0.4 & $1.536 e+09$ \\
\hline 0.5 & $4.894 e+08$ \\
\hline 0.6 & $3.723 e+13$ \\
\hline 0.8 & $3.435 e+10$ \\
\hline 1.0 & $3.354 \mathrm{e}+10$ \\
\hline 1.5 & $4.049 e+10$ \\
\hline 2.0 & $1.705 e+06$ \\
\hline 3.0 & $1.804 e+02$ \\
\hline
\end{tabular}

\begin{tabular}{cc}
$\frac{\text { Fluence Rate }}{\text { MeV/cm } 2 / \mathrm{sec}}$ & $\frac{\text { Fluence Rate }}{\text { MeV/cm } 2 / \mathrm{sec}}$ \\
\hline$\frac{\text { No Buildup }}{4.135 \mathrm{e}-10}$ & $\frac{\text { With Buildup }}{6.057 \mathrm{e}-10}$ \\
$3.361 \mathrm{e}-06$ & $5.954 \mathrm{e}-06$ \\
$7.062 \mathrm{e}-03$ & $1.492 \mathrm{e}-02$ \\
$3.492 \mathrm{e}-01$ & $9.560 \mathrm{e}-01$ \\
$8.268 \mathrm{e}+01$ & $2.690 \mathrm{e}+02$ \\
$3.613 \mathrm{e}+00$ & $1.414 \mathrm{e}+01$ \\
$5.387 \mathrm{e}+02$ & $2.246 \mathrm{e}+03$ \\
$1.812 \mathrm{e}+02$ & $7.410 \mathrm{e}+02$ \\
$6.753 \mathrm{e}+02$ & $2.592 \mathrm{e}+03$ \\
$3.328 \mathrm{e}+02$ & $1.188 \mathrm{e}+03$ \\
$3.577 \mathrm{e}+07$ & $1.191 \mathrm{e}+08$ \\
$5.640 \mathrm{e}+04$ & $1.672 \mathrm{e}+05$ \\
$8.306 \mathrm{e}+04$ & $2.248 \mathrm{e}+05$ \\
$2.082 \mathrm{e}+05$ & $4.782 \mathrm{e}+05$ \\
$1.429 \mathrm{e}+01$ & $2.982 \mathrm{e}+01$ \\
$2.866 \mathrm{e}-03$ & $5.261 \mathrm{e}-03$ \\
$3.612 \mathrm{e}+07$ & $1.200 \mathrm{e}+08$
\end{tabular}

$\frac{\text { Exposure Rate }}{\underline{\mathrm{mR} / \mathrm{hr}}}$

No Buildup

$1.829 \mathrm{e}-12$

$8.954 \mathrm{e}-09$

$1.403 \mathrm{e}-05$

$5.527 \mathrm{e}-04$

$1.265 \mathrm{e}-01$

$5.950 \mathrm{e}-03$

$9.507 \mathrm{e}-01$

$3.438 \mathrm{e}-01$

$1.316 \mathrm{e}+00$

$6.532 \mathrm{e}-01$

$6.983 e+04$

$1.073 e+02$

$1.531 \mathrm{e}+02$

$3.502 \mathrm{e}+02$

$2.210 \mathrm{e}-02$

$3.888 \mathrm{e}-06$

$7.044 e+04$ $\frac{\text { Exposure Rate }}{\mathrm{mR} / \mathrm{hr}}$

With Buildup

2.679e-12

$1.586 \mathrm{e}-08$

$2.963 \mathrm{e}-05$

$1.513 \mathrm{e}-03$

$4.115 \mathrm{e}-01$

$2.328 \mathrm{e}-02$

$3.964 \mathrm{e}+00$

$1.406 \mathrm{e}+00$

$5.050 \mathrm{e}+00$

$2.332 \mathrm{e}+00$

$2.326 \mathrm{e}+05$

$3.181 \mathrm{e}+02$

$4.144 \mathrm{e}+02$

$8.046 \mathrm{e}+02$

$4.612 \mathrm{e}-02$

7.137e-06

$2.341 e+05$

$\begin{array}{cc}\begin{array}{cc}\text { Energy } \\ \text { MeV }\end{array} & \begin{array}{c}\text { Activity } \\ \text { photons } / \mathrm{sec}\end{array} \\ 0.015 & 3.783 \mathrm{e}+10 \\ 0.02 & 2.107 \mathrm{e}+10 \\ 0.03 & 2.512 \mathrm{e}+12 \\ 0.04 & 6.187 \mathrm{e}+11 \\ 0.05 & 5.475 \mathrm{e}+09 \\ 0.06 & 1.646 \mathrm{e}+10 \\ 0.08 & 2.162 \mathrm{e}+09 \\ 0.1 & 3.831 \mathrm{e}+10 \\ 0.15 & 1.224 \mathrm{e}+08 \\ 0.2 & 6.333 \mathrm{e}+09 \\ 0.3 & 7.536 \mathrm{e}+08 \\ 0.4 & 1.536 \mathrm{e}+09 \\ 0.5 & 4.894 \mathrm{e}+08 \\ 0.6 & 3.723 \mathrm{e}+13 \\ 0.8 & 3.435 \mathrm{e}+10 \\ 1.0 & 3.354 \mathrm{e}+10 \\ 1.5 & 4.049 \mathrm{e}+10 \\ 2.0 & 1.705 \mathrm{e}+06 \\ 3.0 & 1.804 \mathrm{e}+02\end{array}$

TOTALS: $\quad 4.060 \mathrm{e}+13$

$\begin{array}{ll}\frac{\text { Energy }}{\text { MeV }} & \begin{array}{c}\text { Activity } \\ \text { photons/sec }\end{array} \\ 0.015 & 3.783 \mathrm{e}+10 \\ 0.02 & 2.107 \mathrm{e}+10 \\ 0.03 & 2.512 \mathrm{e}+12 \\ 0.04 & 6.187 \mathrm{e}+11 \\ 0.05 & 5.475 \mathrm{e}+09 \\ 0.06 & 1.646 \mathrm{e}+10\end{array}$

\section{Results - Dose Point \# 5 - $(55.953,80,0) \mathbf{c m}$}

\begin{tabular}{|c|c|c|}
\hline Fluence Rate & Fluence Rate & Exposure Rate \\
\hline $\mathrm{MeV} / \mathrm{cm}^{2} / \mathrm{sec}$ & $\mathrm{MeV} / \mathrm{cm}^{2} / \mathrm{sec}$ & $\mathrm{mR} / \mathrm{hr}$ \\
\hline No Buildup & $\overline{\text { With Buildup }}$ & No Buildup \\
\hline$\overline{1.434 \mathrm{e}-187}$ & $9.638 \mathrm{e}-23$ & $1.230 \mathrm{e}-188$ \\
\hline $2.036 e-85$ & $6.810 e-23$ & $7.051 \mathrm{e}-87$ \\
\hline $4.928 e-25$ & $1.560 \mathrm{e}-20$ & $4.884 \mathrm{e}-27$ \\
\hline $3.482 \mathrm{e}-10$ & $5.099 \mathrm{e}-10$ & $1.540 \mathrm{e}-12$ \\
\hline $2.717 e-06$ & $4.812 \mathrm{e}-06$ & $7.237 \mathrm{e}-09$ \\
\hline $5.729 e-03$ & $1.211 \mathrm{e}-02$ & $1.138 \mathrm{e}-05$ \\
\hline $2.822 \mathrm{e}-01$ & $7.721 \mathrm{e}-01$ & $4.465 e-04$ \\
\hline $6.649 e+01$ & $2.161 \mathrm{e}+02$ & $1.017 \mathrm{e}-01$ \\
\hline $2.885 e+00$ & $1.126 \mathrm{e}+01$ & $4.752 \mathrm{e}-03$ \\
\hline $4.286 e+02$ & $1.779 e+03$ & $7.565 e-01$ \\
\hline $1.436 \mathrm{e}+02$ & $5.827 e+02$ & $2.724 \mathrm{e}-01$ \\
\hline $5.337 e+02$ & $2.028 e+03$ & $1.040 \mathrm{e}+00$ \\
\hline $2.624 \mathrm{e}+02$ & $9.266 \mathrm{e}+02$ & $5.151 \mathrm{e}-01$ \\
\hline $2.815 e+07$ & $9.265 e+07$ & $5.496 e+04$ \\
\hline $4.424 e+04$ & $1.294 \mathrm{e}+05$ & $8.414 e+01$ \\
\hline $6.495 e+04$ & $1.734 \mathrm{e}+05$ & $1.197 e+02$ \\
\hline $1.617 e+05$ & $3.662 e+05$ & $2.721 e+02$ \\
\hline $1.105 e+01$ & $2.273 e+01$ & $1.709 \mathrm{e}-02$ \\
\hline $2.203 e-03$ & $3.989 e-03$ & $2.989 \mathrm{e}-06$ \\
\hline $2.843 e+07$ & $9.332 e+07$ & $5.543 e+04$ \\
\hline
\end{tabular}

\section{Results - Dose Point \# 6 - $(100.953,80,0) \mathrm{cm}$}

$\begin{array}{ccc}\frac{\text { Fluence Rate }}{\text { MeV/cm } / \mathrm{sec}} & \frac{\text { Fluence Rate }}{\text { MeV/cm } 2 / \mathrm{sec}} & \frac{\text { Exposure Rate }}{\mathrm{mR} / \mathrm{hr}} \\ \frac{\text { No Buildup }}{5.179 \mathrm{e}-188} & \frac{\text { With Buildup }}{3.517 \mathrm{e}-23} & \frac{\text { No Buildup }}{4.442 \mathrm{e}-189} \\ 1.091 \mathrm{e}-85 & 2.485 \mathrm{e}-23 & 3.780 \mathrm{e}-87 \\ 2.682 \mathrm{e}-25 & 5.694 \mathrm{e}-21 & 2.658 \mathrm{e}-27 \\ 1.893 \mathrm{e}-10 & 2.772 \mathrm{e}-10 & 8.371 \mathrm{e}-13 \\ 1.478 \mathrm{e}-06 & 2.619 \mathrm{e}-06 & 3.938 \mathrm{e}-09 \\ 3.104 \mathrm{e}-03 & 6.560 \mathrm{e}-03 & 6.166 \mathrm{e}-06\end{array}$

Exposure Rate $\mathrm{mR} / \mathrm{hr}$

With Buildup

$8.267 \mathrm{e}-24$

$2.359 \mathrm{e}-24$

$1.547 \mathrm{e}-22$

$2.255 \mathrm{e}-12$

$1.282 \mathrm{e}-08$

$2.404 \mathrm{e}-05$

$1.222 \mathrm{e}-03$

$3.306 \mathrm{e}-01$

$1.854 \mathrm{e}-02$

$3.139 \mathrm{e}+00$

$1.105 \mathrm{e}+00$

$3.952 e+00$

$1.819 \mathrm{e}+00$

$1.808 \mathrm{e}+05$

$2.462 \mathrm{e}+02$

$3.196 \mathrm{e}+02$

$6.161 \mathrm{e}+02$

$3.515 \mathrm{e}-02$

5.412e-06

$1.820 \mathrm{e}+05$

Exposure Rate $\mathrm{mR} / \mathrm{hr}$

With Buildup

3.017e-24

$8.608 \mathrm{e}-25$

$5.643 \mathrm{e}-23$

$1.226 \mathrm{e}-12$

$6.975 \mathrm{e}-09$

1.303e-05 
Page : 5

DOS File : Combined Calcine in Batch Tank.ms6

Run Date: April 5, 2005

Run Time: 1:20:38 PM

Duration : 00:01:03

$\begin{array}{ccc}\frac{\text { Energy }}{\text { MeV }} & & \begin{array}{c}\text { Activity } \\ \text { photons } / \mathrm{sec}\end{array} \\ 0.08 & & 2.162 \mathrm{e}+09 \\ 0.1 & & 3.831 \mathrm{e}+10 \\ 0.15 & 1.224 \mathrm{e}+08 \\ 0.2 & 6.333 \mathrm{e}+09 \\ 0.3 & 7.536 \mathrm{e}+08 \\ 0.4 & 1.536 \mathrm{e}+09 \\ 0.5 & 4.894 \mathrm{e}+08 \\ 0.6 & 3.723 \mathrm{e}+13 \\ 0.8 & 3.435 \mathrm{e}+10 \\ 1.0 & 3.354 \mathrm{e}+10 \\ 1.5 & 4.049 \mathrm{e}+10 \\ 2.0 & 1.705 \mathrm{e}+06 \\ 3.0 & 1.804 \mathrm{e}+02\end{array}$

\begin{tabular}{c}
$\frac{\text { Fluence Rate }}{\text { MeV/cm } 2 / \mathrm{sec}}$ \\
\hline$\frac{\text { No Buildup }}{1.506 \mathrm{e}-01}$ \\
$3.461 \mathrm{e}+01$ \\
$1.424 \mathrm{e}+00$ \\
$2.054 \mathrm{e}+02$ \\
$6.683 \mathrm{e}+01$ \\
$2.443 \mathrm{e}+02$ \\
$1.187 \mathrm{e}+02$ \\
$1.262 \mathrm{e}+07$ \\
$1.953 \mathrm{e}+04$ \\
$2.834 \mathrm{e}+04$ \\
$6.906 \mathrm{e}+04$ \\
$4.654 \mathrm{e}+00$ \\
$9.128 \mathrm{e}-04$
\end{tabular}

Fluence Rate

$\mathrm{MeV} / \mathrm{cm}^{2} / \mathrm{sec}$

With Buildup

$4.112 \mathrm{e}-01$

$1.117 \mathrm{e}+02$

$5.424 \mathrm{e}+00$

$8.215 \mathrm{e}+02$

$2.586 e+02$

$8.820 \mathrm{e}+02$

$3.977 \mathrm{e}+02$

$3.939 \mathrm{e}+07$

$5.427 e+04$

$7.199 \mathrm{e}+04$

$1.495 \mathrm{e}+05$

$9.189 \mathrm{e}+00$

$1.595 \mathrm{e}-03$

$1.274 \mathrm{e}+07$

$3.967 \mathrm{e}+07$

\section{Exposure Rate $\mathrm{mR} / \mathrm{hr}$}

No Buildup

2.383e-04

$5.295 \mathrm{e}-02$

2.345e-03

$3.625 \mathrm{e}-01$

$1.268 \mathrm{e}-01$

4.761e-01

2.330e-01

$2.463 e+04$

$3.715 \mathrm{e}+01$

$5.223 e+01$

$1.162 \mathrm{e}+02$

7.197e-03

$1.238 \mathrm{e}-06$

$2.484 \mathrm{e}+04$
Exposure Rate $\mathrm{mR} / \mathrm{hr}$

With Buildup

6.507e-04

$1.708 \mathrm{e}-01$

$8.932 \mathrm{e}-03$

$1.450 \mathrm{e}+00$

4.905e-01

$1.719 \mathrm{e}+00$

7.807e-01

$7.689 \mathrm{e}+04$

$1.032 \mathrm{e}+02$

$1.327 \mathrm{e}+02$

$2.516 \mathrm{e}+02$

$1.421 \mathrm{e}-02$

2.165e-06

$7.738 e+04$ 
Page : 1

DOS File : Combined Calcine in Mixer.ms6

Run Date: April 5, 2005

Run Time: 1:24:23 PM

Duration : 00:01:03
File Ref:

Date:

By:

Checked:

Case Title: Combined Calcine

Description: Combined Calcine in Grout Mixer (3/8" 316 SS) Geometry: 7 - Cylinder Volume - Side Shields

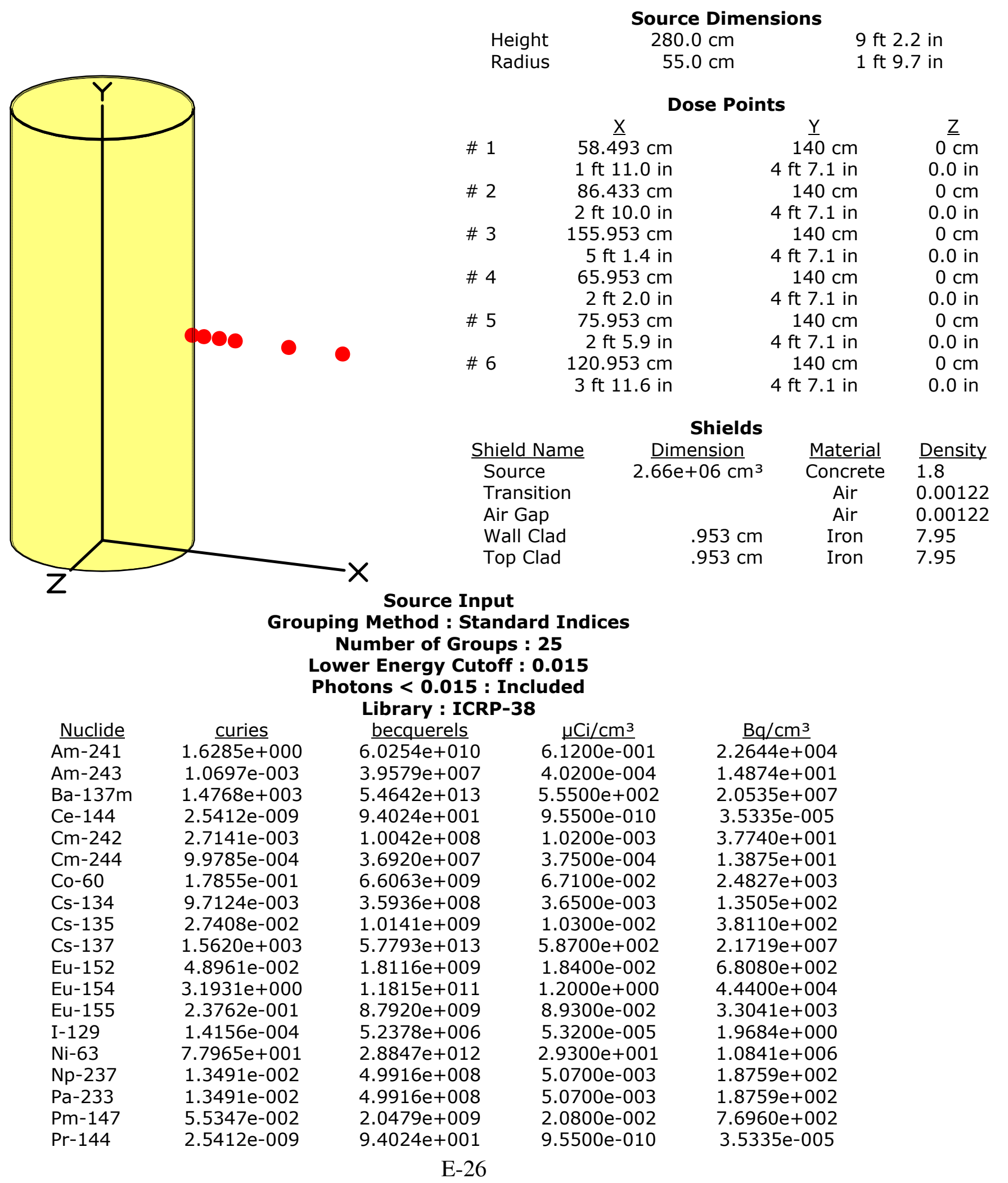


Page : 2

DOS File : Combined Calcine in Mixer.ms6

Run Date: April 5, 2005

Run Time: 1:24:23 PM

Duration : 00:01:03

\begin{tabular}{|c|c|c|c|c|}
\hline Nuclide & curies & becquerels & $\mu \mathrm{Ci} / \mathrm{cm}^{3}$ & $\mathrm{~Bq} / \mathrm{cm}^{3}$ \\
\hline Pu-238 & $1.8414 \mathrm{e}+001$ & $6 . \overline{8130 e+011}$ & $6.9200 \mathrm{e}+000$ & $2.5604 \mathrm{e}+005$ \\
\hline Pu-239 & $4.2043 e-001$ & $1.5556 \mathrm{e}+010$ & $1.5800 \mathrm{e}-001$ & $5.8460 e+003$ \\
\hline Pu-240 & $2.5039 \mathrm{e}-001$ & $9.2646 \mathrm{e}+009$ & $9.4100 \mathrm{e}-002$ & $3.4817 e+003$ \\
\hline Pu-241 & $6.9716 e+000$ & $2.5795 e+011$ & $2.6200 \mathrm{e}+000$ & $9.6940 e+004$ \\
\hline Pu-242 & $3.3794 \mathrm{e}-002$ & $1.2504 \mathrm{e}+009$ & $1.2700 \mathrm{e}-002$ & $4.6990 e+002$ \\
\hline Ru-106 & $9.0472 \mathrm{e}-008$ & $3.3474 \mathrm{e}+003$ & $3.4000 \mathrm{e}-008$ & $1.2580 \mathrm{e}-003$ \\
\hline Sb-125 & $2.5226 \mathrm{e}-002$ & $9.3335 e+008$ & $9.4800 \mathrm{e}-003$ & $3.5076 e+002$ \\
\hline Sb-126 & $2.4853 e-003$ & $9.1956 \mathrm{e}+007$ & $9.3400 \mathrm{e}-004$ & $3.4558 e+001$ \\
\hline Sb-126m & $1.7802 \mathrm{e}-002$ & $6.5866 e+008$ & $6.6900 \mathrm{e}-003$ & $2.4753 e+002$ \\
\hline Sm-151 & $1.5992 \mathrm{e}+001$ & $5.9171 e+011$ & $6.0100 e+000$ & $2.2237 e+005$ \\
\hline Sr-90 & $1.3917 \mathrm{e}+003$ & $5.1492 \mathrm{e}+013$ & $5.2300 \mathrm{e}+002$ & $1.9351 \mathrm{e}+007$ \\
\hline Tc-99 & $6.5991 \mathrm{e}-001$ & $2.4417 e+010$ & $2.4800 \mathrm{e}-001$ & $9.1760 e+003$ \\
\hline Th-230 & $6.9184 \mathrm{e}-005$ & $2.5598 \mathrm{e}+006$ & $2.6000 \mathrm{e}-005$ & $9.6200 e-001$ \\
\hline Th-231 & $6.6789 e-005$ & $2.4712 \mathrm{e}+006$ & $2.5100 \mathrm{e}-005$ & $9.2870 e-001$ \\
\hline$U-232$ & $4.8163 e-005$ & $1.7820 e+006$ & $1.8100 \mathrm{e}-005$ & $6.6970 e-001$ \\
\hline$U-233$ & $3.1931 \mathrm{e}-006$ & $1.1815 \mathrm{e}+005$ & $1.2000 \mathrm{e}-006$ & $4.4400 e-002$ \\
\hline$U-234$ & $1.2746 \mathrm{e}-002$ & $4.7160 e+008$ & $4.7900 \mathrm{e}-003$ & $1.7723 e+002$ \\
\hline$U-235$ & $6.5459 \mathrm{e}-005$ & $2.4220 e+006$ & $2.4600 \mathrm{e}-005$ & $9.1020 \mathrm{e}-001$ \\
\hline$U-236$ & $2.0063 e-004$ & $7.4235 e+006$ & $7.5400 \mathrm{e}-005$ & $2.7898 e+000$ \\
\hline$U-237$ & $1.7402 \mathrm{e}-004$ & $6.4389 e+006$ & $6.5400 \mathrm{e}-005$ & $2.4198 \mathrm{e}+000$ \\
\hline$U-238$ & $2.4481 e-005$ & $9.0578 e+005$ & $9.2000 \mathrm{e}-006$ & $3.4040 e-001$ \\
\hline Y-90 & $1.3917 e+003$ & $5.1492 \mathrm{e}+013$ & $5.2300 e+002$ & $1.9351 \mathrm{e}+007$ \\
\hline
\end{tabular}

$\begin{array}{cc}\frac{\text { Energy }}{\text { MeV }} & \begin{array}{c}\text { Activity } \\ \text { photons/sec }\end{array} \\ 0.015 & 6.055 \mathrm{e}+11 \\ 0.02 & 2.773 \mathrm{e}+10 \\ 0.03 & 3.311 \mathrm{e}+12 \\ 0.04 & 8.153 \mathrm{e}+11 \\ 0.05 & 7.209 \mathrm{e}+09 \\ 0.06 & 2.165 \mathrm{e}+10 \\ 0.08 & 2.857 \mathrm{e}+09 \\ 0.1 & 5.043 \mathrm{e}+10 \\ 0.15 & 1.612 \mathrm{e}+08 \\ 0.2 & 8.336 \mathrm{e}+09 \\ 0.3 & 9.935 \mathrm{e}+08 \\ 0.4 & 2.024 \mathrm{e}+09 \\ 0.5 & 6.443 \mathrm{e}+08 \\ 0.6 & 4.907 \mathrm{e}+13 \\ 0.8 & 4.521 \mathrm{e}+10 \\ 1.0 & 4.416 \mathrm{e}+10 \\ 1.5 & 5.330 \mathrm{e}+10 \\ 2.0 & 2.244 \mathrm{e}+06 \\ 3.0 & 2.378 \mathrm{e}+02\end{array}$

Radial

Integration Parameters

Circumferential

Y Direction (axial) $\quad 24$

\begin{tabular}{|c|c|c|c|}
\hline \multicolumn{4}{|c|}{ Results - Dose Point \# $1-(58.493,140,0) \mathrm{cm}$} \\
\hline Fluence Rate & Fluence Rate & Exposure Rate & Exposure Rate \\
\hline $\mathrm{MeV} / \mathrm{cm}^{2} / \mathrm{sec}$ & $\mathrm{MeV} / \mathrm{cm}^{2} / \mathrm{sec}$ & $\mathrm{mR} / \mathrm{hr}$ & $\mathrm{mR} / \mathrm{hr}$ \\
\hline No Buildup & With Buildup & No Buildup & With Buildup \\
\hline $7.935 e-201$ & $1.271 \mathrm{e}-21$ & $6.806 \mathrm{e}-202$ & $1.090 \mathrm{e}-22$ \\
\hline $7.538 \mathrm{e}-90$ & $9.160 \mathrm{e}-23$ & $2.611 \mathrm{e}-91$ & $3.173 e-24$ \\
\hline $2.154 \mathrm{e}-25$ & $2.418 \mathrm{e}-20$ & $2.135 \mathrm{e}-27$ & $2.396 \mathrm{e}-22$ \\
\hline $7.686 \mathrm{e}-10$ & $2.839 \mathrm{e}-09$ & $3.399 \mathrm{e}-12$ & $1.256 \mathrm{e}-11$ \\
\hline $6.320 \mathrm{e}-06$ & $3.627 e-05$ & $1.684 \mathrm{e}-08$ & $9.661 \mathrm{e}-08$ \\
\hline $1.019 \mathrm{e}-02$ & $7.731 \mathrm{e}-02$ & $2.025 \mathrm{e}-05$ & $1.536 \mathrm{e}-04$ \\
\hline $3.048 \mathrm{e}-01$ & $2.803 e+00$ & $4.823 e-04$ & $4.435 e-03$ \\
\hline $5.122 e+01$ & $4.787 e+02$ & $7.836 e-02$ & $7.323 e-01$ \\
\hline $1.427 \mathrm{e}+00$ & $1.137 \mathrm{e}+01$ & $2.351 \mathrm{e}-03$ & $1.872 \mathrm{e}-02$ \\
\hline $1.770 e+02$ & $1.223 e+03$ & $3.124 \mathrm{e}-01$ & $2.159 e+00$ \\
\hline $5.269 e+01$ & $2.962 e+02$ & $9.995 e-02$ & $5.619 \mathrm{e}-01$ \\
\hline $1.896 \mathrm{e}+02$ & $9.178 e+02$ & $3.694 \mathrm{e}-01$ & $1.788 \mathrm{e}+00$ \\
\hline $9.227 e+01$ & $3.973 e+02$ & $1.811 \mathrm{e}-01$ & $7.798 \mathrm{e}-01$ \\
\hline $9.891 e+06$ & $3.865 e+07$ & $1.931 \mathrm{e}+04$ & $7.543 e+04$ \\
\hline $1.560 \mathrm{e}+04$ & $5.267 e+04$ & $2.966 \mathrm{e}+01$ & $1.002 e+02$ \\
\hline $2.310 e+04$ & $7.009 e+04$ & $4.258 e+01$ & $1.292 \mathrm{e}+02$ \\
\hline $5.910 e+04$ & $1.500 e+05$ & $9.944 e+01$ & $2.524 e+02$ \\
\hline $4.167 e+00$ & $9.530 e+00$ & $6.444 \mathrm{e}-03$ & $1.474 \mathrm{e}-02$ \\
\hline $8.823 e-04$ & $1.771 \mathrm{e}-03$ & $1.197 \mathrm{e}-06$ & $2.403 e-06$ \\
\hline $9.990 e+06$ & $3.892 e+07$ & $1.948 e+04$ & $7.592 e+04$ \\
\hline
\end{tabular}

Results - Dose Point \# 2 - $(86.433,140,0)$ cm 
Page : 3

DOS File : Combined Calcine in Mixer.ms6

Run Date: April 5, 2005

Run Time: 1:24:23 PM

Duration : 00:01:03

$\begin{array}{cc}\begin{array}{cc}\text { Energy } \\ \text { MeV }\end{array} & \begin{array}{c}\text { Activity } \\ \text { photons/sec }\end{array} \\ 0.015 & \\ 0.02 & 6.055 \mathrm{e}+11 \\ 0.03 & 2.773 \mathrm{e}+10 \\ 0.04 & 3.311 \mathrm{e}+12 \\ 0.05 & 8.153 \mathrm{e}+11 \\ 0.06 & 7.209 \mathrm{e}+09 \\ 0.08 & 2.165 \mathrm{e}+10 \\ 0.1 & 2.857 \mathrm{e}+09 \\ 0.15 & 5.043 \mathrm{e}+10 \\ 0.2 & 1.612 \mathrm{e}+08 \\ 0.3 & 8.336 \mathrm{e}+09 \\ 0.4 & 9.935 \mathrm{e}+08 \\ 0.5 & 2.024 \mathrm{e}+09 \\ 0.6 & 6.443 \mathrm{e}+08 \\ 0.8 & 4.907 \mathrm{e}+13 \\ 1.0 & 4.521 \mathrm{e}+10 \\ 1.5 & 4.416 \mathrm{e}+10 \\ 2.0 & 5.330 \mathrm{e}+10 \\ 3.0 & 2.244 \mathrm{e}+06 \\ & 2.378 \mathrm{e}+02\end{array}$

\begin{tabular}{|c|c|c|}
\hline$\frac{\text { Fluence Rate }}{\mathrm{MeV} / \mathrm{cm}^{2} / \mathrm{sec}}$ & $\frac{\text { Fluence Rate }}{\mathrm{MeV} / \mathrm{cm}^{2} / \mathrm{sec}}$ & $\frac{\text { Exposure Rate }}{\mathrm{mR} / \mathrm{hr}}$ \\
\hline No Buildup & With Buildup & No Buildup \\
\hline $4.289 \mathrm{e}-187$ & $6.501 \mathrm{e}-22$ & $3.679 \mathrm{e}-188$ \\
\hline $5.720 e-85$ & $4.685 e-23$ & $1.981 \mathrm{e}-86$ \\
\hline $7.345 e-25$ & $1.237 \mathrm{e}-20$ & $7.280 e-27$ \\
\hline $4.818 \mathrm{e}-10$ & $1.761 \mathrm{e}-09$ & $2.131 \mathrm{e}-12$ \\
\hline $3.210 \mathrm{e}-06$ & $1.846 \mathrm{e}-05$ & $8.550 \mathrm{e}-09$ \\
\hline $5.587 e-03$ & $4.318 \mathrm{e}-02$ & $1.110 \mathrm{e}-05$ \\
\hline $1.884 \mathrm{e}-01$ & $1.766 \mathrm{e}+00$ & $2.981 \mathrm{e}-04$ \\
\hline $3.235 e+01$ & $3.035 e+02$ & $4.950 e-02$ \\
\hline $8.912 \mathrm{e}-01$ & $7.003 e+00$ & $1.468 \mathrm{e}-03$ \\
\hline $1.094 \mathrm{e}+02$ & $7.423 e+02$ & $1.931 \mathrm{e}-01$ \\
\hline $3.223 e+01$ & $1.774 \mathrm{e}+02$ & $6.113 \mathrm{e}-02$ \\
\hline $1.153 e+02$ & $5.459 e+02$ & $2.246 \mathrm{e}-01$ \\
\hline $5.585 e+01$ & $2.353 e+02$ & $1.096 \mathrm{e}-01$ \\
\hline $5.967 e+06$ & $2.282 e+07$ & $1.165 e+04$ \\
\hline $9.355 e+03$ & $3.095 e+04$ & $1.779 e+01$ \\
\hline $1.379 e+04$ & $4.105 e+04$ & $2.543 e+01$ \\
\hline $3.500 e+04$ & $8.735 e+04$ & $5.889 e+01$ \\
\hline $2.455 e+00$ & $5.528 e+00$ & $3.796 e-03$ \\
\hline $5.166 \mathrm{e}-04$ & $1.023 \mathrm{e}-03$ & $7.009 e-07$ \\
\hline $6.025 e+06$ & $2.298 e+07$ & $1.175 e+04$ \\
\hline
\end{tabular}

Exposure Rate $\mathrm{mR} / \mathrm{hr}$

With Buildup

$5.576 \mathrm{e}-23$

$1.623 e-24$

$1.226 \mathrm{e}-22$

$7.788 \mathrm{e}-12$

$4.916 \mathrm{e}-08$

$8.577 \mathrm{e}-05$

$2.795 \mathrm{e}-03$

$4.644 \mathrm{e}-01$

$1.153 \mathrm{e}-02$

$1.310 \mathrm{e}+00$

$3.364 \mathrm{e}-01$

$1.064 \mathrm{e}+00$

$4.619 \mathrm{e}-01$

$4.454 \mathrm{e}+04$

$5.887 e+01$

$7.567 \mathrm{e}+01$

$1.470 \mathrm{e}+02$

$8.548 \mathrm{e}-03$

$1.388 \mathrm{e}-06$

TOTALS: $\quad 5.406 \mathrm{e}+13$

\section{Results - Dose Point \# 3 - $(155.953,140,0) \mathbf{c m}$}

$\begin{array}{cc}\begin{array}{cc}\text { Energy } \\ \text { MeV }\end{array} & \begin{array}{c}\text { Activity } \\ \text { photons/sec }\end{array} \\ 0.015 & 6.055 \mathrm{e}+11 \\ 0.02 & 2.773 \mathrm{e}+10 \\ 0.03 & 3.311 \mathrm{e}+12 \\ 0.04 & 8.153 \mathrm{e}+11 \\ 0.05 & 7.209 \mathrm{e}+09 \\ 0.06 & 2.165 \mathrm{e}+10 \\ 0.08 & 2.857 \mathrm{e}+09 \\ 0.1 & 5.043 \mathrm{e}+10 \\ 0.15 & 1.612 \mathrm{e}+08 \\ 0.2 & 8.336 \mathrm{e}+09 \\ 0.3 & 9.935 \mathrm{e}+08 \\ 0.4 & 2.024 \mathrm{e}+09 \\ 0.5 & 6.443 \mathrm{e}+08 \\ 0.6 & 4.907 \mathrm{e}+13 \\ 0.8 & 4.521 \mathrm{e}+10 \\ 1.0 & 4.416 \mathrm{e}+10 \\ 1.5 & 5.330 \mathrm{e}+10 \\ 2.0 & 2.244 \mathrm{e}+06 \\ 3.0 & 2.378 \mathrm{e}+02\end{array}$

\section{Fluence Rate}

$\mathrm{MeV} / \mathrm{cm}^{2} / \mathrm{sec}$

No Buildup

Fluence Rate

$\mathrm{MeV} / \mathrm{cm}^{2} / \mathrm{sec}$

$1.388 \mathrm{e}-187$

With Buildup

$2.833 \mathrm{e}-85$

$2.447 \mathrm{e}-22$

$1.764 \mathrm{e}-23$

$4.008 \mathrm{e}-25$

$4.656 \mathrm{e}-21$

$9.489 \mathrm{e}-10$

$2.595 \mathrm{e}-10$

$9.983 \mathrm{e}-06$

$1.736 \mathrm{e}-06$

$3.011 \mathrm{e}-03$

$2.328 \mathrm{e}-02$

$1.005 \mathrm{e}-01$

$1.698 \mathrm{e}+01$

$9.404 \mathrm{e}-01$

$1.578 \mathrm{e}+02$

4.506e-01

$3.442 \mathrm{e}+00$

$5.418 \mathrm{e}+01$

$3.543 e+02$

$1.564 \mathrm{e}+01$

$8.253 \mathrm{e}+01$

Exposure Rate $\mathrm{mR} / \mathrm{hr}$

No Buildup

$1.191 \mathrm{e}-188$

$9.815 \mathrm{e}-87$

$3.973 \mathrm{e}-27$

$1.148 \mathrm{e}-12$

4.624e-09

5.980e-06

$1.590 \mathrm{e}-04$

2.597e-02

$7.421 \mathrm{e}-04$

$5.531 \mathrm{e}+01$

$9.563 \mathrm{e}-02$

2.967e-02

$2.509 \mathrm{e}+02 \quad 1.078 \mathrm{e}-01$

$2.658 \mathrm{e}+01$

$5.217 \mathrm{e}-02$

$2.821 \mathrm{e}+06$

$1.073 \mathrm{e}+02$

$5.505 e+03$

$4.375 e+03$

$1.035 e+07$

$8.321 \mathrm{e}+00$

$6.395 \mathrm{e}+03$

$1.391 \mathrm{e}+04$

$1.179 \mathrm{e}+01$

$1.597 \mathrm{e}+04$

$1.832 \mathrm{e}+04$

$3.851 \mathrm{e}+04$

$2.687 \mathrm{e}+01$

$1.108 \mathrm{e}+00$

$2.417 \mathrm{e}+00$

$1.714 \mathrm{e}-03$

2.301e-04

4.427e-04

$3.121 \mathrm{e}-07$

$4.482 \mathrm{e}+04$

TOTALS:

$5.406 e+13$

$2.847 e+06$

$1.042 \mathrm{e}+07$

$5.553 e+03$

Exposure Rate $\mathrm{mR} / \mathrm{hr}$

With Buildup

$2.099 \mathrm{e}-23$

$6.109 \mathrm{e}-25$

$4.614 \mathrm{e}-23$

4.197e-12

2.659e-08

$4.624 \mathrm{e}-05$

$1.488 \mathrm{e}-03$

2.414e-01

5.669e-03

6.252e-01

$1.565 \mathrm{e}-01$

4.889e-01

2.107e-01

$2.019 \mathrm{e}+04$

$2.646 \mathrm{e}+01$

$3.378 \mathrm{e}+01$

$6.479 \mathrm{e}+01$

$3.737 \mathrm{e}-03$

6.007e-07

Results - Dose Point \# 4 - $(65.953,140,0) \mathrm{cm}$

$\begin{array}{cc}\frac{\text { Energy }}{\text { MeV }} & \begin{array}{c}\text { Activity } \\ \text { photons/sec }\end{array} \\ 0.015 & 6.055 \mathrm{e}+11 \\ 0.02 & 2.773 \mathrm{e}+10 \\ 0.03 & 3.311 \mathrm{e}+12\end{array}$

Fluence Rate

$\mathrm{MeV} / \mathrm{cm}^{2} / \mathrm{sec}$

With Buildup

$1.015 \mathrm{e}-21$

$7.314 \mathrm{e}-23$

$1.931 \mathrm{e}-20$
Exposure Rate $\mathrm{mR} / \mathrm{hr}$

No Buildup

6.340e-189

$2.685 \mathrm{e}-86$

$1.319 \mathrm{e}-26$

$2.032 e+04$

Exposure Rate $\mathrm{mR} / \mathrm{hr}$

With Buildup

8.704e-23

$2.533 e-24$

$1.913 \mathrm{e}-22$ 
Page : 4

DOS File : Combined Calcine in Mixer.ms6

Run Date: April 5, 2005

Run Time: 1:24:23 PM

Duration : 00:01:03

$\begin{array}{ccc}\frac{\text { Energy }}{\text { MeV }} & & \begin{array}{c}\text { Activity } \\ \text { photons } / \mathrm{sec}\end{array} \\ 0.04 & & 8.153 \mathrm{e}+11 \\ 0.05 & & 7.209 \mathrm{e}+09 \\ 0.06 & & 2.165 \mathrm{e}+10 \\ 0.08 & & 2.857 \mathrm{e}+09 \\ 0.1 & 5.043 \mathrm{e}+10 \\ 0.15 & 1.612 \mathrm{e}+08 \\ 0.2 & 8.336 \mathrm{e}+09 \\ 0.3 & 9.935 \mathrm{e}+08 \\ 0.4 & 2.024 \mathrm{e}+09 \\ 0.5 & 6.443 \mathrm{e}+08 \\ 0.6 & 4.907 \mathrm{e}+13 \\ 0.8 & 4.521 \mathrm{e}+10 \\ 1.0 & 4.416 \mathrm{e}+10 \\ 1.5 & 5.330 \mathrm{e}+10 \\ 2.0 & 2.244 \mathrm{e}+06 \\ 3.0 & 2.378 \mathrm{e}+02\end{array}$

\begin{tabular}{|c|c|c|}
\hline$\frac{\text { Fluence Rate }}{\mathrm{MeV} / \mathrm{cm}^{2} / \mathrm{sec}}$ & $\frac{\text { Fluence Rate }}{\mathrm{MeV} / \mathrm{cm}^{2} / \mathrm{sec}}$ & $\frac{\text { Exposure Rate }}{\mathrm{mR} / \mathrm{hr}}$ \\
\hline No Buildup & With Buildup & No Buildup \\
\hline $6.346 \mathrm{e}-10$ & $2.314 \mathrm{e}-09$ & $2.807 e-12$ \\
\hline $4.124 \mathrm{e}-06$ & $2.376 \mathrm{e}-05$ & $1.099 \mathrm{e}-08$ \\
\hline $7.390 \mathrm{e}-03$ & $5.731 \mathrm{e}-02$ & $1.468 \mathrm{e}-05$ \\
\hline $2.537 \mathrm{e}-01$ & $2.384 \mathrm{e}+00$ & $4.015 e-04$ \\
\hline $4.391 \mathrm{e}+01$ & $4.134 \mathrm{e}+02$ & $6.718 \mathrm{e}-02$ \\
\hline $1.223 e+00$ & $9.668 e+00$ & $2.014 \mathrm{e}-03$ \\
\hline $1.508 \mathrm{e}+02$ & $1.032 \mathrm{e}+03$ & $2.662 \mathrm{e}-01$ \\
\hline $4.465 e+01$ & $2.482 \mathrm{e}+02$ & $8.470 \mathrm{e}-02$ \\
\hline $1.601 \mathrm{e}+02$ & $7.664 e+02$ & $3.120 \mathrm{e}-01$ \\
\hline $7.776 e+01$ & $3.310 e+02$ & $1.526 \mathrm{e}-01$ \\
\hline $8.321 \mathrm{e}+06$ & $3.216 \mathrm{e}+07$ & $1.624 \mathrm{e}+04$ \\
\hline $1.308 e+04$ & $4.374 e+04$ & $2.488 e+01$ \\
\hline $1.933 e+04$ & $5.814 \mathrm{e}+04$ & $3.564 \mathrm{e}+01$ \\
\hline $4.928 e+04$ & $1.242 \mathrm{e}+05$ & $8.292 e+01$ \\
\hline $3.467 e+00$ & $7.884 e+00$ & $5.362 \mathrm{e}-03$ \\
\hline $7.326 \mathrm{e}-04$ & $1.464 \mathrm{e}-03$ & $9.939 \mathrm{e}-07$ \\
\hline $8.403 e+06$ & $3.239 e+07$ & $1.639 e+04$ \\
\hline
\end{tabular}

Exposure Rate $\mathrm{mR} / \mathrm{hr}$

With Buildup

$1.023 \mathrm{e}-11$

$6.329 \mathrm{e}-08$

$1.138 \mathrm{e}-04$

$3.772 \mathrm{e}-03$

$6.324 \mathrm{e}-01$

$1.592 \mathrm{e}-02$

$1.821 \mathrm{e}+00$

$4.708 \mathrm{e}-01$

$1.493 e+00$

$6.498 \mathrm{e}-01$

$6.277 \mathrm{e}+04$

$8.320 \mathrm{e}+01$

$1.072 \mathrm{e}+02$

$2.090 \mathrm{e}+02$

$1.219 \mathrm{e}-02$

$1.987 \mathrm{e}-06$

TOTALS: $\quad 5.406 \mathrm{e}+13$

Results - Dose Point \# 5 - $(75.953,140,0) \mathbf{c m}$

\begin{tabular}{|c|c|}
\hline$\frac{\text { Energy }}{\mathrm{MeV}}$ & Activity \\
\hline 0.015 & $6.055 e+11$ \\
\hline 0.02 & $2.773 e+10$ \\
\hline 0.03 & $3.311 \mathrm{e}+12$ \\
\hline 0.04 & $8.153 e+11$ \\
\hline 0.05 & $7.209 e+09$ \\
\hline 0.06 & $2.165 e+10$ \\
\hline 0.08 & $2.857 e+09$ \\
\hline 0.1 & $5.043 e+10$ \\
\hline 0.15 & $1.612 e+08$ \\
\hline 0.2 & $8.336 e+09$ \\
\hline 0.3 & $9.935 e+08$ \\
\hline 0.4 & $2.024 \mathrm{e}+09$ \\
\hline 0.5 & $6.443 e+08$ \\
\hline 0.6 & $4.907 e+13$ \\
\hline 0.8 & $4.521 e+10$ \\
\hline 1.0 & $4.416 e+10$ \\
\hline 1.5 & $5.330 e+10$ \\
\hline 2.0 & $2.244 \mathrm{e}+06$ \\
\hline 3.0 & $2.378 e+02$ \\
\hline
\end{tabular}

\begin{tabular}{|c|c|c|}
\hline Fluence Rate & Fluence Rate & Exposure Rate \\
\hline $\mathrm{MeV} / \mathrm{cm}^{2} / \mathrm{sec}$ & $\mathrm{MeV} / \mathrm{cm}^{2} / \mathrm{sec}$ & $\mathrm{mR} / \mathrm{hr}$ \\
\hline No Buildup & With Buildup & No Buildup \\
\hline $4.815 e-187$ & $8.011 \mathrm{e}-22$ & $4.130 \mathrm{e}-188$ \\
\hline $8.822 e-85$ & $5.774 \mathrm{e}-23$ & $3.056 e-86$ \\
\hline $7.990 \mathrm{e}-25$ & $1.524 \mathrm{e}-20$ & $7.918 e-27$ \\
\hline $5.472 \mathrm{e}-10$ & $2.002 \mathrm{e}-09$ & $2.420 \mathrm{e}-12$ \\
\hline $3.691 \mathrm{e}-06$ & $2.121 \mathrm{e}-05$ & $9.833 e-09$ \\
\hline $6.407 e-03$ & $4.952 \mathrm{e}-02$ & $1.273 e-05$ \\
\hline $2.167 e-01$ & $2.033 e+00$ & $3.430 e-04$ \\
\hline $3.733 e+01$ & $3.507 e+02$ & $5.711 \mathrm{e}-02$ \\
\hline $1.032 \mathrm{e}+00$ & $8.129 \mathrm{e}+00$ & $1.700 \mathrm{e}-03$ \\
\hline $1.270 \mathrm{e}+02$ & $8.641 e+02$ & $2.241 \mathrm{e}-01$ \\
\hline $3.747 e+01$ & $2.071 e+02$ & $7.108 \mathrm{e}-02$ \\
\hline $1.342 \mathrm{e}+02$ & $6.387 e+02$ & $2.614 \mathrm{e}-01$ \\
\hline $6.509 e+01$ & $2.756 e+02$ & $1.278 \mathrm{e}-01$ \\
\hline $6.959 e+06$ & $2.676 e+07$ & $1.358 \mathrm{e}+04$ \\
\hline $1.093 e+04$ & $3.635 e+04$ & $2.078 e+01$ \\
\hline $1.613 e+04$ & $4.827 e+04$ & $2.973 e+01$ \\
\hline $4.103 e+04$ & $1.029 e+05$ & $6.902 e+01$ \\
\hline $2.882 e+00$ & $6.525 e+00$ & $4.457 \mathrm{e}-03$ \\
\hline $6.079 \mathrm{e}-04$ & $1.210 \mathrm{e}-03$ & $8.247 e-07$ \\
\hline $7.027 e+06$ & $2.695 e+07$ & $1.370 e+04$ \\
\hline
\end{tabular}

Exposure Rate $\mathrm{mR} / \mathrm{hr}$

With Buildup

$6.871 \mathrm{e}-23$

$2.000 \mathrm{e}-24$

$1.511 \mathrm{e}-22$

$8.855 \mathrm{e}-12$

$5.651 \mathrm{e}-08$

$9.836 \mathrm{e}-05$

$3.218 \mathrm{e}-03$

$5.365 \mathrm{e}-01$

$1.339 \mathrm{e}-02$

$1.525 \mathrm{e}+00$

$3.929 \mathrm{e}-01$

$1.245 \mathrm{e}+00$

$5.411 \mathrm{e}-01$

$5.223 e+04$

$6.914 \mathrm{e}+01$

$8.897 \mathrm{e}+01$

$1.732 \mathrm{e}+02$

$1.009 \mathrm{e}-02$

$1.641 \mathrm{e}-06$

TOTALS: $\quad 5.406 \mathrm{e}+13$

Results - Dose Point \# 6 - $(120.953,140,0) \mathbf{c m}$

$\begin{array}{cc}\frac{\text { Energy }}{\text { MeV }} & \begin{array}{c}\text { Activity } \\ \text { photons } / \mathrm{sec}\end{array} \\ 0.015 & \\ 0.02 & 6.055 \mathrm{e}+11 \\ 0.03 & 2.773 \mathrm{e}+10 \\ 0.04 & 3.311 \mathrm{e}+12 \\ 0.05 & 8.153 \mathrm{e}+11 \\ 0.06 & 7.209 \mathrm{e}+09 \\ & 2.165 \mathrm{e}+10\end{array}$

$\begin{array}{cc}\frac{\text { Fluence Rate }}{\text { MeV/cm } 2 / \mathrm{sec}} & \frac{\text { Fluence Rate }}{\text { MeV/cm } / \mathrm{sec}} \\ \frac{\text { No Buildup }}{1.934 \mathrm{e}-187} & \frac{\text { With Buildup }}{3.766 \mathrm{e}-22} \\ 3.697 \mathrm{e}-85 & 2.714 \mathrm{e}-23 \\ 5.262 \mathrm{e}-25 & 7.165 \mathrm{e}-21 \\ 3.384 \mathrm{e}-10 & 1.237 \mathrm{e}-09 \\ 2.263 \mathrm{e}-06 & 1.301 \mathrm{e}-05 \\ 3.927 \mathrm{e}-03 & 3.036 \mathrm{e}-02\end{array}$

Exposure Rate $\mathrm{mR} / \mathrm{hr}$

No Buildup

$1.659 \mathrm{e}-188$

$1.281 \mathrm{e}-86$

$5.215 \mathrm{e}-27$

$1.496 \mathrm{e}-12$

6.027e-09

7.801e-06

$5.256 e+04$

Exposure Rate $\mathrm{mR} / \mathrm{hr}$

With Buildup

$3.230 \mathrm{e}-23$

$9.402 \mathrm{e}-25$

$7.101 \mathrm{e}-23$

$5.472 \mathrm{e}-12$

$3.466 \mathrm{e}-08$

6.030e-05 
Page : 5

DOS File : Combined Calcine in Mixer.ms6

Run Date: April 5, 2005

Run Time: 1:24:23 PM

Duration : 00:01:03

\begin{tabular}{|c|c|c|}
\hline$\frac{\text { Energy }}{\mathrm{MeV}}$ & $\begin{array}{c}\text { Activity } \\
\text { photons/sec }\end{array}$ & $\frac{\frac{\text { Fluence Rate }}{\text { MeV/cm }{ }^{2} / \mathrm{sec}}}{\text { No Buildup }}$ \\
\hline 0.08 & $2.857 e+09$ & $1.318 \mathrm{e}-01$ \\
\hline 0.1 & $5.043 e+10$ & $2.253 e+01$ \\
\hline 0.15 & $1.612 \mathrm{e}+08$ & $6.130 \mathrm{e}-01$ \\
\hline 0.2 & $8.336 e+09$ & $7.464 e+01$ \\
\hline 0.3 & $9.935 e+08$ & $2.180 e+01$ \\
\hline 0.4 & $2.024 e+09$ & $7.755 e+01$ \\
\hline 0.5 & $6.443 e+08$ & $3.743 e+01$ \\
\hline 0.6 & $4.907 e+13$ & $3.985 e+06$ \\
\hline 0.8 & $4.521 e+10$ & $6.213 e+03$ \\
\hline 1.0 & $4.416 e+10$ & $9.118 \mathrm{e}+03$ \\
\hline 1.5 & $5.330 e+10$ & $2.293 e+04$ \\
\hline 2.0 & $2.244 \mathrm{e}+06$ & $1.598 \mathrm{e}+00$ \\
\hline 3.0 & $2.378 \mathrm{e}+02$ & $3.336 \mathrm{e}-04$ \\
\hline
\end{tabular}

\begin{tabular}{l}
$\frac{\text { Fluence Rate }}{\text { MeV/cm } 2 / \mathrm{sec}}$ \\
\hline With Buildup \\
\hline $1.235 \mathrm{e}+00$ \\
$2.109 \mathrm{e}+02$ \\
$4.768 \mathrm{e}+00$ \\
$4.987 \mathrm{e}+02$ \\
$1.176 \mathrm{e}+02$ \\
$3.596 \mathrm{e}+02$ \\
$1.543 \mathrm{e}+02$ \\
$1.491 \mathrm{e}+07$ \\
$2.011 \mathrm{e}+04$ \\
$2.657 \mathrm{e}+04$ \\
$5.610 \mathrm{e}+04$ \\
$3.532 \mathrm{e}+00$ \\
$6.494 \mathrm{e}-04$
\end{tabular}

$4.023 e+06$

$1.501 \mathrm{e}+07$

\begin{tabular}{c} 
Exposure Rate \\
\hline mR/hr \\
No Buildup \\
\hline $2.086 \mathrm{e}-04$ \\
$3.446 \mathrm{e}-02$ \\
$1.009 \mathrm{e}-03$ \\
$1.317 \mathrm{e}-01$ \\
$4.135 \mathrm{e}-02$ \\
$1.511 \mathrm{e}-01$ \\
$7.347 \mathrm{e}-02$ \\
$7.778 \mathrm{e}+03$ \\
$1.182 \mathrm{e}+01$ \\
$1.681 \mathrm{e}+01$ \\
$3.858 \mathrm{e}+01$ \\
$2.471 \mathrm{e}-03$ \\
$4.526 \mathrm{e}-07$ \\
$7.846 \mathrm{e}+03$
\end{tabular}

Exposure Rate $\mathrm{mR} / \mathrm{hr}$

With Buildup

$1.955 \mathrm{e}-03$

3.227e-01

$7.852 \mathrm{e}-03$

8.802e-01

2.231e-01

7.006e-01

3.028e-01

$2.910 \mathrm{e}+04$

$3.826 \mathrm{e}+01$

$4.897 \mathrm{e}+01$

$9.438 \mathrm{e}+01$

5.461e-03

8.811e-07

$2.928 e+04$ 
Page : 1

DOS File : Combined Calcine in Bulk Vault.ms6

Run Date: May 3, 2005

Run Time: 2:56:35 PM

Duration : 00:01:09
File Ref:

Date:

By

Checked:

Case Title: Combined Calcine

Description: Combined Calcine in Bulk Retrieval Vault ( 2 ft Concrete) Geometry: 7 - Cylinder Volume - Side Shields

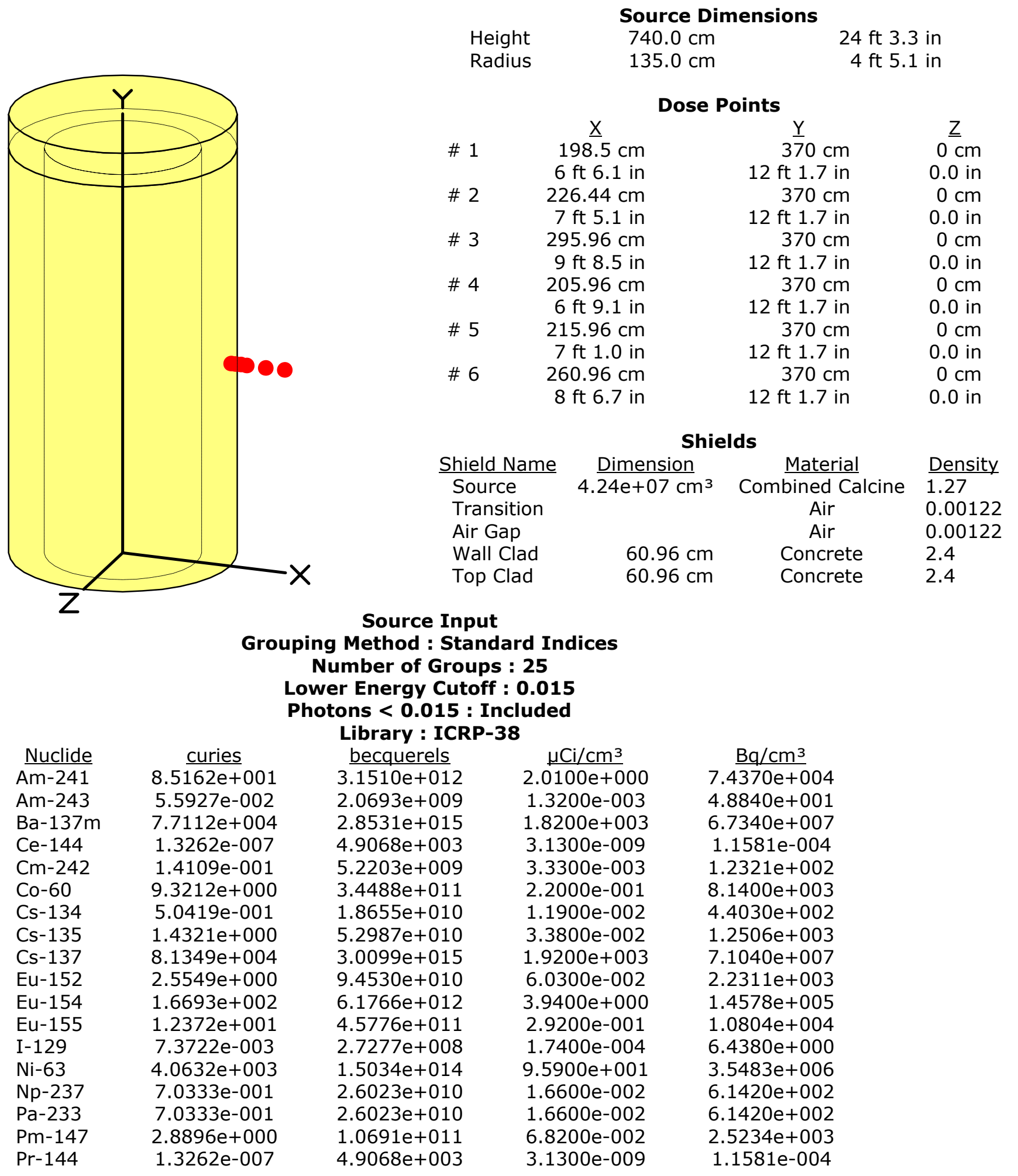


Page : 2

DOS File : Combined Calcine in Bulk Vault.ms6

Run Date: May 3, 2005

Run Time: 2:56:35 PM

Duration : 00:01:09

\begin{tabular}{|c|c|c|c|c|}
\hline Nuclide & curies & becquerels & $\mu \mathrm{Ci} / \mathrm{cm}^{3}$ & $\mathrm{~Bq} / \mathrm{cm}^{3}$ \\
\hline Pu-238 & $9.6 \overline{178 e+002}$ & $3 . \overline{5586 e+013}$ & $2.2700 \mathrm{e}+001$ & $8.3990 e+005$ \\
\hline Pu-239 & $2.1990 \mathrm{e}+001$ & $8.1361 \mathrm{e}+011$ & $5.1900 \mathrm{e}-001$ & $1.9203 e+004$ \\
\hline Pu-240 & $1.3050 \mathrm{e}+001$ & $4.8284 \mathrm{e}+011$ & $3.0800 \mathrm{e}-001$ & $1.1396 \mathrm{e}+004$ \\
\hline Pu-241 & $3.6395 e+002$ & $1.3466 \mathrm{e}+013$ & $8.5900 e+000$ & $3.1783 e+005$ \\
\hline Pu-242 & $1.7668 \mathrm{e}+000$ & $6.5371 \mathrm{e}+010$ & $4.1700 \mathrm{e}-002$ & $1.5429 \mathrm{e}+003$ \\
\hline Ru-106 & $4.7030 \mathrm{e}-006$ & $1.7401 \mathrm{e}+005$ & $1.1100 \mathrm{e}-007$ & $4.1070 \mathrm{e}-003$ \\
\hline Sb- 125 & $1.3177 \mathrm{e}+000$ & $4.8754 \mathrm{e}+010$ & $3.1100 \mathrm{e}-002$ & $1.1507 e+003$ \\
\hline Sb-126 & $1.2965 \mathrm{e}-001$ & $4.7970 e+009$ & $3.0600 \mathrm{e}-003$ & $1.1322 \mathrm{e}+002$ \\
\hline Sb-126m & $9.2788 \mathrm{e}-001$ & $3.4332 \mathrm{e}+010$ & $2.1900 \mathrm{e}-002$ & $8.1030 e+002$ \\
\hline Sm-151 & $8.3467 e+002$ & $3.0883 e+013$ & $1.9700 e+001$ & $7.2890 e+005$ \\
\hline Sr-90 & $7.2451 \mathrm{e}+004$ & $2.6807 e+015$ & $1.7100 \mathrm{e}+003$ & $6.3270 \mathrm{e}+007$ \\
\hline Tc-99 & $3.4488 e+001$ & $1.2761 \mathrm{e}+012$ & $8.1400 \mathrm{e}-001$ & $3.0118 e+004$ \\
\hline Th-230 & $3.6014 \mathrm{e}-003$ & $1.3325 \mathrm{e}+008$ & $8.5000 \mathrm{e}-005$ & $3.1450 \mathrm{e}+000$ \\
\hline Th-231 & $3.4785 e-003$ & $1.2870 \mathrm{e}+008$ & $8.2100 \mathrm{e}-005$ & $3.0377 e+000$ \\
\hline$U-232$ & $2.5167 e-003$ & $9.3119 e+007$ & $5.9400 \mathrm{e}-005$ & $2.1978 \mathrm{e}+000$ \\
\hline$U-233$ & $1.6693 \mathrm{e}-004$ & $6.1766 e+006$ & $3.9400 \mathrm{e}-006$ & $1.4578 \mathrm{e}-001$ \\
\hline$U-234$ & $6.6519 \mathrm{e}-001$ & $2.4612 \mathrm{e}+010$ & $1.5700 \mathrm{e}-002$ & $5.8090 e+002$ \\
\hline$U-235$ & $3.4192 \mathrm{e}-003$ & $1.2651 \mathrm{e}+008$ & $8.0700 \mathrm{e}-005$ & $2.9859 e+000$ \\
\hline$U-236$ & $1.0465 \mathrm{e}-002$ & $3.8721 \mathrm{e}+008$ & $2.4700 \mathrm{e}-004$ & $9.1390 e+000$ \\
\hline$U-237$ & $9.0670 \mathrm{e}-003$ & $3.3548 \mathrm{e}+008$ & $2.1400 \mathrm{e}-004$ & $7.9180 e+000$ \\
\hline$U-238$ & $1.2795 \mathrm{e}-003$ & $4.7343 e+007$ & $3.0200 \mathrm{e}-005$ & $1.1174 \mathrm{e}+000$ \\
\hline Y-90 & $7.2451 \mathrm{e}+004$ & $2.6807 e+015$ & $1.7100 e+003$ & $6.3270 e+007$ \\
\hline
\end{tabular}

$\begin{array}{ccc}\begin{array}{cc}\text { Energy } \\ \underline{\mathrm{MeV}}\end{array} & & \begin{array}{c}\text { Activity } \\ \text { photons } / \mathrm{sec}\end{array} \\ 0.015 & & 3.162 \mathrm{e}+13 \\ 0.02 & & 1.449 \mathrm{e}+12 \\ 0.03 & & 1.729 \mathrm{e}+14 \\ 0.04 & & 4.257 \mathrm{e}+13 \\ 0.05 & & 3.767 \mathrm{e}+11 \\ 0.06 & & 1.132 \mathrm{e}+12 \\ 0.08 & 1.488 \mathrm{e}+11 \\ 0.1 & 2.636 \mathrm{e}+12 \\ 0.15 & 8.425 \mathrm{e}+09 \\ 0.2 & 4.358 \mathrm{e}+11 \\ 0.3 & 5.185 \mathrm{e}+10 \\ 0.4 & 1.057 \mathrm{e}+11 \\ 0.5 & 3.368 \mathrm{e}+10 \\ 0.6 & 2.562 \mathrm{e}+15 \\ 0.8 & 2.363 \mathrm{e}+12 \\ 1.0 & 2.308 \mathrm{e}+12 \\ 1.5 & 2.786 \mathrm{e}+12 \\ 2.0 & 1.173 \mathrm{e}+08 \\ 3.0 & 1.242 \mathrm{e}+04\end{array}$

Radial

Integration Parameters

Y Direction (axial) $\quad 24$

\begin{tabular}{|c|c|c|c|}
\hline \multicolumn{4}{|l|}{ ats - } \\
\hline Fluence Rate & Fluence Rate & Exposure Rate & Exposure Rate \\
\hline $\mathrm{MeV} / \mathrm{cm}^{2} / \mathrm{sec}$ & $\mathrm{MeV} / \mathrm{cm}^{2} / \mathrm{sec}$ & $\mathrm{mR} / \mathrm{hr}$ & $\mathrm{mR} / \mathrm{hr}$ \\
\hline No Buildup & With Buildup & No Buildup & With Buildup \\
\hline $0.000 e+00$ & $5.544 e-21$ & $0.000 e+00$ & $4.755 e-22$ \\
\hline $4.352 e-228$ & $3.224 \mathrm{e}-22$ & $1.507 e-229$ & $1.117 \mathrm{e}-23$ \\
\hline $2.868 e-72$ & $7.389 e-20$ & $2.842 e-74$ & $7.323 e-22$ \\
\hline $2.677 e-35$ & $2.790 \mathrm{e}-20$ & $1.184 \mathrm{e}-37$ & $1.234 \mathrm{e}-22$ \\
\hline $8.139 \mathrm{e}-24$ & $4.355 e-22$ & $2.168 \mathrm{e}-26$ & $1.160 \mathrm{e}-24$ \\
\hline $3.486 \mathrm{e}-17$ & $1.034 \mathrm{e}-16$ & $6.925 e-20$ & $2.054 \mathrm{e}-19$ \\
\hline $9.092 e-13$ & $5.313 e-12$ & $1.439 \mathrm{e}-15$ & $8.408 e-15$ \\
\hline $2.993 e-09$ & $3.141 \mathrm{e}-08$ & $4.578 \mathrm{e}-12$ & $4.805 e-11$ \\
\hline $2.861 \mathrm{e}-09$ & $7.849 \mathrm{e}-08$ & $4.712 \mathrm{e}-12$ & $1.293 \mathrm{e}-10$ \\
\hline $2.577 e-06$ & $1.282 \mathrm{e}-04$ & $4.548 e-09$ & $2.262 \mathrm{e}-07$ \\
\hline $9.337 e-06$ & $5.889 e-04$ & $1.771 \mathrm{e}-08$ & $1.117 \mathrm{e}-06$ \\
\hline $1.753 e-04$ & $1.016 \mathrm{e}-02$ & $3.416 \mathrm{e}-07$ & $1.980 \mathrm{e}-05$ \\
\hline $2.898 \mathrm{e}-04$ & $1.405 \mathrm{e}-02$ & $5.689 e-07$ & $2.758 \mathrm{e}-05$ \\
\hline $8.082 e+01$ & $3.206 e+03$ & $1.578 \mathrm{e}-01$ & $6.258 \mathrm{e}+00$ \\
\hline $5.382 \mathrm{e}-01$ & $1.506 e+01$ & $1.024 \mathrm{e}-03$ & $2.865 e-02$ \\
\hline $2.288 e+00$ & $4.789 e+01$ & $4.217 e-03$ & $8.828 e-02$ \\
\hline $3.382 e+01$ & $4.228 e+02$ & $5.691 \mathrm{e}-02$ & $7.114 \mathrm{e}-01$ \\
\hline $6.987 e-03$ & $6.433 e-02$ & $1.080 \mathrm{e}-05$ & $9.948 e-05$ \\
\hline $5.195 e-06$ & $3.246 \mathrm{e}-05$ & $7.048 \mathrm{e}-09$ & $4.403 e-08$ \\
\hline $1.175 e+02$ & $3.692 e+03$ & $2.199 \mathrm{e}-01$ & $7.086 e+00$ \\
\hline
\end{tabular}

Results - Dose Point \# 2 - $(226.44,370,0) \mathrm{cm}$

E-32 
Page : 3

DOS File : Combined Calcine in Bulk Vault.ms6

Run Date: May 3, 2005

Run Time: 2:56:35 PM

Duration : 00:01:09

\begin{tabular}{|c|c|}
\hline$\frac{\text { Energy }}{\underline{\mathrm{MeV}}}$ & $\begin{array}{c}\text { Activity } \\
\text { photons/sec }\end{array}$ \\
\hline 0.015 & $3.162 e+13$ \\
\hline 0.02 & $1.449 e+12$ \\
\hline 0.03 & $1.729 e+14$ \\
\hline 0.04 & $4.257 e+1$ \\
\hline 0.05 & $3.767 e+1$ \\
\hline 0.06 & $1.132 \mathrm{e}+12$ \\
\hline 0.08 & $1.488 \mathrm{e}+1$ \\
\hline 0.1 & $2.636 \mathrm{e}+1$ \\
\hline 0.15 & $8.425 e+0$ \\
\hline 0.2 & $4.358 e+1$ \\
\hline 0.3 & $5.185 e+1$ \\
\hline 0.4 & $1.057 e+1$ \\
\hline 0.5 & $3.368 e+1$ \\
\hline 0.6 & $2.562 \mathrm{e}+1$ \\
\hline 0.8 & $2.363 e+1$ \\
\hline 1.0 & $2.308 e+1$ \\
\hline 1.5 & $2.786 e+1$ \\
\hline 2.0 & $1.173 e+0$ \\
\hline 3.0 & $1.242 \mathrm{e}+0$ \\
\hline
\end{tabular}

\begin{tabular}{c}
$\frac{\text { Fluence Rate }}{\mathrm{MeV} / \mathrm{cm}^{2} / \mathrm{sec}}$ \\
\hline No Buildup \\
$0.000 \mathrm{e}+00$ \\
$3.517 \mathrm{e}-228$ \\
$2.113 \mathrm{e}-72$ \\
$2.399 \mathrm{e}-35$ \\
$7.350 \mathrm{e}-24$ \\
$3.082 \mathrm{e}-17$ \\
$7.903 \mathrm{e}-13$ \\
$2.593 \mathrm{e}-09$ \\
$2.480 \mathrm{e}-09$ \\
$2.236 \mathrm{e}-06$ \\
$8.109 \mathrm{e}-06$ \\
$1.523 \mathrm{e}-04$ \\
$2.519 \mathrm{e}-04$ \\
$7.026 \mathrm{e}+01$ \\
$4.678 \mathrm{e}-01$ \\
$1.988 \mathrm{e}+00$ \\
$2.938 \mathrm{e}+01$ \\
$6.064 \mathrm{e}-03$ \\
$4.505 \mathrm{e}-06$
\end{tabular}

$1.021 \mathrm{e}+02$

\begin{tabular}{|c|c|}
\hline$\frac{\text { Fluence Rate }}{{\mathrm{MeV} / \mathrm{cm}^{2} / \mathrm{sec}^{2}}}$ & $\frac{\text { Exposure Rate }}{\mathrm{mR} / \mathrm{hr}}$ \\
\hline $\mathrm{MeV} / \mathrm{cm}^{2} / \mathrm{sec}$ & $\mathrm{mR} / \mathrm{hr}$ \\
\hline With Buildup & No Buildup \\
\hline $4.504 \mathrm{e}-21$ & $0.000 \mathrm{e}+00$ \\
\hline $2.620 e-22$ & $1.218 \mathrm{e}-229$ \\
\hline $6.003 e-20$ & $2.094 \mathrm{e}-74$ \\
\hline $2.267 e-20$ & $1.061 \mathrm{e}-37$ \\
\hline $3.553 e-22$ & $1.958 \mathrm{e}-26$ \\
\hline $9.139 \mathrm{e}-17$ & $6.122 \mathrm{e}-20$ \\
\hline $4.616 e-12$ & $1.251 \mathrm{e}-15$ \\
\hline $2.721 \mathrm{e}-08$ & $3.968 \mathrm{e}-12$ \\
\hline $6.806 \mathrm{e}-08$ & $4.084 \mathrm{e}-12$ \\
\hline $1.112 \mathrm{e}-04$ & $3.946 \mathrm{e}-09$ \\
\hline $5.118 e-04$ & $1.538 \mathrm{e}-08$ \\
\hline $8.832 e-03$ & $2.968 \mathrm{e}-07$ \\
\hline $1.222 \mathrm{e}-02$ & $4.945 e-07$ \\
\hline $2.787 e+03$ & $1.371 \mathrm{e}-01$ \\
\hline $1.309 e+01$ & $8.898 e-04$ \\
\hline $4.160 e+01$ & $3.665 e-03$ \\
\hline $3.670 e+02$ & $4.942 \mathrm{e}-02$ \\
\hline $5.580 e-02$ & $9.378 \mathrm{e}-06$ \\
\hline $2.812 \mathrm{e}-05$ & $6.111 \mathrm{e}-09$ \\
\hline $3.209 e+03$ & $1.911 €$ \\
\hline
\end{tabular}

Exposure Rate $\mathrm{mR} / \mathrm{hr}$

With Buildup

$3.863 \mathrm{e}-22$

$9.074 \mathrm{e}-24$

$5.949 \mathrm{e}-22$

$1.002 \mathrm{e}-22$

$9.464 \mathrm{e}-25$

$1.815 \mathrm{e}-19$

$7.305 \mathrm{e}-15$

$4.164 \mathrm{e}-11$

$1.121 \mathrm{e}-10$

$1.963 \mathrm{e}-07$

$9.708 \mathrm{e}-07$

$1.721 \mathrm{e}-05$

$2.398 \mathrm{e}-05$

$5.440 \mathrm{e}+00$

$2.490 \mathrm{e}-02$

$7.669 \mathrm{e}-02$

$6.175 \mathrm{e}-01$

$8.629 \mathrm{e}-05$

$3.816 \mathrm{e}-08$

$6.159 e+00$

\section{Results - Dose Point \# 3 - $(295.96,370,0) \mathrm{cm}$}

$\begin{array}{cc}\begin{array}{cc}\text { Energy } \\ \text { MeV }\end{array} & \begin{array}{c}\text { Activity } \\ \text { photons/sec }\end{array} \\ 0.015 & 3.162 \mathrm{e}+13 \\ 0.02 & 1.449 \mathrm{e}+12 \\ 0.03 & 1.729 \mathrm{e}+14 \\ 0.04 & 4.257 \mathrm{e}+13 \\ 0.05 & 3.767 \mathrm{e}+11 \\ 0.06 & 1.132 \mathrm{e}+12 \\ 0.08 & 1.488 \mathrm{e}+11 \\ 0.1 & 2.636 \mathrm{e}+12 \\ 0.15 & 8.425 \mathrm{e}+09 \\ 0.2 & 4.358 \mathrm{e}+11 \\ 0.3 & 5.185 \mathrm{e}+10 \\ 0.4 & 1.057 \mathrm{e}+11 \\ 0.5 & 3.368 \mathrm{e}+10 \\ 0.6 & 2.562 \mathrm{e}+15 \\ 0.8 & 2.363 \mathrm{e}+12 \\ 1.0 & 2.308 \mathrm{e}+12 \\ 1.5 & 2.786 \mathrm{e}+12 \\ 2.0 & 1.173 \mathrm{e}+08 \\ 3.0 & 1.242 \mathrm{e}+04\end{array}$

$\frac{\frac{\text { Fluence Rate }}{\mathrm{MeV} / \mathrm{cm}^{2} / \mathrm{sec}}}{\text { No Buildup }} \quad$ M

$0.000 e+00$

$1.789 \mathrm{e}-228$

$1.675 \mathrm{e}-72$

$1.830 \mathrm{e}-35$

$5.475 \mathrm{e}-24$

$2.304 \mathrm{e}-17$

$5.940 \mathrm{e}-13$

$1.952 \mathrm{e}-09$

$1.868 \mathrm{e}-09$

$1.684 \mathrm{e}-06$

$6.113 \mathrm{e}-06$

$1.149 \mathrm{e}-04$

Fluence Rate

$\mathrm{MeV} / \mathrm{cm}^{2} / \mathrm{sec}$

With Buildup

$2.938 \mathrm{e}-21$

$1.709 \mathrm{e}-22$

$3.916 \mathrm{e}-20$

$1.479 \mathrm{e}-20$

$2.331 \mathrm{e}-22$

$6.832 \mathrm{e}-17$

$3.471 \mathrm{e}-12$

2.049e-08

$5.127 \mathrm{e}-08$

8.385e-05

$3.860 \mathrm{e}-04$

$6.662 \mathrm{e}-03$

$9.217 \mathrm{e}-03$

$2.103 e+03$

$9.875 e+00$

$3.138 \mathrm{e}+01$

$2.767 \mathrm{e}+02$

$4.205 \mathrm{e}-02$

2.117e-05 $\frac{\text { Exposure Rate }}{\mathrm{mR} / \mathrm{hr}}$

No Buildup

$0.000 \mathrm{e}+00$

$6.196 \mathrm{e}-230$

$1.660 \mathrm{e}-74$

$8.095 \mathrm{e}-38$

$1.458 \mathrm{e}-26$

$4.576 \mathrm{e}-20$

$9.399 \mathrm{e}-16$

$2.986 \mathrm{e}-12$

3.076e-12

2.973e-09

1.160e-08

2.239e-07

3.730e-07

$1.034 \mathrm{e}-01$

$6.713 \mathrm{e}-04$

$2.765 \mathrm{e}-03$

3.727e-02

7.069e-06

4.604e-09

7.701e+01

$2.421 \mathrm{e}+03$

$1.442 \mathrm{e}-01$
Exposure Rate $\mathrm{mR} / \mathrm{hr}$

With Buildup

$2.520 \mathrm{e}-22$

$5.920 \mathrm{e}-24$

$3.881 \mathrm{e}-22$

$6.540 \mathrm{e}-23$

$6.209 \mathrm{e}-25$

$1.357 \mathrm{e}-19$

$5.492 \mathrm{e}-15$

$3.134 \mathrm{e}-11$

$8.443 \mathrm{e}-11$

$1.480 \mathrm{e}-07$

7.321e-07

$1.298 \mathrm{e}-05$

$1.809 \mathrm{e}-05$

$4.104 \mathrm{e}+00$

$1.878 \mathrm{e}-02$

$5.785 \mathrm{e}-02$

4.655e-01

$6.502 \mathrm{e}-05$

2.872e-08

$4.646 e+00$

\section{Results - Dose Point \# 4 - $(205.96,370,0) \mathrm{cm}$}

$\begin{array}{cc}\frac{\text { Energy }}{\underline{\text { MeV }}} & \begin{array}{c}\text { Activity } \\ \text { photons/sec }\end{array} \\ 0.015 & 3.162 \mathrm{e}+13 \\ 0.02 & 1.449 \mathrm{e}+12\end{array}$

Fluence Rate

$\mathrm{MeV} / \mathrm{cm}^{2} / \mathrm{sec}$

With Buildup

$5.230 \mathrm{e}-21$

$3.042 \mathrm{e}-22$

$\frac{\text { Exposure Rate }}{\mathrm{mR} / \mathrm{hr}}$
$\frac{\text { No Buildup }}{0.000 \mathrm{e}+00}$
$1.472 \mathrm{e}-229$

Exposure Rate $\mathrm{mR} / \mathrm{hr}$

With Buildup $4.486 \mathrm{e}-22$ $1.054 \mathrm{e}-23$ 
Page : 4

DOS File : Combined Calcine in Bulk Vault.ms6

Run Date: May 3, 2005

Run Time: 2:56:35 PM

Duration : 00:01:09

$\begin{array}{ccc}\begin{array}{cc}\text { Energy } \\ \text { MeV }\end{array} & \begin{array}{c}\text { Activity } \\ \text { photons/sec }\end{array} \\ 0.03 & & 1.729 \mathrm{e}+14 \\ 0.04 & 4.257 \mathrm{e}+13 \\ 0.05 & 3.767 \mathrm{e}+11 \\ 0.06 & 1.132 \mathrm{e}+12 \\ 0.08 & 1.488 \mathrm{e}+11 \\ 0.1 & 2.636 \mathrm{e}+12 \\ 0.15 & 8.425 \mathrm{e}+09 \\ 0.2 & 4.358 \mathrm{e}+11 \\ 0.3 & 5.185 \mathrm{e}+10 \\ 0.4 & 1.057 \mathrm{e}+11 \\ 0.5 & 3.368 \mathrm{e}+10 \\ 0.6 & 2.562 \mathrm{e}+15 \\ 0.8 & 2.363 \mathrm{e}+12 \\ 1.0 & 2.308 \mathrm{e}+12 \\ 1.5 & 2.786 \mathrm{e}+12 \\ 2.0 & 1.173 \mathrm{e}+08 \\ 3.0 & 1.242 \mathrm{e}+04\end{array}$

\begin{tabular}{cc}
$\frac{\text { Fluence Rate }}{\text { MeV/cm } / \mathrm{sec}}$ & $\frac{\text { Fluence Rate }}{\text { MeV/cm } / \mathrm{sec}}$ \\
\hline \begin{tabular}{c} 
No Buildup \\
\hline $2.576 \mathrm{e}-72$
\end{tabular} & $\begin{array}{c}\text { With Buildup } \\
6.970 \mathrm{e}-20\end{array}$ \\
$2.565 \mathrm{e}-35$ & $2.632 \mathrm{e}-20$ \\
$7.945 \mathrm{e}-24$ & $4.114 \mathrm{e}-22$ \\
$3.390 \mathrm{e}-17$ & $1.005 \mathrm{e}-16$ \\
$8.770 \mathrm{e}-13$ & $5.123 \mathrm{e}-12$ \\
$2.878 \mathrm{e}-09$ & $3.020 \mathrm{e}-08$ \\
$2.748 \mathrm{e}-09$ & $7.538 \mathrm{e}-08$ \\
$2.475 \mathrm{e}-06$ & $1.231 \mathrm{e}-04$ \\
$8.969 \mathrm{e}-06$ & $5.659 \mathrm{e}-04$ \\
$1.685 \mathrm{e}-04$ & $9.765 \mathrm{e}-03$ \\
$2.785 \mathrm{e}-04$ & $1.351 \mathrm{e}-02$ \\
$7.768 \mathrm{e}+01$ & $3.082 \mathrm{e}+03$ \\
$5.174 \mathrm{e}-01$ & $1.448 \mathrm{e}+01$ \\
$2.199 \mathrm{e}+00$ & $4.603 \mathrm{e}+01$ \\
$3.251 \mathrm{e}+01$ & $4.063 \mathrm{e}+02$ \\
$6.714 \mathrm{e}-03$ & $6.180 \mathrm{e}-02$ \\
$4.990 \mathrm{e}-06$ & $3.117 \mathrm{e}-05$ \\
$1.129 \mathrm{e}+02$ & $3.549 \mathrm{e}+03$
\end{tabular}

$\frac{\text { Exposure Rate }}{\mathrm{mR} / \mathrm{hr}}$

No Buildup

$2.553 e-74$

$1.134 \mathrm{e}-37$

$2.117 \mathrm{e}-26$

$6.734 \mathrm{e}-20$

$1.388 \mathrm{e}-15$

$4.403 \mathrm{e}-12$

$4.525 \mathrm{e}-12$

$4.368 \mathrm{e}-09$

$1.701 \mathrm{e}-08$

$3.282 \mathrm{e}-07$

$5.467 \mathrm{e}-07$

$1.516 \mathrm{e}-01$

$9.840 \mathrm{e}-04$

$4.054 \mathrm{e}-03$

$5.469 \mathrm{e}-02$

$1.038 \mathrm{e}-05$

$6.770 \mathrm{e}-09$

2.114e-01
Exposure Rate $\mathrm{mR} / \mathrm{hr}$

With Buildup

$6.908 \mathrm{e}-22$

$1.164 \mathrm{e}-22$

$1.096 \mathrm{e}-24$

$1.997 \mathrm{e}-19$

$8.107 \mathrm{e}-15$

$4.620 \mathrm{e}-11$

$1.241 \mathrm{e}-10$

$2.172 \mathrm{e}-07$

$1.073 \mathrm{e}-06$

$1.903 \mathrm{e}-05$

$2.652 \mathrm{e}-05$

$6.016 \mathrm{e}+00$

$2.754 \mathrm{e}-02$

$8.485 \mathrm{e}-02$

$6.835 \mathrm{e}-01$

$9.557 \mathrm{e}-05$

$4.229 \mathrm{e}-08$

$6.812 \mathrm{e}+00$

Results - Dose Point \# 5 - $(215.96,370,0) \mathbf{c m}$

$\begin{array}{cc}\begin{array}{cc}\text { Energy } \\ \text { MeV }\end{array} & \begin{array}{c}\text { Activity } \\ \text { photons/sec }\end{array} \\ 0.015 & 3.162 \mathrm{e}+13 \\ 0.02 & 1.449 \mathrm{e}+12 \\ 0.03 & 1.729 \mathrm{e}+14 \\ 0.04 & 4.257 \mathrm{e}+13 \\ 0.05 & 3.767 \mathrm{e}+11 \\ 0.06 & 1.132 \mathrm{e}+12 \\ 0.08 & 1.488 \mathrm{e}+11 \\ 0.1 & 2.636 \mathrm{e}+12 \\ 0.15 & 8.425 \mathrm{e}+09 \\ 0.2 & 4.358 \mathrm{e}+11 \\ 0.3 & 5.185 \mathrm{e}+10 \\ 0.4 & 1.057 \mathrm{e}+11 \\ 0.5 & 3.368 \mathrm{e}+10 \\ 0.6 & 2.562 \mathrm{e}+15 \\ 0.8 & 2.363 \mathrm{e}+12 \\ 1.0 & 2.308 \mathrm{e}+12 \\ 1.5 & 2.786 \mathrm{e}+12 \\ 2.0 & 1.173 \mathrm{e}+08 \\ 3.0 & 1.242 \mathrm{e}+04\end{array}$

TOTALS: $\quad 2.823 e+15$

$\begin{array}{cc}\begin{array}{c}\text { Energy } \\ \text { MeV }\end{array} & \begin{array}{c}\text { Activity } \\ \text { photons/sec }\end{array} \\ 0.015 & 3.162 \mathrm{e}+13 \\ 0.02 & 1.449 \mathrm{e}+12 \\ 0.03 & 1.729 \mathrm{e}+14 \\ 0.04 & 4.257 \mathrm{e}+13\end{array}$

\begin{tabular}{|c|c|c|}
\hline Fluence Rate & Fluence Rate & Exposure Rate \\
\hline $\mathrm{MeV} / \mathrm{cm}^{2} / \mathrm{sec}$ & $\mathrm{MeV} / \mathrm{cm}^{2} / \mathrm{sec}$ & $\mathrm{mR} / \mathrm{hr}$ \\
\hline No Buildup & $\overline{\text { With Buildup }}$ & No Buildup \\
\hline $0.000 \mathrm{e}+00$ & $4.853 e-21$ & $0.000 e+00$ \\
\hline $3.927 e-228$ & $2.823 e-22$ & $1.360 \mathrm{e}-229$ \\
\hline $2.297 e-72$ & $6.468 e-20$ & $2.277 e-74$ \\
\hline $2.474 \mathrm{e}-35$ & $2.442 \mathrm{e}-20$ & $1.094 \mathrm{e}-37$ \\
\hline $7.675 e-24$ & $3.823 e-22$ & $2.045 e-26$ \\
\hline $3.243 e-17$ & $9.617 \mathrm{e}-17$ & $6.442 \mathrm{e}-20$ \\
\hline $8.330 \mathrm{e}-13$ & $4.866 \mathrm{e}-12$ & $1.318 \mathrm{e}-15$ \\
\hline $2.731 \mathrm{e}-09$ & $2.866 \mathrm{e}-08$ & $4.179 \mathrm{e}-12$ \\
\hline $2.609 e-09$ & $7.158 \mathrm{e}-08$ & $4.297 e-12$ \\
\hline $2.351 \mathrm{e}-06$ & $1.170 \mathrm{e}-04$ & $4.150 e-09$ \\
\hline $8.526 \mathrm{e}-06$ & $5.381 \mathrm{e}-04$ & $1.617 \mathrm{e}-08$ \\
\hline $1.602 \mathrm{e}-04$ & $9.286 e-03$ & $3.121 \mathrm{e}-07$ \\
\hline $2.649 e-04$ & $1.284 \mathrm{e}-02$ & $5.199 \mathrm{e}-07$ \\
\hline $7.387 e+01$ & $2.931 \mathrm{e}+03$ & $1.442 \mathrm{e}-01$ \\
\hline $4.919 \mathrm{e}-01$ & $1.376 \mathrm{e}+01$ & $9.357 e-04$ \\
\hline $2.091 \mathrm{e}+00$ & $4.376 e+01$ & $3.854 \mathrm{e}-03$ \\
\hline $3.090 \mathrm{e}+01$ & $3.861 e+02$ & $5.198 \mathrm{e}-02$ \\
\hline $6.380 e-03$ & $5.871 \mathrm{e}-02$ & $9.866 e-06$ \\
\hline $4.740 \mathrm{e}-06$ & $2.960 \mathrm{e}-05$ & $6.431 \mathrm{e}-09$ \\
\hline $1.074 \mathrm{e}+02$ & $3.374 \mathrm{e}+03$ & $2.010 \mathrm{e}-01$ \\
\hline
\end{tabular}

\section{Results - Dose Point \# 6 - $(260.96,370,0) \mathrm{cm}$}

\begin{tabular}{|c|c|}
\hline$\frac{\text { Fluence Rate }}{\mathrm{MeV} / \mathrm{cm}^{2} / \mathrm{sec}}$ & $\frac{\text { Fluence Rate }}{\mathrm{MeV} / \mathrm{cm}^{2} / \mathrm{sec}}$ \\
\hline No Buildup & With Buildup \\
\hline $0.000 \mathrm{e}+00$ & $3.598 \mathrm{e}-21$ \\
\hline $2.385 e-228$ & $2.092 \mathrm{e}-22$ \\
\hline $1.856 \mathrm{e}-72$ & $4.795 e-20$ \\
\hline $2.112 \mathrm{e}-35$ & $1.810 \mathrm{e}-20$ \\
\hline
\end{tabular}

\author{
Exposure Rate \\ $\mathrm{mR} / \mathrm{hr}$ \\ No Buildup \\ $0.000 \mathrm{e}+00$ \\ $8.263 e-230$ \\ $1.839 \mathrm{e}-74$ \\ $9.341 \mathrm{e}-38$
}

\section{Exposure Rate $\mathrm{mR} / \mathrm{hr}$ \\ With Buildup \\ $4.163 \mathrm{e}-22$ \\ $9.777 \mathrm{e}-24$ \\ $6.410 \mathrm{e}-22$ \\ $1.080 \mathrm{e}-22$ \\ $1.018 \mathrm{e}-24$ \\ $1.910 \mathrm{e}-19$ \\ $7.700 \mathrm{e}-15$ \\ $4.384 \mathrm{e}-11$ \\ $1.179 \mathrm{e}-10$ \\ 2.064e-07 \\ $1.021 \mathrm{e}-06$ \\ $1.809 \mathrm{e}-05$ \\ 2.521e-05 \\ $5.720 \mathrm{e}+00$ \\ 2.618e-02 \\ 8.066e-02 \\ 6.496e-01 \\ 9.079e-05 \\ 4.016e-08 \\ $6.477 e+00$}

Exposure Rate $\mathrm{mR} / \mathrm{hr}$

With Buildup $3.086 \mathrm{e}-22$

$7.248 \mathrm{e}-24$

$4.752 \mathrm{e}-22$

8.007e-23 
Page : 5

DOS File : Combined Calcine in Bulk Vault.ms6

Run Date: May 3, 2005

Run Time: 2:56:35 PM

Duration : 00:01:09

$\begin{array}{ccc}\begin{array}{cc}\text { Energy } \\ \text { MeV }\end{array} & & \begin{array}{c}\text { Activity } \\ \text { photons/sec }\end{array} \\ 0.05 & & 3.767 \mathrm{e}+11 \\ 0.06 & 1.132 \mathrm{e}+12 \\ 0.08 & 1.488 \mathrm{e}+11 \\ 0.1 & 2.636 \mathrm{e}+12 \\ 0.15 & 8.425 \mathrm{e}+09 \\ 0.2 & 4.358 \mathrm{e}+11 \\ 0.3 & 5.185 \mathrm{e}+10 \\ 0.4 & 1.057 \mathrm{e}+11 \\ 0.5 & 3.368 \mathrm{e}+10 \\ 0.6 & 2.562 \mathrm{e}+15 \\ 0.8 & 2.363 \mathrm{e}+12 \\ 1.0 & 2.308 \mathrm{e}+12 \\ 1.5 & 2.786 \mathrm{e}+12 \\ 2.0 & 1.173 \mathrm{e}+08 \\ 3.0 & 1.242 \mathrm{e}+04\end{array}$

\begin{tabular}{c}
$\frac{\text { Fluence Rate }}{\text { MeV/cm } 2 / \mathrm{sec}}$ \\
\hline$\frac{\text { No Buildup }}{6.282 \mathrm{e}-24}$ \\
$2.634 \mathrm{e}-17$ \\
$6.789 \mathrm{e}-13$ \\
$2.231 \mathrm{e}-09$ \\
$2.135 \mathrm{e}-09$ \\
$1.924 \mathrm{e}-06$ \\
$6.982 \mathrm{e}-06$ \\
$1.312 \mathrm{e}-04$ \\
$2.169 \mathrm{e}-04$ \\
$6.049 \mathrm{e}+01$ \\
$4.028 \mathrm{e}-01$ \\
$1.712 \mathrm{e}+00$ \\
$2.528 \mathrm{e}+01$ \\
$5.217 \mathrm{e}-03$ \\
$3.873 \mathrm{e}-06$
\end{tabular}

Fluence Rate

$\mathrm{MeV} / \mathrm{cm}^{2} / \mathrm{sec}$

With Buildup

$2.846 \mathrm{e}-22$

$7.811 \mathrm{e}-17$

$3.967 \mathrm{e}-12$

2.342e-08

$5.859 \mathrm{e}-08$

$9.577 \mathrm{e}-05$

4.406e-04

$7.605 \mathrm{e}-03$

$1.052 \mathrm{e}-02$

$2.400 \mathrm{e}+03$

$1.127 \mathrm{e}+01$

$3.581 \mathrm{e}+01$

$3.158 \mathrm{e}+02$

$4.799 \mathrm{e}-02$

2.417e-05

$8.789 e+01$

$2.763 e+03$

\section{Exposure Rate \\ $\mathrm{mR} / \mathrm{hr}$}

No Buildup

$1.673 \mathrm{e}-26$

$5.232 \mathrm{e}-20$

$1.074 \mathrm{e}-15$

$3.413 \mathrm{e}-12$

3.515e-12

3.397e-09

$1.324 \mathrm{e}-08$

$2.556 \mathrm{e}-07$

4.258e-07

1.181e-01

7.661e-04

3.155e-03

4.253e-02

$8.068 \mathrm{e}-06$

5.255e-09

$1.645 \mathrm{e}-01$
Exposure Rate $\mathrm{mR} / \mathrm{hr}$

With Buildup

$7.582 \mathrm{e}-25$

$1.551 \mathrm{e}-19$

$6.277 \mathrm{e}-15$

$3.583 e-11$

$9.648 \mathrm{e}-11$

$1.690 \mathrm{e}-07$

8.359e-07

$1.482 \mathrm{e}-05$

$2.065 \mathrm{e}-05$

$4.684 \mathrm{e}+00$

$2.143 \mathrm{e}-02$

6.601e-02

5.313e-01

$7.421 \mathrm{e}-05$

$3.280 \mathrm{e}-08$

$5.303 e+00$ 
Page $: 1$

DOS File : CSSF 1 Calcine in Grout.ms6

Run Date: April 5, 2005

Run Time: 3:08:15 PM

Duration : 00:01:02
File Ref:

Date:

By:

Checked:

Case Title: CSSF 1 Calcine Grout

Description: CSSF 1 Calcine Grouted at 21.5 wt\% in Storage Canister Geometry: 7 - Cylinder Volume - Side Shields

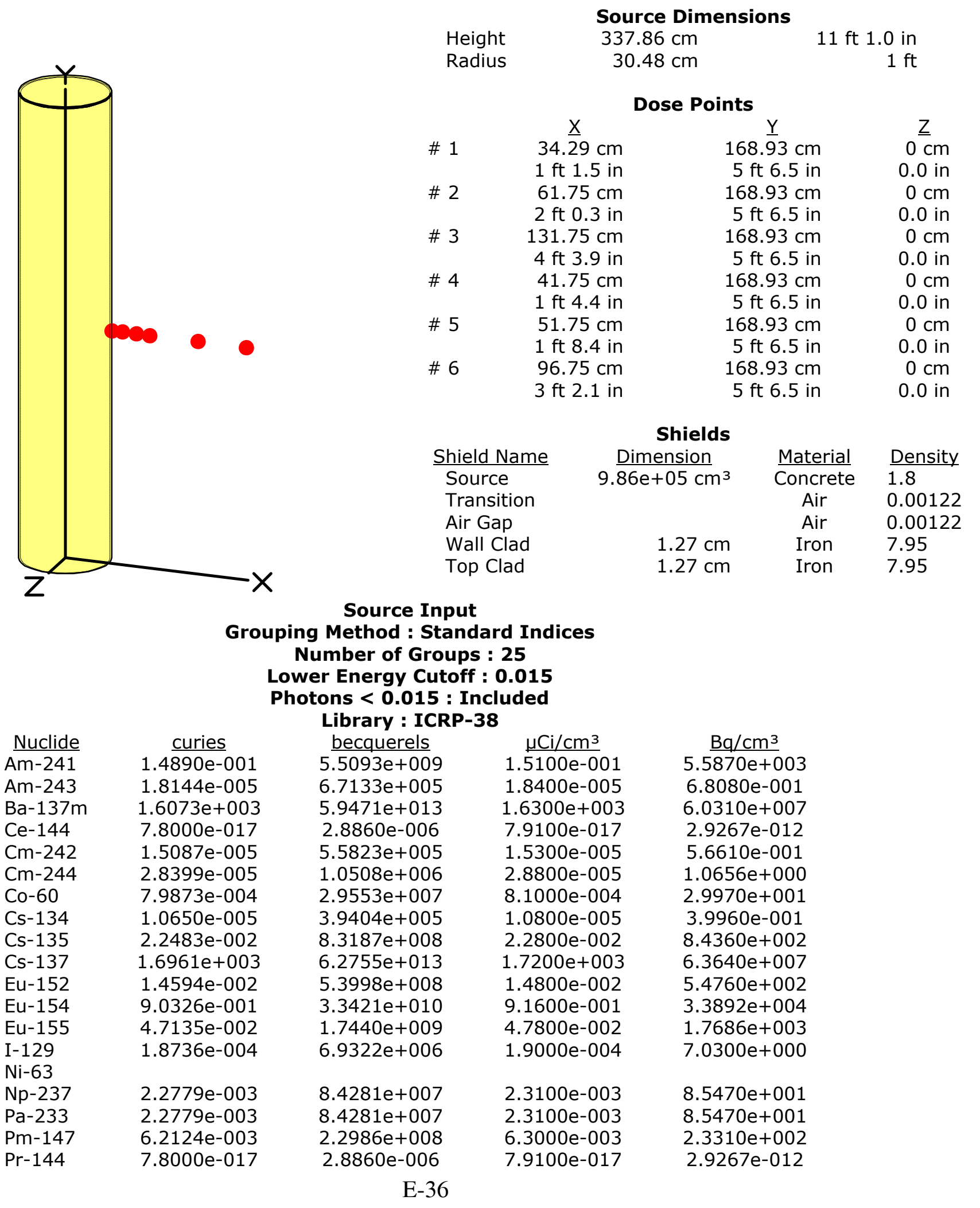


Page : 2

DOS File : CSSF 1 Calcine in Grout.ms6

Run Date: April 5, 2005

Run Time: 3:08:15 PM

Duration : 00:01:02

\begin{tabular}{|c|c|c|c|c|}
\hline Nuclide & curies & becquerels & $\mu \mathrm{Ci} / \mathrm{cm}^{3}$ & $\mathrm{~Bq} / \mathrm{cm}^{3}$ \\
\hline Pu-238 & $6.6 \overline{068 \mathrm{e}-001}$ & $2.4445 e+010$ & $6.7000 \mathrm{e}-001$ & $2.4790 e+004$ \\
\hline Pu-239 & $8.9241 \mathrm{e}-002$ & $3.3019 e+009$ & $9.0500 \mathrm{e}-002$ & $3.3485 e+003$ \\
\hline Pu-240 & $3.5696 \mathrm{e}-002$ & $1.3208 \mathrm{e}+009$ & $3.6200 \mathrm{e}-002$ & $1.3394 \mathrm{e}+003$ \\
\hline Pu-241 & $2.4849 \mathrm{e}-001$ & $9.1943 e+009$ & $2.5200 \mathrm{e}-001$ & $9.3240 e+003$ \\
\hline Pu-242 & $2.0609 \mathrm{e}-005$ & $7.6254 \mathrm{e}+005$ & $2.0900 \mathrm{e}-005$ & $7.7330 e-001$ \\
\hline Ru-106 & $3.9641 \mathrm{e}-013$ & $1.4667 \mathrm{e}-002$ & $4.0200 e-013$ & $1.4874 \mathrm{e}-008$ \\
\hline Sb-125 & $2.5441 \mathrm{e}-004$ & $9.4132 \mathrm{e}+006$ & $2.5800 \mathrm{e}-004$ & $9.5460 e+000$ \\
\hline Sb-126 & $3.2147 e-003$ & $1.1894 \mathrm{e}+008$ & $3.2600 \mathrm{e}-003$ & $1.2062 \mathrm{e}+002$ \\
\hline Sb-126m & $2.2976 \mathrm{e}-002$ & $8.5011 \mathrm{e}+008$ & $2.3300 \mathrm{e}-002$ & $8.6210 e+002$ \\
\hline Sm-151 & $3.4415 e+001$ & $1.2733 \mathrm{e}+012$ & $3.4900 e+001$ & $1.2913 e+006$ \\
\hline Sr-90 & $1.4496 e+003$ & $5.3633 e+013$ & $1.4700 e+003$ & $5.4390 e+007$ \\
\hline Tc-99 & $8.8847 e-001$ & $3.2873 e+010$ & $9.0100 \mathrm{e}-001$ & $3.3337 e+004$ \\
\hline Th-230 & $2.3962 \mathrm{e}-004$ & $8.8659 e+006$ & $2.4300 \mathrm{e}-004$ & $8.9910 e+000$ \\
\hline Th-231 & $4.9798 e-005$ & $1.8425 e+006$ & $5.0500 \mathrm{e}-005$ & $1.8685 e+000$ \\
\hline$U-232$ & $1.6961 \mathrm{e}-007$ & $6.2755 e+003$ & $1.7200 \mathrm{e}-007$ & $6.3640 \mathrm{e}-003$ \\
\hline$U-233$ & $3.2738 \mathrm{e}-007$ & $1.2113 e+004$ & $3.3200 \mathrm{e}-007$ & $1.2284 \mathrm{e}-002$ \\
\hline$U-234$ & $7.1689 \mathrm{e}-003$ & $2.6525 e+008$ & $7.2700 \mathrm{e}-003$ & $2.6899 e+002$ \\
\hline$U-235$ & $4.9798 \mathrm{e}-005$ & $1.8425 e+006$ & $5.0500 \mathrm{e}-005$ & $1.8685 e+000$ \\
\hline$U-236$ & $1.1537 \mathrm{e}-004$ & $4.2688 \mathrm{e}+006$ & $1.1700 \mathrm{e}-004$ & $4.3290 e+000$ \\
\hline$U-237$ & $8.1352 \mathrm{e}-006$ & $3.0100 e+005$ & $8.2500 \mathrm{e}-006$ & $3.0525 e-001$ \\
\hline$U-238$ & $2.8104 \mathrm{e}-006$ & $1.0398 \mathrm{e}+005$ & $2.8500 \mathrm{e}-006$ & $1.0545 e-001$ \\
\hline Y-90 & $1.4496 \mathrm{e}+003$ & $5.3633 e+013$ & $1.4700 e+003$ & $5.4390 e+007$ \\
\hline
\end{tabular}

$\begin{array}{cc}\begin{array}{cc}\text { Energy } \\ \text { MeV }\end{array} & \begin{array}{c}\text { Activity } \\ \text { photons/sec }\end{array} \\ 0.015 & \\ 0.02 & 6.813 \mathrm{e}+09 \\ 0.03 & 3.243 \mathrm{e}+09 \\ 0.04 & 3.601 \mathrm{e}+12 \\ 0.05 & 8.637 \mathrm{e}+11 \\ 0.06 & 1.999 \mathrm{e}+09 \\ 0.08 & 1.992 \mathrm{e}+09 \\ 0.1 & 5.589 \mathrm{e}+08 \\ 0.15 & 1.408 \mathrm{e}+10 \\ 0.2 & 4.191 \mathrm{e}+07 \\ 0.3 & 2.343 \mathrm{e}+09 \\ 0.4 & 2.670 \mathrm{e}+08 \\ 0.5 & 1.143 \mathrm{e}+09 \\ 0.6 & 1.547 \mathrm{e}+08 \\ 0.8 & 5.339 \mathrm{e}+13 \\ 1.0 & 1.277 \mathrm{e}+10 \\ 1.5 & 1.070 \mathrm{e}+10 \\ 2.0 & 1.325 \mathrm{e}+10 \\ 3.0 & 6.170 \mathrm{e}+05 \\ & 1.064 \mathrm{e}+00\end{array}$

Radial

Integration Parameters

Circumferential

Y Direction (axial) $\quad 24$

Results - Dose Point \# 1 - $(34.29,168.93,0) \mathrm{cm}$

\begin{tabular}{|c|c|c|c|}
\hline Fluence Rate & Fluence Rate & Exposure Rate & Exposure Rate \\
\hline $\mathrm{MeV} / \mathrm{cm}^{2} / \mathrm{sec}$ & $\mathrm{MeV} / \mathrm{cm}^{2} / \mathrm{sec}$ & $\mathrm{mR} / \mathrm{hr}$ & $\mathrm{mR} / \mathrm{hr}$ \\
\hline No Buildup & With Buildup & No Buildup & With Buildup \\
\hline $8.522 \mathrm{e}-268$ & $2.201 \mathrm{e}-23$ & $7.310 e-269$ & $1.888 \mathrm{e}-24$ \\
\hline $1.054 \mathrm{e}-119$ & $1.648 \mathrm{e}-23$ & $3.652 \mathrm{e}-121$ & $5.710 e-25$ \\
\hline $2.677 e-34$ & $4.048 e-20$ & $2.653 e-36$ & $4.012 \mathrm{e}-22$ \\
\hline $1.341 \mathrm{e}-13$ & $5.362 \mathrm{e}-13$ & $5.931 \mathrm{e}-16$ & $2.371 \mathrm{e}-15$ \\
\hline $2.495 e-08$ & $1.677 \mathrm{e}-07$ & $6.646 e-11$ & $4.466 e-10$ \\
\hline $1.035 \mathrm{e}-04$ & $9.669 e-04$ & $2.056 \mathrm{e}-07$ & $1.921 \mathrm{e}-06$ \\
\hline $3.265 e-02$ & $3.690 e-01$ & $5.167 e-05$ & $5.839 e-04$ \\
\hline $1.333 e+01$ & $1.506 \mathrm{e}+02$ & $2.039 e-02$ & $2.304 \mathrm{e}-01$ \\
\hline $5.177 \mathrm{e}-01$ & $4.883 e+00$ & $8.525 e-04$ & $8.042 \mathrm{e}-03$ \\
\hline $7.812 e+01$ & $6.309 e+02$ & $1.379 \mathrm{e}-01$ & $1.114 \mathrm{e}+00$ \\
\hline $2.428 e+01$ & $1.562 e+02$ & $4.606 \mathrm{e}-02$ & $2.963 e-01$ \\
\hline $1.910 \mathrm{e}+02$ & $1.042 e+03$ & $3.723 e-01$ & $2.030 e+00$ \\
\hline $4.061 e+01$ & $1.944 \mathrm{e}+02$ & $7.971 \mathrm{e}-02$ & $3.816 \mathrm{e}-01$ \\
\hline $2.011 e+07$ & $8.645 e+07$ & $3.925 e+04$ & $1.687 e+05$ \\
\hline $8.455 e+03$ & $3.089 e+04$ & $1.608 e+01$ & $5.875 e+01$ \\
\hline $1.095 e+04$ & $3.547 e+04$ & $2.018 e+01$ & $6.538 e+01$ \\
\hline $2.953 e+04$ & $7.823 e+04$ & $4.969 e+01$ & $1.316 e+02$ \\
\hline $2.333 e+00$ & $5.484 e+00$ & $3.607 e-03$ & $8.481 \mathrm{e}-03$ \\
\hline $8.088 \mathrm{e}-06$ & $1.641 \mathrm{e}-05$ & $1.097 e-08$ & $2.226 \mathrm{e}-08$ \\
\hline $2.016 e+07$ & $8.659 e+07$ & $3.934 e+04$ & $1.690 e+05$ \\
\hline
\end{tabular}

Results - Dose Point \# 2 - $(61.75,168.93,0) \mathrm{cm}$ 
Page : 3

DOS File : CSSF 1 Calcine in Grout.ms6

Run Date: April 5, 2005

Run Time: 3:08:15 PM

Duration : 00:01:02

$\begin{array}{cc}\begin{array}{cc}\text { Energy } \\ \text { MeV }\end{array} & \begin{array}{c}\text { Activity } \\ \text { photons/sec }\end{array} \\ 0.015 & 6.813 \mathrm{e}+09 \\ 0.02 & 3.243 \mathrm{e}+09 \\ 0.03 & 3.601 \mathrm{e}+12 \\ 0.04 & 8.637 \mathrm{e}+11 \\ 0.05 & 1.999 \mathrm{e}+09 \\ 0.06 & 1.992 \mathrm{e}+09 \\ 0.08 & 5.589 \mathrm{e}+08 \\ 0.1 & 1.408 \mathrm{e}+10 \\ 0.15 & 4.191 \mathrm{e}+07 \\ 0.2 & 2.343 \mathrm{e}+09 \\ 0.3 & 2.670 \mathrm{e}+08 \\ 0.4 & 1.143 \mathrm{e}+09 \\ 0.5 & 1.547 \mathrm{e}+08 \\ 0.6 & 5.339 \mathrm{e}+13 \\ 0.8 & 1.277 \mathrm{e}+10 \\ 1.0 & 1.070 \mathrm{e}+10 \\ 1.5 & 1.325 \mathrm{e}+10 \\ 2.0 & 6.170 \mathrm{e}+05 \\ 3.0 & 1.064 \mathrm{e}+00\end{array}$

\begin{tabular}{|c|c|c|}
\hline$\frac{\text { Fluence Rate }}{\mathrm{MeV} / \mathrm{cm}^{2} / \mathrm{sec}}$ & $\frac{\text { Fluence Rate }}{\mathrm{MeV} / \mathrm{cm}^{2} / \mathrm{sec}}$ & $\frac{\text { Exposure Rate }}{\mathrm{mR} / \mathrm{hr}}$ \\
\hline No Buildup & With Buildup & No Buildup \\
\hline $1.719 \mathrm{e}-250$ & $9.658 e-24$ & $1.475 \mathrm{e}-251$ \\
\hline $2.941 \mathrm{e}-113$ & $7.234 \mathrm{e}-24$ & $1.019 \mathrm{e}-114$ \\
\hline $2.647 e-33$ & $1.777 \mathrm{e}-20$ & $2.623 e-35$ \\
\hline $1.280 \mathrm{e}-13$ & $5.073 e-13$ & $5.660 e-16$ \\
\hline $1.367 \mathrm{e}-08$ & $9.059 \mathrm{e}-08$ & $3.642 e-11$ \\
\hline $4.934 \mathrm{e}-05$ & $4.579 \mathrm{e}-04$ & $9.801 \mathrm{e}-08$ \\
\hline $1.568 \mathrm{e}-02$ & $1.803 e-01$ & $2.481 \mathrm{e}-05$ \\
\hline $6.644 e+00$ & $7.664 e+01$ & $1.017 \mathrm{e}-02$ \\
\hline $2.630 \mathrm{e}-01$ & $2.487 e+00$ & $4.331 \mathrm{e}-04$ \\
\hline $3.960 e+01$ & $3.177 e+02$ & $6.990 e-02$ \\
\hline $1.225 e+01$ & $7.788 e+01$ & $2.323 e-02$ \\
\hline $9.603 e+01$ & $5.173 e+02$ & $1.871 \mathrm{e}-01$ \\
\hline $2.036 e+01$ & $9.625 e+01$ & $3.996 \mathrm{e}-02$ \\
\hline $1.006 e+07$ & $4.272 e+07$ & $1.963 e+04$ \\
\hline $4.215 e+03$ & $1.523 e+04$ & $8.017 e+00$ \\
\hline $5.443 e+03$ & $1.745 e+04$ & $1.003 e+01$ \\
\hline $1.462 \mathrm{e}+04$ & $3.839 e+04$ & $2.459 e+01$ \\
\hline $1.151 \mathrm{e}+00$ & $2.687 e+00$ & $1.781 \mathrm{e}-03$ \\
\hline $3.983 \mathrm{e}-06$ & $8.032 \mathrm{e}-06$ & $5.403 e-09$ \\
\hline $1.008 \mathrm{e}+07$ & $4.279 e+07$ & $1.968 \mathrm{e}+04$ \\
\hline
\end{tabular}

$\frac{\text { Exposure Rate }}{\mathrm{mR} / \mathrm{hr}}$

With Buildup

$8.284 \mathrm{e}-25$

$2.506 \mathrm{e}-25$

$1.761 \mathrm{e}-22$

$2.244 \mathrm{e}-15$

$2.413 e-10$

$9.096 \mathrm{e}-07$

2.853e-04

$1.173 \mathrm{e}-01$

$4.095 \mathrm{e}-03$

$5.607 e-01$

$1.477 \mathrm{e}-01$

$1.008 \mathrm{e}+00$

$1.889 \mathrm{e}-01$

$8.339 \mathrm{e}+04$

$2.896 \mathrm{e}+01$

$3.217 \mathrm{e}+01$

$6.458 \mathrm{e}+01$

$4.156 \mathrm{e}-03$

$1.090 \mathrm{e}-08$

TOTALS:

$5.793 e+13$

Results - Dose Point \# 3 - $(131.75,168.93,0) \mathrm{cm}$

\begin{tabular}{|c|c|}
\hline $\begin{array}{c}\text { Energy } \\
\mathrm{MeV}\end{array}$ & $\begin{array}{c}\text { Activity } \\
\text { photons/sec }\end{array}$ \\
\hline 0.015 & $6.813 e+09$ \\
\hline 0.02 & $3.243 e+09$ \\
\hline 0.03 & $3.601 e+12$ \\
\hline 0.04 & $8.637 e+11$ \\
\hline 0.05 & $1.999 \mathrm{e}+09$ \\
\hline 0.06 & $1.992 \mathrm{e}+09$ \\
\hline 0.08 & $5.589 e+08$ \\
\hline 0.1 & $1.408 \mathrm{e}+10$ \\
\hline 0.15 & $4.191 \mathrm{e}+07$ \\
\hline 0.2 & $2.343 e+09$ \\
\hline 0.3 & $2.670 e+08$ \\
\hline 0.4 & $1.143 e+09$ \\
\hline 0.5 & $1.547 e+08$ \\
\hline 0.6 & $5.339 e+13$ \\
\hline 0.8 & $1.277 \mathrm{e}+10$ \\
\hline 1.0 & $1.070 e+10$ \\
\hline 1.5 & $1.325 e+10$ \\
\hline 2.0 & $6.170 e+05$ \\
\hline 3.0 & $1.064 e+00$ \\
\hline
\end{tabular}

\section{Fluence Rate}

$\mathrm{MeV} / \mathrm{cm}^{2} / \mathrm{sec}$

No Buildup

4.951e-251

$1.136 \mathrm{e}-113$

$1.238 \mathrm{e}-33$

$5.881 \mathrm{e}-14$

$6.256 \mathrm{e}-09$

2.262e-05

$7.165 \mathrm{e}-03$

$3.022 \mathrm{e}+00$

$1.179 \mathrm{e}-01$

$1.758 \mathrm{e}+01$

$5.376 \mathrm{e}+00$

$4.187 \mathrm{e}+01$

$8.829 \mathrm{e}+00$

$4.344 \mathrm{e}+06$

$1.807 \mathrm{e}+03$

$2.318 \mathrm{e}+03$

$6.148 \mathrm{e}+03$

$4.801 \mathrm{e}-01$

$1.641 \mathrm{e}-06$

$4.354 e+06$
Fluence Rate

$\mathrm{MeV} / \mathrm{cm}^{2} / \mathrm{sec}$

With Buildup

$3.269 \mathrm{e}-24$

$2.448 \mathrm{e}-24$

$6.013 \mathrm{e}-21$

$2.331 \mathrm{e}-13$

$4.148 \mathrm{e}-08$

$2.100 \mathrm{e}-04$

$8.242 \mathrm{e}-02$

$3.483 e+01$

$1.104 \mathrm{e}+00$

$1.387 \mathrm{e}+02$

$3.340 \mathrm{e}+01$

$2.198 \mathrm{e}+02$

$4.064 \mathrm{e}+01$

$1.795 \mathrm{e}+07$

$6.346 \mathrm{e}+03$

$7.228 \mathrm{e}+03$

$1.571 \mathrm{e}+04$

$1.091 \mathrm{e}+00$

$3.225 \mathrm{e}-06$

$1.798 e+07$
Exposure Rate $\mathrm{mR} / \mathrm{hr}$

No Buildup

4.247e-252

$3.937 e-115$

$1.227 \mathrm{e}-35$

2.601e-16

$1.666 \mathrm{e}-11$

4.492e-08

$1.134 \mathrm{e}-05$

$4.623 \mathrm{e}-03$

$1.942 \mathrm{e}-04$

3.103e-02

$1.020 \mathrm{e}-02$

8.158e-02

$1.733 \mathrm{e}-02$

$8.478 \mathrm{e}+03$

$3.436 \mathrm{e}+00$

$4.273 \mathrm{e}+00$

$1.034 \mathrm{e}+01$

7.424e-04

2.226e-09

$8.496 e+03$

\section{$8.351 e+04$}

Exposure Rate $\mathrm{mR} / \mathrm{hr}$

With Buildup

$2.804 \mathrm{e}-25$

$8.480 \mathrm{e}-26$

$5.959 \mathrm{e}-23$

$1.031 \mathrm{e}-15$

$1.105 \mathrm{e}-10$

4.172e-07

$1.304 \mathrm{e}-04$

$5.328 \mathrm{e}-02$

$1.818 \mathrm{e}-03$

2.447e-01

$6.336 \mathrm{e}-02$

4.283e-01

7.977e-02

$3.503 e+04$

$1.207 \mathrm{e}+01$

$1.332 \mathrm{e}+01$

$2.643 e+01$

$1.687 \mathrm{e}-03$

4.376e-09

$3.508 \mathrm{e}+04$

\section{Results - Dose Point \# 4 - $(41.75,168.93,0) \mathrm{cm}$}

$\begin{array}{cc}\frac{\text { Energy }}{\text { MeV }} & \begin{array}{c}\text { Activity } \\ \text { photons/sec }\end{array} \\ 0.015 & 6.813 \mathrm{e}+09 \\ 0.02 & 3.243 \mathrm{e}+09 \\ 0.03 & 3.601 \mathrm{e}+12\end{array}$

$\frac{\text { Exposure Rate }}{\mathrm{mR} / \mathrm{hr}}$
No Buildup
$6.056 \mathrm{e}-253$
$6.747 \mathrm{e}-115$
$4.630 \mathrm{e}-35$

$\frac{\text { Exposure Rate }}{\mathrm{mR} / \mathrm{hr}}$
$\frac{\text { With Buildup }}{1.407 \mathrm{e}-24}$
$4.257 \mathrm{e}-25$
$2.991 \mathrm{e}-22$ 
Page : 4

DOS File : CSSF 1 Calcine in Grout.ms6

Run Date: April 5, 2005

Run Time: 3:08:15 PM

Duration : 00:01:02

$\begin{array}{ccc}\frac{\text { Energy }}{\mathrm{MeV}} & & \begin{array}{c}\text { Activity } \\ \text { photons } / \mathrm{sec}\end{array} \\ 0.04 & & 8.637 \mathrm{e}+11 \\ 0.05 & & 1.999 \mathrm{e}+09 \\ 0.06 & & 1.992 \mathrm{e}+09 \\ 0.08 & & 5.589 \mathrm{e}+08 \\ 0.1 & & 1.408 \mathrm{e}+10 \\ 0.15 & 4.191 \mathrm{e}+07 \\ 0.2 & & 2.343 \mathrm{e}+09 \\ 0.3 & 2.670 \mathrm{e}+08 \\ 0.4 & 1.143 \mathrm{e}+09 \\ 0.5 & 1.547 \mathrm{e}+08 \\ 0.6 & 5.339 \mathrm{e}+13 \\ 0.8 & 1.277 \mathrm{e}+10 \\ 1.0 & 1.070 \mathrm{e}+10 \\ 1.5 & 1.325 \mathrm{e}+10 \\ 2.0 & 6.170 \mathrm{e}+05 \\ 3.0 & 1.064 \mathrm{e}+00\end{array}$

\begin{tabular}{|c|c|c|}
\hline Fluence Rate & Fluence Rate & Exposure Rate \\
\hline $\mathrm{MeV} / \mathrm{cm}^{2} / \mathrm{sec}$ & $\mathrm{MeV} / \mathrm{cm}^{2} / \mathrm{sec}$ & $\mathrm{mR} / \mathrm{hr}$ \\
\hline No Buildup & With Buildup & No Buildup \\
\hline $2.229 \mathrm{e}-13$ & $8.826 e-13$ & $9.858 \mathrm{e}-16$ \\
\hline $2.094 \mathrm{e}-08$ & $1.381 \mathrm{e}-07$ & $5.578 \mathrm{e}-11$ \\
\hline $7.246 \mathrm{e}-05$ & $6.718 \mathrm{e}-04$ & $1.439 \mathrm{e}-07$ \\
\hline $2.351 \mathrm{e}-02$ & $2.715 e-01$ & $3.720 e-05$ \\
\hline $1.009 e+01$ & $1.168 \mathrm{e}+02$ & $1.543 \mathrm{e}-02$ \\
\hline $4.036 \mathrm{e}-01$ & $3.833 e+00$ & $6.646 e-04$ \\
\hline $6.102 e+01$ & $4.920 \mathrm{e}+02$ & $1.077 \mathrm{e}-01$ \\
\hline $1.893 e+01$ & $1.211 \mathrm{e}+02$ & $3.591 \mathrm{e}-02$ \\
\hline $1.487 e+02$ & $8.055 e+02$ & $2.898 \mathrm{e}-01$ \\
\hline $3.156 e+01$ & $1.500 e+02$ & $6.196 \mathrm{e}-02$ \\
\hline $1.561 \mathrm{e}+07$ & $6.666 \mathrm{e}+07$ & $3.047 e+04$ \\
\hline $6.552 e+03$ & $2.378 e+04$ & $1.246 e+01$ \\
\hline $8.471 e+03$ & $2.728 e+04$ & $1.561 e+01$ \\
\hline $2.279 e+04$ & $6.011 e+04$ & $3.835 e+01$ \\
\hline $1.798 \mathrm{e}+00$ & $4.213 e+00$ & $2.780 \mathrm{e}-03$ \\
\hline $6.228 \mathrm{e}-06$ & $1.261 \mathrm{e}-05$ & $8.450 e-09$ \\
\hline $1.565 e+07$ & $6.677 e+07$ & $3.054 e+04$ \\
\hline
\end{tabular}

Exposure Rate $\mathrm{mR} / \mathrm{hr}$

With Buildup

$3.903 \mathrm{e}-15$

$3.678 \mathrm{e}-10$

$1.334 \mathrm{e}-06$

$4.296 \mathrm{e}-04$

$1.787 \mathrm{e}-01$

$6.312 \mathrm{e}-03$

$8.683 \mathrm{e}-01$

2.297e-01

$1.570 \mathrm{e}+00$

$2.945 \mathrm{e}-01$

$1.301 \mathrm{e}+05$

$4.524 \mathrm{e}+01$

$5.029 \mathrm{e}+01$

$1.011 \mathrm{e}+02$

$6.515 \mathrm{e}-03$

$1.711 \mathrm{e}-08$

TOTALS: $\quad 5.793 e+13$

Results - Dose Point \# 5 - $(51.75,168.93,0) \mathrm{cm}$

$\begin{array}{cc}\begin{array}{cc}\text { Energy } \\ \text { MeV }\end{array} & \begin{array}{c}\text { Activity } \\ \text { photons } / \mathrm{sec}\end{array} \\ 0.015 & \\ 0.02 & 6.813 \mathrm{e}+09 \\ 0.03 & 3.243 \mathrm{e}+09 \\ 0.04 & 3.601 \mathrm{e}+12 \\ 0.05 & 8.637 \mathrm{e}+11 \\ 0.06 & 1.999 \mathrm{e}+09 \\ 0.08 & 1.992 \mathrm{e}+09 \\ 0.1 & 5.589 \mathrm{e}+08 \\ 0.15 & 1.408 \mathrm{e}+10 \\ 0.2 & 4.191 \mathrm{e}+07 \\ 0.3 & 2.343 \mathrm{e}+09 \\ 0.4 & 2.670 \mathrm{e}+08 \\ 0.5 & 1.143 \mathrm{e}+09 \\ 0.6 & 1.547 \mathrm{e}+08 \\ 0.8 & 5.339 \mathrm{e}+13 \\ 1.0 & 1.277 \mathrm{e}+10 \\ 1.5 & 1.070 \mathrm{e}+10 \\ 2.0 & 1.325 \mathrm{e}+10 \\ 3.0 & 6.170 \mathrm{e}+05 \\ & 1.064 \mathrm{e}+00\end{array}$

\section{Fluence Rate}

$\mathrm{MeV} / \mathrm{cm}^{2} / \mathrm{sec}$

No Buildup

$1.470 \mathrm{e}-250$

$3.823 e-113$

$3.510 \mathrm{e}-33$

$1.491 \mathrm{e}-13$

$1.623 \mathrm{e}-08$

$5.937 \mathrm{e}-05$

$1.888 \mathrm{e}-02$

$8.009 \mathrm{e}+00$

$3.179 \mathrm{e}-01$

$4.793 e+01$

$1.484 \mathrm{e}+01$

$1.164 \mathrm{e}+02$

$2.468 \mathrm{e}+01$

$1.220 \mathrm{e}+07$

$5.115 e+03$

$6.608 \mathrm{e}+03$

$1.776 \mathrm{e}+04$

$1.400 \mathrm{e}+00$

4.847e-06

$1.223 e+07$
Fluence Rate

$\mathrm{MeV} / \mathrm{cm}^{2} / \mathrm{sec}$

With Buildup

$1.224 \mathrm{e}-23$

$9.168 \mathrm{e}-24$

$2.252 \mathrm{e}-20$

5.907e-13

$1.077 \mathrm{e}-07$

$5.511 \mathrm{e}-04$

$2.170 \mathrm{e}-01$

$9.243 e+01$

$3.009 \mathrm{e}+00$

$3.850 \mathrm{e}+02$

$9.450 \mathrm{e}+01$

$6.280 \mathrm{e}+02$

$1.169 \mathrm{e}+02$

$5.191 \mathrm{e}+07$

$1.851 \mathrm{e}+04$

$2.123 e+04$

$4.674 \mathrm{e}+04$

$3.275 e+00$

$9.798 \mathrm{e}-06$

$5.200 \mathrm{e}+07$
Exposure Rate $\mathrm{mR} / \mathrm{hr}$

No Buildup

$1.261 \mathrm{e}-251$

$1.324 \mathrm{e}-114$

$3.479 \mathrm{e}-35$

$6.594 \mathrm{e}-16$

4.323e-11

$1.179 \mathrm{e}-07$

2.987e-05

$1.225 \mathrm{e}-02$

5.236e-04

$8.459 \mathrm{e}-02$

$2.814 \mathrm{e}-02$

2.268e-01

$4.845 \mathrm{e}-02$

$2.381 \mathrm{e}+04$

$9.729 \mathrm{e}+00$

$1.218 \mathrm{e}+01$

$2.988 \mathrm{e}+01$

$2.165 \mathrm{e}-03$

$6.576 \mathrm{e}-09$

$2.386 e+04$

$1.303 e+05$

TOTALS: $\quad 5.793 e+13$

Results - Dose Point \# 6 - $(96.75,168.93,0) \mathrm{cm}$

$\begin{array}{cc}\frac{\text { Energy }}{\text { MeV }} & \begin{array}{c}\text { Activity } \\ \text { photons/sec }\end{array} \\ 0.015 & \\ 0.02 & 6.813 \mathrm{e}+09 \\ 0.03 & 3.243 \mathrm{e}+09 \\ 0.04 & 3.601 \mathrm{e}+12 \\ 0.05 & 8.637 \mathrm{e}+11 \\ 0.06 & 1.999 \mathrm{e}+09 \\ & 1.992 \mathrm{e}+09\end{array}$

\begin{tabular}{c}
$\frac{\text { Fluence Rate }}{\text { MeV/cm } 2 / \mathrm{sec}}$ \\
\hline With Buildup \\
\hline $5.187 \mathrm{e}-24$ \\
$3.884 \mathrm{e}-24$ \\
$9.540 \mathrm{e}-21$ \\
$3.208 \mathrm{e}-13$ \\
$5.702 \mathrm{e}-08$ \\
$2.886 \mathrm{e}-04$
\end{tabular}

Exposure Rate $\mathrm{mR} / \mathrm{hr}$

No Buildup

6.575e-252

$5.275 \mathrm{e}-115$

$1.699 \mathrm{e}-35$

$3.580 \mathrm{e}-16$

2.291e-11

$6.176 \mathrm{e}-08$
Exposure Rate $\mathrm{mR} / \mathrm{hr}$

With Buildup

$1.050 \mathrm{e}-24$

$3.176 \mathrm{e}-25$

$2.231 \mathrm{e}-22$

$2.613 \mathrm{e}-15$

$2.869 \mathrm{e}-10$

$1.095 \mathrm{e}-06$

$3.434 \mathrm{e}-04$

$1.414 \mathrm{e}-01$

$4.956 \mathrm{e}-03$

$6.795 \mathrm{e}-01$

$1.793 \mathrm{e}-01$

$1.224 \mathrm{e}+00$

2.295e-01

$1.013 e+05$

$3.521 \mathrm{e}+01$

$3.913 e+01$

$7.864 \mathrm{e}+01$

5.064e-03

$1.329 \mathrm{e}-08$

$1.015 e+05$

Exposure Rate $\mathrm{mR} / \mathrm{hr}$

With Buildup

4.449e-25

$1.346 \mathrm{e}-25$

$9.455 \mathrm{e}-23$

$1.419 \mathrm{e}-15$

$1.519 \mathrm{e}-10$

5.733e-07 
Page : 5

DOS File : CSSF 1 Calcine in Grout.ms6

Run Date: April 5, 2005

Run Time: 3:08:15 PM

Duration : 00:01:02

\begin{tabular}{|c|c|c|c|}
\hline$\frac{\text { Energy }}{\mathrm{MeV}}$ & $\frac{\text { Activity }}{\text { photons/sec }}$ & 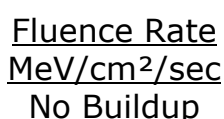 & $\frac{\frac{\text { Fluence Rate }}{\text { MeV/cm } / \mathrm{cm}^{2} / \mathrm{sec}}}{\text { With Buildup }}$ \\
\hline 0.08 & $5.589 e+08$ & $9.856 \mathrm{e}-03$ & $1.133 \mathrm{e}-01$ \\
\hline 0.1 & $1.408 e+10$ & $4.166 \mathrm{e}+00$ & $4.806 e+01$ \\
\hline 0.15 & $4.191 \mathrm{e}+07$ & $1.642 \mathrm{e}-01$ & $1.550 \mathrm{e}+00$ \\
\hline 0.2 & $2.343 e+09$ & $2.466 e+01$ & $1.969 \mathrm{e}+02$ \\
\hline 0.3 & $2.670 e+08$ & $7.599 \mathrm{e}+00$ & $4.794 e+01$ \\
\hline 0.4 & $1.143 e+09$ & $5.944 e+01$ & $3.172 \mathrm{e}+02$ \\
\hline 0.5 & $1.547 e+08$ & $1.258 \mathrm{e}+01$ & $5.885 e+01$ \\
\hline 0.6 & $5.339 e+13$ & $6.204 e+06$ & $2.606 e+07$ \\
\hline 0.8 & $1.277 \mathrm{e}+10$ & $2.592 \mathrm{e}+03$ & $9.254 e+03$ \\
\hline 1.0 & $1.070 e+10$ & $3.339 e+03$ & $1.057 e+04$ \\
\hline 1.5 & $1.325 e+10$ & $8.916 e+03$ & $2.312 e+04$ \\
\hline 2.0 & $6.170 e+05$ & $6.995 \mathrm{e}-01$ & $1.612 \mathrm{e}+00$ \\
\hline 3.0 & $1.064 e+00$ & $2.406 \mathrm{e}-06$ & $4.791 \mathrm{e}-06$ \\
\hline OIALS: & $5.793 e+13$ & $6.219 e+06$ & $2.611 e+07$ \\
\hline
\end{tabular}

\section{Exposure Rate $\mathrm{mR} / \mathrm{hr}$}

No Buildup

$1.560 \mathrm{e}-05$

$6.373 \mathrm{e}-03$

$2.704 \mathrm{e}-04$

$4.352 \mathrm{e}-02$

$1.441 \mathrm{e}-02$

$1.158 \mathrm{e}-01$

2.469e-02

$1.211 \mathrm{e}+04$

$4.930 \mathrm{e}+00$

$6.154 \mathrm{e}+00$

$1.500 \mathrm{e}+01$

$1.082 \mathrm{e}-03$

3. $264 \mathrm{e}-09$

$1.214 \mathrm{e}+04$
Exposure Rate $\mathrm{mR} / \mathrm{hr}$

With Buildup

$1.793 e-04$

$7.352 \mathrm{e}-02$

2.552e-03

$3.475 \mathrm{e}-01$

$9.095 \mathrm{e}-02$

$6.180 \mathrm{e}-01$

$1.155 \mathrm{e}-01$

$5.087 \mathrm{e}+04$

$1.760 \mathrm{e}+01$

$1.949 \mathrm{e}+01$

$3.891 \mathrm{e}+01$

$2.493 \mathrm{e}-03$

6.500e-09

$5.095 e+04$ 
Page $: 1$

DOS File : CSSF 2 Calcine in Grout.ms6

Run Date: April 5, 2005

Run Time: 3:10:00 PM

Duration : 00:01:02
File Ref:

Date:

By:

Checked:

Case Title: CSSF 2 Calcine Grout

Description: CSSF 2 Calcine Grouted at 21.5 wt\% in Storage Canister Geometry: 7 - Cylinder Volume - Side Shields

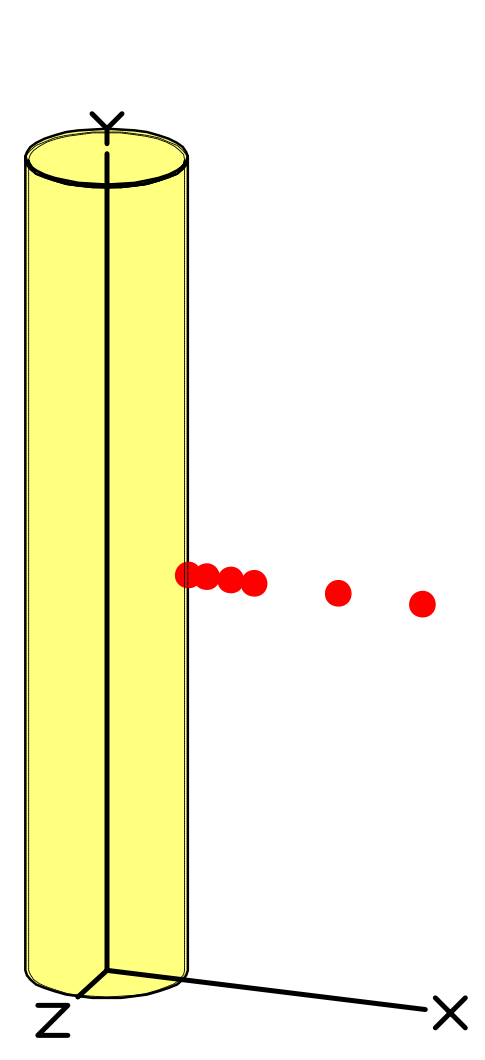

Height
Radius

\section{Source Dimensions}

$337.86 \mathrm{~cm}$

$30.48 \mathrm{~cm}$
$11 \mathrm{ft} 1.0$ in

$1 \mathrm{ft}$

\begin{tabular}{|c|c|c|c|}
\hline \multicolumn{4}{|c|}{ Dose Points } \\
\hline & $\underline{x}$ & $\underline{Y}$ & $\underline{Z}$ \\
\hline \multirow[t]{2}{*}{ \# 1} & $34.29 \mathrm{~cm}$ & $168.93 \mathrm{~cm}$ & $0 \mathrm{Cl}$ \\
\hline & $1 \mathrm{ft} 1.5 \mathrm{in}$ & $5 \mathrm{ft} 6.5$ in & $0.0 \mathrm{i}$ \\
\hline \multirow[t]{2}{*}{ \# 2} & $61.75 \mathrm{~cm}$ & $168.93 \mathrm{~cm}$ & $0 \mathrm{c}$ \\
\hline & $2 \mathrm{ft} 0.3$ in & $5 \mathrm{ft} 6.5 \mathrm{in}$ & 0.0 \\
\hline \multirow[t]{2}{*}{ \# 3} & $131.75 \mathrm{~cm}$ & $168.93 \mathrm{~cm}$ & $0 c$ \\
\hline & $4 \mathrm{ft} 3.9$ in & $5 \mathrm{ft} 6.5 \mathrm{in}$ & 0.0 \\
\hline \multirow[t]{2}{*}{ \# 4} & $41.75 \mathrm{~cm}$ & $168.93 \mathrm{~cm}$ & \\
\hline & $1 \mathrm{ft} 4.4 \mathrm{in}$ & $5 \mathrm{ft} 6.5$ in & 0.0 \\
\hline \multirow[t]{2}{*}{ \# 5} & $51.75 \mathrm{~cm}$ & $168.93 \mathrm{~cm}$ & $0 \mathrm{c}$ \\
\hline & $1 \mathrm{ft} 8.4$ in & $5 \mathrm{ft} 6.5$ in & 0.0 \\
\hline \# 6 & $96.75 \mathrm{~cm}$ & $168.93 \mathrm{~cm}$ & $\begin{array}{lll}0 & 0 \\
0 & 0\end{array}$ \\
\hline
\end{tabular}

\section{Grouping Method : Standard Indices}

Number of Groups : 25

Lower Energy Cutoff : 0.015

Shield Name

Source

Transition

Air Gap

Wall Clad

Top Clad

Shields

Dimension

$9.86 \mathrm{e}+05 \mathrm{~cm}^{3}$

Material

Concrete

Density

1.8

Air

0.00122

$1.27 \mathrm{~cm}$

Air

0.00122

$1.27 \mathrm{~cm}$

Iron

7.95

Iron

7.95

Library : ICRP-38

$\begin{array}{lc}\frac{\text { Nuclide }}{\text { Am-241 }} & \text { curies } \\ \text { Am-243 } & 3.8951 \mathrm{e}-001 \\ \text { Ba-137m } & 6.3231 \mathrm{e}-004 \\ \text { Ce-144 } & 2.77005 \mathrm{e}+002 \\ \mathrm{Cm}-242 & 9.5947 \mathrm{e}-004 \\ \mathrm{Cm}-244 & 3.3330 \mathrm{e}-004 \\ \mathrm{Co}-60 & 8.4113 \mathrm{e}-003 \\ \mathrm{Cs}-134 & 1.4890 \mathrm{e}-004 \\ \mathrm{Cs}-135 & 9.8510 \mathrm{e}-003 \\ \mathrm{Cs}-137 & 6.3307 \mathrm{e}+002 \\ \text { Eu-152 } & 1.5087 \mathrm{e}-002 \\ \mathrm{Eu}-154 & 1.0650 \mathrm{e}+000 \\ \mathrm{Eu}-155 & 6.0743 \mathrm{e}-002 \\ \mathrm{I}-129 & 6.1138 \mathrm{e}-005 \\ \mathrm{Ni}-63 & 2.1398 \mathrm{e}+001 \\ \mathrm{~Np}-237 & 4.7825 \mathrm{e}-004 \\ \mathrm{~Pa}-233 & 4.7825 \mathrm{e}-004 \\ \mathrm{Pm}-147 & 1.9032 \mathrm{e}-002 \\ \mathrm{Pr}-144 & 2.7709 \mathrm{e}-015\end{array}$

becquerels

$1.4412 \mathrm{e}+010$

$1.2296 \mathrm{e}+007$

$2.2220 \mathrm{e}+013$

$1.0252 \mathrm{e}-004$

$3.5500 e+007$

$1.2332 \mathrm{e}+007$

$3.1122 \mathrm{e}+008$

$5.5093 e+006$

$3.6449 e+008$

$2.3424 \mathrm{e}+013$

$5.5823 e+008$

$3.9404 \mathrm{e}+010$

$2.2475 e+009$

$2.2621 \mathrm{e}+006$

$7.9173 e+011$

$1.7695 e+007$

$1.7695 e+007$

$7.0417 e+008$

1.0252e-004 $\mu \mathrm{Ci} / \mathrm{cm}^{3}$

3.9500e-001

3.3700e-004

$6.0900 \mathrm{e}+002$

2.8100e-015

$9.7300 \mathrm{e}-004$

3.3800e-004

8.5300e-003

$1.5100 \mathrm{e}-004$

$9.9900 \mathrm{e}-003$

$6.4200 e+002$

$1.5300 \mathrm{e}-002$

$1.0800 \mathrm{e}+000$

$6.1600 \mathrm{e}-002$

6.2000e-005

$2.1700 e+001$

4.8500e-004

4.8500e-004

$1.9300 \mathrm{e}-002$

2.8100e-015
$\mathrm{Bq} / \mathrm{cm}^{3}$

$1.4615 e+004$

$1.2469 e+001$

$2.2533 e+007$

$1.0397 \mathrm{e}-010$

$3.6001 e+001$

$1.2506 \mathrm{e}+001$

$3.1561 e+002$

$5.5870 \mathrm{e}+000$

$3.6963 e+002$

$2.3754 e+007$

$5.6610 \mathrm{e}+002$

$3.9960 e+004$

$2.2792 e+003$

$2.2940 \mathrm{e}+000$

$8.0290 \mathrm{e}+005$

$1.7945 e+001$

$1.7945 \mathrm{e}+001$

$7.1410 \mathrm{e}+002$

1.0397e-010 
Page : 2

DOS File : CSSF 2 Calcine in Grout.ms6

Run Date: April 5, 2005

Run Time: 3:10:00 PM

Duration : 00:01:02

\begin{tabular}{|c|c|c|c|c|}
\hline Nuclide & curies & becquerels & $\mu \mathrm{Ci} / \mathrm{cm}^{3}$ & $\mathrm{~Bq} / \mathrm{cm}^{3}$ \\
\hline Pu-238 & $3.3428 e+000$ & $1.2369 \mathrm{e}+011$ & $3.3900 \mathrm{e}+000$ & $1.2543 e+005$ \\
\hline Pu-239 & $6.7054 \mathrm{e}-002$ & $2.4810 e+009$ & $6.8000 \mathrm{e}-002$ & $2.5160 e+003$ \\
\hline Pu-240 & $5.6306 \mathrm{e}-002$ & $2.0833 e+009$ & $5.7100 \mathrm{e}-002$ & $2.1127 e+003$ \\
\hline Pu-241 & $1.6961 \mathrm{e}+000$ & $6.2755 e+010$ & $1.7200 e+000$ & $6.3640 e+004$ \\
\hline Pu-242 & $1.3115 \mathrm{e}-004$ & $4.8525 e+006$ & $1.3300 \mathrm{e}-004$ & $4.9210 e+000$ \\
\hline Ru-106 & $1.0453 e-010$ & $3.8674 \mathrm{e}+000$ & $1.0600 \mathrm{e}-010$ & $3.9220 e-006$ \\
\hline Sb-125 & $2.7709 \mathrm{e}-002$ & $1.0252 \mathrm{e}+009$ & $2.8100 \mathrm{e}-002$ & $1.0397 e+003$ \\
\hline Sb-126 & $1.0650 \mathrm{e}-003$ & $3.9404 \mathrm{e}+007$ & $1.0800 \mathrm{e}-003$ & $3.9960 e+001$ \\
\hline Sb-126m & $7.5929 e-003$ & $2.8094 \mathrm{e}+008$ & $7.7000 e-003$ & $2.8490 e+002$ \\
\hline Sm-151 & $9.1115 e+000$ & $3.3712 \mathrm{e}+011$ & $9.2400 e+000$ & $3.4188 e+005$ \\
\hline Sr-90 & $5.5221 \mathrm{e}+002$ & $2.0432 \mathrm{e}+013$ & $5.6000 e+002$ & $2.0720 e+007$ \\
\hline Tc-99 & $2.8794 \mathrm{e}-001$ & $1.0654 \mathrm{e}+010$ & $2.9200 \mathrm{e}-001$ & $1.0804 e+004$ \\
\hline Th-230 & $5.4827 e-005$ & $2.0286 e+006$ & $5.5600 e-005$ & $2.0572 e+000$ \\
\hline Th-231 & $2.2088 \mathrm{e}-005$ & $8.1727 e+005$ & $2.2400 \mathrm{e}-005$ & $8.2880 e-001$ \\
\hline$U-232$ & $3.4020 \mathrm{e}-006$ & $1.2587 e+005$ & $3.4500 \mathrm{e}-006$ & $1.2765 e-001$ \\
\hline$U-233$ & $7.8591 \mathrm{e}-007$ & $2.9079 e+004$ & $7.9700 \mathrm{e}-007$ & $2.9489 e-002$ \\
\hline$U-234$ & $8.1155 e-003$ & $3.0027 e+008$ & $8.2300 \mathrm{e}-003$ & $3.0451 e+002$ \\
\hline U-235 & $2.0708 \mathrm{e}-005$ & $7.6619 e+005$ & $2.1000 \mathrm{e}-005$ & $7.7700 e-001$ \\
\hline$U-236$ & $5.7390 e-005$ & $2.1234 \mathrm{e}+006$ & $5.8200 e-005$ & $2.1534 \mathrm{e}+000$ \\
\hline U-237 & $4.6346 e-005$ & $1.7148 \mathrm{e}+006$ & $4.7000 \mathrm{e}-005$ & $1.7390 e+000$ \\
\hline$U-238$ & $1.2523 \mathrm{e}-006$ & $4.6336 e+004$ & $1.2700 \mathrm{e}-006$ & $4.6990 \mathrm{e}-002$ \\
\hline$Y-90$ & $5.5221 \mathrm{e}+002$ & $2.0432 e+013$ & $5.6000 e+002$ & $2.0720 e+007$ \\
\hline
\end{tabular}

$\begin{array}{cc}\frac{\begin{array}{c}\text { Energy } \\ \text { MeV }\end{array}}{} & \begin{array}{c}\text { Activity } \\ \text { photons/sec }\end{array} \\ 0.015 & \\ 0.02 & 1.032 \mathrm{e}+10 \\ 0.03 & 6.304 \mathrm{e}+09 \\ 0.04 & 1.346 \mathrm{e}+12 \\ 0.05 & 3.292 \mathrm{e}+11 \\ 0.06 & 2.356 \mathrm{e}+09 \\ 0.08 & 5.180 \mathrm{e}+09 \\ 0.1 & 7.149 \mathrm{e}+08 \\ 0.15 & 1.659 \mathrm{e}+10 \\ 0.2 & 5.210 \mathrm{e}+07 \\ 0.3 & 2.828 \mathrm{e}+09 \\ 0.4 & 2.527 \mathrm{e}+08 \\ 0.5 & 9.518 \mathrm{e}+08 \\ 0.6 & 2.866 \mathrm{e}+08 \\ 0.8 & 1.995 \mathrm{e}+13 \\ 1.0 & 1.497 \mathrm{e}+10 \\ 1.5 & 1.282 \mathrm{e}+10 \\ 2.0 & 1.587 \mathrm{e}+10 \\ 3.0 & 7.236 \mathrm{e}+05 \\ & 1.120 \mathrm{e}+01\end{array}$

Radial

Integration Parameters

Circumferential

Y Direction (axial) $\quad 24$

Results - Dose Point \# 1 - $(34.29,168.93,0) \mathrm{cm}$

\begin{tabular}{|c|c|c|c|}
\hline Fluence Rate & Fluence Rate & Exposure Rate & Exposure Rate \\
\hline $\mathrm{MeV} / \mathrm{cm}^{2} / \mathrm{sec}$ & $\mathrm{MeV} / \mathrm{cm}^{2} / \mathrm{sec}$ & $\mathrm{mR} / \mathrm{hr}$ & $\mathrm{mR} / \mathrm{hr}$ \\
\hline No Buildup & With Buildup & No Buildup & With Buildup \\
\hline $1.291 \mathrm{e}-267$ & $3.334 \mathrm{e}-23$ & $1.107 e-268$ & $2.859 \mathrm{e}-24$ \\
\hline $2.049 e-119$ & $3.204 \mathrm{e}-23$ & $7.099 e-121$ & $1.110 \mathrm{e}-24$ \\
\hline $1.001 \mathrm{e}-34$ & $1.513 e-20$ & $9.919 e-37$ & $1.500 e-22$ \\
\hline $5.112 \mathrm{e}-14$ & $2.044 \mathrm{e}-13$ & $2.261 \mathrm{e}-16$ & $9.040 e-16$ \\
\hline $2.940 \mathrm{e}-08$ & $1.976 \mathrm{e}-07$ & $7.832 \mathrm{e}-11$ & $5.264 \mathrm{e}-10$ \\
\hline $2.692 \mathrm{e}-04$ & $2.515 e-03$ & $5.346 e-07$ & $4.994 \mathrm{e}-06$ \\
\hline $4.177 e-02$ & $4.719 \mathrm{e}-01$ & $6.609 e-05$ & $7.468 \mathrm{e}-04$ \\
\hline $1.572 \mathrm{e}+01$ & $1.776 \mathrm{e}+02$ & $2.404 \mathrm{e}-02$ & $2.716 \mathrm{e}-01$ \\
\hline $6.436 \mathrm{e}-01$ & $6.071 e+00$ & $1.060 \mathrm{e}-03$ & 9.997e-03 \\
\hline $9.428 e+01$ & $7.614 e+02$ & $1.664 \mathrm{e}-01$ & $1.344 \mathrm{e}+00$ \\
\hline $2.298 \mathrm{e}+01$ & $1.478 \mathrm{e}+02$ & $4.359 e-02$ & $2.805 e-01$ \\
\hline $1.591 \mathrm{e}+02$ & $8.680 e+02$ & $3.101 \mathrm{e}-01$ & $1.691 \mathrm{e}+00$ \\
\hline $7.521 e+01$ & $3.600 e+02$ & $1.476 \mathrm{e}-01$ & 7.067e-01 \\
\hline $7.513 e+06$ & $3.230 \mathrm{e}+07$ & $1.467 e+04$ & $6.305 e+04$ \\
\hline $9.912 e+03$ & $3.621 e+04$ & $1.885 e+01$ & $6.888 e+01$ \\
\hline $1.312 \mathrm{e}+04$ & $4.249 e+04$ & $2.418 e+01$ & $7.832 e+01$ \\
\hline $3.539 e+04$ & $9.375 e+04$ & $5.954 e+01$ & $1.577 e+02$ \\
\hline $2.736 e+00$ & $6.432 e+00$ & $4.230 \mathrm{e}-03$ & $9.947 e-03$ \\
\hline $8.517 \mathrm{e}-05$ & $1.728 \mathrm{e}-04$ & $1.156 \mathrm{e}-07$ & $2.345 e-07$ \\
\hline $7.572 e+06$ & $3.248 e+07$ & $1.477 e+04$ & $6.336 e+04$ \\
\hline
\end{tabular}

Results - Dose Point \# 2 - $(61.75,168.93,0) \mathrm{cm}$ 
Page : 3

DOS File : CSSF 2 Calcine in Grout.ms6

Run Date: April 5, 2005

Run Time: 3:10:00 PM

Duration : 00:01:02

$\begin{array}{cc}\begin{array}{cc}\text { Energy } \\ \text { MeV }\end{array} & \begin{array}{c}\text { Activity } \\ \text { photons/sec }\end{array} \\ 0.015 & \\ 0.02 & 1.032 \mathrm{e}+10 \\ 0.03 & 6.304 \mathrm{e}+09 \\ 0.04 & 1.346 \mathrm{e}+12 \\ 0.05 & 3.292 \mathrm{e}+11 \\ 0.06 & 2.356 \mathrm{e}+09 \\ 0.08 & 5.180 \mathrm{e}+09 \\ 0.1 & 7.149 \mathrm{e}+08 \\ 0.15 & 1.659 \mathrm{e}+10 \\ 0.2 & 5.210 \mathrm{e}+07 \\ 0.3 & 2.828 \mathrm{e}+09 \\ 0.4 & 2.527 \mathrm{e}+08 \\ 0.5 & 9.518 \mathrm{e}+08 \\ 0.6 & 2.866 \mathrm{e}+08 \\ 0.8 & 1.995 \mathrm{e}+13 \\ 1.0 & 1.497 \mathrm{e}+10 \\ 1.5 & 1.282 \mathrm{e}+10 \\ 2.0 & 1.587 \mathrm{e}+10 \\ 3.0 & 7.236 \mathrm{e}+05 \\ & 1.120 \mathrm{e}+01\end{array}$

\begin{tabular}{c}
$\frac{\text { Fluence Rate }}{\text { MeV/cm } 2 / s e c}$ \\
\hline No Buildup \\
$2.605 \mathrm{e}-250$ \\
$5.718 \mathrm{e}-113$ \\
$9.894 \mathrm{e}-34$ \\
$4.879 \mathrm{e}-14$ \\
$1.611 \mathrm{e}-08$ \\
$1.283 \mathrm{e}-04$ \\
$2.006 \mathrm{e}-02$ \\
$7.833 \mathrm{e}+00$ \\
$3.270 \mathrm{e}-01$ \\
$4.779 \mathrm{e}+01$ \\
$1.159 \mathrm{e}+01$ \\
$7.999 \mathrm{e}+01$ \\
$3.770 \mathrm{e}+01$ \\
$3.759 \mathrm{e}+06$ \\
$4.942 \mathrm{e}+03$ \\
$6.521 \mathrm{e}+03$ \\
$1.752 \mathrm{e}+04$ \\
$1.350 \mathrm{e}+00$ \\
$4.194 \mathrm{e}-05$ \\
$3.788 \mathrm{e}+06$
\end{tabular}

Fluence Rate

$\mathrm{MeV} / \mathrm{cm}^{2} / \mathrm{sec}$

With Buildup

$1.463 \mathrm{e}-23$

$1.406 \mathrm{e}-23$

$6.642 \mathrm{e}-21$

$1.934 \mathrm{e}-13$

$1.068 \mathrm{e}-07$

$1.191 \mathrm{e}-03$

2.306e-01

$9.036 \mathrm{e}+01$

$3.091 \mathrm{e}+00$

$3.834 \mathrm{e}+02$

$7.371 \mathrm{e}+01$

$4.309 \mathrm{e}+02$

$1.783 e+02$

$1.596 \mathrm{e}+07$

$1.785 \mathrm{e}+04$

$2.091 \mathrm{e}+04$

$4.600 \mathrm{e}+04$

$3.152 \mathrm{e}+00$

$8.458 \mathrm{e}-05$

$1.605 e+07$
Exposure Rate $\mathrm{mR} / \mathrm{hr}$

No Buildup

2.234e-251

$1.981 \mathrm{e}-114$

$9.806 \mathrm{e}-36$

$2.158 \mathrm{e}-16$

$4.292 \mathrm{e}-11$

2.549e-07

$3.174 \mathrm{e}-05$

$1.198 \mathrm{e}-02$

5.384e-04

$8.435 \mathrm{e}-02$

2.199e-02

1.559e-01

7.401e-02

$7.336 \mathrm{e}+03$

$9.399 \mathrm{e}+00$

$1.202 \mathrm{e}+01$

$2.947 \mathrm{e}+01$

$2.088 \mathrm{e}-03$

$5.690 \mathrm{e}-08$

$7.387 e+03$
Exposure Rate $\mathrm{mR} / \mathrm{hr}$

With Buildup

$1.255 \mathrm{e}-24$

$4.871 \mathrm{e}-25$

$6.582 \mathrm{e}-23$

8.552e-16

2.844e-10

2.365e-06

3.649e-04

1.382e-01

5.091e-03

6.767e-01

1.398e-01

8.396e-01

3.499e-01

$3.116 \mathrm{e}+04$

$3.395 \mathrm{e}+01$

$3.853 e+01$

$7.739 \mathrm{e}+01$

$4.874 \mathrm{e}-03$

$1.148 \mathrm{e}-07$

$3.131 e+04$

\begin{tabular}{|c|c|}
\hline $\begin{array}{c}\text { Energy } \\
\mathrm{MeV}\end{array}$ & $\begin{array}{c}\text { Activity } \\
\text { photons/sec }\end{array}$ \\
\hline 0.015 & $1.032 \mathrm{e}+10$ \\
\hline 0.02 & $6.304 e+09$ \\
\hline 0.03 & $1.346 \mathrm{e}+12$ \\
\hline 0.04 & $3.292 \mathrm{e}+11$ \\
\hline 0.05 & $2.356 \mathrm{e}+09$ \\
\hline 0.06 & $5.180 e+09$ \\
\hline 0.08 & $7.149 e+08$ \\
\hline 0.1 & $1.659 e+10$ \\
\hline 0.15 & $5.210 \mathrm{e}+07$ \\
\hline 0.2 & $2.828 e+09$ \\
\hline 0.3 & $2.527 e+08$ \\
\hline 0.4 & $9.518 e+08$ \\
\hline 0.5 & $2.866 e+08$ \\
\hline 0.6 & $1.995 e+13$ \\
\hline 0.8 & $1.497 \mathrm{e}+10$ \\
\hline 1.0 & $1.282 e+10$ \\
\hline 1.5 & $1.587 e+10$ \\
\hline 2.0 & $7.236 e+05$ \\
\hline 3.0 & $1.120 \mathrm{e}+01$ \\
\hline
\end{tabular}

TOTALS:

$2.172 e+13$

$\begin{array}{ll}\frac{\text { Energy }}{\text { MeV }} & \begin{array}{c}\text { Activity } \\ \text { photons/sec }\end{array} \\ 0.015 & 1.032 \mathrm{e}+10 \\ 0.02 & 6.304 \mathrm{e}+09 \\ 0.03 & 1.346 \mathrm{e}+12\end{array}$

\section{Results - Dose Point \# 3 - $(131.75,168.93,0) \mathrm{cm}$}

\section{Fluence Rate}

$\mathrm{MeV} / \mathrm{cm}^{2} / \mathrm{sec}$

No Buildup

$7.500 \mathrm{e}-251$

$2.209 \mathrm{e}-113$

$4.627 \mathrm{e}-34$

$2.242 \mathrm{e}-14$

$7.373 \mathrm{e}-09$

$5.882 \mathrm{e}-05$

$9.164 \mathrm{e}-03$

$3.562 \mathrm{e}+00$

$1.466 \mathrm{e}-01$

$2.122 \mathrm{e}+01$

$5.088 \mathrm{e}+00$

$3.488 \mathrm{e}+01$

$1.635 \mathrm{e}+01$

$1.623 \mathrm{e}+06$

$2.118 \mathrm{e}+03$

$2.777 e+03$

$7.368 \mathrm{e}+03$

$5.630 \mathrm{e}-01$

$1.728 \mathrm{e}-05$

$1.635 e+06$
Fluence Rate

$\mathrm{MeV} / \mathrm{cm}^{2} / \mathrm{sec}$

With Buildup

4.951e-24

$4.759 \mathrm{e}-24$

$2.248 \mathrm{e}-21$

$8.885 \mathrm{e}-14$

$4.889 \mathrm{e}-08$

$5.462 \mathrm{e}-04$

$1.054 \mathrm{e}-01$

$4.106 \mathrm{e}+01$

$1.372 \mathrm{e}+00$

$1.673 e+02$

$3.161 \mathrm{e}+01$

$1.831 \mathrm{e}+02$

$7.527 \mathrm{e}+01$

$6.706 \mathrm{e}+06$

$7.440 \mathrm{e}+03$

$8.660 \mathrm{e}+03$

$1.883 e+04$

$1.279 \mathrm{e}+00$

3.396e-05

$6.742 e+06$
Exposure Rate $\mathrm{mR} / \mathrm{hr}$

No Buildup

6.433e-252

$7.653 \mathrm{e}-115$

$4.585 \mathrm{e}-36$

$9.915 \mathrm{e}-17$

$1.964 \mathrm{e}-11$

$1.168 \mathrm{e}-07$

$1.450 \mathrm{e}-05$

$5.450 \mathrm{e}-03$

2.414e-04

$3.745 \mathrm{e}-02$

$9.652 \mathrm{e}-03$

$6.796 \mathrm{e}-02$

3.210e-02

$3.168 \mathrm{e}+03$

$4.029 \mathrm{e}+00$

$5.120 \mathrm{e}+00$

$1.240 \mathrm{e}+01$

8.707e-04

2.344e-08

$3.190 e+03$
Exposure Rate $\mathrm{mR} / \mathrm{hr}$

With Buildup

4.247e-25

$1.649 \mathrm{e}-25$

2.228e-23

$3.929 \mathrm{e}-16$

$1.302 \mathrm{e}-10$

$1.085 \mathrm{e}-06$

$1.668 \mathrm{e}-04$

$6.281 \mathrm{e}-02$

2.260e-03

2.953e-01

5.997e-02

3.567e-01

$1.477 \mathrm{e}-01$

$1.309 e+04$

$1.415 \mathrm{e}+01$

$1.596 \mathrm{e}+01$

$3.168 \mathrm{e}+01$

$1.978 \mathrm{e}-03$

4.608e-08

$1.315 e+04$

\section{Results - Dose Point \# 4 - $(41.75,168.93,0) \mathrm{cm}$}

$\begin{array}{ccc}\frac{\text { Fluence Rate }}{\text { MeV/cm } / \mathrm{sec}} & \frac{\text { Fluence Rate }}{\text { MeV/cm } 2 / \mathrm{sec}} & \begin{array}{c}\text { Exposure Rate } \\ \text { mR/hr }\end{array} \\ \text { No Buildup } & \frac{\text { With Buildup }}{2.485 \mathrm{e}-23} & \frac{\text { No Buildup }}{9.173 \mathrm{e}-253} \\ 1.069 \mathrm{e}-251 & 2.389 \mathrm{e}-23 & 1.312 \mathrm{e}-114 \\ 3.787 \mathrm{e}-113 & 1.128 \mathrm{e}-20 & 1.731 \mathrm{e}-35 \\ 1.746 \mathrm{e}-33 & & \end{array}$

Exposure Rate $\mathrm{mR} / \mathrm{hr}$

With Buildup

$2.132 \mathrm{e}-24$

$8.275 \mathrm{e}-25$

$1.118 \mathrm{e}-22$ 
Page : 4

DOS File : CSSF 2 Calcine in Grout.ms6

Run Date: April 5, 2005

Run Time: 3:10:00 PM

Duration : 00:01:02

$\begin{array}{ccc}\frac{\text { Energy }}{\mathrm{MeV}} & & \begin{array}{c}\text { Activity } \\ \text { photons } / \mathrm{sec}\end{array} \\ 0.04 & & 3.292 \mathrm{e}+11 \\ 0.05 & & 2.356 \mathrm{e}+09 \\ 0.06 & & 5.180 \mathrm{e}+09 \\ 0.08 & & 7.149 \mathrm{e}+08 \\ 0.1 & & 1.659 \mathrm{e}+10 \\ 0.15 & 5.210 \mathrm{e}+07 \\ 0.2 & 2.828 \mathrm{e}+09 \\ 0.3 & 2.527 \mathrm{e}+08 \\ 0.4 & 9.518 \mathrm{e}+08 \\ 0.5 & 2.866 \mathrm{e}+08 \\ 0.6 & 1.995 \mathrm{e}+13 \\ 0.8 & 1.497 \mathrm{e}+10 \\ 1.0 & 1.282 \mathrm{e}+10 \\ 1.5 & 1.587 \mathrm{e}+10 \\ 2.0 & 7.236 \mathrm{e}+05 \\ 3.0 & 1.120 \mathrm{e}+01\end{array}$

\begin{tabular}{|c|c|c|}
\hline Fluence Rate & Fluence Rate & Exposure Rate \\
\hline $\mathrm{MeV} / \mathrm{cm}^{2} / \mathrm{sec}$ & $\mathrm{MeV} / \mathrm{cm}^{2} / \mathrm{sec}$ & $\mathrm{mR} / \mathrm{hr}$ \\
\hline No Buildup & With Buildup & No Buildup \\
\hline $8.497 e-14$ & $3.364 \mathrm{e}-13$ & $3.758 e-16$ \\
\hline $2.468 \mathrm{e}-08$ & $1.627 \mathrm{e}-07$ & $6.574 \mathrm{e}-11$ \\
\hline $1.884 \mathrm{e}-04$ & $1.747 \mathrm{e}-03$ & $3.743 e-07$ \\
\hline $3.007 e-02$ & $3.472 \mathrm{e}-01$ & $4.758 e-05$ \\
\hline $1.189 \mathrm{e}+01$ & $1.377 e+02$ & $1.820 \mathrm{e}-02$ \\
\hline $5.018 \mathrm{e}-01$ & $4.765 e+00$ & $8.263 e-04$ \\
\hline $7.364 \mathrm{e}+01$ & $5.937 e+02$ & $1.300 \mathrm{e}-01$ \\
\hline $1.792 e+01$ & $1.146 \mathrm{e}+02$ & $3.399 \mathrm{e}-02$ \\
\hline $1.239 e+02$ & $6.710 e+02$ & $2.414 \mathrm{e}-01$ \\
\hline $5.846 e+01$ & $2.779 e+02$ & $1.148 \mathrm{e}-01$ \\
\hline $5.834 \mathrm{e}+06$ & $2.491 \mathrm{e}+07$ & $1.139 e+04$ \\
\hline $7.681 e+03$ & $2.788 e+04$ & $1.461 \mathrm{e}+01$ \\
\hline $1.015 e+04$ & $3.268 e+04$ & $1.871 \mathrm{e}+01$ \\
\hline $2.732 e+04$ & $7.203 e+04$ & $4.596 e+01$ \\
\hline $2.109 \mathrm{e}+00$ & $4.941 \mathrm{e}+00$ & $3.261 \mathrm{e}-03$ \\
\hline $6.559 \mathrm{e}-05$ & $1.328 \mathrm{e}-04$ & $8.898 \mathrm{e}-08$ \\
\hline $5.879 e+06$ & $2.504 \mathrm{e}+07$ & $1.147 \mathrm{e}+04$ \\
\hline
\end{tabular}

Exposure Rate $\mathrm{mR} / \mathrm{hr}$

With Buildup

$1.488 \mathrm{e}-15$

$4.335 \mathrm{e}-10$

$3.470 \mathrm{e}-06$

$5.495 \mathrm{e}-04$

2.107e-01

$7.846 \mathrm{e}-03$

$1.048 \mathrm{e}+00$

$2.174 \mathrm{e}-01$

$1.307 \mathrm{e}+00$

$5.455 \mathrm{e}-01$

$4.862 \mathrm{e}+04$

$5.304 \mathrm{e}+01$

$6.025 \mathrm{e}+01$

$1.212 \mathrm{e}+02$

$7.641 \mathrm{e}-03$

$1.801 \mathrm{e}-07$

TOTALS: $\quad 2.172 \mathrm{e}+13$

Results - Dose Point \# 5 - $(51.75,168.93,0) \mathrm{cm}$

$\begin{array}{cc}\begin{array}{cc}\text { Energy } \\ \text { MeV }\end{array} & \begin{array}{c}\text { Activity } \\ \text { photons } / \mathrm{sec}\end{array} \\ 0.015 & \\ 0.02 & 1.032 \mathrm{e}+10 \\ 0.03 & 6.304 \mathrm{e}+09 \\ 0.04 & 1.346 \mathrm{e}+12 \\ 0.05 & 3.292 \mathrm{e}+11 \\ 0.06 & 2.356 \mathrm{e}+09 \\ 0.08 & 5.180 \mathrm{e}+09 \\ 0.1 & 7.149 \mathrm{e}+08 \\ 0.15 & 1.659 \mathrm{e}+10 \\ 0.2 & 5.210 \mathrm{e}+07 \\ 0.3 & 2.828 \mathrm{e}+09 \\ 0.4 & 2.527 \mathrm{e}+08 \\ 0.5 & 9.518 \mathrm{e}+08 \\ 0.6 & 2.866 \mathrm{e}+08 \\ 0.8 & 1.995 \mathrm{e}+13 \\ 1.0 & 1.497 \mathrm{e}+10 \\ 1.5 & 1.282 \mathrm{e}+10 \\ 2.0 & 1.587 \mathrm{e}+10 \\ 3.0 & 7.236 \mathrm{e}+05 \\ & 1.120 \mathrm{e}+01\end{array}$

\section{Fluence Rate}

$\mathrm{MeV} / \mathrm{cm}^{2} / \mathrm{sec}$

No Buildup

$2.227 \mathrm{e}-250$

$7.433 \mathrm{e}-113$

$1.312 \mathrm{e}-33$

$5.683 \mathrm{e}-14$

$1.913 \mathrm{e}-08$

$1.544 \mathrm{e}-04$

$2.414 \mathrm{e}-02$

$9.442 \mathrm{e}+00$

$3.952 \mathrm{e}-01$

$5.784 \mathrm{e}+01$

$1.404 \mathrm{e}+01$

$9.695 \mathrm{e}+01$

$4.571 \mathrm{e}+01$

$4.559 \mathrm{e}+06$

$5.997 \mathrm{e}+03$

$7.917 \mathrm{e}+03$

$2.128 \mathrm{e}+04$

$1.642 \mathrm{e}+00$

5.104e-05

$4.594 e+06$
Fluence Rate

$\mathrm{MeV} / \mathrm{cm}^{2} / \mathrm{sec}$

With Buildup

$1.854 \mathrm{e}-23$

$1.782 \mathrm{e}-23$

$8.417 \mathrm{e}-21$

$2.252 \mathrm{e}-13$

$1.269 \mathrm{e}-07$

$1.433 \mathrm{e}-03$

$2.775 \mathrm{e}-01$

$1.090 \mathrm{e}+02$

$3.741 \mathrm{e}+00$

$4.646 \mathrm{e}+02$

$8.943 e+01$

$5.231 \mathrm{e}+02$

$2.165 \mathrm{e}+02$

$1.940 \mathrm{e}+07$

$2.170 \mathrm{e}+04$

$2.543 e+04$

$5.601 \mathrm{e}+04$

$3.841 \mathrm{e}+00$

$1.032 \mathrm{e}-04$

$1.950 \mathrm{e}+07$
Exposure Rate $\mathrm{mR} / \mathrm{hr}$

No Buildup

1.910e-251

2.575e-114

$1.301 \mathrm{e}-35$

2.513e-16

5.096e-11

3.066e-07

3.821e-05

$1.445 \mathrm{e}-02$

6.509e-04

$1.021 \mathrm{e}-01$

2.663e-02

1.889e-01

8.973e-02

$8.898 \mathrm{e}+03$

$1.141 \mathrm{e}+01$

$1.459 \mathrm{e}+01$

$3.581 \mathrm{e}+01$

$2.539 \mathrm{e}-03$

$6.925 \mathrm{e}-08$

$8.960 e+03$

$4.885 e+04$

Exposure Rate $\mathrm{mR} / \mathrm{hr}$

With Buildup

$1.590 \mathrm{e}-24$

$6.174 \mathrm{e}-25$

8.342e-23

$9.959 \mathrm{e}-16$

$3.381 \mathrm{e}-10$

2.846e-06

4.392e-04

1.667e-01

6.161e-03

8.201e-01

$1.696 \mathrm{e}-01$

$1.019 \mathrm{e}+00$

$4.250 \mathrm{e}-01$

$3.786 e+04$

$4.128 \mathrm{e}+01$

$4.688 \mathrm{e}+01$

$9.424 \mathrm{e}+01$

$5.940 \mathrm{e}-03$

1.400e-07

$3.805 e+04$

\section{Results - Dose Point \# 6 - $(96.75,168.93,0) \mathrm{cm}$}

\begin{tabular}{|c|c|}
\hline$\frac{\text { Energy }}{\text { MeV }}$ & Activity \\
\hline MeV & otons/se \\
\hline 0.015 & $1.032 e+10$ \\
\hline 0.02 & $6.304 e+0$ \\
\hline 0.03 & $1.346 \mathrm{e}+12$ \\
\hline 0.04 & $3.292 e+1$ \\
\hline 0.05 & $2.356 \mathrm{e}+0$ \\
\hline 0.06 & $5.180 \mathrm{e}+0$ \\
\hline
\end{tabular}

\begin{tabular}{c}
$\frac{\text { Fluence Rate }}{\text { MeV/cm }{ }^{2} / \mathrm{sec}}$ \\
\hline With Buildup \\
\hline $7.856 \mathrm{e}-24$ \\
$7.552 \mathrm{e}-24$ \\
$3.566 \mathrm{e}-21$ \\
$1.223 \mathrm{e}-13$ \\
$6.720 \mathrm{e}-08$ \\
$7.506 \mathrm{e}-04$
\end{tabular}

Exposure Rate $\mathrm{mR} / \mathrm{hr}$

No Buildup

9.960e-252

$1.025 \mathrm{e}-114$

$6.352 \mathrm{e}-36$

$1.365 \mathrm{e}-16$

$2.700 \mathrm{e}-11$

$1.606 \mathrm{e}-07$
Exposure Rate $\mathrm{mR} / \mathrm{hr}$

With Buildup

$6.739 \mathrm{e}-25$

$2.616 \mathrm{e}-25$

$3.535 \mathrm{e}-23$

$5.408 \mathrm{e}-16$

$1.790 \mathrm{e}-10$

1.491e-06 
Page : 5

DOS File : CSSF 2 Calcine in Grout.ms6

Run Date: April 5, 2005

Run Time: 3:10:00 PM

Duration : 00:01:02

$\begin{array}{ccc}\frac{\text { Energy }}{\mathrm{MeV}} & & \begin{array}{c}\text { Activity } \\ \text { photons } / \mathrm{sec}\end{array} \\ 0.08 & & 7.149 \mathrm{e}+08 \\ 0.1 & & 1.659 \mathrm{e}+10 \\ 0.15 & & 5.210 \mathrm{e}+07 \\ 0.2 & & 2.828 \mathrm{e}+09 \\ 0.3 & & 2.527 \mathrm{e}+08 \\ 0.4 & & 9.518 \mathrm{e}+08 \\ 0.5 & & 2.866 \mathrm{e}+08 \\ 0.6 & & 1.995 \mathrm{e}+13 \\ 0.8 & & 1.497 \mathrm{e}+10 \\ 1.0 & & 1.282 \mathrm{e}+10 \\ 1.5 & & 1.587 \mathrm{e}+10 \\ 2.0 & 7.236 \mathrm{e}+05 \\ 3.0 & & 1.120 \mathrm{e}+01\end{array}$

\begin{tabular}{c}
$\frac{\text { Fluence Rate }}{\text { MeV/cm } 2 / \mathrm{sec}}$ \\
\hline$\frac{\text { No Buildup }}{1.261 \mathrm{e}-02}$ \\
$4.911 \mathrm{e}+00$ \\
$2.042 \mathrm{e}-01$ \\
$2.976 \mathrm{e}+01$ \\
$7.191 \mathrm{e}+00$ \\
$4.952 \mathrm{e}+01$ \\
$2.329 \mathrm{e}+01$ \\
$2.318 \mathrm{e}+06$ \\
$3.039 \mathrm{e}+03$ \\
$4.000 \mathrm{e}+03$ \\
$1.068 \mathrm{e}+04$ \\
$8.204 \mathrm{e}-01$ \\
$2.534 \mathrm{e}-05$
\end{tabular}

Fluence Rate

$\mathrm{MeV} / \mathrm{cm}^{2} / \mathrm{sec}$

With Buildup

$1.449 \mathrm{e}-01$

$5.665 \mathrm{e}+01$

$1.926 \mathrm{e}+00$

$2.376 \mathrm{e}+02$

$4.537 \mathrm{e}+01$

$2.642 \mathrm{e}+02$

$1.090 \mathrm{e}+02$

$9.739 \mathrm{e}+06$

$1.085 \mathrm{e}+04$

$1.267 \mathrm{e}+04$

$2.771 \mathrm{e}+04$

$1.891 \mathrm{e}+00$

$5.045 \mathrm{e}-05$

$2.336 \mathrm{e}+06$

$9.791 e+06$ $\frac{\text { Exposure Rate }}{\mathrm{mR} / \mathrm{hr}}$

No Buildup

$1.995 \mathrm{e}-05$

$7.514 \mathrm{e}-03$

$3.362 \mathrm{e}-04$

$5.252 \mathrm{e}-02$

$1.364 \mathrm{e}-02$

$9.648 \mathrm{e}-02$

$4.572 \mathrm{e}-02$

$4.525 e+03$

$5.780 \mathrm{e}+00$

$7.373 \mathrm{e}+00$

$1.798 \mathrm{e}+01$

$1.269 \mathrm{e}-03$

3.437e-08

$4.556 e+03$
Exposure Rate $\mathrm{mR} / \mathrm{hr}$

With Buildup

2.294e-04

8.668e-02

$3.172 \mathrm{e}-03$

4.194e-01

8.607e-02

5.148e-01

2.140e-01

$1.901 \mathrm{e}+04$

$2.064 \mathrm{e}+01$

$2.335 \mathrm{e}+01$

$4.662 \mathrm{e}+01$

$2.924 \mathrm{e}-03$

$6.845 \mathrm{e}-08$

$1.910 \mathrm{e}+04$ 
Page $: 1$

DOS File : CSSF 3 Calcine in Grout.ms6

Run Date: April 5, 2005

Run Time: 3:11:44 PM

Duration : 00:01:02
File Ref:

Date:

By:

Checked:

Case Title: CSSF 3 Calcine Grout

Description: CSSF 3 Calcine Grouted at 21.5 wt\% in Storage Canister Geometry: 7 - Cylinder Volume - Side Shields

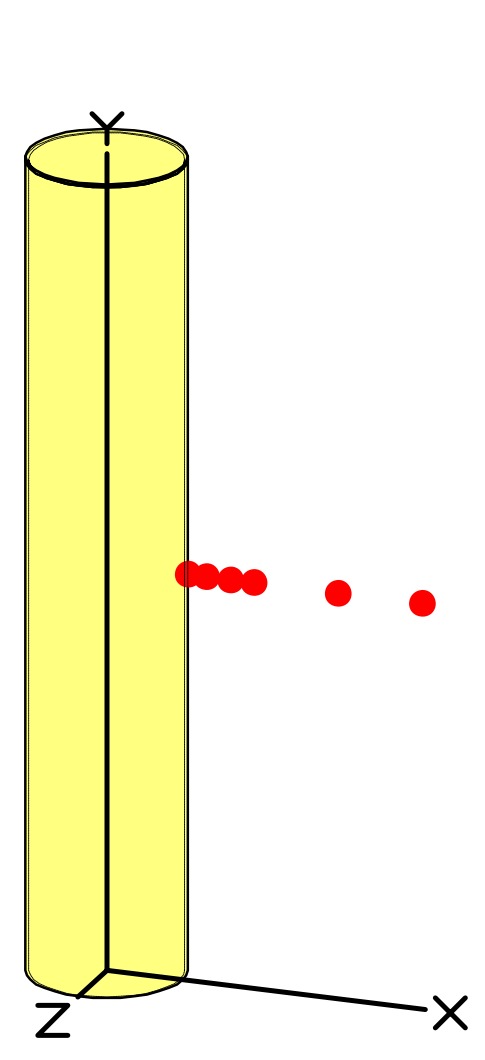

Height
Radius

\section{Source Dimensions}

$337.68 \mathrm{~cm}$

$30.48 \mathrm{~cm}$
$11 \mathrm{ft} 0.9$ in

$1 \mathrm{ft}$

\begin{tabular}{|c|c|c|c|}
\hline \multicolumn{4}{|c|}{ Dose Points } \\
\hline & $\underline{x}$ & $\underline{Y}$ & $\underline{Z}$ \\
\hline \multirow[t]{2}{*}{$\# 1$} & $34.29 \mathrm{~cm}$ & $168.93 \mathrm{~cm}$ & $0 \mathrm{cl}$ \\
\hline & $1 \mathrm{ft} 1.5 \mathrm{in}$ & $5 \mathrm{ft} 6.5$ in & 0.0 \\
\hline \multirow{2}{*}{ \# 2} & $61.75 \mathrm{~cm}$ & $168.93 \mathrm{~cm}$ & $0 \mathrm{c}$ \\
\hline & $2 \mathrm{ft} 0.3$ in & $5 \mathrm{ft} 6.5$ in & 0.0 \\
\hline \multirow[t]{2}{*}{ \# 3} & $131.75 \mathrm{~cm}$ & $168.93 \mathrm{~cm}$ & $0 \mathrm{cr}$ \\
\hline & $4 \mathrm{ft} 3.9$ in & $5 \mathrm{ft} 6.5$ in & 0.0 \\
\hline \multirow[t]{2}{*}{ \# 4} & $41.75 \mathrm{~cm}$ & $168.93 \mathrm{~cm}$ & $0 \mathrm{c}$ \\
\hline & $1 \mathrm{ft} 4.4 \mathrm{in}$ & $5 \mathrm{ft} 6.5$ in & 0.0 \\
\hline \multirow[t]{2}{*}{ \# 5} & $51.75 \mathrm{~cm}$ & $168.93 \mathrm{~cm}$ & $0 \mathrm{c}$ \\
\hline & $1 \mathrm{ft} 8.4$ in & $5 \mathrm{ft} 6.5$ in & 0.0 \\
\hline \multirow[t]{2}{*}{ \# 6} & $96.75 \mathrm{~cm}$ & $168.93 \mathrm{~cm}$ & $0 \mathrm{C}$ \\
\hline & $3 \mathrm{ft} 2.1 \mathrm{in}$ & $5 \mathrm{ft} 6.5 \mathrm{in}$ & \\
\hline
\end{tabular}

\section{Grouping Method : Standard Indices}

Number of Groups : 25

Lower Energy Cutoff : 0.015

Shield Name

Source

Transition

Air Gap

Wall Clad

Top Clad

Shields

Dimension

$9.86 \mathrm{e}+05 \mathrm{~cm}^{3}$

Material

Concrete

Density

1.8

Air

0.00122

$1.27 \mathrm{~cm}$

Air

0.00122

$1.27 \mathrm{~cm}$

Iron

7.95

Iron

7.95

\section{Library : ICRP-38}

\begin{tabular}{lc}
\multicolumn{1}{c}{ Nuclide } & $\underline{\text { curies }}$ \\
Am-241 & $6.1696 \mathrm{e}-001$ \\
Am-243 & $4.0802 \mathrm{e}-004$ \\
Ba-137m & $4.8490 \mathrm{e}+002$ \\
$\mathrm{Ce}-144$ & $3.9718 \mathrm{e}-013$ \\
$\mathrm{Cm}-242$ & $9.8556 \mathrm{e}-004$ \\
$\mathrm{Cm}-244$ & $3.3608 \mathrm{e}-004$ \\
$\mathrm{Co}-60$ & $1.0940 \mathrm{e}-002$ \\
$\mathrm{Cs}-134$ & $2.3654 \mathrm{e}-004$ \\
$\mathrm{Cs}-135$ & $9.7768 \mathrm{e}-003$ \\
$\mathrm{Cs}-137$ & $5.1151 \mathrm{e}+002$ \\
$\mathrm{Eu}-152$ & $1.8726 \mathrm{e}-002$ \\
$\mathrm{Eu}-154$ & $5.9331 \mathrm{e}-001$ \\
$\mathrm{Eu}-155$ & $4.7406 \mathrm{e}-002$ \\
$\mathrm{I}-129$ & $4.4252 \mathrm{e}-005$ \\
$\mathrm{Ni}-63$ & $3.1834 \mathrm{e}+001$ \\
$\mathrm{~Np}-237$ & $2.0303 \mathrm{e}-003$ \\
$\mathrm{~Pa}-233$ & $2.0303 \mathrm{e}-003$ \\
$\mathrm{Pm}-147$ & $1.9613 \mathrm{e}-002$ \\
$\mathrm{Pr}-144$ & $3.9718 \mathrm{e}-013$
\end{tabular}

becquerels

$2.2828 \mathrm{e}+010$

$1.5097 \mathrm{e}+007$

$1.7941 \mathrm{e}+013$

$1.4696 \mathrm{e}-002$

$3.6466 \mathrm{e}+007$

$1.2435 e+007$

$4.0477 e+008$

$8.7518 \mathrm{e}+006$

$3.6174 \mathrm{e}+008$

$1.8926 \mathrm{e}+013$

$6.9285 e+008$

$2.1952 e+010$

$1.7540 \mathrm{e}+009$

$1.6373 e+006$

$1.1778 \mathrm{e}+012$

$7.5120 \mathrm{e}+007$

$7.5120 \mathrm{e}+007$

$7.2567 e+008$

1.4696e-002 $\mu \mathrm{Ci} / \mathrm{cm}^{3}$

6.2600e-001

4.1400e-004

$4.9200 e+002$

4.0300e-013

$1.0000 \mathrm{e}-003$

3.4100e-004

$1.1100 \mathrm{e}-002$

2.4000e-004

$9.9200 \mathrm{e}-003$

$5.1900 \mathrm{e}+002$

1.9000e-002

6.0200e-001

4.8100e-002

4.4900e-005

$3.2300 \mathrm{e}+001$

2.0600e-003

2.0600e-003

$1.9900 \mathrm{e}-002$

$4.0300 \mathrm{e}-013$
$\mathrm{Bq} / \mathrm{cm}^{3}$

$2.3162 e+004$

$1.5318 \mathrm{e}+001$

$1.8204 \mathrm{e}+007$

$1.4911 \mathrm{e}-008$

$3.7000 \mathrm{e}+001$

$1.2617 \mathrm{e}+001$

$4.1070 e+002$

$8.8800 \mathrm{e}+000$

$3.6704 e+002$

$1.9203 e+007$

$7.0300 \mathrm{e}+002$

$2.2274 e+004$

$1.7797 e+003$

$1.6613 e+000$

$1.1951 \mathrm{e}+006$

$7.6220 \mathrm{e}+001$

$7.6220 \mathrm{e}+001$

$7.3630 \mathrm{e}+002$

$1.4911 \mathrm{e}-008$ 
Page : 2

DOS File : CSSF 3 Calcine in Grout.ms6

Run Date: April 5, 2005

Run Time: 3:11:44 PM

Duration : 00:01:02

\begin{tabular}{|c|c|c|c|c|}
\hline Nuclide & curies & becquerels & $\mu \mathrm{Ci} / \mathrm{cm}^{3}$ & $\mathrm{~Bq} / \mathrm{cm}^{3}$ \\
\hline Pu-238 & $1.0743 e+001$ & $3 . \overline{9748 e+011}$ & $1.0900 \mathrm{e}+001$ & $4.0330 e+005$ \\
\hline Pu-239 & $8.6927 e-002$ & $3.2163 e+009$ & $8.8200 \mathrm{e}-002$ & $3.2634 \mathrm{e}+003$ \\
\hline Pu-240 & $1.0743 e-001$ & $3.9748 \mathrm{e}+009$ & $1.0900 \mathrm{e}-001$ & $4.0330 e+003$ \\
\hline Pu-241 & $2.8877 e+000$ & $1.0685 e+011$ & $2.9300 \mathrm{e}+000$ & $1.0841 \mathrm{e}+005$ \\
\hline Pu-242 & $2.7004 \mathrm{e}-004$ & $9.9917 e+006$ & $2.7400 \mathrm{e}-004$ & $1.0138 \mathrm{e}+001$ \\
\hline Ru-106 & $1.2221 \mathrm{e}-010$ & $4.5218 \mathrm{e}+000$ & $1.2400 \mathrm{e}-010$ & $4.5880 e-006$ \\
\hline Sb-125 & $1.1925 \mathrm{e}-003$ & $4.4124 \mathrm{e}+007$ & $1.2100 \mathrm{e}-003$ & $4.4770 e+001$ \\
\hline Sb-126 & $7.8451 e-004$ & $2.9027 e+007$ & $7.9600 \mathrm{e}-004$ & $2.9452 e+001$ \\
\hline Sb-126m & $5.5882 \mathrm{e}-003$ & $2.0676 \mathrm{e}+008$ & $5.6700 \mathrm{e}-003$ & $2.0979 e+002$ \\
\hline Sm-151 & $3.7846 e+000$ & $1.4003 e+011$ & $3.8400 \mathrm{e}+000$ & $1.4208 \mathrm{e}+005$ \\
\hline Sr-90 & $4.5533 e+002$ & $1.6847 e+013$ & $4.6200 e+002$ & $1.7094 \mathrm{e}+007$ \\
\hline Tc-99 & $2.0598 \mathrm{e}-001$ & $7.6214 e+009$ & $2.0900 \mathrm{e}-001$ & $7.7330 e+003$ \\
\hline Th-230 & $7.6578 e-006$ & $2.8334 \mathrm{e}+005$ & $7.7700 \mathrm{e}-006$ & $2.8749 e-001$ \\
\hline Th-231 & $1.9810 \mathrm{e}-005$ & $7.3296 e+005$ & $2.0100 \mathrm{e}-005$ & $7.4370 e-001$ \\
\hline$U-232$ & $2.4245 e-005$ & $8.9706 e+005$ & $2.4600 \mathrm{e}-005$ & $9.1020 \mathrm{e}-001$ \\
\hline$U-233$ & $1.3305 \mathrm{e}-006$ & $4.9229 e+004$ & $1.3500 \mathrm{e}-006$ & $4.9950 e-002$ \\
\hline$U-234$ & $4.2084 \mathrm{e}-003$ & $1.5571 \mathrm{e}+008$ & $4.2700 \mathrm{e}-003$ & $1.5799 \mathrm{e}+002$ \\
\hline U-235 & $1.0743 e-005$ & $3.9748 \mathrm{e}+005$ & $1.0900 \mathrm{e}-005$ & $4.0330 e-001$ \\
\hline$U-236$ & $2.8877 e-005$ & $1.0685 e+006$ & $2.9300 \mathrm{e}-005$ & $1.0841 \mathrm{e}+000$ \\
\hline$U-237$ & $7.0468 \mathrm{e}-005$ & $2.6073 e+006$ & $7.1500 \mathrm{e}-005$ & $2.6455 e+000$ \\
\hline$U-238$ & $9.5600 \mathrm{e}-007$ & $3.5372 \mathrm{e}+004$ & $9.7000 \mathrm{e}-007$ & $3.5890 \mathrm{e}-002$ \\
\hline Y-90 & $4.5533 e+002$ & $1.6847 e+013$ & $4.6200 e+002$ & $1.7094 \mathrm{e}+007$ \\
\hline
\end{tabular}

$\begin{array}{cc}\frac{\text { Energy }}{\text { MeV }} & \begin{array}{c}\text { Activity } \\ \text { photons/sec }\end{array} \\ 0.015 & 2.646 \mathrm{e}+10 \\ 0.02 & 1.220 \mathrm{e}+10 \\ 0.03 & 1.087 \mathrm{e}+12 \\ 0.04 & 2.639 \mathrm{e}+11 \\ 0.05 & 1.401 \mathrm{e}+09 \\ 0.06 & 8.182 \mathrm{e}+09 \\ 0.08 & 5.735 \mathrm{e}+08 \\ 0.1 & 9.516 \mathrm{e}+09 \\ 0.15 & 3.316 \mathrm{e}+07 \\ 0.2 & 1.567 \mathrm{e}+09 \\ 0.3 & 2.746 \mathrm{e}+08 \\ 0.4 & 4.405 \mathrm{e}+08 \\ 0.5 & 1.085 \mathrm{e}+08 \\ 0.6 & 1.611 \mathrm{e}+13 \\ 0.8 & 8.420 \mathrm{e}+09 \\ 1.0 & 7.542 \mathrm{e}+09 \\ 1.5 & 9.164 \mathrm{e}+09 \\ 2.0 & 4.391 \mathrm{e}+05 \\ 3.0 & 1.457 \mathrm{e}+01\end{array}$

Radial

Integration Parameters

Circumferential

Y Direction (axial) $\quad 24$

Results - Dose Point \# 1 - $(34.29,168.93,0) \mathrm{cm}$

\begin{tabular}{|c|c|c|c|}
\hline Fluence Rate & Fluence Rate & Exposure Rate & Exposure Rate \\
\hline $\mathrm{MeV} / \mathrm{cm}^{2} / \mathrm{sec}$ & $\mathrm{MeV} / \mathrm{cm}^{2} / \mathrm{sec}$ & $\mathrm{mR} / \mathrm{hr}$ & $\mathrm{mR} / \mathrm{hr}$ \\
\hline No Buildup & With Buildup & No Buildup & With Buildup \\
\hline $3.427 e-267$ & $8.553 e-23$ & $2.939 \mathrm{e}-268$ & $7.336 e-24$ \\
\hline $4.026 \mathrm{e}-119$ & $6.203 e-23$ & $1.395 \mathrm{e}-120$ & $2.149 \mathrm{e}-24$ \\
\hline $8.118 e-35$ & $1.222 \mathrm{e}-20$ & $8.045 e-37$ & $1.212 \mathrm{e}-22$ \\
\hline $4.105 e-14$ & $1.642 \mathrm{e}-13$ & $1.816 \mathrm{e}-16$ & $7.260 e-16$ \\
\hline $1.750 \mathrm{e}-08$ & $1.176 \mathrm{e}-07$ & $4.663 e-11$ & $3.133 e-10$ \\
\hline $4.254 \mathrm{e}-04$ & $3.974 \mathrm{e}-03$ & $8.450 e-07$ & 7.893e-06 \\
\hline $3.352 \mathrm{e}-02$ & $3.787 e-01$ & $5.304 \mathrm{e}-05$ & $5.993 e-04$ \\
\hline $9.016 e+00$ & $1.019 e+02$ & $1.379 \mathrm{e}-02$ & $1.559 \mathrm{e}-01$ \\
\hline $4.097 e-01$ & $3.865 e+00$ & $6.747 e-04$ & $6.365 e-03$ \\
\hline $5.227 e+01$ & $4.222 e+02$ & $9.226 \mathrm{e}-02$ & $7.451 \mathrm{e}-01$ \\
\hline $2.498 e+01$ & $1.607 e+02$ & $4.739 e-02$ & $3.049 e-01$ \\
\hline $7.369 e+01$ & $4.019 e+02$ & $1.436 \mathrm{e}-01$ & $7.830 e-01$ \\
\hline $2.849 \mathrm{e}+01$ & $1.364 \mathrm{e}+02$ & $5.593 e-02$ & $2.677 \mathrm{e}-01$ \\
\hline $6.070 e+06$ & $2.609 e+07$ & $1.185 e+04$ & $5.093 e+04$ \\
\hline $5.579 \mathrm{e}+03$ & $2.038 e+04$ & $1.061 e+01$ & $3.877 e+01$ \\
\hline $7.721 e+03$ & $2.501 e+04$ & $1.423 e+01$ & $4.611 e+01$ \\
\hline $2.044 \mathrm{e}+04$ & $5.415 e+04$ & $3.440 e+01$ & $9.111 \mathrm{e}+01$ \\
\hline $1.661 \mathrm{e}+00$ & $3.905 e+00$ & $2.569 \mathrm{e}-03$ & $6.039 \mathrm{e}-03$ \\
\hline $1.108 \mathrm{e}-04$ & $2.249 \mathrm{e}-04$ & $1.504 \mathrm{e}-07$ & $3.051 \mathrm{e}-07$ \\
\hline $6.104 e+06$ & $2.619 e+07$ & $1.191 \mathrm{e}+04$ & $5.111 e+04$ \\
\hline
\end{tabular}

Results - Dose Point \# 2 - $(61.75,168.93,0) \mathrm{cm}$ 
Page : 3

DOS File : CSSF 3 Calcine in Grout.ms6

Run Date: April 5, 2005

Run Time: 3:11:44 PM

Duration : 00:01:02

$\begin{array}{cc}\begin{array}{cc}\text { Energy } \\ \underline{\mathrm{MeV}}\end{array} & \begin{array}{c}\text { Activity } \\ \text { photons/sec }\end{array} \\ 0.015 & \\ 0.02 & 2.646 \mathrm{e}+10 \\ 0.03 & 1.220 \mathrm{e}+10 \\ 0.04 & 1.087 \mathrm{e}+12 \\ 0.05 & 2.639 \mathrm{e}+11 \\ 0.06 & 1.401 \mathrm{e}+09 \\ 0.08 & 8.182 \mathrm{e}+09 \\ 0.1 & 5.735 \mathrm{e}+08 \\ 0.15 & 9.516 \mathrm{e}+09 \\ 0.2 & 3.316 \mathrm{e}+07 \\ 0.3 & 1.567 \mathrm{e}+09 \\ 0.4 & 2.746 \mathrm{e}+08 \\ 0.5 & 4.405 \mathrm{e}+08 \\ 0.6 & 1.085 \mathrm{e}+08 \\ 0.8 & 1.611 \mathrm{e}+13 \\ 1.0 & 8.420 \mathrm{e}+09 \\ 1.5 & 7.542 \mathrm{e}+09 \\ 2.0 & 9.164 \mathrm{e}+09 \\ 3.0 & 4.391 \mathrm{e}+05 \\ & 1.457 \mathrm{e}+01\end{array}$

TOTALS:

\begin{tabular}{|c|c|}
\hline$\frac{\text { Energy }}{\mathrm{MeV}}$ & $\begin{array}{c}\text { Activity } \\
\text { photons/sec }\end{array}$ \\
\hline 0.015 & $2.646 e+10$ \\
\hline 0.02 & $1.220 e+10$ \\
\hline 0.03 & $1.087 e+12$ \\
\hline 0.04 & $2.639 e+11$ \\
\hline 0.05 & $1.401 \mathrm{e}+09$ \\
\hline 0.06 & $8.182 e+09$ \\
\hline 0.08 & $5.735 e+08$ \\
\hline 0.1 & $9.516 e+09$ \\
\hline 0.15 & $3.316 e+07$ \\
\hline 0.2 & $1.567 e+09$ \\
\hline 0.3 & $2.746 e+08$ \\
\hline 0.4 & $4.405 e+08$ \\
\hline 0.5 & $1.085 e+08$ \\
\hline 0.6 & $1.611 e+13$ \\
\hline 0.8 & $8.420 e+09$ \\
\hline 1.0 & $7.542 e+09$ \\
\hline 1.5 & $9.164 e+09$ \\
\hline 2.0 & $4.391 e+05$ \\
\hline 3.0 & $1.457 e+01$ \\
\hline
\end{tabular}

TOTALS:

$1.755 e+13$

$\begin{array}{cc}\frac{\text { Energy }}{\text { MeV }} & \begin{array}{c}\text { Activity } \\ \text { photons/sec }\end{array} \\ 0.015 & 2.646 \mathrm{e}+10 \\ 0.02 & 1.220 \mathrm{e}+10 \\ 0.03 & 1.087 \mathrm{e}+12\end{array}$

\begin{tabular}{|c|c|c|}
\hline$\frac{\text { Fluence Rate }}{\mathrm{MeV} / \mathrm{cm}^{2} / \mathrm{sec}}$ & $\frac{\text { Fluence Rate }}{\mathrm{MeV} / \mathrm{cm}^{2} / \mathrm{sec}}$ & $\frac{\text { Exposure Rate }}{\mathrm{mR} / \mathrm{hr}}$ \\
\hline No Buildup & With Buildup & No Buildup \\
\hline $6.684 \mathrm{e}-250$ & $3.753 e-23$ & $5.733 e-251$ \\
\hline $1.107 \mathrm{e}-112$ & $2.722 \mathrm{e}-23$ & $3.834 \mathrm{e}-114$ \\
\hline $7.993 e-34$ & $5.364 \mathrm{e}-21$ & $7.921 \mathrm{e}-36$ \\
\hline $3.913 e-14$ & $1.551 \mathrm{e}-13$ & $1.730 \mathrm{e}-16$ \\
\hline $9.586 \mathrm{e}-09$ & $6.353 e-08$ & $2.554 \mathrm{e}-11$ \\
\hline $2.028 \mathrm{e}-04$ & $1.882 \mathrm{e}-03$ & $4.028 e-07$ \\
\hline $1.610 \mathrm{e}-02$ & $1.851 \mathrm{e}-01$ & $2.548 \mathrm{e}-05$ \\
\hline $4.495 e+00$ & $5.184 e+01$ & $6.876 e-03$ \\
\hline $2.082 \mathrm{e}-01$ & $1.968 \mathrm{e}+00$ & $3.428 e-04$ \\
\hline $2.650 e+01$ & $2.126 \mathrm{e}+02$ & $4.677 e-02$ \\
\hline $1.260 \mathrm{e}+01$ & $8.014 \mathrm{e}+01$ & $2.390 \mathrm{e}-02$ \\
\hline $3.704 e+01$ & $1.995 e+02$ & $7.217 e-02$ \\
\hline $1.428 \mathrm{e}+01$ & $6.753 e+01$ & $2.804 \mathrm{e}-02$ \\
\hline $3.036 e+06$ & $1.290 \mathrm{e}+07$ & $5.926 e+03$ \\
\hline $2.781 e+03$ & $1.005 e+04$ & $5.290 e+00$ \\
\hline $3.839 e+03$ & $1.231 \mathrm{e}+04$ & $7.076 e+00$ \\
\hline $1.012 \mathrm{e}+04$ & $2.657 e+04$ & $1.702 \mathrm{e}+01$ \\
\hline $8.200 e-01$ & $1.914 \mathrm{e}+00$ & $1.268 \mathrm{e}-03$ \\
\hline $5.458 \mathrm{e}-05$ & $1.101 \mathrm{e}-04$ & $7.404 \mathrm{e}-08$ \\
\hline $3.053 e+06$ & $1.295 e+07$ & $5.956 e+03$ \\
\hline
\end{tabular}

Exposure Rate $\mathrm{mR} / \mathrm{hr}$

With Buildup

$3.219 \mathrm{e}-24$

$9.429 \mathrm{e}-25$

$5.317 \mathrm{e}-23$

$6.859 \mathrm{e}-16$

$1.692 \mathrm{e}-10$

$3.738 \mathrm{e}-06$

$2.929 \mathrm{e}-04$

$7.932 \mathrm{e}-02$

$3.241 \mathrm{e}-03$

$3.752 \mathrm{e}-01$

$1.520 \mathrm{e}-01$

$3.887 \mathrm{e}-01$

$1.326 \mathrm{e}-01$

$2.517 \mathrm{e}+04$

$1.911 \mathrm{e}+01$

$2.268 \mathrm{e}+01$

$4.471 \mathrm{e}+01$

$2.959 \mathrm{e}-03$

$1.493 \mathrm{e}-07$

$2.526 e+04$

\section{Results - Dose Point \# 3 - $(131.75,168.93,0) \mathrm{cm}$}

\begin{tabular}{|c|c|c|}
\hline $\begin{array}{l}\text { Fluence Rate } \\
\mathrm{MeV} / \mathrm{cm}^{2} / \mathrm{sec}\end{array}$ & $\begin{array}{l}\text { Fluence Rate } \\
\mathrm{MeV} / \mathrm{cm}^{2} / \mathrm{sec}\end{array}$ & $\frac{\text { Exposure Rate }}{\mathrm{mR} / \mathrm{hr}}$ \\
\hline No Buildup & $\overline{\text { With Buildup }}$ & No Buildup \\
\hline $1.924 \mathrm{e}-250$ & $1.270 \mathrm{e}-23$ & $\overline{1.651 e-251}$ \\
\hline $4.277 e-113$ & $9.211 \mathrm{e}-24$ & $1.481 \mathrm{e}-114$ \\
\hline $3.738 e-34$ & $1.815 e-21$ & $3.704 e-36$ \\
\hline $1.798 \mathrm{e}-14$ & $7.125 e-14$ & $7.951 \mathrm{e}-17$ \\
\hline $4.387 e-09$ & $2.909 e-08$ & $1.169 \mathrm{e}-11$ \\
\hline $9.295 e-05$ & $8.632 \mathrm{e}-04$ & $1.846 \mathrm{e}-07$ \\
\hline $7.356 \mathrm{e}-03$ & $8.462 \mathrm{e}-02$ & $1.164 \mathrm{e}-05$ \\
\hline $2.044 \mathrm{e}+00$ & $2.356 e+01$ & $3.127 e-03$ \\
\hline $9.335 e-02$ & $8.737 e-01$ & $1.537 e-04$ \\
\hline $1.176 \mathrm{e}+01$ & $9.277 e+01$ & $2.076 \mathrm{e}-02$ \\
\hline $5.531 e+00$ & $3.437 e+01$ & $1.049 \mathrm{e}-02$ \\
\hline $1.615 e+01$ & $8.477 e+01$ & $3.146 \mathrm{e}-02$ \\
\hline $6.195 e+00$ & $2.851 \mathrm{e}+01$ & $1.216 \mathrm{e}-02$ \\
\hline $1.311 \mathrm{e}+06$ & $5.417 e+06$ & $2.559 e+03$ \\
\hline $1.192 \mathrm{e}+03$ & $4.187 e+03$ & $2.267 e+00$ \\
\hline $1.635 e+03$ & $5.097 e+03$ & $3.014 \mathrm{e}+00$ \\
\hline $4.255 e+03$ & $1.087 e+04$ & $7.160 e+00$ \\
\hline $3.418 \mathrm{e}-01$ & $7.766 \mathrm{e}-01$ & $5.286 e-04$ \\
\hline $2.248 \mathrm{e}-05$ & $4.419 \mathrm{e}-05$ & $3.050 \mathrm{e}-08$ \\
\hline $1.318 \mathrm{e}+06$ & $5.437 e+06$ & $2.571 \mathrm{e}+03$ \\
\hline
\end{tabular}

Exposure Rate $\mathrm{mR} / \mathrm{hr}$

With Buildup

$1.089 \mathrm{e}-24$

$3.191 \mathrm{e}-25$

$1.799 \mathrm{e}-23$

$3.151 \mathrm{e}-16$

$7.748 \mathrm{e}-11$

$1.715 \mathrm{e}-06$

$1.339 \mathrm{e}-04$

$3.604 \mathrm{e}-02$

$1.439 \mathrm{e}-03$

$1.637 \mathrm{e}-01$

$6.519 \mathrm{e}-02$

$1.652 \mathrm{e}-01$

$5.596 \mathrm{e}-02$

$1.057 \mathrm{e}+04$

$7.964 \mathrm{e}+00$

$9.396 \mathrm{e}+00$

$1.830 \mathrm{e}+01$

$1.201 \mathrm{e}-03$

$5.995 \mathrm{e}-08$

$1.061 \mathrm{e}+04$

\section{Results - Dose Point \# 4 - $(41.75,168.93,0) \mathrm{cm}$}

$\begin{array}{ccc}\frac{\text { Fluence Rate }}{\text { MeV/cm } 2 / \mathrm{sec}} & \frac{\text { Fluence Rate }}{\text { MeV/cm } 2 / \mathrm{sec}} & \begin{array}{c}\text { Exposure Rate } \\ \mathrm{mR} / \mathrm{hr}\end{array} \\ \frac{\text { No Buildup }}{2.758 \mathrm{e}-251} & \frac{\text { With Buildup }}{6.376 \mathrm{e}-23} & \frac{\text { No Buildup }}{2.366 \mathrm{e}-252} \\ 7.345 \mathrm{e}-113 & 4.624 \mathrm{e}-23 & 2.544 \mathrm{e}-114 \\ 1.411 \mathrm{e}-33 & 9.114 \mathrm{e}-21 & 1.398 \mathrm{e}-35\end{array}$

Exposure Rate $\mathrm{mR} / \mathrm{hr}$

With Buildup

$5.469 \mathrm{e}-24$

$1.602 \mathrm{e}-24$

$9.032 \mathrm{e}-23$ 
Page : 4

DOS File : CSSF 3 Calcine in Grout.ms6

Run Date: April 5, 2005

Run Time: 3:11:44 PM

Duration : 00:01:02

$\begin{array}{ccc}\frac{\text { Energy }}{\text { MeV }} & & \begin{array}{c}\text { Activity } \\ \text { photons/sec }\end{array} \\ 0.04 & & 2.639 \mathrm{e}+11 \\ 0.05 & & 1.401 \mathrm{e}+09 \\ 0.06 & & 8.182 \mathrm{e}+09 \\ 0.08 & & 5.735 \mathrm{e}+08 \\ 0.1 & & 9.516 \mathrm{e}+09 \\ 0.15 & & 3.316 \mathrm{e}+07 \\ 0.2 & & 1.567 \mathrm{e}+09 \\ 0.3 & & 2.746 \mathrm{e}+08 \\ 0.4 & & 4.405 \mathrm{e}+08 \\ 0.5 & & 1.085 \mathrm{e}+08 \\ 0.6 & & 1.611 \mathrm{e}+13 \\ 0.8 & & 8.420 \mathrm{e}+09 \\ 1.0 & & 7.542 \mathrm{e}+09 \\ 1.5 & 9.164 \mathrm{e}+09 \\ 2.0 & & 4.391 \mathrm{e}+05 \\ 3.0 & & 1.457 \mathrm{e}+01\end{array}$

TOTALS: $\quad 1.755 \mathrm{e}+13$

$\begin{array}{cc}\begin{array}{cc}\text { Energy } \\ \text { MeV }\end{array} & \begin{array}{c}\text { Activity } \\ \text { photons/sec }\end{array} \\ 0.015 & 2.646 \mathrm{e}+10 \\ 0.02 & 1.220 \mathrm{e}+10 \\ 0.03 & 1.087 \mathrm{e}+12 \\ 0.04 & 2.639 \mathrm{e}+11 \\ 0.05 & 1.401 \mathrm{e}+09 \\ 0.06 & 8.182 \mathrm{e}+09 \\ 0.08 & 5.735 \mathrm{e}+08 \\ 0.1 & 9.516 \mathrm{e}+09 \\ 0.15 & 3.316 \mathrm{e}+07 \\ 0.2 & 1.567 \mathrm{e}+09 \\ 0.3 & 2.746 \mathrm{e}+08 \\ 0.4 & 4.405 \mathrm{e}+08 \\ 0.5 & 1.085 \mathrm{e}+08 \\ 0.6 & 1.611 \mathrm{e}+13 \\ 0.8 & 8.420 \mathrm{e}+09 \\ 1.0 & 7.542 \mathrm{e}+09 \\ 1.5 & 9.164 \mathrm{e}+09 \\ 2.0 & 4.391 \mathrm{e}+05 \\ 3.0 & 1.457 \mathrm{e}+01\end{array}$

TOTALS: $\quad 1.755 \mathrm{e}+13$

$\begin{array}{cc}\frac{\text { Energy }}{\text { MeV }} & \begin{array}{c}\text { Activity } \\ \text { photons/sec }\end{array} \\ 0.015 & \\ 0.02 & 2.646 \mathrm{e}+10 \\ 0.03 & 1.220 \mathrm{e}+10 \\ 0.04 & 1.087 \mathrm{e}+12 \\ 0.05 & 2.639 \mathrm{e}+11 \\ 0.06 & 1.401 \mathrm{e}+09 \\ & 8.182 \mathrm{e}+09\end{array}$

\begin{tabular}{|c|c|c|}
\hline Fluence Rate & Fluence Rate & Exposure Rate \\
\hline $\mathrm{MeV} / \mathrm{cm}^{2} / \mathrm{sec}$ & $\mathrm{MeV} / \mathrm{cm}^{2} / \mathrm{sec}$ & $\underline{\mathrm{mR} / \mathrm{hr}}$ \\
\hline No Buildup & With Buildup & No Buildup \\
\hline $6.813 e-14$ & $2.698 \mathrm{e}-13$ & $3.013 e-16$ \\
\hline $1.468 \mathrm{e}-08$ & $9.681 \mathrm{e}-08$ & $3.911 \mathrm{e}-11$ \\
\hline $2.978 \mathrm{e}-04$ & $2.761 \mathrm{e}-03$ & $5.914 \mathrm{e}-07$ \\
\hline $2.413 e-02$ & $2.787 e-01$ & $3.819 e-05$ \\
\hline $6.824 e+00$ & $7.902 e+01$ & $1.044 \mathrm{e}-02$ \\
\hline $3.195 \mathrm{e}-01$ & $3.034 e+00$ & $5.261 \mathrm{e}-04$ \\
\hline $4.083 e+01$ & $3.292 \mathrm{e}+02$ & $7.206 \mathrm{e}-02$ \\
\hline $1.948 \mathrm{e}+01$ & $1.246 \mathrm{e}+02$ & $3.695 e-02$ \\
\hline $5.736 e+01$ & $3.107 e+02$ & $1.118 \mathrm{e}-01$ \\
\hline $2.215 e+01$ & $1.053 e+02$ & $4.347 e-02$ \\
\hline $4.713 e+06$ & $2.012 \mathrm{e}+07$ & $9.198 e+03$ \\
\hline $4.323 e+03$ & $1.569 e+04$ & $8.223 e+00$ \\
\hline $5.974 e+03$ & $1.924 \mathrm{e}+04$ & $1.101 \mathrm{e}+01$ \\
\hline $1.578 \mathrm{e}+04$ & $4.161 e+04$ & $2.655 e+01$ \\
\hline $1.280 \mathrm{e}+00$ & $3.000 e+00$ & $1.980 \mathrm{e}-03$ \\
\hline $8.535 e-05$ & $1.728 \mathrm{e}-04$ & $1.158 \mathrm{e}-07$ \\
\hline $4.739 e+06$ & $2.020 e+07$ & $9.244 \mathrm{e}+03$ \\
\hline
\end{tabular}

Exposure Rate $\mathrm{mR} / \mathrm{hr}$

With Buildup

$1.193 \mathrm{e}-15$

$2.579 \mathrm{e}-10$

$5.484 \mathrm{e}-06$

$4.410 \mathrm{e}-04$

$1.209 \mathrm{e}-01$

$4.996 \mathrm{e}-03$

$5.810 \mathrm{e}-01$

$2.363 e-01$

$6.054 \mathrm{e}-01$

$2.067 \mathrm{e}-01$

$3.927 \mathrm{e}+04$

$2.985 \mathrm{e}+01$

$3.547 \mathrm{e}+01$

$7.001 \mathrm{e}+01$

$4.639 \mathrm{e}-03$

$2.344 \mathrm{e}-07$

$3.941 \mathrm{e}+04$

Results - Dose Point \# 5 - $(51.75,168.93,0) \mathrm{cm}$
Fluence Rate

$\mathrm{MeV} / \mathrm{cm}^{2} / \mathrm{sec}$

No Buildup

$5.720 \mathrm{e}-250$

$1.439 \mathrm{e}-112$

$1.060 \mathrm{e}-33$

$4.558 \mathrm{e}-14$

$1.138 \mathrm{e}-08$

$2.440 \mathrm{e}-04$

$1.938 \mathrm{e}-02$

$5.418 \mathrm{e}+00$

2.517e-01

$3.207 e+01$

$1.527 \mathrm{e}+01$

$4.489 \mathrm{e}+01$

$1.732 \mathrm{e}+01$

$3.683 e+06$

$3.375 \mathrm{e}+03$

$4.661 \mathrm{e}+03$

$1.229 \mathrm{e}+04$

$9.970 \mathrm{e}-01$

6.642e-05

$3.703 e+06$
Fluence Rate

$\mathrm{MeV} / \mathrm{cm}^{2} / \mathrm{sec}$

With Buildup

4.757e-23

$3.450 \mathrm{e}-23$

$6.799 \mathrm{e}-21$

$1.806 \mathrm{e}-13$

$7.553 \mathrm{e}-08$

$2.265 \mathrm{e}-03$

2.227e-01

$6.252 \mathrm{e}+01$

$2.382 \mathrm{e}+00$

$2.576 \mathrm{e}+02$

$9.723 e+01$

$2.422 \mathrm{e}+02$

$8.203 e+01$

$1.567 \mathrm{e}+07$

$1.497 \mathrm{e}+04$

$3.236 \mathrm{e}+04$

$2.332 \mathrm{e}+00$

$1.343 \mathrm{e}-04$
$1.222 \mathrm{e}+04$

$1.573 e+07$
Exposure Rate $\mathrm{mR} / \mathrm{hr}$

No Buildup

4.906e-251

$4.985 \mathrm{e}-114$

$1.050 \mathrm{e}-35$

2.016e-16

$3.032 \mathrm{e}-11$

4.846e-07

3.066e-05

$8.289 \mathrm{e}-03$

4.144e-04

5.660e-02

$2.896 \mathrm{e}-02$

8.747e-02

3.399e-02

$7.188 \mathrm{e}+03$

$6.420 \mathrm{e}+00$

$8.591 \mathrm{e}+00$

$2.069 \mathrm{e}+01$

$1.542 \mathrm{e}-03$

$9.012 \mathrm{e}-08$

$7.224 \mathrm{e}+03$
Exposure Rate $\mathrm{mR} / \mathrm{hr}$

With Buildup

4.080e-24

$1.195 \mathrm{e}-24$

$6.738 \mathrm{e}-23$

7.987e-16

2.012e-10

4.498e-06

$3.525 \mathrm{e}-04$

$9.565 \mathrm{e}-02$

$3.923 e-03$

4.547e-01

$1.844 \mathrm{e}-01$

4.720e-01

$1.610 \mathrm{e}-01$

$3.059 \mathrm{e}+04$

$2.323 e+01$

$2.760 \mathrm{e}+01$

$5.444 \mathrm{e}+01$

$3.606 \mathrm{e}-03$

$1.822 \mathrm{e}-07$

$3.069 e+04$

\section{Results - Dose Point \# 6 - $(96.75,168.93,0) \mathrm{cm}$}

\begin{tabular}{c}
$\frac{\text { Fluence Rate }}{\text { MeV/cm } 2 / \mathrm{sec}}$ \\
\hline$\frac{\text { No Buildup }}{2.979 \mathrm{e}-250}$ \\
$5.731 \mathrm{e}-113$ \\
$5.178 \mathrm{e}-34$ \\
$2.474 \mathrm{e}-14$ \\
$6.031 \mathrm{e}-09$ \\
$1.278 \mathrm{e}-04$
\end{tabular}

\begin{tabular}{c}
$\frac{\text { Fluence Rate }}{\text { MeV/cm }{ }^{2} / \mathrm{sec}}$ \\
\hline With Buildup \\
\hline $2.015 \mathrm{e}-23$ \\
$1.462 \mathrm{e}-23$ \\
$2.880 \mathrm{e}-21$ \\
$9.806 \mathrm{e}-14$ \\
$3.998 \mathrm{e}-08$ \\
$1.186 \mathrm{e}-03$
\end{tabular}

Exposure Rate $\mathrm{mR} / \mathrm{hr}$

No Buildup

$2.555 \mathrm{e}-251$

$1.985 \mathrm{e}-114$

$5.131 \mathrm{e}-36$

$1.094 \mathrm{e}-16$

$1.607 \mathrm{e}-11$

$2.538 \mathrm{e}-07$

Exposure Rate $\mathrm{mR} / \mathrm{hr}$

With Buildup

$1.729 \mathrm{e}-24$

$5.063 \mathrm{e}-25$

$2.855 e-23$

$4.337 \mathrm{e}-16$

$1.065 \mathrm{e}-10$

2.356e-06 
Page : 5

DOS File : CSSF 3 Calcine in Grout.ms6

Run Date: April 5, 2005

Run Time: 3:11:44 PM

Duration : 00:01:02

$\begin{array}{ccc}\frac{\text { Energy }}{\text { MeV }} & & \begin{array}{c}\text { Activity } \\ \text { photons } / \mathrm{sec}\end{array} \\ 0.08 & & 5.735 \mathrm{e}+08 \\ 0.1 & & 9.516 \mathrm{e}+09 \\ 0.15 & & 3.316 \mathrm{e}+07 \\ 0.2 & & 1.567 \mathrm{e}+09 \\ 0.3 & & 2.746 \mathrm{e}+08 \\ 0.4 & & 4.405 \mathrm{e}+08 \\ 0.5 & & 1.085 \mathrm{e}+08 \\ 0.6 & & 1.611 \mathrm{e}+13 \\ 0.8 & & 8.420 \mathrm{e}+09 \\ 1.0 & & 7.542 \mathrm{e}+09 \\ 1.5 & 9.164 \mathrm{e}+09 \\ 2.0 & & 4.391 \mathrm{e}+05 \\ 3.0 & & 1.457 \mathrm{e}+01\end{array}$

\begin{tabular}{c}
$\frac{\text { Fluence Rate }}{\text { MeV/cm } 2 / \mathrm{sec}}$ \\
\hline$\frac{\text { No Buildup }}{1.012 \mathrm{e}-02}$ \\
$2.818 \mathrm{e}+00$ \\
$1.300 \mathrm{e}-01$ \\
$1.650 \mathrm{e}+01$ \\
$7.818 \mathrm{e}+00$ \\
$2.293 \mathrm{e}+01$ \\
$8.825 \mathrm{e}+00$ \\
$1.873 \mathrm{e}+06$ \\
$1.710 \mathrm{e}+03$ \\
$2.355 \mathrm{e}+03$ \\
$6.172 \mathrm{e}+03$ \\
$4.981 \mathrm{e}-01$ \\
$3.297 \mathrm{e}-05$
\end{tabular}

Fluence Rate

$\mathrm{MeV} / \mathrm{cm}^{2} / \mathrm{sec}$

With Buildup

$1.163 \mathrm{e}-01$

$3.251 \mathrm{e}+01$

$1.227 \mathrm{e}+00$

$1.317 \mathrm{e}+02$

$4.933 \mathrm{e}+01$

$1.223 \mathrm{e}+02$

$4.129 \mathrm{e}+01$

$7.867 \mathrm{e}+06$

$6.106 \mathrm{e}+03$

$7.458 \mathrm{e}+03$

$1.601 \mathrm{e}+04$

$1.148 \mathrm{e}+00$

$6.565 \mathrm{e}-05$

$1.883 \mathrm{e}+06$

$7.897 e+06$ $\frac{\text { Exposure Rate }}{\mathrm{mR} / \mathrm{hr}}$

No Buildup

$1.601 \mathrm{e}-05$

$4.311 \mathrm{e}-03$

$2.141 \mathrm{e}-04$

$2.912 \mathrm{e}-02$

$1.483 \mathrm{e}-02$

$4.467 \mathrm{e}-02$

$1.732 \mathrm{e}-02$

$3.655 e+03$

$3.253 e+00$

$4.340 \mathrm{e}+00$

$1.038 \mathrm{e}+01$

$7.703 e-04$

$4.473 \mathrm{e}-08$

$3.673 e+03$
Exposure Rate $\mathrm{mR} / \mathrm{hr}$

With Buildup

$1.841 \mathrm{e}-04$

$4.973 \mathrm{e}-02$

2.020e-03

2.325e-01

9.357e-02

2.384e-01

8.105e-02

$1.536 \mathrm{e}+04$

$1.161 \mathrm{e}+01$

$1.375 \mathrm{e}+01$

$2.693 e+01$

$1.775 \mathrm{e}-03$

8.906e-08

$1.541 \mathrm{e}+04$ 
Page $: 1$

DOS File : CSSF 4 Calcine in Grout.ms6

Run Date: April 5, 2005

Run Time: 3:13:55 PM

Duration : 00:01:02
File Ref:

Date:

By:

Checked:

Case Title: CSSF 4 Calcine Grout

Description: CSSF 4 Calcine Grouted at 21.5 wt\% in Storage Canister Geometry: 7 - Cylinder Volume - Side Shields

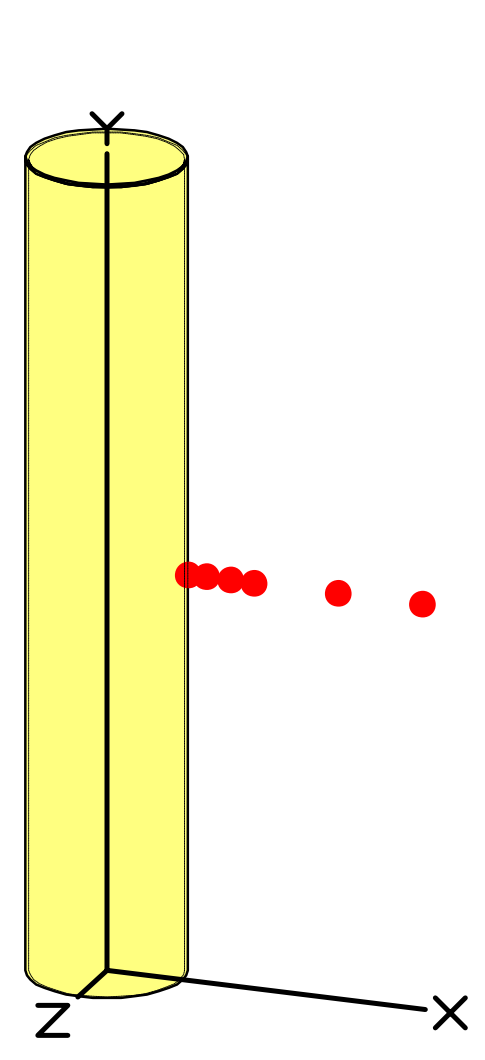

Height
Radius

\section{Source Dimensions}

$337.86 \mathrm{~cm}$

$30.48 \mathrm{~cm}$
$11 \mathrm{ft} 1.0$ in

$1 \mathrm{ft}$

\begin{tabular}{|c|c|c|c|}
\hline \multicolumn{4}{|c|}{ Dose Points } \\
\hline & $\underline{x}$ & $\underline{Y}$ & $\underline{Z}$ \\
\hline \multirow[t]{2}{*}{ \# 1} & $34.29 \mathrm{~cm}$ & $168.93 \mathrm{~cm}$ & $0 \mathrm{Cl}$ \\
\hline & $1 \mathrm{ft} 1.5 \mathrm{in}$ & $5 \mathrm{ft} 6.5$ in & $0.0 \mathrm{i}$ \\
\hline \multirow[t]{2}{*}{ \# 2} & $61.75 \mathrm{~cm}$ & $168.93 \mathrm{~cm}$ & $0 \mathrm{c}$ \\
\hline & $2 \mathrm{ft} 0.3$ in & $5 \mathrm{ft} 6.5 \mathrm{in}$ & 0.0 \\
\hline \multirow[t]{2}{*}{ \# 3} & $131.75 \mathrm{~cm}$ & $168.93 \mathrm{~cm}$ & $0 c$ \\
\hline & $4 \mathrm{ft} 3.9$ in & $5 \mathrm{ft} 6.5 \mathrm{in}$ & 0.0 \\
\hline \multirow[t]{2}{*}{ \# 4} & $41.75 \mathrm{~cm}$ & $168.93 \mathrm{~cm}$ & \\
\hline & $1 \mathrm{ft} 4.4 \mathrm{in}$ & $5 \mathrm{ft} 6.5$ in & 0.0 \\
\hline \multirow[t]{2}{*}{ \# 5} & $51.75 \mathrm{~cm}$ & $168.93 \mathrm{~cm}$ & $0 \mathrm{c}$ \\
\hline & $1 \mathrm{ft} 8.4$ in & $5 \mathrm{ft} 6.5$ in & 0.0 \\
\hline \# 6 & $96.75 \mathrm{~cm}$ & $168.93 \mathrm{~cm}$ & $\begin{array}{lll}0 & 0 \\
0 & 0\end{array}$ \\
\hline
\end{tabular}

\section{Grouping Method : Standard Indices}

Number of Groups : 25

Lower Energy Cutoff : 0.015

Shield Name

Source

Transition

Air Gap

Wall Clad

Top Clad

Shields

Dimension

$9.86 \mathrm{e}+05 \mathrm{~cm}^{3}$

Material

Concrete

Density

1.8

Air

0.00122

$1.27 \mathrm{~cm}$

Air

0.00122

$1.27 \mathrm{~cm}$

Iron

7.95

Iron

7.95

Library : ICRP-38

\begin{tabular}{|c|c|}
\hline Nuclide & curies \\
\hline$A m-241$ & $9.2199 \mathrm{e}-001$ \\
\hline Am-243 & $5.0784 e-004$ \\
\hline $\mathrm{Ba}-137 \mathrm{~m}$ & $6.0842 e+002$ \\
\hline Ce-144 & $1.4002 \mathrm{e}-011$ \\
\hline $\mathrm{Cm}-242$ & $1.1734 \mathrm{e}-003$ \\
\hline $\mathrm{Cm}-244$ & $3.4217 e-004$ \\
\hline Co-60 & $2.1398 \mathrm{e}-002$ \\
\hline Cs-134 & $1.6073 e-003$ \\
\hline Cs-135 & $1.2721 \mathrm{e}-002$ \\
\hline Cs-137 & $6.4392 e+002$ \\
\hline Eu-152 & $2.4159 e-002$ \\
\hline Eu-154 & $2.0116 \mathrm{e}+000$ \\
\hline Eu-155 & $8.6875 e-002$ \\
\hline I-129 & $5.5221 e-005$ \\
\hline $\mathrm{Ni}-63$ & $4.1909 e+001$ \\
\hline $\mathrm{Np}-237$ & $1.0946 \mathrm{e}-002$ \\
\hline Pa-233 & $1.0946 \mathrm{e}-002$ \\
\hline Pm-147 & $2.2483 e-002$ \\
\hline Pr-144 & $1.4002 \mathrm{e}-011$ \\
\hline
\end{tabular}

becquerels

$3.4114 \mathrm{e}+010$

$1.8790 \mathrm{e}+007$

$2.2511 \mathrm{e}+013$

5.1809e-001

$4.3418 e+007$

$1.2660 \mathrm{e}+007$

$7.9173 e+008$

$5.9471 \mathrm{e}+007$

$4.7066 \mathrm{e}+008$

$2.3825 e+013$

$8.9389 e+008$

$7.4430 \mathrm{e}+010$

$3.2144 \mathrm{e}+009$

$2.0432 \mathrm{e}+006$

$1.5506 \mathrm{e}+012$

$4.0499 e+008$

$4.0499 e+008$

$8.3187 e+008$

5.1809e-001 $\mu \mathrm{Ci} / \mathrm{cm}^{3}$

9.3500e-001

5.1500e-004

$6.1700 \mathrm{e}+002$

$1.4200 \mathrm{e}-011$

$1.1900 \mathrm{e}-003$

3.4700e-004

2.1700e-002

$1.6300 \mathrm{e}-003$

1.2900e-002

$6.5300 e+002$

2.4500e-002

$2.0400 \mathrm{e}+000$

8.8100e-002

5.6000e-005

$4.2500 e+001$

$1.1100 \mathrm{e}-002$

$1.1100 \mathrm{e}-002$

2.2800e-002

$1.4200 \mathrm{e}-011$
$\mathrm{Bq} / \mathrm{cm}^{3}$

$3.4595 e+004$

$1.9055 e+001$

$2.2829 \mathrm{e}+007$

5.2540e-007

$4.4030 e+001$

$1.2839 e+001$

$8.0290 e+002$

$6.0310 \mathrm{e}+001$

$4.7730 \mathrm{e}+002$

$2.4161 e+007$

$9.0650 \mathrm{e}+002$

$7.5480 e+004$

$3.2597 e+003$

$2.0720 \mathrm{e}+000$

$1.5725 e+006$

$4.1070 \mathrm{e}+002$

$4.1070 \mathrm{e}+002$

$8.4360 e+002$

$5.2540 \mathrm{e}-007$

E-51 
Page : 2

DOS File : CSSF 4 Calcine in Grout.ms6

Run Date: April 5, 2005

Run Time: 3:13:55 PM

Duration : 00:01:02

\begin{tabular}{|c|c|c|c|c|}
\hline Nuclide & curies & becquerels & $\mu \mathrm{Ci} / \mathrm{cm}^{3}$ & $\underline{\mathrm{Bq} / \mathrm{cm}^{3}}$ \\
\hline Pu-238 & $1.00 \overline{8} \mathrm{e}+001$ & $3 . \overline{7215 e+011}$ & $1.0200 \mathrm{e}+001$ & $3.7740 \mathrm{e}+005$ \\
\hline Pu-239 & $2.4061 \mathrm{e}-001$ & $8.9024 \mathrm{e}+009$ & $2.4400 \mathrm{e}-001$ & $9.0280 e+003$ \\
\hline Pu-240 & $1.3509 \mathrm{e}-001$ & $4.9985 e+009$ & $1.3700 \mathrm{e}-001$ & $5.0690 e+003$ \\
\hline Pu-241 & $3.7570 e+000$ & $1.3901 \mathrm{e}+011$ & $3.8100 e+000$ & $1.4097 e+005$ \\
\hline Pu-242 & $3.4710 \mathrm{e}-004$ & $1.2843 e+007$ & $3.5200 \mathrm{e}-004$ & $1.3024 \mathrm{e}+001$ \\
\hline Ru-106 & $2.5244 \mathrm{e}-009$ & $9.3402 \mathrm{e}+001$ & $2.5600 \mathrm{e}-009$ & $9.4720 \mathrm{e}-005$ \\
\hline Sb-125 & $3.7669 \mathrm{e}-003$ & $1.3937 \mathrm{e}+008$ & $3.8200 \mathrm{e}-003$ & $1.4134 \mathrm{e}+002$ \\
\hline Sb-126 & $9.8017 \mathrm{e}-004$ & $3.6266 \mathrm{e}+007$ & $9.9400 \mathrm{e}-004$ & $3.6778 e+001$ \\
\hline Sb-126m & $7.0308 e-003$ & $2.6014 \mathrm{e}+008$ & $7.1300 \mathrm{e}-003$ & $2.6381 e+002$ \\
\hline Sm-151 & $4.1317 e+000$ & $1.5287 \mathrm{e}+011$ & $4.1900 e+000$ & $1.5503 e+005$ \\
\hline Sr-90 & $5.5517 e+002$ & $2.0541 \mathrm{e}+013$ & $5.6300 e+002$ & $2.0831 e+007$ \\
\hline Tc-99 & $2.5638 \mathrm{e}-001$ & $9.4862 e+009$ & $2.6000 \mathrm{e}-001$ & $9.6200 e+003$ \\
\hline Th-230 & $2.7315 \mathrm{e}-006$ & $1.0106 \mathrm{e}+005$ & $2.7700 \mathrm{e}-006$ & $1.0249 \mathrm{e}-001$ \\
\hline Th-231 & $1.7651 \mathrm{e}-005$ & $6.5309 e+005$ & $1.7900 \mathrm{e}-005$ & $6.6230 \mathrm{e}-001$ \\
\hline$U-232$ & $3.6978 \mathrm{e}-005$ & $1.3682 \mathrm{e}+006$ & $3.7500 \mathrm{e}-005$ & $1.3875 e+000$ \\
\hline$U-233$ & $1.8045 e-006$ & $6.6768 e+004$ & $1.8300 \mathrm{e}-006$ & $6.7710 \mathrm{e}-002$ \\
\hline$U-234$ & $9.0424 \mathrm{e}-003$ & $3.3457 \mathrm{e}+008$ & $9.1700 \mathrm{e}-003$ & $3.3929 e+002$ \\
\hline$U-235$ & $1.7651 \mathrm{e}-005$ & $6.5309 e+005$ & $1.7900 \mathrm{e}-005$ & $6.6230 \mathrm{e}-001$ \\
\hline U-236 & $5.2066 \mathrm{e}-005$ & $1.9264 \mathrm{e}+006$ & $5.2800 \mathrm{e}-005$ & $1.9536 \mathrm{e}+000$ \\
\hline$U-237$ & $9.2199 \mathrm{e}-005$ & $3.4114 \mathrm{e}+006$ & $9.3500 \mathrm{e}-005$ & $3.4595 e+000$ \\
\hline$U-238$ & $1.2622 \mathrm{e}-006$ & $4.6701 e+004$ & $1.2800 \mathrm{e}-006$ & $4.7360 \mathrm{e}-002$ \\
\hline Y-90 & $5.5517 e+002$ & $2.0541 e+013$ & $5.6300 e+002$ & $2.0831 e+007$ \\
\hline
\end{tabular}

$\begin{array}{cc}\frac{\begin{array}{c}\text { Energy } \\ \text { MeV }\end{array}}{} & \begin{array}{c}\text { Activity } \\ \text { photons } / \mathrm{sec}\end{array} \\ 0.015 & \\ 0.02 & 2.671 \mathrm{e}+10 \\ 0.03 & 1.533 \mathrm{e}+10 \\ 0.04 & 1.364 \mathrm{e}+12 \\ 0.05 & 3.416 \mathrm{e}+11 \\ 0.06 & 4.368 \mathrm{e}+09 \\ 0.08 & 1.224 \mathrm{e}+10 \\ 0.1 & 1.087 \mathrm{e}+09 \\ 0.15 & 3.125 \mathrm{e}+10 \\ 0.2 & 9.891 \mathrm{e}+07 \\ 0.3 & 5.200 \mathrm{e}+09 \\ 0.4 & 5.963 \mathrm{e}+08 \\ 0.5 & 9.699 \mathrm{e}+08 \\ 0.6 & 3.551 \mathrm{e}+08 \\ 0.8 & 2.022 \mathrm{e}+13 \\ 1.0 & 2.826 \mathrm{e}+10 \\ 1.5 & 2.433 \mathrm{e}+10 \\ 2.0 & 3.015 \mathrm{e}+10 \\ 3.0 & 1.355 \mathrm{e}+06 \\ & 2.850 \mathrm{e}+01\end{array}$

Radial

Integration Parameters

Y Direction (axial) $\quad 24$

Results - Dose Point \# 1 - $(34.29,168.93,0) \mathrm{cm}$

\begin{tabular}{|c|c|c|c|}
\hline Fluence Rate & Fluence Rate & Exposure Rate & Exposure Rate \\
\hline $\mathrm{MeV} / \mathrm{cm}^{2} / \mathrm{sec}$ & $\mathrm{MeV} / \mathrm{cm}^{2} / \mathrm{sec}$ & $\mathrm{mR} / \mathrm{hr}$ & $\mathrm{mR} / \mathrm{hr}$ \\
\hline No Buildup & With Buildup & No Buildup & With Buildup \\
\hline $3.341 \mathrm{e}-267$ & $8.629 \mathrm{e}-23$ & $2.866 e-268$ & $7.401 \mathrm{e}-24$ \\
\hline $4.985 e-119$ & $7.795 e-23$ & $1.727 e-120$ & $2.700 e-24$ \\
\hline $1.014 \mathrm{e}-34$ & $1.533 e-20$ & $1.005 e-36$ & $1.520 \mathrm{e}-22$ \\
\hline $5.304 \mathrm{e}-14$ & $2.121 \mathrm{e}-13$ & $2.346 \mathrm{e}-16$ & $9.380 e-16$ \\
\hline $5.451 \mathrm{e}-08$ & $3.663 e-07$ & $1.452 \mathrm{e}-10$ & $9.759 \mathrm{e}-10$ \\
\hline $6.359 e-04$ & $5.940 e-03$ & $1.263 e-06$ & $1.180 \mathrm{e}-05$ \\
\hline $6.352 e-02$ & 7.177e-01 & $1.005 e-04$ & $1.136 \mathrm{e}-03$ \\
\hline $2.960 e+01$ & $3.344 \mathrm{e}+02$ & $4.528 \mathrm{e}-02$ & $5.116 \mathrm{e}-01$ \\
\hline $1.222 \mathrm{e}+00$ & $1.152 \mathrm{e}+01$ & $2.012 \mathrm{e}-03$ & $1.898 \mathrm{e}-02$ \\
\hline $1.734 \mathrm{e}+02$ & $1.400 e+03$ & $3.060 e-01$ & $2.471 e+00$ \\
\hline $5.423 e+01$ & $3.489 e+02$ & $1.029 \mathrm{e}-01$ & $6.618 \mathrm{e}-01$ \\
\hline $1.622 e+02$ & $8.845 e+02$ & $3.160 e-01$ & $1.723 e+00$ \\
\hline $9.320 \mathrm{e}+01$ & $4.462 e+02$ & $1.829 \mathrm{e}-01$ & $8.757 e-01$ \\
\hline $7.613 e+06$ & $3.273 e+07$ & $1.486 e+04$ & $6.388 e+04$ \\
\hline $1.872 \mathrm{e}+04$ & $6.838 e+04$ & $3.560 e+01$ & $1.301 \mathrm{e}+02$ \\
\hline $2.490 e+04$ & $8.066 e+04$ & $4.589 e+01$ & $1.487 e+02$ \\
\hline $6.722 e+04$ & $1.781 e+05$ & $1.131 \mathrm{e}+02$ & $2.996 e+02$ \\
\hline $5.123 e+00$ & $1.204 \mathrm{e}+01$ & $7.922 \mathrm{e}-03$ & $1.863 e-02$ \\
\hline $2.167 e-04$ & $4.396 e-04$ & $2.940 \mathrm{e}-07$ & $5.965 e-07$ \\
\hline $7.724 e+06$ & $3.306 e+07$ & $1.506 \mathrm{e}+04$ & $6.447 e+04$ \\
\hline
\end{tabular}

Results - Dose Point \# 2 - $(61.75,168.93,0) \mathrm{cm}$ 
Page : 3

DOS File : CSSF 4 Calcine in Grout.ms6

Run Date: April 5, 2005

Run Time: 3:13:55 PM

Duration : 00:01:02

$\begin{array}{ccc}\frac{\text { Energy }}{\text { MeV }} & & \begin{array}{c}\text { Activity } \\ \text { photons/sec }\end{array} \\ 0.015 & & 2.671 \mathrm{e}+10 \\ 0.02 & & 1.533 \mathrm{e}+10 \\ 0.03 & 1.364 \mathrm{e}+12 \\ 0.04 & 3.416 \mathrm{e}+11 \\ 0.05 & 4.368 \mathrm{e}+09 \\ 0.06 & 1.224 \mathrm{e}+10 \\ 0.08 & 1.087 \mathrm{e}+09 \\ 0.1 & 3.125 \mathrm{e}+10 \\ 0.15 & 9.891 \mathrm{e}+07 \\ 0.2 & 5.200 \mathrm{e}+09 \\ 0.3 & 5.963 \mathrm{e}+08 \\ 0.4 & 9.699 \mathrm{e}+08 \\ 0.5 & 3.551 \mathrm{e}+08 \\ 0.6 & 2.022 \mathrm{e}+13 \\ 0.8 & 2.826 \mathrm{e}+10 \\ 1.0 & 2.433 \mathrm{e}+10 \\ 1.5 & 3.015 \mathrm{e}+10 \\ 2.0 & 1.355 \mathrm{e}+06 \\ 3.0 & 2.850 \mathrm{e}+01\end{array}$

TOTALS:

$2.210 e+13$

\begin{tabular}{|c|c|}
\hline $\begin{array}{c}\text { Energy } \\
\mathrm{MeV}\end{array}$ & $\begin{array}{c}\text { Activity } \\
\text { photons/sec }\end{array}$ \\
\hline 0.015 & $2.671 e+10$ \\
\hline 0.02 & $1.533 e+10$ \\
\hline 0.03 & $1.364 \mathrm{e}+12$ \\
\hline 0.04 & $3.416 \mathrm{e}+11$ \\
\hline 0.05 & $4.368 e+09$ \\
\hline 0.06 & $1.224 \mathrm{e}+10$ \\
\hline 0.08 & $1.087 e+09$ \\
\hline 0.1 & $3.125 \mathrm{e}+10$ \\
\hline 0.15 & $9.891 e+07$ \\
\hline 0.2 & $5.200 e+09$ \\
\hline 0.3 & $5.963 e+08$ \\
\hline 0.4 & $9.699 e+08$ \\
\hline 0.5 & $3.551 e+08$ \\
\hline 0.6 & $2.022 e+13$ \\
\hline 0.8 & $2.826 e+10$ \\
\hline 1.0 & $2.433 e+10$ \\
\hline 1.5 & $3.015 e+10$ \\
\hline 2.0 & $1.355 e+06$ \\
\hline 3.0 & $2.850 e+01$ \\
\hline
\end{tabular}

TOTALS:

$2.210 e+13$

$\begin{array}{cc}\frac{\text { Energy }}{\text { MeV }} & \begin{array}{c}\text { Activity } \\ \text { photons/sec }\end{array} \\ 0.015 & 2.671 \mathrm{e}+10 \\ 0.02 & 1.533 \mathrm{e}+10 \\ 0.03 & 1.364 \mathrm{e}+12\end{array}$

\begin{tabular}{|c|c|c|}
\hline$\frac{\text { Fluence Rate }}{\mathrm{MeV} / \mathrm{cm}^{2} / \mathrm{sec}}$ & $\frac{\text { Fluence Rate }}{\mathrm{MeV} / \mathrm{cm}^{2} / \mathrm{sec}}$ & $\frac{\text { Exposure Rate }}{\mathrm{mR} / \mathrm{hr}}$ \\
\hline No Buildup & With Buildup & No Buildup \\
\hline $6.741 \mathrm{e}-250$ & $3.787 e-23$ & $5.782 \mathrm{e}-251$ \\
\hline $1.391 \mathrm{e}-112$ & $3.421 \mathrm{e}-23$ & $4.818 e-114$ \\
\hline $1.003 e-33$ & $6.729 \mathrm{e}-21$ & $9.936 e-36$ \\
\hline $5.062 e-14$ & $2.006 e-13$ & $2.239 \mathrm{e}-16$ \\
\hline $2.987 \mathrm{e}-08$ & $1.979 \mathrm{e}-07$ & $7.957 \mathrm{e}-11$ \\
\hline $3.031 \mathrm{e}-04$ & $2.813 e-03$ & $6.021 \mathrm{e}-07$ \\
\hline $3.051 \mathrm{e}-02$ & $3.507 e-01$ & $4.827 e-05$ \\
\hline $1.475 e+01$ & $1.702 e+02$ & $2.257 \mathrm{e}-02$ \\
\hline $6.207 e-01$ & $5.868 e+00$ & $1.022 \mathrm{e}-03$ \\
\hline $8.789 e+01$ & $7.051 e+02$ & $1.551 \mathrm{e}-01$ \\
\hline $2.735 e+01$ & $1.739 e+02$ & $5.188 \mathrm{e}-02$ \\
\hline $8.151 e+01$ & $4.391 e+02$ & $1.588 \mathrm{e}-01$ \\
\hline $4.672 e+01$ & $2.209 e+02$ & $9.171 \mathrm{e}-02$ \\
\hline $3.808 e+06$ & $1.617 e+07$ & $7.433 e+03$ \\
\hline $9.330 e+03$ & $3.370 \mathrm{e}+04$ & $1.775 e+01$ \\
\hline $1.238 e+04$ & $3.968 e+04$ & $2.282 e+01$ \\
\hline $3.327 e+04$ & $8.737 e+04$ & $5.598 \mathrm{e}+01$ \\
\hline $2.529 e+00$ & $5.902 e+00$ & $3.911 \mathrm{e}-03$ \\
\hline $1.067 \mathrm{e}-04$ & $2.152 \mathrm{e}-04$ & $1.448 \mathrm{e}-07$ \\
\hline $3.863 e+06$ & $1.634 \mathrm{e}+07$ & $7.530 e+03$ \\
\hline
\end{tabular}

Exposure Rate $\mathrm{mR} / \mathrm{hr}$

With Buildup

$3.248 \mathrm{e}-24$

$1.185 \mathrm{e}-24$

$6.669 \mathrm{e}-23$

$8.874 \mathrm{e}-16$

$5.273 \mathrm{e}-10$

$5.588 \mathrm{e}-06$

$5.549 \mathrm{e}-04$

$2.603 \mathrm{e}-01$

$9.664 \mathrm{e}-03$

$1.244 \mathrm{e}+00$

$3.299 \mathrm{e}-01$

$8.555 \mathrm{e}-01$

$4.336 \mathrm{e}-01$

$3.157 \mathrm{e}+04$

$6.410 \mathrm{e}+01$

$7.315 \mathrm{e}+01$

$1.470 \mathrm{e}+02$

$9.127 \mathrm{e}-03$

2.919e-07

$3.186 \mathrm{e}+04$

\section{Results - Dose Point \# 3 - $(131.75,168.93,0) \mathrm{cm}$}

\begin{tabular}{|c|c|c|}
\hline $\begin{array}{l}\text { Fluence Rate } \\
\mathrm{MeV} / \mathrm{cm}^{2} / \mathrm{sec}\end{array}$ & $\begin{array}{l}\text { Fluence Rate } \\
\mathrm{MeV} / \mathrm{cm}^{2} / \mathrm{sec}\end{array}$ & $\frac{\text { Exposure Rate }}{\mathrm{mR} / \mathrm{hr}}$ \\
\hline No Buildup & $\overline{\text { With Buildup }}$ & No Buildup \\
\hline $1.941 \mathrm{e}-250$ & $1.282 \mathrm{e}-23$ & $1.665 e-251$ \\
\hline $5.374 \mathrm{e}-113$ & $1.158 \mathrm{e}-23$ & $1.862 \mathrm{e}-114$ \\
\hline $4.688 e-34$ & $2.277 e-21$ & $4.646 e-36$ \\
\hline $2.326 \mathrm{e}-14$ & $9.219 e-14$ & $1.029 \mathrm{e}-16$ \\
\hline $1.367 e-08$ & $9.063 e-08$ & $3.641 \mathrm{e}-11$ \\
\hline $1.389 \mathrm{e}-04$ & $1.290 \mathrm{e}-03$ & $2.760 e-07$ \\
\hline $1.394 \mathrm{e}-02$ & $1.603 e-01$ & $2.206 \mathrm{e}-05$ \\
\hline $6.709 e+00$ & $7.732 e+01$ & $1.026 \mathrm{e}-02$ \\
\hline $2.783 e-01$ & $2.605 e+00$ & $4.583 e-04$ \\
\hline $3.902 e+01$ & $3.077 e+02$ & $6.886 \mathrm{e}-02$ \\
\hline $1.201 \mathrm{e}+01$ & $7.460 e+01$ & $2.278 \mathrm{e}-02$ \\
\hline $3.554 \mathrm{e}+01$ & $1.866 e+02$ & $6.925 e-02$ \\
\hline $2.027 e+01$ & $9.327 e+01$ & $3.978 \mathrm{e}-02$ \\
\hline $1.644 \mathrm{e}+06$ & $6.795 e+06$ & $3.210 \mathrm{e}+03$ \\
\hline $3.999 e+03$ & $1.405 e+04$ & $7.606 e+00$ \\
\hline $5.272 e+03$ & $1.644 \mathrm{e}+04$ & $9.718 e+00$ \\
\hline $1.399 \mathrm{e}+04$ & $3.576 e+04$ & $2.355 e+01$ \\
\hline $1.054 \mathrm{e}+00$ & $2.396 e+00$ & $1.630 \mathrm{e}-03$ \\
\hline $4.396 \mathrm{e}-05$ & $8.641 \mathrm{e}-05$ & $5.964 \mathrm{e}-08$ \\
\hline $1.668 \mathrm{e}+06$ & $6.862 e+06$ & $3.251 \mathrm{e}+03$ \\
\hline
\end{tabular}

Exposure Rate $\mathrm{mR} / \mathrm{hr}$

With Buildup

$1.099 \mathrm{e}-24$

$4.010 \mathrm{e}-25$

$2.257 e-23$

4.077e-16

$2.414 \mathrm{e}-10$

$2.563 \mathrm{e}-06$

2.537e-04

1.183e-01

4.290e-03

5.431e-01

$1.415 \mathrm{e}-01$

3.635e-01

$1.831 \mathrm{e}-01$

$1.326 \mathrm{e}+04$

$2.672 \mathrm{e}+01$

$3.030 \mathrm{e}+01$

$6.017 \mathrm{e}+01$

$3.704 \mathrm{e}-03$

$1.172 \mathrm{e}-07$

$1.338 \mathrm{e}+04$

\section{Results - Dose Point \# 4 - $(41.75,168.93,0) \mathrm{cm}$}

\begin{tabular}{ccc}
$\frac{\text { Fluence Rate }}{\text { MeV/cm } / \mathrm{sec}}$ & $\frac{\text { Fluence Rate }}{\text { MeV/cm } 2 / \mathrm{sec}}$ & $\begin{array}{c}\text { Exposure Rate } \\
\text { mR/hr }\end{array}$ \\
\cline { 2 - 3 } No Buildup & $\frac{\text { With Buildup }}{6.433 \mathrm{e}-23}$ & $\frac{\text { No Buildup }}{2.374 \mathrm{e}-252}$ \\
$2.768 \mathrm{e}-251$ & $5.811 \mathrm{e}-23$ & $3.191 \mathrm{e}-114$ \\
$9.212 \mathrm{e}-113$ & $1.143 \mathrm{e}-20$ & $1.754 \mathrm{e}-35$ \\
$1.769 \mathrm{e}-33$ & &
\end{tabular}

Exposure Rate $\mathrm{mR} / \mathrm{hr}$

With Buildup

$5.518 \mathrm{e}-24$

$2.013 e-24$

$1.133 \mathrm{e}-22$ 
Page : 4

DOS File : CSSF 4 Calcine in Grout.ms6

Run Date: April 5, 2005

Run Time: 3:13:55 PM

Duration : 00:01:02

$\begin{array}{ccc}\frac{\text { Energy }}{\mathrm{MeV}} & & \begin{array}{c}\text { Activity } \\ \text { photons } / \mathrm{sec}\end{array} \\ 0.04 & & 3.416 \mathrm{e}+11 \\ 0.05 & & 4.368 \mathrm{e}+09 \\ 0.06 & & 1.224 \mathrm{e}+10 \\ 0.08 & & 1.087 \mathrm{e}+09 \\ 0.1 & 3.125 \mathrm{e}+10 \\ 0.15 & 9.891 \mathrm{e}+07 \\ 0.2 & 5.200 \mathrm{e}+09 \\ 0.3 & 5.963 \mathrm{e}+08 \\ 0.4 & 9.699 \mathrm{e}+08 \\ 0.5 & 3.551 \mathrm{e}+08 \\ 0.6 & 2.022 \mathrm{e}+13 \\ 0.8 & 2.826 \mathrm{e}+10 \\ 1.0 & 2.433 \mathrm{e}+10 \\ 1.5 & 3.015 \mathrm{e}+10 \\ 2.0 & 1.355 \mathrm{e}+06 \\ 3.0 & 2.850 \mathrm{e}+01\end{array}$

\begin{tabular}{|c|c|c|}
\hline Fluence Rate & Fluence Rate & Exposure Rate \\
\hline $\mathrm{MeV} / \mathrm{cm}^{2} / \mathrm{sec}$ & $\mathrm{MeV} / \mathrm{cm}^{2} / \mathrm{sec}$ & $\underline{\mathrm{mR} / \mathrm{hr}}$ \\
\hline No Buildup & $\overline{\text { With Buildup }}$ & No Buildup \\
\hline $8.816 \mathrm{e}-14$ & $3.491 \mathrm{e}-13$ & $3.899 \mathrm{e}-16$ \\
\hline $4.575 e-08$ & $3.017 \mathrm{e}-07$ & $1.219 \mathrm{e}-10$ \\
\hline $4.451 \mathrm{e}-04$ & $4.127 e-03$ & $8.841 \mathrm{e}-07$ \\
\hline $4.573 e-02$ & $5.281 \mathrm{e}-01$ & $7.237 e-05$ \\
\hline $2.240 \mathrm{e}+01$ & $2.593 e+02$ & $3.427 e-02$ \\
\hline $9.525 e-01$ & $9.045 e+00$ & $1.569 \mathrm{e}-03$ \\
\hline $1.354 \mathrm{e}+02$ & $1.092 \mathrm{e}+03$ & $2.390 \mathrm{e}-01$ \\
\hline $4.228 e+01$ & $2.704 e+02$ & $8.021 \mathrm{e}-02$ \\
\hline $1.262 \mathrm{e}+02$ & $6.838 e+02$ & $2.460 \mathrm{e}-01$ \\
\hline $7.245 e+01$ & $3.444 e+02$ & $1.422 \mathrm{e}-01$ \\
\hline $5.911 \mathrm{e}+06$ & $2.524 \mathrm{e}+07$ & $1.154 \mathrm{e}+04$ \\
\hline $1.450 \mathrm{e}+04$ & $5.265 e+04$ & $2.759 e+01$ \\
\hline $1.926 \mathrm{e}+04$ & $6.204 \mathrm{e}+04$ & $3.551 e+01$ \\
\hline $5.188 e+04$ & $1.368 e+05$ & $8.729 e+01$ \\
\hline $3.949 e+00$ & $9.253 e+00$ & $6.106 \mathrm{e}-03$ \\
\hline $1.669 \mathrm{e}-04$ & $3.378 \mathrm{e}-04$ & $2.264 \mathrm{e}-07$ \\
\hline $5.997 e+06$ & $2.549 e+07$ & $1.169 \mathrm{e}+04$ \\
\hline
\end{tabular}

Exposure Rate $\mathrm{mR} / \mathrm{hr}$

With Buildup

$1.544 \mathrm{e}-15$

$8.037 \mathrm{e}-10$

$8.198 \mathrm{e}-06$

$8.357 \mathrm{e}-04$

$3.968 \mathrm{e}-01$

$1.490 \mathrm{e}-02$

$1.927 \mathrm{e}+00$

$5.129 \mathrm{e}-01$

$1.332 \mathrm{e}+00$

$6.760 \mathrm{e}-01$

$4.926 \mathrm{e}+04$

$1.001 \mathrm{e}+02$

$1.144 \mathrm{e}+02$

$2.302 \mathrm{e}+02$

$1.431 \mathrm{e}-02$

$4.583 e-07$

TOTALS: $\quad 2.210 \mathrm{e}+13$

Results - Dose Point \# 5 - $(51.75,168.93,0) \mathrm{cm}$

$\begin{array}{cc}\begin{array}{cc}\text { Energy } \\ \underline{\mathrm{MeV}}\end{array} & \begin{array}{c}\text { Activity } \\ \text { photons/sec }\end{array} \\ 0.015 & \\ 0.02 & 2.671 \mathrm{e}+10 \\ 0.03 & 1.533 \mathrm{e}+10 \\ 0.04 & 1.364 \mathrm{e}+12 \\ 0.05 & 3.416 \mathrm{e}+11 \\ 0.06 & 4.368 \mathrm{e}+09 \\ 0.08 & 1.224 \mathrm{e}+10 \\ 0.1 & 1.087 \mathrm{e}+09 \\ 0.15 & 3.125 \mathrm{e}+10 \\ 0.2 & 9.891 \mathrm{e}+07 \\ 0.3 & 5.200 \mathrm{e}+09 \\ 0.4 & 5.963 \mathrm{e}+08 \\ 0.5 & 9.699 \mathrm{e}+08 \\ 0.6 & 3.551 \mathrm{e}+08 \\ 0.8 & 2.022 \mathrm{e}+13 \\ 1.0 & 2.826 \mathrm{e}+10 \\ 1.5 & 2.433 \mathrm{e}+10 \\ 2.0 & 3.015 \mathrm{e}+10 \\ 3.0 & 1.355 \mathrm{e}+06 \\ & 2.850 \mathrm{e}+01\end{array}$

Fluence Rate

Fluence Rate

$\mathrm{MeV} / \mathrm{cm}^{2} / \mathrm{sec}$

No Buildup

$5.765 \mathrm{e}-250$

$1.808 \mathrm{e}-112$

With Buildup

$4.799 \mathrm{e}-23$

$4.336 \mathrm{e}-23$

$1.330 \mathrm{e}-33$

$8.529 \mathrm{e}-21$

$5.897 \mathrm{e}-14$

$3.546 \mathrm{e}-08$

$2.336 \mathrm{e}-13$

2.353e-07

$3.647 \mathrm{e}-04$

$3.385 \mathrm{e}-03$

$3.672 \mathrm{e}-02$

$4.221 \mathrm{e}-01$

$1.778 \mathrm{e}+01$

$2.052 \mathrm{e}+02$

$7.102 \mathrm{e}+00$

$7.503 e-01$
$1.064 e+02$

$8.545 e+02$

$2.110 \mathrm{e}+02$

$3.313 e+01$

$5.331 e+02$

$9.880 \mathrm{e}+01$

$2.683 e+02$

$5.665 \mathrm{e}+01$

$1.965 \mathrm{e}+07$

$4.098 \mathrm{e}+04$

Exposure Rate $\mathrm{mR} / \mathrm{hr}$

No Buildup

4.944e-251

$6.263 e-114$

$1.318 \mathrm{e}-35$

$2.608 \mathrm{e}-16$

$9.446 \mathrm{e}-11$

7.244e-07

5.811e-05

$2.720 \mathrm{e}-02$

$1.236 \mathrm{e}-03$

$1.877 \mathrm{e}-01$

$6.285 \mathrm{e}-02$

$1.925 \mathrm{e}-01$

$1.112 \mathrm{e}-01$

$1.132 \mathrm{e}+04$

$9.016 \mathrm{e}+03$

$2.154 \mathrm{e}+01$

$1.503 e+04$

$4.043 e+04$

$2.770 \mathrm{e}+01$

$4.827 \mathrm{e}+04$

$6.802 \mathrm{e}+01$

$3.075 \mathrm{e}+00$

$1.064 \mathrm{e}+05$

$4.755 \mathrm{e}-03$

$1.299 \mathrm{e}-04$

2.625e-04

$1.762 \mathrm{e}-07$

$4.686 e+06$

$1.985 \mathrm{e}+07$

$9.133 e+03$

$4.971 e+04$

TOTALS: $\quad 2.210 \mathrm{e}+13$

\section{Results - Dose Point \# 6 - $(96.75,168.93,0) \mathrm{cm}$}

\begin{tabular}{|c|c|}
\hline$\frac{\text { Energy }}{\mathrm{MeV}}$ & $\frac{\text { Activity }}{\text { photons/sec }}$ \\
\hline 0.015 & $2.671 e+10$ \\
\hline 0.02 & $1.533 e+10$ \\
\hline 0.03 & $1.364 \mathrm{e}+12$ \\
\hline 0.04 & $3.416 e+1$ \\
\hline 0.05 & $4.368 e+0$ \\
\hline 0.06 & $1.224 \mathrm{e}+1$ \\
\hline
\end{tabular}

$\frac{\text { Fluence Rate }}{\text { MeV/cm } 2 / \mathrm{sec}}$
$\frac{\text { No Buildup }}{3.005 \mathrm{e}-250}$
$7.201 \mathrm{e}-113$
$6.494 \mathrm{e}-34$
$3.201 \mathrm{e}-14$
$1.879 \mathrm{e}-08$
$1.910 \mathrm{e}-04$

\begin{tabular}{c}
$\frac{\text { Fluence Rate }}{\text { MeV/cm }{ }^{2} / \mathrm{sec}}$ \\
\hline With Buildup \\
\hline $2.033 \mathrm{e}-23$ \\
$1.837 \mathrm{e}-23$ \\
$3.614 \mathrm{e}-21$ \\
$1.269 \mathrm{e}-13$ \\
$1.246 \mathrm{e}-07$ \\
$1.773 \mathrm{e}-03$
\end{tabular}

Exposure Rate $\mathrm{mR} / \mathrm{hr}$

No Buildup

$2.578 \mathrm{e}-251$

$2.494 \mathrm{e}-114$

$6.436 \mathrm{e}-36$

$1.416 \mathrm{e}-16$

$5.006 \mathrm{e}-11$

$3.794 \mathrm{e}-07$

Exposure Rate $\mathrm{mR} / \mathrm{hr}$

With Buildup

4.117e-24

$1.502 \mathrm{e}-24$

$8.453 e-23$

$1.033 \mathrm{e}-15$

$6.269 \mathrm{e}-10$

$6.724 \mathrm{e}-06$

$6.679 \mathrm{e}-04$

3.140e-01

$1.170 \mathrm{e}-02$

$1.508 \mathrm{e}+00$

$4.003 \mathrm{e}-01$

$1.039 \mathrm{e}+00$

5.267e-01

$3.836 \mathrm{e}+04$

$7.794 \mathrm{e}+01$

$8.898 \mathrm{e}+01$

$1.790 \mathrm{e}+02$

$1.112 \mathrm{e}-02$

3.561e-07

$3.871 e+04$

Exposure Rate $\mathrm{mR} / \mathrm{hr}$

With Buildup

$1.744 \mathrm{e}-24$

$6.363 \mathrm{e}-25$

3.581e-23

5.611e-16

$3.319 \mathrm{e}-10$

3.522e-06 
Page : 5

DOS File : CSSF 4 Calcine in Grout.ms6

Run Date: April 5, 2005

Run Time: 3:13:55 PM

Duration : 00:01:02

$\begin{array}{ccc}\frac{\text { Energy }}{\text { MeV }} & & \begin{array}{c}\text { Activity } \\ \text { photons } / \mathrm{sec}\end{array} \\ 0.08 & & 1.087 \mathrm{e}+09 \\ 0.1 & & 3.125 \mathrm{e}+10 \\ 0.15 & & 9.891 \mathrm{e}+07 \\ 0.2 & 5.200 \mathrm{e}+09 \\ 0.3 & & 5.963 \mathrm{e}+08 \\ 0.4 & 9.699 \mathrm{e}+08 \\ 0.5 & 3.551 \mathrm{e}+08 \\ 0.6 & 2.022 \mathrm{e}+13 \\ 0.8 & 2.826 \mathrm{e}+10 \\ 1.0 & 2.433 \mathrm{e}+10 \\ 1.5 & 3.015 \mathrm{e}+10 \\ 2.0 & 1.355 \mathrm{e}+06 \\ 3.0 & 2.850 \mathrm{e}+01\end{array}$

\begin{tabular}{cc}
$\frac{\text { Fluence Rate }}{\text { MeV/cm } 2 / \mathrm{sec}}$ & $\frac{\text { Fluence Rate }}{\text { MeV/cm } 2 / \mathrm{sec}}$ \\
\hline$\frac{\text { No Buildup }}{1.917 \mathrm{e}-02}$ & $\frac{\text { With Buildup }}{2.204 \mathrm{e}-01}$ \\
$9.249 \mathrm{e}+00$ & $1.067 \mathrm{e}+02$ \\
$3.876 \mathrm{e}-01$ & $3.657 \mathrm{e}+00$ \\
$5.472 \mathrm{e}+01$ & $4.369 \mathrm{e}+02$ \\
$1.697 \mathrm{e}+01$ & $1.071 \mathrm{e}+02$ \\
$5.046 \mathrm{e}+01$ & $2.692 \mathrm{e}+02$ \\
$2.887 \mathrm{e}+01$ & $1.351 \mathrm{e}+02$ \\
$2.349 \mathrm{e}+06$ & $9.868 \mathrm{e}+06$ \\
$5.738 \mathrm{e}+03$ & $2.049 \mathrm{e}+04$ \\
$7.593 \mathrm{e}+03$ & $2.405 \mathrm{e}+04$ \\
$2.029 \mathrm{e}+04$ & $5.263 \mathrm{e}+04$ \\
$1.536 \mathrm{e}+00$ & $3.540 \mathrm{e}+00$ \\
$6.445 \mathrm{e}-05$ & $1.283 \mathrm{e}-04$ \\
$2.383 \mathrm{e}+06$ & $9.966 \mathrm{e}+06$
\end{tabular}

$\frac{\text { Exposure Rate }}{\mathrm{mR} / \mathrm{hr}}$

No Buildup

3.034e-05

$1.415 \mathrm{e}-02$

$6.382 \mathrm{e}-04$

$9.658 \mathrm{e}-02$

3.219e-02

$9.831 \mathrm{e}-02$

$5.666 \mathrm{e}-02$

$4.585 \mathrm{e}+03$

$1.091 \mathrm{e}+01$

$1.400 \mathrm{e}+01$

$3.414 \mathrm{e}+01$

$2.376 \mathrm{e}-03$

$8.744 \mathrm{e}-08$

$4.644 \mathrm{e}+03$
Exposure Rate $\mathrm{mR} / \mathrm{hr}$

With Buildup

3.489e-04

$1.632 \mathrm{e}-01$

$6.022 \mathrm{e}-03$

7.712e-01

2.031e-01

5.246e-01

2.651e-01

$1.926 \mathrm{e}+04$

$3.896 \mathrm{e}+01$

$4.433 e+01$

$8.855 \mathrm{e}+01$

$5.475 \mathrm{e}-03$

$1.741 \mathrm{e}-07$

$1.943 \mathrm{e}+04$ 
Page $: 1$

DOS File : CSSF 5 Calcine in Grout.ms6

Run Date: April 5, 2005

Run Time: 3:15:31 PM

Duration : 00:01:02
File Ref:

Date:

By:

Checked:

Case Title: CSSF 5 Calcine Grout

Description: CSSF 5 Calcine Grouted at 21.5 wt\% in Storage Canister Geometry: 7 - Cylinder Volume - Side Shields

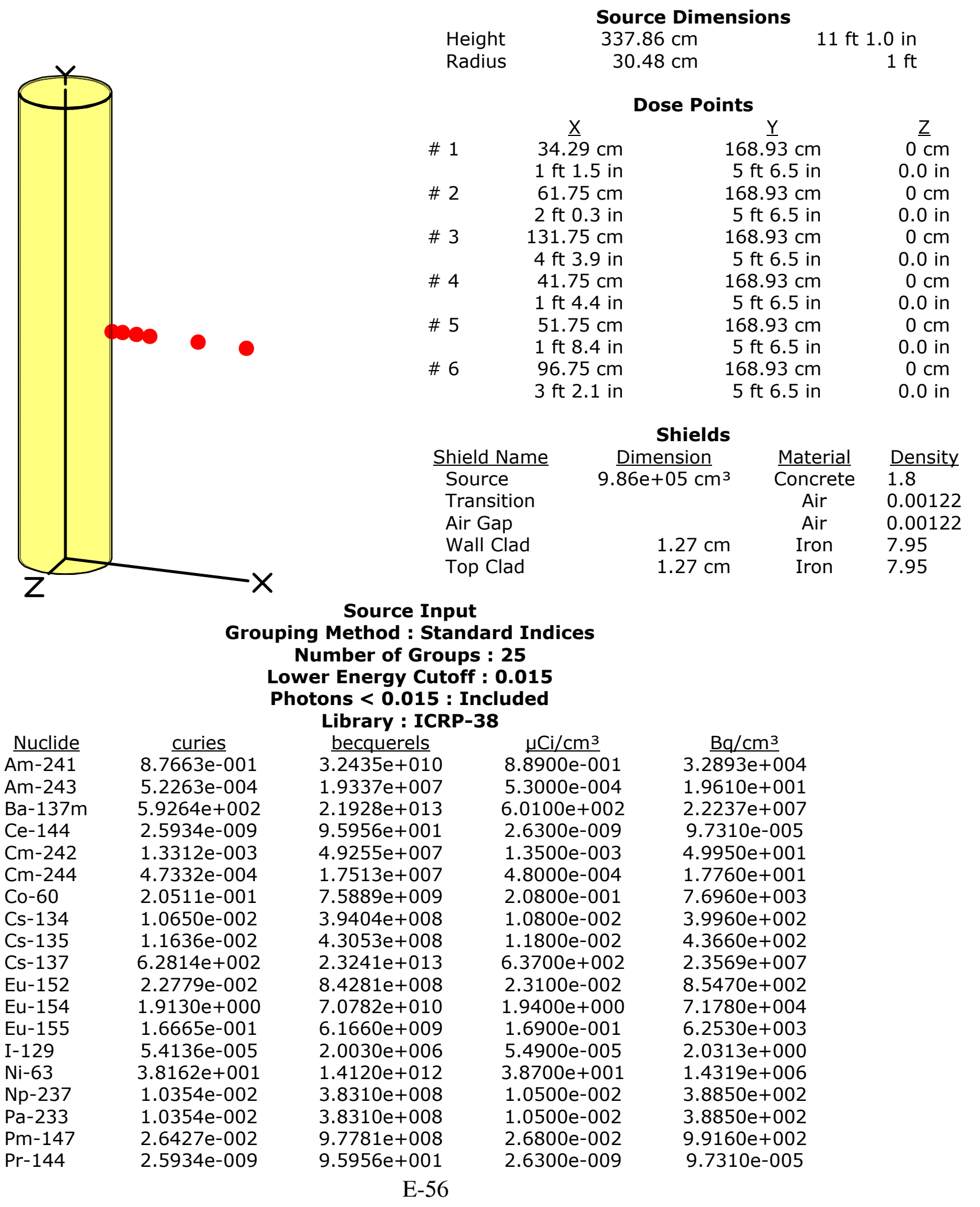


Page : 2

DOS File : CSSF 5 Calcine in Grout.ms6

Run Date: April 5, 2005

Run Time: 3:15:31 PM

Duration : 00:01:02

\begin{tabular}{|c|c|c|c|c|}
\hline Nuclide & curies & becquerels & $\mu \mathrm{Ci} / \mathrm{cm}^{3}$ & $\mathrm{~Bq} / \mathrm{cm}^{3}$ \\
\hline Pu-238 & $7.7 \overline{408 e+000}$ & $2 . \overline{8641 e+011}$ & $7.8500 \mathrm{e}+000$ & $2.9045 e+005$ \\
\hline Pu-239 & $2.5540 \mathrm{e}-001$ & $9.4497 e+009$ & $2.5900 \mathrm{e}-001$ & $9.5830 e+003$ \\
\hline Pu-240 & $1.2030 \mathrm{e}-001$ & $4.4512 \mathrm{e}+009$ & $1.2200 \mathrm{e}-001$ & $4.5140 \mathrm{e}+003$ \\
\hline Pu-241 & $3.4415 e+000$ & $1.2733 \mathrm{e}+011$ & $3.4900 \mathrm{e}+000$ & $1.2913 \mathrm{e}+005$ \\
\hline Pu-242 & $2.9484 \mathrm{e}-004$ & $1.0909 e+007$ & $2.9900 e-004$ & $1.1063 e+001$ \\
\hline Ru-106 & $8.7170 \mathrm{e}-008$ & $3.2253 e+003$ & $8.8400 \mathrm{e}-008$ & $3.2708 \mathrm{e}-003$ \\
\hline Sb-125 & $1.0354 \mathrm{e}-002$ & $3.8310 \mathrm{e}+008$ & $1.0500 \mathrm{e}-002$ & $3.8850 e+002$ \\
\hline Sb-126 & $9.6045 e-004$ & $3.5537 e+007$ & $9.7400 \mathrm{e}-004$ & $3.6038 \mathrm{e}+001$ \\
\hline Sb-126m & $6.8435 \mathrm{e}-003$ & $2.5321 \mathrm{e}+008$ & $6.9400 \mathrm{e}-003$ & $2.5678 \mathrm{e}+002$ \\
\hline Sm-151 & $5.0882 e+000$ & $1.8826 \mathrm{e}+011$ & $5.1600 \mathrm{e}+000$ & $1.9092 \mathrm{e}+005$ \\
\hline Sr-90 & $5.7390 e+002$ & $2.1234 \mathrm{e}+013$ & $5.8200 e+002$ & $2.1534 \mathrm{e}+007$ \\
\hline Tc-99 & $2.5244 \mathrm{e}-001$ & $9.3402 \mathrm{e}+009$ & $2.5600 \mathrm{e}-001$ & $9.4720 e+003$ \\
\hline Th-230 & $1.4890 \mathrm{e}-005$ & $5.5093 e+005$ & $1.5100 \mathrm{e}-005$ & $5.5870 \mathrm{e}-001$ \\
\hline Th-231 & $2.6723 e-005$ & $9.8875 e+005$ & $2.7100 e-005$ & $1.0027 e+000$ \\
\hline$U-232$ & $2.3173 e-005$ & $8.5741 \mathrm{e}+005$ & $2.3500 e-005$ & $8.6950 e-001$ \\
\hline$U-233$ & $1.5284 \mathrm{e}-006$ & $5.6552 e+004$ & $1.5500 \mathrm{e}-006$ & $5.7350 \mathrm{e}-002$ \\
\hline$U-234$ & $2.5737 e-003$ & $9.5227 e+007$ & $2.6100 e-003$ & $9.6570 e+001$ \\
\hline U-235 & $3.3823 e-005$ & $1.2514 \mathrm{e}+006$ & $3.4300 e-005$ & $1.2691 \mathrm{e}+000$ \\
\hline$U-236$ & $1.3312 \mathrm{e}-004$ & $4.9255 e+006$ & $1.3500 \mathrm{e}-004$ & $4.9950 \mathrm{e}+000$ \\
\hline$U-237$ & $8.3916 \mathrm{e}-005$ & $3.1049 e+006$ & $8.5100 \mathrm{e}-005$ & $3.1487 e+000$ \\
\hline$U-238$ & $1.1340 \mathrm{e}-005$ & $4.1958 \mathrm{e}+005$ & $1.1500 \mathrm{e}-005$ & $4.2550 \mathrm{e}-001$ \\
\hline Y-90 & $5.7390 e+002$ & $2.1234 \mathrm{e}+013$ & $5.8200 e+002$ & $2.1534 \mathrm{e}+007$ \\
\hline
\end{tabular}

$\begin{array}{cc}\begin{array}{cc}\text { Energy } \\ \text { MeV }\end{array} & \begin{array}{c}\text { Activity } \\ \text { photons/sec }\end{array} \\ 0.015 & \\ 0.02 & \\ 0.03 & 1.371 \mathrm{e}+10 \\ 0.04 & 1.329 \mathrm{e}+10 \\ 0.05 & 3.329 \mathrm{e}+11 \\ 0.06 & 4.334 \mathrm{e}+09 \\ 0.08 & 1.167 \mathrm{e}+10 \\ 0.1 & 2.000 \mathrm{e}+09 \\ 0.15 & 3.035 \mathrm{e}+10 \\ 0.2 & 9.582 \mathrm{e}+07 \\ 0.3 & 4.963 \mathrm{e}+09 \\ 0.4 & 5.659 \mathrm{e}+08 \\ 0.5 & 1.007 \mathrm{e}+09 \\ 0.6 & 3.684 \mathrm{e}+08 \\ 0.8 & 1.969 \mathrm{e}+13 \\ 1.0 & 2.719 \mathrm{e}+10 \\ 1.5 & 2.998 \mathrm{e}+10 \\ 2.0 & 3.551 \mathrm{e}+10 \\ 3.0 & 1.363 \mathrm{e}+06 \\ & 2.732 \mathrm{e}+02\end{array}$

Radial

Integration Parameters

Circumferential

Y Direction (axial) $\quad 24$

Results - Dose Point \# 1 - $(34.29,168.93,0) \mathrm{cm}$

\begin{tabular}{|c|c|c|c|}
\hline Fluence Rate & Fluence Rate & Exposure Rate & Exposure Rate \\
\hline $\mathrm{MeV} / \mathrm{cm}^{2} / \mathrm{sec}$ & $\mathrm{MeV} / \mathrm{cm}^{2} / \mathrm{sec}$ & $\mathrm{mR} / \mathrm{hr}$ & $\mathrm{mR} / \mathrm{hr}$ \\
\hline No Buildup & With Buildup & No Buildup & With Buildup \\
\hline $2.715 e-267$ & $7.012 \mathrm{e}-23$ & $2.329 e-268$ & $6.014 \mathrm{e}-24$ \\
\hline $4.473 e-119$ & $6.994 \mathrm{e}-23$ & $1.549 \mathrm{e}-120$ & $2.423 e-24$ \\
\hline $9.878 e-35$ & $1.494 \mathrm{e}-20$ & $9.790 e-37$ & $1.480 \mathrm{e}-22$ \\
\hline $5.169 e-14$ & $2.067 e-13$ & $2.286 \mathrm{e}-16$ & $9.140 \mathrm{e}-16$ \\
\hline $5.408 e-08$ & $3.634 \mathrm{e}-07$ & $1.441 \mathrm{e}-10$ & $9.682 \mathrm{e}-10$ \\
\hline $6.065 e-04$ & $5.666 e-03$ & $1.205 e-06$ & $1.125 \mathrm{e}-05$ \\
\hline $1.169 \mathrm{e}-01$ & $1.320 \mathrm{e}+00$ & $1.849 e-04$ & $2.089 e-03$ \\
\hline $2.875 e+01$ & $3.248 e+02$ & $4.398 e-02$ & $4.969 \mathrm{e}-01$ \\
\hline $1.184 \mathrm{e}+00$ & $1.116 \mathrm{e}+01$ & $1.949 \mathrm{e}-03$ & $1.838 \mathrm{e}-02$ \\
\hline $1.655 e+02$ & $1.336 \mathrm{e}+03$ & $2.921 \mathrm{e}-01$ & $2.359 e+00$ \\
\hline $5.146 e+01$ & $3.311 \mathrm{e}+02$ & $9.762 \mathrm{e}-02$ & $6.281 \mathrm{e}-01$ \\
\hline $1.683 e+02$ & $9.179 e+02$ & $3.279 e-01$ & $1.788 \mathrm{e}+00$ \\
\hline $9.670 e+01$ & $4.629 e+02$ & $1.898 \mathrm{e}-01$ & $9.086 \mathrm{e}-01$ \\
\hline $7.416 e+06$ & $3.188 e+07$ & $1.447 e+04$ & $6.223 e+04$ \\
\hline $1.801 \mathrm{e}+04$ & $6.579 e+04$ & $3.425 e+01$ & $1.251 \mathrm{e}+02$ \\
\hline $3.067 e+04$ & $9.936 e+04$ & $5.654 e+01$ & $1.832 \mathrm{e}+02$ \\
\hline $7.919 e+04$ & $2.097 e+05$ & $1.332 e+02$ & $3.529 e+02$ \\
\hline $5.153 e+00$ & $1.212 \mathrm{e}+01$ & $7.969 \mathrm{e}-03$ & $1.874 \mathrm{e}-02$ \\
\hline $2.077 e-03$ & $4.214 \mathrm{e}-03$ & $2.818 \mathrm{e}-06$ & $5.717 \mathrm{e}-06$ \\
\hline $7.544 e+06$ & $3.226 e+07$ & $1.470 \mathrm{e}+04$ & $6.289 e+04$ \\
\hline
\end{tabular}

Results - Dose Point \# 2 - $(61.75,168.93,0) \mathrm{cm}$ 
Page : 3

DOS File : CSSF 5 Calcine in Grout.ms6

Run Date: April 5, 2005

Run Time: 3:15:31 PM

Duration : 00:01:02

$\begin{array}{cc}\begin{array}{cc}\text { Energy } \\ \underline{\mathrm{MeV}}\end{array} & \begin{array}{c}\text { Activity } \\ \text { photons/sec }\end{array} \\ 0.015 & 2.171 \mathrm{e}+10 \\ 0.02 & 1.376 \mathrm{e}+10 \\ 0.03 & 1.329 \mathrm{e}+12 \\ 0.04 & 3.329 \mathrm{e}+11 \\ 0.05 & 4.334 \mathrm{e}+09 \\ 0.06 & 1.167 \mathrm{e}+10 \\ 0.08 & 2.000 \mathrm{e}+09 \\ 0.1 & 3.035 \mathrm{e}+10 \\ 0.15 & 9.582 \mathrm{e}+07 \\ 0.2 & 4.963 \mathrm{e}+09 \\ 0.3 & 5.659 \mathrm{e}+08 \\ 0.4 & 1.007 \mathrm{e}+09 \\ 0.5 & 3.684 \mathrm{e}+08 \\ 0.6 & 1.969 \mathrm{e}+13 \\ 0.8 & 2.719 \mathrm{e}+10 \\ 1.0 & 2.998 \mathrm{e}+10 \\ 1.5 & 3.551 \mathrm{e}+10 \\ 2.0 & 1.363 \mathrm{e}+06 \\ 3.0 & 2.732 \mathrm{e}+02\end{array}$

TOTALS:

\begin{tabular}{|c|c|}
\hline $\begin{array}{c}\text { Energy } \\
\mathrm{MeV}\end{array}$ & $\begin{array}{c}\text { Activity } \\
\text { photons/sec }\end{array}$ \\
\hline 0.015 & $2.171 e+10$ \\
\hline 0.02 & $1.376 e+10$ \\
\hline 0.03 & $1.329 e+12$ \\
\hline 0.04 & $3.329 e+11$ \\
\hline 0.05 & $4.334 \mathrm{e}+09$ \\
\hline 0.06 & $1.167 e+10$ \\
\hline 0.08 & $2.000 e+09$ \\
\hline 0.1 & $3.035 e+10$ \\
\hline 0.15 & $9.582 \mathrm{e}+07$ \\
\hline 0.2 & $4.963 e+09$ \\
\hline 0.3 & $5.659 e+08$ \\
\hline 0.4 & $1.007 e+09$ \\
\hline 0.5 & $3.684 e+08$ \\
\hline 0.6 & $1.969 e+13$ \\
\hline 0.8 & $2.719 e+10$ \\
\hline 1.0 & $2.998 e+10$ \\
\hline 1.5 & $3.551 e+10$ \\
\hline 2.0 & $1.363 e+06$ \\
\hline 3.0 & $2.732 e+02$ \\
\hline
\end{tabular}

TOTALS:

$2.154 \mathrm{e}+13$

$\begin{array}{cc}\frac{\text { Energy }}{\text { MeV }} & \begin{array}{c}\text { Activity } \\ \text { photons/sec }\end{array} \\ 0.015 & 2.171 \mathrm{e}+10 \\ 0.02 & 1.376 \mathrm{e}+10 \\ 0.03 & 1.329 \mathrm{e}+12\end{array}$

\begin{tabular}{|c|c|c|}
\hline$\frac{\text { Fluence Rate }}{\mathrm{MeV} / \mathrm{cm}^{2} / \mathrm{sec}}$ & $\frac{\text { Fluence Rate }}{\mathrm{MeV} / \mathrm{cm}^{2} / \mathrm{sec}}$ & $\frac{\text { Exposure Rate }}{\mathrm{mR} / \mathrm{hr}}$ \\
\hline No Buildup & With Buildup & No Buildup \\
\hline $5.478 \mathrm{e}-250$ & $3.077 e-23$ & $4.699 \mathrm{e}-251$ \\
\hline $1.248 \mathrm{e}-112$ & $3.069 \mathrm{e}-23$ & $4.323 e-114$ \\
\hline $9.766 e-34$ & $6.555 e-21$ & $9.679 e-36$ \\
\hline $4.933 e-14$ & $1.955 e-13$ & $2.182 \mathrm{e}-16$ \\
\hline $2.963 e-08$ & $1.964 \mathrm{e}-07$ & $7.894 \mathrm{e}-11$ \\
\hline $2.891 \mathrm{e}-04$ & $2.683 e-03$ & $5.743 e-07$ \\
\hline $5.612 \mathrm{e}-02$ & $6.451 \mathrm{e}-01$ & $8.881 \mathrm{e}-05$ \\
\hline $1.433 e+01$ & $1.653 e+02$ & $2.192 \mathrm{e}-02$ \\
\hline $6.013 e-01$ & $5.685 e+00$ & $9.902 \mathrm{e}-04$ \\
\hline $8.389 e+01$ & $6.730 e+02$ & $1.481 \mathrm{e}-01$ \\
\hline $2.596 \mathrm{e}+01$ & $1.651 \mathrm{e}+02$ & $4.924 \mathrm{e}-02$ \\
\hline $8.459 e+01$ & $4.557 e+02$ & $1.648 \mathrm{e}-01$ \\
\hline $4.848 e+01$ & $2.292 e+02$ & $9.515 e-02$ \\
\hline $3.710 e+06$ & $1.576 \mathrm{e}+07$ & $7.241 e+03$ \\
\hline $8.978 e+03$ & $3.243 e+04$ & $1.708 e+01$ \\
\hline $1.525 e+04$ & $4.889 e+04$ & $2.811 e+01$ \\
\hline $3.919 e+04$ & $1.029 e+05$ & $6.594 \mathrm{e}+01$ \\
\hline $2.544 \mathrm{e}+00$ & $5.938 e+00$ & $3.934 \mathrm{e}-03$ \\
\hline $1.023 \mathrm{e}-03$ & $2.063 e-03$ & $1.388 \mathrm{e}-06$ \\
\hline $3.773 e+06$ & $1.594 \mathrm{e}+07$ & $7.352 e+03$ \\
\hline
\end{tabular}

Exposure Rate $\mathrm{mR} / \mathrm{hr}$

With Buildup

$2.639 \mathrm{e}-24$

$1.063 \mathrm{e}-24$

$6.497 \mathrm{e}-23$

$8.648 \mathrm{e}-16$

$5.231 \mathrm{e}-10$

$5.330 \mathrm{e}-06$

$1.021 \mathrm{e}-03$

$2.528 \mathrm{e}-01$

$9.362 \mathrm{e}-03$

$1.188 \mathrm{e}+00$

$3.131 \mathrm{e}-01$

$8.878 \mathrm{e}-01$

$4.499 \mathrm{e}-01$

$3.075 e+04$

$6.168 \mathrm{e}+01$

$9.011 \mathrm{e}+01$

$1.732 \mathrm{e}+02$

$9.182 \mathrm{e}-03$

$2.798 \mathrm{e}-06$

$3.108 e+04$

\section{Results - Dose Point \# 3 - $(131.75,168.93,0) \mathrm{cm}$}

\begin{tabular}{|c|c|c|}
\hline $\begin{array}{l}\text { Fluence Rate } \\
\mathrm{MeV} / \mathrm{cm}^{2} / \mathrm{sec}\end{array}$ & $\begin{array}{l}\text { Fluence Rate } \\
\mathrm{MeV} / \mathrm{cm}^{2} / \mathrm{sec}\end{array}$ & $\frac{\text { Exposure Rate }}{\mathrm{mR} / \mathrm{hr}}$ \\
\hline No Buildup & $\overline{\text { With Buildup }}$ & No Buildup \\
\hline $1.577 \mathrm{e}-250$ & $1.041 \mathrm{e}-23$ & $1.353 e-251$ \\
\hline $4.822 \mathrm{e}-113$ & $1.039 e-23$ & $1.670 \mathrm{e}-114$ \\
\hline $4.567 e-34$ & $2.219 \mathrm{e}-21$ & $4.526 \mathrm{e}-36$ \\
\hline $2.267 e-14$ & $8.984 e-14$ & $1.002 \mathrm{e}-16$ \\
\hline $1.356 \mathrm{e}-08$ & $8.991 \mathrm{e}-08$ & $3.612 \mathrm{e}-11$ \\
\hline $1.325 e-04$ & $1.231 \mathrm{e}-03$ & $2.632 \mathrm{e}-07$ \\
\hline $2.564 \mathrm{e}-02$ & $2.950 \mathrm{e}-01$ & $4.058 \mathrm{e}-05$ \\
\hline $6.516 e+00$ & $7.510 e+01$ & $9.968 e-03$ \\
\hline $2.696 \mathrm{e}-01$ & $2.524 \mathrm{e}+00$ & $4.440 e-04$ \\
\hline $3.724 \mathrm{e}+01$ & $2.937 e+02$ & $6.573 e-02$ \\
\hline $1.139 \mathrm{e}+01$ & $7.080 e+01$ & $2.161 \mathrm{e}-02$ \\
\hline $3.688 e+01$ & $1.936 \mathrm{e}+02$ & $7.187 e-02$ \\
\hline $2.103 e+01$ & $9.677 e+01$ & $4.127 e-02$ \\
\hline $1.602 \mathrm{e}+06$ & $6.619 e+06$ & $3.127 e+03$ \\
\hline $3.848 e+03$ & $1.352 \mathrm{e}+04$ & $7.319 e+00$ \\
\hline $6.495 e+03$ & $2.025 e+04$ & $1.197 e+01$ \\
\hline $1.648 e+04$ & $4.213 e+04$ & $2.774 \mathrm{e}+01$ \\
\hline $1.061 \mathrm{e}+00$ & $2.410 e+00$ & $1.640 \mathrm{e}-03$ \\
\hline $4.214 \mathrm{e}-04$ & $8.282 \mathrm{e}-04$ & $5.717 e-07$ \\
\hline $1.629 \mathrm{e}+06$ & $6.696 e+06$ & $3.174 \mathrm{e}+03$ \\
\hline
\end{tabular}

Exposure Rate $\mathrm{mR} / \mathrm{hr}$

With Buildup

$8.933 \mathrm{e}-25$

$3.598 \mathrm{e}-25$

$2.199 \mathrm{e}-23$

$3.973 \mathrm{e}-16$

$2.395 \mathrm{e}-10$

$2.445 \mathrm{e}-06$

$4.668 \mathrm{e}-04$

$1.149 \mathrm{e}-01$

$4.156 \mathrm{e}-03$

$5.184 \mathrm{e}-01$

$1.343 \mathrm{e}-01$

$3.773 \mathrm{e}-01$

$1.900 \mathrm{e}-01$

$1.292 \mathrm{e}+04$

$2.571 \mathrm{e}+01$

$3.733 e+01$

$7.088 \mathrm{e}+01$

$3.727 \mathrm{e}-03$

$1.124 \mathrm{e}-06$

$1.305 e+04$

Results - Dose Point \# 4 - $(41.75,168.93,0) \mathrm{cm}$

$\begin{array}{ccc}\frac{\text { Fluence Rate }}{\text { MeV/cm } / \mathrm{sec}} & \frac{\text { Fluence Rate }}{\text { MeV/cm } 2 / \mathrm{sec}} & \frac{1 \text { Exposure Rate }}{\mathrm{mR} / \mathrm{hr}} \\ \frac{\frac{\text { No Buildup }}{2.249 \mathrm{e}-251}}{\frac{\text { With Buildup }}{5.228 \mathrm{e}-23}} & \frac{\text { No Buildup }}{1.929 \mathrm{e}-252} \\ 8.264 \mathrm{e}-113 & 5.214 \mathrm{e}-23 & 2.863 \mathrm{e}-114 \\ 1.724 \mathrm{e}-33 & 1.114 \mathrm{e}-20 & 1.708 \mathrm{e}-35\end{array}$

Exposure Rate $\mathrm{mR} / \mathrm{hr}$

With Buildup

$4.484 \mathrm{e}-24$

$1.806 \mathrm{e}-24$

$1.104 \mathrm{e}-22$ 
Page : 4

DOS File : CSSF 5 Calcine in Grout.ms6

Run Date: April 5, 2005

Run Time: 3:15:31 PM

Duration : 00:01:02

$\begin{array}{ccc}\frac{\text { Energy }}{\mathrm{MeV}} & & \begin{array}{c}\text { Activity } \\ \text { photons } / \mathrm{sec}\end{array} \\ 0.04 & & 3.329 \mathrm{e}+11 \\ 0.05 & & 4.334 \mathrm{e}+09 \\ 0.06 & & 1.167 \mathrm{e}+10 \\ 0.08 & & 2.000 \mathrm{e}+09 \\ 0.1 & & 3.035 \mathrm{e}+10 \\ 0.15 & 9.582 \mathrm{e}+07 \\ 0.2 & & 4.963 \mathrm{e}+09 \\ 0.3 & 5.659 \mathrm{e}+08 \\ 0.4 & 1.007 \mathrm{e}+09 \\ 0.5 & 3.684 \mathrm{e}+08 \\ 0.6 & 1.969 \mathrm{e}+13 \\ 0.8 & 2.719 \mathrm{e}+10 \\ 1.0 & 2.998 \mathrm{e}+10 \\ 1.5 & 3.551 \mathrm{e}+10 \\ 2.0 & 1.363 \mathrm{e}+06 \\ 3.0 & 2.732 \mathrm{e}+02\end{array}$

\begin{tabular}{|c|c|c|}
\hline$\frac{\text { Fluence Rate }}{\mathrm{MeV} / \mathrm{cm}^{2} / \mathrm{sec}}$ & $\frac{\text { Fluence Rate }}{\mathrm{MeV} / \mathrm{cm}^{2} / \mathrm{sec}}$ & $\frac{\text { Exposure Rate }}{\mathrm{mR} / \mathrm{hr}}$ \\
\hline No Buildup & With Buildup & No Buildup \\
\hline $8.591 \mathrm{e}-14$ & $3.402 \mathrm{e}-13$ & $3.800 \mathrm{e}-16$ \\
\hline $4.539 \mathrm{e}-08$ & $2.993 e-07$ & $1.209 \mathrm{e}-10$ \\
\hline $4.246 e-04$ & $3.937 \mathrm{e}-03$ & $8.433 e-07$ \\
\hline $8.413 e-02$ & $9.715 e-01$ & $1.331 \mathrm{e}-04$ \\
\hline $2.175 e+01$ & $2.519 e+02$ & $3.328 \mathrm{e}-02$ \\
\hline $9.228 \mathrm{e}-01$ & $8.763 e+00$ & $1.520 \mathrm{e}-03$ \\
\hline $1.292 \mathrm{e}+02$ & $1.042 \mathrm{e}+03$ & $2.281 \mathrm{e}-01$ \\
\hline $4.013 e+01$ & $2.566 \mathrm{e}+02$ & $7.612 \mathrm{e}-02$ \\
\hline $1.310 e+02$ & $7.096 e+02$ & $2.553 e-01$ \\
\hline $7.517 e+01$ & $3.573 e+02$ & $1.475 \mathrm{e}-01$ \\
\hline $5.758 e+06$ & $2.458 e+07$ & $1.124 \mathrm{e}+04$ \\
\hline $1.395 e+04$ & $5.066 e+04$ & $2.654 e+01$ \\
\hline $2.373 e+04$ & $7.643 e+04$ & $4.374 \mathrm{e}+01$ \\
\hline $6.112 e+04$ & $1.612 e+05$ & $1.028 e+02$ \\
\hline $3.972 \mathrm{e}+00$ & $9.308 e+00$ & $6.143 e-03$ \\
\hline $1.599 \mathrm{e}-03$ & $3.238 \mathrm{e}-03$ & $2.170 \mathrm{e}-06$ \\
\hline $5.857 e+06$ & $2.487 e+07$ & $1.141 \mathrm{e}+04$ \\
\hline
\end{tabular}

Exposure Rate $\mathrm{mR} / \mathrm{hr}$

With Buildup

$1.505 \mathrm{e}-15$

$7.974 \mathrm{e}-10$

$7.820 \mathrm{e}-06$

$1.537 \mathrm{e}-03$

$3.854 \mathrm{e}-01$

$1.443 \mathrm{e}-02$

$1.839 \mathrm{e}+00$

$4.868 \mathrm{e}-01$

$1.383 e+00$

$7.014 \mathrm{e}-01$

$4.798 \mathrm{e}+04$

$9.636 \mathrm{e}+01$

$1.409 \mathrm{e}+02$

$2.712 \mathrm{e}+02$

$1.439 \mathrm{e}-02$

$4.393 e-06$

TOTALS: $\quad 2.154 \mathrm{e}+13$

\section{Results - Dose Point \# 5 - $(51.75,168.93,0) \mathrm{cm}$}

\begin{tabular}{|c|c|}
\hline $\begin{array}{l}\text { Energy } \\
\underline{\mathrm{MeV}}\end{array}$ & $\begin{array}{c}\text { Activity } \\
\text { photons/sec }\end{array}$ \\
\hline 0.015 & $2.171 \mathrm{e}+10$ \\
\hline 0.02 & $1.376 e+10$ \\
\hline 0.03 & $1.329 \mathrm{e}+12$ \\
\hline 0.04 & $3.329 e+11$ \\
\hline 0.05 & $4.334 e+09$ \\
\hline 0.06 & $1.167 e+10$ \\
\hline 0.08 & $2.000 e+09$ \\
\hline 0.1 & $3.035 e+10$ \\
\hline 0.15 & $9.582 e+07$ \\
\hline 0.2 & $4.963 e+09$ \\
\hline 0.3 & $5.659 e+08$ \\
\hline 0.4 & $1.007 e+09$ \\
\hline 0.5 & $3.684 e+08$ \\
\hline 0.6 & $1.969 e+13$ \\
\hline 0.8 & $2.719 e+10$ \\
\hline 1.0 & $2.998 e+10$ \\
\hline 1.5 & $3.551 e+10$ \\
\hline 2.0 & $1.363 e+06$ \\
\hline 3.0 & $2.732 e+02$ \\
\hline
\end{tabular}

\section{Fluence Rate}

$\mathrm{MeV} / \mathrm{cm}^{2} / \mathrm{sec}$

No Buildup

$4.684 \mathrm{e}-250$

$1.622 \mathrm{e}-112$

$1.295 \mathrm{e}-33$

$5.746 \mathrm{e}-14$

$3.518 \mathrm{e}-08$

$3.479 \mathrm{e}-04$

$6.755 \mathrm{e}-02$

$1.727 e+01$

$7.269 \mathrm{e}-01$

$1.015 \mathrm{e}+02$

$3.144 \mathrm{e}+01$

$1.025 \mathrm{e}+02$

$5.878 \mathrm{e}+01$

$4.499 \mathrm{e}+06$

$1.089 \mathrm{e}+04$

$1.851 \mathrm{e}+04$

$4.762 \mathrm{e}+04$

$3.093 e+00$

$1.245 \mathrm{e}-03$

$4.577 e+06$
Fluence Rate

$\mathrm{MeV} / \mathrm{cm}^{2} / \mathrm{sec}$

With Buildup

$3.900 \mathrm{e}-23$

$3.890 \mathrm{e}-23$

$8.308 \mathrm{e}-21$

2. $277 \mathrm{e}-13$

2.335e-07

$3.229 \mathrm{e}-03$

7.765e-01

$1.993 e+02$

$6.880 \mathrm{e}+00$

$8.156 \mathrm{e}+02$

$2.003 e+02$

$5.532 \mathrm{e}+02$

$2.784 \mathrm{e}+02$

$1.914 \mathrm{e}+07$

$3.943 e+04$

$5.947 \mathrm{e}+04$

$1.253 \mathrm{e}+05$

$7.236 \mathrm{e}+00$

2.516e-03

$1.937 \mathrm{e}+07$
Exposure Rate $\mathrm{mR} / \mathrm{hr}$

No Buildup

4.018e-251

5.619e-114

$1.284 \mathrm{e}-35$

2.541e-16

$9.372 \mathrm{e}-11$

6.910e-07

$1.069 \mathrm{e}-04$

2.642e-02

$1.197 \mathrm{e}-03$

$1.792 \mathrm{e}-01$

5.965e-02

$1.998 \mathrm{e}-01$

$1.154 \mathrm{e}-01$

$8.782 \mathrm{e}+03$

$2.072 \mathrm{e}+01$

$3.413 e+01$

$8.012 \mathrm{e}+01$

$4.784 \mathrm{e}-03$

$1.689 \mathrm{e}-06$

$8.918 \mathrm{e}+03$

$4.850 e+04$

Exposure Rate $\mathrm{mR} / \mathrm{hr}$

With Buildup

$3.345 \mathrm{e}-24$

$1.347 \mathrm{e}-24$

$8.234 \mathrm{e}-23$

$1.007 \mathrm{e}-15$

$6.219 \mathrm{e}-10$

$6.414 \mathrm{e}-06$

$1.229 \mathrm{e}-03$

3.049e-01

$1.133 \mathrm{e}-02$

$1.439 \mathrm{e}+00$

$3.799 \mathrm{e}-01$

$1.078 \mathrm{e}+00$

$5.465 \mathrm{e}-01$

$3.737 \mathrm{e}+04$

$7.500 \mathrm{e}+01$

$1.096 \mathrm{e}+02$

$2.109 \mathrm{e}+02$

$1.119 \mathrm{e}-02$

3.413e-06

$3.777 e+04$

\section{Results - Dose Point \# 6 - $(96.75,168.93,0) \mathrm{cm}$}

$\begin{array}{cc}\frac{\text { Energy }}{\text { MeV }} & \begin{array}{c}\text { Activity } \\ \text { photons/sec }\end{array} \\ 0.015 & \\ 0.02 & 2.171 \mathrm{e}+10 \\ 0.03 & 1.376 \mathrm{e}+10 \\ 0.04 & 1.329 \mathrm{e}+12 \\ 0.05 & 3.329 \mathrm{e}+11 \\ 0.06 & 4.334 \mathrm{e}+09 \\ & 1.167 \mathrm{e}+10\end{array}$

\begin{tabular}{c}
$\frac{\text { Fluence Rate }}{\text { MeV/cm } 2 / s e c}$ \\
\hline With Buildup \\
\hline $1.652 \mathrm{e}-23$ \\
$1.648 \mathrm{e}-23$ \\
$3.520 \mathrm{e}-21$ \\
$1.236 \mathrm{e}-13$ \\
$1.236 \mathrm{e}-07$ \\
$1.691 \mathrm{e}-03$
\end{tabular}

$\frac{\text { Exposure Rate }}{\text { mR/hr }}$
$\frac{\text { No Buildup }}{2.095 \mathrm{e}-251}$
$2.238 \mathrm{e}-114$
$6.270 \mathrm{e}-36$
$1.380 \mathrm{e}-16$
$4.967 \mathrm{e}-11$
$3.619 \mathrm{e}-07$

Exposure Rate $\mathrm{mR} / \mathrm{hr}$

With Buildup

$1.417 \mathrm{e}-24$

$5.709 \mathrm{e}-25$

$3.489 \mathrm{e}-23$

$5.468 \mathrm{e}-16$

$3.292 \mathrm{e}-10$

$3.359 \mathrm{e}-06$ 
Page : 5

DOS File : CSSF 5 Calcine in Grout.ms6

Run Date: April 5, 2005

Run Time: 3:15:31 PM

Duration : 00:01:02

$\begin{array}{cc}\begin{array}{cc}\text { Energy } \\ \text { MeV }\end{array} & \begin{array}{c}\text { Activity } \\ \text { photons } / \mathrm{sec}\end{array} \\ 0.08 & \\ 0.1 & 2.000 \mathrm{e}+09 \\ 0.15 & 3.035 \mathrm{e}+10 \\ 0.2 & 9.582 \mathrm{e}+07 \\ 0.3 & 4.963 \mathrm{e}+09 \\ 0.4 & 5.659 \mathrm{e}+08 \\ 0.5 & 1.007 \mathrm{e}+09 \\ 0.6 & 3.684 \mathrm{e}+08 \\ 0.8 & 1.969 \mathrm{e}+13 \\ 1.0 & 2.719 \mathrm{e}+10 \\ 1.5 & 2.998 \mathrm{e}+10 \\ 2.0 & 3.551 \mathrm{e}+10 \\ 3.0 & 1.363 \mathrm{e}+06 \\ & 2.732 \mathrm{e}+02\end{array}$

\begin{tabular}{c}
$\frac{\text { Fluence Rate }}{\text { MeV/cm } 2 / \mathrm{sec}}$ \\
\hline$\frac{\text { No Buildup }}{3.527 \mathrm{e}-02}$ \\
$8.983 \mathrm{e}+00$ \\
$3.755 \mathrm{e}-01$ \\
$5.223 \mathrm{e}+01$ \\
$1.610 \mathrm{e}+01$ \\
$5.236 \mathrm{e}+01$ \\
$2.995 \mathrm{e}+01$ \\
$2.288 \mathrm{e}+06$ \\
$5.521 \mathrm{e}+03$ \\
$9.354 \mathrm{e}+03$ \\
$2.391 \mathrm{e}+04$ \\
$1.546 \mathrm{e}+00$ \\
$6.178 \mathrm{e}-04$
\end{tabular}

Fluence Rate

$\mathrm{MeV} / \mathrm{cm}^{2} / \mathrm{sec}$

With Buildup

$4.055 \mathrm{e}-01$

$1.036 \mathrm{e}+02$

$3.543 \mathrm{e}+00$

$4.171 \mathrm{e}+02$

$1.016 \mathrm{e}+02$

$2.794 \mathrm{e}+02$

$1.401 \mathrm{e}+02$

$9.612 \mathrm{e}+06$

$1.971 \mathrm{e}+04$

$2.963 e+04$

$6.200 e+04$

$3.561 \mathrm{e}+00$

$1.230 \mathrm{e}-03$

$9.724 \mathrm{e}+06$ $\frac{\text { Exposure Rate }}{\mathrm{mR} / \mathrm{hr}}$

No Buildup

5.581e-05

$1.374 \mathrm{e}-02$

$6.183 \mathrm{e}-04$

$9.219 \mathrm{e}-02$

3.055e-02

$1.020 \mathrm{e}-01$

$5.879 \mathrm{e}-02$

$4.466 \mathrm{e}+03$

$1.050 \mathrm{e}+01$

$1.724 \mathrm{e}+01$

$4.022 \mathrm{e}+01$

$2.390 \mathrm{e}-03$

8.381e-07

$4.534 \mathrm{e}+03$
Exposure Rate $\mathrm{mR} / \mathrm{hr}$

With Buildup

6.418e-04

$1.585 \mathrm{e}-01$

$5.834 \mathrm{e}-03$

7.361e-01

$1.928 \mathrm{e}-01$

5.444e-01

2.751e-01

$1.876 \mathrm{e}+04$

$3.749 \mathrm{e}+01$

$5.461 \mathrm{e}+01$

$1.043 e+02$

5.507e-03

$1.669 \mathrm{e}-06$

$1.896 \mathrm{e}+04$ 
Page $: 1$

DOS File : CSSF 6 Calcine in Grout.ms6

Run Date: April 5, 2005

Run Time: 2:02:52 PM

Duration : 00:01:03
File Ref:

Date:

By:

Checked:

Case Title: CSSF 6 Calcine Grout

Description: CSSF 6 Calcine Grouted at 21.5 wt\% in Storage Canister Geometry: 7 - Cylinder Volume - Side Shields

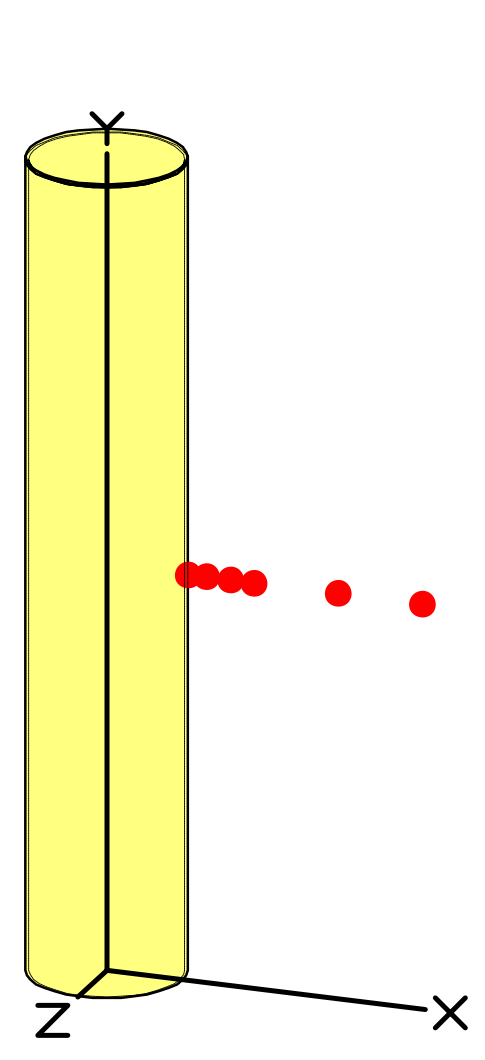

Height
Radius

$\begin{array}{lr} & \\ \text { \# } 1 & \underline{x} \\ & 34.29 \mathrm{~cm} \\ \text { \# } 2 & 1 \mathrm{ft} 1.5 \mathrm{in} \\ & 61.75 \mathrm{~cm} \\ \text { \# } 3 & 2 \mathrm{ft} 0.3 \mathrm{in} \\ & 131.75 \mathrm{~cm} \\ \text { \# 4 } & 4 \mathrm{ft} 3.9 \mathrm{in} \\ & 41.75 \mathrm{~cm} \\ \text { \# 5 } & 1 \mathrm{ft} 4.4 \mathrm{in} \\ & 51.75 \mathrm{~cm} \\ \text { \# 6 } & 1 \mathrm{ft} 8.4 \mathrm{in} \\ & 96.75 \mathrm{~cm} \\ & 3 \mathrm{ft} 2.1 \mathrm{in}\end{array}$

Source Dimensions $337.86 \mathrm{~cm}$

$30.48 \mathrm{~cm}$

$11 \mathrm{ft} 1.0$ in $1 \mathrm{ft}$

Dose Points

$\underline{Y}$
$168.93 \mathrm{~cm}$
$5 \mathrm{ft} 6.5 \mathrm{in}$
$168.93 \mathrm{~cm}$
$5 \mathrm{ft} 6.5 \mathrm{in}$
$168.93 \mathrm{~cm}$
$5 \mathrm{ft} 6.5 \mathrm{in}$
$168.93 \mathrm{~cm}$
$5 \mathrm{ft} 6.5 \mathrm{in}$
$168.93 \mathrm{~cm}$
$5 \mathrm{ft} 6.5 \mathrm{in}$
$168.93 \mathrm{~cm}$
$5 \mathrm{ft} 6.5 \mathrm{in}$

$\underline{Z}$
$0 \mathrm{~cm}$
$0.0 \mathrm{in}$
$0 \mathrm{~cm}$
$0.0 \mathrm{in}$
$0 \mathrm{~cm}$
$0.0 \mathrm{in}$
$0 \mathrm{~cm}$
$0.0 \mathrm{in}$
$0 \mathrm{~cm}$
$0.0 \mathrm{in}$
$0 \mathrm{~cm}$
$0.0 \mathrm{in}$

Shields

Shield Name

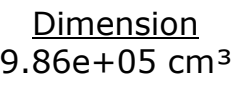

Material

Source

Transition

Air Gap

Wall Clad

Top Clad

$1.27 \mathrm{~cm}$

$1.27 \mathrm{~cm}$

Density

1.8

0.00122

$\begin{array}{ll}\text { Air } & 0.00122 \\ \text { Iron } & 7.95\end{array}$

Iron $\quad 7.95$
Source Input

Grouping Method : Standard Indices

Number of Groups : 25

Lower Energy Cutoff : 0.015

Photons < 0.015 : Included

Library : ICRP-38

$\begin{array}{lc}\frac{\text { Nuclide }}{\text { Am-241 }} & \underline{\text { curies }} \\ \text { Am-243 } & 2.5934 \mathrm{e}-001 \\ \text { Ba-137m } & 2.4849 \mathrm{e}-004 \\ \text { Ce-144 } & 1.8045 \mathrm{e}+002 \\ \mathrm{Cm}-242 & 6.4392 \mathrm{e}-004 \\ \mathrm{Cm}-244 & 3.9049 \mathrm{e}-004 \\ \mathrm{Co}-60 & 4.7530 \mathrm{e}-002 \\ \mathrm{Cs}-134 & 3.9444 \mathrm{e}-003 \\ \mathrm{Cs}-135 & 4.6642 \mathrm{e}-003 \\ \mathrm{Cs}-137 & 2.6822 \mathrm{e}+002 \\ \mathrm{Eu}-152 & 9.6341 \mathrm{e}-003 \\ \mathrm{Eu}-154 & 6.0940 \mathrm{e}-001 \\ \mathrm{Eu}-155 & 7.0407 \mathrm{e}-002 \\ \mathrm{I}-129 & 3.9444 \mathrm{e}-003 \\ \mathrm{Ni}-63 & 1.4693 \mathrm{e}+001 \\ \mathrm{~Np}-237 & 2.6920 \mathrm{e}-003 \\ \mathrm{~Pa}-233 & 2.6920 \mathrm{e}-003 \\ \mathrm{Pm}-147 & 1.5777 \mathrm{e}-002 \\ \mathrm{Pr}-144 & 1.8045 \mathrm{e}-009\end{array}$

becquerels

$9.5956 \mathrm{e}+009$

$9.1943 e+006$

$9.3767 e+012$

$6.6768 \mathrm{e}+001$

$2.3825 e+007$

$1.4448 \mathrm{e}+007$

$1.7586 \mathrm{e}+009$

$1.4594 \mathrm{e}+008$

$1.7258 \mathrm{e}+008$

$9.9240 e+012$

$3.5646 \mathrm{e}+008$

$2.2548 \mathrm{e}+010$

$2.6051 e+009$

$1.4594 \mathrm{e}+008$

$5.4363 e+011$

$9.9605 e+007$

$9.9605 e+007$

$5.8377 e+008$

$6.6768 \mathrm{e}+001$ $\mu \mathrm{Ci} / \mathrm{cm}^{3}$

2.6300e-001

2.5200e-004

$2.5700 \mathrm{e}+002$

$1.8300 \mathrm{e}-009$

$6.5300 \mathrm{e}-004$

3.9600e-004

4.8200e-002

4.0000e-003

$4.7300 \mathrm{e}-003$

$2.7200 e+002$

$9.7700 \mathrm{e}-003$

6.1800e-001

7.1400e-002

4.0000e-003

$1.4900 \mathrm{e}+001$

$2.7300 \mathrm{e}-003$

$2.7300 \mathrm{e}-003$

$1.6000 \mathrm{e}-002$

1.8300e-009
$\mathrm{Bq} / \mathrm{cm}^{3}$

$9.7310 e+003$

$9.3240 \mathrm{e}+000$

$9.5090 \mathrm{e}+006$

6.7710e-005

$2.4161 e+001$

$1.4652 \mathrm{e}+001$

$1.7834 \mathrm{e}+003$

$1.4800 \mathrm{e}+002$

$1.7501 e+002$

$1.0064 \mathrm{e}+007$

$3.6149 \mathrm{e}+002$

$2.2866 \mathrm{e}+004$

$2.6418 \mathrm{e}+003$

$1.4800 \mathrm{e}+002$

$5.5130 \mathrm{e}+005$

$1.0101 e+002$

$1.0101 e+002$

$5.9200 e+002$

$6.7710 \mathrm{e}-005$

E-61 
Page : 2

DOS File : CSSF 6 Calcine in Grout.ms6

Run Date: April 5, 2005

Run Time: 2:02:52 PM

Duration : 00:01:03

\begin{tabular}{lcccc} 
Nuclide & \multicolumn{1}{c}{ curies } & becquerels & $\underline{\mu \mathrm{Ci} / \mathrm{cm}^{3}}$ & $\underline{\mathrm{Bq} / \mathrm{cm}^{3}}$ \\
$\mathrm{Pu}-238$ & $1.9820 \mathrm{e}+000$ & $7.3336 \mathrm{e}+010$ & $2.0100 \mathrm{e}+000$ & $7.4370 \mathrm{e}+004$ \\
$\mathrm{Pu}-239$ & $1.6862 \mathrm{e}-001$ & $6.2390 \mathrm{e}+009$ & $1.7100 \mathrm{e}-001$ & $6.3270 \mathrm{e}+003$ \\
$\mathrm{Pu}-240$ & $4.9699 \mathrm{e}-002$ & $1.8389 \mathrm{e}+009$ & $5.0400 \mathrm{e}-002$ & $1.8648 \mathrm{e}+003$ \\
$\mathrm{Pu}-241$ & $1.4002 \mathrm{e}+000$ & $5.1809 \mathrm{e}+010$ & $1.4200 \mathrm{e}+000$ & $5.2540 \mathrm{e}+004$ \\
$\mathrm{Pu}-242$ & $7.8197 \mathrm{e}-002$ & $2.8933 \mathrm{e}+009$ & $7.9300 \mathrm{e}-002$ & $2.9341 \mathrm{e}+003$ \\
$\mathrm{Ru}-106$ & $7.0900 \mathrm{e}-008$ & $2.6233 \mathrm{e}+003$ & $7.1900 \mathrm{e}-008$ & $2.6603 \mathrm{e}-003$ \\
$\mathrm{Sb}-125$ & $5.6602 \mathrm{e}-003$ & $2.0943 \mathrm{e}+008$ & $5.7400 \mathrm{e}-003$ & $2.1238 \mathrm{e}+002$ \\
$\mathrm{Sb}-126$ & $4.0627 \mathrm{e}-004$ & $1.5032 \mathrm{e}+007$ & $4.1200 \mathrm{e}-004$ & $1.5244 \mathrm{e}+001$ \\
$\mathrm{Sb}-126 \mathrm{~m}$ & $2.8991 \mathrm{e}-003$ & $1.0727 \mathrm{e}+008$ & $2.9400 \mathrm{e}-003$ & $1.0878 \mathrm{e}+002$ \\
$\mathrm{Sm}-151$ & $2.4849 \mathrm{e}+000$ & $9.1943 \mathrm{e}+010$ & $2.5200 \mathrm{e}+000$ & $9.3240 \mathrm{e}+004$ \\
$\mathrm{Sr}-90$ & $2.5244 \mathrm{e}+002$ & $9.3402 \mathrm{e}+012$ & $2.5600 \mathrm{e}+002$ & $9.4720 \mathrm{e}+006$ \\
$\mathrm{Tc}-99$ & $1.0650 \mathrm{e}-001$ & $3.9404 \mathrm{e}+009$ & $1.0800 \mathrm{e}-001$ & $3.9960 \mathrm{e}+003$ \\
$\mathrm{Th}-230$ & $1.0255 \mathrm{e}-005$ & $3.7945 \mathrm{e}+005$ & $1.0400 \mathrm{e}-005$ & $3.8480 \mathrm{e}-001$ \\
$\mathrm{Th}-231$ & $3.2442 \mathrm{e}-005$ & $1.2004 \mathrm{e}+006$ & $3.2900 \mathrm{e}-005$ & $1.2173 \mathrm{e}+000$ \\
$\mathrm{U}-232$ & $5.4432 \mathrm{e}-006$ & $2.0140 \mathrm{e}+005$ & $5.5200 \mathrm{e}-006$ & $2.0424 \mathrm{e}-001$ \\
$\mathrm{U}-233$ & $5.7883 \mathrm{e}-007$ & $2.1417 \mathrm{e}+004$ & $5.8700 \mathrm{e}-007$ & $2.1719 \mathrm{e}-002$ \\
$\mathrm{U}-234$ & $1.3707 \mathrm{e}-003$ & $5.0715 \mathrm{e}+007$ & $1.3900 \mathrm{e}-003$ & $5.1430 \mathrm{e}+001$ \\
$\mathrm{U}-235$ & $3.5105 \mathrm{e}-005$ & $1.2989 \mathrm{e}+006$ & $3.5600 \mathrm{e}-005$ & $1.3172 \mathrm{e}+000$ \\
$\mathrm{U}-236$ & $8.2930 \mathrm{e}-005$ & $3.0684 \mathrm{e}+006$ & $8.4100 \mathrm{e}-005$ & $3.1117 \mathrm{e}+000$ \\
$\mathrm{U}-237$ & $3.6091 \mathrm{e}-005$ & $1.3354 \mathrm{e}+006$ & $3.6600 \mathrm{e}-005$ & $1.3542 \mathrm{e}+000$ \\
$\mathrm{U}-238$ & $3.4809 \mathrm{e}-005$ & $1.2879 \mathrm{e}+006$ & $3.5300 \mathrm{e}-005$ & $1.3061 \mathrm{e}+000$ \\
$\mathrm{Y}-90$ & $2.5244 \mathrm{e}+002$ & $9.3402 \mathrm{e}+012$ & $2.5600 \mathrm{e}+002$ & $9.4720 \mathrm{e}+006$ \\
& & & &
\end{tabular}

$\begin{array}{cc}\frac{\text { Energy }}{\text { MeV }} & \begin{array}{c}\text { Activity } \\ \text { photons/sec }\end{array} \\ 0.015 & 6.271 \mathrm{e}+09 \\ 0.02 & 4.039 \mathrm{e}+09 \\ 0.03 & 5.683 \mathrm{e}+11 \\ 0.04 & 1.406 \mathrm{e}+11 \\ 0.05 & 1.432 \mathrm{e}+09 \\ 0.06 & 3.463 \mathrm{e}+09 \\ 0.08 & 8.346 \mathrm{e}+08 \\ 0.1 & 9.816 \mathrm{e}+09 \\ 0.15 & 3.110 \mathrm{e}+07 \\ 0.2 & 1.595 \mathrm{e}+09 \\ 0.3 & 1.946 \mathrm{e}+08 \\ 0.4 & 3.792 \mathrm{e}+08 \\ 0.5 & 1.274 \mathrm{e}+08 \\ 0.6 & 8.420 \mathrm{e}+12 \\ 0.8 & 8.701 \mathrm{e}+09 \\ 1.0 & 8.931 \mathrm{e}+09 \\ 1.5 & 1.068 \mathrm{e}+10 \\ 2.0 & 4.347 \mathrm{e}+05 \\ 3.0 & 6.331 \mathrm{e}+01\end{array}$

Radial

Integration Parameters

Circumferential

Y Direction (axial) $\quad 24$

Results - Dose Point \# 1 - $(34.29,168.93,0) \mathrm{cm}$

\begin{tabular}{|c|c|c|c|}
\hline Fluence Rate & Fluence Rate & Exposure Rate & Exposure Rate \\
\hline $\mathrm{MeV} / \mathrm{cm}^{2} / \mathrm{sec}$ & $\mathrm{MeV} / \mathrm{cm}^{2} / \mathrm{sec}$ & $\mathrm{mR} / \mathrm{hr}$ & $\mathrm{mR} / \mathrm{hr}$ \\
\hline No Buildup & With Buildup & No Buildup & With Buildup \\
\hline $7.844 \mathrm{e}-268$ & $2.026 \mathrm{e}-23$ & $6.728 e-269$ & $1.738 \mathrm{e}-24$ \\
\hline $1.313 \mathrm{e}-119$ & $2.053 e-23$ & $4.548 e-121$ & $7.112 \mathrm{e}-25$ \\
\hline $4.224 \mathrm{e}-35$ & $6.388 \mathrm{e}-21$ & $4.186 e-37$ & $6.331 \mathrm{e}-23$ \\
\hline $2.184 \mathrm{e}-14$ & $8.731 \mathrm{e}-14$ & $9.657 e-17$ & $3.861 \mathrm{e}-16$ \\
\hline $1.787 \mathrm{e}-08$ & $1.201 \mathrm{e}-07$ & $4.760 \mathrm{e}-11$ & $3.199 \mathrm{e}-10$ \\
\hline $1.799 \mathrm{e}-04$ & $1.681 \mathrm{e}-03$ & $3.574 \mathrm{e}-07$ & $3.339 \mathrm{e}-06$ \\
\hline $4.876 \mathrm{e}-02$ & $5.510 \mathrm{e}-01$ & $7.716 e-05$ & $8.719 e-04$ \\
\hline $9.296 \mathrm{e}+00$ & $1.050 e+02$ & $1.422 \mathrm{e}-02$ & $1.607 e-01$ \\
\hline $3.842 \mathrm{e}-01$ & $3.624 e+00$ & $6.327 e-04$ & $5.968 e-03$ \\
\hline $5.317 e+01$ & $4.294 e+02$ & $9.384 \mathrm{e}-02$ & $7.579 \mathrm{e}-01$ \\
\hline $1.770 \mathrm{e}+01$ & $1.139 \mathrm{e}+02$ & $3.357 e-02$ & $2.160 \mathrm{e}-01$ \\
\hline $6.340 e+01$ & $3.458 e+02$ & $1.235 e-01$ & $6.737 e-01$ \\
\hline $3.344 \mathrm{e}+01$ & $1.601 e+02$ & $6.565 e-02$ & $3.143 e-01$ \\
\hline $3.171 \mathrm{e}+06$ & $1.363 e+07$ & $6.189 e+03$ & $2.661 e+04$ \\
\hline $5.762 e+03$ & $2.105 e+04$ & $1.096 e+01$ & $4.004 e+01$ \\
\hline $9.139 e+03$ & $2.960 e+04$ & $1.685 e+01$ & $5.457 e+01$ \\
\hline $2.380 e+04$ & $6.305 e+04$ & $4.005 e+01$ & $1.061 \mathrm{e}+02$ \\
\hline $1.643 e+00$ & $3.864 e+00$ & $2.541 \mathrm{e}-03$ & $5.975 e-03$ \\
\hline $4.813 e-04$ & $9.765 e-04$ & $6.529 e-07$ & $1.325 \mathrm{e}-06$ \\
\hline $3.210 \mathrm{e}+06$ & $1.375 e+07$ & $6.257 e+03$ & $2.681 e+04$ \\
\hline
\end{tabular}

Results - Dose Point \# 2 - $(61.75,168.93,0) \mathrm{cm}$ 
Page : 3

DOS File : CSSF 6 Calcine in Grout.ms6

Run Date: April 5, 2005

Run Time: 2:02:52 PM

Duration : 00:01:03

$\begin{array}{cc}\begin{array}{cc}\text { Energy } \\ \text { MeV }\end{array} & \begin{array}{c}\text { Activity } \\ \text { photons/sec }\end{array} \\ 0.015 & 6.271 \mathrm{e}+09 \\ 0.02 & 4.039 \mathrm{e}+09 \\ 0.03 & 5.683 \mathrm{e}+11 \\ 0.04 & 1.406 \mathrm{e}+11 \\ 0.05 & 1.432 \mathrm{e}+09 \\ 0.06 & 3.463 \mathrm{e}+09 \\ 0.08 & 8.346 \mathrm{e}+08 \\ 0.1 & 9.816 \mathrm{e}+09 \\ 0.15 & 3.110 \mathrm{e}+07 \\ 0.2 & 1.595 \mathrm{e}+09 \\ 0.3 & 1.946 \mathrm{e}+08 \\ 0.4 & 3.792 \mathrm{e}+08 \\ 0.5 & 1.274 \mathrm{e}+08 \\ 0.6 & 8.420 \mathrm{e}+12 \\ 0.8 & 8.701 \mathrm{e}+09 \\ 1.0 & 8.931 \mathrm{e}+09 \\ 1.5 & 1.068 \mathrm{e}+10 \\ 2.0 & 4.347 \mathrm{e}+05 \\ 3.0 & 6.331 \mathrm{e}+01\end{array}$

\begin{tabular}{c}
$\frac{\text { Fluence Rate }}{\mathrm{MeV} / \mathrm{cm}^{2} / \mathrm{sec}}$ \\
\hline No Buildup \\
$1.583 \mathrm{e}-250$ \\
$3.664 \mathrm{e}-113$ \\
$4.176 \mathrm{e}-34$ \\
$2.084 \mathrm{e}-14$ \\
$9.792 \mathrm{e}-09$ \\
$8.578 \mathrm{e}-05$ \\
$2.342 \mathrm{e}-02$ \\
$4.634 \mathrm{e}+00$ \\
$1.952 \mathrm{e}-01$ \\
$2.695 \mathrm{e}+01$ \\
$8.927 \mathrm{e}+00$ \\
$3.187 \mathrm{e}+01$ \\
$1.677 \mathrm{e}+01$ \\
$1.586 \mathrm{e}+06$ \\
$2.872 \mathrm{e}+03$ \\
$4.544 \mathrm{e}+03$ \\
$1.178 \mathrm{e}+04$ \\
$8.113 \mathrm{e}-01$ \\
$2.370 \mathrm{e}-04$ \\
\end{tabular}

Fluence Rate

$\mathrm{MeV} / \mathrm{cm}^{2} / \mathrm{sec}$

With Buildup

$8.890 \mathrm{e}-24$

$9.010 \mathrm{e}-24$

$2.803 \mathrm{e}-21$

$8.260 \mathrm{e}-14$

$6.489 \mathrm{e}-08$

$7.961 \mathrm{e}-04$

2.692e-01

$5.345 e+01$

$1.845 \mathrm{e}+00$

$2.162 \mathrm{e}+02$

$5.677 \mathrm{e}+01$

$1.717 \mathrm{e}+02$

$7.927 \mathrm{e}+01$

$6.737 \mathrm{e}+06$

$1.038 \mathrm{e}+04$

$1.457 \mathrm{e}+04$

$3.094 \mathrm{e}+04$

$1.893 e+00$

$4.780 \mathrm{e}-04$

$1.605 e+06$

$6.793 e+06$ $\frac{\text { Exposure Rate }}{\mathrm{mR} / \mathrm{hr}}$

No Buildup

1.358e-251

$1.269 \mathrm{e}-114$

$4.139 \mathrm{e}-36$

$9.216 \mathrm{e}-17$

$2.608 \mathrm{e}-11$

$1.704 \mathrm{e}-07$

$3.706 \mathrm{e}-05$

$7.089 \mathrm{e}-03$

$3.214 \mathrm{e}-04$

4.757e-02

$1.693 \mathrm{e}-02$

6.209e-02

3.291e-02

$3.096 \mathrm{e}+03$

$5.464 \mathrm{e}+00$

$8.375 \mathrm{e}+00$

$1.982 \mathrm{e}+01$

$1.255 \mathrm{e}-03$

3.215e-07
$3.130 \mathrm{e}+03$
Exposure Rate $\mathrm{mR} / \mathrm{hr}$

With Buildup

$7.625 \mathrm{e}-25$

$3.121 \mathrm{e}-25$

2.778e-23

$3.653 e-16$

$1.729 \mathrm{e}-10$

$1.581 \mathrm{e}-06$

4.260e-04

8.177e-02

$3.039 \mathrm{e}-03$

3.816e-01

$1.077 \mathrm{e}-01$

3.345e-01

$1.556 \mathrm{e}-01$

$1.315 \mathrm{e}+04$

$1.974 \mathrm{e}+01$

$2.685 e+01$

$5.205 e+01$

$2.928 \mathrm{e}-03$

6.484e-07

$1.325 e+04$

\begin{tabular}{|c|c|}
\hline$\frac{\text { Energy }}{\mathrm{MeV}}$ & $\begin{array}{c}\text { Activity } \\
\text { photons/sec }\end{array}$ \\
\hline 0.015 & $6.271 e+09$ \\
\hline 0.02 & $4.039 e+09$ \\
\hline 0.03 & $5.683 e+11$ \\
\hline 0.04 & $1.406 \mathrm{e}+11$ \\
\hline 0.05 & $1.432 \mathrm{e}+09$ \\
\hline 0.06 & $3.463 e+09$ \\
\hline 0.08 & $8.346 e+08$ \\
\hline 0.1 & $9.816 e+09$ \\
\hline 0.15 & $3.110 \mathrm{e}+07$ \\
\hline 0.2 & $1.595 e+09$ \\
\hline 0.3 & $1.946 \mathrm{e}+08$ \\
\hline 0.4 & $3.792 e+08$ \\
\hline 0.5 & $1.274 \mathrm{e}+08$ \\
\hline 0.6 & $8.420 e+12$ \\
\hline 0.8 & $8.701 e+09$ \\
\hline 1.0 & $8.931 e+09$ \\
\hline 1.5 & $1.068 e+10$ \\
\hline 2.0 & $4.347 e+05$ \\
\hline 3.0 & $6.331 e+01$ \\
\hline
\end{tabular}

TOTALS:

\section{Energy MeV}

0.015

0.02

0.03
$9.185 e+12$

Activity photons/sec

$6.271 e+09$

$4.039 \mathrm{e}+09$

$5.683 e+11$

\section{Results - Dose Point \# 3 - $(131.75,168.93,0) \mathrm{cm}$}

\section{Fluence Rate}

$\mathrm{MeV} / \mathrm{cm}^{2} / \mathrm{sec}$

No Buildup

4.557e-251

$1.415 \mathrm{e}-113$

$1.953 \mathrm{e}-34$

$9.576 \mathrm{e}-15$

4.481e-09

$3.932 \mathrm{e}-05$

$1.070 \mathrm{e}-02$

$2.107 e+00$

8.753e-02

$1.196 \mathrm{e}+01$

$3.919 \mathrm{e}+00$

$1.389 \mathrm{e}+01$

$7.272 \mathrm{e}+00$

$6.850 \mathrm{e}+05$

$1.231 \mathrm{e}+03$

$1.935 \mathrm{e}+03$

$4.955 \mathrm{e}+03$

$3.382 \mathrm{e}-01$

$9.764 \mathrm{e}-05$

$6.931 e+05$
Fluence Rate

$\mathrm{MeV} / \mathrm{cm}^{2} / \mathrm{sec}$

With Buildup

$3.049 \mathrm{e}-24$

$9.487 \mathrm{e}-22$

$3.795 \mathrm{e}-14$

$2.971 \mathrm{e}-08$

3.651e-04

$1.231 \mathrm{e}-01$

$2.429 \mathrm{e}+01$

$8.193 \mathrm{e}-01$

$9.437 \mathrm{e}+01$

$2.435 e+01$

$7.294 \mathrm{e}+01$

$3.347 e+01$

$2.830 \mathrm{e}+06$

$4.325 e+03$

$6.034 \mathrm{e}+03$

$1.266 \mathrm{e}+04$

$7.685 \mathrm{e}-01$

$1.919 \mathrm{e}-04$

$2.854 e+06$
$3.009 \mathrm{e}-24$
Exposure Rate $\mathrm{mR} / \mathrm{hr}$

No Buildup

3.909e-252

$4.903 e-115$

$1.935 \mathrm{e}-36$

$4.235 \mathrm{e}-17$

$1.194 \mathrm{e}-11$

7.809e-08

$1.693 \mathrm{e}-05$

3.224e-03

$1.441 \mathrm{e}-04$

2.112e-02

7.434e-03

$2.707 \mathrm{e}-02$

$1.427 \mathrm{e}-02$

$1.337 \mathrm{e}+03$

$2.342 \mathrm{e}+00$

$3.567 \mathrm{e}+00$

$8.337 \mathrm{e}+00$

$5.230 \mathrm{e}-04$

$1.325 \mathrm{e}-07$

$1.351 \mathrm{e}+03$
Exposure Rate $\mathrm{mR} / \mathrm{hr}$

With Buildup

$2.581 \mathrm{e}-25$

$1.056 \mathrm{e}-25$

$9.403 \mathrm{e}-24$

$1.679 \mathrm{e}-16$

7.915e-11

$7.253 \mathrm{e}-07$

$1.948 \mathrm{e}-04$

3.716e-02

$1.349 \mathrm{e}-03$

$1.666 \mathrm{e}-01$

4.619e-02

$1.421 \mathrm{e}-01$

$6.570 \mathrm{e}-02$

$5.524 \mathrm{e}+03$

$8.226 \mathrm{e}+00$

$1.112 \mathrm{e}+01$

$2.131 \mathrm{e}+01$

$1.188 \mathrm{e}-03$

2.604e-07

$5.565 e+03$
Results - Dose Point \# 4 - $(41.75,168.93,0) \mathrm{cm}$

$\begin{array}{ccc}\frac{\text { Fluence Rate }}{\text { MeV/cm } / \mathrm{sec}} & \frac{\text { Fluence Rate }}{\text { MeV/cm } 2 / \mathrm{sec}} & \begin{array}{c}\text { Exposure Rate } \\ \mathrm{mR} / \mathrm{hr}\end{array} \\ \frac{\underline{\text { No Buildup }}}{6.499 \mathrm{e}-252} & \frac{\text { With Buildup }}{1.510 \mathrm{e}-23} & \frac{\text { No Buildup }}{5.574 \mathrm{e}-253} \\ 2.426 \mathrm{e}-113 & 1.531 \mathrm{e}-23 & 8.404 \mathrm{e}-115 \\ 7.371 \mathrm{e}-34 & 4.762 \mathrm{e}-21 & 7.305 \mathrm{e}-36\end{array}$

Exposure Rate $\mathrm{mR} / \mathrm{hr}$

With Buildup

$1.295 \mathrm{e}-24$

$5.302 \mathrm{e}-25$

$4.720 \mathrm{e}-23$ 
Page : 4

DOS File : CSSF 6 Calcine in Grout.ms6

Run Date: April 5, 2005

Run Time: 2:02:52 PM

Duration : 00:01:03

\begin{tabular}{|c|c|}
\hline$\frac{\text { Energy }}{\text { MeV }}$ & $\frac{\text { Activity }}{\text { photons/sec }}$ \\
\hline 0.04 & $1.406 \mathrm{e}+11$ \\
\hline 0.05 & $1.432 \mathrm{e}+09$ \\
\hline 0.06 & $3.463 e+09$ \\
\hline 0.08 & $8.346 e+08$ \\
\hline 0.1 & $9.816 e+09$ \\
\hline 0.15 & $3.110 e+07$ \\
\hline 0.2 & $1.595 \mathrm{e}+09$ \\
\hline 0.3 & $1.946 \mathrm{e}+08$ \\
\hline 0.4 & $3.792 \mathrm{e}+08$ \\
\hline 0.5 & $1.274 \mathrm{e}+08$ \\
\hline 0.6 & $8.420 \mathrm{e}+12$ \\
\hline 0.8 & $8.701 e+09$ \\
\hline 1.0 & $8.931 \mathrm{e}+09$ \\
\hline 1.5 & $1.068 e+10$ \\
\hline 2.0 & $4.347 e+05$ \\
\hline 3.0 & $6.331 \mathrm{e}+01$ \\
\hline
\end{tabular}

\begin{tabular}{|c|c|}
\hline$\frac{\text { Fluence Rate }}{\mathrm{MeV} / \mathrm{cm}^{2} / \mathrm{sec}}$ & $\frac{\text { Fluence Rate }}{\mathrm{MeV} / \mathrm{cm}^{2} / \mathrm{sec}}$ \\
\hline No Buildup & With Buildup \\
\hline $3.629 \mathrm{e}-14$ & $1.437 \mathrm{e}-13$ \\
\hline $1.500 \mathrm{e}-08$ & $9.891 \mathrm{e}-08$ \\
\hline $1.260 \mathrm{e}-04$ & $1.168 \mathrm{e}-03$ \\
\hline $3.510 \mathrm{e}-02$ & $4.054 \mathrm{e}-01$ \\
\hline $7.035 e+00$ & $8.146 \mathrm{e}+01$ \\
\hline $2.995 \mathrm{e}-01$ & $2.844 \mathrm{e}+00$ \\
\hline $4.153 e+01$ & $3.348 e+02$ \\
\hline $1.380 \mathrm{e}+01$ & $8.825 e+01$ \\
\hline $4.935 e+01$ & $2.673 e+02$ \\
\hline $2.600 \mathrm{e}+01$ & $1.236 \mathrm{e}+02$ \\
\hline $2.462 e+06$ & $1.051 \mathrm{e}+07$ \\
\hline $4.465 e+03$ & $1.621 \mathrm{e}+04$ \\
\hline $7.071 \mathrm{e}+03$ & $2.277 e+04$ \\
\hline $1.837 e+04$ & $4.845 e+04$ \\
\hline $1.267 e+00$ & $2.968 \mathrm{e}+00$ \\
\hline $3.706 \mathrm{e}-04$ & $7.503 e-04$ \\
\hline $2.492 \mathrm{e}+06$ & $1.060 e+07$ \\
\hline
\end{tabular}

$\frac{\text { Exposure Rate }}{\mathrm{mR} / \mathrm{hr}}$

No Buildup

$1.605 \mathrm{e}-16$

$3.995 \mathrm{e}-11$

2.502e-07

$5.555 \mathrm{e}-05$

$1.076 \mathrm{e}-02$

$4.933 \mathrm{e}-04$

$7.329 \mathrm{e}-02$

$2.618 \mathrm{e}-02$

$9.616 \mathrm{e}-02$

$5.103 \mathrm{e}-02$

$4.805 e+03$

$8.493 e+00$

$1.303 \mathrm{e}+01$

$3.091 \mathrm{e}+01$

$1.959 \mathrm{e}-03$

$5.028 \mathrm{e}-07$

$4.858 \mathrm{e}+03$

Results - Dose Point \# 5 - $(51.75,168.93,0) \mathrm{cm}$

\begin{tabular}{|c|c|}
\hline $\begin{array}{l}\text { Energy } \\
\underline{\mathrm{MeV}}\end{array}$ & $\begin{array}{c}\text { Activity } \\
\text { photons/sec }\end{array}$ \\
\hline 0.015 & $6.271 e+09$ \\
\hline 0.02 & $4.039 e+09$ \\
\hline 0.03 & $5.683 e+11$ \\
\hline 0.04 & $1.406 \mathrm{e}+11$ \\
\hline 0.05 & $1.432 e+09$ \\
\hline 0.06 & $3.463 e+09$ \\
\hline 0.08 & $8.346 e+08$ \\
\hline 0.1 & $9.816 e+09$ \\
\hline 0.15 & $3.110 \mathrm{e}+07$ \\
\hline 0.2 & $1.595 e+09$ \\
\hline 0.3 & $1.946 e+08$ \\
\hline 0.4 & $3.792 e+08$ \\
\hline 0.5 & $1.274 \mathrm{e}+08$ \\
\hline 0.6 & $8.420 e+12$ \\
\hline 0.8 & $8.701 e+09$ \\
\hline 1.0 & $8.931 e+09$ \\
\hline 1.5 & $1.068 \mathrm{e}+10$ \\
\hline 2.0 & $4.347 e+05$ \\
\hline 3.0 & $6.331 e+01$ \\
\hline
\end{tabular}

TOTALS: $\quad 9.185 \mathrm{e}+12$

$\begin{array}{cc}\frac{\text { Energy }}{\text { MeV }} & \begin{array}{c}\text { Activity } \\ \text { photons/sec }\end{array} \\ 0.015 & \\ 0.02 & 6.271 \mathrm{e}+09 \\ 0.03 & 4.039 \mathrm{e}+09 \\ 0.04 & 5.683 \mathrm{e}+11 \\ 0.05 & 1.406 \mathrm{e}+11 \\ 0.06 & 1.432 \mathrm{e}+09 \\ & 3.463 \mathrm{e}+09\end{array}$

\begin{tabular}{|c|c|c|}
\hline $\begin{array}{l}\text { Fluence Rate } \\
\mathrm{MeV} / \mathrm{cm}^{2} / \mathrm{sec}\end{array}$ & $\begin{array}{l}\text { Fluence Rate } \\
\mathrm{MeV} / \mathrm{cm}^{2} / \mathrm{sec}\end{array}$ & $\begin{array}{c}\text { Exposure Rate } \\
\mathrm{mR} / \mathrm{hr}\end{array}$ \\
\hline No Buildup & With Buildup & No Buildup \\
\hline $1.353 \mathrm{e}-250$ & $1.127 \mathrm{e}-23$ & $\overline{1.161 \mathrm{e}-251}$ \\
\hline $4.762 \mathrm{e}-113$ & $1.142 \mathrm{e}-23$ & $1.650 \mathrm{e}-114$ \\
\hline $5.539 e-34$ & $3.553 e-21$ & $5.490 e-36$ \\
\hline $2.428 e-14$ & $9.619 \mathrm{e}-14$ & $1.074 \mathrm{e}-16$ \\
\hline $1.163 \mathrm{e}-08$ & $7.714 \mathrm{e}-08$ & $3.097 e-11$ \\
\hline $1.032 \mathrm{e}-04$ & $9.580 e-04$ & $2.050 e-07$ \\
\hline $2.819 \mathrm{e}-02$ & $3.240 \mathrm{e}-01$ & $4.460 \mathrm{e}-05$ \\
\hline $5.585 e+00$ & $6.446 e+01$ & $8.545 e-03$ \\
\hline $2.360 \mathrm{e}-01$ & $2.233 e+00$ & $3.885 e-04$ \\
\hline $3.262 e+01$ & $2.620 e+02$ & $5.757 e-02$ \\
\hline $1.081 e+01$ & $6.888 e+01$ & $2.051 \mathrm{e}-02$ \\
\hline $3.862 e+01$ & $2.084 e+02$ & $7.526 e-02$ \\
\hline $2.033 e+01$ & $9.629 e+01$ & $3.990 e-02$ \\
\hline $1.924 \mathrm{e}+06$ & $8.186 e+06$ & $3.755 e+03$ \\
\hline $3.486 e+03$ & $1.262 e+04$ & $6.630 e+00$ \\
\hline $5.516 e+03$ & $1.772 \mathrm{e}+04$ & $1.017 e+01$ \\
\hline $1.432 e+04$ & $3.767 e+04$ & $2.409 e+01$ \\
\hline $9.865 e-01$ & $2.307 e+00$ & $1.525 \mathrm{e}-03$ \\
\hline $2.884 \mathrm{e}-04$ & $5.830 e-04$ & $3.913 e-07$ \\
\hline $1.947 e+06$ & $8.255 e+06$ & $3.796 \mathrm{e}+03$ \\
\hline
\end{tabular}

\section{Results - Dose Point \# 6 - $(96.75,168.93,0) \mathrm{cm}$}

\begin{tabular}{c}
$\frac{\text { Fluence Rate }}{\text { MeV/cm } 2 / \mathrm{sec}}$ \\
\hline No Buildup \\
\hline $7.056 \mathrm{e}-251$ \\
$1.897 \mathrm{e}-113$ \\
$2.705 \mathrm{e}-34$ \\
$1.318 \mathrm{e}-14$ \\
$6.161 \mathrm{e}-09$ \\
$5.405 \mathrm{e}-05$
\end{tabular}

$\frac{\text { Exposure Rate }}{\text { mR/hr }}$
No Buildup
$6.052 \mathrm{e}-252$
$6.570 \mathrm{e}-115$
$2.681 \mathrm{e}-36$
$5.829 \mathrm{e}-17$
$1.641 \mathrm{e}-11$
$1.074 \mathrm{e}-07$

\begin{tabular}{c}
$\frac{\text { Exposure Rate }}{\mathrm{mR} / \mathrm{hr}}$ \\
With Buildup \\
\hline $6.356 \mathrm{e}-16$ \\
$2.635 \mathrm{e}-10$ \\
$2.320 \mathrm{e}-06$ \\
$6.415 \mathrm{e}-04$ \\
$1.246 \mathrm{e}-01$ \\
$4.684 \mathrm{e}-03$ \\
$5.910 \mathrm{e}-01$ \\
$1.674 \mathrm{e}-01$ \\
$5.209 \mathrm{e}-01$ \\
$2.426 \mathrm{e}-01$ \\
$2.052 \mathrm{e}+04$ \\
$3.083 \mathrm{e}+01$ \\
$4.198 \mathrm{e}+01$ \\
$8.151 \mathrm{e}+01$ \\
$4.590 \mathrm{e}-03$ \\
$1.018 \mathrm{e}-06$ \\
$2.067 \mathrm{e}+04$
\end{tabular}

Exposure Rate $\mathrm{mR} / \mathrm{hr}$

With Buildup

9.664e-25

$3.955 \mathrm{e}-25$

$3.521 \mathrm{e}-23$

$4.254 \mathrm{e}-16$

$2.055 \mathrm{e}-10$

$1.903 e-06$

$5.127 \mathrm{e}-04$

$9.861 \mathrm{e}-02$

$3.678 \mathrm{e}-03$

$4.625 \mathrm{e}-01$

1.307e-01

4.061e-01

$1.890 \mathrm{e}-01$

$1.598 \mathrm{e}+04$

$2.400 \mathrm{e}+01$

$3.266 \mathrm{e}+01$

$6.338 \mathrm{e}+01$

3.568e-03

7.910e-07

$1.610 \mathrm{e}+04$
Exposure Rate $\mathrm{mR} / \mathrm{hr}$

With Buildup

$4.095 \mathrm{e}-25$

$1.676 \mathrm{e}-25$

$1.492 \mathrm{e}-23$

$2.310 \mathrm{e}-16$

$1.088 \mathrm{e}-10$

9.966e-07 
Page : 5

DOS File : CSSF 6 Calcine in Grout.ms6

Run Date: April 5, 2005

Run Time: 2:02:52 PM

Duration : 00:01:03

\begin{tabular}{|c|c|}
\hline Energy & Activity \\
\hline $\mathrm{MeV}$ & photons/sec \\
\hline 0.08 & $8.346 e+08$ \\
\hline 0.1 & $9.816 e+09$ \\
\hline 0.15 & $3.110 e+07$ \\
\hline 0.2 & $1.595 \mathrm{e}+09$ \\
\hline 0.3 & $1.946 e+08$ \\
\hline 0.4 & $3.792 e+08$ \\
\hline 0.5 & $1.274 \mathrm{e}+08$ \\
\hline 0.6 & $8.420 e+12$ \\
\hline 0.8 & $8.701 e+09$ \\
\hline 1.0 & $8.931 e+09$ \\
\hline 1.5 & $1.068 \mathrm{e}+10$ \\
\hline 2.0 & $4.347 e+05$ \\
\hline 3.0 & $6.331 e+01$ \\
\hline
\end{tabular}

\begin{tabular}{c}
$\frac{\text { Fluence Rate }}{\text { MeV/cm } 2 / \mathrm{sec}}$ \\
\hline$\frac{\text { No Buildup }}{1.472 \mathrm{e}-02}$ \\
$2.905 \mathrm{e}+00$ \\
$1.219 \mathrm{e}-01$ \\
$1.678 \mathrm{e}+01$ \\
$5.539 \mathrm{e}+00$ \\
$1.973 \mathrm{e}+01$ \\
$1.036 \mathrm{e}+01$ \\
$9.784 \mathrm{e}+05$ \\
$1.766 \mathrm{e}+03$ \\
$2.787 \mathrm{e}+03$ \\
$7.186 \mathrm{e}+03$ \\
$4.929 \mathrm{e}-01$ \\
$1.432 \mathrm{e}-04$
\end{tabular}

Fluence Rate

$\mathrm{MeV} / \mathrm{cm}^{2} / \mathrm{sec}$

With Buildup

1.692e-01

$3.351 \mathrm{e}+01$

$1.150 \mathrm{e}+00$

$1.340 \mathrm{e}+02$

$3.495 \mathrm{e}+01$

$1.053 \mathrm{e}+02$

$4.847 \mathrm{e}+01$

$4.110 \mathrm{e}+06$

$6.307 e+03$

$8.827 \mathrm{e}+03$

$1.864 \mathrm{e}+04$

$1.136 \mathrm{e}+00$

2.851e-04

$9.902 \mathrm{e}+05$ $\frac{\text { Exposure Rate }}{\mathrm{mR} / \mathrm{hr}}$

No Buildup

2.329e-05

$4.445 \mathrm{e}-03$

$2.007 \mathrm{e}-04$

$2.962 \mathrm{e}-02$

$1.051 \mathrm{e}-02$

$3.844 \mathrm{e}-02$

$2.033 \mathrm{e}-02$

$1.910 \mathrm{e}+03$

$3.360 \mathrm{e}+00$

$5.137 \mathrm{e}+00$

$1.209 \mathrm{e}+01$

$7.621 \mathrm{e}-04$

$1.942 \mathrm{e}-07$

$1.930 e+03$
Exposure Rate $\mathrm{mR} / \mathrm{hr}$

With Buildup

2.678e-04

$5.127 \mathrm{e}-02$

$1.894 \mathrm{e}-03$

2.365e-01

$6.629 \mathrm{e}-02$

2.051e-01

9.514e-02

$8.022 \mathrm{e}+03$

$1.200 \mathrm{e}+01$

$1.627 \mathrm{e}+01$

$3.136 \mathrm{e}+01$

$1.756 \mathrm{e}-03$

3.868e-07

$8.083 e+03$ 
Page : 1

DOS File : Combined Calcine in Grout.ms6

Run Date: April 5, 2005

Run Time: 3:17:39 PM

Duration : 00:01:02
File Ref:

Date:

By:

Checked:

\section{Case Title: Grouted Calcine \\ Description: Combined Calcine Grouted at $\mathbf{2 1 . 5}$ wt\% in Storage Canister Geometry: 7 - Cylinder Volume - Side Shields}

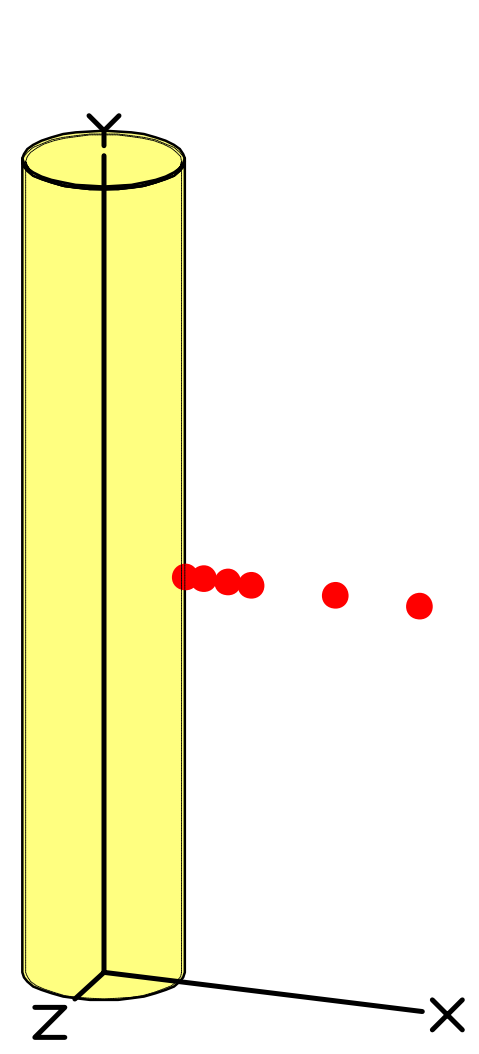

Height
Radius

$\begin{array}{lr} & \\ \text { \# } 1 & \underline{x} \\ & 34.29 \mathrm{~cm} \\ \text { \# } 2 & 1 \mathrm{ft} 1.5 \mathrm{in} \\ & 61.75 \mathrm{~cm} \\ \text { \# } 3 & 2 \mathrm{ft} 0.3 \mathrm{in} \\ & 131.75 \mathrm{~cm} \\ \text { \# 4 } & 4 \mathrm{ft} 3.9 \mathrm{in} \\ & 41.75 \mathrm{~cm} \\ \text { \# 5 } & 1 \mathrm{ft} 4.4 \mathrm{in} \\ & 51.75 \mathrm{~cm} \\ \text { \# 6 } & 1 \mathrm{ft} 8.4 \mathrm{in} \\ & 96.75 \mathrm{~cm} \\ & 3 \mathrm{ft} 2.1 \mathrm{in}\end{array}$

Source Dimensions $337.86 \mathrm{~cm}$

$30.48 \mathrm{~cm}$

$11 \mathrm{ft} 1.0 \mathrm{in}$

$1 \mathrm{ft}$

\section{Dose Points}

$\frac{Y}{9} \mathrm{~cm}$
$168.93 \mathrm{ct} 6.5 \mathrm{in}$
$5 \mathrm{ft}$
$168.93 \mathrm{~cm}$
$5 \mathrm{ft} 6.5 \mathrm{in}$
$168.93 \mathrm{~cm}$
$5 \mathrm{ft} 6.5 \mathrm{in}$
$168.93 \mathrm{~cm}$
$5 \mathrm{ft} 6.5 \mathrm{in}$
$168.93 \mathrm{~cm}$
$5 \mathrm{ft} 6.5 \mathrm{in}$
$168.93 \mathrm{~cm}$
$5 \mathrm{ft} 6.5 \mathrm{in}$

$\underline{Z}$
$0 \mathrm{~cm}$
$0.0 \mathrm{in}$
$0 \mathrm{~cm}$
$0.0 \mathrm{in}$
$0 \mathrm{~cm}$
$0.0 \mathrm{in}$
$0 \mathrm{~cm}$
$0.0 \mathrm{in}$
$0 \mathrm{~cm}$
$0.0 \mathrm{in}$
$0 \mathrm{~cm}$
$0.0 \mathrm{in}$

Shields

Shield Name

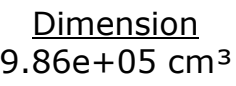

Material

Source

Transition

Air Gap

Wall Clad

Top Clad

$1.27 \mathrm{~cm}$

$1.27 \mathrm{~cm}$

Density

1.8

0.00122

0.00122

$\begin{array}{ll}\text { Air } & 0.001 \\ \text { Iron } & 7.95\end{array}$

Iron $\quad 7.95$

Source Input

Grouping Method : Standard Indices

Number of Groups : 25

Lower Energy Cutoff : 0.015

Photons < 0.015 : Included

Library : ICRP-38

\begin{tabular}{|c|c|}
\hline Nuclide & curies \\
\hline $\mathrm{Am}-241$ & $6.0349 \mathrm{e}-001$ \\
\hline Am-243 & $3.9641 e-004$ \\
\hline Ba-137m & $5.4728 e+002$ \\
\hline $\mathrm{Ce}-144$ & $9.4172 \mathrm{e}-010$ \\
\hline $\mathrm{Cm}-242$ & $1.0058 e-003$ \\
\hline $\mathrm{Cm}-244$ & $3.6978 \mathrm{e}-004$ \\
\hline Co-60 & $6.6167 e-002$ \\
\hline Cs-134 & $3.5992 \mathrm{e}-003$ \\
\hline Cs-135 & $1.0157 \mathrm{e}-002$ \\
\hline Cs-137 & $5.7883 e+002$ \\
\hline Eu-152 & $1.8144 \mathrm{e}-002$ \\
\hline Eu-154 & $1.1833 e+000$ \\
\hline Eu-155 & $8.8058 e-002$ \\
\hline I-129 & $5.2460 \mathrm{e}-005$ \\
\hline $\mathrm{Ni}-63$ & $2.8892 e+001$ \\
\hline $\mathrm{Np}-237$ & $4.9995 e-003$ \\
\hline Pa-233 & $4.9995 e-003$ \\
\hline Pm-147 & $2.0511 \mathrm{e}-002$ \\
\hline Pr-144 & $9.4172 \mathrm{e}-010$ \\
\hline
\end{tabular}

becquerels

2.2329e+010

$1.4667 e+007$

$2.0249 e+013$

$3.4843 e+001$

$3.7215 e+007$

$1.3682 \mathrm{e}+007$

$2.4482 e+009$

$1.3317 \mathrm{e}+008$

$3.7580 e+008$

$2.1417 e+013$

$6.7133 e+008$

$4.3782 e+010$

$3.2581 e+009$

$1.9410 \mathrm{e}+006$

$1.0690 \mathrm{e}+012$

$1.8498 \mathrm{e}+008$

$1.8498 \mathrm{e}+008$

$7.5889 e+008$

$3.4843 e+001$ $\mu \mathrm{Ci} / \mathrm{cm}^{3}$

6.1200e-001

4.0200e-004

$5.5500 e+002$

$9.5500 \mathrm{e}-010$

$1.0200 \mathrm{e}-003$

3.7500e-004

6.7100e-002

3.6500e-003

$1.0300 \mathrm{e}-002$

$5.8700 e+002$

$1.8400 \mathrm{e}-002$

$1.2000 \mathrm{e}+000$

8.9300e-002

5.3200e-005

$2.9300 e+001$

5.0700e-003

5.0700e-003

2.0800e-002

$9.5500 \mathrm{e}-010$
$\mathrm{Bq} / \mathrm{cm}^{3}$

2. $2644 \mathrm{e}+004$

$1.4874 e+001$

$2.0535 e+007$

3.5335e-005

$3.7740 e+001$

$1.3875 e+001$

$2.4827 e+003$

$1.3505 e+002$

$3.8110 \mathrm{e}+002$

$2.1719 \mathrm{e}+007$

$6.8080 e+002$

$4.4400 \mathrm{e}+004$

$3.3041 e+003$

$1.9684 \mathrm{e}+000$

$1.0841 \mathrm{e}+006$

$1.8759 e+002$

$1.8759 e+002$

$7.6960 \mathrm{e}+002$

3.5335e-005 
Page : 2

DOS File : Combined Calcine in Grout.ms6

Run Date: April 5, 2005

Run Time: 3:17:39 PM

Duration : 00:01:02

\begin{tabular}{|c|c|c|c|c|}
\hline Nuclide & curies & becquerels & $\mu \mathrm{Ci} / \mathrm{cm}^{3}$ & $\mathrm{~Bq} / \mathrm{cm}^{3}$ \\
\hline Pu-238 & $6.8237 \mathrm{e}+000$ & $2.5248 \mathrm{e}+011$ & $6.9200 \mathrm{e}+000$ & $2.5604 \mathrm{e}+005$ \\
\hline Pu-239 & $1.5580 \mathrm{e}-001$ & $5.7647 e+009$ & $1.5800 \mathrm{e}-001$ & $5.8460 e+003$ \\
\hline Pu-240 & $9.2791 \mathrm{e}-002$ & $3.4333 e+009$ & $9.4100 \mathrm{e}-002$ & $3.4817 e+003$ \\
\hline Pu-241 & $2.5836 \mathrm{e}+000$ & $9.5592 \mathrm{e}+010$ & $2.6200 e+000$ & $9.6940 e+004$ \\
\hline Pu-242 & $1.2523 e-002$ & $4.6336 e+008$ & $1.2700 \mathrm{e}-002$ & $4.6990 e+002$ \\
\hline Ru-106 & $3.3527 e-008$ & $1.2405 e+003$ & $3.4000 e-008$ & $1.2580 \mathrm{e}-003$ \\
\hline Sb-125 & $9.3481 \mathrm{e}-003$ & $3.4588 \mathrm{e}+008$ & $9.4800 \mathrm{e}-003$ & $3.5076 e+002$ \\
\hline Sb-126 & $9.2101 \mathrm{e}-004$ & $3.4077 e+007$ & $9.3400 \mathrm{e}-004$ & $3.4558 e+001$ \\
\hline Sb-126m & $6.5969 \mathrm{e}-003$ & $2.4409 e+008$ & $6.6900 \mathrm{e}-003$ & $2.4753 e+002$ \\
\hline Sm-151 & $5.9264 \mathrm{e}+000$ & $2.1928 \mathrm{e}+011$ & $6.0100 e+000$ & $2.2237 e+005$ \\
\hline Sr-90 & $5.1573 e+002$ & $1.9082 \mathrm{e}+013$ & $5.2300 e+002$ & $1.9351 e+007$ \\
\hline Tc-99 & $2.4455 e-001$ & $9.0484 e+009$ & $2.4800 \mathrm{e}-001$ & $9.1760 e+003$ \\
\hline Th-230 & $2.5638 \mathrm{e}-005$ & $9.4862 e+005$ & $2.6000 \mathrm{e}-005$ & $9.6200 \mathrm{e}-001$ \\
\hline Th-231 & $2.4751 \mathrm{e}-005$ & $9.1578 \mathrm{e}+005$ & $2.5100 \mathrm{e}-005$ & $9.2870 \mathrm{e}-001$ \\
\hline$U-232$ & $1.7848 \mathrm{e}-005$ & $6.6038 e+005$ & $1.8100 \mathrm{e}-005$ & $6.6970 \mathrm{e}-001$ \\
\hline$U-233$ & $1.1833 \mathrm{e}-006$ & $4.3782 e+004$ & $1.2000 \mathrm{e}-006$ & $4.4400 \mathrm{e}-002$ \\
\hline$U-234$ & $4.7234 \mathrm{e}-003$ & $1.7476 \mathrm{e}+008$ & $4.7900 \mathrm{e}-003$ & $1.7723 e+002$ \\
\hline$U-235$ & $2.4258 \mathrm{e}-005$ & $8.9754 \mathrm{e}+005$ & $2.4600 \mathrm{e}-005$ & $9.1020 \mathrm{e}-001$ \\
\hline$U-236$ & $7.4351 \mathrm{e}-005$ & $2.7510 \mathrm{e}+006$ & $7.5400 \mathrm{e}-005$ & $2.7898 e+000$ \\
\hline$U-237$ & $6.4490 \mathrm{e}-005$ & $2.3861 \mathrm{e}+006$ & $6.5400 \mathrm{e}-005$ & $2.4198 e+000$ \\
\hline$U-238$ & $9.0720 e-006$ & $3.3567 e+005$ & $9.2000 \mathrm{e}-006$ & $3.4040 \mathrm{e}-001$ \\
\hline Y-90 & $5.1573 e+002$ & $1.9082 e+013$ & $5.2300 e+002$ & $1.9351 e+007$ \\
\hline
\end{tabular}

$\begin{array}{cc}\frac{\text { Energy }}{\text { MeV }} & \begin{array}{c}\text { Activity } \\ \text { photons/sec }\end{array} \\ 0.015 & 1.847 \mathrm{e}+10 \\ 0.02 & 1.028 \mathrm{e}+10 \\ 0.03 & 1.227 \mathrm{e}+12 \\ 0.04 & 3.021 \mathrm{e}+11 \\ 0.05 & 2.671 \mathrm{e}+09 \\ 0.06 & 8.024 \mathrm{e}+09 \\ 0.08 & 1.059 \mathrm{e}+09 \\ 0.1 & 1.869 \mathrm{e}+10 \\ 0.15 & 5.974 \mathrm{e}+07 \\ 0.2 & 3.089 \mathrm{e}+09 \\ 0.3 & 3.682 \mathrm{e}+08 \\ 0.4 & 7.502 \mathrm{e}+08 \\ 0.5 & 2.388 \mathrm{e}+08 \\ 0.6 & 1.818 \mathrm{e}+13 \\ 0.8 & 1.675 \mathrm{e}+10 \\ 1.0 & 1.636 \mathrm{e}+10 \\ 1.5 & 1.975 \mathrm{e}+10 \\ 2.0 & 8.316 \mathrm{e}+05 \\ 3.0 & 8.813 \mathrm{e}+01\end{array}$

Radial

Integration Parameters

Y Direction (axial) $\quad 24$

Results - Dose Point \# 1 - $(34.29,168.93,0) \mathbf{c m}$

\begin{tabular}{|c|c|c|c|}
\hline Fluence Rate & Fluence Rate & Exposure Rate & Exposure Rate \\
\hline $\mathrm{MeV} / \mathrm{cm}^{2} / \mathrm{sec}$ & $\mathrm{MeV} / \mathrm{cm}^{2} / \mathrm{sec}$ & $\mathrm{mR} / \mathrm{hr}$ & $\mathrm{mR} / \mathrm{hr}$ \\
\hline No Buildup & With Buildup & No Buildup & With Buildup \\
\hline $2.311 \mathrm{e}-267$ & $5.968 e-23$ & $1.982 \mathrm{e}-268$ & $5.119 e-24$ \\
\hline $3.341 \mathrm{e}-119$ & $5.225 e-23$ & $1.157 \mathrm{e}-120$ & $1.810 \mathrm{e}-24$ \\
\hline $9.120 e-35$ & $1.379 \mathrm{e}-20$ & $9.039 e-37$ & $1.367 \mathrm{e}-22$ \\
\hline $4.691 \mathrm{e}-14$ & $1.876 \mathrm{e}-13$ & $2.075 e-16$ & $8.296 e-16$ \\
\hline $3.333 e-08$ & $2.240 \mathrm{e}-07$ & $8.880 e-11$ & $5.968 e-10$ \\
\hline $4.169 e-04$ & $3.895 e-03$ & $8.281 \mathrm{e}-07$ & $7.736 e-06$ \\
\hline $6.186 \mathrm{e}-02$ & $6.990 e-01$ & $9.789 e-05$ & $1.106 \mathrm{e}-03$ \\
\hline $1.770 \mathrm{e}+01$ & $2.000 e+02$ & $2.708 e-02$ & $3.059 \mathrm{e}-01$ \\
\hline $7.379 e-01$ & $6.961 e+00$ & $1.215 \mathrm{e}-03$ & $1.146 \mathrm{e}-02$ \\
\hline $1.030 \mathrm{e}+02$ & $8.319 e+02$ & $1.818 \mathrm{e}-01$ & $1.468 \mathrm{e}+00$ \\
\hline $3.348 e+01$ & $2.154 \mathrm{e}+02$ & $6.351 \mathrm{e}-02$ & $4.086 \mathrm{e}-01$ \\
\hline $1.254 \mathrm{e}+02$ & $6.841 e+02$ & $2.444 \mathrm{e}-01$ & $1.333 e+00$ \\
\hline $6.267 e+01$ & $3.000 e+02$ & $1.230 \mathrm{e}-01$ & $5.889 e-01$ \\
\hline $6.847 e+06$ & $2.944 \mathrm{e}+07$ & $1.337 e+04$ & $5.746 e+04$ \\
\hline $1.109 e+04$ & $4.053 e+04$ & $2.110 e+01$ & $7.710 e+01$ \\
\hline $1.675 e+04$ & $5.425 e+04$ & $3.087 e+01$ & $9.999 e+01$ \\
\hline $4.405 e+04$ & $1.167 e+05$ & $7.410 e+01$ & $1.963 e+02$ \\
\hline $3.144 \mathrm{e}+00$ & $7.392 e+00$ & $4.862 e-03$ & $1.143 e-02$ \\
\hline $6.700 e-04$ & $1.359 \mathrm{e}-03$ & $9.090 \mathrm{e}-07$ & $1.844 \mathrm{e}-06$ \\
\hline $6.920 e+06$ & $2.965 e+07$ & $1.349 \mathrm{e}+04$ & $5.784 e+04$ \\
\hline
\end{tabular}

Results - Dose Point \# 2 - $(61.75,168.93,0) \mathrm{cm}$ 
Page : 3

DOS File : Combined Calcine in Grout.ms6

Run Date: April 5, 2005

Run Time: 3:17:39 PM

Duration : 00:01:02

$\begin{array}{cc}\frac{\text { Energy }}{\underline{\mathrm{MeV}}} & \begin{array}{c}\text { Activity } \\ \text { photons/sec }\end{array} \\ 0.015 & \\ 0.02 & 1.847 \mathrm{e}+10 \\ 0.03 & 1.028 \mathrm{e}+10 \\ 0.04 & 1.227 \mathrm{e}+12 \\ 0.05 & 3.021 \mathrm{e}+11 \\ 0.06 & 2.671 \mathrm{e}+09 \\ 0.08 & 8.024 \mathrm{e}+09 \\ 0.1 & 1.059 \mathrm{e}+09 \\ 0.15 & 1.869 \mathrm{e}+10 \\ 0.2 & 5.974 \mathrm{e}+07 \\ 0.3 & 3.089 \mathrm{e}+09 \\ 0.4 & 3.682 \mathrm{e}+08 \\ 0.5 & 7.502 \mathrm{e}+08 \\ 0.6 & 2.388 \mathrm{e}+08 \\ 0.8 & 1.818 \mathrm{e}+13 \\ 1.0 & 1.675 \mathrm{e}+10 \\ 1.5 & 1.636 \mathrm{e}+10 \\ 2.0 & 1.975 \mathrm{e}+10 \\ 3.0 & 8.316 \mathrm{e}+05 \\ & 8.813 \mathrm{e}+01\end{array}$

\begin{tabular}{c}
$\frac{\text { Fluence Rate }}{\text { MeV/cm } 2 / s e c}$ \\
\hline No Buildup \\
\hline $4.662 \mathrm{e}-250$ \\
$9.323 \mathrm{e}-113$ \\
$9.017 \mathrm{e}-34$ \\
$4.477 \mathrm{e}-14$ \\
$1.827 \mathrm{e}-08$ \\
$1.987 \mathrm{e}-04$ \\
$2.971 \mathrm{e}-02$ \\
$8.822 \mathrm{e}+00$ \\
$3.749 \mathrm{e}-01$ \\
$5.222 \mathrm{e}+01$ \\
$1.689 \mathrm{e}+01$ \\
$6.305 \mathrm{e}+01$ \\
$3.142 \mathrm{e}+01$ \\
$3.425 \mathrm{e}+06$ \\
$5.531 \mathrm{e}+03$ \\
$8.325 \mathrm{e}+03$ \\
$2.180 \mathrm{e}+04$ \\
$1.552 \mathrm{e}+00$ \\
$3.299 \mathrm{e}-04$ \\
\end{tabular}

Fluence Rate

$\mathrm{MeV} / \mathrm{cm}^{2} / \mathrm{sec}$

With Buildup

$2.619 \mathrm{e}-23$

$2.293 \mathrm{e}-23$

$6.053 \mathrm{e}-21$

$1.775 \mathrm{e}-13$

$1.210 \mathrm{e}-07$

$1.844 \mathrm{e}-03$

$3.415 \mathrm{e}-01$

$1.018 \mathrm{e}+02$

$3.544 \mathrm{e}+00$

$4.189 \mathrm{e}+02$

$1.074 \mathrm{e}+02$

$3.396 \mathrm{e}+02$

$1.485 \mathrm{e}+02$

$1.455 \mathrm{e}+07$

$1.998 \mathrm{e}+04$

$2.669 \mathrm{e}+04$

$5.725 e+04$

$3.622 \mathrm{e}+00$

6.654e-04

$3.461 e+06$

$1.465 e+07$
Exposure Rate $\mathrm{mR} / \mathrm{hr}$

No Buildup

3.999e-251

$3.230 \mathrm{e}-114$

$8.936 \mathrm{e}-36$

$1.980 \mathrm{e}-16$

4.866e-11

3.948e-07

4.701e-05

1.350e-02

6.174e-04

$9.216 \mathrm{e}-02$

3.203e-02

$1.229 \mathrm{e}-01$

6.167e-02

$6.686 e+03$

$1.052 \mathrm{e}+01$

$1.535 \mathrm{e}+01$

$3.668 \mathrm{e}+01$

$2.400 \mathrm{e}-03$

4.476e-07
$6.749 e+03$
Exposure Rate $\mathrm{mR} / \mathrm{hr}$

With Buildup

2.246e-24

$7.942 \mathrm{e}-25$

$5.998 \mathrm{e}-23$

$7.848 \mathrm{e}-16$

$3.224 \mathrm{e}-10$

3.664e-06

5.404e-04

$1.557 \mathrm{e}-01$

5.837e-03

7.393e-01

2.037e-01

6.618e-01

2.916e-01

$2.840 \mathrm{e}+04$

$3.800 \mathrm{e}+01$

$4.920 \mathrm{e}+01$

$9.632 \mathrm{e}+01$

$5.602 \mathrm{e}-03$

$9.027 \mathrm{e}-07$

$2.858 e+04$

\begin{tabular}{|c|c|}
\hline$\frac{\text { Energy }}{\mathrm{MeV}}$ & $\begin{array}{c}\text { Activity } \\
\text { photons/sec }\end{array}$ \\
\hline 0.015 & $1.847 e+10$ \\
\hline 0.02 & $1.028 e+10$ \\
\hline 0.03 & $1.227 e+12$ \\
\hline 0.04 & $3.021 \mathrm{e}+11$ \\
\hline 0.05 & $2.671 \mathrm{e}+09$ \\
\hline 0.06 & $8.024 e+09$ \\
\hline 0.08 & $1.059 e+09$ \\
\hline 0.1 & $1.869 e+10$ \\
\hline 0.15 & $5.974 \mathrm{e}+07$ \\
\hline 0.2 & $3.089 e+09$ \\
\hline 0.3 & $3.682 \mathrm{e}+08$ \\
\hline 0.4 & $7.502 e+08$ \\
\hline 0.5 & $2.388 e+08$ \\
\hline 0.6 & $1.818 e+13$ \\
\hline 0.8 & $1.675 \mathrm{e}+10$ \\
\hline 1.0 & $1.636 e+10$ \\
\hline 1.5 & $1.975 e+10$ \\
\hline 2.0 & $8.316 e+05$ \\
\hline 3.0 & $8.813 e+01$ \\
\hline
\end{tabular}

TOTALS:

$1.983 e+13$

$\begin{array}{ll}\frac{\text { Energy }}{\text { MeV }} & \begin{array}{c}\text { Activity } \\ \text { photons/sec }\end{array} \\ 0.015 & 1.847 \mathrm{e}+10 \\ 0.02 & 1.028 \mathrm{e}+10 \\ 0.03 & 1.227 \mathrm{e}+12\end{array}$

\section{Results - Dose Point \# 3 - $(131.75,168.93,0) \mathrm{cm}$}

\section{Fluence Rate}

$\mathrm{MeV} / \mathrm{cm}^{2} / \mathrm{sec}$

No Buildup

$1.343 e-250$

$3.602 \mathrm{e}-113$

$4.216 \mathrm{e}-34$

$2.057 \mathrm{e}-14$

$8.359 \mathrm{e}-09$

$9.110 \mathrm{e}-05$

$1.357 \mathrm{e}-02$

$4.012 \mathrm{e}+00$

$1.681 \mathrm{e}-01$

$2.318 \mathrm{e}+01$

$7.413 \mathrm{e}+00$

$2.749 \mathrm{e}+01$

$1.363 \mathrm{e}+01$

$1.479 \mathrm{e}+06$

$2.371 \mathrm{e}+03$

$3.546 e+03$

$9.169 \mathrm{e}+03$

$6.471 \mathrm{e}-01$

$1.359 \mathrm{e}-04$

$1.494 \mathrm{e}+06$
Fluence Rate

$\mathrm{MeV} / \mathrm{cm}^{2} / \mathrm{sec}$

With Buildup

$8.863 \mathrm{e}-24$

$7.760 \mathrm{e}-24$

$2.048 \mathrm{e}-21$

$8.154 \mathrm{e}-14$

$5.542 \mathrm{e}-08$

8.460e-04

$1.561 \mathrm{e}-01$

$4.624 \mathrm{e}+01$

$1.574 \mathrm{e}+00$

$1.828 \mathrm{e}+02$

$4.606 \mathrm{e}+01$

$1.443 e+02$

$6.272 \mathrm{e}+01$

$6.112 \mathrm{e}+06$

$8.327 e+03$

$1.106 \mathrm{e}+04$

$2.343 e+04$

$1.470 \mathrm{e}+00$

2.672e-04

$6.155 e+06$
Exposure Rate $\mathrm{mR} / \mathrm{hr}$

No Buildup

1.152e-251

$1.248 \mathrm{e}-114$

$4.179 \mathrm{e}-36$

9.098e-17

2.227e-11

1.809e-07

2.148e-05

$6.138 \mathrm{e}-03$

2.768e-04

4.091e-02

$1.406 \mathrm{e}-02$

5.357e-02

$2.675 \mathrm{e}-02$

$2.887 e+03$

$4.509 \mathrm{e}+00$

$6.536 \mathrm{e}+00$

$1.543 \mathrm{e}+01$

$1.001 \mathrm{e}-03$

$1.844 \mathrm{e}-07$

$2.914 \mathrm{e}+03$
Exposure Rate $\mathrm{mR} / \mathrm{hr}$

With Buildup

$7.602 \mathrm{e}-25$

$2.688 \mathrm{e}-25$

$2.030 \mathrm{e}-23$

$3.606 \mathrm{e}-16$

$1.476 \mathrm{e}-10$

$1.680 \mathrm{e}-06$

2.471e-04

7.074e-02

2.591e-03

3.227e-01

8.736e-02

2.812e-01

$1.231 \mathrm{e}-01$

$1.193 e+04$

$1.584 \mathrm{e}+01$

$2.038 \mathrm{e}+01$

$3.942 \mathrm{e}+01$

2.274e-03

3.625e-07

$1.201 \mathrm{e}+04$

\section{Results - Dose Point \# 4 - $(41.75,168.93,0) \mathrm{cm}$}

\begin{tabular}{|c|c|c|}
\hline Fluence Rate & Fluence Rate & Exposure Rate \\
\hline $\mathrm{MeV} / \mathrm{cm}^{2} / \mathrm{sec}$ & $\mathrm{MeV} / \mathrm{cm}^{2} / \mathrm{sec}$ & $\mathrm{mR} / \mathrm{hr}$ \\
\hline No Buildup & With Buildup & No Buildup \\
\hline $1.914 \mathrm{e}-251$ & $4.449 \mathrm{e}-23$ & $1.642 \mathrm{e}-252$ \\
\hline $6.174 \mathrm{e}-113$ & $3.895 e-23$ & $2.139 e-114$ \\
\hline $1.591 \mathrm{e}-33$ & $1.028 \mathrm{e}-20$ & $1.577 e-35$ \\
\hline
\end{tabular}

Exposure Rate $\mathrm{mR} / \mathrm{hr}$

With Buildup

$3.816 \mathrm{e}-24$

$1.349 \mathrm{e}-24$

$1.019 \mathrm{e}-22$ 
Page : 4

DOS File : Combined Calcine in Grout.ms6

Run Date: April 5, 2005

Run Time: 3:17:39 PM

Duration : 00:01:02

$\begin{array}{ccc}\begin{array}{cc}\text { Energy } \\ \text { MeV }\end{array} & \begin{array}{c}\text { Activity } \\ \text { photons/sec }\end{array} \\ 0.04 & & 3.021 \mathrm{e}+11 \\ 0.05 & & 2.671 \mathrm{e}+09 \\ 0.06 & & 8.024 \mathrm{e}+09 \\ 0.08 & 1.059 \mathrm{e}+09 \\ 0.1 & 1.869 \mathrm{e}+10 \\ 0.15 & 5.974 \mathrm{e}+07 \\ 0.2 & 3.089 \mathrm{e}+09 \\ 0.3 & 3.682 \mathrm{e}+08 \\ 0.4 & 7.502 \mathrm{e}+08 \\ 0.5 & 2.388 \mathrm{e}+08 \\ 0.6 & 1.818 \mathrm{e}+13 \\ 0.8 & 1.675 \mathrm{e}+10 \\ 1.0 & 1.636 \mathrm{e}+10 \\ 1.5 & 1.975 \mathrm{e}+10 \\ 2.0 & 8.316 \mathrm{e}+05 \\ 3.0 & 8.813 \mathrm{e}+01\end{array}$

\begin{tabular}{|c|c|c|}
\hline Fluence Rate & Fluence Rate & Exposure Rate \\
\hline $\mathrm{MeV} / \mathrm{cm}^{2} / \mathrm{sec}$ & $\mathrm{MeV} / \mathrm{cm}^{2} / \mathrm{sec}$ & $\mathrm{mR} / \mathrm{hr}$ \\
\hline No Buildup & With Buildup & No Buildup \\
\hline $7.797 e-14$ & $3.087 e-13$ & $3.448 \mathrm{e}-16$ \\
\hline $2.798 \mathrm{e}-08$ & $1.845 \mathrm{e}-07$ & $7.453 e-11$ \\
\hline $2.918 \mathrm{e}-04$ & $2.706 \mathrm{e}-03$ & $5.797 e-07$ \\
\hline $4.453 e-02$ & $5.143 e-01$ & $7.047 e-05$ \\
\hline $1.339 \mathrm{e}+01$ & $1.551 \mathrm{e}+02$ & $2.049 \mathrm{e}-02$ \\
\hline $5.753 e-01$ & $5.463 e+00$ & $9.474 \mathrm{e}-04$ \\
\hline $8.045 e+01$ & $6.487 e+02$ & $1.420 \mathrm{e}-01$ \\
\hline $2.610 e+01$ & $1.669 e+02$ & $4.952 \mathrm{e}-02$ \\
\hline $9.765 e+01$ & $5.289 e+02$ & $1.903 \mathrm{e}-01$ \\
\hline $4.872 e+01$ & $2.316 \mathrm{e}+02$ & $9.562 \mathrm{e}-02$ \\
\hline $5.316 \mathrm{e}+06$ & $2.270 e+07$ & $1.038 \mathrm{e}+04$ \\
\hline $8.597 e+03$ & $3.121 \mathrm{e}+04$ & $1.635 e+01$ \\
\hline $1.296 \mathrm{e}+04$ & $4.173 e+04$ & $2.388 e+01$ \\
\hline $3.399 e+04$ & $8.965 e+04$ & $5.719 e+01$ \\
\hline $2.423 e+00$ & $5.679 e+00$ & $3.748 \mathrm{e}-03$ \\
\hline $5.159 e-04$ & $1.044 \mathrm{e}-03$ & $7.000 e-07$ \\
\hline $5.372 e+06$ & $2.286 e+07$ & $1.047 e+04$ \\
\hline
\end{tabular}

Exposure Rate $\mathrm{mR} / \mathrm{hr}$

With Buildup

$1.365 \mathrm{e}-15$

$4.915 \mathrm{e}-10$

$5.375 \mathrm{e}-06$

$8.138 \mathrm{e}-04$

$2.373 e-01$

$8.996 \mathrm{e}-03$

$1.145 \mathrm{e}+00$

$3.167 \mathrm{e}-01$

$1.031 \mathrm{e}+00$

$4.546 \mathrm{e}-01$

$4.431 \mathrm{e}+04$

$5.936 \mathrm{e}+01$

$7.692 \mathrm{e}+01$

$1.508 \mathrm{e}+02$

$8.781 \mathrm{e}-03$

$1.417 \mathrm{e}-06$

TOTALS: $\quad 1.983 \mathrm{e}+13$

Results - Dose Point \# 5 - $(51.75,168.93,0) \mathrm{cm}$

$\begin{array}{cc}\begin{array}{cc}\text { Energy } \\ \underline{\mathrm{MeV}}\end{array} & \begin{array}{c}\text { Activity } \\ \text { photons/sec }\end{array} \\ 0.015 & \\ 0.02 & 1.847 \mathrm{e}+10 \\ 0.03 & 1.028 \mathrm{e}+10 \\ 0.04 & 1.227 \mathrm{e}+12 \\ 0.05 & 3.021 \mathrm{e}+11 \\ 0.06 & 2.671 \mathrm{e}+09 \\ 0.08 & 8.024 \mathrm{e}+09 \\ 0.1 & 1.059 \mathrm{e}+09 \\ 0.15 & 1.869 \mathrm{e}+10 \\ 0.2 & 5.974 \mathrm{e}+07 \\ 0.3 & 3.089 \mathrm{e}+09 \\ 0.4 & 3.682 \mathrm{e}+08 \\ 0.5 & 7.502 \mathrm{e}+08 \\ 0.6 & 2.388 \mathrm{e}+08 \\ 0.8 & 1.818 \mathrm{e}+13 \\ 1.0 & 1.675 \mathrm{e}+10 \\ 1.5 & 1.636 \mathrm{e}+10 \\ 2.0 & 1.975 \mathrm{e}+10 \\ 3.0 & 8.316 \mathrm{e}+05 \\ & 8.813 \mathrm{e}+01\end{array}$

$\underline{\text { Fluence Rate }}$

No Buildup

3.987e-250

$1.212 \mathrm{e}-112$

$1.196 \mathrm{e}-33$

$5.215 \mathrm{e}-14$

$2.169 \mathrm{e}-08$

$2.391 \mathrm{e}-04$

$3.576 \mathrm{e}-02$

$1.063 e+01$

$4.532 \mathrm{e}-01$

$6.319 \mathrm{e}+01$

$2.046 \mathrm{e}+01$

$7.642 \mathrm{e}+01$

$3.809 \mathrm{e}+01$

$4.154 \mathrm{e}+06$

$6.712 \mathrm{e}+03$

$1.011 \mathrm{e}+04$

$2.649 \mathrm{e}+04$

$1.887 \mathrm{e}+00$

4.015e-04

$4.198 \mathrm{e}+06$
Fluence Rate

$\mathrm{MeV} / \mathrm{cm}^{2} / \mathrm{sec}$

With Buildup

$3.319 \mathrm{e}-23$

$2.906 \mathrm{e}-23$

$7.671 \mathrm{e}-21$

$2.066 \mathrm{e}-13$

$1.439 \mathrm{e}-07$

$2.220 \mathrm{e}-03$

$4.110 \mathrm{e}-01$

$1.227 \mathrm{e}+02$

$4.290 \mathrm{e}+00$

$5.076 \mathrm{e}+02$

$1.303 e+02$

$4.124 \mathrm{e}+02$

$1.804 \mathrm{e}+02$

$1.768 \mathrm{e}+07$

$2.429 e+04$

$3.247 \mathrm{e}+04$

$6.971 \mathrm{e}+04$

$4.414 \mathrm{e}+00$

8.116e-04

$1.781 \mathrm{e}+07$
Exposure Rate $\mathrm{mR} / \mathrm{hr}$

No Buildup

3.419e-251

$4.198 \mathrm{e}-114$

$1.185 \mathrm{e}-35$

2.307e-16

5.777e-11

4.749e-07

5.659e-05

$1.627 \mathrm{e}-02$

7.463e-04

$1.115 \mathrm{e}-01$

3.880e-02

$1.489 \mathrm{e}-01$

$7.477 \mathrm{e}-02$

$8.109 e+03$

$1.277 \mathrm{e}+01$

$1.863 e+01$

$4.457 \mathrm{e}+01$

$2.918 \mathrm{e}-03$

5.448e-07

$8.185 e+03$

$4.460 e+04$

Exposure Rate $\mathrm{mR} / \mathrm{hr}$

With Buildup

2.847e-24

$1.007 \mathrm{e}-24$

$7.602 e-23$

$9.139 \mathrm{e}-16$

$3.833 \mathrm{e}-10$

$4.409 \mathrm{e}-06$

$6.505 \mathrm{e}-04$

$1.877 \mathrm{e}-01$

7.064e-03

8.959e-01

2.472e-01

8.035e-01

3.542e-01

$3.450 \mathrm{e}+04$

$4.620 \mathrm{e}+01$

$5.985 e+01$

$1.173 e+02$

6.826e-03

1.101e-06

$3.473 e+04$

\section{Results - Dose Point \# 6 - $(96.75,168.93,0) \mathrm{cm}$}

$\begin{array}{cc}\frac{\text { Energy }}{\text { MeV }} & \begin{array}{c}\text { Activity } \\ \text { photons/sec }\end{array} \\ 0.015 & \\ 0.02 & 1.847 \mathrm{e}+10 \\ 0.03 & 1.028 \mathrm{e}+10 \\ 0.04 & 1.227 \mathrm{e}+12 \\ 0.05 & 3.021 \mathrm{e}+11 \\ 0.06 & 2.671 \mathrm{e}+09 \\ & 8.024 \mathrm{e}+09\end{array}$

\begin{tabular}{c}
$\frac{\text { Fluence Rate }}{\text { MeV/cm } 2 / \mathrm{sec}}$ \\
\hline$\frac{\text { With Buildup }}{1.406 \mathrm{e}-23}$ \\
$1.231 \mathrm{e}-23$ \\
$3.250 \mathrm{e}-21$ \\
$1.122 \mathrm{e}-13$ \\
$7.618 \mathrm{e}-08$ \\
$1.163 \mathrm{e}-03$
\end{tabular}

$\frac{\text { Exposure Rate }}{\text { mR/hr }}$
No Buildup
$1.783 \mathrm{e}-251$
$1.672 \mathrm{e}-114$
$5.789 \mathrm{e}-36$
$1.252 \mathrm{e}-16$
$3.061 \mathrm{e}-11$
$2.487 \mathrm{e}-07$

Exposure Rate $\mathrm{mR} / \mathrm{hr}$

With Buildup

$1.206 \mathrm{e}-24$

$4.265 \mathrm{e}-25$

$3.221 \mathrm{e}-23$

$4.963 \mathrm{e}-16$

$2.029 \mathrm{e}-10$

$2.309 \mathrm{e}-06$ 
Page : 5

DOS File : Combined Calcine in Grout.ms6

Run Date: April 5, 2005

Run Time: 3:17:39 PM

Duration : 00:01:02

$\begin{array}{ccccc}\frac{\text { Energy }}{\text { MeV }} & \begin{array}{c}\text { Activity } \\ \text { photons } / \mathrm{sec}\end{array} & \frac{\text { Fluence Rate }}{\text { MeV/cm } 2 / \mathrm{sec}} & \frac{\text { Fluence Rate }}{\text { MeV/cm } 2 / \mathrm{sec}} \\ 0.08 & 1.059 \mathrm{e}+09 & \frac{\text { No Buildup }}{1.867 \mathrm{e}-02} & \frac{\text { With Buildup }^{2}}{2.147 \mathrm{e}-01} \\ 0.1 & 1.869 \mathrm{e}+10 & 5.531 \mathrm{e}+00 & 6.381 \mathrm{e}+01 \\ 0.15 & 5.974 \mathrm{e}+07 & 2.341 \mathrm{e}-01 & 2.209 \mathrm{e}+00 \\ 0.2 & 3.089 \mathrm{e}+09 & 3.251 \mathrm{e}+01 & 2.596 \mathrm{e}+02 \\ 0.3 & 3.682 \mathrm{e}+08 & 1.048 \mathrm{e}+01 & 6.610 \mathrm{e}+01 \\ 0.4 & 7.502 \mathrm{e}+08 & 3.903 \mathrm{e}+01 & 2.083 \mathrm{e}+02 \\ 0.5 & 2.388 \mathrm{e}+08 & 1.941 \mathrm{e}+01 & 9.083 \mathrm{e}+01 \\ 0.6 & 1.818 \mathrm{e}+13 & 2.113 \mathrm{e}+06 & 8.875 \mathrm{e}+06 \\ 0.8 & 1.675 \mathrm{e}+10 & 3.401 \mathrm{e}+03 & 1.214 \mathrm{e}+04 \\ 1.0 & 1.636 \mathrm{e}+10 & 5.106 \mathrm{e}+03 & 1.617 \mathrm{e}+04 \\ 1.5 & 1.975 \mathrm{e}+10 & 1.330 \mathrm{e}+04 & 3.449 \mathrm{e}+04 \\ 2.0 & 8.316 \mathrm{e}+05 & 9.429 \mathrm{e}-01 & 2.173 \mathrm{e}+00 \\ 3.0 & 8.813 \mathrm{e}+01 & 1.993 \mathrm{e}-04 & 3.969 \mathrm{e}-04 \\ & & & \\ \text { TOTALS: } & 1.983 \mathrm{e}+13 & 2.135 \mathrm{e}+06 & 8.939 \mathrm{e}+06\end{array}$

$\frac{\text { Exposure Rate }}{\mathrm{mR} / \mathrm{hr}}$

No Buildup

2.955e-05

$8.462 \mathrm{e}-03$

$3.855 \mathrm{e}-04$

$5.738 \mathrm{e}-02$

1.987e-02

$7.605 \mathrm{e}-02$

$3.810 \mathrm{e}-02$

$4.124 \mathrm{e}+03$

$6.470 e+00$

$9.413 e+00$

$2.237 e+01$

$1.458 \mathrm{e}-03$

2.704e-07

$4.162 \mathrm{e}+03$
Exposure Rate $\mathrm{mR} / \mathrm{hr}$

With Buildup

3.397e-04

$9.762 \mathrm{e}-02$

$3.637 \mathrm{e}-03$

4.582e-01

$1.254 \mathrm{e}-01$

4.058e-01

$1.783 \mathrm{e}-01$

$1.732 \mathrm{e}+04$

$2.310 \mathrm{e}+01$

$2.981 \mathrm{e}+01$

$5.802 \mathrm{e}+01$

$3.360 \mathrm{e}-03$

5.384e-07

$1.744 \mathrm{e}+04$ 
Page : 1

DOS File : Combined Calcine Grout in Canister - Top - No Lid.ms6

Run Date: April 5, 2005

Run Time: 2:39:39 PM

Duration : 00:01:05
File Ref:

Date:

By:

Checked:

Case Title: Combined Calcine Grt

Description: Radiation measured from above open canister.

Geometry: 8 - Cylinder Volume - End Shields

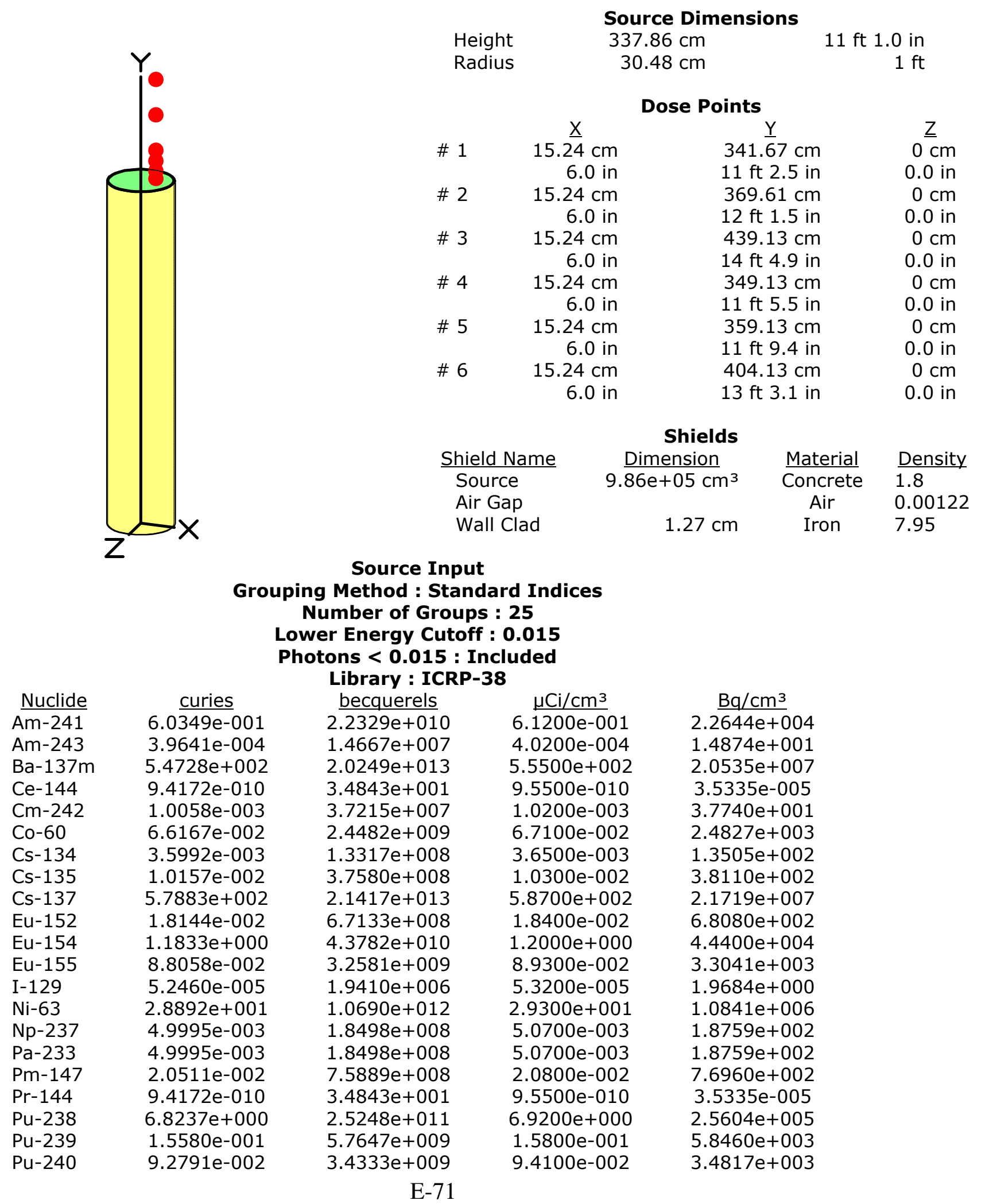


Page : : 2

DOS File : Combined Calcine Grout in Canister - Top - No Lid.ms6

Run Date: April 5, 2005

Run Time: 2:39:39 PM

Duration : 00:01:05

$\begin{array}{lc}\begin{array}{cc}\text { Nuclide } \\ \text { Pu-241 }\end{array} & 2.5836 \mathrm{e}+000 \\ \text { Pu-242 } & 1.2523 \mathrm{e}-002 \\ \text { Ru-106 } & 3.3527 \mathrm{e}-008 \\ \mathrm{Sb}-125 & 9.3481 \mathrm{e}-003 \\ \mathrm{Sb}-126 & 9.2101 \mathrm{e}-004 \\ \mathrm{Sb}-126 \mathrm{~m} & 6.5969 \mathrm{e}-003 \\ \mathrm{Sm}-151 & 5.9264 \mathrm{e}+000 \\ \mathrm{Sr}-90 & 5.1573 \mathrm{e}+002 \\ \text { Tc-99 } & 2.4455 \mathrm{e}-001 \\ \text { Th-230 } & 2.5638 \mathrm{e}-005 \\ \text { Th-231 } & 2.4751 \mathrm{e}-005 \\ \mathrm{U}-232 & 1.7848 \mathrm{e}-005 \\ \mathrm{U}-233 & 1.1833 \mathrm{e}-006 \\ \mathrm{U}-234 & 4.7234 \mathrm{e}-003 \\ \mathrm{U}-235 & 2.4258 \mathrm{e}-005 \\ \mathrm{U}-236 & 7.4351 \mathrm{e}-005 \\ \mathrm{U}-237 & 6.4490 \mathrm{e}-005 \\ \mathrm{U}-238 & 9.0720 \mathrm{e}-006 \\ \mathrm{Y}-90 & 5.1573 \mathrm{e}+002\end{array}$

$\underline{\text { becquerels }}$
$9.5592 \mathrm{e}+010$
$4.6336 \mathrm{e}+008$
$1.2405 \mathrm{e}+003$
$3.4588 \mathrm{e}+008$
$3.4077 \mathrm{e}+007$
$2.4409 \mathrm{e}+008$
$2.1928 \mathrm{e}+011$
$1.9082 \mathrm{e}+013$
$9.0484 \mathrm{e}+009$
$9.4862 \mathrm{e}+005$
$9.1578 \mathrm{e}+005$
$6.6038 \mathrm{e}+005$
$4.3782 \mathrm{e}+004$
$1.7476 \mathrm{e}+008$
$8.9754 \mathrm{e}+005$
$2.7510 \mathrm{e}+006$
$2.3861 \mathrm{e}+006$
$3.3567 \mathrm{e}+005$
$1.9082 \mathrm{e}+013$

Buildup

The material reference is : Source
$\mathrm{Bq} / \mathrm{cm}^{3}$

$9.6940 \mathrm{e}+004$

$4.6990 \mathrm{e}+002$

$1.2580 \mathrm{e}-003$

$3.5076 \mathrm{e}+002$

$3.4558 \mathrm{e}+001$

$2.4753 e+002$

$2.2237 e+005$

$1.9351 \mathrm{e}+007$

$9.1760 \mathrm{e}+003$

$9.6200 \mathrm{e}-001$

$9.2870 \mathrm{e}-001$

$6.6970 \mathrm{e}-001$

4.4400e-002

$1.7723 e+002$

$9.1020 \mathrm{e}-001$

$2.7898 \mathrm{e}+000$

$2.4198 \mathrm{e}+000$

3.4040e-001

$1.9351 \mathrm{e}+007$

$\begin{array}{ccc}\begin{array}{cc}\text { Energy } \\ \text { MeV }\end{array} & \begin{array}{c}\text { Activity } \\ \text { photons/sec }\end{array} \\ 0.015 & & 2.244 \mathrm{e}+11 \\ 0.02 & & 1.028 \mathrm{e}+10 \\ 0.03 & & 1.227 \mathrm{e}+12 \\ 0.04 & & 3.021 \mathrm{e}+11 \\ 0.05 & & 2.671 \mathrm{e}+09 \\ 0.06 & & 8.024 \mathrm{e}+09 \\ 0.08 & & 1.059 \mathrm{e}+09 \\ 0.1 & 1.869 \mathrm{e}+10 \\ 0.15 & 5.974 \mathrm{e}+07 \\ 0.2 & 3.089 \mathrm{e}+09 \\ 0.3 & 3.682 \mathrm{e}+08 \\ 0.4 & 7.502 \mathrm{e}+08 \\ 0.5 & 2.388 \mathrm{e}+08 \\ 0.6 & 1.818 \mathrm{e}+13 \\ 0.8 & 1.675 \mathrm{e}+10 \\ 1.0 & 1.636 \mathrm{e}+10 \\ 1.5 & 1.975 \mathrm{e}+10 \\ 2.0 & 8.316 \mathrm{e}+05 \\ 3.0 & 8.813 \mathrm{e}+01\end{array}$

TOTALS: $\frac{\text { Energy }}{\text { MeV }}$

0.015
$2.003 e+13$

Activity photons/sec

$2.244 \mathrm{e}+11$
Radial

Integration Parameters

Circumferential 24

Y Direction (axial)
24

24

Results - Dose Point \# 1 - $(15.24,341.67,0) \mathrm{cm}$

\begin{tabular}{|c|c|c|c|}
\hline$\frac{\text { Fluence Rate }}{\mathrm{MeV} / \mathrm{cm}^{2} / \mathrm{sec}}$ & $\frac{\text { Fluence Rate }}{\mathrm{MeV} / \mathrm{cm}^{2} / \mathrm{sec}}$ & $\frac{\text { Exposure Rate }}{\mathrm{mR} / \mathrm{hr}}$ & $\frac{\text { Exposure Rate }}{\mathrm{mR} / \mathrm{hr}}$ \\
\hline No Buildup & With Buildup & No Buildup & With Buildup \\
\hline $2.043 e-03$ & $2.224 \mathrm{e}-03$ & $1.753 e-04$ & $1.907 e-04$ \\
\hline $2.117 \mathrm{e}-01$ & $2.435 \mathrm{e}-01$ & $7.335 e-03$ & $8.434 \mathrm{e}-03$ \\
\hline $3.019 e+03$ & $4.005 e+03$ & $2.992 \mathrm{e}+01$ & $3.970 e+01$ \\
\hline $3.755 e+03$ & $5.902 e+03$ & $1.661 \mathrm{e}+01$ & $2.610 e+01$ \\
\hline $7.584 e+01$ & $1.383 e+02$ & $2.020 \mathrm{e}-01$ & $3.685 e-01$ \\
\hline $3.819 e+02$ & $8.325 e+02$ & $7.586 \mathrm{e}-01$ & $1.654 \mathrm{e}+00$ \\
\hline $9.461 e+01$ & $2.445 e+02$ & $1.497 e-01$ & $3.869 \mathrm{e}-01$ \\
\hline $2.464 e+03$ & $6.950 e+03$ & $3.770 e+00$ & $1.063 e+01$ \\
\hline $1.436 \mathrm{e}+01$ & $4.222 e+01$ & $2.365 e-02$ & $6.953 e-02$ \\
\hline $1.103 e+03$ & $3.148 e+03$ & $1.947 e+00$ & $5.556 e+00$ \\
\hline $2.276 \mathrm{e}+02$ & $5.951 \mathrm{e}+02$ & $4.318 \mathrm{e}-01$ & $1.129 \mathrm{e}+00$ \\
\hline $6.862 e+02$ & $1.659 e+03$ & $1.337 e+00$ & $3.233 e+00$ \\
\hline $2.966 \mathrm{e}+02$ & $6.741 e+02$ & $5.822 \mathrm{e}-01$ & $1.323 e+00$ \\
\hline $2.905 e+07$ & $6.251 e+07$ & $5.670 e+04$ & $1.220 \mathrm{e}+05$ \\
\hline $3.994 \mathrm{e}+04$ & $7.924 \mathrm{e}+04$ & $7.596 e+01$ & $1.507 e+02$ \\
\hline $5.334 \mathrm{e}+04$ & $9.977 e+04$ & $9.832 e+01$ & $1.839 \mathrm{e}+02$ \\
\hline $1.140 \mathrm{e}+05$ & $1.924 \mathrm{e}+05$ & $1.918 \mathrm{e}+02$ & $3.237 e+02$ \\
\hline $7.171 \mathrm{e}+00$ & $1.140 \mathrm{e}+01$ & $1.109 \mathrm{e}-02$ & $1.762 \mathrm{e}-02$ \\
\hline $1.322 \mathrm{e}-03$ & $1.940 \mathrm{e}-03$ & $1.793 \mathrm{e}-06$ & $2.632 \mathrm{e}-06$ \\
\hline $2.927 e+07$ & $6.291 e+07$ & $5.712 e+04$ & $1.228 \mathrm{e}+05$ \\
\hline
\end{tabular}

Results - Dose Point \# 2 - $(15.24,369.61,0) \mathrm{cm}$

\begin{tabular}{|c|c|c|}
\hline $\begin{array}{l}\text { Fluence Rate } \\
\mathrm{MeV} / \mathrm{cm}^{2} / \mathrm{sec}\end{array}$ & $\begin{array}{l}\text { Fluence Rate } \\
\mathrm{MeV} / \mathrm{cm}^{2} / \mathrm{sec}\end{array}$ & $\begin{array}{c}\text { Exposure Rate } \\
\mathrm{mR} / \mathrm{hr}\end{array}$ \\
\hline No Buildup & With Buildup & No Buildup \\
\hline $1.772 \mathrm{e}-03$ & $1.929 \mathrm{e}-03$ & $1.520 \mathrm{e}-04$ \\
\hline
\end{tabular}

Exposure Rate $\mathrm{mR} / \mathrm{hr}$

With Buildup $1.654 \mathrm{e}-04$ 
Page : 3

DOS File : Combined Calcine Grout in Canister - Top - No Lid.ms6

Run Date: April 5, 2005

Run Time: 2:39:39 PM

Duration : 00:01:05

$\begin{array}{ccc}\begin{array}{cc}\text { Energy } \\ \text { MeV }\end{array} & \begin{array}{c}\text { Activity } \\ \text { photons/sec }\end{array} \\ 0.02 & & 1.028 \mathrm{e}+10 \\ 0.03 & 1.227 \mathrm{e}+12 \\ 0.04 & 3.021 \mathrm{e}+11 \\ 0.05 & 2.671 \mathrm{e}+09 \\ 0.06 & 8.024 \mathrm{e}+09 \\ 0.08 & 1.059 \mathrm{e}+09 \\ 0.1 & 1.869 \mathrm{e}+10 \\ 0.15 & 5.974 \mathrm{e}+07 \\ 0.2 & 3.089 \mathrm{e}+09 \\ 0.3 & 3.682 \mathrm{e}+08 \\ 0.4 & 7.502 \mathrm{e}+08 \\ 0.5 & 2.388 \mathrm{e}+08 \\ 0.6 & 1.818 \mathrm{e}+13 \\ 0.8 & 1.675 \mathrm{e}+10 \\ 1.0 & 1.636 \mathrm{e}+10 \\ 1.5 & 1.975 \mathrm{e}+10 \\ 2.0 & 8.316 \mathrm{e}+05 \\ 3.0 & 8.813 \mathrm{e}+01\end{array}$

\begin{tabular}{l}
$\frac{\text { Fluence Rate }}{\text { MeV/cm } 2 / \mathrm{sec}}$ \\
\hline$\frac{\text { No Buildup }}{1.580 \mathrm{e}-01}$ \\
$1.499 \mathrm{e}+03$ \\
$1.384 \mathrm{e}+03$ \\
$2.428 \mathrm{e}+01$ \\
$1.153 \mathrm{e}+02$ \\
$2.733 \mathrm{e}+01$ \\
$7.007 \mathrm{e}+02$ \\
$4.024 \mathrm{e}+00$ \\
$3.069 \mathrm{e}+02$ \\
$6.285 \mathrm{e}+01$ \\
$1.885 \mathrm{e}+02$ \\
$8.121 \mathrm{e}+01$ \\
$7.933 \mathrm{e}+06$ \\
$1.087 \mathrm{e}+04$ \\
$1.448 \mathrm{e}+04$ \\
$3.086 \mathrm{e}+04$ \\
$1.940 \mathrm{e}+00$ \\
$3.582 \mathrm{e}-04$ \\
$7.993 \mathrm{e}+06$
\end{tabular}

$\begin{array}{cc}\frac{\text { Fluence Rate }}{\text { MeV/cm } / \mathrm{sec}} & \frac{\text { Exposure Rate }}{\mathrm{mR} / \mathrm{hr}} \\ \frac{\text { With Buildup }}{\text { Wo Buildup }} & 5.473 \mathrm{e}-03 \\ 1.813 \mathrm{e}-01 & 1.486 \mathrm{e}+01 \\ 1.946 \mathrm{e}+03 & 6.120 \mathrm{e}+00 \\ 2.052 \mathrm{e}+03 & 6.469 \mathrm{e}-02 \\ 4.058 \mathrm{e}+01 & 2.289 \mathrm{e}-01 \\ 2.316 \mathrm{e}+02 & 4.325 \mathrm{e}-02 \\ 6.576 \mathrm{e}+01 & 1.072 \mathrm{e}+00 \\ 1.863 \mathrm{e}+03 & 6.627 \mathrm{e}-03 \\ 1.131 \mathrm{e}+01 & 5.418 \mathrm{e}-01 \\ 8.424 \mathrm{e}+02 & 1.192 \mathrm{e}-01 \\ 1.592 \mathrm{e}+02 & 3.673 \mathrm{e}-01 \\ 4.443 \mathrm{e}+02 & 1.594 \mathrm{e}-01 \\ 1.807 \mathrm{e}+02 & 1.548 \mathrm{e}+04 \\ 1.677 \mathrm{e}+07 & 2.067 \mathrm{e}+01 \\ 2.130 \mathrm{e}+04 & 2.669 \mathrm{e}+01 \\ 2.687 \mathrm{e}+04 & 5.191 \mathrm{e}+01 \\ 5.201 \mathrm{e}+04 & 3.000 \mathrm{e}-03 \\ 3.092 \mathrm{e}+00 & 4.860 \mathrm{e}-07 \\ 5.298 \mathrm{e}-04 & \\ 1.688 \mathrm{e}+07 & 1.561 \mathrm{e}+04\end{array}$

$\frac{\text { Exposure Rate }}{\mathrm{mR} / \mathrm{hr}}$

With Buildup

$6.279 \mathrm{e}-03$

$1.929 \mathrm{e}+01$

$9.076 \mathrm{e}+00$

$1.081 \mathrm{e}-01$

$4.600 \mathrm{e}-01$

$1.041 \mathrm{e}-01$

$2.850 \mathrm{e}+00$

$1.863 \mathrm{e}-02$

$1.487 \mathrm{e}+00$

$3.020 \mathrm{e}-01$

$8.657 \mathrm{e}-01$

$3.546 \mathrm{e}-01$

$3.273 e+04$

$4.051 \mathrm{e}+01$

$4.952 \mathrm{e}+01$

$8.751 \mathrm{e}+01$

$4.781 \mathrm{e}-03$

$7.188 \mathrm{e}-07$

TOTALS: $\quad 2.003 e+13$

Results - Dose Point \# 3 - (15.24,439.13,0) cm

$\begin{array}{cc}\frac{\begin{array}{c}\text { Energy } \\ \text { MeV }\end{array}}{} & \begin{array}{c}\text { Activity } \\ \text { photons } / \mathrm{sec}\end{array} \\ 0.015 & \\ 0.02 & 2.244 \mathrm{e}+11 \\ 0.03 & 1.028 \mathrm{e}+10 \\ 0.04 & 1.227 \mathrm{e}+12 \\ 0.05 & 3.021 \mathrm{e}+11 \\ 0.06 & 2.671 \mathrm{e}+09 \\ 0.08 & 8.024 \mathrm{e}+09 \\ 0.1 & 1.059 \mathrm{e}+09 \\ 0.15 & 1.869 \mathrm{e}+10 \\ 0.2 & 5.974 \mathrm{e}+07 \\ 0.3 & 3.089 \mathrm{e}+09 \\ 0.4 & 3.682 \mathrm{e}+08 \\ 0.5 & 7.502 \mathrm{e}+08 \\ 0.6 & 2.388 \mathrm{e}+08 \\ 0.8 & 1.818 \mathrm{e}+13 \\ 1.0 & 1.675 \mathrm{e}+10 \\ 1.5 & 1.636 \mathrm{e}+10 \\ 2.0 & 1.975 \mathrm{e}+10 \\ 3.0 & 8.316 \mathrm{e}+05 \\ & 8.813 \mathrm{e}+01\end{array}$

Fluence Rate

Fluence Rate Exposure Rate

$\mathrm{MeV} / \mathrm{cm}^{2} / \mathrm{sec}$

No Buildup

$6.495 \mathrm{e}-04$

$\mathrm{mR} / \mathrm{hr}$

$4.184 \mathrm{e}-02$

$2.795 e+02$

$2.330 \mathrm{e}+02$

$4.040 \mathrm{e}+00$

$1.924 \mathrm{e}+01$

$4.602 \mathrm{e}+00$

$1.186 \mathrm{e}+02$

$6.869 \mathrm{e}-01$

$5.267 \mathrm{e}+01$

$\mathrm{MeV} / \mathrm{cm}^{2} / \mathrm{sec}$

With Buildup

7.067e-04

No Buildup

$4.784 \mathrm{e}-02$

5.571e-05

$3.584 \mathrm{e}+02$

$3.404 \mathrm{e}+02$

$1.449 \mathrm{e}-03$

$2.770 \mathrm{e}+00$

$1.031 \mathrm{e}+00$

$6.754 \mathrm{e}+00 \quad 1.076 \mathrm{e}-02$

$3.882 \mathrm{e}+01 \quad 3.822 \mathrm{e}-02$

$1.133 \mathrm{e}+01 \quad 7.282 \mathrm{e}-03$

$3.257 \mathrm{e}+02 \quad 1.815 \mathrm{e}-01$

$2.011 \mathrm{e}+00 \quad 1.131 \mathrm{e}-03$

$1.512 \mathrm{e}+02 \quad 9.296 \mathrm{e}-02$

$1.087 e+01$

$2.893 e+01$

$2.062 \mathrm{e}-02$

$\begin{array}{lll}3.283 e+01 & 8.146 e+01 & 6.397 e-02\end{array}$

$1.422 \mathrm{e}+01 \quad 3.334 \mathrm{e}+01 \quad 2.792 \mathrm{e}-02$

$1.396 \mathrm{e}+06 \quad 3.112 \mathrm{e}+06 \quad 2.726 \mathrm{e}+03$

$1.930 \mathrm{e}+03 \quad 3.990 \mathrm{e}+03 \quad 3.672 \mathrm{e}+00$

$2.593 e+03 \quad 5.073 e+03 \quad 4.780 e+00$

$\begin{array}{lll}5.621 \mathrm{e}+03 & 9.978 \mathrm{e}+03 & 9.457 \mathrm{e}+00\end{array}$

3.583e-01 $\quad 6.009 \mathrm{e}-01 \quad 5.541 \mathrm{e}-04$

$6.756 \mathrm{e}-05$

$1.050 \mathrm{e}-04$

$9.166 \mathrm{e}-08$

$3.294 \mathrm{e}+04$

TOTALS: $\quad 2.003 e+13$

$1.407 \mathrm{e}+06$

$3.133 e+06$

$2.748 e+03$

Exposure Rate $\mathrm{mR} / \mathrm{hr}$

With Buildup

$6.061 \mathrm{e}-05$

$1.657 \mathrm{e}-03$

$3.552 \mathrm{e}+00$

$1.505 \mathrm{e}+00$

$1.799 \mathrm{e}-02$

$7.711 \mathrm{e}-02$

$1.793 \mathrm{e}-02$

$4.983 \mathrm{e}-01$

3.311e-03

$2.669 \mathrm{e}-01$

$5.488 \mathrm{e}-02$

$1.587 \mathrm{e}-01$

$6.545 \mathrm{e}-02$

$6.075 e+03$

$7.589 e+00$

$9.351 \mathrm{e}+00$

$1.679 \mathrm{e}+01$

$9.292 \mathrm{e}-04$

$1.425 \mathrm{e}-07$

$6.115 e+03$

\section{Results - Dose Point \# 4 - (15.24,349.13,0) cm}

\begin{tabular}{|c|c|}
\hline$\frac{\text { Energy }}{\underline{\mathrm{MeV}}}$ & $\frac{\text { Activity }}{\text { photons/sec }}$ \\
\hline 0.015 & $2.244 \mathrm{e}$ \\
\hline 0.02 & $1.028 e+10$ \\
\hline 0.03 & $1.227 e+12$ \\
\hline & \\
\hline
\end{tabular}

Fluence Rate

$\mathrm{MeV} / \mathrm{cm}^{2} / \mathrm{sec}$

With Buildup

2.190e-03

$2.401 \mathrm{e}-01$

$3.618 \mathrm{e}+03$

$4.674 \mathrm{e}+03$
Exposure Rate $\mathrm{mR} / \mathrm{hr}$

No Buildup

$1.726 \mathrm{e}-04$

$7.232 \mathrm{e}-03$

$2.719 \mathrm{e}+01$

$1.347 \mathrm{e}+01$
Exposure Rate $\mathrm{mR} / \mathrm{hr}$

With Buildup

$1.879 \mathrm{e}-04$

8.315e-03

$3.586 \mathrm{e}+01$

$2.067 \mathrm{e}+01$ 
Page : 4

DOS File : Combined Calcine Grout in Canister - Top - No Lid.ms6

Run Date: April 5, 2005

Run Time: 2:39:39 PM

Duration : 00:01:05

$\begin{array}{ccc}\begin{array}{cc}\text { Energy } \\ \text { MeV }\end{array} & & \begin{array}{c}\text { Activity } \\ \text { photons/sec }\end{array} \\ 0.05 & & 2.671 \mathrm{e}+09 \\ 0.06 & & 8.024 \mathrm{e}+09 \\ 0.08 & & 1.059 \mathrm{e}+09 \\ 0.1 & 1.869 \mathrm{e}+10 \\ 0.15 & 5.974 \mathrm{e}+07 \\ 0.2 & 3.089 \mathrm{e}+09 \\ 0.3 & 3.682 \mathrm{e}+08 \\ 0.4 & 7.502 \mathrm{e}+08 \\ 0.5 & 2.388 \mathrm{e}+08 \\ 0.6 & 1.818 \mathrm{e}+13 \\ 0.8 & 1.675 \mathrm{e}+10 \\ 1.0 & 1.636 \mathrm{e}+10 \\ 1.5 & 1.975 \mathrm{e}+10 \\ 2.0 & 8.316 \mathrm{e}+05 \\ 3.0 & 8.813 \mathrm{e}+01\end{array}$

TOTALS: $\quad 2.003 e+13$

$\begin{array}{cc}\begin{array}{cc}\text { Energy } \\ \text { MeV }\end{array} & \begin{array}{c}\text { Activity } \\ \text { photons/sec }\end{array} \\ 0.015 & 2.244 \mathrm{e}+11 \\ 0.02 & 1.028 \mathrm{e}+10 \\ 0.03 & 1.227 \mathrm{e}+12 \\ 0.04 & 3.021 \mathrm{e}+11 \\ 0.05 & 2.671 \mathrm{e}+09 \\ 0.06 & 8.024 \mathrm{e}+09 \\ 0.08 & 1.059 \mathrm{e}+09 \\ 0.1 & 1.869 \mathrm{e}+10 \\ 0.15 & 5.974 \mathrm{e}+07 \\ 0.2 & 3.089 \mathrm{e}+09 \\ 0.3 & 3.682 \mathrm{e}+08 \\ 0.4 & 7.502 \mathrm{e}+08 \\ 0.5 & 2.388 \mathrm{e}+08 \\ 0.6 & 1.818 \mathrm{e}+13 \\ 0.8 & 1.675 \mathrm{e}+10 \\ 1.0 & 1.636 \mathrm{e}+10 \\ 1.5 & 1.975 \mathrm{e}+10 \\ 2.0 & 8.316 \mathrm{e}+05 \\ 3.0 & 8.813 \mathrm{e}+01\end{array}$

TOTALS: $\quad 2.003 e+13$

$\begin{array}{cc}\frac{\text { Energy }}{\text { MeV }} & \begin{array}{c}\text { Activity } \\ \text { photons/sec }\end{array} \\ 0.015 & \\ 0.02 & 2.244 \mathrm{e}+11 \\ 0.03 & 1.028 \mathrm{e}+10 \\ 0.04 & 1.227 \mathrm{e}+12 \\ 0.05 & 3.021 \mathrm{e}+11 \\ 0.06 & 2.671 \mathrm{e}+09 \\ 0.08 & 8.024 \mathrm{e}+09 \\ & 1.059 \mathrm{e}+09\end{array}$

\begin{tabular}{ccc}
$\frac{\text { Fluence Rate }}{\text { MeV/cm } / \mathrm{sec}}$ & $\frac{\text { Fluence Rate }}{\text { MeV/cm } / \mathrm{sec}}$ & $\begin{array}{c}\text { Exposure Rate } \\
\text { mR/hr } \\
\text { No Buildup }\end{array}$ \\
\hline$\frac{\text { No Buildup }}{5.706 e+01}$ & $\frac{\text { With Buildup }}{9.921 \mathrm{e}+01}$ & $1.520 \mathrm{e}-01$ \\
$2.763 \mathrm{e}+02$ & $5.719 \mathrm{e}+02$ & $5.487 \mathrm{e}-01$ \\
$6.618 \mathrm{e}+01$ & $1.619 \mathrm{e}+02$ & $1.047 \mathrm{e}-01$ \\
$1.701 \mathrm{e}+03$ & $4.559 \mathrm{e}+03$ & $2.602 \mathrm{e}+00$ \\
$9.785 \mathrm{e}+00$ & $2.755 \mathrm{e}+01$ & $1.611 \mathrm{e}-02$ \\
$7.467 \mathrm{e}+02$ & $2.050 \mathrm{e}+03$ & $1.318 \mathrm{e}+00$ \\
$1.529 \mathrm{e}+02$ & $3.868 \mathrm{e}+02$ & $2.901 \mathrm{e}-01$ \\
$4.587 \mathrm{e}+02$ & $1.077 \mathrm{e}+03$ & $8.937 \mathrm{e}-01$ \\
$1.975 \mathrm{e}+02$ & $4.374 \mathrm{e}+02$ & $3.878 \mathrm{e}-01$ \\
$1.929 \mathrm{e}+07$ & $4.054 \mathrm{e}+07$ & $3.765 \mathrm{e}+04$ \\
$2.640 \mathrm{e}+04$ & $5.135 \mathrm{e}+04$ & $5.022 \mathrm{e}+01$ \\
$3.514 \mathrm{e}+04$ & $6.461 \mathrm{e}+04$ & $6.478 \mathrm{e}+01$ \\
$7.469 \mathrm{e}+04$ & $1.245 \mathrm{e}+05$ & $1.257 \mathrm{e}+02$ \\
$4.683 \mathrm{e}+00$ & $7.370 \mathrm{e}+00$ & $7.241 \mathrm{e}-03$ \\
$8.602 \mathrm{e}-04$ & $1.255 \mathrm{e}-03$ & $1.167 \mathrm{e}-06$ \\
$1.943 \mathrm{e}+07$ & $4.080 \mathrm{e}+07$ & $3.794 \mathrm{e}+04$
\end{tabular}

Exposure Rate $\mathrm{mR} / \mathrm{hr}$

With Buildup

$2.643 e-01$

$1.136 \mathrm{e}+00$

$2.561 \mathrm{e}-01$

$6.975 \mathrm{e}+00$

$4.537 \mathrm{e}-02$

$3.618 \mathrm{e}+00$

$7.338 \mathrm{e}-01$

$2.099 \mathrm{e}+00$

$8.585 \mathrm{e}-01$

$7.912 \mathrm{e}+04$

$9.766 \mathrm{e}+01$

$1.191 \mathrm{e}+02$

$2.095 \mathrm{e}+02$

$1.140 \mathrm{e}-02$

$1.703 \mathrm{e}-06$

$7.962 \mathrm{e}+04$

\section{Results - Dose Point \# 5 - $(15.24,359.13,0) \mathrm{cm}$}

\begin{tabular}{|c|c|c|}
\hline Fluence Rate & Fluence Rate & Exposure Rate \\
\hline $\mathrm{MeV} / \mathrm{cm}^{2} / \mathrm{sec}$ & $\mathrm{MeV} / \mathrm{cm}^{2} / \mathrm{sec}$ & $\mathrm{mR} / \mathrm{hr}$ \\
\hline No Buildup & With Buildup & No Buildup \\
\hline $1.942 \mathrm{e}-03$ & $2.114 \mathrm{e}-03$ & $1.666 \mathrm{e}-04$ \\
\hline $1.889 \mathrm{e}-01$ & $2.170 \mathrm{e}-01$ & $6.544 \mathrm{e}-03$ \\
\hline $2.079 e+03$ & $2.717 e+03$ & $2.061 e+01$ \\
\hline $2.058 e+03$ & $3.091 \mathrm{e}+03$ & $9.102 e+00$ \\
\hline $3.682 e+01$ & $6.219 e+01$ & $9.807 e-02$ \\
\hline $1.754 \mathrm{e}+02$ & $3.550 e+02$ & $3.485 e-01$ \\
\hline $4.165 e+01$ & $1.004 \mathrm{e}+02$ & $6.591 \mathrm{e}-02$ \\
\hline $1.068 \mathrm{e}+03$ & $2.835 e+03$ & $1.633 e+00$ \\
\hline $6.129 e+00$ & $1.716 \mathrm{e}+01$ & $1.009 \mathrm{e}-02$ \\
\hline $4.672 e+02$ & $1.277 e+03$ & $8.246 e-01$ \\
\hline $9.560 e+01$ & $2.410 e+02$ & $1.813 e-01$ \\
\hline $2.866 e+02$ & $6.714 e+02$ & $5.584 \mathrm{e}-01$ \\
\hline $1.234 \mathrm{e}+02$ & $2.727 e+02$ & $2.422 \mathrm{e}-01$ \\
\hline $1.204 \mathrm{e}+07$ & $2.529 e+07$ & $2.351 e+04$ \\
\hline $1.648 \mathrm{e}+04$ & $3.206 e+04$ & $3.134 \mathrm{e}+01$ \\
\hline $2.193 e+04$ & $4.038 e+04$ & $4.043 e+01$ \\
\hline $4.663 e+04$ & $7.795 e+04$ & $7.846 e+01$ \\
\hline $2.926 e+00$ & $4.623 e+00$ & $4.525 \mathrm{e}-03$ \\
\hline $5.384 \mathrm{e}-04$ & $7.894 \mathrm{e}-04$ & $7.304 \mathrm{e}-07$ \\
\hline $1.214 \mathrm{e}+07$ & $2.545 e+07$ & $2.369 e+04$ \\
\hline
\end{tabular}

Exposure Rate $\mathrm{mR} / \mathrm{hr}$

With Buildup

$1.813 \mathrm{e}-04$

7.516e-03

$2.693 e+01$

$1.367 \mathrm{e}+01$

1.657e-01

7.050e-01

$1.589 \mathrm{e}-01$

$4.337 \mathrm{e}+00$

2.826e-02

$2.253 \mathrm{e}+00$

$4.571 \mathrm{e}-01$

$1.308 \mathrm{e}+00$

5.352e-01

$4.936 \mathrm{e}+04$

$6.098 \mathrm{e}+01$

$7.444 \mathrm{e}+01$

$1.312 \mathrm{e}+02$

$7.149 \mathrm{e}-03$

$1.071 \mathrm{e}-06$

$4.967 e+04$

\section{Results - Dose Point \# 6 - $(15.24,404.13,0) \mathrm{cm}$}

\begin{tabular}{ccc}
$\frac{\text { Fluence Rate }}{\text { MeV/cm } / \mathrm{sec}}$ & $\frac{\text { Fluence Rate }}{\text { MeV/cm } 2 / \mathrm{sec}}$ & $\begin{array}{c}\text { Exposure Rate } \\
\mathrm{mR} / \mathrm{hr}\end{array}$ \\
\cline { 3 - 4 } No Buildup & $\frac{\text { With Buildup }}{1.201 \mathrm{e}-03}$ & $\frac{\text { No Buildup }}{9.464 \mathrm{e}-05}$ \\
$1.103 \mathrm{e}-03$ & $9.066 \mathrm{e}-02$ & $2.743 \mathrm{e}-03$ \\
$7.920 \mathrm{e}-02$ & $7.408 \mathrm{e}+02$ & $5.712 \mathrm{e}+00$ \\
$5.764 \mathrm{e}+02$ & $7.160 \mathrm{e}+02$ & $2.164 \mathrm{e}+00$ \\
$4.894 \mathrm{e}+02$ & $1.412 \mathrm{e}+01$ & $2.259 \mathrm{e}-02$ \\
$8.478 \mathrm{e}+00$ & $8.083 \mathrm{e}+01$ & $7.999 \mathrm{e}-02$ \\
$4.027 \mathrm{e}+01$ & $2.333 \mathrm{e}+01$ & $1.518 \mathrm{e}-02$ \\
$9.590 \mathrm{e}+00$ & &
\end{tabular}

Exposure Rate $\underline{\mathrm{mR} / \mathrm{hr}}$

With Buildup

$1.030 \mathrm{e}-04$

$3.140 \mathrm{e}-03$

$7.342 \mathrm{e}+00$

$3.167 \mathrm{e}+00$

$3.761 \mathrm{e}-02$

$1.605 \mathrm{e}-01$

$3.692 \mathrm{e}-02$ 
Page : 5

DOS File : Combined Calcine Grout in Canister - Top - No Lid.ms6

Run Date: April 5, 2005

Run Time: 2:39:39 PM

Duration : 00:01:05

\begin{tabular}{|c|c|c|c|c|c|}
\hline 0.1 & $1.869 \mathrm{e}+10$ & $\frac{\text { No Buildup }}{2.466 \mathrm{e}+02}$ & $\frac{\text { With Buildup }}{6.669 e+02}$ & $\frac{\text { No Buildup }}{3.772 \mathrm{e}-01}$ & $\frac{\text { With Buildup }}{1.020 \mathrm{e}+00}$ \\
\hline 0.15 & $5.974 e+07$ & $1.422 \mathrm{e}+00$ & $4.086 e+00$ & $2.342 \mathrm{e}-03$ & $6.729 \mathrm{e}-03$ \\
\hline 0.2 & $3.089 e+09$ & $1.088 e+02$ & $3.059 e+02$ & $1.920 \mathrm{e}-01$ & $5.399 e-01$ \\
\hline 0.3 & $3.682 e+08$ & $2.237 e+01$ & $5.822 e+01$ & $4.244 \mathrm{e}-02$ & $1.104 \mathrm{e}-01$ \\
\hline 0.4 & $7.502 e+08$ & $6.735 e+01$ & $1.633 e+02$ & $1.312 \mathrm{e}-01$ & $3.182 \mathrm{e}-01$ \\
\hline 0.5 & $2.388 e+08$ & $2.910 e+01$ & $6.666 e+01$ & $5.712 e-02$ & $1.308 \mathrm{e}-01$ \\
\hline 0.6 & $1.818 e+13$ & $2.851 e+06$ & $6.208 e+06$ & $5.564 e+03$ & $1.212 \mathrm{e}+04$ \\
\hline 0.8 & $1.675 e+10$ & $3.924 \mathrm{e}+03$ & $7.927 e+03$ & $7.464 e+00$ & $1.508 e+01$ \\
\hline 1.0 & $1.636 e+10$ & $5.253 e+03$ & $1.005 e+04$ & $9.682 e+00$ & $1.852 \mathrm{e}+01$ \\
\hline 1.5 & $1.975 e+10$ & $1.130 \mathrm{e}+04$ & $1.962 e+04$ & $1.902 \mathrm{e}+01$ & $3.302 \mathrm{e}+01$ \\
\hline 2.0 & $8.316 e+05$ & $7.162 \mathrm{e}-01$ & $1.175 e+00$ & $1.108 \mathrm{e}-03$ & $1.817 \mathrm{e}-03$ \\
\hline 3.0 & $8.813 e+01$ & $1.338 \mathrm{e}-04$ & $2.037 e-04$ & $1.816 \mathrm{e}-07$ & $2.764 \mathrm{e}-07$ \\
\hline TOTALS: & $2.003 e+13$ & $2.873 e+06$ & $6.248 e+06$ & $5.609 e+03$ & $1.220 e+04$ \\
\hline
\end{tabular}


Page : 1

DOS File : Combined Calcine Grout in Canister - Top - with Lid.ms6

Run Date: April 5, 2005

Run Time: 2:42:18 PM

Duration : 00:01:06
File Ref:

Date:

By:

Checked:

Case Title: Combined Calcine Grt

Description: Radiation measured from above closed canister.

Geometry: 8 - Cylinder Volume - End Shields

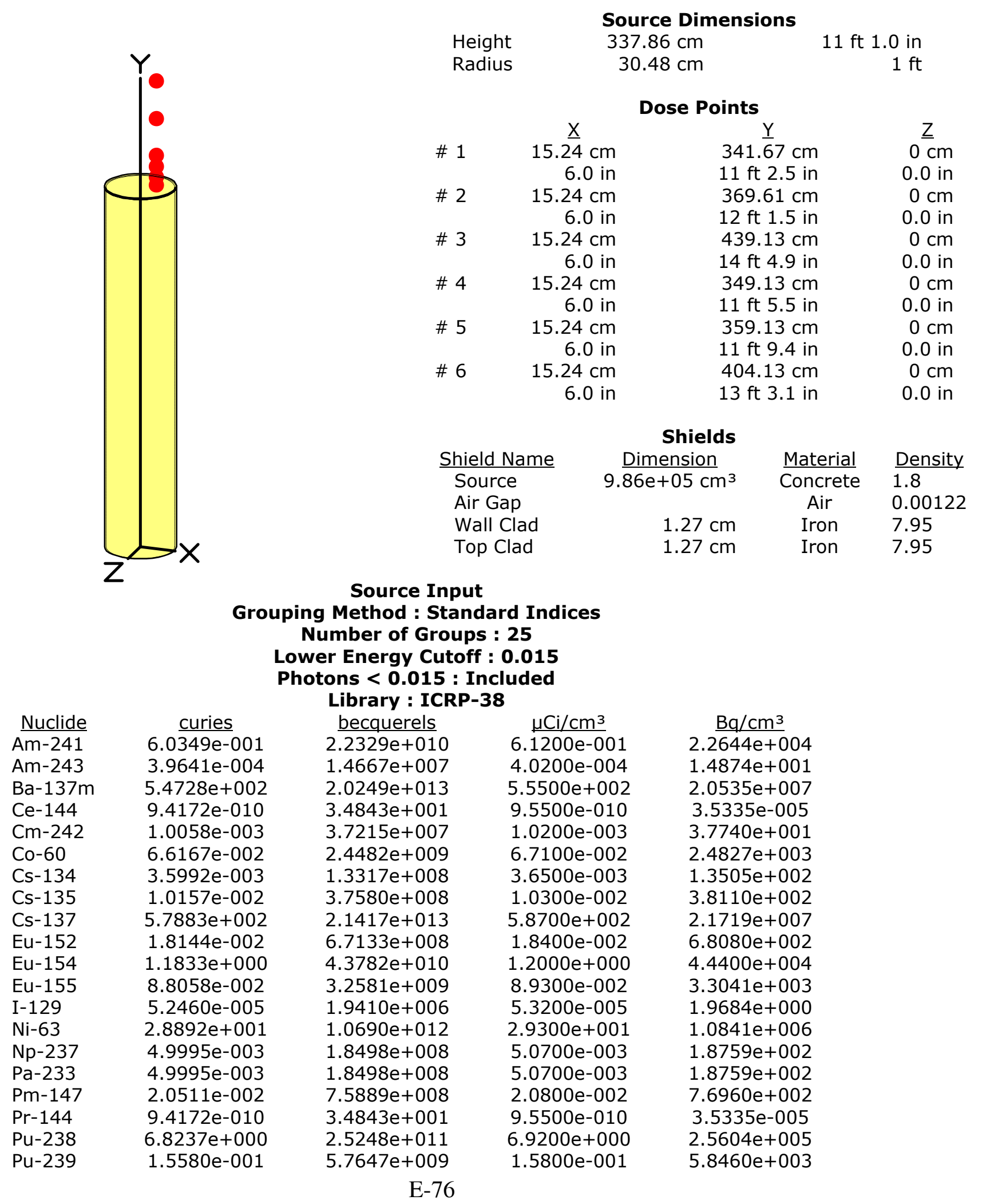


Page : 2

DOS File : Combined Calcine Grout in Canister - Top - with Lid.ms6

Run Date: April 5, 2005

Run Time: 2:42:18 PM

Duration : 00:01:06

$\begin{array}{lc}\text { Nuclide } & \text { curies } \\ \text { Pu-240 } & 9.2791 \mathrm{e}-002 \\ \text { Pu-241 } & 2.5836 \mathrm{e}+000 \\ \text { Pu-242 } & 1.2523 \mathrm{e}-002 \\ \text { Ru-106 } & 3.3527 \mathrm{e}-008 \\ \mathrm{Sb}-125 & 9.3481 \mathrm{e}-003 \\ \mathrm{Sb}-126 & 9.2101 \mathrm{e}-004 \\ \mathrm{Sb}-126 \mathrm{~m} & 6.5969 \mathrm{e}-003 \\ \mathrm{Sm}-151 & 5.9264 \mathrm{e}+000 \\ \mathrm{Sr}-90 & 5.1573 \mathrm{e}+002 \\ \text { Tc-99 } & 2.4455 \mathrm{e}-001 \\ \text { Th-230 } & 2.5638 \mathrm{e}-005 \\ \text { Th-231 } & 2.4751 \mathrm{e}-005 \\ \mathrm{U}-232 & 1.7848 \mathrm{e}-005 \\ \mathrm{U}-233 & 1.1833 \mathrm{e}-006 \\ \mathrm{U}-234 & 4.7234 \mathrm{e}-003 \\ \mathrm{U}-235 & 2.4258 \mathrm{e}-005 \\ \mathrm{U}-236 & 7.4351 \mathrm{e}-005 \\ \mathrm{U}-237 & 6.4490 \mathrm{e}-005 \\ \mathrm{U}-238 & 9.0720 \mathrm{e}-006 \\ \mathrm{Y}-90 & 5.1573 \mathrm{e}+002\end{array}$

becquerels

$3.4333 e+009$

$9.5592 \mathrm{e}+010$

$4.6336 \mathrm{e}+008$

$1.2405 \mathrm{e}+003$

$3.4588 \mathrm{e}+008$

$3.4077 \mathrm{e}+007$

$2.4409 e+008$

$2.1928 \mathrm{e}+011$

$1.9082 \mathrm{e}+013$

$9.0484 \mathrm{e}+009$

$9.4862 e+005$

$9.1578 \mathrm{e}+005$

$6.6038 \mathrm{e}+005$

$4.3782 \mathrm{e}+004$

$1.7476 \mathrm{e}+008$

$8.9754 \mathrm{e}+005$

$2.7510 \mathrm{e}+006$

$2.3861 \mathrm{e}+006$

$3.3567 e+005$

$1.9082 \mathrm{e}+013$ $\mu \mathrm{Ci} / \mathrm{cm}^{3}$
$9.4100 \mathrm{e}-002$
$2.6200 \mathrm{e}+000$
$1.2700 \mathrm{e}-002$
$3.4000 \mathrm{e}-008$
$9.4800 \mathrm{e}-003$
$9.3400 \mathrm{e}-004$
$6.6900 \mathrm{e}-003$
$6.0100 \mathrm{e}+000$
$5.2300 \mathrm{e}+002$
$2.4800 \mathrm{e}-001$
$2.6000 \mathrm{e}-005$
$2.5100 \mathrm{e}-005$
$1.8100 \mathrm{e}-005$
$1.2000 \mathrm{e}-006$
$4.7900 \mathrm{e}-003$
$2.4600 \mathrm{e}-005$
$7.5400 \mathrm{e}-005$
$6.5400 \mathrm{e}-005$
$9.2000 \mathrm{e}-006$
$5.2300 \mathrm{e}+002$

$\mathrm{Bq} / \mathrm{cm}^{3}$

$3.4817 \mathrm{e}+003$

$9.6940 \mathrm{e}+004$

$4.6990 \mathrm{e}+002$

$1.2580 \mathrm{e}-003$

$3.5076 \mathrm{e}+002$

$3.4558 \mathrm{e}+001$

$2.4753 e+002$

$2.2237 e+005$

$1.9351 \mathrm{e}+007$

$9.1760 \mathrm{e}+003$

$9.6200 \mathrm{e}-001$

$9.2870 \mathrm{e}-001$

6.6970e-001

4.4400e-002

$1.7723 e+002$

9.1020e-001

$2.7898 \mathrm{e}+000$

$2.4198 \mathrm{e}+000$

$3.4040 \mathrm{e}-001$

$1.9351 \mathrm{e}+007$

\section{Buildup}

\section{The material reference is: Source}

\section{Integration Parameters}

$\begin{array}{ll}\text { Radial } & 24 \\ \text { Circumferential } & 24 \\ \text { Y Direction (axial) } & 24\end{array}$

$\begin{array}{cc}\frac{\begin{array}{c}\text { Energy } \\ \text { MeV }\end{array}}{} & \begin{array}{c}\text { Activity } \\ \text { photons/sec }\end{array} \\ 0.015 & 2.244 \mathrm{e}+11 \\ 0.02 & 1.028 \mathrm{e}+10 \\ 0.03 & 1.227 \mathrm{e}+12 \\ 0.04 & 3.021 \mathrm{e}+11 \\ 0.05 & 2.671 \mathrm{e}+09 \\ 0.06 & 8.024 \mathrm{e}+09 \\ 0.08 & 1.059 \mathrm{e}+09 \\ 0.1 & 1.869 \mathrm{e}+10 \\ 0.15 & 5.974 \mathrm{e}+07 \\ 0.2 & 3.089 \mathrm{e}+09 \\ 0.3 & 3.682 \mathrm{e}+08 \\ 0.4 & 7.502 \mathrm{e}+08 \\ 0.5 & 2.388 \mathrm{e}+08 \\ 0.6 & 1.818 \mathrm{e}+13 \\ 0.8 & 1.675 \mathrm{e}+10 \\ 1.0 & 1.636 \mathrm{e}+10 \\ 1.5 & 1.975 \mathrm{e}+10 \\ 2.0 & 8.316 \mathrm{e}+05 \\ 3.0 & 8.813 \mathrm{e}+01\end{array}$

TOTALS:

$2.003 e+13$
Activity photons/sec

$2.244 \mathrm{e}+11$
Results - Dose Point \# 1 - $(15.24,341.67,0) \mathrm{cm}$

\begin{tabular}{|c|c|c|}
\hline$\frac{\text { Fluence Rate }}{\mathrm{MeV} / \mathrm{cm}^{2} / \mathrm{sec}}$ & $\frac{\text { Fluence Rate }}{\mathrm{MeV} / \mathrm{cm}^{2} / \mathrm{sec}}$ & $\frac{\text { Exposure Rate }}{\mathrm{mR} / \mathrm{hr}}$ \\
\hline $\mathrm{MeV} / \mathrm{cm}^{2} / \mathrm{sec}$ & $\mathrm{MeV} / \mathrm{cm}^{2} / \mathrm{sec}$ & $\mathrm{mR} / \mathrm{hr}$ \\
\hline No Buildup & With Buildup & No Buildup \\
\hline $2.742 \mathrm{e}-252$ & $5.337 \mathrm{e}-22$ & $2.352 \mathrm{e}-253$ \\
\hline $1.473 e-113$ & $3.846 e-23$ & $5.103 e-115$ \\
\hline $1.449 \mathrm{e}-33$ & $1.015 \mathrm{e}-20$ & $1.436 \mathrm{e}-35$ \\
\hline $8.945 e-14$ & $3.547 e-13$ & $3.956 e-16$ \\
\hline $3.715 e-08$ & $2.462 \mathrm{e}-07$ & $9.895 e-11$ \\
\hline $4.080 e-04$ & $3.791 \mathrm{e}-03$ & $8.105 e-07$ \\
\hline $6.227 e-02$ & $7.188 \mathrm{e}-01$ & $9.854 \mathrm{e}-05$ \\
\hline $1.885 e+01$ & $2.192 e+02$ & $2.884 \mathrm{e}-02$ \\
\hline $8.249 \mathrm{e}-01$ & $7.884 e+00$ & $1.358 \mathrm{e}-03$ \\
\hline $1.163 e+02$ & $9.392 e+02$ & $2.053 e-01$ \\
\hline $3.791 e+01$ & $2.401 \mathrm{e}+02$ & $7.192 \mathrm{e}-02$ \\
\hline $1.418 \mathrm{e}+02$ & $7.539 e+02$ & $2.762 \mathrm{e}-01$ \\
\hline $7.056 e+01$ & $3.271 \mathrm{e}+02$ & $1.385 \mathrm{e}-01$ \\
\hline $7.677 e+06$ & $3.179 e+07$ & $1.499 e+04$ \\
\hline $1.232 \mathrm{e}+04$ & $4.305 e+04$ & $2.344 \mathrm{e}+01$ \\
\hline $1.842 e+04$ & $5.679 e+04$ & $3.396 e+01$ \\
\hline $4.736 e+04$ & $1.186 \mathrm{e}+05$ & $7.968 e+01$ \\
\hline $3.314 \mathrm{e}+00$ & $7.347 e+00$ & $5.125 e-03$ \\
\hline $6.849 e-04$ & $1.312 \mathrm{e}-03$ & $9.292 \mathrm{e}-07$ \\
\hline $7.756 e+06$ & $3.201 \mathrm{e}+07$ & $1.512 \mathrm{e}+04$ \\
\hline
\end{tabular}

Results - Dose Point \# 2 - $(15.24,369.61,0) \mathrm{cm}$

\begin{tabular}{|c|c|c|}
\hline Fluence Rate & Fluence Rate & Exposure Rate \\
\hline $\mathrm{MeV} / \mathrm{cm}^{2} / \mathrm{sec}$ & $\mathrm{MeV} / \mathrm{cm}^{2} / \mathrm{sec}$ & $\mathrm{mR} / \mathrm{hr}$ \\
\hline$\frac{\text { No Buildup }}{1.902 \mathrm{e}-252}$ & $\frac{\text { With Buildup }}{1.859 \mathrm{e}-22}$ & $\frac{\text { No Buildup }}{1.631 \mathrm{e}-253}$ \\
\hline
\end{tabular}

Exposure Rate $\mathrm{mR} / \mathrm{hr}$ With Buildup $4.577 \mathrm{e}-23$ $1.332 \mathrm{e}-24$ $1.006 \mathrm{e}-22$ $1.569 \mathrm{e}-15$

$6.560 \mathrm{e}-10$ 7.529e-06 $1.137 \mathrm{e}-03$ 3.353e-01 $1.298 \mathrm{e}-02$ $1.658 \mathrm{e}+00$ $4.554 \mathrm{e}-01$ $1.469 \mathrm{e}+00$ 6.421e-01 $6.206 \mathrm{e}+04$ $8.188 \mathrm{e}+01$ $1.047 \mathrm{e}+02$ $1.995 e+02$ $1.136 \mathrm{e}-02$ 1.779e-06

$6.245 e+04$

$\frac{\frac{\text { Exposure Rate }}{\mathrm{mR/hr}}}{\frac{\text { With Buildup }}{1.594 \mathrm{e}-23}}$


Page : 3

DOS File : Combined Calcine Grout in Canister - Top - with Lid.ms6

Run Date: April 5, 2005

Run Time: 2:42:18 PM

Duration : 00:01:06

$\begin{array}{ccc}\begin{array}{cc}\text { Energy } \\ \text { MeV }\end{array} & \begin{array}{c}\text { Activity } \\ \text { photons/sec }\end{array} \\ 0.02 & & 1.028 \mathrm{e}+10 \\ 0.03 & 1.227 \mathrm{e}+12 \\ 0.04 & 3.021 \mathrm{e}+11 \\ 0.05 & 2.671 \mathrm{e}+09 \\ 0.06 & 8.024 \mathrm{e}+09 \\ 0.08 & 1.059 \mathrm{e}+09 \\ 0.1 & 1.869 \mathrm{e}+10 \\ 0.15 & 5.974 \mathrm{e}+07 \\ 0.2 & 3.089 \mathrm{e}+09 \\ 0.3 & 3.682 \mathrm{e}+08 \\ 0.4 & 7.502 \mathrm{e}+08 \\ 0.5 & 2.388 \mathrm{e}+08 \\ 0.6 & 1.818 \mathrm{e}+13 \\ 0.8 & 1.675 \mathrm{e}+10 \\ 1.0 & 1.636 \mathrm{e}+10 \\ 1.5 & 1.975 \mathrm{e}+10 \\ 2.0 & 8.316 \mathrm{e}+05 \\ 3.0 & 8.813 \mathrm{e}+01\end{array}$

\begin{tabular}{|c|c|}
\hline$\frac{\text { Fluence Rate }}{\mathrm{MeV} / \mathrm{cm}^{2} / \mathrm{sec}}$ & $\frac{\text { Fluence Rate }}{\mathrm{MeV} / \mathrm{cm}^{2} / \mathrm{sec}}$ \\
\hline $\mathrm{MeV} / \mathrm{cm}^{2} / \mathrm{sec}$ & $\mathrm{MeV} / \mathrm{cm}^{2} / \mathrm{sec}$ \\
\hline No Buildup & With Buildup \\
\hline $1.237 \mathrm{e}-113$ & $1.340 \mathrm{e}-23$ \\
\hline $1.480 \mathrm{e}-33$ & $3.537 e-21$ \\
\hline $8.864 \mathrm{e}-14$ & $3.514 \mathrm{e}-13$ \\
\hline $3.559 \mathrm{e}-08$ & $2.355 \mathrm{e}-07$ \\
\hline $3.705 e-04$ & $3.413 e-03$ \\
\hline $4.910 \mathrm{e}-02$ & $5.470 \mathrm{e}-01$ \\
\hline $1.307 e+01$ & $1.412 \mathrm{e}+02$ \\
\hline $4.684 \mathrm{e}-01$ & $3.876 e+00$ \\
\hline $6.008 e+01$ & $4.083 e+02$ \\
\hline $1.790 \mathrm{e}+01$ & $9.428 \mathrm{e}+01$ \\
\hline $6.365 e+01$ & $2.823 e+02$ \\
\hline $3.057 e+01$ & $1.191 \mathrm{e}+02$ \\
\hline $3.233 e+06$ & $1.133 \mathrm{e}+07$ \\
\hline $4.969 \mathrm{e}+03$ & $1.490 \mathrm{e}+04$ \\
\hline $7.185 e+03$ & $1.926 \mathrm{e}+04$ \\
\hline $1.745 e+04$ & $3.896 e+04$ \\
\hline $1.180 \mathrm{e}+00$ & $2.374 \mathrm{e}+00$ \\
\hline $2.352 \mathrm{e}-04$ & $4.176 \mathrm{e}-04$ \\
\hline $3.263 e+06$ & $1.141 \mathrm{e}+07$ \\
\hline
\end{tabular}
$\frac{\text { Exposure Rate }}{\mathrm{mR} / \mathrm{hr}}$

No Buildup

$4.284 \mathrm{e}-115$

$1.466 \mathrm{e}-35$

$3.920 \mathrm{e}-16$

$9.482 \mathrm{e}-11$

$7.359 \mathrm{e}-07$

$7.770 \mathrm{e}-05$

$1.999 \mathrm{e}-02$

$7.714 \mathrm{e}-04$

$1.060 \mathrm{e}-01$

3.395e-02

$1.240 \mathrm{e}-01$

$6.000 \mathrm{e}-02$

$6.311 e+03$

$9.451 \mathrm{e}+00$

$1.324 \mathrm{e}+01$

$2.935 \mathrm{e}+01$

$1.825 \mathrm{e}-03$

3.191e-07

$6.364 \mathrm{e}+03$ $\frac{\text { Exposure Rate }}{\mathrm{mR} / \mathrm{hr}}$

With Buildup

4.640e-25

$3.505 e-23$

$1.554 \mathrm{e}-15$

$6.274 \mathrm{e}-10$

$6.779 \mathrm{e}-06$

8.657e-04

2.160e-01

$6.383 \mathrm{e}-03$

7.207e-01

$1.788 \mathrm{e}-01$

5.501e-01

2.337e-01

$2.212 e+04$

$2.834 \mathrm{e}+01$

$3.551 \mathrm{e}+01$

$6.555 \mathrm{e}+01$

3.671e-03

5.666e-07

$2.225 e+04$

$\begin{array}{cc}\begin{array}{cc}\text { Energy } \\ \text { MeV }\end{array} & \begin{array}{c}\text { Activity } \\ \text { photons/sec }\end{array} \\ 0.015 & 2.244 \mathrm{e}+11 \\ 0.02 & 1.028 \mathrm{e}+10 \\ 0.03 & 1.227 \mathrm{e}+12 \\ 0.04 & 3.021 \mathrm{e}+11 \\ 0.05 & 2.671 \mathrm{e}+09 \\ 0.06 & 8.024 \mathrm{e}+09 \\ 0.08 & 1.059 \mathrm{e}+09 \\ 0.1 & 1.869 \mathrm{e}+10 \\ 0.15 & 5.974 \mathrm{e}+07 \\ 0.2 & 3.089 \mathrm{e}+09 \\ 0.3 & 3.682 \mathrm{e}+08 \\ 0.4 & 7.502 \mathrm{e}+08 \\ 0.5 & 2.388 \mathrm{e}+08 \\ 0.6 & 1.818 \mathrm{e}+13 \\ 0.8 & 1.675 \mathrm{e}+10 \\ 1.0 & 1.636 \mathrm{e}+10 \\ 1.5 & 1.975 \mathrm{e}+10 \\ 2.0 & 8.316 \mathrm{e}+05 \\ 3.0 & 8.813 \mathrm{e}+01\end{array}$

TOTALS: $\quad 2.003 e+13$

$\begin{array}{cc}\frac{\text { Energy }}{\text { MeV }} & \begin{array}{c}\text { Activity } \\ \text { photons/sec }\end{array} \\ 0.015 & 2.244 \mathrm{e}+11 \\ 0.02 & 1.028 \mathrm{e}+10 \\ 0.03 & 1.227 \mathrm{e}+12 \\ 0.04 & 3.021 \mathrm{e}+11\end{array}$

\section{Results - Dose Point \# 3 - $(15.24,439.13,0) \mathrm{cm}$}

\begin{tabular}{c}
$\frac{\text { Fluence Rate }}{\text { MeV/cm } / \mathrm{sec}}$ \\
\hline No Buildup \\
\hline $1.672 \mathrm{e}-252$ \\
$1.149 \mathrm{e}-113$ \\
$1.238 \mathrm{e}-33$ \\
$5.916 \mathrm{e}-14$ \\
$1.851 \mathrm{e}-08$ \\
$1.548 \mathrm{e}-04$ \\
$1.507 \mathrm{e}-02$ \\
$3.381 \mathrm{e}+00$ \\
$1.027 \mathrm{e}-01$ \\
$1.252 \mathrm{e}+01$ \\
$3.601 \mathrm{e}+00$ \\
$1.263 \mathrm{e}+01$ \\
$6.020 \mathrm{e}+00$ \\
$6.337 \mathrm{e}+05$ \\
$9.687 \mathrm{e}+02$ \\
$1.398 \mathrm{e}+03$ \\
$3.395 \mathrm{e}+03$ \\
$2.307 \mathrm{e}-01$ \\
$4.648 \mathrm{e}-05$ \\
\\
$6.395 \mathrm{e}+05$
\end{tabular}

\section{Fluence Rate}

$\mathrm{MeV} / \mathrm{cm}^{2} / \mathrm{sec}$

With Buildup

$5.694 \mathrm{e}-23$

$4.104 \mathrm{e}-24$

$1.083 \mathrm{e}-21$

$2.341 \mathrm{e}-13$

$1.211 \mathrm{e}-07$

$1.390 \mathrm{e}-03$

$1.592 \mathrm{e}-01$

$3.407 e+01$

$7.926 \mathrm{e}-01$

$8.022 \mathrm{e}+01$

$1.818 \mathrm{e}+01$

$5.428 \mathrm{e}+01$

$2.290 \mathrm{e}+01$

$2.183 e+06$

$2.883 e+03$

$3.745 e+03$

$7.662 \mathrm{e}+03$

$4.718 \mathrm{e}-01$

8.435e-05

$2.198 e+06$
Exposure Rate $\mathrm{mR} / \mathrm{hr}$

No Buildup

1.434e-253

$3.980 \mathrm{e}-115$

$1.227 \mathrm{e}-35$

2.616e-16

4.932e-11

3.074e-07

2.385e-05

$5.173 \mathrm{e}-03$

$1.692 \mathrm{e}-04$

2.210e-02

$6.831 \mathrm{e}-03$

2.461e-02

$1.182 \mathrm{e}-02$

$1.237 \mathrm{e}+03$

$1.843 \mathrm{e}+00$

$2.576 \mathrm{e}+00$

$5.712 \mathrm{e}+00$

3.567e-04

6.305e-08

$1.247 \mathrm{e}+03$
Exposure Rate $\mathrm{mR} / \mathrm{hr}$

With Buildup

$4.884 \mathrm{e}-24$

$1.421 \mathrm{e}-25$

$1.074 \mathrm{e}-23$

$1.035 \mathrm{e}-15$

$3.226 \mathrm{e}-10$

2.760e-06

2.519e-04

5.213e-02

$1.305 \mathrm{e}-03$

$1.416 \mathrm{e}-01$

3.449e-02

$1.058 \mathrm{e}-01$

4.495e-02

$4.262 \mathrm{e}+03$

$5.484 \mathrm{e}+00$

$6.904 \mathrm{e}+00$

$1.289 \mathrm{e}+01$

$7.296 \mathrm{e}-04$

$1.144 \mathrm{e}-07$

$4.287 e+03$

\section{Results - Dose Point \# 4 - $(15.24,349.13,0) \mathrm{cm}$}

\begin{tabular}{c}
$\frac{\text { Fluence Rate }}{\text { MeV/cm } 2 / \mathrm{sec}}$ \\
\hline$\frac{\text { No Buildup }}{1.913 \mathrm{e}-252}$ \\
$1.253 \mathrm{e}-113$ \\
$1.491 \mathrm{e}-33$ \\
$8.956 \mathrm{e}-14$
\end{tabular}

Fluence Rate

$\mathrm{MeV} / \mathrm{cm}^{2} / \mathrm{sec}$

With Buildup

$3.670 \mathrm{e}-22$

$2.645 \mathrm{e}-23$

$6.982 \mathrm{e}-21$

$3.551 \mathrm{e}-13$
Exposure Rate $\mathrm{mR} / \mathrm{hr}$

No Buildup

$1.641 \mathrm{e}-253$

$4.342 \mathrm{e}-115$

$1.478 \mathrm{e}-35$

3.961e-16
Exposure Rate $\mathrm{mR} / \mathrm{hr}$

With Buildup

$3.148 \mathrm{e}-23$

$9.162 \mathrm{e}-25$

$6.920 \mathrm{e}-23$

$1.571 \mathrm{e}-15$ 
Page : 4

DOS File : Combined Calcine Grout in Canister - Top - with Lid.ms6

Run Date: April 5, 2005

Run Time: 2:42:18 PM

Duration : 00:01:06

$\begin{array}{ccc}\begin{array}{cc}\text { Energy } \\ \text { MeV }\end{array} & & \begin{array}{c}\text { Activity } \\ \text { photons/sec }\end{array} \\ 0.05 & & 2.671 \mathrm{e}+09 \\ 0.06 & 8.024 \mathrm{e}+09 \\ 0.08 & 1.059 \mathrm{e}+09 \\ 0.1 & 1.869 \mathrm{e}+10 \\ 0.15 & 5.974 \mathrm{e}+07 \\ 0.2 & 3.089 \mathrm{e}+09 \\ 0.3 & 3.682 \mathrm{e}+08 \\ 0.4 & 7.502 \mathrm{e}+08 \\ 0.5 & 2.388 \mathrm{e}+08 \\ 0.6 & 1.818 \mathrm{e}+13 \\ 0.8 & 1.675 \mathrm{e}+10 \\ 1.0 & 1.636 \mathrm{e}+10 \\ 1.5 & 1.975 \mathrm{e}+10 \\ 2.0 & 8.316 \mathrm{e}+05 \\ 3.0 & 8.813 \mathrm{e}+01\end{array}$

\begin{tabular}{cc}
$\frac{\text { Fluence Rate }}{\text { MeV/cm } 2 / \mathrm{sec}}$ & $\frac{\text { Fluence Rate }}{\text { MeV/cm }{ }^{2} / \mathrm{sec}}$ \\
\hline $\begin{array}{c}\text { No Buildup } \\
\text { 3.698e-08 }\end{array}$ & $\frac{\text { With Buildup }}{2.452 \mathrm{e}-07}$ \\
$4.072 \mathrm{e}-04$ & $3.783 \mathrm{e}-03$ \\
$6.174 \mathrm{e}-02$ & $7.100 \mathrm{e}-01$ \\
$1.836 \mathrm{e}+01$ & $2.102 \mathrm{e}+02$ \\
$7.664 \mathrm{e}-01$ & $6.987 \mathrm{e}+00$ \\
$1.049 \mathrm{e}+02$ & $7.936 \mathrm{e}+02$ \\
$3.306 \mathrm{e}+01$ & $1.938 \mathrm{e}+02$ \\
$1.211 \mathrm{e}+02$ & $5.942 \mathrm{e}+02$ \\
$5.934 \mathrm{e}+01$ & $2.539 \mathrm{e}+02$ \\
$6.373 \mathrm{e}+06$ & $2.439 \mathrm{e}+07$ \\
$1.002 \mathrm{e}+04$ & $3.246 \mathrm{e}+04$ \\
$1.473 \mathrm{e}+04$ & $4.229 \mathrm{e}+04$ \\
$3.675 \mathrm{e}+04$ & $8.645 \mathrm{e}+04$ \\
$2.523 \mathrm{e}+00$ & $5.290 \mathrm{e}+00$ \\
$5.099 \mathrm{e}-04$ & $9.327 \mathrm{e}-04$ \\
& \\
$6.435 \mathrm{e}+06$ & $2.455 \mathrm{e}+07$
\end{tabular}

$\frac{\text { Exposure Rate }}{\mathrm{mR} / \mathrm{hr}}$

No Buildup

$9.852 \mathrm{e}-11$

$8.087 \mathrm{e}-07$

$9.770 \mathrm{e}-05$

$2.809 \mathrm{e}-02$

$1.262 \mathrm{e}-03$

$1.851 \mathrm{e}-01$

$6.271 \mathrm{e}-02$

$2.359 \mathrm{e}-01$

$1.165 \mathrm{e}-01$

$1.244 \mathrm{e}+04$

$1.906 \mathrm{e}+01$

$2.716 \mathrm{e}+01$

$6.183 e+01$

$3.901 \mathrm{e}-03$

$6.918 \mathrm{e}-07$

TOTALS: $\quad 2.003 e+13$

$\begin{array}{cc}\frac{\text { Energy }}{\text { MeV }} & \begin{array}{c}\text { Activity } \\ \text { photons/sec }\end{array} \\ 0.015 & 2.244 \mathrm{e}+11 \\ 0.02 & 1.028 \mathrm{e}+10 \\ 0.03 & 1.227 \mathrm{e}+12 \\ 0.04 & 3.021 \mathrm{e}+11 \\ 0.05 & 2.671 \mathrm{e}+09 \\ 0.06 & 8.024 \mathrm{e}+09 \\ 0.08 & 1.059 \mathrm{e}+09 \\ 0.1 & 1.869 \mathrm{e}+10 \\ 0.15 & 5.974 \mathrm{e}+07 \\ 0.2 & 3.089 \mathrm{e}+09 \\ 0.3 & 3.682 \mathrm{e}+08 \\ 0.4 & 7.502 \mathrm{e}+08 \\ 0.5 & 2.388 \mathrm{e}+08 \\ 0.6 & 1.818 \mathrm{e}+13 \\ 0.8 & 1.675 \mathrm{e}+10 \\ 1.0 & 1.636 \mathrm{e}+10 \\ 1.5 & 1.975 \mathrm{e}+10 \\ 2.0 & 8.316 \mathrm{e}+05 \\ 3.0 & 8.813 \mathrm{e}+01\end{array}$

TOTALS: $\quad 2.003 e+13$

\begin{tabular}{|c|c|}
\hline$\frac{\text { Energy }}{\mathrm{MeV}}$ & $\begin{array}{l}\text { Activity } \\
\text { photons/sec }\end{array}$ \\
\hline 0.015 & $2.244 \mathrm{e}+11$ \\
\hline 0.02 & $1.028 e+10$ \\
\hline 0.03 & $1.227 e+12$ \\
\hline 0.04 & $3.021 e+1$ \\
\hline 0.05 & $2.671 \mathrm{e}+0$ \\
\hline 0.06 & $8.024 e+0$ \\
\hline 0.08 & $1.059 \mathrm{e}+0$ \\
\hline
\end{tabular}

\section{Results - Dose Point \# 5 - $(15.24,359.13,0) \mathrm{cm}$}

\section{Fluence Rate}

$\mathrm{MeV} / \mathrm{cm}^{2} / \mathrm{sec}$

No Buildup

$1.952 \mathrm{e}-252$

$1.247 \mathrm{e}-113$

$1.486 \mathrm{e}-33$

$8.929 \mathrm{e}-14$

$3.678 \mathrm{e}-08$

$3.991 \mathrm{e}-04$

$5.704 \mathrm{e}-02$

$1.601 \mathrm{e}+01$

$6.149 \mathrm{e}-01$

$8.105 e+01$

$2.469 \mathrm{e}+01$

$8.879 \mathrm{e}+01$

$4.295 e+01$

$4.568 \mathrm{e}+06$

$7.075 e+03$

$1.029 \mathrm{e}+04$

$2.518 \mathrm{e}+04$

$1.710 \mathrm{e}+00$

$3.419 \mathrm{e}-04$

$4.611 \mathrm{e}+06$

\section{Fluence Rate}

$\mathrm{MeV} / \mathrm{cm}^{2} / \mathrm{sec}$

With Buildup

2.527e-22

$1.821 \mathrm{e}-23$

$4.808 \mathrm{e}-21$

$3.540 \mathrm{e}-13$

$2.438 \mathrm{e}-07$

3.697e-03

6.453e-01

$1.776 \mathrm{e}+02$

$5.300 \mathrm{e}+00$

$5.748 \mathrm{e}+02$

$1.353 \mathrm{e}+02$

$4.084 \mathrm{e}+02$

$1.729 \mathrm{e}+02$

$2.175 \mathrm{e}+04$

$2.817 \mathrm{e}+04$

$5.709 \mathrm{e}+04$

$3.478 \mathrm{e}+00$

6.112e-04

$1.660 e+07$
$1.649 \mathrm{e}+07$
Exposure Rate $\mathrm{mR} / \mathrm{hr}$

No Buildup

1.674e-253

$4.320 \mathrm{e}-115$

$1.473 \mathrm{e}-35$

3.949e-16

$9.798 \mathrm{e}-11$

7.927e-07

$9.026 \mathrm{e}-05$

$2.450 \mathrm{e}-02$

$1.013 \mathrm{e}-03$

$4.684 \mathrm{e}-02$

$1.730 \mathrm{e}-01$

$8.431 \mathrm{e}-02$

$8.917 \mathrm{e}+03$

$1.346 \mathrm{e}+01$

$1.896 \mathrm{e}+01$

$4.237 \mathrm{e}+01$

$2.645 \mathrm{e}-03$

$4.639 \mathrm{e}-07$

$8.992 e+03$
$1.430 \mathrm{e}-01$

\section{Results - Dose Point \# 6 - $(15.24,404.13,0) \mathrm{cm}$}

$\begin{array}{ccc}\frac{\text { Fluence Rate }}{\text { MeV/cm } 2 / \mathrm{sec}} & \frac{\text { Fluence Rate }}{\text { MeV/cm } / \mathrm{sec}} & \begin{array}{c}\text { Exposure Rate } \\ \text { mR/hr }\end{array} \\ \frac{n}{\text { No Buildup }} & \frac{\text { With Buildup }}{9.186 \mathrm{e}-23} & \frac{\text { No Buildup }}{1.531 \mathrm{e}-253} \\ 1.785 \mathrm{e}-252 & 6.620 \mathrm{e}-24 & 4.154 \mathrm{e}-115 \\ 1.199 \mathrm{e}-113 & 1.748 \mathrm{e}-21 & 1.403 \mathrm{e}-35 \\ 1.415 \mathrm{e}-33 & 3.017 \mathrm{e}-13 & 3.369 \mathrm{e}-16 \\ 7.616 \mathrm{e}-14 & 1.759 \mathrm{e}-07 & 7.127 \mathrm{e}-11 \\ 2.676 \mathrm{e}-08 & 2.234 \mathrm{e}-03 & 4.890 \mathrm{e}-07 \\ 2.462 \mathrm{e}-04 & 2.886 \mathrm{e}-01 & 4.252 \mathrm{e}-05 \\ 2.687 \mathrm{e}-02 & & \end{array}$
$\frac{\text { Exposure Rate }}{\mathrm{mR} / \mathrm{hr}}$

With Buildup

$6.533 e-10$

$7.514 \mathrm{e}-06$

$1.123 \mathrm{e}-03$

3.216e-01

$1.151 \mathrm{e}-02$

$1.401 \mathrm{e}+00$

$3.676 \mathrm{e}-01$

$1.158 \mathrm{e}+00$

$4.984 \mathrm{e}-01$

$4.761 \mathrm{e}+04$

$6.174 \mathrm{e}+01$

$7.795 e+01$

$1.455 \mathrm{e}+02$

$8.180 \mathrm{e}-03$

$1.265 \mathrm{e}-06$

$4.790 e+04$

Exposure Rate $\mathrm{mR} / \mathrm{hr}$

With Buildup

$2.168 \mathrm{e}-23$

$6.308 \mathrm{e}-25$

$4.765 \mathrm{e}-23$

$1.566 \mathrm{e}-15$

$6.495 \mathrm{e}-10$

7.343e-06

$1.021 \mathrm{e}-03$

2.717e-01

$8.727 \mathrm{e}-03$

$1.014 \mathrm{e}+00$

2.567e-01

7.957e-01

3.393e-01

$3.220 \mathrm{e}+04$

$4.138 \mathrm{e}+01$

$5.193 e+01$

$9.605 e+01$

$5.379 \mathrm{e}-03$

8.293e-07

$3.239 e+04$

$\frac{\text { Exposure Rate }}{\mathrm{mR} / \mathrm{hr}}$

With Buildup

$7.879 \mathrm{e}-24$

$2.293 e-25$

$1.732 \mathrm{e}-23$

$1.334 \mathrm{e}-15$

4.687e-10

4.437e-06

4.567e-04 
Page : 5

DOS File : Combined Calcine Grout in Canister - Top - with Lid.ms6

Run Date: April 5, 2005

Run Time: 2:42:18 PM

Duration : 00:01:06

\begin{tabular}{|c|c|c|c|c|c|}
\hline$\frac{\text { Energy }}{\underline{\mathrm{MeV}}}$ & $\begin{array}{c}\text { Activity } \\
\text { photons/sec }\end{array}$ & $\begin{array}{l}\text { Fluence Rate } \\
\mathrm{MeV} / \mathrm{cm}^{2} / \mathrm{sec}\end{array}$ & $\begin{array}{l}\text { Fluence Rate } \\
\mathrm{MeV} / \mathrm{cm}^{2} / \mathrm{sec}\end{array}$ & $\frac{\text { Exposure Rate }}{\mathrm{mR} / \mathrm{hr}}$ & $\frac{\text { Exposure Rate }}{\frac{\mathrm{mR} / \mathrm{hr}}{\text { With Buildup }}}$ \\
\hline 0.1 & $1.869 \mathrm{e}+10$ & $\frac{\text { No Buildup }}{6.345 e+00}$ & $\frac{\text { With Buildup }}{6.507 e+01}$ & $\frac{\text { No Buildup }}{9.707 e-03}$ & $\frac{\text { With Buildup }}{9.955 \mathrm{e}-02}$ \\
\hline 0.15 & $5.974 \mathrm{e}+07$ & $2.010 \mathrm{e}-01$ & $1.570 \mathrm{e}+00$ & $3.310 \mathrm{e}-04$ & $2.586 \mathrm{e}-03$ \\
\hline 0.2 & $3.089 e+09$ & $2.477 e+01$ & $1.599 e+02$ & $4.372 \mathrm{e}-02$ & $2.822 \mathrm{e}-01$ \\
\hline 0.3 & $3.682 e+08$ & $7.170 e+00$ & $3.627 e+01$ & $1.360 \mathrm{e}-02$ & $6.880 e-02$ \\
\hline 0.4 & $7.502 e+08$ & $2.519 e+01$ & $1.081 \mathrm{e}+02$ & $4.907 e-02$ & $2.106 \mathrm{e}-01$ \\
\hline 0.5 & $2.388 e+08$ & $1.201 \mathrm{e}+01$ & $4.553 e+01$ & $2.357 e-02$ & $8.937 e-02$ \\
\hline 0.6 & $1.818 e+13$ & $1.264 \mathrm{e}+06$ & $4.333 e+06$ & $2.467 e+03$ & $8.457 e+03$ \\
\hline 0.8 & $1.675 e+10$ & $1.931 \mathrm{e}+03$ & $5.703 e+03$ & $3.672 e+00$ & $1.085 e+01$ \\
\hline 1.0 & $1.636 e+10$ & $2.782 e+03$ & $7.386 e+03$ & $5.128 e+00$ & $1.362 \mathrm{e}+01$ \\
\hline 1.5 & $1.975 e+10$ & $6.731 \mathrm{e}+03$ & $1.502 e+04$ & $1.133 e+01$ & $2.526 e+01$ \\
\hline 2.0 & $8.316 e+05$ & $4.555 e-01$ & $9.199 \mathrm{e}-01$ & $7.044 e-04$ & $1.422 \mathrm{e}-03$ \\
\hline 3.0 & $8.813 e+01$ & $9.116 e-05$ & $1.632 \mathrm{e}-04$ & $1.237 \mathrm{e}-07$ & $2.214 \mathrm{e}-07$ \\
\hline TOTALS: & $2.003 e+13$ & $1.276 \mathrm{e}+06$ & $4.361 e+06$ & $2.488 e+03$ & $8.507 e+03$ \\
\hline
\end{tabular}

